\title{
ATF-1 Power Histories
}

\author{
B. J. Curnutt
}

December 2017

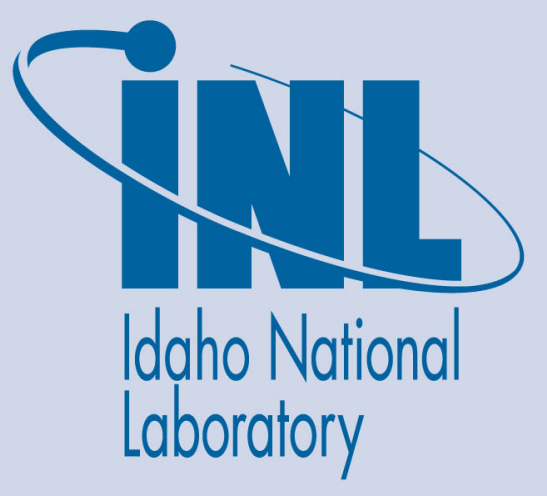

The INL is a U.S. Department of Energy National Laboratory operated by Battelle Energy Alliance 


\title{
ATF-1 Power Histories
}

\author{
B. J. Curnutt
}

December 2017

\section{Idaho National Laboratory Idaho Falls, Idaho 83415}

http://www.inl.gov

$$
\text { Prepared for the }
$$

U.S. Department of Energy

Under DOE Idaho Operations Office

Contract DE-AC07-05ID14517 
TEM-10300-1

$03 / 01 / 2012$

TECHNICAL EVALUATION

Page 1 of 110

Rev. 03

Title: $\quad$ ATF-1 Power Histories

$\begin{array}{lllll}\text { TEV No.: } & 3119 & \text { Rev. No.: } & 0 & \text { Project No.: } 31418 \quad \text { Date: } 12 / 01 / 2017\end{array}$

\begin{tabular}{|c|c|c|}
\hline 1. Quality Level (QL) No. & 3 & Professional Engineer's Stamp \\
\hline 2. QL Determination No. & $\begin{array}{c}\text { MSA-000305 } \\
\text { Revision } 3\end{array}$ & $\mathrm{~N} / \mathrm{A}$ \\
\hline 3. Engineering Job (EJ) No. & NA & See LWP-10010 for requirements. \\
\hline 4. SSC ID & NA & \\
\hline 5. Building & NA & \\
\hline 6. Site Area & NA & \\
\hline
\end{tabular}

7. Introduction:

ATF-1 experiment power histories are tabulated in Appendix A of this Technical Evaluation (TEV). The current revision of this TEV (Revision 0) includes power histories through Advanced Test Reactor (ATR) Cycle 162A. The Cycle 162A power histories are based off projection calculations while all other cycles are based off as-run (core follow) simulations. No calculations are documented in this TEV. This TEV serves as a single source for ATF-1 experiment power history information and compiles results from multiple ATF-1 as-run and projection Engineering Calculations and Analysis Reports (ECARs), and will be updated after each cycle specific as-run and projection ECAR is released.

8. Revision:

Revision 0 is a new document.

9. Conclusions/Recommendations:

The tables in Appendix A of this TEV summarize the following results for each ATF-1 experiment:

- Cumulative Effective Full Power Days (EFPD)

- $\quad{ }^{235} \mathrm{U}$ Burnup (GWd/MTU)

- Fission Density (fissions $/ \mathrm{cm}^{3}$ )

- Linear Heat Generation Rate (LHGR W/cm)

- Thermal Flux $\left(\mathrm{n} / \mathrm{cm}^{2 *} \mathrm{~s}<0.625 \mathrm{eV}\right)$

- Fast Flux (n/cm $\left.{ }^{2 *} \mathrm{~s}>1.0 \mathrm{MeV}\right)$

- Total Flux $\left(\mathrm{n} / \mathrm{cm}^{2 *} \mathrm{~s}\right)$

- Fuel Centerline Temperature $\left({ }^{\circ} \mathrm{C}\right)$

- Peak Internal Clad Temperature (PICT, ${ }^{\circ} \mathrm{C}$ )

The power histories for the Oak Ridge National Laboratory (ORNL) Fuel Chemical Clad Interaction (FCCI) experiments (ATF-73 through ATF-75) do no include fuel centerline temperatures or PICT, 
TEM-10300-1

$03 / 01 / 2012$

TECHNICAL EVALUATION

Page 2 of 110

Rev. 03

Title: $\quad$ ATF-1 Power Histories

$\begin{array}{llllll}\text { TEV No.: } & 3119 & \text { Rev. No.: } & 0 & \text { Project No.: } & 31418 \quad \text { Date: } 12 / 01 / 2017\end{array}$

as these results are not applicable to this experiment design. If necessary, the results for the $\mathrm{FCCl}$ experiments may be updated to include the temperatures at desired fuel-cladding interaction sites.

The results documented in Appendix A of this TEV are available in the spreadsheet shown. Note that this file has also been archived as an 'additional information' attachment to this TEV on EDMS.

//fswcb1/projects/usuf/irradiation testing/LWR ATF/ATF-1_Neutronics/TEV-

3119_ATF-1_Histories

1258104320275573 Database_162A.xlsx 
TEM-10300-1

$03 / 01 / 2012$

Rev. 03

Title: $\quad$ ATF-1 Power Histories

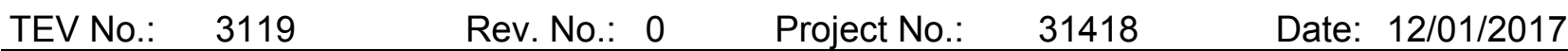

\section{CONTENTS}

PROJECT ROLES AND RESPONSIBILITIES

INTRODUCTION 7

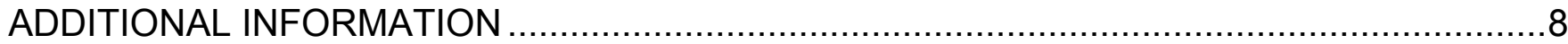

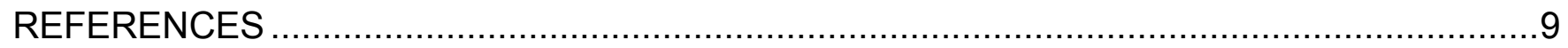

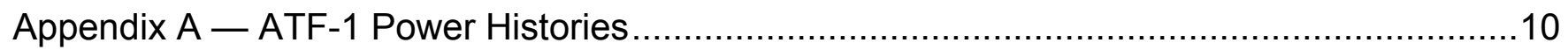

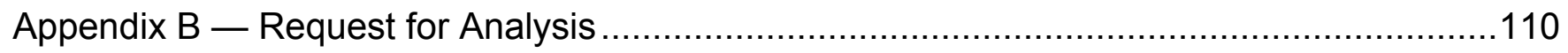

\section{List of Tables}

Table 1. ATF-1 Experiment Dimensions and Compositions ........................................................ 8

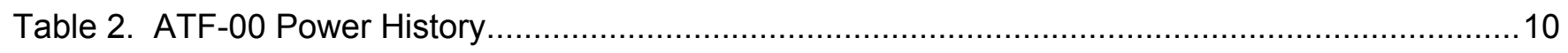

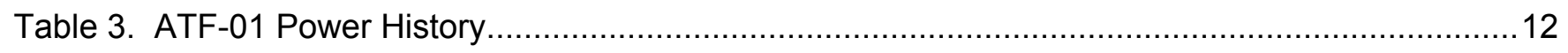

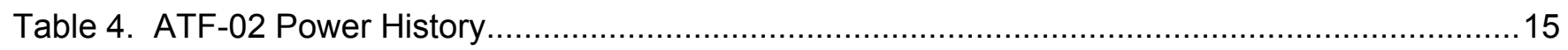

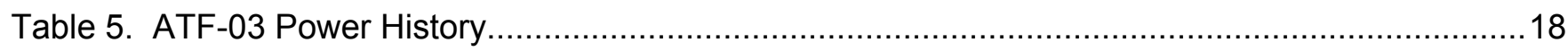

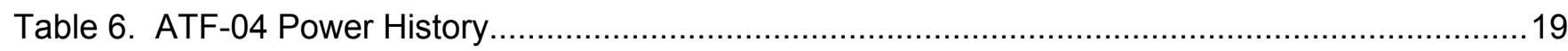

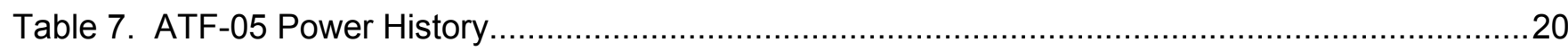

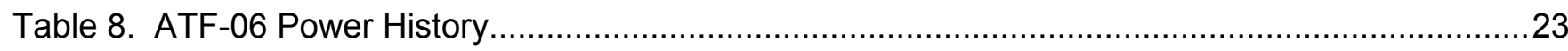

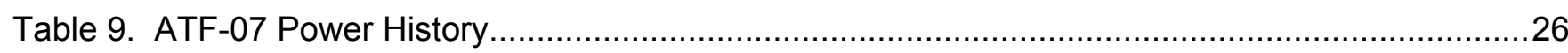

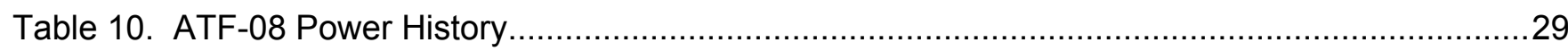

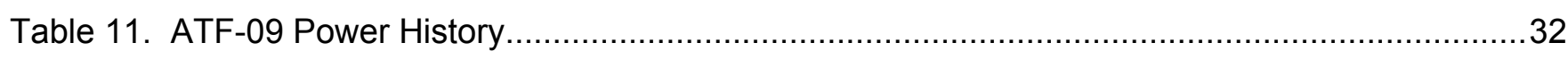

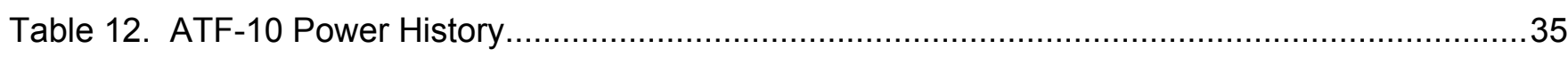

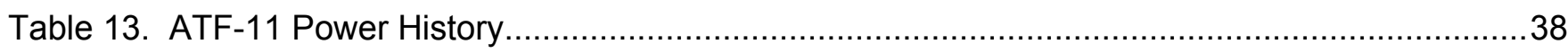

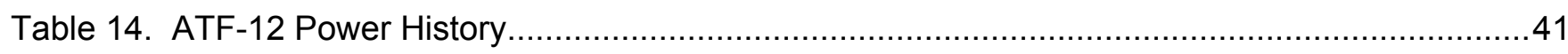

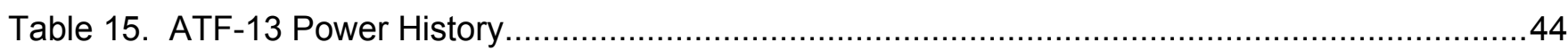

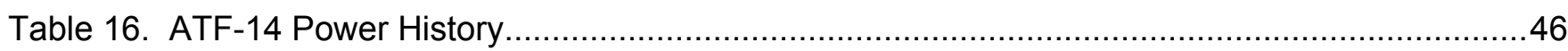

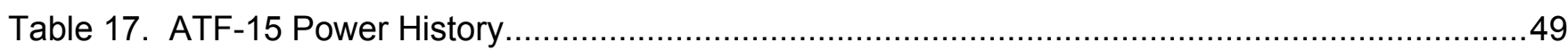

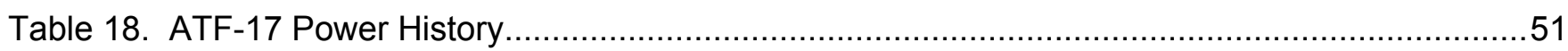

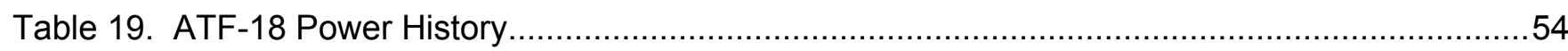

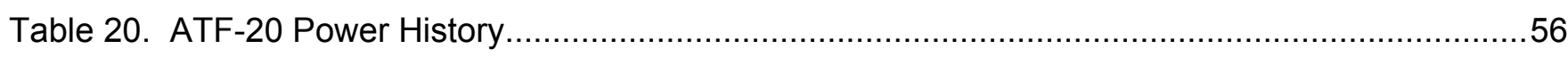

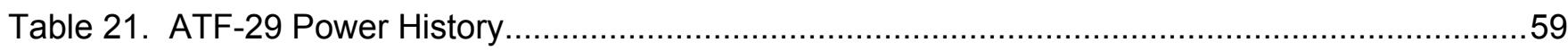

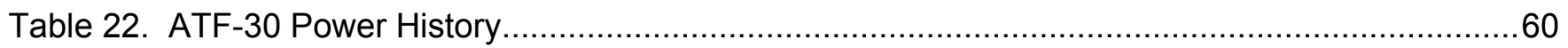


TEM-10300-1

$03 / 01 / 2012$

Rev. 03

Title: $\quad$ ATF-1 Power Histories

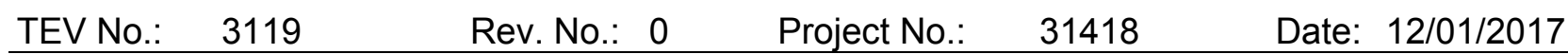

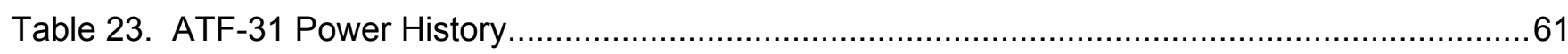

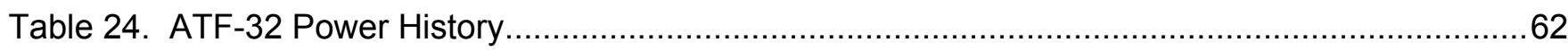

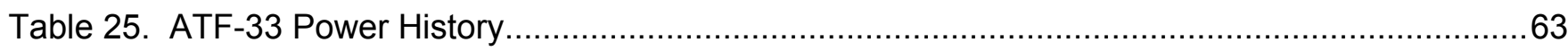

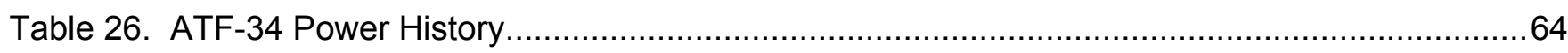

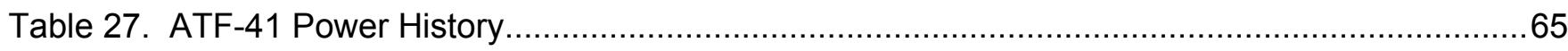

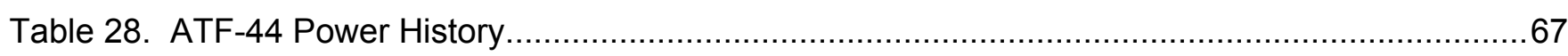

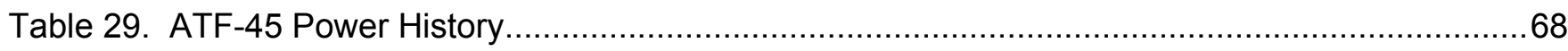

Table 30. ATF-73 (Pellet 1 - Top) Power History .............................................................. 70

Table 31. ATF-73 (Pellet 2) Power History ................................................................. 71

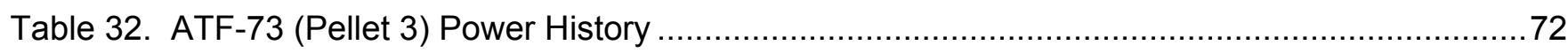

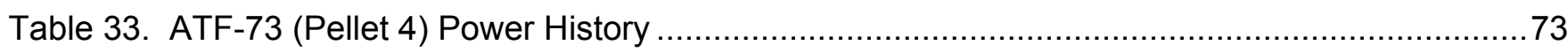

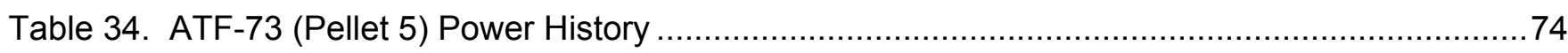

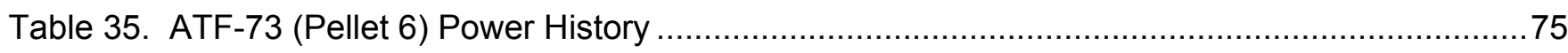

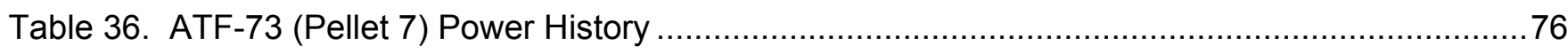

Table 37. ATF-73 (Pellet 8) Power History ................................................................... 77

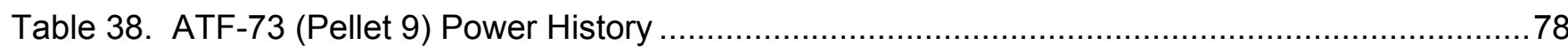

Table 39. ATF-73 (Pellet 10) Power History ................................................................... 79

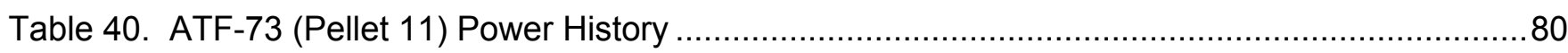

Table 41. ATF-73 (Pellet 12) Power History ................................................................. 81

Table 42. ATF-73 (Pellet 13 - Bottom) Power History........................................................... 82

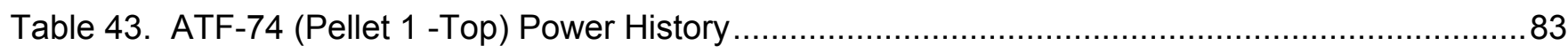

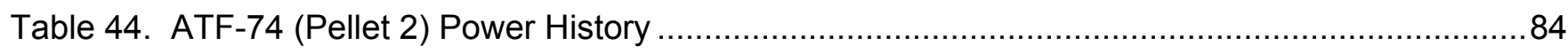

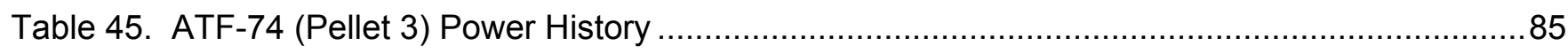

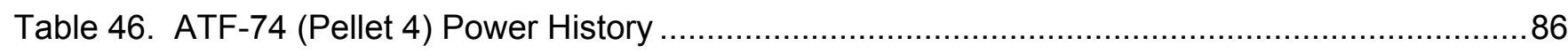

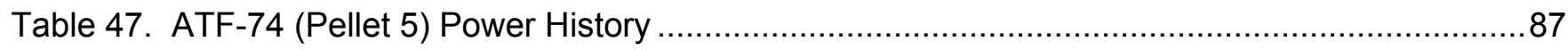

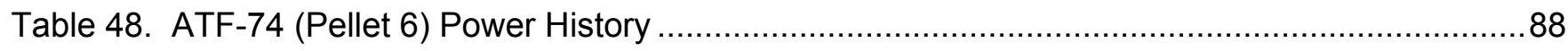

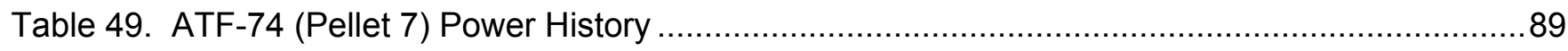

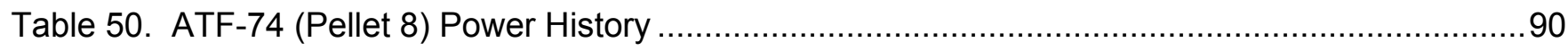

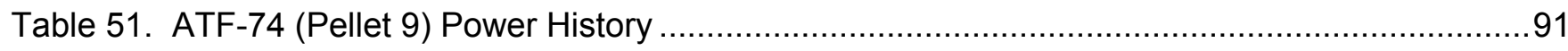

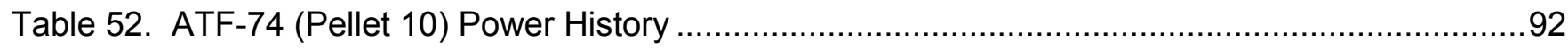

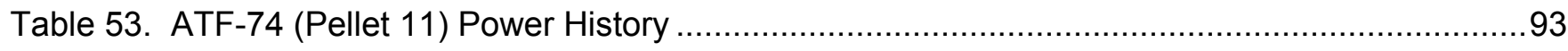

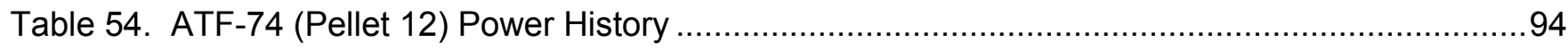

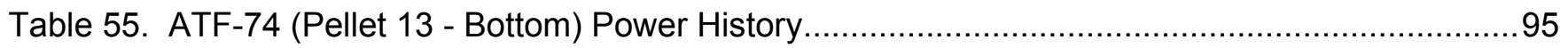


TEM-10300-1

$03 / 01 / 2012$

TECHNICAL EVALUATION

Page 5 of 110

Rev. 03

Title: $\quad$ ATF-1 Power Histories

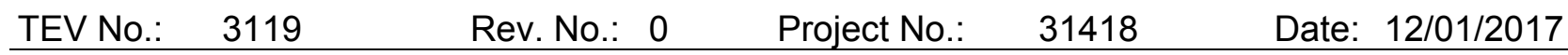

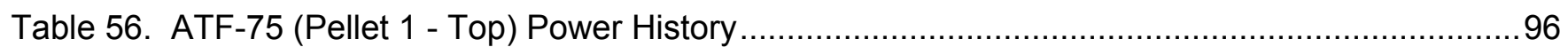

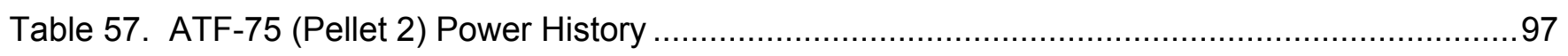

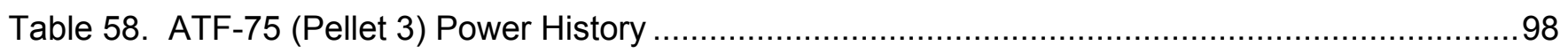

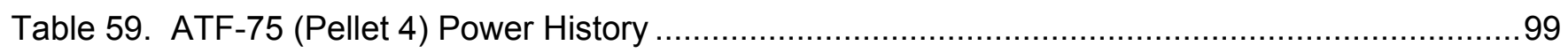

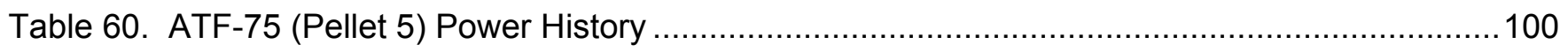

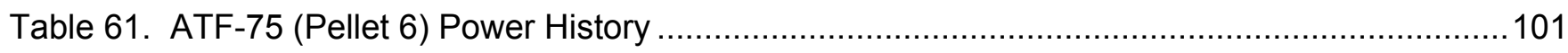

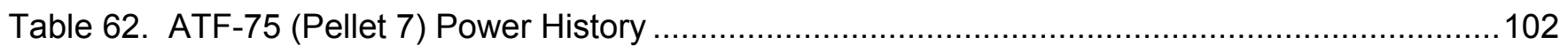

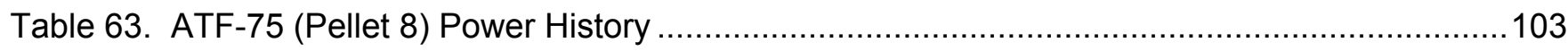

Table 64. ATF-75 (Pellet 9) Power History .................................................................. 104

Table 65. ATF-75 (Pellet 10) Power History .................................................................... 105

Table 66. ATF-75 (Pellet 11) Power History ........................................................................ 106

Table 67. ATF-75 (Pellet 12) Power History ..................................................................... 107

Table 68. ATF-75 (Pellet 13 - Bottom) Power History ............................................................. 108 
TEM-10300-1

$03 / 01 / 2012$

TECHNICAL EVALUATION

Page 6 of 110

Rev. 03

Title: $\quad$ ATF-1 Power Histories

\begin{tabular}{llllll} 
TEV No.: & 3119 & Rev. No.: 0 & Project No.: $31418 \quad$ Date: $12 / 01 / 2017$ \\
\hline
\end{tabular}

PROJECT ROLES AND RESPONSIBILITIES

\begin{tabular}{l|l|l|l}
\multicolumn{1}{c|}{ Project Role } & \multicolumn{1}{|c|}{ Name (Typed) } & Organization & Pages covered (if applicable) \\
\hline Performer & B. J. Curnutt & C130 & All \\
Checker $^{\mathrm{a}}$ & J. R. Mitchell & All \\
Independent Reviewer $^{\mathrm{b}}$ & N/A & & All \\
CUl Reviewer & B. P. Durtschi & C660 & All \\
Manager & M. A. Lillo & All \\
Requestore & G. M. Core & C630 & All \\
Nuclear Safetye & N/A & & All \\
Document Ownere & G. L. Povirk & &
\end{tabular}

\section{Responsibilities}
a. Confirmation of completeness, mathematical accuracy, and correctness of data and appropriateness of assumptions.
b. Concurrence of method or approach. See definition, LWP-10106.
c. Concurrence with the document's markings in accordance with LWP-11202.
d. Concurrence of procedure compliance. Concurrence with method/approach and conclusion.
e.Concurrence with the document's assumptions and input information. See definition of Acceptance, LWP-10200.

NOTE: $\quad$ Delete or mark "N/A" for project roles not engaged. Include ALL personnel and their roles listed above in the eCR system. The list of the roles above is not all inclusive. If needed, the list can be extended or reduced. 
TEM-10300-1

$03 / 01 / 2012$

TECHNICAL EVALUATION

Page 7 of 110

Rev. 03

Title: $\quad$ ATF-1 Power Histories

$\begin{array}{llllll}\text { TEV No.: } & 3119 & \text { Rev. No.: } & 0 & \text { Project No.: } 31418 \quad \text { Date: } 12 / 01 / 2017\end{array}$

\section{INTRODUCTION}

ATF-1 calculated power histories for irradiation during Advanced Test Reactor (ATR) Cycles 157C through 162A are tabulated in Appendix A of this Technical Evaluation (TEV). The ATR Cycle 162A power histories are based off projection calculations while all previous cycles are based off as-run (core follow) simulations. This TEV will serve as the single source for ATF-1 experiment power history information and will be updated after each cycle of operation.

Table 2 through Table 68 in Appendix A summarize the following results for each ATF-1 experiment:

- Cumulative Effective Full Power Days (EFPD)

- ${ }^{235} \mathrm{U}$ Burnup (GWd/MTU)

- Fission Density (fissions $/ \mathrm{cm}^{3}$ )

- Linear Heat Generation Rate (LHGR W/cm)

- Thermal Flux $\left(\mathrm{n} / \mathrm{cm}^{2 *} \mathrm{~s}<0.625 \mathrm{eV}\right)$

- Fast Flux $\left(\mathrm{n} / \mathrm{cm}^{2 *} \mathrm{~s}>1.0 \mathrm{MeV}\right)$

- Total Flux $\left(\mathrm{n} / \mathrm{cm}^{2 *} \mathrm{~s}\right)$

- Fuel Centerline Temperature $\left({ }^{\circ} \mathrm{C}\right)$

- Peak Internal Clad Temperature (PICT, $\left.{ }^{\circ} \mathrm{C}\right)$

The power histories for the Oak Ridge National Laboratory (ORNL) Fuel Chemical Clad Interaction (FCCl) experiments (ATF-73 through ATF-75) do not include fuel centerline temperatures or Peak Internal Clad Temperature (PICT), as these results are not applicable to this experiment design. If necessary, the results for the $\mathrm{FCCl}$ experiments may be updated to include the temperatures at desired fuel-clad interaction sites. Due to the unique design of the $\mathrm{FCCl}$ experiments relative to the other ATF1 experiments, the power histories for each individual fuel pellet in the $\mathrm{FCCl}$ experiments are provided. Pellets are labeled top to bottom, with the top pellet oriented upward in the core, $1-13$. 
TEM-10300-1

$03 / 01 / 2012$

TECHNICAL EVALUATION

Page 8 of 110

Rev. 03

Title: $\quad$ ATF-1 Power Histories

$\begin{array}{llllll}\text { TEV No.: } & 3119 & \text { Rev. No.: } & 0 & \text { Project No.: } 31418 \quad \text { Date: } 12 / 01 / 2017\end{array}$

\section{ADDITIONAL INFORMATION}

The ATF-1 power histories tabulated in Appendix A of this TEV were calculated and summarized in Reference 1.

The ATR as-operated lobe power histories (Cycles 157C through 161A) are documented in Reference 2. The projected lobe powers for Cycle 162A are documented in Section 4 of Reference 1.

The ATF-1 experiment dimensions and material compositions are provided in the References summarized in Table 1.

Table 1. ATF-1 Experiment Dimensions and Compositions

\begin{tabular}{c|c|c}
\hline Vendor/Experiment Design & Capsule IDs & Reference \\
\hline AREVA & ATF-00 through ATF-05 & Reference 6 \\
\hline $\begin{array}{c}\text { General Electric (GE) } \\
\text { Companghouse Electric }\end{array}$ & ATF-06 through ATF-09 & Refence 6 \\
\hline $\begin{array}{c}\text { Oak Ridge National Laboratory } 6 \\
\text { Loss of Coolant Accident } \\
\text { (ORNL-LOCA) }\end{array}$ & ATF-17, ATF-18, and ATF-20 through ATF-15 & Reference 6 \\
\hline $\begin{array}{c}\text { Westinghouse Electric } \\
\text { Company (WEC-1B) }\end{array}$ & ATF-29 through ATF-34 & Reference 5 \\
\hline $\begin{array}{c}\text { Los Alamos National Laboratory } \\
\text { (LANL) }\end{array}$ & ATF-41, ATF-44, and ATF-45 & Reference 4 \\
\hline $\begin{array}{c}\text { Oak Ridge National Laboratory } \\
\text { Fuel Chemical Clad Interaction } \\
\text { (ORNL-FCCI) }\end{array}$ & ATF-73 through ATF-75 & Reference 3 \\
\hline
\end{tabular}

\section{ATF-13 Pellet Misload}

Neutron radiography during Post Irradiation Examination (PIE) of ATF-13 indicated a pellet misload from that which was actually modeled. An enriched pellet was erroneously loaded below the lower DU pellet. The erroneous loading, from top to bottom, is two DU pellets followed by a stack up of 11 enriched $\mathrm{U}_{3} \mathrm{Si}_{2}$ pellets, then a single $\mathrm{DU}$ pellet above one enriched pellet.

Each pellet was not explicitly modeled in MCNP. The twelve enriched pellets were modeled as a single volume; therefore, the fluxes and LHGRs tallied and documented in this ECAR are values averaged over the entire enriched cell. Given the LHGR results are an average over the entire enriched stack up, and not pellet specific, it was expected and clearly shown in Reference 1 that the as-modeled average LHGR and flux results are essentially identical to those from a model of the misloaded experiment. The LHGR and flux results from the misloaded enriched pellet located below the lower DU pellet were found to be substantially different; therefore, the results documented in this TEV DO NOT APPLY to the misloaded enriched pellet in ATF-13. 
TEM-10300-1

$03 / 01 / 2012$

TECHNICAL EVALUATION

Page 9 of 110

Rev. 03

Title: $\quad$ ATF-1 Power Histories

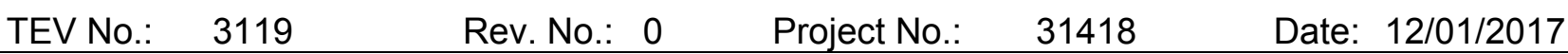

\section{REFERENCES}

1. B. J. Curnutt, "ATR Cycle 160B Through 161A As-Run Analysis and Cycle 162A Bounding Physics Evaluation of the Accident Tolerant Fuels (ATF-1-21-4, ATF-1-23-2, and ATF-1-24-6 Basket Configurations," ECAR 3773, Revision 1, October 2017.

2. M. K. Morrison, Advanced Test Reactor Power History Through Cycle 161A-1, RE-12-17, June 2017.

3. C. T. Woolum, As-Built Dimensions of Capsules, Rodlets, and Rodlet Internals for the ORNLFCCI Accident Tolerant Fuels (ATF-1) Experiment, TEV-2877, Revision 0, November 2016.

4. C. T. Woolum, "As-Built Dimensions of Capsules, Rodlets, and Fuel Pellets for the LANL-1 Accident Tolerant Fuels (ATF-1) Experiment," TEV-2796, Revision 1, November 2016.

5. G. M. Core, "As-Built Dimensions of Capsules, Rodlets, and Fuel Pellets for the Second Westinghouse Accident Tolerant Fuels (ATF-1) Experiment (ATF-1WB)," TEV-2779, Revision 0 August 2016.

6. C. T. Woolum, "As-Built Dimensions for Accident Tolerant Fuels (ATF-1) with AREVA, GE, Westinghouse, and ORNL Capsules, Rodlets, and Fuel Pellets, TEV-2451 Revision 0, May 2015. 
TEM-10300-1

$03 / 01 / 2012$

Rev. 03

Title: $\quad$ ATF-1 Power Histories

TEV No.: $3119 \quad$ Rev. No.: $0 \quad$ Project No.:

\section{Appendix A}

\section{ATF-1 Power Histories}

The results documented in Appendix A of this TEV are available in the spreadsheet shown. Note that this file has also been archived as an 'additional information' attachment to this TEV on EDMS.

//fswcb1/projects/usuf/irradiation testing/LWR ATF/ATF-1_Neutronics/TEV-3119_ATF-1_Histories

1258104320275573 Database_162A.xlsx

\section{ATF-1 Power Histories}

Table 2. ATF-00 Power History

\begin{tabular}{|c|c|c|c|c|c|c|c|c|c|c|}
\hline Cycle & Critical Dates & EFPD & $\begin{array}{c}\text { Burnup } \\
\text { (GWd/MTU) }\end{array}$ & $\begin{array}{l}\text { LHGR } \\
\text { (W/cm) }\end{array}$ & $\begin{array}{l}\text { Thermal Flux } \\
\left(\mathrm{n} / \mathrm{cm}^{2 *} \mathrm{~s}\right)\end{array}$ & $\begin{array}{c}\text { Fast Flux } \\
\left(\mathrm{n} / \mathrm{cm}^{2 *} \mathrm{~s}>1\right. \\
\mathrm{MeV})\end{array}$ & $\begin{array}{l}\text { Total Flux } \\
\left(\mathrm{n} / \mathrm{cm}^{2 *} \mathrm{~s}\right)\end{array}$ & $\begin{array}{c}\text { Fission Density } \\
\left(\text { Fissions } / \mathrm{cm}^{3}\right)\end{array}$ & $\begin{array}{c}\text { Fuel Centerline } \\
\text { Temperature } \\
\left({ }^{\circ} \mathrm{C}\right)\end{array}$ & $\mathrm{PICT}\left({ }^{\circ} \mathrm{C}\right)$ \\
\hline \multirow[t]{2}{*}{$157 \mathrm{C}$} & BOC $(2 / 10 / 2015,0600)$ & 0.0 & 0.00 & 329.4 & $4.16150 \mathrm{E}+13$ & $2.29916 \mathrm{E}+13$ & $9.76392 \mathrm{E}+13$ & $0.00000 \mathrm{E}+00$ & 1476.5 & 360.1 \\
\hline & EOC $(2 / 15 / 2015,0800)$ & 5.4 & 0.40 & 333.2 & $4.25601 \mathrm{E}+13$ & $2.32156 \mathrm{E}+13$ & $9.91808 \mathrm{E}+13$ & $1.01772 \mathrm{E}+19$ & 1495.0 & 363.0 \\
\hline \multirow[t]{13}{*}{ 157D } & \multicolumn{10}{|c|}{ Cycle 157D Outage - 104 Day, 3 Hour Down Time } \\
\hline & BOC $(5 / 30 / 2015,1100)$ & 5.4 & 0.40 & 285.0 & $3.63128 \mathrm{E}+13$ & $1.99583 \mathrm{E}+13$ & $8.48195 \mathrm{E}+13$ & $1.01772 \mathrm{E}+19$ & 1262.7 & 327.1 \\
\hline & & 8.4 & 0.61 & 307.1 & $3.90791 \mathrm{E}+13$ & $2.15061 \mathrm{E}+13$ & $9.16634 \mathrm{E}+13$ & $1.52959 \mathrm{E}+19$ & 1369.6 & 343.7 \\
\hline & & 15.4 & 1.09 & 309.3 & $3.95190 \mathrm{E}+13$ & $2.17563 \mathrm{E}+13$ & $9.30586 \mathrm{E}+13$ & $2.75205 \mathrm{E}+19$ & 1380.1 & 345.3 \\
\hline & & 22.4 & 1.57 & 307.0 & $3.96217 \mathrm{E}+13$ & $2.15503 E+13$ & $9.26586 \mathrm{E}+13$ & $3.96248 \mathrm{E}+19$ & 1369.3 & 343.6 \\
\hline & & 29.4 & 2.06 & 309.2 & $4.02697 \mathrm{E}+13$ & $2.17638 \mathrm{E}+13$ & $9.37430 \mathrm{E}+13$ & $5.19096 \mathrm{E}+19$ & 1379.8 & 345.2 \\
\hline & & 36.4 & 2.55 & 317.1 & $4.17802 \mathrm{E}+13$ & $2.24745 \mathrm{E}+13$ & $9.71848 \mathrm{E}+13$ & $6.44354 \mathrm{E}+19$ & 1417.5 & 351.1 \\
\hline & & 43.4 & 3.05 & 317.3 & $4.22509 \mathrm{E}+13$ & $2.24701 \mathrm{E}+13$ & $9.77579 \mathrm{E}+13$ & $7.69009 \mathrm{E}+19$ & 1418.8 & 351.3 \\
\hline & & 50.4 & 3.55 & 328.5 & $4.40805 \mathrm{E}+13$ & $2.31118 \mathrm{E}+13$ & $1.01151 \mathrm{E}+14$ & $8.97278 \mathrm{E}+19$ & 1472.6 & 359.5 \\
\hline & & 57.4 & 4.07 & 331.6 & $4.49978 \mathrm{E}+13$ & $2.34362 E+13$ & $1.02864 \mathrm{E}+14$ & $1.02675 \mathrm{E}+20$ & 1487.1 & 361.8 \\
\hline & & 60.4 & 4.28 & 330.9 & $4.54526 \mathrm{E}+13$ & $2.33176 \mathrm{E}+13$ & $1.03400 \mathrm{E}+14$ & $1.08035 \mathrm{E}+20$ & 1484.0 & 361.3 \\
\hline & \multicolumn{10}{|c|}{ Mid-Cycle 157D Outage - 14 Day, 10 Hour Down Time $(7 / 23 / 2015,1800$ - 8/7/2015, 0400) } \\
\hline & EOC $(8 / 12 / 2015,0400)$ & 65.1 & 4.63 & 331.5 & $4.59923 \mathrm{E}+13$ & $2.34293 \mathrm{E}+13$ & $1.04116 \mathrm{E}+14$ & 1.16887E+20 & 1486.8 & 361.7 \\
\hline
\end{tabular}


TEM-10300-1

$03 / 01 / 2012$

Rev. 03

TECHNICAL EVALUATION

Page 11 of 110

Title: $\quad$ ATF-1 Power Histories

\begin{tabular}{lllll} 
& TEV No.: 3119 & Rev. No.: & 0 & Project No.: \\
\hline
\end{tabular}

Table 2. ATF-00 Power History

\begin{tabular}{|c|c|c|c|c|c|c|c|c|c|c|}
\hline Cycle & Critical Dates & EFPD & $\begin{array}{c}\text { Burnup } \\
\text { (GWd/MTU) }\end{array}$ & $\begin{array}{l}\text { LHGR } \\
(\mathrm{W} / \mathrm{cm})\end{array}$ & $\begin{array}{l}\text { Thermal Flux } \\
\left(\mathrm{n} / \mathrm{cm}^{2 *} \mathrm{~s}\right)\end{array}$ & $\begin{array}{c}\text { Fast Flux } \\
\left(\mathrm{n} / \mathrm{cm}^{2 *} \mathrm{~s}>1\right. \\
\mathrm{MeV})\end{array}$ & $\begin{array}{l}\text { Total Flux } \\
\left(\mathbf{n} / \mathrm{cm}^{2 *} \mathbf{s}\right)\end{array}$ & $\begin{array}{c}\text { Fission Density } \\
\text { (Fissions/cm }{ }^{3} \text { ) }\end{array}$ & $\begin{array}{c}\text { Fuel Centerline } \\
\text { Temperature } \\
\left({ }^{\circ} \mathrm{C}\right)\end{array}$ & $\operatorname{PICT}\left({ }^{\circ} \mathrm{C}\right)$ \\
\hline \multirow[t]{8}{*}{$158 \mathrm{~A}$} & \multicolumn{10}{|c|}{ Cycle 158A Outage - 91 Day, 11 Hour Down Time } \\
\hline & BOC $(11 / 11 / 2015,1500)$ & 65.1 & 4.63 & 333.2 & $4.60454 \mathrm{E}+13$ & $2.35449 \mathrm{E}+13$ & $1.04337 \mathrm{E}+14$ & $1.16887 \mathrm{E}+20$ & 1494.9 & 363.0 \\
\hline & & 75.1 & 5.36 & 327.3 & $4.53500 \mathrm{E}+13$ & $2.31771 \mathrm{E}+13$ & $1.02900 \mathrm{E}+14$ & $1.35435 E+20$ & 1466.5 & 358.6 \\
\hline & & 85.1 & 6.06 & 317.9 & $4.48150 \mathrm{E}+13$ & $2.26774 \mathrm{E}+13$ & $1.01253 \mathrm{E}+14$ & $1.52959 \mathrm{E}+20$ & 1421.6 & 351.7 \\
\hline & & 95.1 & 6.77 & 319.4 & $4.57104 \mathrm{E}+13$ & $2.26969 \mathrm{E}+13$ & $1.02611 \mathrm{E}+14$ & $1.71085 \mathrm{E}+20$ & 1428.9 & 352.8 \\
\hline & & 105.1 & 7.50 & 325.4 & $4.72804 \mathrm{E}+13$ & $2.31034 \mathrm{E}+13$ & $1.05136 \mathrm{E}+14$ & $1.89392 \mathrm{E}+20$ & 1457.5 & 357.2 \\
\hline & & 117.1 & 8.38 & 328.8 & $4.85328 \mathrm{E}+13$ & $2.33759 \mathrm{E}+13$ & $1.06755 \mathrm{E}+14$ & $2.11553 E+20$ & 1473.9 & 359.7 \\
\hline & $\operatorname{EOC}^{*}(1 / 2 / 2016,0400)$ & 117.3 & 8.39 & 323.5 & $4.87584 \mathrm{E}+13$ & $2.30640 \mathrm{E}+13$ & $1.06409 \mathrm{E}+14$ & $2.11854 \mathrm{E}+20$ & 1448.7 & 355.9 \\
\hline
\end{tabular}

ATF-00 was discharged for PIE during the Cycle 158B outage. 
TEM-10300-1

$03 / 01 / 2012$

Rev. 03

Title: $\quad$ ATF-1 Power Histories

$\begin{array}{lllll}\text { TEV No.: } & 3119 & \text { Rev. No.: } & 0 & \text { Project No.: }\end{array}$

Table 3. ATF-01 Power History

\begin{tabular}{|c|c|c|c|c|c|c|c|c|c|c|}
\hline Cycle & Critical Dates & EFPD & $\begin{array}{l}\text { Burnup } \\
\text { (GWd/MTU) }\end{array}$ & $\begin{array}{l}\text { LHGR } \\
(\mathrm{W} / \mathrm{cm})\end{array}$ & $\begin{array}{l}\text { Thermal Flux } \\
\left(\mathrm{n} / \mathrm{cm}^{2 *} \mathrm{~s}\right)\end{array}$ & $\begin{array}{c}\text { Fast Flux } \\
\left(\mathrm{n} / \mathrm{cm}^{2 *} s>1\right. \\
\mathrm{MeV})\end{array}$ & $\begin{array}{l}\text { Total Flux } \\
\left(\mathrm{n} / \mathrm{cm}^{2 *} \mathrm{~s}\right)\end{array}$ & $\begin{array}{c}\text { Fission Density } \\
\left(\text { Fissions } / \mathrm{cm}^{3}\right)\end{array}$ & $\begin{array}{c}\text { Fuel Centerline } \\
\text { Temperature } \\
\left({ }^{\circ} \mathrm{C}\right)\end{array}$ & PICT $\left({ }^{\circ} \mathrm{C}\right)$ \\
\hline \multirow[t]{2}{*}{$157 C$} & BOC $(2 / 10 / 2015,0600)$ & 0.0 & 0.00 & 328.9 & $4.19056 \mathrm{E}+13$ & $2.29526 \mathrm{E}+13$ & $9.80330 \mathrm{E}+13$ & $0.00000 \mathrm{E}+00$ & 1538.1 & 391.7 \\
\hline & EOC $(2 / 15 / 2015,0800)$ & 5.4 & 0.41 & 333.6 & $4.28011 \mathrm{E}+13$ & $2.33065 \mathrm{E}+13$ & $9.96535 \mathrm{E}+13$ & $1.02374 \mathrm{E}+19$ & 1561.1 & 395.5 \\
\hline \multirow[t]{13}{*}{ 157D } & \multicolumn{10}{|c|}{ Cycle 157D Outage - 104 Day, 3 Hour Down Time } \\
\hline & BOC $(5 / 30 / 2015,1100)$ & 5.4 & 0.41 & 282.0 & $3.62657 \mathrm{E}+13$ & $1.99026 \mathrm{E}+13$ & $8.44703 \mathrm{E}+13$ & $1.02374 \mathrm{E}+19$ & 1303.9 & 352.9 \\
\hline & & 8.4 & 0.61 & 306.7 & $3.93691 \mathrm{E}+13$ & $2.12920 \mathrm{E}+13$ & $9.14791 \mathrm{E}+13$ & $1.52959 \mathrm{E}+19$ & 1428.2 & 373.6 \\
\hline & & 15.4 & 1.10 & 305.2 & $3.94287 \mathrm{E}+13$ & $2.15902 E+13$ & $9.24171 \mathrm{E}+13$ & $2.74001 \mathrm{E}+19$ & 1420.9 & 372.4 \\
\hline & & 22.4 & 1.58 & 304.5 & $3.98316 \mathrm{E}+13$ & $2.13302 E+13$ & $9.23683 \mathrm{E}+13$ & $3.95645 \mathrm{E}+19$ & 1417.2 & 371.8 \\
\hline & & 29.4 & 2.06 & 305.9 & $4.01296 \mathrm{E}+13$ & $2.15614 \mathrm{E}+13$ & $9.33074 \mathrm{E}+13$ & $5.16688 \mathrm{E}+19$ & 1424.2 & 372.9 \\
\hline & & 36.4 & 2.56 & 314.8 & $4.17403 E+13$ & $2.24274 \mathrm{E}+13$ & $9.68865 \mathrm{E}+13$ & $6.40741 \mathrm{E}+19$ & 1468.7 & 380.3 \\
\hline & & 43.4 & 3.06 & 315.4 & $4.22548 \mathrm{E}+13$ & $2.23100 E+13$ & $9.75163 \mathrm{E}+13$ & 7.64794E+19 & 1471.6 & 380.8 \\
\hline & & 50.4 & 3.57 & 324.9 & $4.39665 \mathrm{E}+13$ & $2.30542 \mathrm{E}+13$ & $1.00746 \mathrm{E}+14$ & $8.92460 \mathrm{E}+19$ & 1518.4 & 388.5 \\
\hline & & 57.4 & 4.08 & 329.6 & $4.51703 E+13$ & $2.32773 E+13$ & $1.02895 \mathrm{E}+14$ & $1.02133 \mathrm{E}+20$ & 1541.6 & 392.3 \\
\hline & & 60.4 & 4.30 & 327.3 & $4.53723 E+13$ & $2.31153 E+13$ & $1.02775 \mathrm{E}+14$ & $1.07493 \mathrm{E}+20$ & 1530.5 & 390.5 \\
\hline & \multicolumn{10}{|c|}{ Mid-Cycle 157D Outage - 14 Day, 10 Hour Down Time (7/23/2015, 1800 - 8/7/2015, 0400) } \\
\hline & EOC $(8 / 12 / 2015,0400)$ & 65.1 & 4.65 & 331.0 & $4.64228 \mathrm{E}+13$ & $2.32935 \mathrm{E}+13$ & $1.04339 \mathrm{E}+14$ & $1.16345 \mathrm{E}+20$ & 1548.5 & 393.5 \\
\hline \multirow[t]{8}{*}{$158 \mathrm{~A}$} & \multicolumn{10}{|c|}{ Cycle 158A Outage - 91 Day, 11 Hour Down Time } \\
\hline & BOC $(11 / 11 / 2015,1500)$ & 65.1 & 4.65 & 330.1 & $4.61435 \mathrm{E}+13$ & $2.34161 \mathrm{E}+13$ & $1.04078 \mathrm{E}+14$ & $1.16345 \mathrm{E}+20$ & 1544.0 & 392.7 \\
\hline & & 75.1 & 5.36 & 325.3 & $4.55498 \mathrm{E}+13$ & $2.30535 \mathrm{E}+13$ & $1.02821 \mathrm{E}+14$ & $1.34230 \mathrm{E}+20$ & 1520.3 & 388.8 \\
\hline & & 85.1 & 6.06 & 313.8 & $4.46677 \mathrm{E}+13$ & $2.23225 E+13$ & $1.00736 \mathrm{E}+14$ & $1.51514 \mathrm{E}+20$ & 1463.7 & 379.5 \\
\hline & & 95.1 & 6.77 & 317.8 & $4.59707 \mathrm{E}+13$ & $2.26276 \mathrm{E}+13$ & $1.02607 \mathrm{E}+14$ & $1.69519 \mathrm{E}+20$ & 1483.6 & 382.8 \\
\hline & & 105.1 & 7.50 & 322.7 & $4.74664 \mathrm{E}+13$ & $2.30155 \mathrm{E}+13$ & 1.04976E+14 & $1.87766 \mathrm{E}+20$ & 1507.7 & 386.7 \\
\hline & & 117.1 & 8.38 & 326.1 & $4.85997 \mathrm{E}+13$ & $2.31702 \mathrm{E}+13$ & $1.06575 \mathrm{E}+14$ & $2.09806 \mathrm{E}+20$ & 1524.5 & 389.5 \\
\hline & EOC $(1 / 2 / 2016,0400)$ & 117.3 & 8.40 & 321.0 & $4.88207 E+13$ & $2.28731 \mathrm{E}+13$ & $1.06218 \mathrm{E}+14$ & $2.10108 \mathrm{E}+20$ & 1499.2 & 385.3 \\
\hline \multirow[t]{5}{*}{$158 \mathrm{~B}$} & \multicolumn{10}{|c|}{ Cycle 158B Outage - 39 Day, 2 Hour Down Time } \\
\hline & BOC $(2 / 10 / 2016,0600)$ & 118.3 & 8.45 & 286.8 & $4.34082 \mathrm{E}+13$ & $1.85988 \mathrm{E}+13$ & $8.80276 \mathrm{E}+13$ & $2.11493 \mathrm{E}+20$ & 1328.6 & 357.0 \\
\hline & & 119.3 & 8.51 & 320.1 & $4.85112 \mathrm{E}+13$ & $2.04156 \mathrm{E}+13$ & $9.83399 \mathrm{E}+13$ & $2.12998 \mathrm{E}+20$ & 1494.9 & 384.6 \\
\hline & & 122.3 & 8.72 & 324.8 & $4.93509 \mathrm{E}+13$ & $2.09298 \mathrm{E}+13$ & $1.00245 \mathrm{E}+14$ & $2.18237 \mathrm{E}+20$ & 1518.2 & 388.5 \\
\hline & & 127.3 & 9.07 & 318.1 & $4.86504 \mathrm{E}+13$ & $2.02254 \mathrm{E}+13$ & $9.83377 \mathrm{E}+13$ & $2.26969 \mathrm{E}+20$ & 1485.1 & 383.0 \\
\hline
\end{tabular}


TEM-10300-1

$03 / 01 / 2012$

Rev. 03

Title: $\quad$ ATF-1 Power Histories

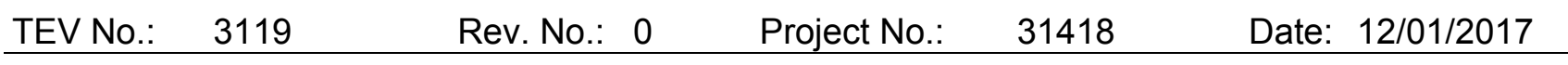

Table 3. ATF-01 Power History

\begin{tabular}{|c|c|c|c|c|c|c|c|c|c|c|}
\hline Cycle & Critical Dates & EFPD & $\begin{array}{c}\text { Burnup } \\
\text { (GWd/MTU) }\end{array}$ & $\begin{array}{c}\text { LHGR } \\
(\mathrm{W} / \mathrm{cm})\end{array}$ & $\begin{array}{l}\text { Thermal Flux } \\
\left(\mathbf{n} / \mathrm{cm}^{2 *} \mathrm{~s}\right)\end{array}$ & $\begin{array}{c}\text { Fast Flux } \\
\left(\mathrm{n} / \mathrm{cm}^{2 *} \mathrm{~s}>1\right. \\
\mathrm{MeV})\end{array}$ & $\begin{array}{l}\text { Total Flux } \\
\left(\mathrm{n} / \mathrm{cm}^{2 *} \mathrm{~s}\right)\end{array}$ & $\begin{array}{l}\text { Fission Density } \\
(\text { Fissions/cm³) }\end{array}$ & $\begin{array}{l}\text { Fuel Centerline } \\
\text { Temperature } \\
\left({ }^{\circ} \mathrm{C}\right)\end{array}$ & $\mathrm{PICT}\left({ }^{\circ} \mathrm{C}\right)$ \\
\hline & & 132.3 & 9.43 & 328.6 & $5.07356 \mathrm{E}+13$ & $2.11446 \mathrm{E}+13$ & $1.02446 \mathrm{E}+14$ & $2.35942 \mathrm{E}+20$ & 1536.6 & 391.5 \\
\hline & & 137.3 & 9.78 & 318.1 & $4.94676 \mathrm{E}+13$ & $2.05029 E+13$ & $9.94962 \mathrm{E}+13$ & $2.44614 \mathrm{E}+20$ & 1484.7 & 382.9 \\
\hline & & 147.3 & 10.52 & 333.4 & $5.23717 \mathrm{E}+13$ & $2.15015 \mathrm{E}+13$ & $1.05200 \mathrm{E}+14$ & $2.63282 \mathrm{E}+20$ & 1560.4 & 395.4 \\
\hline & & 157.3 & 11.26 & 328.3 & $5.23696 \mathrm{E}+13$ & $2.10220 \mathrm{E}+13$ & $1.03936 \mathrm{E}+14$ & $2.81649 \mathrm{E}+20$ & 1535.1 & 391.2 \\
\hline & EOC $(4 / 1 / 2016,0900)$ & 168.7 & 12.10 & 321.5 & $5.34315 E+13$ & $2.09657 \mathrm{E}+13$ & 1.04793E+14 & $3.02726 \mathrm{E}+20$ & 1501.7 & 385.7 \\
\hline \multirow[t]{7}{*}{$159 A$} & \multicolumn{10}{|c|}{ Cycle 159A Outage - 77 Day, 2 Hour Down Time } \\
\hline & $\operatorname{BOC}(6 / 17 / 2016,1100)$ & 169.7 & 12.12 & 135.3 & $2.24154 \mathrm{E}+13$ & $8.73419 \mathrm{E}+12$ & $4.40615 \mathrm{E}+13$ & $3.03388 \mathrm{E}+20$ & 523.8 & 220.3 \\
\hline & & 170.7 & 12.15 & 140.6 & $2.33184 \mathrm{E}+13$ & $9.05996 \mathrm{E}+12$ & $4.58136 \mathrm{E}+13$ & $3.03991 \mathrm{E}+20$ & 553.2 & 225.3 \\
\hline & & 174.6 & 12.26 & 120.5 & $2.00357 \mathrm{E}+13$ & $7.73583 \mathrm{E}+12$ & $3.92044 \mathrm{E}+13$ & $3.06821 \mathrm{E}+20$ & 440.8 & 205.9 \\
\hline & \multicolumn{10}{|c|}{ Mid-Cycle 159A Outage - 3 Day, 21 Hour Down Time (6/23/2016, 1000 - 6/27/2016, 0700) } \\
\hline & & 176.0 & 12.29 & 174.5 & $2.93925 \mathrm{E}+13$ & $1.16091 \mathrm{E}+13$ & $5.96705 \mathrm{E}+13$ & $3.07483 \mathrm{E}+20$ & 739.2 & 257.4 \\
\hline & EOC $(6 / 28 / 2016,1800)$ & 176.2 & 12.30 & 191.7 & $3.23394 \mathrm{E}+13$ & $1.28777 \mathrm{E}+13$ & $6.74108 \mathrm{E}+13$ & $3.07724 \mathrm{E}+20$ & 832.3 & 273.3 \\
\hline $160 \mathrm{~A}$ & \multicolumn{10}{|c|}{ Cycle 160A Outage - 80 Day, 1 Hour Down Time } \\
\hline & $\operatorname{BOC}(9 / 16 / 2016,1900)$ & 177.2 & 12.38 & 319.4 & $5.32350 \mathrm{E}+13$ & $2.07524 \mathrm{E}+13$ & $1.04207 \mathrm{E}+14$ & $3.09832 \mathrm{E}+20$ & 1491.3 & 384.0 \\
\hline & & 179.2 & 12.53 & 317.0 & $5.30294 \mathrm{E}+13$ & $2.07675 \mathrm{E}+13$ & $1.03954 \mathrm{E}+14$ & $3.13445 \mathrm{E}+20$ & 1479.2 & 382.0 \\
\hline & & 186.2 & 13.01 & 317.3 & $5.31914 \mathrm{E}+13$ & $2.06310 \mathrm{E}+13$ & $1.04346 \mathrm{E}+14$ & $3.25489 \mathrm{E}+20$ & 1481.0 & 382.3 \\
\hline & & 196.2 & 13.71 & 314.1 & $5.33709 \mathrm{E}+13$ & $2.04044 \mathrm{E}+13$ & $1.04046 \mathrm{E}+14$ & $3.43013 \mathrm{E}+20$ & 1465.3 & 379.7 \\
\hline & & 206.2 & 14.40 & 309.1 & $5.36591 \mathrm{E}+13$ & $2.03955 \mathrm{E}+13$ & $1.03815 \mathrm{E}+14$ & $3.60236 \mathrm{E}+20$ & 1440.4 & 375.6 \\
\hline & & 216.2 & 15.09 & 311.3 & $5.50867 \mathrm{E}+13$ & $2.03848 \mathrm{E}+13$ & $1.05772 \mathrm{E}+14$ & $3.77519 \mathrm{E}+20$ & 1451.0 & 377.4 \\
\hline & & 222.2 & 15.49 & 301.3 & $5.42256 \mathrm{E}+13$ & $1.97981 \mathrm{E}+13$ & $1.03600 \mathrm{E}+14$ & $3.87636 \mathrm{E}+20$ & 1401.1 & 369.1 \\
\hline & & 223.8 & 15.60 & 282.4 & $5.13623 \mathrm{E}+13$ & $1.84406 \mathrm{E}+13$ & $9.75564 \mathrm{E}+13$ & $3.90346 \mathrm{E}+20$ & 1306.4 & 353.3 \\
\hline & & 226.2 & 15.74 & 276.6 & $5.05538 \mathrm{E}+13$ & $1.83344 \mathrm{E}+13$ & $9.64759 \mathrm{E}+13$ & $3.93959 \mathrm{E}+20$ & 1276.6 & 348.4 \\
\hline & EOC $(11 / 8 / 2016,1100)$ & 228.9 & 15.90 & 263.1 & $4.85674 \mathrm{E}+13$ & $1.73313 E+13$ & $9.28213 \mathrm{E}+13$ & $3.97753 \mathrm{E}+20$ & 1207.6 & 336.8 \\
\hline \multirow[t]{6}{*}{$160 \mathrm{~B}$} & \multicolumn{10}{|c|}{ Cycle 160B Outage - 42 Day, 1 Hour Down Time Outage } \\
\hline & $\operatorname{BOC}(12 / 20 / 2016,1200)$ & 229.9 & 15.95 & 290.5 & $5.35675 \mathrm{E}+13$ & $1.91078 \mathrm{E}+13$ & $1.02287 \mathrm{E}+14$ & $3.99078 \mathrm{E}+20$ & 1347.2 & 360.1 \\
\hline & & 231.9 & 16.08 & 290.3 & $5.36484 \mathrm{E}+13$ & $1.91490 \mathrm{E}+13$ & $1.02460 \mathrm{E}+14$ & $4.02330 \mathrm{E}+20$ & 1346.3 & 360.0 \\
\hline & & 238.9 & 16.52 & 287.0 & $5.32997 \mathrm{E}+13$ & 1.89057E+13 & $1.01235 \mathrm{E}+14$ & $4.13290 \mathrm{E}+20$ & 1329.4 & 357.2 \\
\hline & & 248.9 & 17.13 & 273.9 & $5.15512 \mathrm{E}+13$ & $1.80595 \mathrm{E}+13$ & $9.77562 \mathrm{E}+13$ & $4.28646 \mathrm{E}+20$ & 1263.1 & 346.1 \\
\hline & & 258.1 & 17.67 & 265.2 & $5.16782 \mathrm{E}+13$ & $1.74760 \mathrm{E}+13$ & $9.69189 \mathrm{E}+13$ & $4.42195 \mathrm{E}+20$ & 1218.7 & 338.7 \\
\hline
\end{tabular}


TEM-10300-1

03/01/2012

Rev. 03

Title: $\quad$ ATF-1 Power Histories

$\begin{array}{lllll}\text { TEV No.: } & 3119 & \text { Rev. No.: } & 0 & \text { Project No.: }\end{array}$

Table 3. ATF-01 Power History

\begin{tabular}{|c|c|c|c|c|c|c|c|c|c|c|}
\hline Cycle & Critical Dates & EFPD & $\begin{array}{l}\text { Burnup } \\
\text { (GWd/MTU) }\end{array}$ & $\begin{array}{l}\text { LHGR } \\
(\mathrm{W} / \mathrm{cm})\end{array}$ & $\begin{array}{l}\text { Thermal Flux } \\
\left(\mathbf{n} / \mathrm{cm}^{2 *} \mathrm{~s}\right)\end{array}$ & $\begin{array}{c}\text { Fast Flux } \\
\left(\mathrm{n} / \mathrm{cm}^{2 *} \mathrm{~s}>1\right. \\
\mathrm{MeV})\end{array}$ & $\begin{array}{l}\text { Total Flux } \\
\left(\mathrm{n} / \mathrm{cm}^{2 *} \mathrm{~s}\right)\end{array}$ & $\begin{array}{l}\text { Fission Density } \\
\text { (Fissions/(cm }{ }^{3} \text { ) }\end{array}$ & $\begin{array}{l}\text { Fuel Centerline } \\
\text { Temperature } \\
\left({ }^{\circ} \mathrm{C}\right)\end{array}$ & $\mathrm{PICT}\left({ }^{\circ} \mathrm{C}\right)$ \\
\hline & \multicolumn{10}{|c|}{ Mid-Cycle 160B Outage - 5 Day, 14 Hour Down Time } \\
\hline & & 268.9 & 18.32 & 270.5 & $5.30232 \mathrm{E}+13$ & $1.79249 \mathrm{E}+13$ & $9.88843 E+13$ & $4.58334 \mathrm{E}+20$ & 1245.6 & 343.2 \\
\hline & & 278.9 & 18.90 & 258.5 & $5.15486 \mathrm{E}+13$ & $1.74719 \mathrm{E}+13$ & $9.83743 \mathrm{E}+13$ & $4.72908 \mathrm{E}+20$ & 1184.0 & 332.8 \\
\hline & $\operatorname{EOC}(2 / 23 / 2017,1400)$ & 288.4 & 19.48 & 270.4 & $5.58047 \mathrm{E}+13$ & $1.81352 \mathrm{E}+13$ & $1.03758 \mathrm{E}+14$ & $4.87481 \mathrm{E}+20$ & 1245.2 & 343.1 \\
\hline \multirow[t]{9}{*}{$161 \mathrm{~A}$} & \multicolumn{10}{|c|}{ Cycle 161A Outage - 95 Day, 13 Hour Down Time } \\
\hline & $\mathrm{BOC}(5 / 30 / 2017,0300)$ & 290.1 & 19.57 & 220.7 & $4.53742 \mathrm{E}+13$ & $1.47075 \mathrm{E}+13$ & $8.36143 \mathrm{E}+13$ & $4.89769 \mathrm{E}+20$ & 987.0 & 299.6 \\
\hline & & 290.4 & 19.58 & 197.9 & $4.08490 \mathrm{E}+13$ & $1.32625 \mathrm{E}+13$ & $7.52197 \mathrm{E}+13$ & $4.90010 \mathrm{E}+20$ & 865.9 & 279.0 \\
\hline & & 292.4 & 19.68 & 202.6 & $4.18700 \mathrm{E}+13$ & $1.36382 \mathrm{E}+13$ & $7.69968 \mathrm{E}+13$ & $4.92419 \mathrm{E}+20$ & 891.1 & 283.3 \\
\hline & & 294.4 & 19.78 & 205.6 & $4.25100 \mathrm{E}+13$ & $1.38262 \mathrm{E}+13$ & $7.82987 \mathrm{E}+13$ & $4.94828 \mathrm{E}+20$ & 906.8 & 286.0 \\
\hline & & 296.4 & 19.87 & 204.5 & $4.25542 \mathrm{E}+13$ & $1.37368 \mathrm{E}+13$ & $7.79612 \mathrm{E}+13$ & $4.97297 \mathrm{E}+20$ & 900.9 & 285.0 \\
\hline & & 298.4 & 19.97 & 210.1 & $4.37191 \mathrm{E}+13$ & $1.40881 \mathrm{E}+13$ & $8.00622 \mathrm{E}+13$ & $4.99766 \mathrm{E}+20$ & 930.6 & 290.0 \\
\hline & & 300.4 & 20.07 & 213.7 & $4.46350 \mathrm{E}+13$ & $1.42959 \mathrm{E}+13$ & $8.15814 \mathrm{E}+13$ & $5.02235 \mathrm{E}+20$ & 949.9 & 293.3 \\
\hline & $\operatorname{EOC}(6 / 11 / 2017,1600)$ & 300.9 & 20.09 & 217.5 & $4.57229 \mathrm{E}+13$ & 1.46087E+13 & $8.33799 \mathrm{E}+13$ & $5.02777 \mathrm{E}+20$ & 970.1 & 296.8 \\
\hline \multirow[t]{8}{*}{$162 \mathrm{~A}$} & \multicolumn{10}{|c|}{ Cycle 162A Outage } \\
\hline & $\mathrm{BOC}$ & 301.9 & 20.17 & 297.3 & $6.07881 \mathrm{E}+13$ & $1.94962 \mathrm{E}+13$ & $1.11421 \mathrm{E}+14$ & $5.04644 \mathrm{E}+20$ & 1381.3 & 365.8 \\
\hline & & 303.9 & 20.29 & 282.5 & $5.85138 \mathrm{E}+13$ & $1.87762 \mathrm{E}+13$ & $1.07544 \mathrm{E}+14$ & $5.07775 \mathrm{E}+20$ & 1306.6 & 353.4 \\
\hline & & 330.9 & 21.92 & 281.2 & $5.99351 \mathrm{E}+13$ & $1.85959 \mathrm{E}+13$ & $1.08637 \mathrm{E}+14$ & $5.48424 \mathrm{E}+20$ & 1300.2 & 352.3 \\
\hline & & 340.9 & 22.51 & 278.8 & $6.03309 \mathrm{E}+13$ & $1.83115 \mathrm{E}+13$ & $1.08824 \mathrm{E}+14$ & $5.63177 \mathrm{E}+20$ & 1287.8 & 350.2 \\
\hline & & 350.9 & 23.13 & 281.6 & $6.31849 \mathrm{E}+13$ & $1.89546 \mathrm{E}+13$ & $1.13153 E+14$ & $5.78714 \mathrm{E}+20$ & 1302.2 & 352.6 \\
\hline & & 359.9 & 23.66 & 271.0 & $6.19262 \mathrm{E}+13$ & $1.81151 \mathrm{E}+13$ & $1.10812 \mathrm{E}+14$ & $5.91963 \mathrm{E}+20$ & 1248.4 & 343.6 \\
\hline & EOC & 362.9 & 23.84 & 265.8 & $6.22670 \mathrm{E}+13$ & $1.78231 \mathrm{E}+13$ & $1.10704 \mathrm{E}+14$ & $5.96539 \mathrm{E}+20$ & 1221.7 & 339.2 \\
\hline
\end{tabular}


TEM-10300-1

$03 / 01 / 2012$

Rev. 03

Title: $\quad$ ATF-1 Power Histories

\begin{tabular}{lllll} 
TEV No.: & 3119 & Rev. No.: & 0 & Project No.: \\
\hline
\end{tabular}

Table 4. ATF-02 Power History

\begin{tabular}{|c|c|c|c|c|c|c|c|c|c|c|}
\hline Cycle & Critical Dates & EFPD & $\begin{array}{c}\text { Burnup } \\
\text { (GWd/MTU) }\end{array}$ & $\begin{array}{l}\text { LHGR } \\
(\mathrm{W} / \mathrm{cm})\end{array}$ & $\begin{array}{l}\text { Thermal Flux } \\
\left(\mathrm{n} / \mathrm{cm}^{2 *} \mathrm{~s}\right)\end{array}$ & $\begin{array}{c}\text { Fast Flux } \\
\left(\mathrm{n} / \mathrm{cm}^{2 *} \mathrm{~s}>1\right. \\
\mathrm{MeV})\end{array}$ & $\begin{array}{l}\text { Total Flux } \\
\left(\mathrm{n} / \mathrm{cm}^{2 *} \mathrm{~s}\right)\end{array}$ & $\begin{array}{c}\text { Fission Density } \\
\text { (Fissions/cm³) }\end{array}$ & $\begin{array}{c}\text { Fuel Centerline } \\
\text { Temperature } \\
\left({ }^{\circ} \mathrm{C}\right)\end{array}$ & $\mathrm{PICT}\left({ }^{\circ} \mathrm{C}\right)$ \\
\hline \multirow[t]{2}{*}{$157 \mathrm{C}$} & $\mathrm{BOC}(2 / 10 / 2015,0600)$ & 0.0 & 0.00 & 397.6 & $5.87400 \mathrm{E}+13$ & $2.82076 \mathrm{E}+13$ & $1.28827 E+14$ & $0.00000 \mathrm{E}+00$ & 1796.5 & 447.9 \\
\hline & EOC $(2 / 15 / 2015,0800)$ & 5.4 & 0.59 & 403.2 & $6.02479 \mathrm{E}+13$ & $2.86737 \mathrm{E}+13$ & $1.31244 \mathrm{E}+14$ & 1.27064E+19 & 1821.4 & 451.9 \\
\hline \multirow[t]{13}{*}{ 157D } & \multicolumn{10}{|c|}{ Cycle 157D Outage - 104 Day, 3 Hour Down Time } \\
\hline & $\mathrm{BOC}(5 / 30 / 2015,1100)$ & 5.4 & 0.59 & 341.8 & $5.10648 \mathrm{E}+13$ & $2.42448 \mathrm{E}+13$ & $1.10932 \mathrm{E}+14$ & 1.27064E+19 & 1544.0 & 405.6 \\
\hline & & 8.4 & 0.87 & 369.9 & $5.52898 \mathrm{E}+13$ & $2.61121 \mathrm{E}+13$ & $1.20282 E+14$ & $1.87284 \mathrm{E}+19$ & 1672.0 & 427.2 \\
\hline & & 15.4 & 1.54 & 368.3 & $5.52452 \mathrm{E}+13$ & $2.62257 E+13$ & $1.20895 E+14$ & $3.30006 \mathrm{E}+19$ & 1664.8 & 426.0 \\
\hline & & 22.4 & 2.19 & 362.9 & $5.52482 \mathrm{E}+13$ & $2.58992 \mathrm{E}+13$ & $1.20124 \mathrm{E}+14$ & $4.70920 \mathrm{E}+19$ & 1640.0 & 421.8 \\
\hline & & 29.4 & 2.85 & 364.5 & $5.62768 \mathrm{E}+13$ & $2.60067 E+13$ & $1.21303 E+14$ & $6.12437 E+19$ & 1647.3 & 423.1 \\
\hline & & 36.4 & 3.52 & 376.5 & $5.89328 \mathrm{E}+13$ & $2.68666 \mathrm{E}+13$ & $1.26209 E+14$ & $7.57568 \mathrm{E}+19$ & 1701.6 & 432.2 \\
\hline & & 43.4 & 4.20 & 375.2 & $5.96413 \mathrm{E}+13$ & $2.67975 E+13$ & $1.27020 \mathrm{E}+14$ & $9.02096 \mathrm{E}+19$ & 1695.7 & 431.2 \\
\hline & & 50.4 & 4.90 & 381.7 & $6.13783 \mathrm{E}+13$ & $2.73280 \mathrm{E}+13$ & $1.29944 \mathrm{E}+14$ & $1.05385 \mathrm{E}+20$ & 1725.1 & 436.1 \\
\hline & & 57.4 & 5.61 & 383.3 & $6.26546 \mathrm{E}+13$ & $2.75264 \mathrm{E}+13$ & $1.31934 \mathrm{E}+14$ & $1.20681 \mathrm{E}+20$ & 1732.0 & 437.2 \\
\hline & & 60.4 & 5.92 & 375.3 & $6.23574 \mathrm{E}+13$ & $2.70134 \mathrm{E}+13$ & $1.30461 \mathrm{E}+14$ & $1.27305 E+20$ & 1696.2 & 431.2 \\
\hline & \multicolumn{10}{|c|}{ Mid-Cycle 157D Outage - 14 Day, 10 Hour Down Time $(7 / 23 / 2015,1800-8 / 7 / 2015,0400)$} \\
\hline & EOC $(8 / 12 / 2015,0400)$ & 65.1 & 6.38 & 376.7 & $6.35970 \mathrm{E}+13$ & $2.71109 \mathrm{E}+13$ & $1.31976 \mathrm{E}+14$ & $1.37181 \mathrm{E}+20$ & 1702.6 & 432.3 \\
\hline \multirow[t]{10}{*}{ 158B } & \multicolumn{10}{|c|}{ ATF-02 Withheld from Cycle 158A - 182 Day, 2 Hour Down Time } \\
\hline & $\mathrm{BOC}(2 / 10 / 2016,0600)$ & 66.1 & 6.44 & 252.6 & $4.24237 \mathrm{E}+13$ & $1.82762 \mathrm{E}+13$ & $8.79948 \mathrm{E}+13$ & $1.38386 \mathrm{E}+20$ & 1130.6 & 332.9 \\
\hline & & 67.1 & 6.50 & 280.8 & $4.73433 \mathrm{E}+13$ & $2.01998 \mathrm{E}+13$ & $9.89695 \mathrm{E}+13$ & $1.39771 \mathrm{E}+20$ & 1262.8 & 356.6 \\
\hline & & 70.1 & 6.72 & 284.4 & $4.81632 \mathrm{E}+13$ & $2.06537 E+13$ & $1.00795 E+14$ & $1.44408 \mathrm{E}+20$ & 1279.5 & 359.5 \\
\hline & & 75.1 & 7.08 & 279.1 & $4.74483 \mathrm{E}+13$ & $2.01633 E+13$ & $9.88876 \mathrm{E}+13$ & $1.52296 \mathrm{E}+20$ & 1254.8 & 355.1 \\
\hline & & 80.1 & 7.46 & 290.7 & $5.00020 \mathrm{E}+13$ & $2.09036 \mathrm{E}+13$ & $1.03357 E+14$ & $1.60426 \mathrm{E}+20$ & 1308.5 & 364.7 \\
\hline & & 85.1 & 7.83 & 281.1 & $4.87084 \mathrm{E}+13$ & $2.04816 \mathrm{E}+13$ & $1.00954 \mathrm{E}+14$ & $1.68315 \mathrm{E}+20$ & 1264.0 & 356.8 \\
\hline & & 95.1 & 8.59 & 293.8 & $5.12850 \mathrm{E}+13$ & $2.13642 \mathrm{E}+13$ & $1.05362 \mathrm{E}+14$ & $1.84635 \mathrm{E}+20$ & 1322.9 & 367.2 \\
\hline & & 105.1 & 9.33 & 287.4 & $5.09403 E+13$ & $2.05445 E+13$ & $1.03610 \mathrm{E}+14$ & $2.00533 E+20$ & 1293.2 & 361.9 \\
\hline & EOC $(4 / 1 / 2016,0900)$ & 116.5 & 10.19 & 281.6 & $5.20076 \mathrm{E}+13$ & $2.04939 E+13$ & $1.04777 \mathrm{E}+14$ & $2.19080 \mathrm{E}+20$ & 1266.3 & 357.2 \\
\hline \multirow[t]{3}{*}{$159 \mathrm{~A}$} & \multicolumn{10}{|c|}{ Cycle 159A Outage - 77 Day, 2 Hour Down Time } \\
\hline & $\operatorname{BOC}(6 / 17 / 2016,1100)$ & 117.5 & 10.22 & 118.5 & $2.18021 \mathrm{E}+13$ & $8.76738 \mathrm{E}+12$ & $4.40173 E+13$ & $2.19622 \mathrm{E}+20$ & 484.7 & 211.5 \\
\hline & & 118.5 & 10.25 & 123.7 & $2.28130 \mathrm{E}+13$ & $9.13679 \mathrm{E}+12$ & $4.60874 \mathrm{E}+13$ & $2.20225 E+20$ & 510.2 & 216.5 \\
\hline
\end{tabular}


TEM-10300-1

$03 / 01 / 2012$

Rev. 03

Title: $\quad$ ATF-1 Power Histories

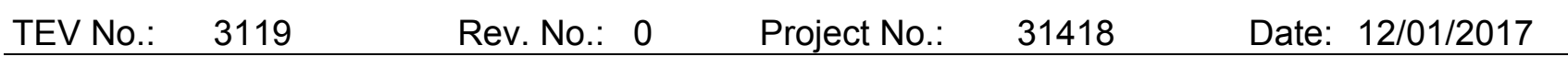

Table 4. ATF-02 Power History

\begin{tabular}{|c|c|c|c|c|c|c|c|c|c|c|}
\hline Cycle & Critical Dates & EFPD & $\begin{array}{c}\text { Burnup } \\
\text { (GWd/MTU) }\end{array}$ & $\begin{array}{l}\text { LHGR } \\
(\mathrm{W} / \mathrm{cm})\end{array}$ & $\begin{array}{l}\text { Thermal Flux } \\
\left(\mathrm{n} / \mathrm{cm}^{2 *} \mathrm{~s}\right)\end{array}$ & $\begin{array}{c}\text { Fast Flux } \\
\left(\mathrm{n} / \mathrm{cm}^{2 *} \mathrm{~s}>1\right. \\
\mathrm{MeV})\end{array}$ & $\begin{array}{l}\text { Total Flux } \\
\left(\mathrm{n} / \mathrm{cm}^{2 *} \mathrm{~s}\right)\end{array}$ & $\begin{array}{l}\text { Fission Density } \\
\text { (Fissions/cm³) }\end{array}$ & $\begin{array}{c}\text { Fuel Centerline } \\
\text { Temperature } \\
\left({ }^{\circ} \mathrm{C}\right)\end{array}$ & $\mathrm{PICT}\left({ }^{\circ} \mathrm{C}\right)$ \\
\hline & & 122.4 & 10.36 & 105.6 & $1.96027 \mathrm{E}+13$ & $7.63556 \mathrm{E}+12$ & $3.92603 \mathrm{E}+13$ & $2.22754 \mathrm{E}+20$ & 421.2 & 199.1 \\
\hline & \multicolumn{10}{|c|}{ Mid-Cycle 159A Outage - 3 Day, 21 Hour Down Time (6/23/2016, 1000 - 6/27/2016, 0700) } \\
\hline & & 123.8 & 10.39 & 152.6 & $2.83953 E+13$ & $1.13748 \mathrm{E}+13$ & $5.93813 \mathrm{E}+13$ & $2.23356 \mathrm{E}+20$ & 651.5 & 243.7 \\
\hline & EOC $(6 / 28 / 2016,1800)$ & 124.0 & 10.40 & 166.3 & $3.11815 \mathrm{E}+13$ & $1.26898 \mathrm{E}+13$ & $6.70192 \mathrm{E}+13$ & $2.23537 \mathrm{E}+20$ & 718.3 & 256.4 \\
\hline \multirow[t]{11}{*}{$160 \mathrm{~A}$} & \multicolumn{10}{|c|}{ Cycle 160A Outage - 80 Day, 1 Hour Down Time } \\
\hline & BOC $(9 / 16 / 2016,1900)$ & 125.0 & 10.46 & 278.5 & $5.16739 \mathrm{E}+13$ & $2.02547 \mathrm{E}+13$ & $1.03714 \mathrm{E}+14$ & $2.24861 \mathrm{E}+20$ & 1251.7 & 354.6 \\
\hline & & 127.0 & 10.61 & 277.0 & $5.15119 \mathrm{E}+13$ & $2.00412 \mathrm{E}+13$ & $1.03393 \mathrm{E}+14$ & $2.28053 \mathrm{E}+20$ & 1244.7 & 353.3 \\
\hline & & 134.0 & 11.11 & 276.4 & $5.16537 \mathrm{E}+13$ & $2.01599 \mathrm{E}+13$ & $1.03848 \mathrm{E}+14$ & $2.38893 \mathrm{E}+20$ & 1242.2 & 352.9 \\
\hline & & 144.0 & 11.82 & 273.4 & $5.17849 \mathrm{E}+13$ & $2.00787 \mathrm{E}+13$ & $1.03654 \mathrm{E}+14$ & $2.54008 \mathrm{E}+20$ & 1228.2 & 350.4 \\
\hline & & 154.0 & 12.52 & 273.0 & $5.25720 \mathrm{E}+13$ & $2.01803 E+13$ & $1.04286 \mathrm{E}+14$ & $2.69123 \mathrm{E}+20$ & 1226.3 & 350.1 \\
\hline & & 164.0 & 13.23 & 274.8 & $5.39827 \mathrm{E}+13$ & $2.03022 \mathrm{E}+13$ & $1.06716 \mathrm{E}+14$ & $2.84299 \mathrm{E}+20$ & 1234.8 & 351.6 \\
\hline & & 170.0 & 13.65 & 263.3 & $5.26141 \mathrm{E}+13$ & $1.94473 \mathrm{E}+13$ & 1.03157E+14 & $2.93392 \mathrm{E}+20$ & 1180.6 & 341.9 \\
\hline & & 171.6 & 13.76 & 246.6 & $4.98393 E+13$ & $1.81600 \mathrm{E}+13$ & $9.70364 \mathrm{E}+13$ & $2.95861 \mathrm{E}+20$ & 1102.1 & 327.7 \\
\hline & & 174.0 & 13.92 & 241.3 & $4.89429 \mathrm{E}+13$ & $1.77204 \mathrm{E}+13$ & $9.55029 \mathrm{E}+13$ & $2.99113 \mathrm{E}+20$ & 1077.2 & 323.2 \\
\hline & EOC $(11 / 8 / 2016,1100)$ & 176.7 & 14.07 & 229.9 & $4.70701 \mathrm{E}+13$ & $1.70132 \mathrm{E}+13$ & $9.21349 \mathrm{E}+13$ & $3.02485 \mathrm{E}+20$ & 1023.1 & 313.3 \\
\hline \multirow[t]{10}{*}{$160 \mathrm{~B}$} & \multicolumn{10}{|c|}{ Cycle 160B Outage - 42 Day, 1 Hour Down Time Outage } \\
\hline & $\mathrm{BOC}(12 / 20 / 2016,1200)$ & 177.7 & 14.13 & 242.3 & $4.95478 \mathrm{E}+13$ & 1.85467E+13 & $9.92743 \mathrm{E}+13$ & $3.03629 \mathrm{E}+20$ & 1081.9 & 324.0 \\
\hline & & 179.7 & 14.26 & 241.5 & $4.95628 \mathrm{E}+13$ & $1.85035 \mathrm{E}+13$ & $9.93290 \mathrm{E}+13$ & $3.06460 \mathrm{E}+20$ & 1078.3 & 323.4 \\
\hline & & 186.7 & 14.70 & 237.5 & $4.89876 \mathrm{E}+13$ & $1.82730 \mathrm{E}+13$ & $9.78828 \mathrm{E}+13$ & $3.15914 \mathrm{E}+20$ & 1059.1 & 319.9 \\
\hline & & 196.7 & 15.29 & 228.2 & $4.75838 \mathrm{E}+13$ & $1.74769 \mathrm{E}+13$ & $9.48257 \mathrm{E}+13$ & $3.28681 \mathrm{E}+20$ & 1015.2 & 311.8 \\
\hline & & 205.9 & 15.84 & 221.5 & $4.76673 \mathrm{E}+13$ & $1.70569 \mathrm{E}+13$ & 9.40347E+13 & $3.40484 \mathrm{E}+20$ & 983.3 & 306.0 \\
\hline & \multicolumn{10}{|c|}{ Mid-Cycle 160B Outage - 5 Day, 14 Hour Down Time } \\
\hline & & 216.7 & 16.50 & 224.9 & $4.88201 \mathrm{E}+13$ & $1.74330 \mathrm{E}+13$ & $9.59784 \mathrm{E}+13$ & $3.54575 \mathrm{E}+20$ & 999.5 & 309.0 \\
\hline & & 226.7 & 17.06 & 215.0 & $4.73352 \mathrm{E}+13$ & $1.70060 \mathrm{E}+13$ & $9.52206 \mathrm{E}+13$ & $3.66619 \mathrm{E}+20$ & 952.2 & 300.2 \\
\hline & EOC $(2 / 23 / 2017,1400)$ & 236.2 & 17.62 & 225.4 & $5.11749 \mathrm{E}+13$ & $1.77142 \mathrm{E}+13$ & $1.00230 \mathrm{E}+14$ & $3.78724 \mathrm{E}+20$ & 1001.6 & 309.4 \\
\hline $161 \mathrm{~A}$ & \multicolumn{10}{|c|}{ Cycle 161A Outage - 95 Day, 13 Hour Down Time } \\
\hline & BOC $(5 / 30 / 2017,0300)$ & 237.9 & 17.71 & 178.9 & $4.05804 \mathrm{E}+13$ & $1.38491 \mathrm{E}+13$ & $7.84890 \mathrm{E}+13$ & $3.80711 \mathrm{E}+20$ & 779.2 & 268.0 \\
\hline & & 238.2 & 17.72 & 161.1 & $3.65805 E+13$ & $1.25116 \mathrm{E}+13$ & $7.08951 \mathrm{E}+13$ & $3.80952 \mathrm{E}+20$ & 693.0 & 251.6 \\
\hline & & 240.2 & 17.82 & 164.9 & $3.75398 \mathrm{E}+13$ & $1.28596 \mathrm{E}+13$ & $7.28404 \mathrm{E}+13$ & $3.83059 \mathrm{E}+20$ & 711.3 & 255.1 \\
\hline
\end{tabular}


TEM-10300-1

03/01/2012

TECHNICAL EVALUATION

Page 17 of 110

Rev. 03

ATF-1 Power Histories

$\begin{array}{lllll}\text { TEV No.: } 3119 \quad \text { Rev. No.: } & 0 & \text { Project No.: } & 31418\end{array}$

Table 4. ATF-02 Power History

\begin{tabular}{|c|c|c|c|c|c|c|c|c|c|c|}
\hline Cycle & Critical Dates & EFPD & $\begin{array}{c}\text { Burnup } \\
\text { (GWd/MTU) }\end{array}$ & $\begin{array}{l}\text { LHGR } \\
(\mathrm{W} / \mathrm{cm})\end{array}$ & $\begin{array}{l}\text { Thermal Flux } \\
\left(\mathrm{n} / \mathrm{cm}^{2 *} \mathrm{~s}\right)\end{array}$ & $\begin{array}{c}\text { Fast Flux } \\
\left(\mathrm{n} / \mathrm{cm}^{2 *} \mathrm{~s}>1\right. \\
\mathrm{MeV})\end{array}$ & $\begin{array}{l}\text { Total Flux } \\
\left(\mathrm{n} / \mathrm{cm}^{2 *} \mathrm{~s}\right)\end{array}$ & $\begin{array}{c}\text { Fission Density } \\
\left(\text { Fissions } / \mathrm{cm}^{3}\right)\end{array}$ & $\begin{array}{l}\text { Fuel Centerline } \\
\text { Temperature } \\
\left({ }^{\circ} \mathrm{C}\right)\end{array}$ & PICT $\left({ }^{\circ} \mathrm{C}\right)$ \\
\hline & & 242.2 & 17.92 & 167.3 & $3.82077 \mathrm{E}+13$ & $1.30157 \mathrm{E}+13$ & $7.40285 \mathrm{E}+13$ & $3.85167 \mathrm{E}+20$ & 723.2 & 257.4 \\
\hline & & 244.2 & 18.02 & 166.6 & $3.81485 E+13$ & $1.29223 E+13$ & $7.34862 E+13$ & $3.87275 \mathrm{E}+20$ & 719.4 & 256.7 \\
\hline & & 246.2 & 18.11 & 170.0 & $3.89711 \mathrm{E}+13$ & $1.32215 \mathrm{E}+13$ & $7.51351 \mathrm{E}+13$ & $3.89383 E+20$ & 736.2 & 259.8 \\
\hline & & 248.2 & 18.22 & 173.0 & $3.98123 E+13$ & $1.34222 \mathrm{E}+13$ & $7.65381 \mathrm{E}+13$ & $3.91550 \mathrm{E}+20$ & 750.8 & 262.6 \\
\hline & EOC $(6 / 11 / 2017,1600)$ & 248.7 & 18.23 & 176.4 & $4.06215 E+13$ & $1.36061 \mathrm{E}+13$ & $7.81268 \mathrm{E}+13$ & $3.91912 \mathrm{E}+20$ & 767.0 & 265.7 \\
\hline \multirow[t]{10}{*}{$162 \mathrm{~A}$} & \multicolumn{10}{|c|}{ Cycle 162A Outage } \\
\hline & $\mathrm{BOC}$ & 249.7 & 18.29 & 252.9 & $5.67337 \mathrm{E}+13$ & $1.90104 \mathrm{E}+13$ & $1.09389 \mathrm{E}+14$ & $3.93056 \mathrm{E}+20$ & 1132.0 & 333.1 \\
\hline & & 251.7 & 18.41 & 238.5 & $5.42648 \mathrm{E}+13$ & 1.82057E+13 & $1.04669 \mathrm{E}+14$ & $3.95766 \mathrm{E}+20$ & 1064.1 & 320.8 \\
\hline & & 258.7 & 18.83 & 237.9 & $5.43364 \mathrm{E}+13$ & $1.82319 \mathrm{E}+13$ & $1.04805 \mathrm{E}+14$ & $4.04859 \mathrm{E}+20$ & 1060.9 & 320.2 \\
\hline & & 268.7 & 19.43 & 239.5 & $5.46434 \mathrm{E}+13$ & $1.79789 \mathrm{E}+13$ & $1.04621 \mathrm{E}+14$ & $4.17565 \mathrm{E}+20$ & 1068.5 & 321.6 \\
\hline & & 278.7 & 20.01 & 237.3 & $5.53721 \mathrm{E}+13$ & $1.80353 \mathrm{E}+13$ & $1.05433 \mathrm{E}+14$ & $4.30151 \mathrm{E}+20$ & 1058.3 & 319.7 \\
\hline & & 288.7 & 20.62 & 236.6 & $5.60846 \mathrm{E}+13$ & $1.78421 \mathrm{E}+13$ & $1.06113 \mathrm{E}+14$ & $4.43219 \mathrm{E}+20$ & 1055.0 & 319.1 \\
\hline & & 298.7 & 21.24 & 239.2 & $5.86848 E+13$ & $1.83656 \mathrm{E}+13$ & 1.10199E+14 & $4.56528 \mathrm{E}+20$ & 1067.1 & 321.3 \\
\hline & & 307.7 & 21.76 & 230.3 & $5.73579 \mathrm{E}+13$ & $1.77188 \mathrm{E}+13$ & $1.07934 \mathrm{E}+14$ & $4.67729 \mathrm{E}+20$ & 1025.3 & 313.7 \\
\hline & EOC & 310.7 & 21.92 & 226.2 & $5.76596 \mathrm{E}+13$ & $1.74274 \mathrm{E}+13$ & $1.07809 \mathrm{E}+14$ & $4.71222 \mathrm{E}+20$ & 1005.4 & 310.1 \\
\hline
\end{tabular}


TEM-10300-1

$03 / 01 / 2012$

Rev. 03

TECHNICAL EVALUATION

Page 18 of 110

Title: $\quad$ ATF-1 Power Histories

$\begin{array}{lllll}\text { TEV No.: } & 3119 & \text { Rev. No.: } & 0 & \text { Project No.: }\end{array}$

Table 5. ATF-03 Power History

\begin{tabular}{|c|c|c|c|c|c|c|c|c|c|c|}
\hline Cycle & Critical Dates & EFPD & $\begin{array}{c}\text { Burnup } \\
\text { (GWd/MTU) }\end{array}$ & $\begin{array}{c}\text { LHGR } \\
(\mathrm{W} / \mathrm{cm})\end{array}$ & $\begin{array}{l}\text { Thermal Flux } \\
\left(\mathrm{n} / \mathrm{cm}^{2 *} \mathrm{~s}\right)\end{array}$ & $\begin{array}{c}\text { Fast Flux } \\
\left(\mathrm{n} / \mathrm{cm}^{2 *} \mathrm{~s}>1\right. \\
\mathrm{MeV})\end{array}$ & $\begin{array}{l}\text { Total Flux } \\
\left(\mathrm{n} / \mathrm{cm}^{2 *} \mathrm{~s}\right)\end{array}$ & $\begin{array}{l}\text { Fission Density } \\
\text { (Fissions/cm³) }\end{array}$ & $\begin{array}{c}\text { Fuel Centerline } \\
\text { Temperature } \\
\left({ }^{\circ} \mathrm{C}\right)\end{array}$ & $\mathrm{PICT}\left({ }^{\circ} \mathrm{C}\right)$ \\
\hline \multirow[t]{2}{*}{$157 \mathrm{C}$} & BOC $(2 / 10 / 2015,0600)$ & 0.0 & 0.00 & 395.4 & $5.82731 \mathrm{E}+13$ & $2.81429 \mathrm{E}+13$ & $1.28367 \mathrm{E}+14$ & $0.00000 \mathrm{E}+00$ & 1748.7 & 400.6 \\
\hline & EOC $(2 / 15 / 2015,0800)$ & 5.4 & 0.59 & 401.2 & $5.97438 \mathrm{E}+13$ & $2.86128 \mathrm{E}+13$ & $1.30817 \mathrm{E}+14$ & $1.25860 \mathrm{E}+19$ & 1775.1 & 404.5 \\
\hline \multirow[t]{13}{*}{ 157D } & \multicolumn{10}{|c|}{ Cycle 157D Outage - 104 Day, 3 Hour Down Time } \\
\hline & $\operatorname{BOC}(5 / 30 / 2015,1100)$ & 5.4 & 0.59 & 341.2 & $5.06671 \mathrm{E}+13$ & $2.43656 \mathrm{E}+13$ & $1.10696 \mathrm{E}+14$ & $1.25860 \mathrm{E}+19$ & 1501.2 & 363.5 \\
\hline & & 8.4 & 0.87 & 370.3 & $5.50740 \mathrm{E}+13$ & $2.63120 \mathrm{E}+13$ & $1.20337 \mathrm{E}+14$ & $1.86080 \mathrm{E}+19$ & 1633.9 & 383.3 \\
\hline & & 15.4 & 1.53 & 369.3 & $5.53300 \mathrm{E}+13$ & $2.65321 \mathrm{E}+13$ & $1.21086 \mathrm{E}+14$ & $3.28801 \mathrm{E}+19$ & 1629.1 & 382.6 \\
\hline & & 22.4 & 2.19 & 364.7 & $5.53630 \mathrm{E}+13$ & $2.60977 \mathrm{E}+13$ & $1.20319 \mathrm{E}+14$ & $4.70318 \mathrm{E}+19$ & 1608.1 & 379.5 \\
\hline & & 29.4 & 2.84 & 363.5 & $5.59858 \mathrm{E}+13$ & $2.60617 \mathrm{E}+13$ & $1.21095 \mathrm{E}+14$ & $6.10029 \mathrm{E}+19$ & 1602.6 & 378.7 \\
\hline & & 36.4 & 3.51 & 374.3 & $5.84538 \mathrm{E}+13$ & $2.68944 \mathrm{E}+13$ & $1.25731 \mathrm{E}+14$ & 7.53954E+19 & 1651.8 & 386.0 \\
\hline & & 43.4 & 4.17 & 374.2 & $5.92343 E+13$ & $2.68695 \mathrm{E}+13$ & $1.26801 \mathrm{E}+14$ & 8.97278E+19 & 1651.6 & 386.0 \\
\hline & & 50.4 & 4.88 & 379.6 & $6.09594 \mathrm{E}+13$ & $2.73019 \mathrm{E}+13$ & $1.29261 \mathrm{E}+14$ & $1.04843 \mathrm{E}+20$ & 1675.9 & 389.6 \\
\hline & & 57.4 & 5.58 & 382.0 & $6.21504 \mathrm{E}+13$ & $2.74789 \mathrm{E}+13$ & $1.31813 \mathrm{E}+14$ & $1.20018 \mathrm{E}+20$ & 1687.1 & 391.3 \\
\hline & & 60.4 & 5.89 & 375.3 & $6.20567 \mathrm{E}+13$ & 2.69797E+13 & $1.30259 \mathrm{E}+14$ & $1.26582 \mathrm{E}+20$ & 1656.6 & 386.7 \\
\hline & \multicolumn{10}{|c|}{ Mid-Cycle 157D Outage - 14 Day, 10 Hour Down Time $(7 / 23 / 2015,1800$ - 8/7/2015, 0400) } \\
\hline & $\mathrm{EOC}^{\dagger}(8 / 12 / 2015,0400)$ & 65.1 & 6.34 & 375.0 & $6.29634 \mathrm{E}+13$ & $2.69455 \mathrm{E}+13$ & $1.31273 \mathrm{E}+14$ & $1.36398 \mathrm{E}+20$ & 1654.9 & 386.5 \\
\hline
\end{tabular}

ATF-03 was discharged for PIE following Cycle 157D irradiation prior to the generation of LHGR dependent temperature relationships. 
TEM-10300-1

$03 / 01 / 2012$

Rev. 03

Title: $\quad$ ATF-1 Power Histories

$\begin{array}{lllll}\text { TEV No.: } & 3119 & \text { Rev. No.: } & 0 & \text { Project No.: }\end{array}$

Table 6. ATF-04 Power History

\begin{tabular}{|c|c|c|c|c|c|c|c|c|c|c|}
\hline Cycle & Critical Dates & EFPD & $\begin{array}{c}\text { Burnup } \\
\text { (GWd/MTU) }\end{array}$ & $\begin{array}{c}\text { LHGR } \\
(\mathrm{W} / \mathrm{cm})\end{array}$ & $\begin{array}{l}\text { Thermal Flux } \\
\left(\mathrm{n} / \mathrm{cm}^{2 *} \mathrm{~s}\right)\end{array}$ & $\begin{array}{c}\text { Fast Flux } \\
\left(\mathrm{n} / \mathrm{cm}^{2 *} \mathrm{~s}>1\right. \\
\mathrm{MeV})\end{array}$ & $\begin{array}{l}\text { Total Flux } \\
\left(\mathrm{n} / \mathrm{cm}^{2 *} \mathrm{~s}\right)\end{array}$ & $\begin{array}{l}\text { Fission Density } \\
\text { (Fissions/cm³) }\end{array}$ & $\begin{array}{c}\text { Fuel Centerline } \\
\text { Temperature } \\
\left({ }^{\circ} \mathrm{C}\right)\end{array}$ & $\mathrm{PICT}\left({ }^{\circ} \mathrm{C}\right)$ \\
\hline \multirow[t]{2}{*}{$157 \mathrm{C}$} & BOC $(2 / 10 / 2015,0600)$ & 0.0 & 0.00 & 308.4 & $4.42556 \mathrm{E}+13$ & $2.19585 E+13$ & $9.85835 \mathrm{E}+13$ & $0.00000 \mathrm{E}+00$ & 1147.0 & 378.2 \\
\hline & EOC $(2 / 15 / 2015,0800)$ & 5.4 & 0.44 & 313.0 & $4.54146 \mathrm{E}+13$ & $2.22518 \mathrm{E}+13$ & $1.00195 \mathrm{E}+14$ & $9.69542 \mathrm{E}+18$ & 1163.3 & 381.9 \\
\hline \multirow[t]{13}{*}{ 157D } & \multicolumn{10}{|c|}{ Cycle 157D Outage - 104 Day, 3 Hour Down Time } \\
\hline & $\operatorname{BOC}(5 / 30 / 2015,1100)$ & 5.4 & 0.44 & 265.5 & $3.84547 \mathrm{E}+13$ & $1.87003 \mathrm{E}+13$ & $8.46558 \mathrm{E}+13$ & $9.69542 \mathrm{E}+18$ & 997.4 & 342.9 \\
\hline & & 8.4 & 0.66 & 288.1 & $4.17514 \mathrm{E}+13$ & $2.04524 \mathrm{E}+13$ & $9.23636 \mathrm{E}+13$ & $1.45130 \mathrm{E}+19$ & 1076.1 & 361.7 \\
\hline & & 15.4 & 1.19 & 291.7 & $4.25636 \mathrm{E}+13$ & $2.06289 E+13$ & $9.39089 E+13$ & $2.61957 \mathrm{E}+19$ & 1088.7 & 364.6 \\
\hline & & 22.4 & 1.72 & 288.7 & $4.25669 \mathrm{E}+13$ & $2.06076 \mathrm{E}+13$ & $9.41889 E+13$ & $3.78182 \mathrm{E}+19$ & 1078.3 & 362.2 \\
\hline & & 29.4 & 2.24 & 289.0 & $4.29878 \mathrm{E}+13$ & $2.06739 \mathrm{E}+13$ & $9.44116 \mathrm{E}+13$ & $4.93202 \mathrm{E}+19$ & 1079.2 & 362.4 \\
\hline & & 36.4 & 2.78 & 296.6 & $4.47047 \mathrm{E}+13$ & $2.12388 \mathrm{E}+13$ & $9.78088 \mathrm{E}+13$ & $6.11835 \mathrm{E}+19$ & 1105.8 & 368.6 \\
\hline & & 43.4 & 3.32 & 298.8 & $4.54548 \mathrm{E}+13$ & $2.13223 E+13$ & $9.88474 \mathrm{E}+13$ & $7.30469 \mathrm{E}+19$ & 1113.4 & 370.4 \\
\hline & & 50.4 & 3.87 & 305.5 & $4.69618 \mathrm{E}+13$ & $2.18352 \mathrm{E}+13$ & $1.01654 \mathrm{E}+14$ & $8.51511 \mathrm{E}+19$ & 1136.8 & 375.8 \\
\hline & & 57.4 & 4.43 & 308.4 & $4.80520 \mathrm{E}+13$ & $2.20208 E+13$ & $1.03346 \mathrm{E}+14$ & $9.73155 \mathrm{E}+19$ & 1147.1 & 378.2 \\
\hline & & 60.4 & 4.65 & 307.6 & $4.83315 \mathrm{E}+13$ & $2.21201 \mathrm{E}+13$ & $1.03750 \mathrm{E}+14$ & $1.02314 \mathrm{E}+20$ & 1144.4 & 377.6 \\
\hline & \multicolumn{10}{|c|}{ Mid-Cycle 157D Outage - 14 Day, 10 Hour Down Time $(7 / 23 / 2015,1800-8 / 7 / 2015,0400)$} \\
\hline & EOC $(8 / 12 / 2015,0400)$ & 65.1 & 5.03 & 309.6 & $4.93630 \mathrm{E}+13$ & $2.21742 \mathrm{E}+13$ & $1.04933 \mathrm{E}+14$ & $1.10684 \mathrm{E}+20$ & 1151.4 & 379.2 \\
\hline \multirow[t]{8}{*}{$158 \mathrm{~A}$} & \multicolumn{10}{|c|}{ Cycle 158A Outage - 91 Day, 11 Hour Down Time } \\
\hline & $\mathrm{BOC}(11 / 11 / 2015,1500)$ & 65.1 & 5.03 & 309.7 & $4.92932 \mathrm{E}+13$ & $2.22904 \mathrm{E}+13$ & $1.05220 \mathrm{E}+14$ & 1.10684E+20 & 1151.6 & 379.2 \\
\hline & & 75.1 & 5.80 & 304.0 & $4.83212 \mathrm{E}+13$ & $2.19912 \mathrm{E}+13$ & $1.03821 \mathrm{E}+14$ & $1.27546 \mathrm{E}+20$ & 1131.6 & 374.6 \\
\hline & & 85.1 & 6.55 & 296.8 & $4.81067 \mathrm{E}+13$ & $2.15029 E+13$ & $1.02964 \mathrm{E}+14$ & $1.44046 \mathrm{E}+20$ & 1106.6 & 368.8 \\
\hline & & 95.1 & 7.30 & 298.2 & $4.91862 \mathrm{E}+13$ & $2.16859 E+13$ & $1.04370 \mathrm{E}+14$ & $1.60547 \mathrm{E}+20$ & 1111.3 & 369.9 \\
\hline & & 105.1 & 8.06 & 302.6 & $5.07420 \mathrm{E}+13$ & $2.18675 E+13$ & $1.06409 \mathrm{E}+14$ & 1.77227E+20 & 1126.9 & 373.5 \\
\hline & & 117.1 & 8.98 & 305.4 & $5.21317 \mathrm{E}+13$ & $2.21699 \mathrm{E}+13$ & $1.08466 \mathrm{E}+14$ & $1.97461 \mathrm{E}+20$ & 1136.6 & 375.8 \\
\hline & $\mathrm{EOC} \ddagger(1 / 2 / 2016,0400)$ & 117.3 & 8.99 & 300.1 & $5.23950 \mathrm{E}+13$ & $2.18539 E+13$ & $1.08155 E+14$ & $1.97702 \mathrm{E}+20$ & 1118.1 & 371.5 \\
\hline
\end{tabular}

\#ATF-04 was discharged for PIE following Cycle 158A irradiation. 
TEM-10300-1

$03 / 01 / 2012$

Rev. 03

Title: $\quad$ ATF-1 Power Histories

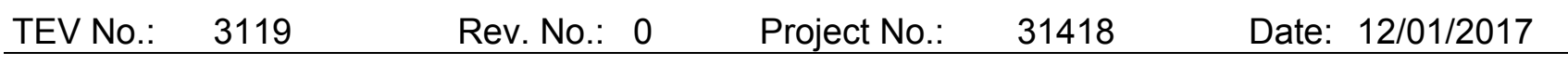

Table 7. ATF-05 Power History

\begin{tabular}{|c|c|c|c|c|c|c|c|c|c|c|}
\hline Cycle & Critical Dates & EFPD & $\begin{array}{c}\text { Burnup } \\
\text { (GWd/MTU) }\end{array}$ & $\begin{array}{l}\text { LHGR } \\
(\mathrm{W} / \mathrm{cm})\end{array}$ & $\begin{array}{l}\text { Thermal Flux } \\
\left(\mathrm{n} / \mathrm{cm}^{2 *} \mathrm{~s}\right)\end{array}$ & $\begin{array}{c}\text { Fast Flux } \\
\left(\mathrm{n} / \mathrm{cm}^{2 *} \mathrm{~s}>1\right. \\
\mathrm{MeV})\end{array}$ & $\begin{array}{l}\text { Total Flux } \\
\left(\mathrm{n} / \mathrm{cm}^{2 *} \mathrm{~s}\right)\end{array}$ & $\begin{array}{l}\text { Fission Density } \\
\text { (Fissions/cm³) }\end{array}$ & $\begin{array}{c}\text { Fuel Centerline } \\
\text { Temperature } \\
\left({ }^{\circ} \mathrm{C}\right)\end{array}$ & $\mathrm{PICT}\left({ }^{\circ} \mathrm{C}\right)$ \\
\hline \multirow[t]{2}{*}{$157 \mathrm{C}$} & BOC $(2 / 10 / 2015,0600)$ & 0.0 & 0.00 & 309.8 & $4.43032 \mathrm{E}+13$ & $2.18585 E+13$ & $9.84569 \mathrm{E}+13$ & $0.00000 \mathrm{E}+00$ & 1105.9 & 374.5 \\
\hline & EOC $(2 / 15 / 2015,0800)$ & 5.4 & 0.44 & 314.6 & $4.53979 \mathrm{E}+13$ & $2.22600 \mathrm{E}+13$ & $1.00285 \mathrm{E}+14$ & $9.63520 \mathrm{E}+18$ & 1122.4 & 378.3 \\
\hline \multirow[t]{13}{*}{ 157D } & \multicolumn{10}{|c|}{ Cycle 157D Outage - 104 Day, 3 Hour Down Time } \\
\hline & $\mathrm{BOC}(5 / 30 / 2015,1100)$ & 5.4 & 0.44 & 267.7 & $3.85925 \mathrm{E}+13$ & $1.88266 \mathrm{E}+13$ & $8.48387 \mathrm{E}+13$ & $9.63520 \mathrm{E}+18$ & 963.9 & 340.5 \\
\hline & & 8.4 & 0.65 & 287.6 & $4.15239 \mathrm{E}+13$ & $2.05084 \mathrm{E}+13$ & $9.22776 \mathrm{E}+13$ & $1.44528 \mathrm{E}+19$ & 1030.6 & 356.7 \\
\hline & & 15.4 & 1.18 & 291.6 & $4.22686 \mathrm{E}+13$ & $2.08753 E+13$ & $9.38854 \mathrm{E}+13$ & $2.61355 \mathrm{E}+19$ & 1044.3 & 360.0 \\
\hline & & 22.4 & 1.70 & 288.3 & $4.23720 \mathrm{E}+13$ & $2.05978 \mathrm{E}+13$ & $9.35749 \mathrm{E}+13$ & $3.76375 \mathrm{E}+19$ & 1033.1 & 357.3 \\
\hline & & 29.4 & 2.23 & 289.5 & $4.28567 \mathrm{E}+13$ & $2.06892 E+13$ & $9.44043 E+13$ & $4.92600 \mathrm{E}+19$ & 1037.1 & 358.2 \\
\hline & & 36.4 & 2.76 & 297.0 & $4.44272 \mathrm{E}+13$ & $2.12853 E+13$ & $9.76048 \mathrm{E}+13$ & $6.10631 \mathrm{E}+19$ & 1062.4 & 364.3 \\
\hline & & 43.4 & 3.30 & 298.6 & $4.51517 \mathrm{E}+13$ & $2.13548 \mathrm{E}+13$ & $9.85046 \mathrm{E}+13$ & $7.29264 \mathrm{E}+19$ & 1067.8 & 365.6 \\
\hline & & 50.4 & 3.85 & 307.6 & $4.70841 \mathrm{E}+13$ & $2.19708 \mathrm{E}+13$ & $1.01881 \mathrm{E}+14$ & $8.50909 \mathrm{E}+19$ & 1098.4 & 372.7 \\
\hline & & 57.4 & 4.40 & 310.1 & $4.80407 \mathrm{E}+13$ & $2.21887 E+13$ & $1.03546 \mathrm{E}+14$ & $9.73155 \mathrm{E}+19$ & 1107.0 & 374.7 \\
\hline & & 60.4 & 4.63 & 309.8 & $4.84616 \mathrm{E}+13$ & $2.22093 E+13$ & $1.04189 \mathrm{E}+14$ & $1.02374 \mathrm{E}+20$ & 1106.0 & 374.5 \\
\hline & \multicolumn{10}{|c|}{ Mid-Cycle 157D Outage - 14 Day, 10 Hour Down Time $(7 / 23 / 2015,1800-8 / 7 / 2015,0400)$} \\
\hline & EOC $(8 / 12 / 2015,0400)$ & 65.1 & 5.01 & 311.5 & $4.93785 \mathrm{E}+13$ & $2.24053 E+13$ & $1.05265 \mathrm{E}+14$ & $1.10745 \mathrm{E}+20$ & 1111.6 & 375.8 \\
\hline \multirow[t]{8}{*}{$158 \mathrm{~A}$} & \multicolumn{10}{|c|}{ Cycle 158A Outage - 91 Day, 11 Hour Down Time } \\
\hline & $\operatorname{BOC}(11 / 11 / 2015,1500)$ & 65.1 & 5.01 & 312.0 & $4.93945 \mathrm{E}+13$ & $2.23630 \mathrm{E}+13$ & $1.05299 \mathrm{E}+14$ & $1.10745 \mathrm{E}+20$ & 1113.4 & 376.2 \\
\hline & & 75.1 & 5.78 & 307.3 & $4.86889 \mathrm{E}+13$ & $2.19315 E+13$ & $1.04210 \mathrm{E}+14$ & $1.27787 \mathrm{E}+20$ & 1097.3 & 372.5 \\
\hline & & 85.1 & 6.53 & 298.8 & $4.82014 \mathrm{E}+13$ & $2.15061 \mathrm{E}+13$ & $1.02890 \mathrm{E}+14$ & $1.44347 \mathrm{E}+20$ & 1068.7 & 365.8 \\
\hline & & 95.1 & 7.28 & 300.8 & $4.94144 \mathrm{E}+13$ & $2.16817 E+13$ & $1.04411 \mathrm{E}+14$ & $1.61028 \mathrm{E}+20$ & 1075.4 & 367.3 \\
\hline & & 105.1 & 8.04 & 304.2 & $5.07164 \mathrm{E}+13$ & $2.18805 E+13$ & $1.06335 \mathrm{E}+14$ & $1.77830 \mathrm{E}+20$ & 1086.7 & 370.0 \\
\hline & & 117.1 & 8.96 & 308.0 & $5.22780 \mathrm{E}+13$ & $2.22958 \mathrm{E}+13$ & $1.08396 \mathrm{E}+14$ & $1.98184 \mathrm{E}+20$ & 1100.0 & 373.1 \\
\hline & EOC $(1 / 2 / 2016,0400)$ & 117.3 & 8.97 & 302.6 & $5.25237 \mathrm{E}+13$ & $2.19741 \mathrm{E}+13$ & $1.08097 E+14$ & $1.98425 \mathrm{E}+20$ & 1081.4 & 368.8 \\
\hline \multirow[t]{5}{*}{$158 \mathrm{~B}$} & \multicolumn{10}{|c|}{ Cycle 158B Outage - 39 Day, 2 Hour Down Time } \\
\hline & BOC $(2 / 10 / 2016,0600)$ & 118.3 & 9.03 & 245.5 & $4.24652 \mathrm{E}+13$ & $1.79406 \mathrm{E}+13$ & $8.78051 \mathrm{E}+13$ & $1.99629 \mathrm{E}+20$ & 889.8 & 322.0 \\
\hline & & 119.3 & 9.08 & 272.4 & $4.72284 \mathrm{E}+13$ & 1.99219E+13 & $9.81954 \mathrm{E}+13$ & $2.00894 \mathrm{E}+20$ & 979.7 & 344.4 \\
\hline & & 122.3 & 9.28 & 277.4 & $4.84343 \mathrm{E}+13$ & $2.01595 \mathrm{E}+13$ & $1.00276 \mathrm{E}+14$ & $2.05350 \mathrm{E}+20$ & 996.5 & 348.5 \\
\hline & & 127.3 & 9.63 & 271.6 & $4.75080 \mathrm{E}+13$ & $1.96739 \mathrm{E}+13$ & $9.82039 \mathrm{E}+13$ & $2.12998 \mathrm{E}+20$ & 977.1 & 343.7 \\
\hline
\end{tabular}


TEM-10300-1

$03 / 01 / 2012$

Rev. 03

Title: $\quad$ ATF-1 Power Histories

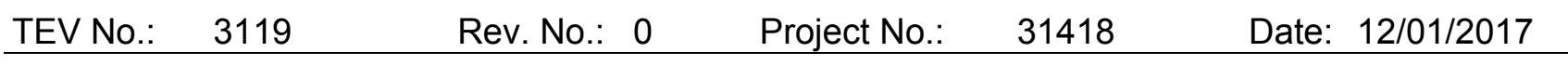

Table 7. ATF-05 Power History

\begin{tabular}{|c|c|c|c|c|c|c|c|c|c|c|}
\hline Cycle & Critical Dates & EFPD & $\begin{array}{l}\text { Burnup } \\
\text { (GWd/MTU) }\end{array}$ & $\begin{array}{l}\text { LHGR } \\
(\mathrm{W} / \mathrm{cm})\end{array}$ & $\begin{array}{l}\text { Thermal Flux } \\
\left(\mathbf{n} / \mathrm{cm}^{2 *} \mathbf{s}\right)\end{array}$ & $\begin{array}{c}\text { Fast Flux } \\
\left(\mathrm{n} / \mathrm{cm}^{2 *} \mathrm{~s}>1\right. \\
\mathrm{MeV})\end{array}$ & $\begin{array}{l}\text { Total Flux } \\
\left(\mathrm{n} / \mathrm{cm}^{2 *} \mathbf{s}\right)\end{array}$ & 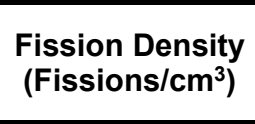 & $\begin{array}{c}\text { Fuel Centerline } \\
\text { Temperature } \\
\left({ }^{\circ} \mathrm{C}\right)\end{array}$ & $\mathrm{PICT}\left({ }^{\circ} \mathrm{C}\right)$ \\
\hline & & 132.3 & 9.99 & 283.9 & $5.01120 \mathrm{E}+13$ & $2.04099 \mathrm{E}+13$ & $1.02805 \mathrm{E}+14$ & $2.20887 \mathrm{E}+20$ & 1018.2 & 353.7 \\
\hline & & 137.3 & 10.33 & 274.0 & $4.89216 \mathrm{E}+13$ & $1.99840 \mathrm{E}+13$ & $1.00215 \mathrm{E}+14$ & $2.28475 \mathrm{E}+20$ & 985.0 & 345.7 \\
\hline & & 147.3 & 11.04 & 286.3 & $5.13248 \mathrm{E}+13$ & $2.08036 \mathrm{E}+13$ & $1.04937 \mathrm{E}+14$ & $2.44192 \mathrm{E}+20$ & 1026.3 & 355.7 \\
\hline & & 157.3 & 11.73 & 279.0 & $5.10179 \mathrm{E}+13$ & $2.03989 \mathrm{E}+13$ & $1.03353 \mathrm{E}+14$ & $2.59548 \mathrm{E}+20$ & 1001.8 & 349.7 \\
\hline & EOC $(4 / 1 / 2016,0900)$ & 168.7 & 12.55 & 277.4 & $5.25512 \mathrm{E}+13$ & $2.04075 \mathrm{E}+13$ & $1.05218 \mathrm{E}+14$ & $2.77494 \mathrm{E}+20$ & 996.5 & 348.4 \\
\hline \multirow[t]{6}{*}{$159 \mathrm{~A}$} & \multicolumn{10}{|c|}{ Cycle 159A Outage - 77 Day, 2 Hour Down Time } \\
\hline & $\operatorname{BOC}(6 / 17 / 2016,1100)$ & 169.7 & 12.57 & 116.0 & $2.20065 \mathrm{E}+13$ & $8.42861 \mathrm{E}+12$ & $4.39577 \mathrm{E}+13$ & $2.78036 \mathrm{E}+20$ & 469.4 & 206.0 \\
\hline & & 174.6 & 12.71 & 103.6 & $1.96983 \mathrm{E}+13$ & $7.63819 \mathrm{E}+12$ & $3.93839 \mathrm{E}+13$ & $2.81047 \mathrm{E}+20$ & 430.2 & 194.3 \\
\hline & \multicolumn{10}{|c|}{ Mid-Cycle 159A Outage - 3 Day, 21 Hour Down Time $(6 / 23 / 2016,1000$ - 6/27/2016, 0700) } \\
\hline & & 176.0 & 12.73 & 150.7 & $2.87434 \mathrm{E}+13$ & $1.13507 \mathrm{E}+13$ & $5.93999 \mathrm{E}+13$ & $2.81649 \mathrm{E}+20$ & 580.1 & 238.4 \\
\hline & EOC $(6 / 28 / 2016,1800)$ & 176.2 & 12.74 & 166.2 & $3.19437 \mathrm{E}+13$ & $1.27300 \mathrm{E}+13$ & $6.75297 \mathrm{E}+13$ & $2.81769 \mathrm{E}+20$ & 630.1 & 252.6 \\
\hline $160 \mathrm{~A}$ & \multicolumn{10}{|c|}{ Cycle 160A Outage - 80 Day, 1 Hour Down Time } \\
\hline & BOC $(9 / 16 / 2016,1900)$ & 177.2 & 12.80 & 273.7 & $5.21284 \mathrm{E}+13$ & $2.01131 \mathrm{E}+13$ & 1.04197E+14 & $2.83094 \mathrm{E}+20$ & 983.8 & 345.4 \\
\hline & & 179.2 & 12.94 & 270.9 & $5.17974 \mathrm{E}+13$ & $1.99510 \mathrm{E}+13$ & $1.03763 \mathrm{E}+14$ & $2.86165 \mathrm{E}+20$ & 974.5 & 343.1 \\
\hline & & 186.2 & 13.41 & 270.7 & $5.19414 \mathrm{E}+13$ & $1.98340 \mathrm{E}+13$ & $1.03868 \mathrm{E}+14$ & $2.96704 \mathrm{E}+20$ & 974.0 & 343.0 \\
\hline & & 216.2 & 15.43 & 269.0 & $5.41755 \mathrm{E}+13$ & $1.99042 \mathrm{E}+13$ & $1.06132 \mathrm{E}+14$ & $3.41207 \mathrm{E}+20$ & 968.1 & 341.5 \\
\hline & & 222.2 & 15.82 & 255.6 & $5.26365 \mathrm{E}+13$ & $1.90901 \mathrm{E}+13$ & $1.02858 \mathrm{E}+14$ & $3.49938 \mathrm{E}+20$ & 923.4 & 330.4 \\
\hline & & 223.8 & 15.93 & 241.1 & $5.02397 \mathrm{E}+13$ & $1.78851 \mathrm{E}+13$ & $9.75121 \mathrm{E}+13$ & 3.52347E+20 & 875.1 & 318.2 \\
\hline & & 226.2 & 16.07 & 237.8 & $4.95336 \mathrm{E}+13$ & $1.77130 \mathrm{E}+13$ & $9.64377 \mathrm{E}+13$ & $3.55479 \mathrm{E}+20$ & 864.2 & 315.4 \\
\hline & $\operatorname{EOC}(11 / 8 / 2016,1100)$ & 228.9 & 16.22 & 226.7 & $4.77562 E+13$ & $1.69666 \mathrm{E}+13$ & $9.32071 \mathrm{E}+13$ & $3.58791 \mathrm{E}+20$ & 827.5 & 306.0 \\
\hline \multirow[t]{6}{*}{$160 \mathrm{~B}$} & \multicolumn{10}{|c|}{ Cycle 160B Outage - 42 Day, 1 Hour Down Time Outage } \\
\hline & $\mathrm{BOC}(12 / 20 / 2016,1200)$ & 229.9 & 16.27 & 235.3 & $4.94735 \mathrm{E}+13$ & $1.81270 \mathrm{E}+13$ & $9.86549 \mathrm{E}+13$ & $3.59875 \mathrm{E}+20$ & 855.9 & 313.3 \\
\hline & & 231.9 & 16.39 & 234.6 & $4.94593 \mathrm{E}+13$ & $1.81173 \mathrm{E}+13$ & $9.87022 \mathrm{E}+13$ & $3.62585 \mathrm{E}+20$ & 853.5 & 312.7 \\
\hline & & 238.9 & 16.81 & 232.7 & $4.93299 \mathrm{E}+13$ & $1.78936 \mathrm{E}+13$ & $9.78136 \mathrm{E}+13$ & $3.71738 \mathrm{E}+20$ & 847.3 & 311.1 \\
\hline & & 248.9 & 17.37 & 222.9 & $4.78662 \mathrm{E}+13$ & $1.71419 \mathrm{E}+13$ & $9.48477 \mathrm{E}+13$ & $3.84083 \mathrm{E}+20$ & 815.0 & 302.7 \\
\hline & & 258.1 & 17.88 & 216.3 & $4.79521 \mathrm{E}+13$ & $1.67735 \mathrm{E}+13$ & $9.41059 \mathrm{E}+13$ & $3.95525 \mathrm{E}+20$ & 793.2 & 297.0 \\
\hline
\end{tabular}


TEM-10300-1

03/01/2012

Rev. 03

Title: $\quad$ ATF-1 Power Histories

$\begin{array}{lllll}\text { TEV No.: } & 3119 & \text { Rev. No.: } & 0 & \text { Project No.: }\end{array}$

Table 7. ATF-05 Power History

\begin{tabular}{|c|c|c|c|c|c|c|c|c|c|c|}
\hline Cycle & Critical Dates & EFPD & $\begin{array}{c}\text { Burnup } \\
\text { (GWd/MTU) }\end{array}$ & $\begin{array}{c}\text { LHGR } \\
(\mathrm{W} / \mathrm{cm})\end{array}$ & $\begin{array}{l}\text { Thermal Flux } \\
\left(\mathrm{n} / \mathrm{cm}^{2 *} \mathrm{~s}\right)\end{array}$ & $\begin{array}{c}\text { Fast Flux } \\
\left(\mathrm{n} / \mathrm{cm}^{2 *} \mathrm{~s}>1\right. \\
\mathrm{MeV})\end{array}$ & $\begin{array}{l}\text { Total Flux } \\
\left(\mathrm{n} / \mathrm{cm}^{2 *} \mathrm{~s}\right)\end{array}$ & $\begin{array}{c}\text { Fission Density } \\
\left(\text { Fissions } / \mathrm{cm}^{3}\right)\end{array}$ & $\begin{array}{c}\text { Fuel Centerline } \\
\text { Temperature } \\
\left({ }^{\circ} \mathrm{C}\right)\end{array}$ & PICT $\left({ }^{\circ} \mathrm{C}\right)$ \\
\hline & \multicolumn{10}{|c|}{ Mid-Cycle 160B Outage - 5 Day, 14 Hour Down Time } \\
\hline & & 268.9 & 18.50 & 220.8 & $4.92202 \mathrm{E}+13$ & $1.70725 \mathrm{E}+13$ & $9.60666 \mathrm{E}+13$ & $4.09135 \mathrm{E}+20$ & 808.1 & 300.9 \\
\hline & & 278.9 & 19.03 & 210.0 & $4.75705 \mathrm{E}+13$ & $1.66399 \mathrm{E}+13$ & $9.50957 \mathrm{E}+13$ & $4.20817 \mathrm{E}+20$ & 772.6 & 291.5 \\
\hline & EOC $(2 / 23 / 2017,1400)$ & 288.4 & 19.55 & 220.1 & $5.13893 \mathrm{E}+13$ & $1.73558 \mathrm{E}+13$ & $1.00160 \mathrm{E}+14$ & $4.32500 \mathrm{E}+20$ & 805.8 & 300.3 \\
\hline \multirow[t]{9}{*}{$161 \mathrm{~A}$} & \multicolumn{10}{|c|}{ Cycle 161A Outage - 95 Day, 13 Hour Down Time } \\
\hline & BOC $(5 / 30 / 2017,0300)$ & 290.1 & 19.64 & 174.4 & $4.06234 \mathrm{E}+13$ & $1.37155 E+13$ & $7.82592 \mathrm{E}+13$ & $4.34427 \mathrm{E}+20$ & 656.4 & 260.0 \\
\hline & & 290.4 & 19.65 & 157.3 & $3.67274 \mathrm{E}+13$ & $1.21855 \mathrm{E}+13$ & $7.04961 \mathrm{E}+13$ & $4.34608 \mathrm{E}+20$ & 601.2 & 244.4 \\
\hline & & 292.4 & 19.74 & 161.3 & $3.77088 \mathrm{E}+13$ & $1.26963 E+13$ & $7.28729 E+13$ & $4.36655 E+20$ & 614.2 & 248.1 \\
\hline & & 294.4 & 19.83 & 163.8 & $3.84459 \mathrm{E}+13$ & $1.27172 \mathrm{E}+13$ & $7.37635 \mathrm{E}+13$ & $4.38703 E+20$ & 622.1 & 250.4 \\
\hline & & 296.4 & 19.93 & 162.2 & $3.81510 \mathrm{E}+13$ & $1.28180 \mathrm{E}+13$ & $7.34690 \mathrm{E}+13$ & $4.40750 \mathrm{E}+20$ & 617.0 & 248.9 \\
\hline & & 298.4 & 20.02 & 166.4 & $3.92964 \mathrm{E}+13$ & $1.30183 E+13$ & $7.54363 E+13$ & $4.42798 \mathrm{E}+20$ & 630.5 & 252.7 \\
\hline & & 300.4 & 20.11 & 168.9 & $3.99865 \mathrm{E}+13$ & $1.32019 E+13$ & $7.66708 \mathrm{E}+13$ & $4.44905 E+20$ & 638.6 & 255.0 \\
\hline & $\operatorname{EOC}(6 / 11 / 2017,1600)$ & 300.9 & 20.13 & 171.8 & $4.08737 \mathrm{E}+13$ & $1.34418 E+13$ & $7.79738 \mathrm{E}+13$ & $4.45267 \mathrm{E}+20$ & 647.9 & 257.6 \\
\hline \multirow[t]{10}{*}{$162 \mathrm{~A}$} & \multicolumn{10}{|c|}{ Cycle 162A Outage } \\
\hline & $\mathrm{BOC}$ & 301.9 & 20.18 & 246.1 & $5.67659 \mathrm{E}+13$ & $1.86899 \mathrm{E}+13$ & $1.08938 \mathrm{E}+14$ & $4.46351 \mathrm{E}+20$ & 891.7 & 322.4 \\
\hline & & 303.9 & 20.30 & 233.6 & $5.45834 \mathrm{E}+13$ & $1.79920 \mathrm{E}+13$ & $1.04870 \mathrm{E}+14$ & $4.48940 \mathrm{E}+20$ & 850.2 & 311.8 \\
\hline & & 310.9 & 20.70 & 233.2 & $5.47257 \mathrm{E}+13$ & $1.79891 \mathrm{E}+13$ & $1.04997 E+14$ & $4.57792 \mathrm{E}+20$ & 848.8 & 311.5 \\
\hline & & 320.9 & 21.28 & 233.1 & $5.47018 \mathrm{E}+13$ & $1.77700 \mathrm{E}+13$ & $1.04424 \mathrm{E}+14$ & $4.70619 E+20$ & 848.5 & 311.4 \\
\hline & & 330.9 & 21.85 & 231.0 & $5.55355 \mathrm{E}+13$ & $1.77681 \mathrm{E}+13$ & $1.05229 \mathrm{E}+14$ & $4.83386 \mathrm{E}+20$ & 841.6 & 309.6 \\
\hline & & 340.9 & 22.42 & 229.6 & $5.59865 \mathrm{E}+13$ & $1.75405 \mathrm{E}+13$ & $1.05491 \mathrm{E}+14$ & $4.95972 \mathrm{E}+20$ & 837.1 & 308.4 \\
\hline & & 350.9 & 23.00 & 233.4 & $5.88065 \mathrm{E}+13$ & $1.82058 E+13$ & $1.10222 \mathrm{E}+14$ & $5.08799 \mathrm{E}+20$ & 849.6 & 311.7 \\
\hline & & 359.9 & 23.49 & 224.5 & $5.74786 \mathrm{E}+13$ & $1.75204 \mathrm{E}+13$ & $1.07906 \mathrm{E}+14$ & $5.19578 \mathrm{E}+20$ & 820.2 & 304.1 \\
\hline & EOC & 362.9 & 23.64 & 220.6 & $5.78154 \mathrm{E}+13$ & $1.72427 E+13$ & $1.07744 \mathrm{E}+14$ & $5.22950 \mathrm{E}+20$ & 807.5 & 300.7 \\
\hline
\end{tabular}


TEM-10300-1

$03 / 01 / 2012$

Rev. 03

Title: $\quad$ ATF-1 Power Histories

\begin{tabular}{lllll} 
TEV No.: & 3119 & Rev. No.: & 0 & Project No.: \\
\hline
\end{tabular}

Table 8. ATF-06 Power History

\begin{tabular}{|c|c|c|c|c|c|c|c|c|c|c|}
\hline Cycle & Critical Dates & EFPD & $\begin{array}{l}\text { Burnup } \\
\text { (GWd/MTU) }\end{array}$ & $\begin{array}{c}\text { LHGR } \\
(\mathrm{W} / \mathrm{cm})\end{array}$ & $\begin{array}{l}\text { Thermal Flux } \\
\left(\mathrm{n} / \mathrm{cm}^{2 *} \mathrm{~s}\right)\end{array}$ & $\begin{array}{c}\text { Fast Flux } \\
\left(\mathrm{n} / \mathrm{cm}^{2 *} \mathrm{~s}>1\right. \\
\mathrm{MeV})\end{array}$ & $\begin{array}{l}\text { Total Flux } \\
\left(\mathrm{n} / \mathrm{cm}^{2 *} \mathrm{~s}\right)\end{array}$ & $\begin{array}{l}\text { Fission Density } \\
\left(\text { Fissions } / \mathrm{cm}^{3}\right)\end{array}$ & $\begin{array}{c}\text { Fuel Centerline } \\
\text { Temperature } \\
\left({ }^{\circ} \mathrm{C}\right)\end{array}$ & $\mathrm{PICT}\left({ }^{\circ} \mathrm{C}\right)$ \\
\hline \multirow[t]{2}{*}{$157 \mathrm{C}$} & BOC $(2 / 10 / 2015,0600)$ & 0.0 & 0.00 & 279.4 & $3.61853 \mathrm{E}+13$ & $1.96765 E+13$ & $8.50784 \mathrm{E}+13$ & $0.00000 \mathrm{E}+00$ & 1341.2 & 385.8 \\
\hline & EOC $(2 / 15 / 2015,0800)$ & 5.4 & 0.36 & 290.4 & $3.77759 \mathrm{E}+13$ & $2.03371 \mathrm{E}+13$ & $8.77882 E+13$ & $9.03300 \mathrm{E}+18$ & 1396.6 & 394.5 \\
\hline \multirow[t]{13}{*}{ 157D } & \multicolumn{10}{|c|}{ Cycle 157D Outage - 104 Day, 3 Hour Down Time } \\
\hline & $\operatorname{BOC}(5 / 30 / 2015,1100)$ & 5.4 & 0.36 & 236.0 & $3.07754 \mathrm{E}+13$ & $1.65398 \mathrm{E}+13$ & $7.10765 E+13$ & $9.03300 \mathrm{E}+18$ & 1123.1 & 349.5 \\
\hline & & 8.4 & 0.54 & 267.4 & $3.47342 \mathrm{E}+13$ & $1.86357 E+13$ & $8.03864 E+13$ & $1.34893 \mathrm{E}+19$ & 1280.9 & 376.1 \\
\hline & & 15.4 & 0.95 & 266.7 & $3.48056 \mathrm{E}+13$ & $1.86553 \mathrm{E}+13$ & $8.04174 \mathrm{E}+13$ & $2.37267 \mathrm{E}+19$ & 1277.4 & 375.5 \\
\hline & & 22.4 & 1.36 & 265.2 & $3.48553 E+13$ & $1.86008 \mathrm{E}+13$ & $8.05316 E+13$ & $3.39039 \mathrm{E}+19$ & 1269.9 & 374.3 \\
\hline & & 29.4 & 1.77 & 269.7 & $3.58098 \mathrm{E}+13$ & $1.88885 E+13$ & $8.22649 E+13$ & $4.42015 \mathrm{E}+19$ & 1292.4 & 377.9 \\
\hline & & 36.4 & 2.21 & 273.4 & $3.66144 \mathrm{E}+13$ & $1.92394 \mathrm{E}+13$ & $8.39788 E+13$ & $5.51615 \mathrm{E}+19$ & 1311.3 & 381.0 \\
\hline & & 43.4 & 2.67 & 285.3 & $3.85019 \mathrm{E}+13$ & $2.00359 \mathrm{E}+13$ & $8.79229 \mathrm{E}+13$ & $6.66033 \mathrm{E}+19$ & 1371.1 & 390.5 \\
\hline & & 50.4 & 3.12 & 286.2 & $3.89630 \mathrm{E}+13$ & $2.01253 \mathrm{E}+13$ & $8.85830 \mathrm{E}+13$ & $7.79849 \mathrm{E}+19$ & 1375.5 & 391.2 \\
\hline & & 57.4 & 3.59 & 293.9 & $4.04522 \mathrm{E}+13$ & $2.07424 \mathrm{E}+13$ & $9.17658 E+13$ & 8.96074E+19 & 1414.5 & 397.3 \\
\hline & & 60.4 & 3.78 & 295.1 & $4.09848 \mathrm{E}+13$ & $2.08532 E+13$ & $9.27577 E+13$ & $9.44852 \mathrm{E}+19$ & 1420.1 & 398.1 \\
\hline & \multicolumn{10}{|c|}{ Mid-Cycle 157D Outage - 14 Day, 10 Hour Down Time $(7 / 23 / 2015,1800-8 / 7 / 2015,0400)$} \\
\hline & EOC $(8 / 12 / 2015,0400)$ & 65.1 & 4.10 & 298.3 & $4.18051 \mathrm{E}+13$ & $2.10385 E+13$ & $9.37867 \mathrm{E}+13$ & $1.02494 \mathrm{E}+20$ & 1436.4 & 400.7 \\
\hline \multirow[t]{8}{*}{$158 \mathrm{~A}$} & \multicolumn{10}{|c|}{ Cycle 158A Outage - 91 Day, 11 Hour Down Time } \\
\hline & $\mathrm{BOC}(11 / 11 / 2015,1500)$ & 65.1 & 4.10 & 224.5 & $3.17479 \mathrm{E}+13$ & $1.64268 \mathrm{E}+13$ & $7.66419 \mathrm{E}+13$ & $1.02494 \mathrm{E}+20$ & 1065.1 & 339.4 \\
\hline & & 75.1 & 4.61 & 230.2 & $3.25153 \mathrm{E}+13$ & $1.68291 \mathrm{E}+13$ & $7.82048 \mathrm{E}+13$ & $1.15141 \mathrm{E}+20$ & 1093.8 & 344.5 \\
\hline & & 85.1 & 5.16 & 240.4 & $3.44024 \mathrm{E}+13$ & $1.77725 \mathrm{E}+13$ & 8.20119E+13 & $1.28811 \mathrm{E}+20$ & 1144.8 & 353.3 \\
\hline & & 95.1 & 5.76 & 266.1 & $3.84133 \mathrm{E}+13$ & $1.93305 E+13$ & $8.87910 \mathrm{E}+13$ & $1.43745 \mathrm{E}+20$ & 1274.4 & 375.0 \\
\hline & & 105.1 & 6.37 & 272.1 & $3.97845 \mathrm{E}+13$ & $1.95536 \mathrm{E}+13$ & $9.08111 \mathrm{E}+13$ & $1.58981 \mathrm{E}+20$ & 1304.8 & 379.9 \\
\hline & & 117.1 & 7.11 & 278.3 & $4.12014 \mathrm{E}+13$ & $2.02484 \mathrm{E}+13$ & $9.35253 E+13$ & $1.77589 \mathrm{E}+20$ & 1335.9 & 384.9 \\
\hline & $\operatorname{EOC}(1 / 2 / 2016,0400)$ & 117.3 & 7.12 & 279.1 & $4.20113 \mathrm{E}+13$ & $2.02784 \mathrm{E}+13$ & $9.46274 \mathrm{E}+13$ & $1.77890 \mathrm{E}+20$ & 1339.8 & 385.6 \\
\hline \multirow[t]{5}{*}{$158 \mathrm{~B}$} & \multicolumn{10}{|c|}{ Cycle 158B Outage - 39 Day, 2 Hour Down Time } \\
\hline & BOC $(2 / 10 / 2016,0600)$ & 118.3 & 7.17 & 255.1 & $3.82390 \mathrm{E}+13$ & $1.83262 \mathrm{E}+13$ & $8.61542 \mathrm{E}+13$ & $1.79094 \mathrm{E}+20$ & 1219.0 & 365.8 \\
\hline & & 119.3 & 7.22 & 234.0 & $3.52338 \mathrm{E}+13$ & $1.71314 \mathrm{E}+13$ & $8.06333 E+13$ & $1.80238 \mathrm{E}+20$ & 1112.8 & 347.8 \\
\hline & & 122.3 & 7.37 & 225.6 & $3.41114 \mathrm{E}+13$ & $1.65012 \mathrm{E}+13$ & $7.77748 \mathrm{E}+13$ & $1.84032 \mathrm{E}+20$ & 1070.3 & 340.3 \\
\hline & & 127.3 & 7.63 & 231.1 & $3.51648 \mathrm{E}+13$ & $1.69159 \mathrm{E}+13$ & $7.96828 \mathrm{E}+13$ & $1.90657 \mathrm{E}+20$ & 1098.3 & 345.2 \\
\hline
\end{tabular}


TEM-10300-1

$03 / 01 / 2012$

Rev. 03

Title: $\quad$ ATF-1 Power Histories

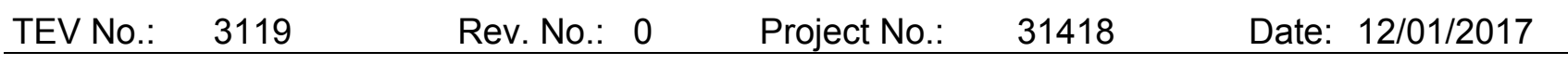

Table 8. ATF-06 Power History

\begin{tabular}{|c|c|c|c|c|c|c|c|c|c|c|}
\hline Cycle & Critical Dates & EFPD & $\begin{array}{l}\text { Burnup } \\
\text { (GWd/MTU) }\end{array}$ & $\begin{array}{l}\text { LHGR } \\
(\mathrm{W} / \mathrm{cm})\end{array}$ & $\begin{array}{l}\text { Thermal Flux } \\
\left(\mathbf{n} / \mathrm{cm}^{2 *} \mathbf{s}\right)\end{array}$ & $\begin{array}{c}\text { Fast Flux } \\
\left(\mathrm{n} / \mathrm{cm}^{2 *} \mathrm{~s}>1\right. \\
\mathrm{MeV})\end{array}$ & $\begin{array}{l}\text { Total Flux } \\
\left(\mathrm{n} / \mathrm{cm}^{2 *} \mathbf{s}\right)\end{array}$ & 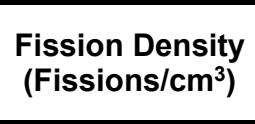 & $\begin{array}{c}\text { Fuel Centerline } \\
\text { Temperature } \\
\left({ }^{\circ} \mathrm{C}\right)\end{array}$ & $\mathrm{PICT}\left({ }^{\circ} \mathrm{C}\right)$ \\
\hline & & 132.3 & 7.91 & 238.9 & $3.65881 \mathrm{E}+13$ & $1.76258 \mathrm{E}+13$ & $8.28620 \mathrm{E}+13$ & $1.97461 \mathrm{E}+20$ & 1137.6 & 352.0 \\
\hline & & 137.3 & 8.18 & 243.7 & $3.75331 \mathrm{E}+13$ & $1.77539 \mathrm{E}+13$ & $8.35563 \mathrm{E}+13$ & $2.04387 \mathrm{E}+20$ & 1161.8 & 356.2 \\
\hline & & 147.3 & 8.77 & 264.0 & $4.06074 \mathrm{E}+13$ & $1.90307 \mathrm{E}+13$ & $8.98553 \mathrm{E}+13$ & $2.19080 \mathrm{E}+20$ & 1263.9 & 373.3 \\
\hline & & 157.3 & 9.37 & 267.5 & $4.16651 \mathrm{E}+13$ & $1.91923 \mathrm{E}+13$ & $9.14995 \mathrm{E}+13$ & $2.34015 \mathrm{E}+20$ & 1281.6 & 376.2 \\
\hline & EOC $(4 / 1 / 2016,0900)$ & 168.7 & 10.07 & 276.7 & $4.44616 \mathrm{E}+13$ & 1.99777E+13 & $9.52156 \mathrm{E}+13$ & $2.51539 \mathrm{E}+20$ & 1327.7 & 383.6 \\
\hline \multirow[t]{6}{*}{$159 \mathrm{~A}$} & \multicolumn{10}{|c|}{ Cycle 159A Outage - 77 Day, 2 Hour Down Time } \\
\hline & $\operatorname{BOC}(6 / 17 / 2016,1100)$ & 169.7 & 10.10 & 127.9 & $2.04682 E+13$ & $9.14972 \mathrm{E}+12$ & $4.33149 \mathrm{E}+13$ & $2.52141 \mathrm{E}+20$ & 577.5 & 246.0 \\
\hline & & 174.6 & 10.22 & 106.9 & $1.71120 \mathrm{E}+13$ & $7.61458 \mathrm{E}+12$ & $3.63705 \mathrm{E}+13$ & $2.55273 \mathrm{E}+20$ & 471.3 & 223.7 \\
\hline & \multicolumn{10}{|c|}{ Mid-Cycle 159A Outage - 3 Day, 21 Hour Down Time $(6 / 23 / 2016,1000$ - 6/27/2016, 0700) } \\
\hline & & 176.0 & 10.25 & 204.8 & $3.31266 \mathrm{E}+13$ & $1.48862 \mathrm{E}+13$ & $7.13415 \mathrm{E}+13$ & $2.55995 \mathrm{E}+20$ & 965.5 & 321.5 \\
\hline & EOC $(6 / 28 / 2016,1800)$ & 176.2 & 10.26 & 284.6 & $4.60275 \mathrm{E}+13$ & $2.05611 \mathrm{E}+13$ & $9.88419 \mathrm{E}+13$ & $2.56296 \mathrm{E}+20$ & 1367.4 & 389.9 \\
\hline $160 \mathrm{~A}$ & \multicolumn{10}{|c|}{ Cycle 160A Outage - 80 Day, 1 Hour Down Time } \\
\hline & BOC $(9 / 16 / 2016,1900)$ & 177.2 & 10.31 & 271.4 & 4.39766E+13 & $1.97354 \mathrm{E}+13$ & $9.56839 \mathrm{E}+13$ & $2.57561 \mathrm{E}+20$ & 1301.2 & 379.3 \\
\hline & & 179.2 & 10.43 & 262.2 & $4.25630 \mathrm{E}+13$ & $1.93026 \mathrm{E}+13$ & $9.32036 \mathrm{E}+13$ & $2.60572 \mathrm{E}+20$ & 1254.7 & 371.8 \\
\hline & & 186.2 & 10.86 & 271.3 & $4.41021 \mathrm{E}+13$ & $1.95182 \mathrm{E}+13$ & $9.57809 \mathrm{E}+13$ & $2.71231 \mathrm{E}+20$ & 1300.5 & 379.2 \\
\hline & & 216.2 & 12.84 & 297.3 & $5.04841 \mathrm{E}+13$ & $2.15829 \mathrm{E}+13$ & $1.06277 \mathrm{E}+14$ & $3.20611 \mathrm{E}+20$ & 1431.4 & 399.9 \\
\hline & & 222.2 & 13.25 & 309.1 & $5.33507 \mathrm{E}+13$ & $2.23940 \mathrm{E}+13$ & $1.11259 \mathrm{E}+14$ & $3.30969 \mathrm{E}+20$ & 1490.6 & 408.9 \\
\hline & & 223.8 & 13.37 & 311.6 & $5.43840 \mathrm{E}+13$ & $2.24341 \mathrm{E}+13$ & 1.12456E+14 & $3.33860 \mathrm{E}+20$ & 1503.6 & 410.8 \\
\hline & & 226.2 & 13.53 & 317.2 & $5.54209 \mathrm{E}+13$ & $2.29456 \mathrm{E}+13$ & 1.14360E+14 & $3.37955 \mathrm{E}+20$ & 1531.4 & 414.9 \\
\hline & $\operatorname{EOC}(11 / 8 / 2016,1100)$ & 228.9 & 13.71 & 310.0 & $5.48821 \mathrm{E}+13$ & $2.25474 \mathrm{E}+13$ & 1.12379E+14 & $3.42411 \mathrm{E}+20$ & 1495.1 & 409.6 \\
\hline \multirow[t]{6}{*}{$160 \mathrm{~B}$} & \multicolumn{10}{|c|}{ Cycle 160B Outage - 42 Day, 1 Hour Down Time Outage } \\
\hline & $\mathrm{BOC}(12 / 20 / 2016,1200)$ & 229.9 & 13.76 & 285.5 & $5.05178 \mathrm{E}+13$ & $1.88353 \mathrm{E}+13$ & $9.90471 \mathrm{E}+13$ & $3.43736 \mathrm{E}+20$ & 1372.1 & 390.7 \\
\hline & & 231.9 & 13.89 & 287.2 & 5.10117E+13 & $1.90701 \mathrm{E}+13$ & $1.00351 \mathrm{E}+14$ & $3.46988 \mathrm{E}+20$ & 1380.7 & 392.0 \\
\hline & & 238.9 & 14.34 & 290.3 & $5.17137 \mathrm{E}+13$ & $1.90830 \mathrm{E}+13$ & $1.00215 \mathrm{E}+14$ & $3.58068 \mathrm{E}+20$ & 1396.3 & 394.4 \\
\hline & & 248.9 & 15.00 & 297.4 & $5.35724 \mathrm{E}+13$ & $1.96637 \mathrm{E}+13$ & $1.03522 \mathrm{E}+14$ & $3.74629 \mathrm{E}+20$ & 1431.8 & 399.9 \\
\hline & & 258.1 & 15.63 & 296.0 & $5.53288 \mathrm{E}+13$ & $1.95820 \mathrm{E}+13$ & 1.05067E+14 & $3.90286 \mathrm{E}+20$ & 1425.0 & 398.9 \\
\hline
\end{tabular}


TEM-10300-1

$03 / 01 / 2012$

Rev. 03

TECHNICAL EVALUATION

Page 25 of 110

Title: $\quad$ ATF-1 Power Histories

$\begin{array}{lllll}\text { TEV No.: } & 3119 & \text { Rev. No.: } & 0 & \text { Project No.: }\end{array}$

Table 8. ATF-06 Power History

\begin{tabular}{|c|c|c|c|c|c|c|c|c|c|c|}
\hline Cycle & Critical Dates & EFPD & $\begin{array}{c}\text { Burnup } \\
\text { (GWd/MTU) }\end{array}$ & $\begin{array}{l}\text { LHGR } \\
\text { (W/cm) }\end{array}$ & $\begin{array}{l}\text { Thermal Flux } \\
\left(\mathrm{n} / \mathrm{cm}^{2 *} \mathrm{~s}\right)\end{array}$ & $\begin{array}{c}\text { Fast Flux } \\
\left(\mathrm{n} / \mathrm{cm}^{2 *} \mathrm{~s}>1\right. \\
\mathrm{MeV})\end{array}$ & $\begin{array}{l}\text { Total Flux } \\
\left(\mathrm{n} / \mathrm{cm}^{2 *} \mathrm{~s}\right)\end{array}$ & $\begin{array}{l}\text { Fission Density } \\
\left.\text { (Fissions } / \mathrm{cm}^{3}\right)\end{array}$ & $\begin{array}{c}\text { Fuel Centerline } \\
\text { Temperature } \\
\left({ }^{\circ} \mathrm{C}\right)\end{array}$ & $\mathrm{PICT}\left({ }^{\circ} \mathrm{C}\right)$ \\
\hline & \multicolumn{10}{|c|}{ Mid-Cycle 160B Outage - 5 Day, 14 Hour Down Time } \\
\hline & & 268.9 & 16.38 & 310.3 & $5.83745 \mathrm{E}+13$ & $2.04756 \mathrm{E}+13$ & $1.10082 \mathrm{E}+14$ & $4.09014 \mathrm{E}+20$ & 1496.8 & 409.8 \\
\hline & & 278.9 & 17.04 & 303.1 & $5.78732 \mathrm{E}+13$ & $1.99894 \mathrm{E}+13$ & $1.08475 E+14$ & $4.25695 \mathrm{E}+20$ & 1460.5 & 404.3 \\
\hline & EOC $(2 / 23 / 2017,1400)$ & 288.4 & 17.68 & 298.6 & $5.92089 \mathrm{E}+13$ & $1.98728 \mathrm{E}+13$ & $1.09589 E+14$ & $4.41653 \mathrm{E}+20$ & 1438.0 & 400.9 \\
\hline \multirow[t]{9}{*}{$161 \mathrm{~A}$} & \multicolumn{10}{|c|}{ Cycle 161A Outage - 95 Day, 13 Hour Down Time } \\
\hline & BOC $(5 / 30 / 2017,0300)$ & 290.1 & 17.78 & 250.0 & $4.95666 \mathrm{E}+13$ & $1.66061 \mathrm{E}+13$ & $9.16610 \mathrm{E}+13$ & $4.44183 \mathrm{E}+20$ & 1193.3 & 361.5 \\
\hline & & 290.4 & 17.80 & 232.2 & $4.61761 \mathrm{E}+13$ & $1.54949 \mathrm{E}+13$ & $8.53209 E+13$ & $4.44544 \mathrm{E}+20$ & 1103.9 & 346.2 \\
\hline & & 292.4 & 17.91 & 233.9 & $4.65262 \mathrm{E}+13$ & $1.55216 \mathrm{E}+13$ & $8.59421 \mathrm{E}+13$ & $4.47254 \mathrm{E}+20$ & 1112.5 & 347.7 \\
\hline & & 294.4 & 18.02 & 232.9 & $4.64418 \mathrm{E}+13$ & $1.55792 \mathrm{E}+13$ & $8.59108 E+13$ & $4.49964 \mathrm{E}+20$ & 1107.1 & 346.8 \\
\hline & & 296.4 & 18.12 & 237.1 & $4.74024 \mathrm{E}+13$ & $1.57642 \mathrm{E}+13$ & $8.74421 \mathrm{E}+13$ & $4.52674 \mathrm{E}+20$ & 1128.2 & 350.4 \\
\hline & & 298.4 & 18.24 & 239.5 & $4.80751 \mathrm{E}+13$ & $1.59530 \mathrm{E}+13$ & $8.84477 \mathrm{E}+13$ & $4.55444 \mathrm{E}+20$ & 1140.5 & 352.6 \\
\hline & & 300.4 & 18.35 & 241.0 & $4.85382 \mathrm{E}+13$ & $1.61041 \mathrm{E}+13$ & $8.93295 E+13$ & $4.58214 \mathrm{E}+20$ & 1148.3 & 353.9 \\
\hline & EOC§ $(6 / 11 / 2017,1600)$ & 300.9 & 18.37 & 244.2 & $4.93429 \mathrm{E}+13$ & $1.62880 \mathrm{E}+13$ & $9.04484 \mathrm{E}+13$ & $4.58756 \mathrm{E}+20$ & 1164.1 & 356.6 \\
\hline
\end{tabular}

$\S$ ATF-06 was discharged for PIE following Cycle 161A irradiation. 
TEM-10300-1

$03 / 01 / 2012$

Rev. 03

Title: $\quad$ ATF-1 Power Histories

\begin{tabular}{lllll} 
TEV No.: & 3119 & Rev. No.: & 0 & Project No.: \\
\hline
\end{tabular}

Table 9. ATF-07 Power History

\begin{tabular}{|c|c|c|c|c|c|c|c|c|c|c|}
\hline Cycle & Critical Dates & EFPD & $\begin{array}{c}\text { Burnup } \\
\text { (GWd/MTU) }\end{array}$ & $\begin{array}{l}\text { LHGR } \\
(\mathrm{W} / \mathrm{cm})\end{array}$ & $\begin{array}{l}\text { Thermal Flux } \\
\left(\mathrm{n} / \mathrm{cm}^{2 *} \mathrm{~s}\right)\end{array}$ & $\begin{array}{c}\text { Fast Flux } \\
\left(\mathrm{n} / \mathrm{cm}^{2 *} \mathrm{~s}>1\right. \\
\mathrm{MeV})\end{array}$ & $\begin{array}{l}\text { Total Flux } \\
\left(\mathrm{n} / \mathrm{cm}^{2 *} \mathrm{~s}\right)\end{array}$ & $\begin{array}{l}\text { Fission Density } \\
\text { (Fissions/cm³) }\end{array}$ & $\begin{array}{c}\text { Fuel Centerline } \\
\text { Temperature } \\
\left({ }^{\circ} \mathrm{C}\right)\end{array}$ & PICT $\left({ }^{\circ} \mathrm{C}\right)$ \\
\hline \multirow[t]{2}{*}{$157 \mathrm{C}$} & BOC $(2 / 10 / 2015,0600)$ & 0.0 & 0.00 & 283.6 & $3.66547 \mathrm{E}+13$ & $1.99526 \mathrm{E}+13$ & $8.61525 E+13$ & $0.00000 \mathrm{E}+00$ & 1354.7 & 373.5 \\
\hline & EOC $(2 / 15 / 2015,0800)$ & 5.4 & 0.37 & 295.8 & $3.85405 \mathrm{E}+13$ & $2.06664 \mathrm{E}+13$ & $8.93277 E+13$ & $9.15344 \mathrm{E}+18$ & 1415.5 & 382.6 \\
\hline \multirow[t]{13}{*}{ 157D } & \multicolumn{10}{|c|}{ Cycle 157D Outage - 104 Day, 3 Hour Down Time } \\
\hline & BOC $(5 / 30 / 2015,1100)$ & 5.4 & 0.37 & 238.5 & $3.10234 \mathrm{E}+13$ & $1.65283 E+13$ & $7.14775 \mathrm{E}+13$ & $9.15344 \mathrm{E}+18$ & 1128.8 & 338.1 \\
\hline & & 8.4 & 0.55 & 271.2 & $3.53047 \mathrm{E}+13$ & $1.88811 \mathrm{E}+13$ & $8.17212 E+13$ & $1.36699 \mathrm{E}+19$ & 1292.3 & 364.0 \\
\hline & & 15.4 & 0.96 & 272.0 & $3.54756 \mathrm{E}+13$ & $1.88988 \mathrm{E}+13$ & $8.20536 \mathrm{E}+13$ & $2.40880 \mathrm{E}+19$ & 1296.3 & 364.6 \\
\hline & & 22.4 & 1.38 & 268.9 & $3.53304 \mathrm{E}+13$ & $1.89424 \mathrm{E}+13$ & $8.18796 E+13$ & $3.43856 \mathrm{E}+19$ & 1280.7 & 362.2 \\
\hline & & 29.4 & 1.82 & 274.4 & $3.64489 E+13$ & $1.92400 \mathrm{E}+13$ & $8.37060 E+13$ & $4.54059 \mathrm{E}+19$ & 1308.4 & 366.4 \\
\hline & & 36.4 & 2.26 & 277.0 & $3.71368 \mathrm{E}+13$ & $1.95242 E+13$ & $8.52369 E+13$ & $5.65466 \mathrm{E}+19$ & 1321.5 & 368.5 \\
\hline & & 43.4 & 2.72 & 289.9 & $3.91888 \mathrm{E}+13$ & $2.02128 \mathrm{E}+13$ & $8.91294 E+13$ & $6.81088 \mathrm{E}+19$ & 1386.2 & 378.2 \\
\hline & & 50.4 & 3.19 & 291.4 & $3.97115 \mathrm{E}+13$ & $2.03521 \mathrm{E}+13$ & $8.98919 E+13$ & 7.97313E+19 & 1393.8 & 379.4 \\
\hline & & 57.4 & 3.66 & 298.1 & $4.10159 \mathrm{E}+13$ & $2.09755 E+13$ & $9.30735 E+13$ & $9.15344 \mathrm{E}+19$ & 1427.2 & 384.3 \\
\hline & & 60.4 & 3.86 & 298.5 & $4.14909 \mathrm{E}+13$ & $2.10957 E+13$ & $9.37494 \mathrm{E}+13$ & $9.64122 \mathrm{E}+19$ & 1429.2 & 384.6 \\
\hline & \multicolumn{10}{|c|}{ Mid-Cycle 157D Outage - 14 Day, 10 Hour Down Time $(7 / 23 / 2015,1800-8 / 7 / 2015,0400)$} \\
\hline & EOC $(8 / 12 / 2015,0400)$ & 65.1 & 4.18 & 301.3 & $4.22843 \mathrm{E}+13$ & $2.13107 \mathrm{E}+13$ & $9.49488 \mathrm{E}+13$ & $1.04542 \mathrm{E}+20$ & 1443.4 & 386.7 \\
\hline \multirow[t]{8}{*}{$158 \mathrm{~A}$} & \multicolumn{10}{|c|}{ Cycle 158A Outage - 91 Day, 11 Hour Down Time } \\
\hline & $\mathrm{BOC}(11 / 11 / 2015,1500)$ & 65.1 & 4.18 & 227.0 & $3.22262 \mathrm{E}+13$ & $1.66680 \mathrm{E}+13$ & $7.76276 \mathrm{E}+13$ & $1.04542 \mathrm{E}+20$ & 1071.8 & 328.7 \\
\hline & & 75.1 & 4.69 & 233.2 & $3.31033 E+13$ & $1.70738 \mathrm{E}+13$ & $7.92463 E+13$ & $1.17309 \mathrm{E}+20$ & 1102.4 & 333.8 \\
\hline & & 85.1 & 5.25 & 242.6 & $3.47460 \mathrm{E}+13$ & $1.78581 \mathrm{E}+13$ & $8.24535 E+13$ & $1.31159 \mathrm{E}+20$ & 1149.5 & 341.4 \\
\hline & & 95.1 & 5.84 & 266.1 & $3.84801 \mathrm{E}+13$ & $1.95947 \mathrm{E}+13$ & $8.95641 \mathrm{E}+13$ & $1.46034 \mathrm{E}+20$ & 1267.0 & 360.1 \\
\hline & & 105.1 & 6.45 & 275.1 & $4.03414 \mathrm{E}+13$ & $1.97836 \mathrm{E}+13$ & $9.20713 E+13$ & $1.61390 \mathrm{E}+20$ & 1311.9 & 367.0 \\
\hline & & 117.1 & 7.21 & 280.9 & $4.16312 \mathrm{E}+13$ & $2.02773 E+13$ & $9.41699 \mathrm{E}+13$ & $1.80178 \mathrm{E}+20$ & 1341.1 & 371.4 \\
\hline & $\operatorname{EOC}(1 / 2 / 2016,0400)$ & 117.3 & 7.22 & 281.7 & $4.24822 \mathrm{E}+13$ & $2.03990 E+13$ & $9.54082 \mathrm{E}+13$ & $1.80419 \mathrm{E}+20$ & 1344.8 & 372.0 \\
\hline \multirow[t]{5}{*}{$158 \mathrm{~B}$} & \multicolumn{10}{|c|}{ Cycle 158B Outage - 39 Day, 2 Hour Down Time } \\
\hline & BOC $(2 / 10 / 2016,0600)$ & 118.3 & 7.27 & 255.7 & $3.85434 \mathrm{E}+13$ & $1.85404 \mathrm{E}+13$ & $8.68688 \mathrm{E}+13$ & $1.81684 \mathrm{E}+20$ & 1215.0 & 351.9 \\
\hline & & 119.3 & 7.31 & 236.4 & $3.56414 \mathrm{E}+13$ & $1.71989 \mathrm{E}+13$ & $8.10675 E+13$ & $1.82768 \mathrm{E}+20$ & 1118.3 & 336.4 \\
\hline & & 122.3 & 7.46 & 228.3 & $3.44610 \mathrm{E}+13$ & $1.66320 \mathrm{E}+13$ & $7.83665 \mathrm{E}+13$ & $1.86622 \mathrm{E}+20$ & 1078.2 & 329.8 \\
\hline & & 127.3 & 7.73 & 234.4 & $3.55563 \mathrm{E}+13$ & $1.72522 \mathrm{E}+13$ & $8.06022 \mathrm{E}+13$ & $1.93306 \mathrm{E}+20$ & 1108.4 & 334.8 \\
\hline
\end{tabular}


TEM-10300-1

$03 / 01 / 2012$

Rev. 03

Title: $\quad$ ATF-1 Power Histories

$\begin{array}{lllll}\text { TEV No.: } & 3119 & \text { Rev. No.: } & 0 & \text { Project No.: } 31418 \quad \text { Date: } 12 / 01 / 2017\end{array}$

Table 9. ATF-07 Power History

\begin{tabular}{|c|c|c|c|c|c|c|c|c|c|c|}
\hline Cycle & Critical Dates & EFPD & $\begin{array}{c}\text { Burnup } \\
\text { (GWd/MTU) }\end{array}$ & $\begin{array}{c}\text { LHGR } \\
(\mathrm{W} / \mathrm{cm})\end{array}$ & $\begin{array}{l}\text { Thermal Flux } \\
\left(\mathrm{n} / \mathrm{cm}^{2 *} \mathrm{~s}\right)\end{array}$ & $\begin{array}{c}\text { Fast Flux } \\
\left(\mathrm{n} / \mathrm{cm}^{2 *} \mathrm{~s}>1\right. \\
\mathrm{MeV})\end{array}$ & $\begin{array}{l}\text { Total Flux } \\
\left(\mathrm{n} / \mathrm{cm}^{2 *} \mathrm{~s}\right)\end{array}$ & $\begin{array}{l}\text { Fission Density } \\
\left(\text { Fissions } / \mathrm{cm}^{3}\right)\end{array}$ & $\begin{array}{l}\text { Fuel Centerline } \\
\text { Temperature } \\
\left({ }^{\circ} \mathrm{C}\right)\end{array}$ & PICT $\left({ }^{\circ} \mathrm{C}\right)$ \\
\hline & & 132.3 & 8.01 & 243.6 & $3.71610 \mathrm{E}+13$ & $1.79682 \mathrm{E}+13$ & $8.37691 \mathrm{E}+13$ & $2.00171 \mathrm{E}+20$ & 1154.3 & 342.2 \\
\hline & & 137.3 & 8.28 & 243.7 & $3.75214 \mathrm{E}+13$ & $1.76248 \mathrm{E}+13$ & $8.39465 E+13$ & $2.07097 \mathrm{E}+20$ & 1154.7 & 342.3 \\
\hline & & 147.3 & 8.88 & 267.4 & $4.11767 \mathrm{E}+13$ & $1.94489 \mathrm{E}+13$ & $9.10138 E+13$ & $2.21911 \mathrm{E}+20$ & 1273.6 & 361.1 \\
\hline & & 157.3 & 9.47 & 268.3 & $4.18968 \mathrm{E}+13$ & $1.95355 \mathrm{E}+13$ & $9.22324 \mathrm{E}+13$ & $2.36785 \mathrm{E}+20$ & 1278.1 & 361.8 \\
\hline & EOC $(4 / 1 / 2016,0900)$ & 168.7 & 10.17 & 278.8 & $4.48180 \mathrm{E}+13$ & $2.00899 \mathrm{E}+13$ & $9.61503 E+13$ & $2.54369 \mathrm{E}+20$ & 1330.5 & 369.8 \\
\hline \multirow[t]{7}{*}{$159 A$} & \multicolumn{10}{|c|}{ Cycle 159A Outage - 77 Day, 2 Hour Down Time } \\
\hline & $\mathrm{BOC}(6 / 17 / 2016,1100)$ & 169.7 & 10.20 & 128.8 & $2.06620 \mathrm{E}+13$ & $9.27864 \mathrm{E}+12$ & $4.38422 \mathrm{E}+13$ & $2.54971 \mathrm{E}+20$ & 583.7 & 241.9 \\
\hline & & 170.7 & 10.22 & 129.6 & $2.08509 \mathrm{E}+13$ & $9.41490 \mathrm{E}+12$ & $4.43248 E+13$ & $2.55574 \mathrm{E}+20$ & 587.7 & 242.7 \\
\hline & & 174.6 & 10.33 & 108.8 & 1.75057E+13 & $7.62730 \mathrm{E}+12$ & $3.69042 \mathrm{E}+13$ & $2.58163 \mathrm{E}+20$ & 484.8 & 222.8 \\
\hline & \multicolumn{10}{|c|}{ Mid-Cycle 159A Outage - 3 Day, 21 Hour Down Time $(6 / 23 / 2016,1000$ - 6/27/2016, 0700) } \\
\hline & & 176.0 & 10.35 & 206.4 & $3.34725 \mathrm{E}+13$ & $1.49191 \mathrm{E}+13$ & $7.20710 \mathrm{E}+13$ & $2.58886 \mathrm{E}+20$ & 968.8 & 311.4 \\
\hline & EOC $(6 / 28 / 2016,1800)$ & 176.2 & 10.37 & 286.9 & $4.65878 \mathrm{E}+13$ & $2.07767 \mathrm{E}+13$ & $9.99557 \mathrm{E}+13$ & $2.59187 \mathrm{E}+20$ & 1371.3 & 376.0 \\
\hline $160 \mathrm{~A}$ & \multicolumn{10}{|c|}{ Cycle 160A Outage - 80 Day, 1 Hour Down Time } \\
\hline & $\operatorname{BOC}(9 / 16 / 2016,1900)$ & 177.2 & 10.42 & 275.2 & $4.45516 \mathrm{E}+13$ & $2.02074 \mathrm{E}+13$ & $9.73118 \mathrm{E}+13$ & $2.60452 \mathrm{E}+20$ & 1312.7 & 367.1 \\
\hline & & 179.2 & 10.54 & 264.1 & $4.29534 \mathrm{E}+13$ & $1.93551 \mathrm{E}+13$ & $9.42272 \mathrm{E}+13$ & $2.63463 \mathrm{E}+20$ & 1256.8 & 358.5 \\
\hline & & 186.2 & 10.97 & 274.3 & $4.47960 \mathrm{E}+13$ & $1.99864 \mathrm{E}+13$ & $9.75982 \mathrm{E}+13$ & $2.74182 \mathrm{E}+20$ & 1308.0 & 366.4 \\
\hline & & 196.2 & 11.61 & 284.4 & $4.69507 \mathrm{E}+13$ & $2.09079 \mathrm{E}+13$ & $1.01189 \mathrm{E}+14$ & $2.90260 \mathrm{E}+20$ & 1358.6 & 374.1 \\
\hline & & 206.2 & 12.31 & 314.4 & $5.24932 \mathrm{E}+13$ & $2.27109 \mathrm{E}+13$ & $1.10495 E+14$ & $3.07724 \mathrm{E}+20$ & 1509.2 & 396.2 \\
\hline & & 216.2 & 12.98 & 299.9 & $5.11758 \mathrm{E}+13$ & $2.17101 \mathrm{E}+13$ & $1.07292 \mathrm{E}+14$ & $3.24465 E+20$ & 1436.2 & 385.6 \\
\hline & & 222.2 & 13.39 & 311.6 & $5.41398 \mathrm{E}+13$ & $2.26115 \mathrm{E}+13$ & $1.12330 \mathrm{E}+14$ & $3.34823 E+20$ & 1494.9 & 394.2 \\
\hline & & 223.8 & 13.51 & 313.8 & $5.50415 \mathrm{E}+13$ & $2.28385 \mathrm{E}+13$ & $1.13565 E+14$ & $3.37834 \mathrm{E}+20$ & 1505.9 & 395.8 \\
\hline & & 226.2 & 13.67 & 317.2 & $5.57048 \mathrm{E}+13$ & $2.31758 \mathrm{E}+13$ & $1.15205 E+14$ & $3.41869 \mathrm{E}+20$ & 1523.1 & 398.2 \\
\hline & EOC (11/8/2016, 1100) & 228.9 & 13.85 & 312.9 & $5.55299 \mathrm{E}+13$ & $2.27430 \mathrm{E}+13$ & 1.14083E+14 & $3.46325 E+20$ & 1501.5 & 395.1 \\
\hline \multirow[t]{6}{*}{$160 \mathrm{~B}$} & \multicolumn{10}{|c|}{ Cycle 160B Outage - 42 Day, 1 Hour Down Time Outage } \\
\hline & $\operatorname{BOC}(12 / 20 / 2016,1200)$ & 229.9 & 13.93 & 319.8 & $5.66809 \mathrm{E}+13$ & $2.37102 \mathrm{E}+13$ & $1.18930 \mathrm{E}+14$ & $3.48373 \mathrm{E}+20$ & 1536.4 & 400.1 \\
\hline & & 231.9 & 14.07 & 321.1 & $5.71256 \mathrm{E}+13$ & $2.38595 \mathrm{E}+13$ & $1.19813 E+14$ & $3.51865 E+20$ & 1542.7 & 401.0 \\
\hline & & 238.9 & 14.58 & 325.0 & $5.79652 \mathrm{E}+13$ & $2.39216 \mathrm{E}+13$ & $1.20392 \mathrm{E}+14$ & $3.64632 \mathrm{E}+20$ & 1562.6 & 403.8 \\
\hline & & 248.9 & 15.33 & 335.9 & $6.07087 \mathrm{E}+13$ & $2.46480 \mathrm{E}+13$ & $1.24899 \mathrm{E}+14$ & $3.83421 \mathrm{E}+20$ & 1617.1 & 411.4 \\
\hline & & 258.1 & 16.01 & 332.3 & $6.26123 \mathrm{E}+13$ & $2.44363 \mathrm{E}+13$ & $1.26196 \mathrm{E}+14$ & $4.00403 E+20$ & 1599.1 & 408.9 \\
\hline
\end{tabular}


TEM-10300-1

03/01/2012

Rev. 03

Title: $\quad$ ATF-1 Power Histories

$\begin{array}{lllll}\text { TEV No.: } & 3119 & \text { Rev. No.: } & 0 & \text { Project No.: }\end{array}$

Table 9. ATF-07 Power History

\begin{tabular}{|c|c|c|c|c|c|c|c|c|c|c|}
\hline Cycle & Critical Dates & EFPD & $\begin{array}{c}\text { Burnup } \\
\text { (GWd/MTU) }\end{array}$ & $\begin{array}{l}\text { LHGR } \\
(\mathrm{W} / \mathrm{cm})\end{array}$ & $\begin{array}{l}\text { Thermal Flux } \\
\left(\mathrm{n} / \mathrm{cm}^{2 *} \mathrm{~s}\right)\end{array}$ & $\begin{array}{c}\text { Fast Flux } \\
\left(\mathrm{n} / \mathrm{cm}^{2 *} \mathrm{~s}>1\right. \\
\mathrm{MeV})\end{array}$ & $\begin{array}{c}\text { Total Flux } \\
\left(\mathrm{n} / \mathrm{cm}^{2 *} \mathrm{~s}\right)\end{array}$ & 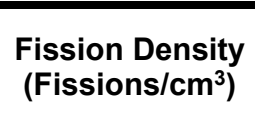 & $\begin{array}{l}\text { Fuel Centerline } \\
\text { Temperature } \\
\left({ }^{\circ} \mathrm{C}\right)\end{array}$ & PICT $\left({ }^{\circ} \mathrm{C}\right)$ \\
\hline & \multicolumn{10}{|c|}{ Mid-Cycle 160B Outage - 5 Day, 14 Hour Down Time } \\
\hline & & 268.9 & 16.83 & 345.9 & $6.56075 \mathrm{E}+13$ & $2.54792 \mathrm{E}+13$ & $1.31504 \mathrm{E}+14$ & $4.20817 \mathrm{E}+20$ & 1667.4 & 418.3 \\
\hline & & 278.9 & 17.58 & 338.4 & $6.52001 \mathrm{E}+13$ & $2.48618 \mathrm{E}+13$ & $1.29563 \mathrm{E}+14$ & $4.39486 \mathrm{E}+20$ & 1629.8 & 413.2 \\
\hline & $\operatorname{EOC}(2 / 23 / 2017,1400)$ & 288.4 & 18.30 & 335.3 & $6.76126 \mathrm{E}+13$ & $2.47567 \mathrm{E}+13$ & $1.31674 \mathrm{E}+14$ & $4.57491 \mathrm{E}+20$ & 1614.0 & 411.0 \\
\hline \multirow[t]{9}{*}{$161 \mathrm{~A}$} & \multicolumn{10}{|c|}{ Cycle 161A Outage - 95 Day, 13 Hour Down Time } \\
\hline & $\mathrm{BOC}(5 / 30 / 2017,0300)$ & 290.1 & 18.41 & 278.6 & $5.60594 \mathrm{E}+13$ & $2.07070 \mathrm{E}+13$ & $1.09466 \mathrm{E}+14$ & $4.60201 \mathrm{E}+20$ & 1329.6 & 369.7 \\
\hline & & 290.4 & 18.42 & 261.0 & $5.26648 \mathrm{E}+13$ & $1.92153 \mathrm{E}+13$ & $1.02635 \mathrm{E}+14$ & $4.60623 \mathrm{E}+20$ & 1241.5 & 356.1 \\
\hline & & 292.4 & 18.54 & 261.8 & $5.28926 \mathrm{E}+13$ & $1.93940 \mathrm{E}+13$ & $1.03249 \mathrm{E}+14$ & $4.63513 \mathrm{E}+20$ & 1245.6 & 356.7 \\
\hline & & 294.4 & 18.65 & 259.5 & $5.26462 \mathrm{E}+13$ & $1.91831 \mathrm{E}+13$ & $1.02633 \mathrm{E}+14$ & $4.66404 \mathrm{E}+20$ & 1234.1 & 354.9 \\
\hline & & 296.4 & 18.77 & 265.2 & $5.40116 \mathrm{E}+13$ & $1.97087 \mathrm{E}+13$ & $1.04960 \mathrm{E}+14$ & $4.69415 \mathrm{E}+20$ & 1262.6 & 359.4 \\
\hline & & 298.4 & 18.89 & 268.9 & $5.48735 \mathrm{E}+13$ & $1.99059 \mathrm{E}+13$ & $1.06424 \mathrm{E}+14$ & $4.72366 \mathrm{E}+20$ & 1281.0 & 362.2 \\
\hline & & 300.4 & 19.01 & 271.6 & $5.56171 \mathrm{E}+13$ & $2.00660 \mathrm{E}+13$ & $1.07578 \mathrm{E}+14$ & $4.75437 \mathrm{E}+20$ & 1294.2 & 364.3 \\
\hline & $\operatorname{EOC}(6 / 11 / 2017,1600)$ & 300.9 & 19.04 & 273.9 & $5.64602 \mathrm{E}+13$ & $2.03094 \mathrm{E}+13$ & $1.09186 \mathrm{E}+14$ & $4.76039 \mathrm{E}+20$ & 1306.2 & 366.1 \\
\hline \multirow[t]{8}{*}{$162 \mathrm{~A}$} & \multicolumn{10}{|c|}{ Cycle 162A Outage } \\
\hline & $\mathrm{BOC}$ & 301.9 & 19.12 & 303.0 & $6.34470 \mathrm{E}+13$ & $2.29400 \mathrm{E}+13$ & $1.23673 E+14$ & $4.78026 \mathrm{E}+20$ & 1451.6 & 387.9 \\
\hline & & 303.9 & 19.24 & 280.7 & $5.80836 \mathrm{E}+13$ & $2.07559 \mathrm{E}+13$ & $1.13734 \mathrm{E}+14$ & $4.81098 \mathrm{E}+20$ & 1340.0 & 371.3 \\
\hline & & 330.9 & 20.92 & 281.3 & $5.94975 \mathrm{E}+13$ & $2.05407 \mathrm{E}+13$ & $1.14381 \mathrm{E}+14$ & $5.23191 \mathrm{E}+20$ & 1343.0 & 371.7 \\
\hline & & 340.9 & 21.51 & 276.3 & $5.96298 \mathrm{E}+13$ & $2.02871 \mathrm{E}+13$ & $1.14140 \mathrm{E}+14$ & $5.37945 \mathrm{E}+20$ & 1318.1 & 367.9 \\
\hline & & 350.9 & 22.14 & 277.4 & $6.18157 \mathrm{E}+13$ & $2.05894 \mathrm{E}+13$ & $1.17061 \mathrm{E}+14$ & $5.53482 \mathrm{E}+20$ & 1323.3 & 368.7 \\
\hline & & 359.9 & 22.69 & 281.9 & $6.48011 \mathrm{E}+13$ & $2.11817 \mathrm{E}+13$ & $1.21418 \mathrm{E}+14$ & $5.67393 E+20$ & 1345.8 & 372.2 \\
\hline & EOC & 362.9 & 22.89 & 276.1 & $6.48820 \mathrm{E}+13$ & $2.07720 \mathrm{E}+13$ & $1.20713 \mathrm{E}+14$ & $5.72210 \mathrm{E}+20$ & 1317.1 & 367.8 \\
\hline
\end{tabular}


TEM-10300-1

$03 / 01 / 2012$

Rev. 03

Title: $\quad$ ATF-1 Power Histories

\begin{tabular}{lllll} 
TEV No.: & 3119 & Rev. No.: & 0 & Project No.: \\
\hline
\end{tabular}

Table 10. ATF-08 Power History

\begin{tabular}{|c|c|c|c|c|c|c|c|c|c|c|}
\hline Cycle & Critical Dates & EFPD & $\begin{array}{c}\text { Burnup } \\
\text { (GWd/MTU) }\end{array}$ & $\begin{array}{l}\text { LHGR } \\
(\mathrm{W} / \mathrm{cm})\end{array}$ & $\begin{array}{l}\text { Thermal Flux } \\
\left(\mathrm{n} / \mathrm{cm}^{2 *} \mathrm{~s}\right)\end{array}$ & $\begin{array}{c}\text { Fast Flux } \\
\left(\mathrm{n} / \mathrm{cm}^{2 *} \mathrm{~s}>1\right. \\
\mathrm{MeV})\end{array}$ & $\begin{array}{l}\text { Total Flux } \\
\left(\mathrm{n} / \mathrm{cm}^{2 *} \mathrm{~s}\right)\end{array}$ & $\begin{array}{l}\text { Fission Density } \\
\left(\text { Fissions } / \mathrm{cm}^{3}\right)\end{array}$ & $\begin{array}{c}\text { Fuel Centerline } \\
\text { Temperature } \\
\left({ }^{\circ} \mathrm{C}\right)\end{array}$ & PICT $\left({ }^{\circ} \mathrm{C}\right)$ \\
\hline \multirow[t]{2}{*}{$157 \mathrm{C}$} & BOC $(2 / 10 / 2015,0600)$ & 0.0 & 0.00 & 273.3 & $3.54013 E+13$ & $2.04703 E+13$ & $8.69853 E+13$ & $0.00000 \mathrm{E}+00$ & 1324.0 & 401.8 \\
\hline & EOC (2/15/2015, 0800) & 5.4 & 0.35 & 284.3 & $3.71006 \mathrm{E}+13$ & $2.12741 \mathrm{E}+13$ & $9.01168 \mathrm{E}+13$ & $8.79212 \mathrm{E}+18$ & 1379.9 & 411.1 \\
\hline \multirow[t]{13}{*}{$157 \mathrm{D}$} & \multicolumn{10}{|c|}{ Cycle 157D Outage - 104 Day, 3 Hour Down Time } \\
\hline & $\mathrm{BOC}(5 / 30 / 2015,1100)$ & 5.4 & 0.35 & 232.2 & $3.01984 \mathrm{E}+13$ & $1.74344 \mathrm{E}+13$ & $7.33555 \mathrm{E}+13$ & $8.79212 \mathrm{E}+18$ & 1116.3 & 365.6 \\
\hline & & 8.4 & 0.53 & 265.2 & $3.44983 E+13$ & $1.98174 \mathrm{E}+13$ & $8.37632 \mathrm{E}+13$ & $1.31882 \mathrm{E}+19$ & 1282.8 & 394.8 \\
\hline & & 15.4 & 0.93 & 262.4 & $3.43209 \mathrm{E}+13$ & $1.95481 \mathrm{E}+13$ & $8.29758 \mathrm{E}+13$ & $2.32449 \mathrm{E}+19$ & 1269.0 & 392.5 \\
\hline & & 22.4 & 1.33 & 262.0 & $3.45238 \mathrm{E}+13$ & $1.95922 \mathrm{E}+13$ & $8.34357 \mathrm{E}+13$ & $3.32414 \mathrm{E}+19$ & 1267.0 & 392.1 \\
\hline & & 29.4 & 1.74 & 266.2 & $3.54056 \mathrm{E}+13$ & $1.99399 \mathrm{E}+13$ & $8.49919 E+13$ & $4.34186 \mathrm{E}+19$ & 1288.0 & 395.7 \\
\hline & & 36.4 & 2.17 & 269.5 & $3.61281 \mathrm{E}+13$ & $2.01664 \mathrm{E}+13$ & $8.65441 \mathrm{E}+13$ & $5.42582 \mathrm{E}+19$ & 1305.1 & 398.6 \\
\hline & & 43.4 & 2.62 & 280.1 & $3.78630 \mathrm{E}+13$ & $2.09206 \mathrm{E}+13$ & $9.02011 \mathrm{E}+13$ & $6.54591 \mathrm{E}+19$ & 1358.7 & 407.6 \\
\hline & & 50.4 & 3.07 & 281.6 & $3.84393 E+13$ & $2.11124 \mathrm{E}+13$ & $9.12768 \mathrm{E}+13$ & $7.66601 \mathrm{E}+19$ & 1366.1 & 408.8 \\
\hline & & 57.4 & 3.53 & 289.8 & $3.99043 \mathrm{E}+13$ & $2.19039 \mathrm{E}+13$ & $9.49293 E+13$ & $8.81621 \mathrm{E}+19$ & 1407.7 & 415.7 \\
\hline & & 60.4 & 3.72 & 291.0 & $4.03889 E+13$ & $2.17927 E+13$ & $9.53466 \mathrm{E}+13$ & $9.29195 E+19$ & 1413.5 & 416.7 \\
\hline & \multicolumn{10}{|c|}{ Mid-Cycle 157D Outage - 14 Day, 10 Hour Down Time $(7 / 23 / 2015,1800$ - 8/7/2015, 0400) } \\
\hline & EOC $(8 / 12 / 2015,0400)$ & 65.1 & 4.03 & 293.3 & $4.11010 \mathrm{E}+13$ & $2.19068 \mathrm{E}+13$ & $9.63426 \mathrm{E}+13$ & $1.00869 \mathrm{E}+20$ & 1425.3 & 418.6 \\
\hline \multirow[t]{8}{*}{$158 \mathrm{~A}$} & \multicolumn{10}{|c|}{ Cycle 158A Outage - 91 Day, 11 Hour Down Time } \\
\hline & $\operatorname{BOC}(11 / 11 / 2015,1500)$ & 65.1 & 4.03 & 219.4 & $3.10253 \mathrm{E}+13$ & $1.72018 \mathrm{E}+13$ & $7.84923 \mathrm{E}+13$ & $1.00869 \mathrm{E}+20$ & 1051.3 & 353.8 \\
\hline & & 75.1 & 4.53 & 225.3 & $3.18022 \mathrm{E}+13$ & $1.76139 \mathrm{E}+13$ & $8.00280 \mathrm{E}+13$ & $1.13214 \mathrm{E}+20$ & 1081.2 & 359.3 \\
\hline & & 85.1 & 5.07 & 235.9 & $3.37925 \mathrm{E}+13$ & $1.84230 \mathrm{E}+13$ & $8.39689 \mathrm{E}+13$ & 1.26643E+20 & 1134.9 & 369.0 \\
\hline & & 95.1 & 5.65 & 259.9 & $3.75160 \mathrm{E}+13$ & $2.00201 \mathrm{E}+13$ & $9.06495 \mathrm{E}+13$ & $1.41216 \mathrm{E}+20$ & 1256.2 & 390.3 \\
\hline & & 105.1 & 6.24 & 262.7 & $3.83468 \mathrm{E}+13$ & $2.01534 \mathrm{E}+13$ & $9.16764 \mathrm{E}+13$ & $1.55910 \mathrm{E}+20$ & 1270.6 & 392.7 \\
\hline & & 117.1 & 6.96 & 266.8 & $3.93874 \mathrm{E}+13$ & $2.04139 \mathrm{E}+13$ & $9.28515 \mathrm{E}+13$ & $1.73915 E+20$ & 1291.0 & 396.2 \\
\hline & EOC $(1 / 2 / 2016,0400)$ & 117.3 & 6.97 & 267.3 & $4.02294 \mathrm{E}+13$ & $2.04640 \mathrm{E}+13$ & $9.40979 \mathrm{E}+13$ & $1.74156 \mathrm{E}+20$ & 1293.8 & 396.7 \\
\hline \multirow[t]{5}{*}{$158 \mathrm{~B}$} & \multicolumn{10}{|c|}{ Cycle 158B Outage - 39 Day, 2 Hour Down Time } \\
\hline & BOC $(2 / 10 / 2016,0600)$ & 118.3 & 7.01 & 250.1 & $3.76357 \mathrm{E}+13$ & $1.93512 \mathrm{E}+13$ & $8.90821 \mathrm{E}+13$ & $1.75300 \mathrm{E}+20$ & 1206.4 & 381.6 \\
\hline & & 119.3 & 7.06 & 229.5 & $3.45092 \mathrm{E}+13$ & $1.78350 \mathrm{E}+13$ & $8.20460 \mathrm{E}+13$ & 1.76384E+20 & 1102.6 & 363.2 \\
\hline & & 122.3 & 7.20 & 222.7 & $3.34635 \mathrm{E}+13$ & $1.73915 \mathrm{E}+13$ & $7.99813 \mathrm{E}+13$ & $1.80118 \mathrm{E}+20$ & 1068.1 & 356.9 \\
\hline & & 127.3 & 7.47 & 229.3 & $3.45713 \mathrm{E}+13$ & $1.79605 \mathrm{E}+13$ & $8.24418 \mathrm{E}+13$ & $1.86682 \mathrm{E}+20$ & 1101.4 & 362.9 \\
\hline
\end{tabular}


TEM-10300-1

$03 / 01 / 2012$

Rev. 03

Title: $\quad$ ATF-1 Power Histories

\begin{tabular}{lllll} 
TEV No.: & 3119 & Rev. No.: & 0 & Project No.: \\
\hline
\end{tabular}

Table 10. ATF-08 Power History

\begin{tabular}{|c|c|c|c|c|c|c|c|c|c|c|}
\hline Cycle & Critical Dates & EFPD & $\begin{array}{c}\text { Burnup } \\
\text { (GWd/MTU) }\end{array}$ & $\begin{array}{l}\text { LHGR } \\
\text { (W/cm) }\end{array}$ & $\begin{array}{l}\text { Thermal Flux } \\
\left(\mathrm{n} / \mathrm{cm}^{2 *} \mathrm{~s}\right)\end{array}$ & $\begin{array}{c}\text { Fast Flux } \\
\left(\mathrm{n} / \mathrm{cm}^{2 *} \mathrm{~s}>1\right. \\
\mathrm{MeV})\end{array}$ & $\begin{array}{l}\text { Total Flux } \\
\left(\mathrm{n} / \mathrm{cm}^{2 *} \mathrm{~s}\right)\end{array}$ & $\begin{array}{l}\text { Fission Density } \\
\text { (Fissions/cm }\end{array}$ & $\begin{array}{c}\text { Fuel Centerline } \\
\text { Temperature } \\
\left({ }^{\circ} \mathrm{C}\right)\end{array}$ & PICT $\left({ }^{\circ} \mathrm{C}\right)$ \\
\hline & & 132.3 & 7.74 & 238.1 & $3.61983 \mathrm{E}+13$ & $1.84690 \mathrm{E}+13$ & $8.53433 \mathrm{E}+13$ & $1.93427 \mathrm{E}+20$ & 1145.8 & 370.9 \\
\hline & & 137.3 & 8.01 & 240.8 & $3.67717 \mathrm{E}+13$ & $1.84498 \mathrm{E}+13$ & $8.56317 \mathrm{E}+13$ & $2.00292 \mathrm{E}+20$ & 1159.6 & 373.4 \\
\hline & & 147.3 & 8.59 & 260.0 & $3.98435 \mathrm{E}+13$ & $2.01174 \mathrm{E}+13$ & $9.23357 \mathrm{E}+13$ & $2.14745 \mathrm{E}+20$ & 1256.7 & 390.3 \\
\hline & & 157.3 & 9.17 & 261.6 & $4.06486 \mathrm{E}+13$ & $2.02628 \mathrm{E}+13$ & $9.33506 \mathrm{E}+13$ & $2.29378 \mathrm{E}+20$ & 1264.9 & 391.7 \\
\hline & EOC $(4 / 1 / 2016,0900)$ & 168.7 & 9.86 & 266.0 & $4.27211 \mathrm{E}+13$ & $2.04492 \mathrm{E}+13$ & $9.55355 \mathrm{E}+13$ & $2.46601 \mathrm{E}+20$ & 1287.2 & 395.6 \\
\hline \multirow[t]{7}{*}{$159 \mathrm{~A}$} & \multicolumn{10}{|c|}{ Cycle 159A Outage - 77 Day, 2 Hour Down Time } \\
\hline & $\operatorname{BOC}(6 / 17 / 2016,1100)$ & 169.7 & 9.89 & 126.7 & $2.02375 E+13$ & $9.71019 \mathrm{E}+12$ & $4.47664 \mathrm{E}+13$ & $2.47203 E+20$ & 582.5 & 261.8 \\
\hline & & 170.7 & 9.91 & 129.0 & $2.05303 E+13$ & $9.83762 \mathrm{E}+12$ & $4.53561 \mathrm{E}+13$ & $2.47805 \mathrm{E}+20$ & 594.4 & 264.3 \\
\hline & & 174.6 & 10.01 & 106.6 & $1.69318 \mathrm{E}+13$ & $8.16361 \mathrm{E}+12$ & $3.74572 \mathrm{E}+13$ & $2.50335 \mathrm{E}+20$ & 480.7 & 240.3 \\
\hline & \multicolumn{10}{|c|}{ Mid-Cycle 159A Outage - 3 Day, 21 Hour Down Time (6/23/2016, 1000 - 6/27/2016, 0700) } \\
\hline & & 176.0 & 10.04 & 201.2 & $3.23629 \mathrm{E}+13$ & $1.55053 \mathrm{E}+13$ & $7.27531 \mathrm{E}+13$ & $2.51057 \mathrm{E}+20$ & 959.4 & 336.7 \\
\hline & EOC $(6 / 28 / 2016,1800)$ & 176.2 & 10.05 & 280.2 & $4.50594 \mathrm{E}+13$ & $2.15554 \mathrm{E}+13$ & $1.00751 \mathrm{E}+14$ & $2.51358 \mathrm{E}+20$ & 1358.9 & 407.7 \\
\hline $160 \mathrm{~A}$ & \multicolumn{10}{|c|}{ Cycle 160A Outage - 80 Day, 1 Hour Down Time } \\
\hline & $\operatorname{BOC}(9 / 16 / 2016,1900)$ & 177.2 & 10.10 & 269.2 & $4.33684 \mathrm{E}+13$ & $2.09902 E+13$ & $9.93022 \mathrm{E}+13$ & $2.52623 \mathrm{E}+20$ & 1303.4 & 398.3 \\
\hline & & 179.2 & 10.23 & 259.1 & $4.19132 \mathrm{E}+13$ & $2.03908 \mathrm{E}+13$ & $9.62564 \mathrm{E}+13$ & $2.55634 \mathrm{E}+20$ & 1252.2 & 389.6 \\
\hline & & 186.2 & 10.64 & 267.3 & $4.33076 \mathrm{E}+13$ & $2.09152 \mathrm{E}+13$ & $9.86194 \mathrm{E}+13$ & $2.66052 \mathrm{E}+20$ & 1293.7 & 396.7 \\
\hline & & 196.2 & 11.28 & 280.5 & $4.58149 \mathrm{E}+13$ & $2.18089 \mathrm{E}+13$ & $1.02804 \mathrm{E}+14$ & $2.81950 \mathrm{E}+20$ & 1360.5 & 407.9 \\
\hline & & 206.2 & 11.96 & 306.2 & $5.07579 \mathrm{E}+13$ & $2.37270 \mathrm{E}+13$ & 1.12353E+14 & $2.99053 \mathrm{E}+20$ & 1490.3 & 429.1 \\
\hline & & 216.2 & 12.61 & 288.7 & $4.87670 \mathrm{E}+13$ & $2.25079 \mathrm{E}+13$ & $1.07360 \mathrm{E}+14$ & $3.15252 \mathrm{E}+20$ & 1402.1 & 414.8 \\
\hline & & 222.2 & 13.01 & 298.1 & $5.10727 \mathrm{E}+13$ & $2.31516 \mathrm{E}+13$ & $1.11252 \mathrm{E}+14$ & $3.25248 \mathrm{E}+20$ & 1449.4 & 422.5 \\
\hline & & 223.8 & 13.13 & 301.7 & $5.21411 \mathrm{E}+13$ & $2.34632 \mathrm{E}+13$ & 1.13104E+14 & $3.28139 E+20$ & 1467.5 & 425.4 \\
\hline & & 226.2 & 13.28 & 307.7 & $5.33287 \mathrm{E}+13$ & $2.38359 \mathrm{E}+13$ & $1.14975 \mathrm{E}+14$ & $3.32113 E+20$ & 1497.9 & 430.3 \\
\hline & EOC $(11 / 8 / 2016,1100)$ & 228.9 & 13.46 & 301.3 & $5.27393 E+13$ & $2.33919 \mathrm{E}+13$ & $1.13269 \mathrm{E}+14$ & $3.36389 \mathrm{E}+20$ & 1465.8 & 425.1 \\
\hline \multirow[t]{6}{*}{ 160B } & \multicolumn{10}{|c|}{ Cycle 160B Outage - 42 Day, 1 Hour Down Time Outage } \\
\hline & BOC $(12 / 20 / 2016,1200)$ & 229.9 & 13.51 & 285.5 & $4.96528 \mathrm{E}+13$ & $1.90538 \mathrm{E}+13$ & $9.84823 \mathrm{E}+13$ & $3.37714 \mathrm{E}+20$ & 1385.6 & 412.1 \\
\hline & & 231.9 & 13.64 & 286.6 & $5.01139 \mathrm{E}+13$ & $1.90887 \mathrm{E}+13$ & $9.94197 \mathrm{E}+13$ & $3.40966 \mathrm{E}+20$ & 1391.4 & 413.0 \\
\hline & & 238.9 & 14.08 & 289.0 & $5.06278 \mathrm{E}+13$ & $1.91961 \mathrm{E}+13$ & $9.97403 \mathrm{E}+13$ & $3.52046 \mathrm{E}+20$ & 1403.4 & 415.0 \\
\hline & & 248.9 & 14.76 & 305.2 & $5.41406 \mathrm{E}+13$ & $2.02439 \mathrm{E}+13$ & $1.05669 \mathrm{E}+14$ & $3.68968 \mathrm{E}+20$ & 1485.2 & 428.3 \\
\hline & & 258.1 & 15.40 & 308.5 & $5.67465 \mathrm{E}+13$ & $2.05451 \mathrm{E}+13$ & $1.09279 \mathrm{E}+14$ & $3.85047 \mathrm{E}+20$ & 1502.3 & 431.0 \\
\hline
\end{tabular}


TEM-10300-1

$03 / 01 / 2012$

Rev. 03

TECHNICAL EVALUATION

Page 31 of 110

Title: $\quad$ ATF-1 Power Histories

\begin{tabular}{lllll} 
& TEV No.: 3119 & Rev. No.: & 0 & Project No.: \\
\hline
\end{tabular}

Table 10. ATF-08 Power History

\begin{tabular}{|c|c|c|c|c|c|c|c|c|c|c|}
\hline Cycle & Critical Dates & EFPD & $\begin{array}{c}\text { Burnup } \\
\text { (GWd/MTU) }\end{array}$ & $\begin{array}{l}\text { LHGR } \\
(\mathrm{W} / \mathrm{cm})\end{array}$ & $\begin{array}{l}\text { Thermal Flux } \\
\left(\mathrm{n} / \mathrm{cm}^{2 *} \mathrm{~s}\right)\end{array}$ & $\begin{array}{c}\text { Fast Flux } \\
\left(\mathrm{n} / \mathrm{cm}^{2 *} \mathrm{~s}>1\right. \\
\mathrm{MeV})\end{array}$ & $\begin{array}{l}\text { Total Flux } \\
\left(\mathrm{n} / \mathrm{cm}^{2 *} \mathrm{~s}\right)\end{array}$ & $\begin{array}{c}\text { Fission Density } \\
\text { (Fissions/cm³) }\end{array}$ & $\begin{array}{c}\text { Fuel Centerline } \\
\text { Temperature } \\
\left({ }^{\circ} \mathrm{C}\right)\end{array}$ & $\operatorname{PICT}\left({ }^{\circ} \mathrm{C}\right)$ \\
\hline & \multicolumn{10}{|c|}{ Mid-Cycle 160B Outage - 5 Day, 14 Hour Down Time } \\
\hline & & 268.9 & 16.19 & 329.6 & $6.10307 \mathrm{E}+13$ & $2.18393 \mathrm{E}+13$ & $1.16521 \mathrm{E}+14$ & $4.04678 \mathrm{E}+20$ & 1608.7 & 447.5 \\
\hline & & 278.9 & 16.92 & 325.6 & $6.12403 \mathrm{E}+13$ & $2.14826 \mathrm{E}+13$ & $1.15908 \mathrm{E}+14$ & 4.22925E+20 & 1588.4 & 444.4 \\
\hline & $\operatorname{EOC}(2 / 23 / 2017,1400)$ & 288.4 & 17.59 & 322.0 & $6.31549 \mathrm{E}+13$ & $2.14765 \mathrm{E}+13$ & 1.17769E+14 & 4.39847E+20 & 1570.2 & 441.6 \\
\hline \multirow[t]{9}{*}{$161 \mathrm{~A}$} & \multicolumn{10}{|c|}{ Cycle 161A Outage - 95 Day, 13 Hour Down Time } \\
\hline & BOC $(5 / 30 / 2017,0300)$ & 290.1 & 17.70 & 275.7 & $5.37580 \mathrm{E}+13$ & $1.81425 \mathrm{E}+13$ & $1.00046 \mathrm{E}+14$ & 4.42557E+20 & 1336.0 & 403.8 \\
\hline & & 290.4 & 17.71 & 254.3 & $4.99263 \mathrm{E}+13$ & $1.70320 \mathrm{E}+13$ & $9.32446 \mathrm{E}+13$ & $4.42858 \mathrm{E}+20$ & 1227.8 & 385.3 \\
\hline & & 292.4 & 17.83 & 255.8 & $5.02764 \mathrm{E}+13$ & $1.70635 \mathrm{E}+13$ & $9.38428 \mathrm{E}+13$ & $4.45809 \mathrm{E}+20$ & 1235.3 & 386.7 \\
\hline & & 294.4 & 17.95 & 254.7 & $5.03266 \mathrm{E}+13$ & $1.69342 \mathrm{E}+13$ & $9.37506 \mathrm{E}+13$ & $4.48699 \mathrm{E}+20$ & 1230.1 & 385.7 \\
\hline & & 296.4 & 18.06 & 257.6 & $5.10109 \mathrm{E}+13$ & $1.72285 \mathrm{E}+13$ & $9.50580 \mathrm{E}+13$ & $4.51590 \mathrm{E}+20$ & 1244.7 & 388.3 \\
\hline & & 298.4 & 18.18 & 261.7 & $5.19539 \mathrm{E}+13$ & $1.73938 \mathrm{E}+13$ & $9.63758 \mathrm{E}+13$ & $4.54541 \mathrm{E}+20$ & 1265.1 & 391.8 \\
\hline & & 300.4 & 18.30 & 264.4 & $5.27022 \mathrm{E}+13$ & $1.75938 \mathrm{E}+13$ & $9.76877 \mathrm{E}+13$ & $4.57552 \mathrm{E}+20$ & 1278.9 & 394.2 \\
\hline & $\operatorname{EOC}^{\star *}(6 / 11 / 2017,1600)$ & 300.9 & 18.33 & 267.9 & $5.36474 \mathrm{E}+13$ & $1.77428 \mathrm{E}+13$ & $9.92188 \mathrm{E}+13$ & $4.58154 \mathrm{E}+20$ & 1296.5 & 397.2 \\
\hline
\end{tabular}

${ }^{* *}$ ATF-08 was discharged for PIE following Cycle 161A irradiation. 
TEM-10300-1

$03 / 01 / 2012$

Rev. 03

Title: $\quad$ ATF-1 Power Histories

\begin{tabular}{lllll} 
TEV No.: & 3119 & Rev. No.: & 0 & Project No.: \\
\hline
\end{tabular}

Table 11. ATF-09 Power History

\begin{tabular}{|c|c|c|c|c|c|c|c|c|c|c|}
\hline Cycle & Critical Dates & EFPD & $\begin{array}{c}\text { Burnup } \\
\text { (GWd/MTU) }\end{array}$ & $\begin{array}{l}\text { LHGR } \\
(\mathrm{W} / \mathrm{cm})\end{array}$ & $\begin{array}{l}\text { Thermal Flux } \\
\left(\mathrm{n} / \mathrm{cm}^{2 *} \mathrm{~s}\right)\end{array}$ & $\begin{array}{c}\text { Fast Flux } \\
\left(\mathrm{n} / \mathrm{cm}^{2 *} \mathrm{~s}>1\right. \\
\mathrm{MeV})\end{array}$ & $\begin{array}{l}\text { Total Flux } \\
\left(\mathrm{n} / \mathrm{cm}^{2 *} \mathrm{~s}\right)\end{array}$ & $\begin{array}{l}\text { Fission Density } \\
\text { (Fissions } / \mathrm{cm}^{3} \text { ) }\end{array}$ & $\begin{array}{c}\text { Fuel Centerline } \\
\text { Temperature } \\
\left({ }^{\circ} \mathrm{C}\right)\end{array}$ & PICT $\left({ }^{\circ} \mathrm{C}\right)$ \\
\hline \multirow[t]{2}{*}{$157 \mathrm{C}$} & BOC $(2 / 10 / 2015,0600)$ & 0.0 & 0.00 & 275.2 & $3.56706 \mathrm{E}+13$ & $2.06256 \mathrm{E}+13$ & $8.75926 \mathrm{E}+13$ & $0.00000 \mathrm{E}+00$ & 1309.2 & 386.2 \\
\hline & EOC (2/15/2015, 0800) & 5.4 & 0.35 & 285.6 & $3.72892 \mathrm{E}+13$ & $2.13207 \mathrm{E}+13$ & $9.03467 \mathrm{E}+13$ & $8.85234 \mathrm{E}+18$ & 1361.2 & 394.8 \\
\hline \multirow[t]{13}{*}{$157 \mathrm{D}$} & \multicolumn{10}{|c|}{ Cycle 157D Outage - 104 Day, 3 Hour Down Time } \\
\hline & $\mathrm{BOC}(5 / 30 / 2015,1100)$ & 5.4 & 0.35 & 232.3 & $3.02550 \mathrm{E}+13$ & $1.74768 \mathrm{E}+13$ & $7.33663 \mathrm{E}+13$ & $8.85234 \mathrm{E}+18$ & 1094.8 & 348.7 \\
\hline & & 8.4 & 0.53 & 266.6 & $3.46545 \mathrm{E}+13$ & $1.99226 \mathrm{E}+13$ & $8.38934 \mathrm{E}+13$ & $1.32484 \mathrm{E}+19$ & 1266.1 & 378.9 \\
\hline & & 15.4 & 0.93 & 263.1 & $3.44046 \mathrm{E}+13$ & $1.96085 \mathrm{E}+13$ & $8.34810 \mathrm{E}+13$ & $2.33654 \mathrm{E}+19$ & 1248.7 & 375.9 \\
\hline & & 22.4 & 1.34 & 262.9 & $3.46297 \mathrm{E}+13$ & $1.96465 \mathrm{E}+13$ & $8.33981 \mathrm{E}+13$ & $3.34221 \mathrm{E}+19$ & 1247.7 & 375.7 \\
\hline & & 29.4 & 1.77 & 269.0 & $3.57158 \mathrm{E}+13$ & $2.01643 E+13$ & $8.58527 \mathrm{E}+13$ & $4.42015 \mathrm{E}+19$ & 1277.9 & 380.9 \\
\hline & & 36.4 & 2.20 & 270.2 & $3.62286 \mathrm{E}+13$ & $2.03149 \mathrm{E}+13$ & $8.68403 E+13$ & $5.51013 E+19$ & 1283.9 & 381.9 \\
\hline & & 43.4 & 2.65 & 281.1 & $3.80732 \mathrm{E}+13$ & $2.11671 \mathrm{E}+13$ & $9.07787 \mathrm{E}+13$ & $6.63022 \mathrm{E}+19$ & 1338.4 & 391.1 \\
\hline & & 50.4 & 3.10 & 282.4 & $3.85501 \mathrm{E}+13$ & $2.13516 \mathrm{E}+13$ & $9.18085 \mathrm{E}+13$ & 7.75634E+19 & 1345.0 & 392.2 \\
\hline & & 57.4 & 3.57 & 291.4 & $4.00860 \mathrm{E}+13$ & $2.20410 \mathrm{E}+13$ & $9.55690 \mathrm{E}+13$ & $8.91256 \mathrm{E}+19$ & 1389.8 & 399.5 \\
\hline & & 60.4 & 3.76 & 292.6 & $4.06284 \mathrm{E}+13$ & $2.20860 E+13$ & $9.60055 \mathrm{E}+13$ & $9.38830 \mathrm{E}+19$ & 1395.9 & 400.5 \\
\hline & \multicolumn{10}{|c|}{ Mid-Cycle 157D Outage - 14 Day, 10 Hour Down Time $(7 / 23 / 2015,1800$ - 8/7/2015, 0400) } \\
\hline & EOC $(8 / 12 / 2015,0400)$ & 65.1 & 4.07 & 295.2 & $4.14524 \mathrm{E}+13$ & $2.21232 \mathrm{E}+13$ & $9.69029 \mathrm{E}+13$ & $1.01772 \mathrm{E}+20$ & 1409.1 & 402.6 \\
\hline \multirow[t]{8}{*}{$158 \mathrm{~A}$} & \multicolumn{10}{|c|}{ Cycle 158A Outage - 91 Day, 11 Hour Down Time } \\
\hline & $\operatorname{BOC}(11 / 11 / 2015,1500)$ & 65.1 & 4.07 & 219.0 & $3.10725 \mathrm{E}+13$ & $1.73249 \mathrm{E}+13$ & $7.88290 \mathrm{E}+13$ & $1.01772 \mathrm{E}+20$ & 1028.6 & 336.5 \\
\hline & & 75.1 & 4.57 & 224.8 & $3.18320 \mathrm{E}+13$ & $1.76978 \mathrm{E}+13$ & $8.04178 \mathrm{E}+13$ & 1.14117E+20 & 1057.2 & 341.8 \\
\hline & & 85.1 & 5.10 & 235.4 & $3.36963 \mathrm{E}+13$ & $1.83619 \mathrm{E}+13$ & $8.38399 \mathrm{E}+13$ & $1.27546 \mathrm{E}+20$ & 1110.2 & 351.5 \\
\hline & & 95.1 & 5.69 & 260.5 & $3.76810 \mathrm{E}+13$ & $1.98743 \mathrm{E}+13$ & $9.08441 \mathrm{E}+13$ & 1.42179E+20 & 1235.8 & 373.7 \\
\hline & & 105.1 & 6.28 & 263.3 & $3.85380 \mathrm{E}+13$ & $2.03235 \mathrm{E}+13$ & $9.20596 \mathrm{E}+13$ & $1.56933 \mathrm{E}+20$ & 1249.6 & 376.1 \\
\hline & & 117.1 & 7.00 & 267.5 & $3.95364 \mathrm{E}+13$ & $2.05271 \mathrm{E}+13$ & $9.35012 \mathrm{E}+13$ & 1.74939E+20 & 1270.6 & 379.7 \\
\hline & EOC $(1 / 2 / 2016,0400)$ & 117.3 & 7.01 & 268.5 & $4.03556 \mathrm{E}+13$ & $2.05856 \mathrm{E}+13$ & $9.46650 \mathrm{E}+13$ & $1.75240 \mathrm{E}+20$ & 1275.6 & 380.5 \\
\hline \multirow[t]{5}{*}{$158 \mathrm{~B}$} & \multicolumn{10}{|c|}{ Cycle 158B Outage - 39 Day, 2 Hour Down Time } \\
\hline & BOC $(2 / 10 / 2016,0600)$ & 118.3 & 7.06 & 251.6 & $3.77506 \mathrm{E}+13$ & $1.94585 \mathrm{E}+13$ & $8.93041 \mathrm{E}+13$ & $1.76384 \mathrm{E}+20$ & 1191.4 & 366.0 \\
\hline & & 119.3 & 7.10 & 228.2 & $3.44369 \mathrm{E}+13$ & $1.78522 \mathrm{E}+13$ & $8.21661 \mathrm{E}+13$ & $1.77468 \mathrm{E}+20$ & 1074.4 & 345.0 \\
\hline & & 122.3 & 7.25 & 223.6 & $3.36857 \mathrm{E}+13$ & $1.74480 \mathrm{E}+13$ & $8.02634 \mathrm{E}+13$ & $1.81202 E+20$ & 1051.5 & 340.8 \\
\hline & & 127.3 & 7.51 & 228.7 & $3.47012 \mathrm{E}+13$ & $1.79099 \mathrm{E}+13$ & $8.22213 E+13$ & 1.87706E+20 & 1076.7 & 345.4 \\
\hline
\end{tabular}


TEM-10300-1

$03 / 01 / 2012$

Rev. 03

Title: $\quad$ ATF-1 Power Histories

\begin{tabular}{lllll} 
TEV No.: & 3119 & Rev. No.: & 0 & Project No.: \\
\hline
\end{tabular}

Table 11. ATF-09 Power History

\begin{tabular}{|c|c|c|c|c|c|c|c|c|c|c|}
\hline Cycle & Critical Dates & EFPD & $\begin{array}{c}\text { Burnup } \\
\text { (GWd/MTU) }\end{array}$ & $\begin{array}{l}\text { LHGR } \\
(\mathrm{W} / \mathrm{cm})\end{array}$ & $\begin{array}{l}\text { Thermal Flux } \\
\left(\mathrm{n} / \mathrm{cm}^{2 *} \mathrm{~s}\right)\end{array}$ & $\begin{array}{c}\text { Fast Flux } \\
\left(\mathrm{n} / \mathrm{cm}^{2 *} \mathrm{~s}>1\right. \\
\mathrm{MeV})\end{array}$ & $\begin{array}{l}\text { Total Flux } \\
\left(\mathrm{n} / \mathrm{cm}^{2 *} \mathrm{~s}\right)\end{array}$ & $\begin{array}{l}\text { Fission Density } \\
\text { (Fissions } / \mathrm{cm}^{3} \text { ) }\end{array}$ & $\begin{array}{c}\text { Fuel Centerline } \\
\text { Temperature } \\
\left({ }^{\circ} \mathrm{C}\right)\end{array}$ & PICT $\left({ }^{\circ} \mathrm{C}\right)$ \\
\hline & & 132.3 & 7.78 & 235.3 & $3.58960 \mathrm{E}+13$ & $1.84919 \mathrm{E}+13$ & $8.47021 \mathrm{E}+13$ & $1.94390 \mathrm{E}+20$ & 1110.0 & 351.4 \\
\hline & & 137.3 & 8.05 & 240.4 & $3.67007 \mathrm{E}+13$ & $1.86092 \mathrm{E}+13$ & $8.58434 \mathrm{E}+13$ & $2.01195 \mathrm{E}+20$ & 1135.0 & 356.0 \\
\hline & & 147.3 & 8.63 & 260.7 & $3.99765 \mathrm{E}+13$ & $2.00791 \mathrm{E}+13$ & $9.26049 \mathrm{E}+13$ & $2.15708 \mathrm{E}+20$ & 1236.5 & 373.8 \\
\hline & & 157.3 & 9.21 & 263.4 & $4.10141 \mathrm{E}+13$ & $2.02306 \mathrm{E}+13$ & $9.37494 \mathrm{E}+13$ & $2.30342 \mathrm{E}+20$ & 1250.0 & 376.1 \\
\hline & EOC $(4 / 1 / 2016,0900)$ & 168.7 & 9.91 & 267.7 & $4.29769 \mathrm{E}+13$ & $2.05926 \mathrm{E}+13$ & $9.59255 \mathrm{E}+13$ & $2.47625 \mathrm{E}+20$ & 1271.4 & 379.8 \\
\hline \multirow[t]{7}{*}{$159 \mathrm{~A}$} & \multicolumn{10}{|c|}{ Cycle 159A Outage - 77 Day, 2 Hour Down Time } \\
\hline & BOC $(6 / 17 / 2016,1100)$ & 169.7 & 9.93 & 127.9 & $2.04437 \mathrm{E}+13$ & $9.82518 \mathrm{E}+12$ & $4.51270 \mathrm{E}+13$ & $2.48227 \mathrm{E}+20$ & 573.8 & 245.2 \\
\hline & & 170.7 & 9.95 & 129.1 & $2.05623 \mathrm{E}+13$ & $9.87082 \mathrm{E}+12$ & $4.53847 \mathrm{E}+13$ & $2.48829 \mathrm{E}+20$ & 579.7 & 246.5 \\
\hline & & 174.6 & 10.05 & 104.9 & $1.67538 \mathrm{E}+13$ & $8.12429 \mathrm{E}+12$ & $3.73001 \mathrm{E}+13$ & $2.51298 \mathrm{E}+20$ & 458.8 & 220.0 \\
\hline & \multicolumn{10}{|c|}{ Mid-Cycle 159A Outage - 3 Day, 21 Hour Down Time (6/23/2016, 1000 - 6/27/2016, 0700) } \\
\hline & & 176.0 & 10.08 & 201.0 & $3.23688 \mathrm{E}+13$ & $1.57181 \mathrm{E}+13$ & $7.31339 \mathrm{E}+13$ & $2.52021 \mathrm{E}+20$ & 938.5 & 319.5 \\
\hline & EOC $(6 / 28 / 2016,1800)$ & 176.2 & 10.09 & 279.3 & $4.50353 \mathrm{E}+13$ & $2.19366 \mathrm{E}+13$ & $1.01487 \mathrm{E}+14$ & $2.52322 \mathrm{E}+20$ & 1329.7 & 389.6 \\
\hline $160 \mathrm{~A}$ & \multicolumn{10}{|c|}{ Cycle 160A Outage - 80 Day, 1 Hour Down Time } \\
\hline & BOC $(9 / 16 / 2016,1900)$ & 177.2 & 10.14 & 271.6 & $4.37062 \mathrm{E}+13$ & $2.13428 \mathrm{E}+13$ & $9.98933 \mathrm{E}+13$ & $2.53586 \mathrm{E}+20$ & 1291.1 & 383.1 \\
\hline & & 179.2 & 10.27 & 261.3 & $4.21911 \mathrm{E}+13$ & $2.04401 \mathrm{E}+13$ & $9.62385 \mathrm{E}+13$ & $2.56658 \mathrm{E}+20$ & 1239.8 & 374.4 \\
\hline & & 186.2 & 10.68 & 268.1 & $4.34076 \mathrm{E}+13$ & $2.10131 \mathrm{E}+13$ & $9.90751 \mathrm{E}+13$ & $2.67076 \mathrm{E}+20$ & 1273.5 & 380.2 \\
\hline & & 196.2 & 11.32 & 280.4 & $4.58539 \mathrm{E}+13$ & $2.17790 \mathrm{E}+13$ & $1.02889 \mathrm{E}+14$ & $2.82974 \mathrm{E}+20$ & 1335.1 & 390.5 \\
\hline & & 206.2 & 12.00 & 303.7 & $5.04666 \mathrm{E}+13$ & $2.36399 \mathrm{E}+13$ & $1.11958 \mathrm{E}+14$ & $2.99896 \mathrm{E}+20$ & 1451.2 & 409.4 \\
\hline & & 216.2 & 12.65 & 290.1 & 4.88777E+13 & $2.24928 \mathrm{E}+13$ & $1.07291 \mathrm{E}+14$ & $3.16155 \mathrm{E}+20$ & 1383.4 & 398.5 \\
\hline & & 222.2 & 13.05 & 299.4 & $5.13049 \mathrm{E}+13$ & $2.31191 \mathrm{E}+13$ & $1.11591 \mathrm{E}+14$ & $3.26152 \mathrm{E}+20$ & 1429.9 & 406.0 \\
\hline & & 223.8 & 13.16 & 302.5 & $5.24457 \mathrm{E}+13$ & $2.34122 \mathrm{E}+13$ & $1.13235 \mathrm{E}+14$ & $3.29042 \mathrm{E}+20$ & 1445.4 & 408.5 \\
\hline & & 226.2 & 13.32 & 307.9 & $5.35919 \mathrm{E}+13$ & $2.37995 \mathrm{E}+13$ & $1.15083 \mathrm{E}+14$ & $3.33017 \mathrm{E}+20$ & 1472.5 & 412.8 \\
\hline & EOC $(11 / 8 / 2016,1100)$ & 228.9 & 13.50 & 302.8 & $5.31576 \mathrm{E}+13$ & $2.35287 \mathrm{E}+13$ & $1.13539 \mathrm{E}+14$ & $3.37413 E+20$ & 1447.1 & 408.7 \\
\hline \multirow[t]{6}{*}{$160 \mathrm{~B}$} & \multicolumn{10}{|c|}{ Cycle 160B Outage - 42 Day, 1 Hour Down Time Outage } \\
\hline & BOC $(12 / 20 / 2016,1200)$ & 229.9 & 13.58 & 329.6 & $5.77303 E+13$ & $2.41097 \mathrm{E}+13$ & $1.20546 \mathrm{E}+14$ & $3.39460 \mathrm{E}+20$ & 1580.7 & 429.4 \\
\hline & & 231.9 & 13.73 & 330.6 & $5.80906 \mathrm{E}+13$ & $2.41137 \mathrm{E}+13$ & $1.21168 \mathrm{E}+14$ & $3.43134 \mathrm{E}+20$ & 1585.7 & 430.2 \\
\hline & & 238.9 & 14.25 & 332.5 & $5.86234 \mathrm{E}+13$ & $2.43313 \mathrm{E}+13$ & $1.21705 \mathrm{E}+14$ & $3.56201 \mathrm{E}+20$ & 1595.5 & 431.6 \\
\hline & & 248.9 & 15.02 & 343.6 & $6.14152 \mathrm{E}+13$ & $2.49845 \mathrm{E}+13$ & $1.25978 \mathrm{E}+14$ & $3.75351 \mathrm{E}+20$ & 1651.1 & 439.9 \\
\hline & & 258.1 & 15.71 & 338.3 & $6.29831 \mathrm{E}+13$ & $2.46737 \mathrm{E}+13$ & $1.26902 \mathrm{E}+14$ & $3.92634 \mathrm{E}+20$ & 1624.5 & 436.0 \\
\hline
\end{tabular}


TEM-10300-1

03/01/2012

Rev. 03

Title:

ATF-1 Power Histories

$\begin{array}{lllll}\text { TEV No.: } & 3119 & \text { Rev. No.: } & 0 & \text { Project No.: }\end{array} 31418$

Table 11. ATF-09 Power History

\begin{tabular}{|c|c|c|c|c|c|c|c|c|c|c|}
\hline Cycle & Critical Dates & EFPD & $\begin{array}{c}\text { Burnup } \\
\text { (GWd/MTU) }\end{array}$ & $\begin{array}{l}\text { LHGR } \\
(\mathrm{W} / \mathrm{cm})\end{array}$ & $\begin{array}{l}\text { Thermal Flux } \\
\left(\mathrm{n} / \mathrm{cm}^{2 *} \mathbf{s}\right)\end{array}$ & $\begin{array}{c}\text { Fast Flux } \\
\left(\mathrm{n} / \mathrm{cm}^{2 *} \mathrm{~s}>1\right. \\
\mathrm{MeV})\end{array}$ & $\begin{array}{c}\text { Total Flux } \\
\left(\mathrm{n} / \mathrm{cm}^{2 *} \mathrm{~s}\right)\end{array}$ & $\begin{array}{c}\text { Fission Density } \\
\left(\text { Fissions } / \mathrm{cm}^{3}\right)\end{array}$ & $\begin{array}{l}\text { Fuel Centerline } \\
\text { Temperature } \\
\left({ }^{\circ} \mathrm{C}\right)\end{array}$ & PICT $\left({ }^{\circ} \mathrm{C}\right)$ \\
\hline & \multicolumn{10}{|c|}{ Mid-Cycle 160B Outage - 5 Day, 14 Hour Down Time } \\
\hline & & 268.9 & 16.54 & 353.2 & $6.62262 \mathrm{E}+13$ & $2.57544 \mathrm{E}+13$ & $1.32686 \mathrm{E}+14$ & $4.13350 \mathrm{E}+20$ & 1698.8 & 446.8 \\
\hline & & 278.9 & 17.29 & 344.2 & $6.55937 \mathrm{E}+13$ & $2.51800 \mathrm{E}+13$ & $1.30585 \mathrm{E}+14$ & $4.32319 \mathrm{E}+20$ & 1653.9 & 440.3 \\
\hline & EOC $(2 / 23 / 2017,1400)$ & 288.4 & 18.03 & 343.0 & $6.84329 \mathrm{E}+13$ & $2.50809 \mathrm{E}+13$ & $1.32992 \mathrm{E}+14$ & $4.50747 \mathrm{E}+20$ & 1648.0 & 439.4 \\
\hline \multirow[t]{9}{*}{$161 \mathrm{~A}$} & \multicolumn{10}{|c|}{ Cycle 161A Outage - 95 Day, 13 Hour Down Time } \\
\hline & $\mathrm{BOC}(5 / 30 / 2017,0300)$ & 290.1 & 18.14 & 286.9 & $5.69315 \mathrm{E}+13$ & $2.08042 \mathrm{E}+13$ & $1.10749 \mathrm{E}+14$ & $4.53517 \mathrm{E}+20$ & 1367.3 & 395.8 \\
\hline & & 290.4 & 18.16 & 264.6 & $5.27902 \mathrm{E}+13$ & $1.94925 \mathrm{E}+13$ & $1.03200 \mathrm{E}+14$ & $4.53938 \mathrm{E}+20$ & 1256.2 & 377.2 \\
\hline & & 292.4 & 18.28 & 267.6 & $5.35177 \mathrm{E}+13$ & $1.95401 \mathrm{E}+13$ & $1.04147 \mathrm{E}+14$ & $4.56889 \mathrm{E}+20$ & 1271.0 & 379.7 \\
\hline & & 294.4 & 18.40 & 265.3 & $5.31438 \mathrm{E}+13$ & $1.95284 \mathrm{E}+13$ & $1.03661 \mathrm{E}+14$ & $4.59900 \mathrm{E}+20$ & 1259.5 & 377.8 \\
\hline & & 296.4 & 18.52 & 271.7 & $5.46970 \mathrm{E}+13$ & $1.97695 \mathrm{E}+13$ & $1.05945 \mathrm{E}+14$ & $4.62911 \mathrm{E}+20$ & 1291.4 & 383.2 \\
\hline & & 298.4 & 18.64 & 274.9 & $5.55980 \mathrm{E}+13$ & $2.00350 \mathrm{E}+13$ & $1.07444 \mathrm{E}+14$ & $4.65922 \mathrm{E}+20$ & 1307.7 & 385.9 \\
\hline & & 300.4 & 18.76 & 277.3 & $5.63006 \mathrm{E}+13$ & $2.02741 \mathrm{E}+13$ & $1.08768 \mathrm{E}+14$ & $4.69054 \mathrm{E}+20$ & 1319.5 & 387.9 \\
\hline & $\operatorname{EOC}(6 / 11 / 2017,1600)$ & 300.9 & 18.79 & 279.2 & $5.70106 \mathrm{E}+13$ & $2.05056 \mathrm{E}+13$ & $1.09807 \mathrm{E}+14$ & $4.69656 \mathrm{E}+20$ & 1329.1 & 389.5 \\
\hline \multirow[t]{10}{*}{$162 \mathrm{~A}$} & \multicolumn{10}{|c|}{ Cycle 162A Outage } \\
\hline & $\mathrm{BOC}$ & 301.9 & 18.87 & 310.2 & $6.41615 \mathrm{E}+13$ & $2.30323 \mathrm{E}+13$ & $1.24781 \mathrm{E}+14$ & $4.71703 \mathrm{E}+20$ & 1484.0 & 414.6 \\
\hline & & 303.9 & 19.00 & 286.0 & $5.85504 \mathrm{E}+13$ & $2.11130 \mathrm{E}+13$ & $1.14787 \mathrm{E}+14$ & $4.74835 \mathrm{E}+20$ & 1363.1 & 395.1 \\
\hline & & 310.9 & 19.44 & 288.0 & $5.85201 \mathrm{E}+13$ & $2.09766 \mathrm{E}+13$ & $1.14355 \mathrm{E}+14$ & $4.86036 \mathrm{E}+20$ & 1373.0 & 396.8 \\
\hline & & 320.9 & 20.10 & 293.6 & $6.05220 \mathrm{E}+13$ & $2.14359 \mathrm{E}+13$ & $1.17564 \mathrm{E}+14$ & $5.02355 \mathrm{E}+20$ & 1400.9 & 401.3 \\
\hline & & 330.9 & 20.71 & 284.9 & $5.96430 \mathrm{E}+13$ & $2.07200 \mathrm{E}+13$ & $1.14835 \mathrm{E}+14$ & $5.17591 \mathrm{E}+20$ & 1357.4 & 394.2 \\
\hline & & 340.9 & 21.33 & 280.0 & $5.97996 \mathrm{E}+13$ & $2.04412 \mathrm{E}+13$ & $1.14647 \mathrm{E}+14$ & $5.33128 \mathrm{E}+20$ & 1333.1 & 390.2 \\
\hline & & 350.9 & 21.96 & 282.9 & $6.23173 \mathrm{E}+13$ & $2.08200 \mathrm{E}+13$ & $1.18108 \mathrm{E}+14$ & $5.48905 \mathrm{E}+20$ & 1347.4 & 392.6 \\
\hline & & 359.9 & 22.52 & 285.7 & $6.50557 \mathrm{E}+13$ & $2.12953 \mathrm{E}+13$ & $1.22043 \mathrm{E}+14$ & $5.62997 \mathrm{E}+20$ & 1361.5 & 394.9 \\
\hline & EOC & 362.9 & 22.72 & 280.0 & $6.51517 \mathrm{E}+13$ & $2.07896 \mathrm{E}+13$ & $1.21093 \mathrm{E}+14$ & $5.67814 \mathrm{E}+20$ & 1333.3 & 390.2 \\
\hline
\end{tabular}


TEM-10300-1

03/01/2012

Rev. 03

Title: $\quad$ ATF-1 Power Histories

\begin{tabular}{lllll} 
TEV No.: & 3119 & Rev. No.: & 0 & Project No.: \\
\hline
\end{tabular}

Table 12. ATF-10 Power History

\begin{tabular}{|c|c|c|c|c|c|c|c|c|c|c|}
\hline Cycle & Critical Dates & EFPD & $\begin{array}{c}\text { Burnup } \\
\text { (GWd/MTU) }\end{array}$ & $\begin{array}{l}\text { LHGR } \\
(\mathrm{W} / \mathrm{cm})\end{array}$ & $\begin{array}{l}\text { Thermal Flux } \\
\left(\mathrm{n} / \mathrm{cm}^{2 *} \mathrm{~s}\right)\end{array}$ & $\begin{array}{c}\text { Fast Flux } \\
\left(\mathrm{n} / \mathrm{cm}^{2 *} \mathrm{~s}>1\right. \\
\mathrm{MeV})\end{array}$ & $\begin{array}{l}\text { Total Flux } \\
\left(\mathrm{n} / \mathrm{cm}^{2 *} \mathrm{~s}\right)\end{array}$ & $\begin{array}{l}\text { Fission Density } \\
\left(\text { Fissions } / \mathrm{cm}^{3}\right)\end{array}$ & $\begin{array}{c}\text { Fuel Centerline } \\
\text { Temperature } \\
\left({ }^{\circ} \mathrm{C}\right)\end{array}$ & PICT $\left({ }^{\circ} \mathrm{C}\right)$ \\
\hline \multirow[t]{2}{*}{$157 \mathrm{C}$} & BOC $(2 / 10 / 2015,0600)$ & 0.0 & 0.00 & 261.0 & $2.68221 \mathrm{E}+13$ & $1.61986 \mathrm{E}+13$ & $6.37269 \mathrm{E}+13$ & $0.00000 \mathrm{E}+00$ & 698.1 & 338.7 \\
\hline & EOC (2/15/2015, 0800) & 5.4 & 0.30 & 264.7 & $2.73577 \mathrm{E}+13$ & $1.64671 \mathrm{E}+13$ & $6.48173 \mathrm{E}+13$ & $8.49102 \mathrm{E}+18$ & 704.3 & 342.0 \\
\hline \multirow[t]{13}{*}{$157 \mathrm{D}$} & \multicolumn{10}{|c|}{ Cycle 157D Outage - 104 Day, 3 Hour Down Time } \\
\hline & $\mathrm{BOC}(5 / 30 / 2015,1100)$ & 5.4 & 0.30 & 203.8 & $2.11934 \mathrm{E}+13$ & $1.41281 \mathrm{E}+13$ & $5.42397 \mathrm{E}+13$ & $8.49102 E+18$ & 597.2 & 287.1 \\
\hline & & 8.4 & 0.43 & 219.0 & $2.27144 \mathrm{E}+13$ & $1.53081 \mathrm{E}+13$ & $5.87153 E+13$ & $1.22849 \mathrm{E}+19$ & 625.2 & 301.2 \\
\hline & & 15.4 & 0.74 & 224.1 & $2.33506 \mathrm{E}+13$ & $1.55054 \mathrm{E}+13$ & $5.98540 \mathrm{E}+13$ & $2.11974 \mathrm{E}+19$ & 634.3 & 305.9 \\
\hline & & 22.4 & 1.06 & 223.8 & $2.33337 \mathrm{E}+13$ & $1.55773 \mathrm{E}+13$ & $5.99953 E+13$ & $3.01702 E+19$ & 633.6 & 305.5 \\
\hline & & 29.4 & 1.37 & 225.7 & $2.37611 \mathrm{E}+13$ & $1.57486 \mathrm{E}+13$ & $6.10544 \mathrm{E}+13$ & $3.90828 E+19$ & 637.1 & 307.3 \\
\hline & & 36.4 & 1.69 & 233.2 & $2.46727 \mathrm{E}+13$ & $1.62132 \mathrm{E}+13$ & $6.30319 E+13$ & $4.82964 \mathrm{E}+19$ & 650.4 & 314.1 \\
\hline & & 43.4 & 2.02 & 235.9 & $2.50883 E+13$ & $1.63487 \mathrm{E}+13$ & $6.38003 E+13$ & $5.75703 E+19$ & 655.2 & 316.5 \\
\hline & & 50.4 & 2.35 & 244.5 & $2.61873 E+13$ & $1.70210 \mathrm{E}+13$ & $6.62068 \mathrm{E}+13$ & $6.70851 \mathrm{E}+19$ & 670.1 & 324.2 \\
\hline & & 57.4 & 2.69 & 247.6 & $2.66648 \mathrm{E}+13$ & $1.71206 \mathrm{E}+13$ & $6.75396 \mathrm{E}+13$ & $7.68407 \mathrm{E}+19$ & 675.5 & 327.0 \\
\hline & & 60.4 & 2.84 & 250.8 & $2.71687 E+13$ & $1.73491 \mathrm{E}+13$ & $6.82187 E+13$ & $8.11163 E+19$ & 680.9 & 329.8 \\
\hline & \multicolumn{10}{|c|}{ Mid-Cycle 157D Outage - 14 Day, 10 Hour Down Time $(7 / 23 / 2015,1800-8 / 7 / 2015,0400)$} \\
\hline & EOC $(8 / 12 / 2015,0400)$ & 65.1 & 3.07 & 251.3 & $2.74238 \mathrm{E}+13$ & $1.74640 \mathrm{E}+13$ & $6.88090 \mathrm{E}+13$ & $8.76803 \mathrm{E}+19$ & 681.7 & 330.2 \\
\hline \multirow[t]{8}{*}{$158 \mathrm{~A}$} & \multicolumn{10}{|c|}{ Cycle 158A Outage - 91 Day, 11 Hour Down Time } \\
\hline & $\operatorname{BOC}(11 / 11 / 2015,1500)$ & 65.1 & 3.07 & 248.7 & $2.71116 \mathrm{E}+13$ & $1.72550 \mathrm{E}+13$ & $6.79694 \mathrm{E}+13$ & $8.76803 \mathrm{E}+19$ & 677.3 & 327.9 \\
\hline & & 75.1 & 3.55 & 243.3 & $2.65168 \mathrm{E}+13$ & $1.69833 \mathrm{E}+13$ & $6.68986 \mathrm{E}+13$ & 1.01350E+20 & 668.1 & 323.1 \\
\hline & & 85.1 & 4.02 & 237.8 & $2.62117 \mathrm{E}+13$ & $1.65386 \mathrm{E}+13$ & $6.54284 \mathrm{E}+13$ & 1.14719E+20 & 658.5 & 318.2 \\
\hline & & 95.1 & 4.49 & 239.3 & $2.65765 \mathrm{E}+13$ & $1.66836 \mathrm{E}+13$ & $6.64708 \mathrm{E}+13$ & $1.28148 \mathrm{E}+20$ & 661.1 & 319.5 \\
\hline & & 105.1 & 4.97 & 247.0 & $2.77174 \mathrm{E}+13$ & $1.74314 \mathrm{E}+13$ & $6.91606 \mathrm{E}+13$ & 1.41999E+20 & 674.5 & 326.5 \\
\hline & & 117.1 & 5.57 & 255.4 & $2.89079 \mathrm{E}+13$ & $1.77691 \mathrm{E}+13$ & $7.11883 E+13$ & $1.59222 \mathrm{E}+20$ & 688.8 & 333.9 \\
\hline & EOC $(1 / 2 / 2016,0400)$ & 117.3 & 5.58 & 253.2 & $2.90008 \mathrm{E}+13$ & 1.76457E+13 & $7.10389 \mathrm{E}+13$ & $1.59463 E+20$ & 685.0 & 331.9 \\
\hline \multirow[t]{5}{*}{$158 \mathrm{~B}$} & \multicolumn{10}{|c|}{ Cycle 158B Outage - 39 Day, 2 Hour Down Time } \\
\hline & BOC $(2 / 10 / 2016,0600)$ & 118.3 & 5.62 & 203.5 & $2.32778 \mathrm{E}+13$ & $1.40691 \mathrm{E}+13$ & $5.63807 \mathrm{E}+13$ & $1.60426 \mathrm{E}+20$ & 596.7 & 286.9 \\
\hline & & 119.3 & 5.66 & 224.0 & $2.56630 \mathrm{E}+13$ & $1.56901 \mathrm{E}+13$ & $6.25846 \mathrm{E}+13$ & 1.61570E+20 & 634.0 & 305.7 \\
\hline & & 122.3 & 5.79 & 230.7 & $2.64216 \mathrm{E}+13$ & $1.62814 \mathrm{E}+13$ & $6.46310 \mathrm{E}+13$ & 1.65545E+20 & 646.0 & 311.8 \\
\hline & & 127.3 & 6.01 & 225.5 & $2.58648 \mathrm{E}+13$ & $1.57627 \mathrm{E}+13$ & $6.30253 E+13$ & 1.71627E+20 & 636.7 & 307.1 \\
\hline
\end{tabular}


TEM-10300-1

$03 / 01 / 2012$

Rev. 03

Title: $\quad$ ATF-1 Power Histories

\begin{tabular}{lllll} 
TEV No.: & 3119 & Rev. No.: & 0 & Project No.: \\
\hline
\end{tabular}

Table 12. ATF-10 Power History

\begin{tabular}{|c|c|c|c|c|c|c|c|c|c|c|}
\hline Cycle & Critical Dates & EFPD & $\begin{array}{c}\text { Burnup } \\
\text { (GWd/MTU) }\end{array}$ & $\begin{array}{c}\text { LHGR } \\
(\mathrm{W} / \mathrm{cm})\end{array}$ & $\begin{array}{l}\text { Thermal Flux } \\
\left(\mathrm{n} / \mathrm{cm}^{2 *} \mathrm{~s}\right)\end{array}$ & $\begin{array}{c}\text { Fast Flux } \\
\left(\mathrm{n} / \mathrm{cm}^{2 *} \mathrm{~s}>1\right. \\
\mathrm{MeV})\end{array}$ & $\begin{array}{l}\text { Total Flux } \\
\left(\mathrm{n} / \mathrm{cm}^{2 *} \mathrm{~s}\right)\end{array}$ & $\begin{array}{l}\text { Fission Density } \\
\left(\text { Fissions } / \mathrm{cm}^{3}\right)\end{array}$ & $\begin{array}{l}\text { Fuel Centerline } \\
\text { Temperature } \\
\left({ }^{\circ} \mathrm{C}\right)\end{array}$ & PICT $\left({ }^{\circ} \mathrm{C}\right)$ \\
\hline & & 132.3 & 6.25 & 231.9 & $2.68705 \mathrm{E}+13$ & $1.62254 \mathrm{E}+13$ & $6.57294 \mathrm{E}+13$ & $1.78432 \mathrm{E}+20$ & 648.1 & 312.9 \\
\hline & & 137.3 & 6.46 & 228.3 & $2.64945 \mathrm{E}+13$ & $1.58333 \mathrm{E}+13$ & $6.42974 \mathrm{E}+13$ & $1.84574 \mathrm{E}+20$ & 641.7 & 309.6 \\
\hline & & 147.3 & 6.93 & 237.5 & $2.76109 \mathrm{E}+13$ & $1.65782 \mathrm{E}+13$ & $6.70132 \mathrm{E}+13$ & $1.97883 \mathrm{E}+20$ & 657.9 & 317.9 \\
\hline & & 157.3 & 7.39 & 237.9 & $2.79478 \mathrm{E}+13$ & $1.66050 \mathrm{E}+13$ & $6.70813 \mathrm{E}+13$ & $2.11192 \mathrm{E}+20$ & 658.7 & 318.3 \\
\hline & EOC $(4 / 1 / 2016,0900)$ & 168.7 & 7.94 & 240.6 & $2.88103 E+13$ & $1.68250 \mathrm{E}+13$ & $6.87258 \mathrm{E}+13$ & $2.26909 \mathrm{E}+20$ & 663.3 & 320.7 \\
\hline $159 \mathrm{~A}$ & \multicolumn{10}{|c|}{ Cycle 159A Outage - 77 Day, 2 Hour Down Time } \\
\hline & $\operatorname{BOC}(6 / 17 / 2016,1100)$ & 169.7 & 7.96 & 103.6 & $1.24882 \mathrm{E}+13$ & $7.22598 \mathrm{E}+12$ & $2.96291 \mathrm{E}+13$ & $2.27451 \mathrm{E}+20$ & 395.2 & 188.8 \\
\hline & & 170.7 & 7.98 & 107.7 & 1.29295E+13 & $7.45748 \mathrm{E}+12$ & $3.08054 \mathrm{E}+13$ & $2.27933 \mathrm{E}+20$ & 404.1 & 193.1 \\
\hline & & 174.6 & 8.04 & 92.7 & $1.11433 \mathrm{E}+13$ & $6.52652 \mathrm{E}+12$ & $2.65132 \mathrm{E}+13$ & $2.29679 \mathrm{E}+20$ & 371.3 & 177.5 \\
\hline & \multicolumn{10}{|c|}{ Mid-Cycle 159A Outage - 3 Day, 21 Hour Down Time (6/23/2016, 1000 - 6/27/2016, 0700) } \\
\hline & & 176.0 & 8.06 & 133.7 & $1.62208 \mathrm{E}+13$ & $9.33915 \mathrm{E}+12$ & $3.95597 \mathrm{E}+13$ & $2.30221 \mathrm{E}+20$ & 459.2 & 219.4 \\
\hline & EOC $(6 / 28 / 2016,1800)$ & 176.2 & 8.06 & 147.8 & $1.79684 \mathrm{E}+13$ & $1.06619 \mathrm{E}+13$ & $4.54704 \mathrm{E}+13$ & $2.30402 E+20$ & 488.2 & 233.4 \\
\hline $160 \mathrm{~A}$ & \multicolumn{10}{|c|}{ Cycle 160A Outage - 80 Day, 1 Hour Down Time } \\
\hline & $\operatorname{BOC}(9 / 16 / 2016,1900)$ & 177.2 & 8.11 & 236.6 & $2.84252 \mathrm{E}+13$ & $1.64223 \mathrm{E}+13$ & $6.77262 \mathrm{E}+13$ & $2.31546 \mathrm{E}+20$ & 656.5 & 317.2 \\
\hline & & 179.2 & 8.21 & 235.1 & $2.83150 \mathrm{E}+13$ & $1.63824 \mathrm{E}+13$ & $6.75315 \mathrm{E}+13$ & $2.34436 \mathrm{E}+20$ & 653.8 & 315.8 \\
\hline & & 186.2 & 8.53 & 236.3 & $2.85716 \mathrm{E}+13$ & $1.65794 \mathrm{E}+13$ & $6.80370 \mathrm{E}+13$ & $2.43590 \mathrm{E}+20$ & 655.9 & 316.9 \\
\hline & & 196.2 & 8.99 & 235.8 & $2.87264 \mathrm{E}+13$ & $1.65916 \mathrm{E}+13$ & $6.80834 \mathrm{E}+13$ & $2.56778 \mathrm{E}+20$ & 655.1 & 316.5 \\
\hline & & 206.2 & 9.45 & 238.4 & $2.92805 \mathrm{E}+13$ & $1.68954 \mathrm{E}+13$ & $6.95366 \mathrm{E}+13$ & $2.69966 \mathrm{E}+20$ & 659.4 & 318.7 \\
\hline & & 216.2 & 9.92 & 241.5 & $3.00485 \mathrm{E}+13$ & $1.72247 \mathrm{E}+13$ & $7.12693 \mathrm{E}+13$ & $2.83395 E+20$ & 664.9 & 321.5 \\
\hline & & 222.2 & 10.20 & 239.0 & $3.00402 \mathrm{E}+13$ & $1.67576 \mathrm{E}+13$ & $7.02962 \mathrm{E}+13$ & $2.91465 \mathrm{E}+20$ & 660.5 & 319.3 \\
\hline & & 223.8 & 10.28 & 223.8 & $2.83389 \mathrm{E}+13$ & $1.59289 \mathrm{E}+13$ & $6.62995 \mathrm{E}+13$ & $2.93813 \mathrm{E}+20$ & 633.7 & 305.5 \\
\hline & & 226.2 & 10.39 & 222.1 & $2.80200 \mathrm{E}+13$ & $1.57672 \mathrm{E}+13$ & $6.59043 \mathrm{E}+13$ & $2.96945 \mathrm{E}+20$ & 630.6 & 304.0 \\
\hline & EOC $(11 / 8 / 2016,1100)$ & 228.9 & 10.51 & 211.0 & $2.67303 \mathrm{E}+13$ & $1.50726 \mathrm{E}+13$ & $6.30987 \mathrm{E}+13$ & $3.00257 E+20$ & 610.4 & 293.8 \\
\hline \multirow[t]{6}{*}{$160 \mathrm{~B}$} & \multicolumn{10}{|c|}{ Cycle 160B Outage - 42 Day, 1 Hour Down Time Outage } \\
\hline & BOC $(12 / 20 / 2016,1200)$ & 229.9 & 10.55 & 224.2 & $2.84703 \mathrm{E}+13$ & $1.58107 \mathrm{E}+13$ & $6.66544 \mathrm{E}+13$ & $3.01281 \mathrm{E}+20$ & 634.3 & 305.9 \\
\hline & & 231.9 & 10.64 & 224.0 & $2.84625 \mathrm{E}+13$ & $1.58057 \mathrm{E}+13$ & $6.67581 \mathrm{E}+13$ & $3.04051 \mathrm{E}+20$ & 634.0 & 305.7 \\
\hline & & 238.9 & 10.94 & 221.0 & $2.82064 \mathrm{E}+13$ & $1.55654 \mathrm{E}+13$ & $6.58743 \mathrm{E}+13$ & $3.12662 \mathrm{E}+20$ & 628.8 & 303.0 \\
\hline & & 248.9 & 11.36 & 211.3 & $2.71984 \mathrm{E}+13$ & $1.49174 \mathrm{E}+13$ & $6.33244 \mathrm{E}+13$ & $3.24586 \mathrm{E}+20$ & 611.1 & 294.1 \\
\hline & & 258.1 & 11.75 & 210.6 & $2.76008 \mathrm{E}+13$ & $1.48862 \mathrm{E}+13$ & $6.37564 \mathrm{E}+13$ & $3.35666 \mathrm{E}+20$ & 609.7 & 293.4 \\
\hline
\end{tabular}


TEM-10300-1

03/01/2012

Rev. 03

Title:

ATF-1 Power Histories

$\begin{array}{lllll}\text { TEV No.: } & 3119 & \text { Rev. No.: } & 0 & \text { Project No.: }\end{array} 31418$

Table 12. ATF-10 Power History

\begin{tabular}{|c|c|c|c|c|c|c|c|c|c|c|}
\hline Cycle & Critical Dates & EFPD & $\begin{array}{c}\text { Burnup } \\
\text { (GWd/MTU) }\end{array}$ & $\begin{array}{l}\text { LHGR } \\
(\mathrm{W} / \mathrm{cm})\end{array}$ & $\begin{array}{l}\text { Thermal Flux } \\
\left(\mathrm{n} / \mathrm{cm}^{2 *} \mathbf{s}\right)\end{array}$ & $\begin{array}{c}\text { Fast Flux } \\
\left(\mathrm{n} / \mathrm{cm}^{2 *} \mathrm{~s}>1\right. \\
\mathrm{MeV})\end{array}$ & $\begin{array}{l}\text { Total Flux } \\
\left(\mathrm{n} / \mathrm{cm}^{2 *} \mathbf{s}\right)\end{array}$ & 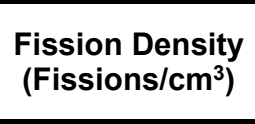 & $\begin{array}{c}\text { Fuel Centerline } \\
\text { Temperature } \\
\left({ }^{\circ} \mathrm{C}\right)\end{array}$ & $\operatorname{PICT}\left({ }^{\circ} \mathrm{C}\right)$ \\
\hline & \multicolumn{10}{|c|}{ Mid-Cycle 160B Outage - 5 Day, 14 Hour Down Time } \\
\hline & & 268.9 & 12.20 & 215.0 & $2.82563 \mathrm{E}+13$ & $1.51879 \mathrm{E}+13$ & $6.52056 \mathrm{E}+13$ & $3.48493 \mathrm{E}+20$ & 617.8 & 297.5 \\
\hline & & 278.9 & 12.61 & 211.6 & $2.80803 \mathrm{E}+13$ & $1.51990 \mathrm{E}+13$ & $6.61314 \mathrm{E}+13$ & $3.60296 \mathrm{E}+20$ & 611.6 & 294.4 \\
\hline & $\operatorname{EOC}(2 / 23 / 2017,1400)$ & 288.4 & 13.03 & 224.2 & $3.02563 \mathrm{E}+13$ & $1.60523 \mathrm{E}+13$ & $6.98319 \mathrm{E}+13$ & $3.72280 \mathrm{E}+20$ & 634.4 & 305.9 \\
\hline \multirow[t]{9}{*}{$161 \mathrm{~A}$} & \multicolumn{10}{|c|}{ Cycle 161A Outage - 95 Day, 13 Hour Down Time } \\
\hline & $\mathrm{BOC}(5 / 30 / 2017,0300)$ & 290.1 & 13.09 & 192.7 & $2.59914 \mathrm{E}+13$ & $1.37360 \mathrm{E}+13$ & $5.94094 \mathrm{E}+13$ & $3.73846 \mathrm{E}+20$ & 576.4 & 276.7 \\
\hline & & 290.4 & 13.09 & 170.7 & $2.31170 \mathrm{E}+13$ & $1.21403 \mathrm{E}+13$ & $5.26857 \mathrm{E}+13$ & $3.74087 \mathrm{E}+20$ & 534.0 & 255.8 \\
\hline & & 292.4 & 13.17 & 175.8 & $2.37361 \mathrm{E}+13$ & $1.24705 \mathrm{E}+13$ & $5.43799 \mathrm{E}+13$ & $3.76315 \mathrm{E}+20$ & 544.0 & 260.7 \\
\hline & & 294.4 & 13.25 & 178.0 & $2.40495 \mathrm{E}+13$ & $1.26955 \mathrm{E}+13$ & $5.51149 \mathrm{E}+13$ & $3.78663 \mathrm{E}+20$ & 548.3 & 262.8 \\
\hline & & 296.4 & 13.33 & 177.6 & $2.40386 \mathrm{E}+13$ & $1.25902 \mathrm{E}+13$ & $5.47890 \mathrm{E}+13$ & $3.80952 \mathrm{E}+20$ & 547.4 & 262.4 \\
\hline & & 298.4 & 13.42 & 183.4 & $2.48901 \mathrm{E}+13$ & $1.29494 \mathrm{E}+13$ & $5.64520 \mathrm{E}+13$ & $3.83300 \mathrm{E}+20$ & 558.7 & 268.0 \\
\hline & & 300.4 & 13.50 & 187.6 & $2.54922 \mathrm{E}+13$ & $1.32406 \mathrm{E}+13$ & $5.79667 \mathrm{E}+13$ & $3.85709 \mathrm{E}+20$ & 566.7 & 271.9 \\
\hline & $\operatorname{EOC}(6 / 11 / 2017,1600)$ & 300.9 & 13.52 & 190.6 & $2.59666 \mathrm{E}+13$ & $1.36670 \mathrm{E}+13$ & $5.91667 \mathrm{E}+13$ & $3.86191 \mathrm{E}+20$ & 572.5 & 274.8 \\
\hline \multirow[t]{8}{*}{$162 \mathrm{~A}$} & \multicolumn{10}{|c|}{ Cycle 162A Outage } \\
\hline & $\mathrm{BOC}$ & 301.9 & 13.56 & 240.6 & $3.19463 E+13$ & $1.65836 \mathrm{E}+13$ & $7.25094 \mathrm{E}+13$ & $3.87275 \mathrm{E}+20$ & 663.3 & 320.7 \\
\hline & & 303.9 & 13.65 & 227.8 & $3.06049 \mathrm{E}+13$ & $1.60826 \mathrm{E}+13$ & $6.96405 \mathrm{E}+13$ & $3.89985 \mathrm{E}+20$ & 640.9 & 309.2 \\
\hline & & 330.9 & 14.80 & 229.3 & $3.11163 \mathrm{E}+13$ & $1.60603 \mathrm{E}+13$ & $7.02075 \mathrm{E}+13$ & $4.22925 \mathrm{E}+20$ & 643.4 & 310.5 \\
\hline & & 340.9 & 15.24 & 231.5 & $3.15902 \mathrm{E}+13$ & $1.59270 \mathrm{E}+13$ & $7.05066 \mathrm{E}+13$ & $4.35270 \mathrm{E}+20$ & 647.4 & 312.5 \\
\hline & & 350.9 & 15.70 & 234.1 & $3.27978 \mathrm{E}+13$ & $1.64109 \mathrm{E}+13$ & $7.32052 \mathrm{E}+13$ & $4.48458 \mathrm{E}+20$ & 651.9 & 314.9 \\
\hline & & 359.9 & 16.09 & 227.2 & $3.20722 \mathrm{E}+13$ & $1.60871 \mathrm{E}+13$ & $7.17649 \mathrm{E}+13$ & $4.59659 \mathrm{E}+20$ & 639.7 & 308.6 \\
\hline & EOC & 362.9 & 16.22 & 225.9 & $3.23364 \mathrm{E}+13$ & $1.60542 E+13$ & $7.20324 \mathrm{E}+13$ & $4.63393 \mathrm{E}+20$ & 637.5 & 307.5 \\
\hline
\end{tabular}


TEM-10300-1

$03 / 01 / 2012$

Rev. 03

Title: $\quad$ ATF-1 Power Histories

\begin{tabular}{lllll} 
TEV No.: & 3119 & Rev. No.: & 0 & Project No.: \\
\hline
\end{tabular}

Table 13. ATF-11 Power History

\begin{tabular}{|c|c|c|c|c|c|c|c|c|c|c|}
\hline Cycle & Critical Dates & EFPD & $\begin{array}{l}\text { Burnup } \\
\text { (GWd/MTU) }\end{array}$ & $\begin{array}{l}\text { LHGR } \\
(\mathrm{W} / \mathrm{cm})\end{array}$ & $\begin{array}{l}\text { Thermal Flux } \\
\left(\mathbf{n} / \mathrm{cm}^{2 *} \mathbf{s}\right)\end{array}$ & $\begin{array}{c}\text { Fast Flux } \\
\left(\mathrm{n} / \mathrm{cm}^{2 *} \mathrm{~s}>1\right. \\
\mathrm{MeV})\end{array}$ & $\begin{array}{l}\text { Total Flux } \\
\left(\mathrm{n} / \mathrm{cm}^{2 *} \mathbf{s}\right)\end{array}$ & $\begin{array}{l}\text { Fission Density } \\
\text { (Fissions/cm }\end{array}$ & $\begin{array}{c}\text { Fuel Centerline } \\
\text { Temperature } \\
\left({ }^{\circ} \mathrm{C}\right)\end{array}$ & PICT $\left({ }^{\circ} \mathrm{C}\right)$ \\
\hline \multirow[t]{12}{*}{ 157D } & $\mathrm{BOC}(5 / 30 / 2015,1100)$ & 0.0 & 0.00 & 206.5 & $2.12949 E+13$ & $1.43074 \mathrm{E}+13$ & $5.48688 \mathrm{E}+13$ & $0.00000 \mathrm{E}+00$ & 579.4 & 256.9 \\
\hline & & 3.0 & 0.14 & 224.4 & $2.31374 \mathrm{E}+13$ & $1.53391 \mathrm{E}+13$ & $5.92665 \mathrm{E}+13$ & $3.91430 \mathrm{E}+18$ & 610.2 & 271.2 \\
\hline & & 10.0 & 0.45 & 225.8 & $2.34967 \mathrm{E}+13$ & $1.54564 \mathrm{E}+13$ & $6.02803 E+13$ & $1.28871 \mathrm{E}+19$ & 612.7 & 272.4 \\
\hline & & 17.0 & 0.77 & 226.0 & $2.35793 \mathrm{E}+13$ & $1.56061 \mathrm{E}+13$ & $6.05427 \mathrm{E}+13$ & $2.19201 \mathrm{E}+19$ & 613.0 & 272.5 \\
\hline & & 24.0 & 1.09 & 227.7 & $2.38570 \mathrm{E}+13$ & $1.57189 \mathrm{E}+13$ & $6.12561 \mathrm{E}+13$ & $3.09531 \mathrm{E}+19$ & 615.9 & 273.9 \\
\hline & & 31.0 & 1.41 & 236.4 & $2.49908 \mathrm{E}+13$ & $1.62932 \mathrm{E}+13$ & $6.34222 \mathrm{E}+13$ & $4.02872 \mathrm{E}+19$ & 630.4 & 280.7 \\
\hline & & 38.0 & 1.74 & 237.7 & $2.52605 \mathrm{E}+13$ & $1.64334 \mathrm{E}+13$ & $6.40669 \mathrm{E}+13$ & $4.96815 \mathrm{E}+19$ & 632.6 & 281.8 \\
\hline & & 45.0 & 2.08 & 245.8 & $2.62530 \mathrm{E}+13$ & $1.68923 \mathrm{E}+13$ & $6.65301 \mathrm{E}+13$ & $5.93769 \mathrm{E}+19$ & 645.9 & 288.1 \\
\hline & & 52.0 & 2.42 & 249.3 & $2.67867 \mathrm{E}+13$ & $1.72622 \mathrm{E}+13$ & $6.78812 \mathrm{E}+13$ & $6.91326 \mathrm{E}+19$ & 651.5 & 290.9 \\
\hline & & 55.0 & 2.57 & 250.0 & $2.70665 \mathrm{E}+13$ & $1.74015 \mathrm{E}+13$ & $6.81733 \mathrm{E}+13$ & $7.34082 E+19$ & 652.8 & 291.4 \\
\hline & \multicolumn{10}{|c|}{ Mid-Cycle 157D Outage - 14 Day, 10 Hour Down Time $(7 / 23 / 2015,1800-8 / 7 / 2015,0400)$} \\
\hline & EOC $(8 / 12 / 2015,0400)$ & 59.7 & 2.80 & 253.8 & $2.77057 \mathrm{E}+13$ & $1.76389 \mathrm{E}+13$ & $6.93437 \mathrm{E}+13$ & $7.99722 \mathrm{E}+19$ & 658.8 & 294.3 \\
\hline \multirow[t]{8}{*}{$158 \mathrm{~A}$} & \multicolumn{10}{|c|}{ Cycle 158A Outage - 91 Day, 11 Hour Down Time } \\
\hline & $\operatorname{BOC}(11 / 11 / 2015,1500)$ & 59.7 & 2.80 & 250.5 & $2.71487 \mathrm{E}+13$ & $1.75103 \mathrm{E}+13$ & $6.83943 \mathrm{E}+13$ & $7.99722 \mathrm{E}+19$ & 653.5 & 291.8 \\
\hline & & 69.7 & 3.29 & 245.6 & $2.66795 \mathrm{E}+13$ & $1.71466 \mathrm{E}+13$ & $6.73544 \mathrm{E}+13$ & $9.37625 E+19$ & 645.6 & 288.0 \\
\hline & & 79.7 & 3.76 & 239.2 & $2.62849 \mathrm{E}+13$ & $1.66626 \mathrm{E}+13$ & $6.61651 \mathrm{E}+13$ & $1.07192 \mathrm{E}+20$ & 635.0 & 282.9 \\
\hline & & 89.7 & 4.23 & 240.6 & $2.66506 \mathrm{E}+13$ & $1.68814 \mathrm{E}+13$ & $6.68670 \mathrm{E}+13$ & $1.20681 \mathrm{E}+20$ & 637.4 & 284.1 \\
\hline & & 99.7 & 4.72 & 249.1 & $2.78339 \mathrm{E}+13$ & $1.73811 \mathrm{E}+13$ & $6.93493 \mathrm{E}+13$ & $1.34652 \mathrm{E}+20$ & 651.2 & 290.7 \\
\hline & & 111.7 & 5.33 & 256.3 & $2.89654 \mathrm{E}+13$ & $1.78525 \mathrm{E}+13$ & $7.14742 \mathrm{E}+13$ & $1.51935 \mathrm{E}+20$ & 662.9 & 296.3 \\
\hline & $\operatorname{EOC}(1 / 2 / 2016,0400)$ & 111.9 & 5.34 & 254.0 & $2.90451 \mathrm{E}+13$ & $1.77250 \mathrm{E}+13$ & $7.13040 \mathrm{E}+13$ & $1.52176 \mathrm{E}+20$ & 659.2 & 294.5 \\
\hline \multirow[t]{9}{*}{ 158B } & \multicolumn{10}{|c|}{ Cycle 158B Outage - 39 Day, 2 Hour Down Time } \\
\hline & BOC $(2 / 10 / 2016,0600)$ & 112.9 & 5.37 & 202.5 & $2.31424 \mathrm{E}+13$ & $1.42534 \mathrm{E}+13$ & $5.65577 \mathrm{E}+13$ & $1.53200 \mathrm{E}+20$ & 572.2 & 253.5 \\
\hline & & 113.9 & 5.41 & 227.4 & $2.59924 \mathrm{E}+13$ & $1.57178 \mathrm{E}+13$ & $6.34317 \mathrm{E}+13$ & $1.54284 \mathrm{E}+20$ & 615.3 & 273.6 \\
\hline & & 116.9 & 5.55 & 229.6 & $2.62123 \mathrm{E}+13$ & 1.60797E+13 & $6.48569 \mathrm{E}+13$ & $1.58198 \mathrm{E}+20$ & 619.1 & 275.4 \\
\hline & & 121.9 & 5.76 & 227.7 & $2.60671 \mathrm{E}+13$ & $1.56173 \mathrm{E}+13$ & $6.36818 \mathrm{E}+13$ & $1.64340 \mathrm{E}+20$ & 615.9 & 273.9 \\
\hline & & 126.9 & 5.98 & 234.9 & $2.70149 \mathrm{E}+13$ & $1.62399 \mathrm{E}+13$ & $6.61146 \mathrm{E}+13$ & $1.70663 \mathrm{E}+20$ & 627.9 & 279.6 \\
\hline & & 131.9 & 6.20 & 227.4 & $2.62656 \mathrm{E}+13$ & $1.59743 \mathrm{E}+13$ & $6.42712 \mathrm{E}+13$ & $1.76806 \mathrm{E}+20$ & 615.3 & 273.6 \\
\hline & & 141.9 & 6.67 & 238.6 & $2.76741 \mathrm{E}+13$ & $1.64680 \mathrm{E}+13$ & $6.72319 \mathrm{E}+13$ & $1.90175 \mathrm{E}+20$ & 634.1 & 282.5 \\
\hline & & 151.9 & 7.14 & 240.7 & $2.82942 \mathrm{E}+13$ & $1.66913 \mathrm{E}+13$ & $6.77946 \mathrm{E}+13$ & $2.03664 \mathrm{E}+20$ & 637.5 & 284.1 \\
\hline
\end{tabular}


TEM-10300-1

$03 / 01 / 2012$

Rev. 03

Title: $\quad$ ATF-1 Power Histories

\begin{tabular}{lllll} 
TEV No.: & 3119 & Rev. No.: & 0 & Project No.: \\
\hline
\end{tabular}

Table 13. ATF-11 Power History

\begin{tabular}{|c|c|c|c|c|c|c|c|c|c|c|}
\hline Cycle & Critical Dates & EFPD & $\begin{array}{c}\text { Burnup } \\
\text { (GWd/MTU) }\end{array}$ & $\begin{array}{l}\text { LHGR } \\
(\mathrm{W} / \mathrm{cm})\end{array}$ & $\begin{array}{l}\text { Thermal Flux } \\
\left(\mathrm{n} / \mathrm{cm}^{2 *} \mathrm{~s}\right)\end{array}$ & $\begin{array}{c}\text { Fast Flux } \\
\left(\mathrm{n} / \mathrm{cm}^{2 *} \mathrm{~s}>1\right. \\
\mathrm{MeV})\end{array}$ & $\begin{array}{l}\text { Total Flux } \\
\left(\mathrm{n} / \mathrm{cm}^{2 *} \mathrm{~s}\right)\end{array}$ & $\begin{array}{c}\text { Fission Density } \\
\text { (Fissions } / \mathrm{cm}^{3} \text { ) }\end{array}$ & $\begin{array}{c}\text { Fuel Centerline } \\
\text { Temperature } \\
\left({ }^{\circ} \mathrm{C}\right)\end{array}$ & $\mathrm{PICT}\left({ }^{\circ} \mathrm{C}\right)$ \\
\hline & EOC (4/1/2016, 0900) & 163.3 & 7.70 & 241.2 & $2.89444 \mathrm{E}+13$ & 1.67047E+13 & $6.87520 \mathrm{E}+13$ & $2.19502 E+20$ & 638.3 & 284.5 \\
\hline \multirow[t]{7}{*}{$159 \mathrm{~A}$} & \multicolumn{10}{|c|}{ Cycle 159A Outage - 77 Day, 2 Hour Down Time } \\
\hline & $\mathrm{BOC}(6 / 17 / 2016,1100)$ & 164.3 & 7.71 & 105.0 & $1.25608 \mathrm{E}+13$ & $7.28560 \mathrm{E}+12$ & $2.98619 \mathrm{E}+13$ & 2.19984E+20 & 384.6 & 170.3 \\
\hline & & 165.3 & 7.73 & 108.6 & $1.30538 \mathrm{E}+13$ & $7.53821 \mathrm{E}+12$ & $3.09637 \mathrm{E}+13$ & $2.20465 E+20$ & 392.1 & 173.5 \\
\hline & & 169.2 & 7.79 & 93.3 & $1.11892 \mathrm{E}+13$ & $6.53940 \mathrm{E}+12$ & $2.67891 \mathrm{E}+13$ & $2.22272 \mathrm{E}+20$ & 360.1 & 159.8 \\
\hline & \multicolumn{10}{|c|}{ Mid-Cycle 159A Outage - 3 Day, 21 Hour Down Time (6/23/2016, 1000 - 6/27/2016, 0700) } \\
\hline & & 170.6 & 7.81 & 136.4 & $1.64975 \mathrm{E}+13$ & $9.83888 \mathrm{E}+12$ & $4.04335 \mathrm{E}+13$ & $2.22814 \mathrm{E}+20$ & 448.3 & 197.9 \\
\hline & EOC $(6 / 28 / 2016,1800)$ & 170.8 & 7.82 & 151.9 & $1.84647 \mathrm{E}+13$ & $1.09402 \mathrm{E}+13$ & $4.63572 \mathrm{E}+13$ & $2.22995 \mathrm{E}+20$ & 478.8 & 211.3 \\
\hline \multirow[t]{11}{*}{$160 \mathrm{~A}$} & \multicolumn{10}{|c|}{ Cycle 160A Outage - 80 Day, 1 Hour Down Time } \\
\hline & $\mathrm{BOC}(9 / 16 / 2016,1900)$ & 171.8 & 7.86 & 237.7 & $2.84801 \mathrm{E}+13$ & 1.66129E+13 & $6.79736 \mathrm{E}+13$ & $2.24139 \mathrm{E}+20$ & 632.6 & 281.8 \\
\hline & & 173.8 & 7.96 & 236.3 & $2.83868 \mathrm{E}+13$ & $1.64606 \mathrm{E}+13$ & $6.76922 \mathrm{E}+13$ & $2.27029 \mathrm{E}+20$ & 630.3 & 280.7 \\
\hline & & 180.8 & 8.28 & 237.6 & $2.86692 \mathrm{E}+13$ & $1.65924 \mathrm{E}+13$ & $6.83243 E+13$ & $2.36243 E+20$ & 632.4 & 281.7 \\
\hline & & 190.8 & 8.75 & 236.4 & $2.87252 \mathrm{E}+13$ & $1.63898 \mathrm{E}+13$ & $6.78040 \mathrm{E}+13$ & $2.49491 \mathrm{E}+20$ & 630.3 & 280.7 \\
\hline & & 200.8 & 9.21 & 240.0 & $2.94452 \mathrm{E}+13$ & $1.67805 \mathrm{E}+13$ & $6.94233 \mathrm{E}+13$ & $2.62800 \mathrm{E}+20$ & 636.4 & 283.6 \\
\hline & & 210.8 & 9.69 & 243.5 & $3.02300 \mathrm{E}+13$ & $1.72639 \mathrm{E}+13$ & $7.13469 \mathrm{E}+13$ & $2.76289 \mathrm{E}+20$ & 642.1 & 286.3 \\
\hline & & 216.8 & 9.97 & 240.8 & $3.02131 \mathrm{E}+13$ & $1.68504 \mathrm{E}+13$ & $7.02939 \mathrm{E}+13$ & $2.84419 \mathrm{E}+20$ & 637.6 & 284.2 \\
\hline & & 218.4 & 10.06 & 226.9 & $2.86648 \mathrm{E}+13$ & $1.57755 \mathrm{E}+13$ & $6.64941 \mathrm{E}+13$ & $2.86828 E+20$ & 614.4 & 273.2 \\
\hline & & 220.8 & 10.17 & 221.5 & $2.79424 \mathrm{E}+13$ & $1.55837 \mathrm{E}+13$ & $6.54477 \mathrm{E}+13$ & $2.89959 \mathrm{E}+20$ & 605.3 & 268.9 \\
\hline & EOC $(11 / 8 / 2016,1100)$ & 223.5 & 10.28 & 211.7 & $2.67745 \mathrm{E}+13$ & $1.49859 \mathrm{E}+13$ & $6.29958 E+13$ & $2.93271 \mathrm{E}+20$ & 588.4 & 261.0 \\
\hline \multirow[t]{9}{*}{$160 \mathrm{~B}$} & \multicolumn{10}{|c|}{ Cycle 160B Outage - 42 Day, 1 Hour Down Time Outage } \\
\hline & $\mathrm{BOC}(12 / 20 / 2016,1200)$ & 224.5 & 10.32 & 226.9 & $2.86718 \mathrm{E}+13$ & $1.59659 \mathrm{E}+13$ & $6.72186 \mathrm{E}+13$ & $2.94355 E+20$ & 614.5 & 273.3 \\
\hline & & 226.5 & 10.42 & 225.9 & $2.85663 \mathrm{E}+13$ & $1.59079 \mathrm{E}+13$ & $6.70902 \mathrm{E}+13$ & $2.97125 \mathrm{E}+20$ & 612.8 & 272.5 \\
\hline & & 233.5 & 10.72 & 221.4 & $2.82304 \mathrm{E}+13$ & $1.55846 \mathrm{E}+13$ & $6.58704 \mathrm{E}+13$ & $3.05737 \mathrm{E}+20$ & 605.1 & 268.9 \\
\hline & & 243.5 & 11.14 & 212.9 & $2.72743 \mathrm{E}+13$ & $1.50270 \mathrm{E}+13$ & $6.36090 \mathrm{E}+13$ & $3.17721 \mathrm{E}+20$ & 590.6 & 262.1 \\
\hline & & 252.7 & 11.53 & 211.5 & $2.75958 \mathrm{E}+13$ & $1.49486 \mathrm{E}+13$ & $6.38914 \mathrm{E}+13$ & $3.28861 \mathrm{E}+20$ & 588.1 & 260.9 \\
\hline & \multicolumn{10}{|c|}{ Mid-Cycle 160B Outage - 5 Day, 14 Hour Down Time } \\
\hline & & 263.5 & 11.99 & 217.9 & $2.84608 \mathrm{E}+13$ & $1.52850 \mathrm{E}+13$ & $6.56976 \mathrm{E}+13$ & $3.41869 \mathrm{E}+20$ & 599.1 & 266.0 \\
\hline & & 273.5 & 12.41 & 214.1 & $2.82666 \mathrm{E}+13$ & $1.52895 \mathrm{E}+13$ & $6.66699 \mathrm{E}+13$ & $3.53793 E+20$ & 592.6 & 263.0 \\
\hline
\end{tabular}


TEM-10300-1

03/01/2012

TECHNICAL EVALUATION

Page 40 of 110

Rev. 03

Title: $\quad$ ATF-1 Power Histories

$\begin{array}{lllll}\text { TEV No.: } & 3119 & \text { Rev. No.: } & 0 & \text { Project No.: }\end{array}$

Table 13. ATF-11 Power History

\begin{tabular}{|c|c|c|c|c|c|c|c|c|c|c|}
\hline Cycle & Critical Dates & EFPD & $\begin{array}{l}\text { Burnup } \\
\text { (GWd/MTU) }\end{array}$ & $\begin{array}{l}\text { LHGR } \\
(\mathrm{W} / \mathrm{cm})\end{array}$ & $\begin{array}{l}\text { Thermal Flux } \\
\left(\mathrm{n} / \mathrm{cm}^{2 *} \mathrm{~s}\right)\end{array}$ & $\begin{array}{c}\text { Fast Flux } \\
\left(\mathrm{n} / \mathrm{cm}^{2 *} \mathrm{~s}>1\right. \\
\mathrm{MeV})\end{array}$ & $\begin{array}{l}\text { Total Flux } \\
\left(\mathrm{n} / \mathrm{cm}^{2 *} \mathrm{~s}\right)\end{array}$ & $\begin{array}{l}\text { Fission Density } \\
\text { (Fissions/cm³) }\end{array}$ & $\begin{array}{c}\text { Fuel Centerline } \\
\text { Temperature } \\
\left({ }^{\circ} \mathrm{C}\right)\end{array}$ & $\mathrm{PICT}\left({ }^{\circ} \mathrm{C}\right)$ \\
\hline & EOC $(2 / 23 / 2017,1400)$ & 283.0 & 12.83 & 226.9 & $3.04892 \mathrm{E}+13$ & $1.61212 \mathrm{E}+13$ & $7.02533 \mathrm{E}+13$ & $3.65897 \mathrm{E}+20$ & 614.4 & 273.2 \\
\hline \multirow[t]{9}{*}{$161 \mathrm{~A}$} & \multicolumn{10}{|c|}{ Cycle 161A Outage - 95 Day, 13 Hour Down Time } \\
\hline & BOC $(5 / 30 / 2017,0300)$ & 284.7 & 12.88 & 193.9 & $2.61178 \mathrm{E}+13$ & $1.38059 \mathrm{E}+13$ & $5.96015 \mathrm{E}+13$ & $3.67462 \mathrm{E}+20$ & 557.0 & 246.6 \\
\hline & & 285.0 & 12.89 & 171.8 & $2.31335 \mathrm{E}+13$ & $1.21264 \mathrm{E}+13$ & $5.29002 E+13$ & $3.67703 \mathrm{E}+20$ & 516.5 & 228.2 \\
\hline & & 287.0 & 12.97 & 177.0 & $2.38566 \mathrm{E}+13$ & $1.25775 \mathrm{E}+13$ & $5.46289 \mathrm{E}+13$ & $3.69931 \mathrm{E}+20$ & 526.2 & 232.5 \\
\hline & & 289.0 & 13.05 & 179.4 & $2.42558 \mathrm{E}+13$ & $1.27231 \mathrm{E}+13$ & $5.52763 E+13$ & $3.72280 \mathrm{E}+20$ & 530.5 & 234.5 \\
\hline & & 291.0 & 13.13 & 179.8 & $2.43517 \mathrm{E}+13$ & $1.27765 \mathrm{E}+13$ & $5.54883 \mathrm{E}+13$ & $3.74568 \mathrm{E}+20$ & 531.4 & 234.9 \\
\hline & & 293.0 & 13.22 & 183.4 & $2.48695 \mathrm{E}+13$ & $1.29822 \mathrm{E}+13$ & $5.66937 \mathrm{E}+13$ & $3.76917 \mathrm{E}+20$ & 537.9 & 237.8 \\
\hline & & 295.0 & 13.30 & 188.7 & $2.56378 \mathrm{E}+13$ & $1.33491 \mathrm{E}+13$ & $5.83726 \mathrm{E}+13$ & $3.79326 \mathrm{E}+20$ & 547.6 & 242.3 \\
\hline & $\operatorname{EOC}(6 / 11 / 2017,1600)$ & 295.5 & 13.32 & 193.0 & $2.62038 \mathrm{E}+13$ & $1.36208 \mathrm{E}+13$ & $5.94270 \mathrm{E}+13$ & $3.79808 E+20$ & 555.3 & 245.8 \\
\hline \multirow[t]{10}{*}{$162 \mathrm{~A}$} & \multicolumn{10}{|c|}{ Cycle 162A Outage } \\
\hline & $\mathrm{BOC}$ & 296.5 & 13.36 & 241.9 & $3.20481 \mathrm{E}+13$ & $1.67104 \mathrm{E}+13$ & $7.26668 \mathrm{E}+13$ & $3.80892 \mathrm{E}+20$ & 639.4 & 285.1 \\
\hline & & 298.5 & 13.45 & 229.9 & $3.08311 \mathrm{E}+13$ & $1.61301 \mathrm{E}+13$ & $7.00795 E+13$ & $3.83662 E+20$ & 619.6 & 275.7 \\
\hline & & 305.5 & 13.76 & 228.8 & $3.07364 \mathrm{E}+13$ & $1.59780 \mathrm{E}+13$ & $6.98131 \mathrm{E}+13$ & $3.92333 E+20$ & 617.7 & 274.7 \\
\hline & & 315.5 & 14.18 & 225.7 & $3.01065 \mathrm{E}+13$ & $1.54770 \mathrm{E}+13$ & $6.79694 \mathrm{E}+13$ & $4.04498 \mathrm{E}+20$ & 612.4 & 272.3 \\
\hline & & 325.5 & 14.62 & 232.5 & $3.14585 \mathrm{E}+13$ & $1.60128 \mathrm{E}+13$ & $7.05376 \mathrm{E}+13$ & $4.16903 \mathrm{E}+20$ & 623.9 & 277.7 \\
\hline & & 335.5 & 15.05 & 232.7 & $3.16922 \mathrm{E}+13$ & $1.60077 \mathrm{E}+13$ & $7.08367 E+13$ & $4.29308 \mathrm{E}+20$ & 624.3 & 277.9 \\
\hline & & 345.5 & 15.52 & 236.8 & $3.31265 \mathrm{E}+13$ & $1.65714 \mathrm{E}+13$ & $7.37776 \mathrm{E}+13$ & $4.42617 \mathrm{E}+20$ & 631.0 & 281.1 \\
\hline & & 354.5 & 15.91 & 229.8 & $3.23120 \mathrm{E}+13$ & $1.61472 \mathrm{E}+13$ & $7.22676 \mathrm{E}+13$ & $4.53818 \mathrm{E}+20$ & 619.4 & 275.5 \\
\hline & EOC & 357.5 & 16.04 & 228.6 & $3.26005 \mathrm{E}+13$ & $1.61182 \mathrm{E}+13$ & $7.24869 E+13$ & $4.57552 \mathrm{E}+20$ & 617.4 & 274.6 \\
\hline
\end{tabular}


TEM-10300-1

$03 / 01 / 2012$

Rev. 03

Title: $\quad$ ATF-1 Power Histories

$\begin{array}{lllll}\text { TEV No.: } & 3119 & \text { Rev. No.: } & 0 & \text { Project No.: }\end{array} 31418$

Table 14. ATF-12 Power History

\begin{tabular}{|c|c|c|c|c|c|c|c|c|c|c|}
\hline Cycle & Critical Dates & EFPD & $\begin{array}{c}\text { Burnup } \\
\text { (GWd/MTU) }\end{array}$ & $\begin{array}{l}\text { LHGR } \\
(\mathrm{W} / \mathrm{cm})\end{array}$ & $\begin{array}{l}\text { Thermal Flux } \\
\left(\mathrm{n} / \mathrm{cm}^{2 *} \mathrm{~s}\right)\end{array}$ & $\begin{array}{c}\text { Fast Flux } \\
\left(\mathrm{n} / \mathrm{cm}^{2 *} \mathrm{~s}>1\right. \\
\mathrm{MeV})\end{array}$ & $\begin{array}{l}\text { Total Flux } \\
\left(\mathrm{n} / \mathrm{cm}^{2 *} \mathrm{~s}\right)\end{array}$ & $\begin{array}{l}\text { Fission Density } \\
\left(\text { Fissions } / \mathrm{cm}^{3}\right)\end{array}$ & $\begin{array}{c}\text { Fuel Centerline } \\
\text { Temperature } \\
\left({ }^{\circ} \mathrm{C}\right)\end{array}$ & PICT $\left({ }^{\circ} \mathrm{C}\right)$ \\
\hline \multirow[t]{2}{*}{$157 \mathrm{C}$} & BOC $(2 / 10 / 2015,0600)$ & 0.0 & 0.00 & 254.0 & $2.61036 \mathrm{E}+13$ & $1.57324 \mathrm{E}+13$ & $6.20242 E+13$ & $0.00000 \mathrm{E}+00$ & 686.3 & 335.6 \\
\hline & EOC $(2 / 15 / 2015,0800)$ & 5.4 & 0.29 & 257.8 & $2.66016 \mathrm{E}+13$ & $1.60305 E+13$ & $6.30423 \mathrm{E}+13$ & $8.31036 \mathrm{E}+18$ & 692.5 & 338.7 \\
\hline \multirow[t]{13}{*}{$157 \mathrm{D}$} & \multicolumn{10}{|c|}{ Cycle 157D Outage - 104 Day, 3 Hour Down Time } \\
\hline & $\operatorname{BOC}(5 / 30 / 2015,1100)$ & 5.4 & 0.29 & 216.5 & $2.23587 \mathrm{E}+13$ & $1.37005 E+13$ & $5.33636 \mathrm{E}+13$ & $8.31036 \mathrm{E}+18$ & 621.6 & 302.5 \\
\hline & & 8.4 & 0.43 & 237.2 & $2.44768 \mathrm{E}+13$ & $1.46833 E+13$ & $5.79139 \mathrm{E}+13$ & $1.24053 \mathrm{E}+19$ & 657.8 & 320.9 \\
\hline & & 15.4 & 0.77 & 240.6 & $2.49378 \mathrm{E}+13$ & $1.50125 \mathrm{E}+13$ & $5.93116 \mathrm{E}+13$ & $2.19201 \mathrm{E}+19$ & 663.7 & 323.9 \\
\hline & & 22.4 & 1.10 & 240.9 & $2.51177 \mathrm{E}+13$ & $1.50287 \mathrm{E}+13$ & $5.95301 \mathrm{E}+13$ & $3.14951 \mathrm{E}+19$ & 664.2 & 324.2 \\
\hline & & 29.4 & 1.44 & 242.4 & $2.53659 \mathrm{E}+13$ & $1.50181 \mathrm{E}+13$ & $5.98884 \mathrm{E}+13$ & $4.10700 \mathrm{E}+19$ & 666.8 & 325.5 \\
\hline & & 36.4 & 1.78 & 249.8 & $2.63571 \mathrm{E}+13$ & $1.56363 E+13$ & $6.22463 \mathrm{E}+13$ & $5.08859 \mathrm{E}+19$ & 679.3 & 331.9 \\
\hline & & 43.4 & 2.13 & 250.9 & $2.66189 \mathrm{E}+13$ & $1.57190 \mathrm{E}+13$ & $6.27653 \mathrm{E}+13$ & $6.07620 \mathrm{E}+19$ & 681.1 & 332.8 \\
\hline & & 50.4 & 2.48 & 259.7 & $2.77067 \mathrm{E}+13$ & $1.62996 \mathrm{E}+13$ & $6.50065 \mathrm{E}+13$ & $7.09392 \mathrm{E}+19$ & 695.7 & 340.4 \\
\hline & & 57.4 & 2.84 & 263.7 & $2.84481 \mathrm{E}+13$ & $1.64211 \mathrm{E}+13$ & $6.63271 \mathrm{E}+13$ & $8.12368 \mathrm{E}+19$ & 702.3 & 343.8 \\
\hline & & 60.4 & 3.00 & 266.1 & $2.88528 \mathrm{E}+13$ & $1.65486 \mathrm{E}+13$ & $6.67282 E+13$ & $8.58135 \mathrm{E}+19$ & 706.2 & 345.9 \\
\hline & \multicolumn{10}{|c|}{ Mid-Cycle 157D Outage - 14 Day, 10 Hour Down Time $(7 / 23 / 2015,1800$ - 8/7/2015, 0400) } \\
\hline & EOC $(8 / 12 / 2015,0400)$ & 65.1 & 3.26 & 265.8 & $2.90774 \mathrm{E}+13$ & $1.66109 \mathrm{E}+13$ & $6.73276 \mathrm{E}+13$ & $9.32808 \mathrm{E}+19$ & 705.7 & 345.6 \\
\hline \multirow[t]{8}{*}{$158 \mathrm{~A}$} & \multicolumn{10}{|c|}{ Cycle 158A Outage - 91 Day, 11 Hour Down Time } \\
\hline & $\operatorname{BOC}(11 / 11 / 2015,1500)$ & 65.1 & 3.26 & 265.3 & $2.88994 \mathrm{E}+13$ & $1.66328 \mathrm{E}+13$ & $6.70087 \mathrm{E}+13$ & $9.32808 \mathrm{E}+19$ & 704.7 & 345.1 \\
\hline & & 75.1 & 3.77 & 261.2 & $2.84495 \mathrm{E}+13$ & $1.64300 \mathrm{E}+13$ & $6.62801 \mathrm{E}+13$ & $1.07854 \mathrm{E}+20$ & 698.1 & 341.6 \\
\hline & & 85.1 & 4.28 & 255.9 & $2.82776 \mathrm{E}+13$ & $1.61001 \mathrm{E}+13$ & $6.56922 \mathrm{E}+13$ & $1.22186 \mathrm{E}+20$ & 689.5 & 337.2 \\
\hline & & 95.1 & 4.78 & 258.2 & $2.87572 E+13$ & $1.62863 E+13$ & $6.65465 \mathrm{E}+13$ & $1.36639 \mathrm{E}+20$ & 693.3 & 339.2 \\
\hline & & 105.1 & 5.30 & 264.3 & $2.97122 \mathrm{E}+13$ & $1.66025 \mathrm{E}+13$ & $6.82129 \mathrm{E}+13$ & $1.51333 \mathrm{E}+20$ & 703.2 & 344.3 \\
\hline & & 117.1 & 5.93 & 270.3 & $3.06378 \mathrm{E}+13$ & $1.69946 \mathrm{E}+13$ & $6.97352 \mathrm{E}+13$ & $1.69519 \mathrm{E}+20$ & 712.9 & 349.4 \\
\hline & $\operatorname{EOC}(1 / 2 / 2016,0400)$ & 117.3 & 5.94 & 267.8 & $3.07457 \mathrm{E}+13$ & $1.68435 E+13$ & $6.95983 E+13$ & $1.69760 \mathrm{E}+20$ & 708.8 & 347.2 \\
\hline \multirow[t]{5}{*}{$158 \mathrm{~B}$} & \multicolumn{10}{|c|}{ Cycle 158B Outage - 39 Day, 2 Hour Down Time } \\
\hline & BOC $(2 / 10 / 2016,0600)$ & 118.3 & 5.98 & 214.0 & $2.46240 \mathrm{E}+13$ & $1.33584 \mathrm{E}+13$ & $5.52480 \mathrm{E}+13$ & $1.70844 \mathrm{E}+20$ & 617.1 & 300.2 \\
\hline & & 119.3 & 6.02 & 238.0 & $2.74550 \mathrm{E}+13$ & $1.49546 \mathrm{E}+13$ & $6.21175 \mathrm{E}+13$ & $1.71988 \mathrm{E}+20$ & 659.3 & 321.6 \\
\hline & & 122.3 & 6.16 & 245.4 & $2.83160 \mathrm{E}+13$ & $1.53329 \mathrm{E}+13$ & $6.40046 \mathrm{E}+13$ & $1.76144 \mathrm{E}+20$ & 671.8 & 328.1 \\
\hline & & 127.3 & 6.41 & 239.5 & $2.77293 \mathrm{E}+13$ & $1.49981 \mathrm{E}+13$ & $6.28016 \mathrm{E}+13$ & $1.83189 \mathrm{E}+20$ & 661.8 & 322.9 \\
\hline
\end{tabular}


TEM-10300-1

$03 / 01 / 2012$

Rev. 03

Title: $\quad$ ATF-1 Power Histories

\begin{tabular}{lllll} 
TEV No.: & 3119 & Rev. No.: & 0 & Project No.: \\
\hline
\end{tabular}

Table 14. ATF-12 Power History

\begin{tabular}{|c|c|c|c|c|c|c|c|c|c|c|}
\hline Cycle & Critical Dates & EFPD & $\begin{array}{c}\text { Burnup } \\
\text { (GWd/MTU) }\end{array}$ & $\begin{array}{l}\text { LHGR } \\
(\mathrm{W} / \mathrm{cm})\end{array}$ & $\begin{array}{l}\text { Thermal Flux } \\
\left(\mathrm{n} / \mathrm{cm}^{2 *} \mathrm{~s}\right)\end{array}$ & $\begin{array}{c}\text { Fast Flux } \\
\left(\mathrm{n} / \mathrm{cm}^{2 *} \mathrm{~s}>1\right. \\
\mathrm{MeV})\end{array}$ & $\begin{array}{l}\text { Total Flux } \\
\left(\mathrm{n} / \mathrm{cm}^{2 *} \mathrm{~s}\right)\end{array}$ & $\begin{array}{l}\text { Fission Density } \\
\text { (Fissions } / \mathrm{cm}^{3} \text { ) }\end{array}$ & $\begin{array}{c}\text { Fuel Centerline } \\
\text { Temperature } \\
\left({ }^{\circ} \mathrm{C}\right)\end{array}$ & PICT $\left({ }^{\circ} \mathrm{C}\right)$ \\
\hline & & 132.3 & 6.66 & 250.0 & $2.90057 \mathrm{E}+13$ & $1.58043 \mathrm{E}+13$ & $6.58343 \mathrm{E}+13$ & $1.90476 \mathrm{E}+20$ & 679.6 & 332.1 \\
\hline & & 137.3 & 6.92 & 244.8 & $2.86168 \mathrm{E}+13$ & $1.55497 \mathrm{E}+13$ & $6.47709 \mathrm{E}+13$ & $1.97642 \mathrm{E}+20$ & 670.8 & 327.5 \\
\hline & & 147.3 & 7.41 & 255.2 & $2.99882 \mathrm{E}+13$ & $1.60354 \mathrm{E}+13$ & $6.72986 \mathrm{E}+13$ & $2.11854 \mathrm{E}+20$ & 688.2 & 336.5 \\
\hline & & 157.3 & 7.90 & 249.3 & $2.95010 \mathrm{E}+13$ & $1.57939 \mathrm{E}+13$ & $6.62397 \mathrm{E}+13$ & $2.25705 \mathrm{E}+20$ & 678.5 & 331.5 \\
\hline & EOC $(4 / 1 / 2016,0900)$ & 168.7 & 8.47 & 250.9 & $3.03763 E+13$ & $1.59519 \mathrm{E}+13$ & $6.74149 \mathrm{E}+13$ & $2.42145 \mathrm{E}+20$ & 681.1 & 332.9 \\
\hline \multirow[t]{7}{*}{$159 \mathrm{~A}$} & \multicolumn{10}{|c|}{ Cycle 159A Outage - 77 Day, 2 Hour Down Time } \\
\hline & BOC $(6 / 17 / 2016,1100)$ & 169.7 & 8.49 & 109.8 & $1.32662 \mathrm{E}+13$ & $6.99830 \mathrm{E}+12$ & $2.96078 \mathrm{E}+13$ & $2.42747 \mathrm{E}+20$ & 411.3 & 199.2 \\
\hline & & 170.7 & 8.51 & 113.6 & $1.37653 \mathrm{E}+13$ & $7.20340 \mathrm{E}+12$ & $3.06218 \mathrm{E}+13$ & $2.43289 \mathrm{E}+20$ & 419.4 & 203.0 \\
\hline & & 174.6 & 8.60 & 96.8 & $1.17531 \mathrm{E}+13$ & $6.08498 \mathrm{E}+12$ & $2.59973 \mathrm{E}+13$ & $2.45698 \mathrm{E}+20$ & 383.1 & 185.7 \\
\hline & \multicolumn{10}{|c|}{ Mid-Cycle 159A Outage - 3 Day, 21 Hour Down Time (6/23/2016, 1000 - 6/27/2016, 0700) } \\
\hline & & 176.0 & 8.62 & 141.0 & $1.73083 \mathrm{E}+13$ & $9.04627 \mathrm{E}+12$ & $3.94127 \mathrm{E}+13$ & $2.46300 \mathrm{E}+20$ & 476.7 & 230.7 \\
\hline & $\mathrm{EOC}(6 / 28 / 2016,1800)$ & 176.2 & 8.62 & 155.9 & $1.92759 \mathrm{E}+13$ & $1.00524 \mathrm{E}+13$ & $4.47133 \mathrm{E}+13$ & $2.46480 \mathrm{E}+20$ & 506.9 & 245.5 \\
\hline $160 \mathrm{~A}$ & \multicolumn{10}{|c|}{ Cycle 160A Outage - 80 Day, 1 Hour Down Time } \\
\hline & BOC $(9 / 16 / 2016,1900)$ & 177.2 & 8.67 & 288.7 & $3.50708 \mathrm{E}+13$ & $1.84353 \mathrm{E}+13$ & $7.81469 \mathrm{E}+13$ & $2.47866 \mathrm{E}+20$ & 742.0 & 364.7 \\
\hline & & 179.2 & 8.79 & 279.4 & $3.40809 \mathrm{E}+13$ & $1.76395 \mathrm{E}+13$ & $7.59687 \mathrm{E}+13$ & $2.51178 \mathrm{E}+20$ & 727.3 & 356.9 \\
\hline & & 186.2 & 9.19 & 286.9 & $3.50359 \mathrm{E}+13$ & $1.80932 \mathrm{E}+13$ & $7.79607 \mathrm{E}+13$ & $2.62680 \mathrm{E}+20$ & 739.1 & 363.2 \\
\hline & & 196.2 & 9.77 & 293.5 & $3.61563 E+13$ & $1.86173 \mathrm{E}+13$ & $7.97206 \mathrm{E}+13$ & $2.79180 \mathrm{E}+20$ & 749.3 & 368.6 \\
\hline & & 206.2 & 10.35 & 296.2 & $3.68748 \mathrm{E}+13$ & $1.86476 \mathrm{E}+13$ & $8.10698 \mathrm{E}+13$ & $2.95801 \mathrm{E}+20$ & 753.4 & 370.7 \\
\hline & & 216.2 & 10.93 & 295.5 & $3.72746 \mathrm{E}+13$ & $1.87939 \mathrm{E}+13$ & $8.20419 \mathrm{E}+13$ & $3.12361 \mathrm{E}+20$ & 752.3 & 370.1 \\
\hline & & 222.2 & 11.31 & 315.8 & $4.04030 \mathrm{E}+13$ & $2.01112 \mathrm{E}+13$ & $8.78103 \mathrm{E}+13$ & $3.23261 \mathrm{E}+20$ & 782.5 & 386.4 \\
\hline & & 223.8 & 11.41 & 287.4 & $3.70873 E+13$ & $1.83422 \mathrm{E}+13$ & $8.00652 E+13$ & $3.26152 \mathrm{E}+20$ & 739.9 & 363.6 \\
\hline & & 226.2 & 11.55 & 286.0 & $3.70474 \mathrm{E}+13$ & $1.82628 \mathrm{E}+13$ & $8.00805 \mathrm{E}+13$ & $3.30066 \mathrm{E}+20$ & 737.8 & 362.4 \\
\hline & EOC $(11 / 8 / 2016,1100)$ & 228.9 & 11.69 & 274.7 & $3.57456 \mathrm{E}+13$ & $1.75512 \mathrm{E}+13$ & $7.76072 \mathrm{E}+13$ & $3.34161 \mathrm{E}+20$ & 720.0 & 353.1 \\
\hline \multirow[t]{6}{*}{$160 \mathrm{~B}$} & \multicolumn{10}{|c|}{ Cycle 160B Outage - 42 Day, 1 Hour Down Time Outage } \\
\hline & BOC $(12 / 20 / 2016,1200)$ & 229.9 & 11.74 & 289.1 & $3.78315 \mathrm{E}+13$ & $2.03526 \mathrm{E}+13$ & $8.82972 \mathrm{E}+13$ & $3.35546 \mathrm{E}+20$ & 742.6 & 365.0 \\
\hline & & 231.9 & 11.85 & 281.8 & $3.69640 \mathrm{E}+13$ & $1.98427 \mathrm{E}+13$ & $8.62784 \mathrm{E}+13$ & $3.38798 \mathrm{E}+20$ & 731.2 & 359.0 \\
\hline & & 238.9 & 12.24 & 278.9 & $3.67159 \mathrm{E}+13$ & $1.96276 \mathrm{E}+13$ & $8.54356 \mathrm{E}+13$ & $3.49818 \mathrm{E}+20$ & 726.7 & 356.6 \\
\hline & & 248.9 & 12.78 & 275.6 & $3.65701 \mathrm{E}+13$ & $1.92786 \mathrm{E}+13$ & $8.47128 \mathrm{E}+13$ & $3.65234 \mathrm{E}+20$ & 721.4 & 353.8 \\
\hline & & 258.1 & 13.29 & 275.0 & $3.73580 \mathrm{E}+13$ & $1.93586 \mathrm{E}+13$ & $8.58147 \mathrm{E}+13$ & $3.79687 E+20$ & 720.4 & 353.3 \\
\hline
\end{tabular}


TEM-10300-1

03/01/2012

TECHNICAL EVALUATION

Page 43 of 110

Rev. 03

ATF-1 Power Histories

$\begin{array}{lllll}\text { TEV No.: } & 3119 & \text { Rev. No.: } & 0 & \text { Project No.: } \\ & 31418\end{array}$

Table 14. ATF-12 Power History

\begin{tabular}{|c|c|c|c|c|c|c|c|c|c|c|}
\hline Cycle & Critical Dates & EFPD & $\begin{array}{c}\text { Burnup } \\
\text { (GWd/MTU) }\end{array}$ & $\begin{array}{l}\text { LHGR } \\
(\mathrm{W} / \mathrm{cm})\end{array}$ & $\begin{array}{l}\text { Thermal Flux } \\
\left(\mathrm{n} / \mathrm{cm}^{2 *} \mathrm{~s}\right)\end{array}$ & $\begin{array}{c}\text { Fast Flux } \\
\left(\mathrm{n} / \mathrm{cm}^{2 *} \mathrm{~s}>1\right. \\
\mathrm{MeV})\end{array}$ & $\begin{array}{l}\text { Total Flux } \\
\left(\mathrm{n} / \mathrm{cm}^{2 *} \mathrm{~s}\right)\end{array}$ & $\begin{array}{c}\text { Fission Density } \\
\left(\text { Fissions } / \mathrm{cm}^{3}\right)\end{array}$ & $\begin{array}{c}\text { Fuel Centerline } \\
\text { Temperature } \\
\left({ }^{\circ} \mathrm{C}\right)\end{array}$ & $\mathrm{PICT}\left({ }^{\circ} \mathrm{C}\right)$ \\
\hline & \multicolumn{10}{|c|}{ Mid-Cycle 160B Outage - 5 Day, 14 Hour Down Time } \\
\hline & & 268.9 & 13.89 & 283.0 & $3.86284 \mathrm{E}+13$ & $2.00243 E+13$ & $8.86391 \mathrm{E}+13$ & $3.97091 \mathrm{E}+20$ & 733.1 & 360.0 \\
\hline & & 278.9 & 14.45 & 289.1 & $3.98678 \mathrm{E}+13$ & $2.04488 \mathrm{E}+13$ & $9.08826 \mathrm{E}+13$ & $4.13049 \mathrm{E}+20$ & 742.5 & 364.9 \\
\hline & EOC $(2 / 23 / 2017,1400)$ & 288.4 & 15.00 & 295.5 & $4.18213 E+13$ & $2.08920 E+13$ & $9.37923 E+13$ & $4.28766 \mathrm{E}+20$ & 752.3 & 370.2 \\
\hline \multirow[t]{10}{*}{$162 \mathrm{~A}$} & \multicolumn{10}{|c|}{ Withheld from Cycle 161A PALM } \\
\hline & $\mathrm{BOC}$ & 289.4 & 15.04 & 252.6 & $3.62946 \mathrm{E}+13$ & $1.82557 \mathrm{E}+13$ & $8.22129 E+13$ & $4.29971 \mathrm{E}+20$ & 684.0 & 334.3 \\
\hline & & 291.4 & 15.14 & 241.7 & $3.36729 \mathrm{E}+13$ & $1.68725 \mathrm{E}+13$ & $7.63277 \mathrm{E}+13$ & $4.32741 \mathrm{E}+20$ & 665.6 & 324.9 \\
\hline & & 298.4 & 15.48 & 245.3 & $3.45328 \mathrm{E}+13$ & $1.74330 \mathrm{E}+13$ & $7.85021 \mathrm{E}+13$ & $4.42497 \mathrm{E}+20$ & 671.6 & 328.0 \\
\hline & & 308.4 & 15.98 & 252.6 & $3.63533 \mathrm{E}+13$ & $1.80657 E+13$ & $8.16911 \mathrm{E}+13$ & $4.56769 \mathrm{E}+20$ & 683.9 & 334.3 \\
\hline & & 318.4 & 16.47 & 247.5 & $3.60806 \mathrm{E}+13$ & $1.77975 \mathrm{E}+13$ & $8.10033 E+13$ & $4.70740 \mathrm{E}+20$ & 675.4 & 329.9 \\
\hline & & 328.4 & 16.97 & 251.0 & $3.75436 \mathrm{E}+13$ & $1.81771 \mathrm{E}+13$ & $8.34426 \mathrm{E}+13$ & $4.84952 E+20$ & 681.2 & 332.9 \\
\hline & & 338.4 & 17.46 & 248.3 & $3.72767 \mathrm{E}+13$ & $1.79263 \mathrm{E}+13$ & $8.25657 E+13$ & $4.98923 \mathrm{E}+20$ & 676.8 & 330.6 \\
\hline & & 347.4 & 17.92 & 255.4 & $3.93864 \mathrm{E}+13$ & $1.86961 \mathrm{E}+13$ & $8.67134 E+13$ & $5.12171 \mathrm{E}+20$ & 688.6 & 336.7 \\
\hline & EOC & 350.4 & 18.07 & 254.4 & $3.99654 \mathrm{E}+13$ & $1.86178 \mathrm{E}+13$ & $8.73965 E+13$ & $5.16387 \mathrm{E}+20$ & 686.9 & 335.9 \\
\hline
\end{tabular}


TEM-10300-1

$03 / 01 / 2012$

Rev. 03

Title:

ATF-1 Power Histories

\begin{tabular}{lllll} 
TEV No.: & 3119 & Rev. No.: & 0 & Project No.: \\
\hline
\end{tabular}

Date: $12 / 01 / 2017$

The results documented in Table 15 DO NOT APPLY to the misloaded enriched pellet in ATF-13.

Table 15. ATF-13 Power History

\begin{tabular}{|c|c|c|c|c|c|c|c|c|c|c|}
\hline Cycle & Critical Dates & EFPD & $\begin{array}{l}\text { Burnup } \\
\text { (GWd/MTU) }\end{array}$ & $\begin{array}{c}\text { LHGR } \\
(\mathrm{W} / \mathrm{cm})\end{array}$ & $\begin{array}{l}\text { Thermal Flux } \\
\left(\mathrm{n} / \mathrm{cm}^{2 *} \mathrm{~s}\right)\end{array}$ & $\begin{array}{c}\text { Fast Flux } \\
\left(\mathrm{n} / \mathrm{cm}^{2 *} s>1\right. \\
\mathrm{MeV})\end{array}$ & $\begin{array}{l}\text { Total Flux } \\
\left(\mathrm{n} / \mathrm{cm}^{2 *} \mathrm{~s}\right)\end{array}$ & $\begin{array}{l}\text { Fission Density } \\
\text { (Fissions/cm }\end{array}$ & $\begin{array}{l}\text { Fuel Centerline } \\
\text { Temperature } \\
\left({ }^{\circ} \mathrm{C}\right)\end{array}$ & $\mathrm{PICT}\left({ }^{\circ} \mathrm{C}\right)$ \\
\hline \multirow[t]{2}{*}{$157 \mathrm{C}$} & BOC $(2 / 10 / 2015,0600)$ & 0.0 & 0.00 & 467.8 & $4.84993 \mathrm{E}+13$ & $3.22515 \mathrm{E}+13$ & $1.24189 \mathrm{E}+14$ & $0.00000 \mathrm{E}+00$ & 945.4 & 445.6 \\
\hline & EOC $(2 / 15 / 2015,0800)$ & 5.4 & 0.52 & 472.2 & $4.94263 E+13$ & $3.26452 E+13$ & $1.25858 \mathrm{E}+14$ & 1.47539E+19 & 950.6 & 448.5 \\
\hline \multirow[t]{13}{*}{ 157D } & \multicolumn{10}{|c|}{ Cycle 157D Outage - 104 Day, 3 Hour Down Time } \\
\hline & $\operatorname{BOC}(5 / 30 / 2015,1100)$ & 5.4 & 0.52 & 403.8 & $4.21560 \mathrm{E}+13$ & $2.78514 \mathrm{E}+13$ & $1.06830 \mathrm{E}+14$ & $1.47539 \mathrm{E}+19$ & 868.6 & 402.8 \\
\hline & & 8.4 & 0.79 & 435.0 & $4.54068 \mathrm{E}+13$ & $3.02968 \mathrm{E}+13$ & $1.16105 \mathrm{E}+14$ & $2.23416 \mathrm{E}+19$ & 906.7 & 423.9 \\
\hline & & 15.4 & 1.39 & 433.4 & $4.56461 \mathrm{E}+13$ & $3.01510 \mathrm{E}+13$ & $1.16678 \mathrm{E}+14$ & $3.96248 \mathrm{E}+19$ & 904.8 & 422.8 \\
\hline & & 22.4 & 2.00 & 427.8 & $4.54671 \mathrm{E}+13$ & $2.95845 \mathrm{E}+13$ & 1.15283E+14 & $5.67272 \mathrm{E}+19$ & 898.0 & 419.1 \\
\hline & & 29.4 & 2.60 & 432.6 & $4.65389 \mathrm{E}+13$ & $2.99491 \mathrm{E}+13$ & $1.17330 \mathrm{E}+14$ & $7.38899 \mathrm{E}+19$ & 903.8 & 422.3 \\
\hline & & 36.4 & 3.22 & 445.0 & $4.83366 \mathrm{E}+13$ & $3.08469 E+13$ & $1.21293 \mathrm{E}+14$ & $9.15344 \mathrm{E}+19$ & 918.6 & 430.6 \\
\hline & & 43.4 & 3.84 & 445.5 & $4.89632 \mathrm{E}+13$ & $3.10589 \mathrm{E}+13$ & $1.22284 \mathrm{E}+14$ & $1.09179 \mathrm{E}+20$ & 919.2 & 430.9 \\
\hline & & 50.4 & 4.48 & 457.5 & $5.08002 E+13$ & $3.16607 E+13$ & $1.25989 \mathrm{E}+14$ & $1.27185 \mathrm{E}+20$ & 933.4 & 438.8 \\
\hline & & 57.4 & 5.11 & 459.8 & $5.17102 \mathrm{E}+13$ & $3.19413 E+13$ & $1.27265 \mathrm{E}+14$ & $1.45251 \mathrm{E}+20$ & 936.0 & 440.3 \\
\hline & & 60.4 & 5.38 & 454.4 & $5.17848 \mathrm{E}+13$ & $3.16206 \mathrm{E}+13$ & $1.26786 \mathrm{E}+14$ & $1.53019 \mathrm{E}+20$ & 929.8 & 436.8 \\
\hline & \multicolumn{10}{|c|}{ Mid-Cycle 157D Outage - 14 Day, 10 Hour Down Time (7/23/2015, 1800 - 8/7/2015, 0400) } \\
\hline & EOC $(8 / 12 / 2015,0400)$ & 65.1 & 5.82 & 456.5 & $5.26536 \mathrm{E}+13$ & $3.16360 \mathrm{E}+13$ & $1.27650 \mathrm{E}+14$ & $1.65485 \mathrm{E}+20$ & 932.2 & 438.2 \\
\hline \multirow[t]{8}{*}{$158 \mathrm{~A}$} & \multicolumn{10}{|c|}{ Cycle 158A Outage - 91 Day, 11 Hour Down Time } \\
\hline & $\operatorname{BOC}(11 / 11 / 2015,1500)$ & 65.1 & 5.82 & 468.6 & $5.39904 \mathrm{E}+13$ & $3.25788 \mathrm{E}+13$ & 1.30769E+14 & $1.65485 \mathrm{E}+20$ & 946.4 & 446.2 \\
\hline & & 75.1 & 6.73 & 458.6 & $5.28976 \mathrm{E}+13$ & $3.18898 \mathrm{E}+13$ & $1.28672 \mathrm{E}+14$ & $1.91199 \mathrm{E}+20$ & 934.7 & 439.5 \\
\hline & & 85.1 & 7.61 & 444.8 & $5.24015 \mathrm{E}+13$ & $3.11095 \mathrm{E}+13$ & $1.26719 \mathrm{E}+14$ & $2.16190 \mathrm{E}+20$ & 918.3 & 430.4 \\
\hline & & 95.1 & 8.49 & 445.8 & $5.34202 E+13$ & $3.10830 \mathrm{E}+13$ & $1.27809 \mathrm{E}+14$ & $2.41181 \mathrm{E}+20$ & 919.6 & 431.1 \\
\hline & & 105.1 & 9.37 & 450.7 & $5.49446 \mathrm{E}+13$ & 3.15437E+13 & $1.29960 \mathrm{E}+14$ & $2.66353 \mathrm{E}+20$ & 925.4 & 434.4 \\
\hline & & 117.1 & 10.43 & 453.4 & $5.63337 \mathrm{E}+13$ & $3.16194 \mathrm{E}+13$ & $1.31500 \mathrm{E}+14$ & $2.96463 \mathrm{E}+20$ & 928.6 & 436.1 \\
\hline & EOC $(1 / 2 / 2016,0400)$ & 117.3 & 10.44 & 445.8 & $5.67266 \mathrm{E}+13$ & $3.11475 \mathrm{E}+13$ & $1.31094 \mathrm{E}+14$ & $2.96824 \mathrm{E}+20$ & 919.6 & 431.1 \\
\hline \multirow[t]{4}{*}{$158 \mathrm{~B}$} & \multicolumn{10}{|c|}{ Cycle 158B Outage - 39 Day, 2 Hour Down Time } \\
\hline & BOC $(2 / 10 / 2016,0600)$ & 118.3 & 10.53 & 374.1 & $4.74922 \mathrm{E}+13$ & $2.64676 \mathrm{E}+13$ & $1.10168 \mathrm{E}+14$ & $2.99233 E+20$ & 831.3 & 382.3 \\
\hline & & 119.3 & 10.62 & 413.6 & $5.26017 \mathrm{E}+13$ & $2.90224 \mathrm{E}+13$ & $1.22013 \mathrm{E}+14$ & $3.01823 E+20$ & 880.7 & 409.4 \\
\hline & & 122.3 & 10.87 & 418.5 & $5.33041 \mathrm{E}+13$ & $2.93809 \mathrm{E}+13$ & $1.23755 \mathrm{E}+14$ & $3.09049 \mathrm{E}+20$ & 886.6 & 412.7 \\
\hline
\end{tabular}


TEM-10300-1

$03 / 01 / 2012$

Rev. 03

Title: $\quad$ ATF-1 Power Histories

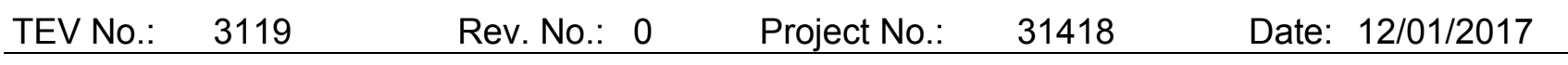

Table 15. ATF-13 Power History

\begin{tabular}{|c|c|c|c|c|c|c|c|c|c|c|}
\hline Cycle & Critical Dates & EFPD & $\begin{array}{c}\text { Burnup } \\
\text { (GWd/MTU) }\end{array}$ & $\begin{array}{c}\text { LHGR } \\
(\mathrm{W} / \mathrm{cm})\end{array}$ & $\begin{array}{l}\text { Thermal Flux } \\
\left(\mathrm{n} / \mathrm{cm}^{2 *} \mathrm{~s}\right)\end{array}$ & $\begin{array}{c}\text { Fast Flux } \\
\left(\mathrm{n} / \mathrm{cm}^{2 *} \mathrm{~s}>1\right. \\
\mathrm{MeV})\end{array}$ & $\begin{array}{l}\text { Total Flux } \\
\left(\mathrm{n} / \mathrm{cm}^{2 *} \mathrm{~s}\right)\end{array}$ & $\begin{array}{l}\text { Fission Density } \\
\left(\text { Fissions } / \mathrm{cm}^{3}\right)\end{array}$ & $\begin{array}{c}\text { Fuel Centerline } \\
\text { Temperature } \\
\left({ }^{\circ} \mathrm{C}\right)\end{array}$ & $\mathrm{PICT}\left({ }^{\circ} \mathrm{C}\right)$ \\
\hline & & 127.3 & 11.28 & 410.1 & $5.27394 \mathrm{E}+13$ & $2.88654 \mathrm{E}+13$ & $1.22102 \mathrm{E}+14$ & $3.20672 \mathrm{E}+20$ & 876.4 & 407.1 \\
\hline & & 132.3 & 11.70 & 424.8 & $5.50680 \mathrm{E}+13$ & $2.98124 \mathrm{E}+13$ & $1.26432 \mathrm{E}+14$ & $3.32595 \mathrm{E}+20$ & 894.3 & 417.0 \\
\hline & & 137.3 & 12.11 & 407.8 & $5.32749 \mathrm{E}+13$ & $2.88815 E+13$ & $1.22706 \mathrm{E}+14$ & $3.44097 \mathrm{E}+20$ & 873.6 & 405.5 \\
\hline & & 147.3 & 12.94 & 427.6 & $5.64024 \mathrm{E}+13$ & $3.03549 E+13$ & $1.29044 \mathrm{E}+14$ & $3.67884 \mathrm{E}+20$ & 897.7 & 418.9 \\
\hline & & 157.3 & 13.77 & 420.5 & $5.66711 \mathrm{E}+13$ & $2.96218 \mathrm{E}+13$ & $1.28025 E+14$ & $3.91249 \mathrm{E}+20$ & 889.1 & 414.1 \\
\hline & EOC $(4 / 1 / 2016,0900)$ & 168.7 & 14.70 & 406.4 & $5.70131 \mathrm{E}+13$ & $2.89322 \mathrm{E}+13$ & $1.26727 E+14$ & $4.17806 \mathrm{E}+20$ & 871.9 & 404.6 \\
\hline \multirow[t]{7}{*}{$159 \mathrm{~A}$} & \multicolumn{10}{|c|}{ Cycle 159A Outage - 77 Day, 2 Hour Down Time } \\
\hline & $\operatorname{BOC}(6 / 17 / 2016,1100)$ & 169.7 & 14.73 & 167.9 & $2.35342 \mathrm{E}+13$ & $1.18566 \mathrm{E}+13$ & $5.21099 E+13$ & $4.18589 \mathrm{E}+20$ & 542.9 & 230.7 \\
\hline & & 170.7 & 14.76 & 175.0 & $2.45309 \mathrm{E}+13$ & $1.22975 E+13$ & $5.41805 E+13$ & $4.19432 \mathrm{E}+20$ & 553.8 & 236.3 \\
\hline & & 174.6 & 14.88 & 150.1 & $2.11032 \mathrm{E}+13$ & $1.06789 \mathrm{E}+13$ & $4.67086 \mathrm{E}+13$ & $4.22805 \mathrm{E}+20$ & 515.6 & 216.9 \\
\hline & \multicolumn{10}{|c|}{ Mid-Cycle 159A Outage - 3 Day, 21 Hour Down Time (6/23/2016, 1000 - 6/27/2016, 0700) } \\
\hline & & 176.0 & 14.91 & 215.8 & $3.05054 \mathrm{E}+13$ & $1.54792 \mathrm{E}+13$ & $6.97963 \mathrm{E}+13$ & $4.23648 \mathrm{E}+20$ & 614.5 & 267.5 \\
\hline & EOC $(6 / 28 / 2016,1800)$ & 176.2 & 14.92 & 237.9 & $3.37237 \mathrm{E}+13$ & $1.73610 \mathrm{E}+13$ & $7.90789 \mathrm{E}+13$ & $4.23889 \mathrm{E}+20$ & 646.5 & 284.1 \\
\hline $160 \mathrm{~A}$ & \multicolumn{10}{|c|}{ Cycle 160A Outage - 80 Day, 1 Hour Down Time } \\
\hline & $\operatorname{BOC}(9 / 16 / 2016,1900)$ & 177.2 & 15.00 & 411.6 & $5.81256 \mathrm{E}+13$ & $2.91495 \mathrm{E}+13$ & $1.28402 \mathrm{E}+14$ & $4.26418 \mathrm{E}+20$ & 878.3 & 408.1 \\
\hline & & 179.2 & 15.16 & 404.6 & $5.74027 \mathrm{E}+13$ & $2.87885 E+13$ & $1.27196 \mathrm{E}+14$ & $4.30814 \mathrm{E}+20$ & 869.6 & 403.3 \\
\hline & & 186.2 & 15.71 & 402.7 & $5.71880 \mathrm{E}+13$ & $2.86476 \mathrm{E}+13$ & $1.26440 \mathrm{E}+14$ & $4.46411 \mathrm{E}+20$ & 867.2 & 402.0 \\
\hline & & 196.2 & 16.49 & 397.3 & $5.72733 \mathrm{E}+13$ & $2.84374 \mathrm{E}+13$ & $1.25925 \mathrm{E}+14$ & $4.68512 \mathrm{E}+20$ & 860.6 & 398.4 \\
\hline & & 206.2 & 17.24 & 386.2 & $5.66622 \mathrm{E}+13$ & $2.74635 E+13$ & $1.23536 \mathrm{E}+14$ & $4.90010 \mathrm{E}+20$ & 846.6 & 390.7 \\
\hline & & 216.2 & 18.00 & 388.7 & $5.81633 \mathrm{E}+13$ & $2.80151 \mathrm{E}+13$ & $1.25683 E+14$ & $5.11569 \mathrm{E}+20$ & 849.7 & 392.4 \\
\hline & & 222.2 & 18.45 & 369.3 & $5.64453 \mathrm{E}+13$ & $2.63096 \mathrm{E}+13$ & $1.20700 \mathrm{E}+14$ & $5.24215 \mathrm{E}+20$ & 825.1 & 379.0 \\
\hline & & 223.8 & 18.56 & 345.0 & $5.33514 \mathrm{E}+13$ & $2.45861 \mathrm{E}+13$ & $1.13560 \mathrm{E}+14$ & $5.27407 \mathrm{E}+20$ & 793.6 & 362.0 \\
\hline & & 226.2 & 18.71 & 341.6 & $5.30752 \mathrm{E}+13$ & $2.45853 \mathrm{E}+13$ & $1.12786 \mathrm{E}+14$ & $5.31743 \mathrm{E}+20$ & 789.2 & 359.6 \\
\hline & $\mathrm{EOC}^{\dagger \dagger}(11 / 8 / 2016,1100)$ & 228.9 & 18.87 & 325.6 & $5.10610 \mathrm{E}+13$ & $2.33930 E+13$ & $1.08608 E+14$ & $5.36380 \mathrm{E}+20$ & 768.1 & 348.2 \\
\hline
\end{tabular}

${ }^{\dagger}$ ATF-13 was discharged for PIE following Cycle 160A irradiation. 
TEM-10300-1

$03 / 01 / 2012$

Rev. 03

Title: $\quad$ ATF-1 Power Histories

\begin{tabular}{lllll} 
TEV No.: & 3119 & Rev. No.: & 0 & Project No.: \\
\hline
\end{tabular}

Table 16. ATF-14 Power History

\begin{tabular}{|c|c|c|c|c|c|c|c|c|c|c|}
\hline Cycle & Critical Dates & EFPD & $\begin{array}{c}\text { Burnup } \\
\text { (GWd/MTU) }\end{array}$ & $\begin{array}{l}\text { LHGR } \\
(\mathrm{W} / \mathrm{cm})\end{array}$ & $\begin{array}{l}\text { Thermal Flux } \\
\left(\mathrm{n} / \mathrm{cm}^{2 *} \mathrm{~s}\right)\end{array}$ & $\begin{array}{c}\text { Fast Flux } \\
\left(\mathrm{n} / \mathrm{cm}^{2 *} \mathrm{~s}>1\right. \\
\mathrm{MeV})\end{array}$ & $\begin{array}{l}\text { Total Flux } \\
\left(\mathrm{n} / \mathrm{cm}^{2 *} \mathrm{~s}\right)\end{array}$ & $\begin{array}{l}\text { Fission Density } \\
\left(\text { Fissions } / \mathrm{cm}^{3}\right)\end{array}$ & $\begin{array}{c}\text { Fuel Centerline } \\
\text { Temperature } \\
\left({ }^{\circ} \mathrm{C}\right)\end{array}$ & PICT $\left({ }^{\circ} \mathrm{C}\right)$ \\
\hline \multirow[t]{2}{*}{$157 \mathrm{C}$} & BOC $(2 / 10 / 2015,0600)$ & 0.0 & 0.00 & 467.6 & $4.83991 \mathrm{E}+13$ & $3.21796 \mathrm{E}+13$ & $1.23985 E+14$ & $0.00000 \mathrm{E}+00$ & 986.3 & 494.7 \\
\hline & EOC $(2 / 15 / 2015,0800)$ & 5.4 & 0.52 & 473.5 & $4.94705 \mathrm{E}+13$ & $3.24277 E+13$ & $1.25599 \mathrm{E}+14$ & $1.48141 \mathrm{E}+19$ & 993.5 & 498.7 \\
\hline \multirow[t]{13}{*}{$157 \mathrm{D}$} & \multicolumn{10}{|c|}{ Cycle 157D Outage - 104 Day, 3 Hour Down Time } \\
\hline & $\operatorname{BOC}(5 / 30 / 2015,1100)$ & 5.4 & 0.52 & 403.8 & $4.20210 \mathrm{E}+13$ & $2.77615 \mathrm{E}+13$ & $1.06852 \mathrm{E}+14$ & $1.48141 \mathrm{E}+19$ & 905.8 & 449.7 \\
\hline & & 8.4 & 0.79 & 435.0 & $4.53652 \mathrm{E}+13$ & $2.99454 \mathrm{E}+13$ & $1.15638 \mathrm{E}+14$ & $2.24621 \mathrm{E}+19$ & 945.9 & 472.0 \\
\hline & & 15.4 & 1.39 & 437.1 & $4.58092 \mathrm{E}+13$ & $3.03043 E+13$ & $1.17102 \mathrm{E}+14$ & $3.98054 \mathrm{E}+19$ & 948.6 & 473.5 \\
\hline & & 22.4 & 2.00 & 431.1 & $4.56635 \mathrm{E}+13$ & $2.98203 E+13$ & $1.16016 \mathrm{E}+14$ & $5.69681 \mathrm{E}+19$ & 940.9 & 469.2 \\
\hline & & 29.4 & 2.60 & 434.3 & $4.66267 \mathrm{E}+13$ & $2.99332 E+13$ & 1.17245E+14 & $7.42513 \mathrm{E}+19$ & 945.0 & 471.5 \\
\hline & & 36.4 & 3.22 & 444.9 & $4.83073 \mathrm{E}+13$ & $3.10328 E+13$ & $1.21574 \mathrm{E}+14$ & $9.18355 \mathrm{E}+19$ & 958.4 & 479.0 \\
\hline & & 43.4 & 3.84 & 445.8 & $4.89430 \mathrm{E}+13$ & $3.11520 \mathrm{E}+13$ & $1.22464 \mathrm{E}+14$ & $1.09480 \mathrm{E}+20$ & 959.5 & 479.6 \\
\hline & & 50.4 & 4.47 & 456.3 & $5.05704 \mathrm{E}+13$ & $3.17023 E+13$ & $1.25553 \mathrm{E}+14$ & $1.27426 \mathrm{E}+20$ & 972.5 & 486.9 \\
\hline & & 57.4 & 5.10 & 459.1 & $5.15722 \mathrm{E}+13$ & $3.19461 \mathrm{E}+13$ & $1.27328 \mathrm{E}+14$ & $1.45431 \mathrm{E}+20$ & 976.0 & 488.9 \\
\hline & & 60.4 & 5.37 & 454.7 & $5.16806 \mathrm{E}+13$ & $3.15657 E+13$ & $1.26327 E+14$ & $1.53260 \mathrm{E}+20$ & 970.4 & 485.8 \\
\hline & \multicolumn{10}{|c|}{ Mid-Cycle 157D Outage - 14 Day, 10 Hour Down Time $(7 / 23 / 2015,1800-8 / 7 / 2015,0400)$} \\
\hline & EOC $(8 / 12 / 2015,0400)$ & 65.1 & 5.81 & 455.7 & $5.25431 \mathrm{E}+13$ & $3.17176 \mathrm{E}+13$ & 1.27543E+14 & 1.65725E+20 & 971.7 & 486.5 \\
\hline \multirow[t]{8}{*}{$158 \mathrm{~A}$} & \multicolumn{10}{|c|}{ Cycle 158A Outage - 91 Day, 11 Hour Down Time } \\
\hline & $\operatorname{BOC}(11 / 11 / 2015,1500)$ & 65.1 & 5.81 & 468.4 & $5.38053 \mathrm{E}+13$ & $3.27546 \mathrm{E}+13$ & $1.30693 \mathrm{E}+14$ & $1.65725 \mathrm{E}+20$ & 987.3 & 495.2 \\
\hline & & 75.1 & 6.71 & 460.1 & $5.27918 \mathrm{E}+13$ & $3.21537 \mathrm{E}+13$ & $1.28563 E+14$ & $1.91500 \mathrm{E}+20$ & 977.2 & 489.5 \\
\hline & & 85.1 & 7.59 & 445.4 & $5.23055 \mathrm{E}+13$ & $3.11412 \mathrm{E}+13$ & $1.26299 \mathrm{E}+14$ & $2.16551 \mathrm{E}+20$ & 958.9 & 479.3 \\
\hline & & 95.1 & 8.46 & 446.8 & $5.33203 E+13$ & $3.13694 \mathrm{E}+13$ & $1.27953 E+14$ & $2.41542 \mathrm{E}+20$ & 960.6 & 480.3 \\
\hline & & 105.1 & 9.35 & 450.8 & $5.47970 \mathrm{E}+13$ & $3.16763 E+13$ & $1.29863 E+14$ & $2.66775 \mathrm{E}+20$ & 965.7 & 483.1 \\
\hline & & 117.1 & 10.40 & 454.1 & $5.62608 \mathrm{E}+13$ & $3.18370 \mathrm{E}+13$ & $1.31673 E+14$ & $2.96885 \mathrm{E}+20$ & 969.8 & 485.4 \\
\hline & $\operatorname{EOC}(1 / 2 / 2016,0400)$ & 117.3 & 10.42 & 446.4 & $5.66516 \mathrm{E}+13$ & $3.13548 E+13$ & $1.31253 E+14$ & $2.97306 \mathrm{E}+20$ & 960.2 & 480.0 \\
\hline \multirow[t]{5}{*}{$158 \mathrm{~B}$} & \multicolumn{10}{|c|}{ Cycle 158B Outage - 39 Day, 2 Hour Down Time } \\
\hline & BOC $(2 / 10 / 2016,0600)$ & 118.3 & 10.50 & 375.4 & $4.74203 E+13$ & $2.61442 \mathrm{E}+13$ & $1.09405 E+14$ & $2.99715 \mathrm{E}+20$ & 868.1 & 428.9 \\
\hline & & 119.3 & 10.59 & 415.3 & $5.26626 \mathrm{E}+13$ & $2.89973 E+13$ & $1.22008 \mathrm{E}+14$ & $3.02244 \mathrm{E}+20$ & 920.6 & 458.0 \\
\hline & & 122.3 & 10.85 & 419.4 & $5.34331 \mathrm{E}+13$ & $2.96992 \mathrm{E}+13$ & $1.23993 E+14$ & $3.09471 \mathrm{E}+20$ & 926.0 & 461.0 \\
\hline & & 127.3 & 11.25 & 409.4 & $5.24828 \mathrm{E}+13$ & $2.87891 \mathrm{E}+13$ & $1.21746 \mathrm{E}+14$ & $3.21033 E+20$ & 913.0 & 453.7 \\
\hline
\end{tabular}


TEM-10300-1

$03 / 01 / 2012$

Rev. 03

Title: $\quad$ ATF-1 Power Histories

\begin{tabular}{lllll} 
TEV No.: & 3119 & Rev. No.: & 0 & Project No.: \\
\hline
\end{tabular}

Table 16. ATF-14 Power History

\begin{tabular}{|c|c|c|c|c|c|c|c|c|c|c|}
\hline Cycle & Critical Dates & EFPD & $\begin{array}{c}\text { Burnup } \\
\text { (GWd/MTU) }\end{array}$ & $\begin{array}{l}\text { LHGR } \\
(\mathrm{W} / \mathrm{cm})\end{array}$ & $\begin{array}{l}\text { Thermal Flux } \\
\left(\mathrm{n} / \mathrm{cm}^{2 *} \mathrm{~s}\right)\end{array}$ & $\begin{array}{c}\text { Fast Flux } \\
\left(\mathrm{n} / \mathrm{cm}^{2 *} \mathrm{~s}>1\right. \\
\mathrm{MeV})\end{array}$ & $\begin{array}{l}\text { Total Flux } \\
\left(\mathrm{n} / \mathrm{cm}^{2 *} \mathrm{~s}\right)\end{array}$ & $\begin{array}{l}\text { Fission Density } \\
\text { (Fissions } / \mathrm{cm}^{3} \text { ) }\end{array}$ & $\begin{array}{c}\text { Fuel Centerline } \\
\text { Temperature } \\
\left({ }^{\circ} \mathrm{C}\right)\end{array}$ & PICT $\left({ }^{\circ} \mathrm{C}\right)$ \\
\hline & & 132.3 & 11.66 & 419.3 & $5.42037 \mathrm{E}+13$ & $2.96102 E+13$ & $1.25268 \mathrm{E}+14$ & $3.32836 \mathrm{E}+20$ & 925.9 & 460.9 \\
\hline & & 137.3 & 12.07 & 409.9 & $5.34953 E+13$ & $2.89307 E+13$ & $1.22564 \mathrm{E}+14$ & $3.44398 \mathrm{E}+20$ & 913.7 & 454.1 \\
\hline & & 147.3 & 12.90 & 426.0 & $5.60310 \mathrm{E}+13$ & $3.00084 \mathrm{E}+13$ & $1.28135 E+14$ & $3.68125 \mathrm{E}+20$ & 934.4 & 465.6 \\
\hline & & 157.3 & 13.72 & 421.2 & $5.65798 \mathrm{E}+13$ & $2.95211 \mathrm{E}+13$ & $1.27609 \mathrm{E}+14$ & $3.91550 \mathrm{E}+20$ & 928.4 & 462.2 \\
\hline & EOC $(4 / 1 / 2016,0900)$ & 168.7 & 14.66 & 409.3 & $5.73208 \mathrm{E}+13$ & $2.88851 \mathrm{E}+13$ & $1.26791 \mathrm{E}+14$ & $4.18228 \mathrm{E}+20$ & 912.9 & 453.7 \\
\hline \multirow[t]{7}{*}{$159 \mathrm{~A}$} & \multicolumn{10}{|c|}{ Cycle 159A Outage - 77 Day, 2 Hour Down Time } \\
\hline & $\mathrm{BOC}(6 / 17 / 2016,1100)$ & 169.7 & 14.68 & 168.6 & $2.35072 \mathrm{E}+13$ & $1.19202 \mathrm{E}+13$ & $5.19632 \mathrm{E}+13$ & $4.19011 \mathrm{E}+20$ & 559.5 & 262.8 \\
\hline & & 170.7 & 14.71 & 174.7 & $2.44010 \mathrm{E}+13$ & $1.22937 \mathrm{E}+13$ & $5.41330 \mathrm{E}+13$ & $4.19854 \mathrm{E}+20$ & 569.4 & 268.0 \\
\hline & & 174.6 & 14.83 & 151.6 & $2.11702 \mathrm{E}+13$ & $1.07073 \mathrm{E}+13$ & $4.69252 \mathrm{E}+13$ & $4.23286 \mathrm{E}+20$ & 531.4 & 247.9 \\
\hline & \multicolumn{10}{|c|}{ Mid-Cycle 159A Outage - 3 Day, 21 Hour Down Time (6/23/2016, 1000 - 6/27/2016, 0700) } \\
\hline & & 176.0 & 14.86 & 218.2 & $3.08068 \mathrm{E}+13$ & $1.58061 \mathrm{E}+13$ & $7.07247 \mathrm{E}+13$ & $4.24129 \mathrm{E}+20$ & 639.0 & 305.0 \\
\hline & EOC $(6 / 28 / 2016,1800)$ & 176.2 & 14.87 & 240.4 & $3.41839 \mathrm{E}+13$ & $1.77446 \mathrm{E}+13$ & $8.04618 \mathrm{E}+13$ & $4.24431 \mathrm{E}+20$ & 673.4 & 323.3 \\
\hline $160 \mathrm{~A}$ & \multicolumn{10}{|c|}{ Cycle 160A Outage - 80 Day, 1 Hour Down Time } \\
\hline & $\operatorname{BOC}(9 / 16 / 2016,1900)$ & 177.2 & 14.96 & 411.7 & $5.79625 \mathrm{E}+13$ & $2.91682 E+13$ & $1.28186 \mathrm{E}+14$ & $4.26960 \mathrm{E}+20$ & 916.0 & 455.4 \\
\hline & & 179.2 & 15.12 & 406.0 & $5.73571 \mathrm{E}+13$ & $2.86903 E+13$ & $1.26976 \mathrm{E}+14$ & $4.31416 \mathrm{E}+20$ & 908.6 & 451.3 \\
\hline & & 186.2 & 15.66 & 402.1 & $5.70800 \mathrm{E}+13$ & $2.84248 \mathrm{E}+13$ & $1.26018 \mathrm{E}+14$ & $4.46953 \mathrm{E}+20$ & 903.5 & 448.5 \\
\hline & & 196.2 & 16.44 & 396.4 & $5.71389 \mathrm{E}+13$ & $2.83498 E+13$ & $1.25603 E+14$ & $4.69054 \mathrm{E}+20$ & 896.1 & 444.3 \\
\hline & & 206.2 & 17.20 & 390.0 & $5.71428 \mathrm{E}+13$ & $2.76906 \mathrm{E}+13$ & $1.24492 \mathrm{E}+14$ & $4.90673 E+20$ & 887.6 & 439.6 \\
\hline & & 216.2 & 17.95 & 390.9 & $5.84244 \mathrm{E}+13$ & $2.79364 \mathrm{E}+13$ & $1.25850 \mathrm{E}+14$ & $5.12352 \mathrm{E}+20$ & 888.8 & 440.3 \\
\hline & & 222.2 & 18.40 & 369.4 & $5.65072 \mathrm{E}+13$ & $2.66119 E+13$ & $1.21355 E+14$ & $5.24998 \mathrm{E}+20$ & 859.9 & 424.4 \\
\hline & & 223.8 & 18.51 & 345.5 & $5.34514 \mathrm{E}+13$ & $2.47886 \mathrm{E}+13$ & $1.13938 \mathrm{E}+14$ & $5.28190 \mathrm{E}+20$ & 827.2 & 406.5 \\
\hline & & 226.2 & 18.66 & 342.2 & $5.30903 E+13$ & $2.43786 \mathrm{E}+13$ & $1.12602 \mathrm{E}+14$ & $5.32525 \mathrm{E}+20$ & 822.5 & 403.9 \\
\hline & EOC $(11 / 8 / 2016,1100)$ & 228.9 & 18.82 & 325.4 & $5.09442 \mathrm{E}+13$ & $2.33316 \mathrm{E}+13$ & $1.08646 \mathrm{E}+14$ & $5.37162 \mathrm{E}+20$ & 798.9 & 391.1 \\
\hline \multirow[t]{6}{*}{$160 \mathrm{~B}$} & \multicolumn{10}{|c|}{ Cycle 160B Outage - 42 Day, 1 Hour Down Time Outage } \\
\hline & $\mathrm{BOC}(12 / 20 / 2016,1200)$ & 229.9 & 18.91 & 411.1 & $6.38648 \mathrm{E}+13$ & $2.63893 E+13$ & $1.27715 E+14$ & $5.39692 \mathrm{E}+20$ & 915.3 & 455.0 \\
\hline & & 231.9 & 19.07 & 409.6 & $6.38608 \mathrm{E}+13$ & $2.62898 E+13$ & $1.27724 \mathrm{E}+14$ & $5.44088 \mathrm{E}+20$ & 913.3 & 453.9 \\
\hline & & 238.9 & 19.63 & 402.9 & $6.31529 \mathrm{E}+13$ & $2.58752 \mathrm{E}+13$ & $1.25885 E+14$ & $5.60046 \mathrm{E}+20$ & 904.6 & 449.1 \\
\hline & & 248.9 & 20.37 & 384.9 & $6.12902 \mathrm{E}+13$ & $2.48543 E+13$ & $1.21858 E+14$ & $5.81304 \mathrm{E}+20$ & 880.8 & 435.9 \\
\hline & & 258.1 & 21.05 & 368.5 & $6.11022 \mathrm{E}+13$ & $2.40138 \mathrm{E}+13$ & $1.19986 E+14$ & $6.00634 \mathrm{E}+20$ & 858.7 & 423.8 \\
\hline
\end{tabular}


TEM-10300-1

03/01/2012

Rev. 03

Title: $\quad$ ATF-1 Power Histories

$\begin{array}{lllll}\text { TEV No.: } & 3119 & \text { Rev. No.: } & 0 & \text { Project No.: }\end{array}$

Table 16. ATF-14 Power History

\begin{tabular}{|c|c|c|c|c|c|c|c|c|c|c|}
\hline Cycle & Critical Dates & EFPD & $\begin{array}{c}\begin{array}{c}\text { Burnup } \\
\text { (GWd/MTU) }\end{array} \\
\text { (GW }\end{array}$ & $\begin{array}{l}\text { LHGR } \\
(\mathrm{W} / \mathrm{cm})\end{array}$ & $\begin{array}{l}\text { Thermal Flux } \\
\left(\mathrm{n} / \mathrm{cm}^{2 *} \mathrm{~s}\right)\end{array}$ & $\begin{array}{c}\text { Fast Flux } \\
\left(\mathrm{n} / \mathrm{cm}^{2 *} \mathrm{~s}>1\right. \\
\mathrm{MeV})\end{array}$ & $\begin{array}{l}\text { Total Flux } \\
\left(\mathrm{n} / \mathrm{cm}^{2 *} \mathbf{s}\right)\end{array}$ & $\begin{array}{l}\text { Fission Density } \\
\text { (Fissions/cm }{ }^{3} \text { ) }\end{array}$ & $\begin{array}{c}\text { Fuel Centerline } \\
\text { Temperature } \\
\left({ }^{\circ} \mathrm{C}\right)\end{array}$ & PICT $\left({ }^{\circ} \mathrm{C}\right)$ \\
\hline & \multicolumn{10}{|c|}{ Mid-Cycle 160B Outage - 5 Day, 14 Hour Down Time } \\
\hline & & 268.9 & 21.84 & 373.1 & $6.23178 \mathrm{E}+13$ & $2.41451 \mathrm{E}+13$ & $1.21456 \mathrm{E}+14$ & $6.23277 \mathrm{E}+20$ & 864.9 & 427.2 \\
\hline & & 278.9 & 22.52 & 351.7 & $5.98990 \mathrm{E}+13$ & $2.32472 \mathrm{E}+13$ & $1.18933 E+14$ & $6.42668 \mathrm{E}+20$ & 835.8 & 411.2 \\
\hline & $\operatorname{EOC}(2 / 23 / 2017,1400)$ & 288.4 & 23.21 & 366.4 & $6.49938 \mathrm{E}+13$ & $2.40698 \mathrm{E}+13$ & $1.25369 \mathrm{E}+14$ & $6.62179 \mathrm{E}+20$ & 855.8 & 422.2 \\
\hline \multirow[t]{9}{*}{$161 \mathrm{~A}$} & \multicolumn{10}{|c|}{ Cycle 161A Outage - 95 Day, 13 Hour Down Time } \\
\hline & $\mathrm{BOC}(5 / 30 / 2017,0300)$ & 290.1 & 23.30 & 283.4 & $5.00779 \mathrm{E}+13$ & $1.85124 \mathrm{E}+13$ & $9.55644 \mathrm{E}+13$ & $6.65009 \mathrm{E}+20$ & 738.1 & 358.1 \\
\hline & & 290.4 & 23.32 & 254.9 & $4.52357 \mathrm{E}+13$ & $1.66644 \mathrm{E}+13$ & $8.62155 E+13$ & $6.65311 \mathrm{E}+20$ & 695.5 & 335.2 \\
\hline & & 292.4 & 23.42 & 259.7 & 4.60937E+13 & $1.71111 \mathrm{E}+13$ & $8.81578 \mathrm{E}+13$ & $6.68261 \mathrm{E}+20$ & 702.8 & 339.1 \\
\hline & & 294.4 & 23.52 & 263.2 & $4.69126 \mathrm{E}+13$ & $1.72481 \mathrm{E}+13$ & $8.95543 E+13$ & $6.71212 \mathrm{E}+20$ & 708.0 & 341.9 \\
\hline & & 296.4 & 23.63 & 264.1 & $4.71450 \mathrm{E}+13$ & $1.73419 \mathrm{E}+13$ & $8.97496 \mathrm{E}+13$ & $6.74223 E+20$ & 709.5 & 342.7 \\
\hline & & 298.4 & 23.73 & 270.4 & $4.84681 \mathrm{E}+13$ & $1.76537 \mathrm{E}+13$ & $9.18044 \mathrm{E}+13$ & $6.77234 \mathrm{E}+20$ & 718.9 & 347.7 \\
\hline & & 300.4 & 23.84 & 273.7 & $4.92623 \mathrm{E}+13$ & $1.79836 \mathrm{E}+13$ & $9.33431 \mathrm{E}+13$ & $6.80305 E+20$ & 723.8 & 350.4 \\
\hline & $\operatorname{EOC}(6 / 11 / 2017,1600)$ & 300.9 & 23.86 & 276.9 & $5.00557 \mathrm{E}+13$ & $1.81617 \mathrm{E}+13$ & $9.45173 E+13$ & $6.80908 \mathrm{E}+20$ & 728.6 & 353.0 \\
\hline \multirow[t]{10}{*}{$162 \mathrm{~A}$} & \multicolumn{10}{|c|}{ Cycle 162A Outage } \\
\hline & $\mathrm{BOC}$ & 301.9 & 23.95 & 417.1 & $7.33584 \mathrm{E}+13$ & $2.66874 \mathrm{E}+13$ & $1.39849 \mathrm{E}+14$ & $6.83316 \mathrm{E}+20$ & 923.0 & 459.3 \\
\hline & & 303.9 & 24.09 & 393.9 & $7.04098 \mathrm{E}+13$ & $2.57074 \mathrm{E}+13$ & $1.34592 \mathrm{E}+14$ & $6.87411 \mathrm{E}+20$ & 892.8 & 442.5 \\
\hline & & 310.9 & 24.62 & 393.5 & $7.06903 \mathrm{E}+13$ & $2.56374 \mathrm{E}+13$ & $1.34783 E+14$ & $7.02466 \mathrm{E}+20$ & 892.2 & 442.2 \\
\hline & & 320.9 & 25.35 & 392.2 & $7.07128 \mathrm{E}+13$ & $2.51346 \mathrm{E}+13$ & $1.33614 \mathrm{E}+14$ & $7.23483 E+20$ & 890.5 & 441.3 \\
\hline & & 330.9 & 26.09 & 389.7 & $7.23415 \mathrm{E}+13$ & $2.52513 \mathrm{E}+13$ & $1.35737 \mathrm{E}+14$ & $7.44379 \mathrm{E}+20$ & 887.2 & 439.5 \\
\hline & & 340.9 & 26.81 & 387.1 & $7.33033 \mathrm{E}+13$ & $2.49237 \mathrm{E}+13$ & $1.36097 \mathrm{E}+14$ & $7.64914 \mathrm{E}+20$ & 883.8 & 437.5 \\
\hline & & 350.9 & 27.55 & 388.5 & $7.66625 \mathrm{E}+13$ & $2.54644 \mathrm{E}+13$ & $1.41522 \mathrm{E}+14$ & $7.86293 \mathrm{E}+20$ & 885.6 & 438.6 \\
\hline & & 359.9 & 28.19 & 370.3 & 7.47217E+13 & $2.42494 \mathrm{E}+13$ & $1.37560 \mathrm{E}+14$ & $8.04539 \mathrm{E}+20$ & 861.2 & 425.1 \\
\hline & EOC & 362.9 & 28.40 & 361.6 & $7.52161 \mathrm{E}+13$ & $2.38204 \mathrm{E}+13$ & $1.37311 \mathrm{E}+14$ & $8.10320 \mathrm{E}+20$ & 849.4 & 418.6 \\
\hline
\end{tabular}


TEM-10300-1

$03 / 01 / 2012$

Rev. 03

Title: $\quad$ ATF-1 Power Histories

\begin{tabular}{lllll} 
TEV No.: & 3119 & Rev. No.: & 0 & Project No.: \\
\hline
\end{tabular}

Table 17. ATF-15 Power History

\begin{tabular}{|c|c|c|c|c|c|c|c|c|c|c|}
\hline Cycle & Critical Dates & EFPD & $\begin{array}{c}\text { Burnup } \\
\text { (GWd/MTU) }\end{array}$ & $\begin{array}{l}\text { LHGR } \\
(\mathrm{W} / \mathrm{cm})\end{array}$ & $\begin{array}{l}\text { Thermal Flux } \\
\left(\mathrm{n} / \mathrm{cm}^{2 *} \mathrm{~s}\right)\end{array}$ & $\begin{array}{c}\text { Fast Flux } \\
\left(\mathrm{n} / \mathrm{cm}^{2 *} \mathrm{~s}>1\right. \\
\mathrm{MeV})\end{array}$ & $\begin{array}{l}\text { Total Flux } \\
\left(\mathrm{n} / \mathrm{cm}^{2 *} \mathrm{~s}\right)\end{array}$ & $\begin{array}{l}\text { Fission Density } \\
\left(\text { Fissions } / \mathrm{cm}^{3}\right)\end{array}$ & $\begin{array}{c}\text { Fuel Centerline } \\
\text { Temperature } \\
\left({ }^{\circ} \mathrm{C}\right)\end{array}$ & PICT $\left({ }^{\circ} \mathrm{C}\right)$ \\
\hline \multirow[t]{2}{*}{$157 \mathrm{C}$} & BOC $(2 / 10 / 2015,0600)$ & 0.0 & 0.00 & 507.1 & $5.20467 \mathrm{E}+13$ & $3.14625 E+13$ & $1.23266 \mathrm{E}+14$ & $0.00000 \mathrm{E}+00$ & 1292.1 & 490.3 \\
\hline & EOC (2/15/2015, 0800) & 5.4 & 0.56 & 513.3 & $5.32487 \mathrm{E}+13$ & $3.18012 \mathrm{E}+13$ & $1.25011 \mathrm{E}+14$ & 1.59583E+19 & 1298.5 & 494.5 \\
\hline \multirow[t]{13}{*}{$157 \mathrm{D}$} & \multicolumn{10}{|c|}{ Cycle 157D Outage - 104 Day, 3 Hour Down Time } \\
\hline & $\mathrm{BOC}(5 / 30 / 2015,1100)$ & 5.4 & 0.56 & 437.7 & $4.53182 \mathrm{E}+13$ & $2.70619 \mathrm{E}+13$ & $1.06106 \mathrm{E}+14$ & $1.59583 E+19$ & 1215.5 & 443.1 \\
\hline & & 8.4 & 0.85 & 472.1 & $4.90497 \mathrm{E}+13$ & $2.92589 \mathrm{E}+13$ & $1.15347 \mathrm{E}+14$ & $2.42084 \mathrm{E}+19$ & 1254.7 & 466.8 \\
\hline & & 15.4 & 1.51 & 474.2 & $4.95022 \mathrm{E}+13$ & $2.93480 \mathrm{E}+13$ & $1.16475 \mathrm{E}+14$ & $4.29369 \mathrm{E}+19$ & 1257.0 & 468.2 \\
\hline & & 22.4 & 2.16 & 467.7 & $4.95197 \mathrm{E}+13$ & $2.90659 \mathrm{E}+13$ & $1.15666 \mathrm{E}+14$ & $6.14846 \mathrm{E}+19$ & 1249.9 & 463.8 \\
\hline & & 29.4 & 2.81 & 470.0 & $5.02779 \mathrm{E}+13$ & $2.93810 \mathrm{E}+13$ & 1.17193E+14 & $8.00926 \mathrm{E}+19$ & 1252.4 & 465.4 \\
\hline & & 36.4 & 3.48 & 483.1 & $5.23948 \mathrm{E}+13$ & $3.01864 \mathrm{E}+13$ & $1.21168 \mathrm{E}+14$ & $9.90619 \mathrm{E}+19$ & 1266.7 & 474.2 \\
\hline & & 43.4 & 4.15 & 482.3 & $5.29310 \mathrm{E}+13$ & $3.00891 \mathrm{E}+13$ & $1.21626 \mathrm{E}+14$ & $1.18031 \mathrm{E}+20$ & 1265.8 & 473.7 \\
\hline & & 50.4 & 4.82 & 493.1 & $5.48384 \mathrm{E}+13$ & $3.06854 \mathrm{E}+13$ & $1.25208 \mathrm{E}+14$ & $1.37302 \mathrm{E}+20$ & 1277.4 & 480.9 \\
\hline & & 57.4 & 5.51 & 497.9 & $5.60783 \mathrm{E}+13$ & $3.10933 E+13$ & $1.27061 \mathrm{E}+14$ & $1.56753 \mathrm{E}+20$ & 1282.5 & 484.2 \\
\hline & & 60.4 & 5.80 & 493.6 & $5.61926 \mathrm{E}+13$ & $3.07230 \mathrm{E}+13$ & $1.26624 \mathrm{E}+14$ & $1.65183 E+20$ & 1278.0 & 481.3 \\
\hline & \multicolumn{10}{|c|}{ Mid-Cycle 157D Outage - 14 Day, 10 Hour Down Time $(7 / 23 / 2015,1800$ - 8/7/2015, 0400) } \\
\hline & EOC $(8 / 12 / 2015,0400)$ & 65.1 & 6.27 & 493.2 & $5.71128 \mathrm{E}+13$ & $3.08396 \mathrm{E}+13$ & $1.27614 \mathrm{E}+14$ & $1.78613 \mathrm{E}+20$ & 1277.5 & 481.0 \\
\hline \multirow[t]{8}{*}{$158 \mathrm{~A}$} & \multicolumn{10}{|c|}{ Cycle 158A Outage - 91 Day, 11 Hour Down Time } \\
\hline & $\operatorname{BOC}(11 / 11 / 2015,1500)$ & 65.1 & 6.27 & 502.4 & $5.80935 \mathrm{E}+13$ & $3.13604 \mathrm{E}+13$ & $1.29805 \mathrm{E}+14$ & $1.78613 \mathrm{E}+20$ & 1287.3 & 487.2 \\
\hline & & 75.1 & 7.24 & 492.2 & $5.69742 \mathrm{E}+13$ & $3.07392 \mathrm{E}+13$ & $1.27871 \mathrm{E}+14$ & $2.06013 E+20$ & 1276.4 & 480.3 \\
\hline & & 85.1 & 8.17 & 479.0 & $5.66978 \mathrm{E}+13$ & $3.00903 E+13$ & $1.26572 \mathrm{E}+14$ & $2.32750 \mathrm{E}+20$ & 1262.3 & 471.4 \\
\hline & & 95.1 & 9.11 & 478.7 & $5.77376 \mathrm{E}+13$ & $3.00958 \mathrm{E}+13$ & $1.27696 \mathrm{E}+14$ & $2.59307 \mathrm{E}+20$ & 1261.9 & 471.2 \\
\hline & & 105.1 & 10.05 & 482.1 & $5.93203 E+13$ & $3.03418 \mathrm{E}+13$ & $1.29517 \mathrm{E}+14$ & $2.86045 \mathrm{E}+20$ & 1265.7 & 473.6 \\
\hline & & 117.1 & 11.19 & 484.4 & $6.07938 \mathrm{E}+13$ & $3.04419 E+13$ & $1.31133 \mathrm{E}+14$ & $3.18564 \mathrm{E}+20$ & 1268.1 & 475.1 \\
\hline & EOC $(1 / 2 / 2016,0400)$ & 117.3 & 11.21 & 474.8 & $6.11654 \mathrm{E}+13$ & $2.99881 \mathrm{E}+13$ & $1.30686 \mathrm{E}+14$ & $3.19046 \mathrm{E}+20$ & 1257.6 & 468.6 \\
\hline \multirow[t]{5}{*}{$158 \mathrm{~B}$} & \multicolumn{10}{|c|}{ Cycle 158B Outage - 39 Day, 2 Hour Down Time } \\
\hline & BOC $(2 / 10 / 2016,0600)$ & 118.3 & 11.29 & 396.9 & $5.09944 \mathrm{E}+13$ & $2.49297 \mathrm{E}+13$ & $1.08387 \mathrm{E}+14$ & $3.21515 E+20$ & 1166.1 & 414.6 \\
\hline & & 119.3 & 11.39 & 442.3 & $5.71996 \mathrm{E}+13$ & $2.79096 \mathrm{E}+13$ & $1.22433 E+14$ & $3.24224 \mathrm{E}+20$ & 1221.0 & 446.4 \\
\hline & & 122.3 & 11.65 & 443.9 & $5.74587 \mathrm{E}+13$ & $2.80006 \mathrm{E}+13$ & $1.23118 \mathrm{E}+14$ & $3.31812 \mathrm{E}+20$ & 1222.8 & 447.4 \\
\hline & & 127.3 & 12.08 & 435.1 & $5.67917 \mathrm{E}+13$ & $2.77906 \mathrm{E}+13$ & $1.21929 E+14$ & $3.44037 \mathrm{E}+20$ & 1212.5 & 441.3 \\
\hline
\end{tabular}


TEM-10300-1

$03 / 01 / 2012$

Rev. 03

Title: $\quad$ ATF-1 Power Histories

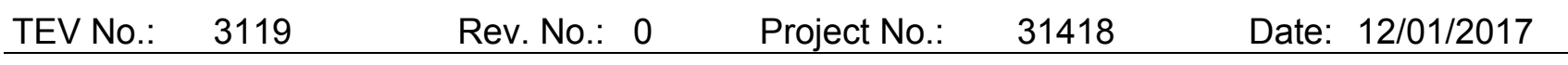

Table 17. ATF-15 Power History

\begin{tabular}{|c|c|c|c|c|c|c|c|c|c|c|}
\hline Cycle & Critical Dates & EFPD & $\begin{array}{l}\text { Burnup } \\
\text { (GWd/MTU) }\end{array}$ & $\begin{array}{l}\text { LHGR } \\
(\mathrm{W} / \mathrm{cm})\end{array}$ & $\begin{array}{l}\text { Thermal Flux } \\
\left(\mathbf{n} / \mathrm{cm}^{2 *} \mathbf{s}\right)\end{array}$ & $\begin{array}{c}\text { Fast Flux } \\
\left(\mathrm{n} / \mathrm{cm}^{2 *} \mathrm{~s}>1\right. \\
\mathrm{MeV})\end{array}$ & $\begin{array}{l}\text { Total Flux } \\
\left(\mathrm{n} / \mathrm{cm}^{2 *} \mathrm{~s}\right)\end{array}$ & $\begin{array}{l}\text { Fission Density } \\
\left(\text { Fissions } / \mathbf{c m}^{3}\right)\end{array}$ & $\begin{array}{c}\text { Fuel Centerline } \\
\text { Temperature } \\
\left({ }^{\circ} \mathrm{C}\right)\end{array}$ & PICT $\left({ }^{\circ} \mathrm{C}\right)$ \\
\hline & & 132.3 & 12.52 & 450.7 & $5.92618 \mathrm{E}+13$ & $2.85080 \mathrm{E}+13$ & $1.26184 \mathrm{E}+14$ & $3.56623 \mathrm{E}+20$ & 1230.6 & 452.1 \\
\hline & & 137.3 & 12.95 & 433.5 & $5.76533 \mathrm{E}+13$ & $2.75099 \mathrm{E}+13$ & $1.22461 \mathrm{E}+14$ & $3.68727 \mathrm{E}+20$ & 1210.6 & 440.2 \\
\hline & & 147.3 & 13.83 & 454.4 & $6.10727 \mathrm{E}+13$ & $2.88867 \mathrm{E}+13$ & $1.28779 \mathrm{E}+14$ & $3.93839 \mathrm{E}+20$ & 1234.9 & 454.7 \\
\hline & & 157.3 & 14.69 & 443.5 & $6.09881 \mathrm{E}+13$ & $2.84526 \mathrm{E}+13$ & $1.27611 \mathrm{E}+14$ & $4.18348 \mathrm{E}+20$ & 1222.4 & 447.2 \\
\hline & EOC $(4 / 1 / 2016,0900)$ & 168.7 & 15.68 & 432.1 & $6.20267 \mathrm{E}+13$ & $2.75739 \mathrm{E}+13$ & $1.26564 \mathrm{E}+14$ & $4.46411 \mathrm{E}+20$ & 1208.9 & 439.2 \\
\hline \multirow[t]{7}{*}{$159 \mathrm{~A}$} & \multicolumn{10}{|c|}{ Cycle 159A Outage - 77 Day, 2 Hour Down Time } \\
\hline & $\mathrm{BOC}(6 / 17 / 2016,1100)$ & 169.7 & 15.71 & 176.1 & $2.52670 \mathrm{E}+13$ & $1.11955 \mathrm{E}+13$ & $5.15101 \mathrm{E}+13$ & $4.47194 \mathrm{E}+20$ & 840.4 & 248.0 \\
\hline & & 170.7 & 15.74 & 185.4 & $2.65647 \mathrm{E}+13$ & $1.18011 \mathrm{E}+13$ & $5.42987 \mathrm{E}+13$ & $4.48097 \mathrm{E}+20$ & 856.2 & 255.5 \\
\hline & & 174.6 & 15.86 & 157.1 & $2.25907 \mathrm{E}+13$ & $1.00717 \mathrm{E}+13$ & $4.63403 \mathrm{E}+13$ & $4.51650 \mathrm{E}+20$ & 807.9 & 232.8 \\
\hline & \multicolumn{10}{|c|}{ Mid-Cycle 159A Outage - 3 Day, 21 Hour Down Time $(6 / 23 / 2016,1000-6 / 27 / 2016,0700)$} \\
\hline & & 176.0 & 15.89 & 228.1 & $3.30582 \mathrm{E}+13$ & $1.48867 \mathrm{E}+13$ & $7.00732 \mathrm{E}+13$ & $4.52553 \mathrm{E}+20$ & 926.0 & 289.1 \\
\hline & EOC $(6 / 28 / 2016,1800)$ & 176.2 & 15.90 & 248.6 & $3.61792 \mathrm{E}+13$ & $1.65480 \mathrm{E}+13$ & $7.89098 \mathrm{E}+13$ & $4.52854 \mathrm{E}+20$ & 958.2 & 304.9 \\
\hline $160 \mathrm{~A}$ & \multicolumn{10}{|c|}{ Cycle 160A Outage - 80 Day, 1 Hour Down Time } \\
\hline & BOC $(9 / 16 / 2016,1900)$ & 177.2 & 16.00 & 431.7 & $6.22682 \mathrm{E}+13$ & $2.76625 \mathrm{E}+13$ & $1.27516 \mathrm{E}+14$ & $4.55504 \mathrm{E}+20$ & 1208.5 & 439.0 \\
\hline & & 179.2 & 16.16 & 426.8 & $6.17616 \mathrm{E}+13$ & $2.73342 \mathrm{E}+13$ & $1.26463 \mathrm{E}+14$ & $4.60141 \mathrm{E}+20$ & 1202.7 & 435.6 \\
\hline & & 186.2 & 16.75 & 425.2 & $6.18450 \mathrm{E}+13$ & $2.70698 \mathrm{E}+13$ & $1.26247 \mathrm{E}+14$ & $4.77063 \mathrm{E}+20$ & 1200.8 & 434.5 \\
\hline & & 216.2 & 19.16 & 407.4 & $6.27914 \mathrm{E}+13$ & $2.62747 \mathrm{E}+13$ & $1.25051 \mathrm{E}+14$ & $5.45593 \mathrm{E}+20$ & 1179.1 & 422.0 \\
\hline & & 222.2 & 19.62 & 389.0 & $6.13275 \mathrm{E}+13$ & $2.47620 \mathrm{E}+13$ & $1.20756 \mathrm{E}+14$ & $5.58781 \mathrm{E}+20$ & 1156.1 & 408.9 \\
\hline & & 223.8 & 19.74 & 362.3 & $5.79231 \mathrm{E}+13$ & $2.30852 \mathrm{E}+13$ & $1.13472 \mathrm{E}+14$ & $5.62154 \mathrm{E}+20$ & 1121.5 & 389.7 \\
\hline & & 226.2 & 19.90 & 357.1 & $5.72016 \mathrm{E}+13$ & $2.28580 \mathrm{E}+13$ & $1.12347 \mathrm{E}+14$ & $5.66610 \mathrm{E}+20$ & 1114.6 & 386.0 \\
\hline & 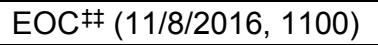 & 228.9 & 20.09 & 338.5 & $5.47334 \mathrm{E}+13$ & $2.18219 \mathrm{E}+13$ & $1.08043 \mathrm{E}+14$ & $5.72030 \mathrm{E}+20$ & 1089.4 & 372.4 \\
\hline
\end{tabular}

$\$$ ATF-15 was discharged for PIE following Cycle 160A irradiation. 
TEM-10300-1

$03 / 01 / 2012$

Rev. 03

Title: $\quad$ ATF-1 Power Histories

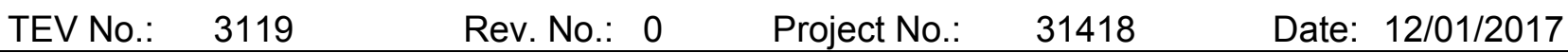

Table 18. ATF-17 Power History

\begin{tabular}{|c|c|c|c|c|c|c|c|c|c|c|}
\hline Cycle & Critical Dates & EFPD & $\begin{array}{c}\text { Burnup } \\
\text { (GWd/MTU) }\end{array}$ & $\begin{array}{l}\text { LHGR } \\
(\mathrm{W} / \mathrm{cm})\end{array}$ & $\begin{array}{l}\text { Thermal Flux } \\
\left(\mathbf{n} / \mathrm{cm}^{2 *} \mathbf{s}\right)\end{array}$ & $\begin{array}{c}\text { Fast Flux } \\
\left(\mathrm{n} / \mathrm{cm}^{2 *} \mathrm{~s}>1\right. \\
\mathrm{MeV})\end{array}$ & $\begin{array}{l}\text { Total Flux } \\
\left(\mathrm{n} / \mathrm{cm}^{2 *} \mathrm{~s}\right)\end{array}$ & $\begin{array}{l}\text { Fission Density } \\
\left(\text { Fissions } / \mathrm{cm}^{3}\right)\end{array}$ & $\begin{array}{c}\text { Fuel Centerline } \\
\text { Temperature } \\
\left({ }^{\circ} \mathrm{C}\right)\end{array}$ & $\mathrm{PICT}\left({ }^{\circ} \mathrm{C}\right)$ \\
\hline \multirow[t]{11}{*}{ 157D } & $\operatorname{BOC}(5 / 30 / 2015,1100)$ & 0.0 & 0.00 & 148.2 & $1.94331 \mathrm{E}+13$ & $1.01789 \mathrm{E}+13$ & $4.42596 \mathrm{E}+13$ & $0.00000 \mathrm{E}+00$ & 817.1 & 331.9 \\
\hline & & 3.0 & 0.13 & 171.7 & $2.24509 \mathrm{E}+13$ & $1.17280 \mathrm{E}+13$ & $5.12610 \mathrm{E}+13$ & $3.13144 \mathrm{E}+18$ & 940.9 & 362.8 \\
\hline & & 10.0 & 0.41 & 170.5 & $2.22999 \mathrm{E}+13$ & $1.17684 \mathrm{E}+13$ & $5.10977 \mathrm{E}+13$ & $1.01772 \mathrm{E}+19$ & 934.3 & 361.2 \\
\hline & & 17.0 & 0.70 & 171.4 & $2.26264 \mathrm{E}+13$ & $1.17763 \mathrm{E}+13$ & $5.14369 \mathrm{E}+13$ & $1.72229 \mathrm{E}+19$ & 939.5 & 362.4 \\
\hline & & 24.0 & 0.99 & 175.1 & $2.31342 \mathrm{E}+13$ & $1.21580 \mathrm{E}+13$ & $5.28611 \mathrm{E}+13$ & $2.43891 \mathrm{E}+19$ & 959.1 & 367.1 \\
\hline & & 31.0 & 1.28 & 177.3 & $2.35668 \mathrm{E}+13$ & $1.21038 \mathrm{E}+13$ & $5.32724 \mathrm{E}+13$ & 3.16757E+19 & 970.5 & 369.8 \\
\hline & & 38.0 & 1.59 & 184.8 & $2.46553 \mathrm{E}+13$ & $1.26370 \mathrm{E}+13$ & $5.59568 \mathrm{E}+13$ & $3.91430 \mathrm{E}+19$ & 1010.6 & 379.1 \\
\hline & & 52.0 & 2.21 & 194.7 & $2.63104 \mathrm{E}+13$ & $1.33848 \mathrm{E}+13$ & $5.93784 \mathrm{E}+13$ & $5.44389 \mathrm{E}+19$ & 1064.0 & 391.2 \\
\hline & & 55.0 & 2.35 & 197.4 & $2.68863 \mathrm{E}+13$ & $1.36499 \mathrm{E}+13$ & $6.07026 \mathrm{E}+13$ & $5.79316 \mathrm{E}+19$ & 1078.5 & 394.4 \\
\hline & \multicolumn{10}{|c|}{ Mid-Cycle 157D Outage - 14 Day, 10 Hour Down Time $(7 / 23 / 2015,1800$ - 8/7/2015, 0400) } \\
\hline & EOC $(8 / 12 / 2015,0400)$ & 59.7 & 2.56 & 199.1 & $2.73525 \mathrm{E}+13$ & $1.37561 \mathrm{E}+13$ & $6.11961 \mathrm{E}+13$ & $6.31708 \mathrm{E}+19$ & 1087.9 & 396.5 \\
\hline \multirow[t]{7}{*}{$158 \mathrm{~A}$} & \multicolumn{10}{|c|}{ Cycle 158A Outage - 91 Day, 11 Hour Down Time } \\
\hline & $\operatorname{BOC}(11 / 11 / 2015,1500)$ & 59.7 & 2.56 & 146.8 & $2.02875 \mathrm{E}+13$ & $1.05210 \mathrm{E}+13$ & $4.88062 \mathrm{E}+13$ & $6.31708 \mathrm{E}+19$ & 809.6 & 329.9 \\
\hline & & 69.7 & 2.90 & 150.2 & $2.07007 \mathrm{E}+13$ & $1.07131 \mathrm{E}+13$ & $4.95703 \mathrm{E}+13$ & $7.15414 \mathrm{E}+19$ & 827.4 & 334.5 \\
\hline & & 79.7 & 3.27 & 161.5 & $2.24381 \mathrm{E}+13$ & $1.15053 \mathrm{E}+13$ & $5.31338 \mathrm{E}+13$ & $8.05141 \mathrm{E}+19$ & 886.8 & 349.6 \\
\hline & & 89.7 & 3.68 & 178.3 & $2.49168 \mathrm{E}+13$ & $1.25937 \mathrm{E}+13$ & $5.76869 \mathrm{E}+13$ & $9.08118 \mathrm{E}+19$ & 975.8 & 371.1 \\
\hline & & 111.7 & 4.64 & 194.0 & $2.75300 \mathrm{E}+13$ & $1.35487 \mathrm{E}+13$ & $6.25962 \mathrm{E}+13$ & $1.14478 \mathrm{E}+20$ & 1060.3 & 390.4 \\
\hline & $\operatorname{EOC}(1 / 2 / 2016,0400)$ & 111.9 & 4.65 & 196.9 & $2.82372 \mathrm{E}+13$ & $1.37753 \mathrm{E}+13$ & $6.38270 \mathrm{E}+13$ & $1.14659 \mathrm{E}+20$ & 1076.0 & 393.9 \\
\hline \multirow[t]{7}{*}{ 158B } & \multicolumn{10}{|c|}{ Cycle 158B Outage - 39 Day, 2 Hour Down Time } \\
\hline & $\operatorname{BOC}(2 / 10 / 2016,0600)$ & 112.9 & 4.68 & 165.6 & $2.37981 \mathrm{E}+13$ & $1.18066 \mathrm{E}+13$ & $5.43021 \mathrm{E}+13$ & $1.15502 \mathrm{E}+20$ & 908.4 & 354.9 \\
\hline & & 113.9 & 4.71 & 153.6 & $2.20671 \mathrm{E}+13$ & $1.07888 \mathrm{E}+13$ & $5.03172 \mathrm{E}+13$ & $1.16164 \mathrm{E}+20$ & 845.2 & 339.1 \\
\hline & & 116.9 & 4.82 & 146.3 & $2.11989 \mathrm{E}+13$ & $1.03786 \mathrm{E}+13$ & $4.83529 \mathrm{E}+13$ & $1.18874 \mathrm{E}+20$ & 807.2 & 329.3 \\
\hline & & 121.9 & 4.99 & 150.8 & $2.18076 \mathrm{E}+13$ & $1.06713 \mathrm{E}+13$ & $4.97238 \mathrm{E}+13$ & $1.23090 \mathrm{E}+20$ & 830.8 & 335.4 \\
\hline & & 126.9 & 5.17 & 157.9 & $2.28599 \mathrm{E}+13$ & $1.11517 \mathrm{E}+13$ & $5.18288 \mathrm{E}+13$ & $1.27486 \mathrm{E}+20$ & 867.8 & 344.8 \\
\hline & & 131.9 & 5.35 & 163.6 & $2.38018 \mathrm{E}+13$ & $1.15059 \mathrm{E}+13$ & $5.37238 \mathrm{E}+13$ & $1.32002 \mathrm{E}+20$ & 898.0 & 352.3 \\
\hline
\end{tabular}


TEM-10300-1

$03 / 01 / 2012$

Rev. 03

Title: $\quad$ ATF-1 Power Histories

\begin{tabular}{lllll} 
TEV No.: & 3119 & Rev. No.: & 0 & Project No.: \\
\hline
\end{tabular}

Table 18. ATF-17 Power History

\begin{tabular}{|c|c|c|c|c|c|c|c|c|c|c|}
\hline Cycle & Critical Dates & EFPD & $\begin{array}{c}\text { Burnup } \\
\text { (GWd/MTU) }\end{array}$ & $\begin{array}{c}\text { LHGR } \\
(\mathrm{W} / \mathrm{cm})\end{array}$ & $\begin{array}{l}\text { Thermal Flux } \\
\left(\mathrm{n} / \mathrm{cm}^{2 *} \mathrm{~s}\right)\end{array}$ & $\begin{array}{c}\text { Fast Flux } \\
\left(\mathrm{n} / \mathrm{cm}^{2 *} \mathrm{~s}>1\right. \\
\mathrm{MeV})\end{array}$ & $\begin{array}{l}\text { Total Flux } \\
\left(\mathrm{n} / \mathrm{cm}^{2 *} \mathrm{~s}\right)\end{array}$ & $\begin{array}{l}\text { Fission Density } \\
\text { (Fissions/cm }\end{array}$ & $\begin{array}{c}\text { Fuel Centerline } \\
\text { Temperature } \\
\left({ }^{\circ} \mathrm{C}\right)\end{array}$ & $\operatorname{PICT}\left({ }^{\circ} \mathrm{C}\right)$ \\
\hline & & 141.9 & 5.77 & 178.3 & $2.59769 \mathrm{E}+13$ & $1.26652 \mathrm{E}+13$ & $5.77624 \mathrm{E}+13$ & $1.42300 \mathrm{E}+20$ & 976.2 & 371.2 \\
\hline & & 151.9 & 6.20 & 183.2 & $2.68571 \mathrm{E}+13$ & $1.27588 \mathrm{E}+13$ & $5.94777 \mathrm{E}+13$ & $1.52778 \mathrm{E}+20$ & 1002.2 & 377.2 \\
\hline & EOC $(4 / 1 / 2016,0900)$ & 163.3 & 6.68 & 195.4 & $2.92076 \mathrm{E}+13$ & $1.36346 \mathrm{E}+13$ & $6.34995 \mathrm{E}+13$ & $1.64822 \mathrm{E}+20$ & 1067.6 & 392.0 \\
\hline \multirow[t]{7}{*}{$159 \mathrm{~A}$} & \multicolumn{10}{|c|}{ Cycle 159A Outage - 77 Day, 2 Hour Down Time } \\
\hline & $\operatorname{BOC}(6 / 17 / 2016,1100)$ & 164.3 & 6.70 & 90.7 & $1.35016 \mathrm{E}+13$ & $6.27214 \mathrm{E}+12$ & $2.92385 \mathrm{E}+13$ & $1.65244 \mathrm{E}+20$ & 523.1 & 248.3 \\
\hline & & 165.3 & 6.72 & 91.7 & $1.36822 \mathrm{E}+13$ & $6.48943 \mathrm{E}+12$ & $2.98615 \mathrm{E}+13$ & $1.65725 \mathrm{E}+20$ & 527.9 & 249.8 \\
\hline & & 169.2 & 6.78 & 76.6 & $1.14901 \mathrm{E}+13$ & $5.36147 \mathrm{E}+12$ & $2.48499 \mathrm{E}+13$ & $1.67171 \mathrm{E}+20$ & 453.1 & 226.2 \\
\hline & \multicolumn{10}{|c|}{ Mid-Cycle 159A Outage - 3 Day, 21 Hour Down Time $(6 / 23 / 2016,1000$ - 6/27/2016, 0700) } \\
\hline & & 170.6 & 6.80 & 148.6 & $2.23182 \mathrm{E}+13$ & $1.01981 \mathrm{E}+13$ & $4.83469 \mathrm{E}+13$ & $1.67652 \mathrm{E}+20$ & 819.2 & 332.4 \\
\hline & EOC $(6 / 28 / 2016,1800)$ & 170.8 & 6.81 & 206.1 & $3.10328 \mathrm{E}+13$ & $1.42470 \mathrm{E}+13$ & $6.71802 \mathrm{E}+13$ & 1.67893E+20 & 1126.1 & 404.7 \\
\hline $160 \mathrm{~A}$ & \multicolumn{10}{|c|}{ Cycle 160A Outage - 80 Day, 1 Hour Down Time } \\
\hline & BOC $(9 / 16 / 2016,1900)$ & 171.8 & 6.84 & 183.1 & $2.75191 \mathrm{E}+13$ & $1.29716 \mathrm{E}+13$ & $6.11474 \mathrm{E}+13$ & $1.68736 \mathrm{E}+20$ & 1001.5 & 377.1 \\
\hline & & 173.8 & 6.94 & 175.0 & $2.63795 \mathrm{E}+13$ & 1.22637E+13 & $5.84576 \mathrm{E}+13$ & $1.71025 E+20$ & 958.2 & 366.9 \\
\hline & & 180.8 & 7.23 & 183.4 & $2.75648 \mathrm{E}+13$ & $1.29706 \mathrm{E}+13$ & $6.10722 \mathrm{E}+13$ & $1.78311 \mathrm{E}+20$ & 1003.5 & 377.5 \\
\hline & & 190.8 & 7.69 & 198.0 & $2.99351 \mathrm{E}+13$ & $1.38538 \mathrm{E}+13$ & $6.57442 \mathrm{E}+13$ & $1.89512 \mathrm{E}+20$ & 1081.7 & 395.1 \\
\hline & & 200.8 & 8.18 & 218.1 & $3.32847 \mathrm{E}+13$ & $1.52908 \mathrm{E}+13$ & $7.20658 \mathrm{E}+13$ & $2.01617 \mathrm{E}+20$ & 1191.2 & 418.2 \\
\hline & & 210.8 & 8.66 & 213.5 & $3.29577 \mathrm{E}+13$ & $1.48823 \mathrm{E}+13$ & $7.12841 \mathrm{E}+13$ & $2.13540 \mathrm{E}+20$ & 1166.2 & 413.1 \\
\hline & & 216.8 & 8.97 & 223.8 & $3.50812 \mathrm{E}+13$ & $1.56332 \mathrm{E}+13$ & $7.53635 \mathrm{E}+13$ & $2.21068 \mathrm{E}+20$ & 1222.8 & 424.6 \\
\hline & & 218.4 & 9.06 & 226.6 & $3.58360 \mathrm{E}+13$ & $1.58997 \mathrm{E}+13$ & $7.63616 \mathrm{E}+13$ & $2.23476 \mathrm{E}+20$ & 1237.9 & 427.6 \\
\hline & & 220.8 & 9.19 & 229.6 & $3.63663 \mathrm{E}+13$ & $1.61151 \mathrm{E}+13$ & $7.74598 \mathrm{E}+13$ & $2.26668 \mathrm{E}+20$ & 1254.6 & 430.9 \\
\hline & EOC $(11 / 8 / 2016,1100)$ & 223.5 & 9.34 & 227.4 & $3.60900 \mathrm{E}+13$ & $1.59109 \mathrm{E}+13$ & $7.63629 \mathrm{E}+13$ & $2.30161 \mathrm{E}+20$ & 1242.8 & 428.6 \\
\hline \multirow[t]{6}{*}{ 160B } & \multicolumn{10}{|c|}{ Cycle 160B Outage - 42 Day, 1 Hour Down Time Outage } \\
\hline & BOC $(12 / 20 / 2016,1200)$ & 224.5 & 9.37 & 180.1 & $2.85926 \mathrm{E}+13$ & $1.27463 \mathrm{E}+13$ & $6.15438 \mathrm{E}+13$ & $2.31004 \mathrm{E}+20$ & 985.5 & 373.3 \\
\hline & & 226.5 & 9.46 & 180.1 & $2.86399 \mathrm{E}+13$ & $1.28245 \mathrm{E}+13$ & $6.21274 \mathrm{E}+13$ & $2.33353 E+20$ & 985.5 & 373.3 \\
\hline & & 233.5 & 9.76 & 181.4 & $2.89304 \mathrm{E}+13$ & $1.28774 \mathrm{E}+13$ & $6.19399 \mathrm{E}+13$ & $2.40519 \mathrm{E}+20$ & 992.8 & 375.0 \\
\hline & & 243.5 & 10.20 & 195.7 & $3.14870 \mathrm{E}+13$ & $1.38403 \mathrm{E}+13$ & $6.70810 \mathrm{E}+13$ & $2.51539 \mathrm{E}+20$ & 1069.2 & 392.3 \\
\hline & & 252.7 & 10.64 & 204.5 & $3.35709 \mathrm{E}+13$ & $1.45131 \mathrm{E}+13$ & 7.07837E+13 & $2.62258 \mathrm{E}+20$ & 1117.0 & 402.7 \\
\hline
\end{tabular}


TEM-10300-1

03/01/2012

Rev. 03

Title: $\quad$ ATF-1 Power Histories

$\begin{array}{lllll}\text { TEV No.: } & 3119 & \text { Rev. No.: } & 0 & \text { Project No.: }\end{array}$

Table 18. ATF-17 Power History

\begin{tabular}{|c|c|c|c|c|c|c|c|c|c|c|}
\hline Cycle & Critical Dates & EFPD & $\begin{array}{c}\begin{array}{c}\text { Burnup } \\
\text { (GWd/MTU) }\end{array}\end{array}$ & $\begin{array}{l}\text { LHGR } \\
(\mathrm{W} / \mathrm{cm})\end{array}$ & $\begin{array}{l}\text { Thermal Flux } \\
\left(\mathrm{n} / \mathrm{cm}^{2 *} \mathrm{~s}\right)\end{array}$ & $\begin{array}{c}\text { Fast Flux } \\
\left(\mathrm{n} / \mathrm{cm}^{2 *} \mathrm{~s}>1\right. \\
\mathrm{MeV})\end{array}$ & $\begin{array}{c}\text { Total Flux } \\
\left(\mathrm{n} / \mathrm{cm}^{2 *} \mathrm{~s}\right)\end{array}$ & $\begin{array}{l}\text { Fission Density } \\
\left(\text { Fissions/cm } \mathbf{c m}^{3}\right)\end{array}$ & $\begin{array}{l}\text { Fuel Centerline } \\
\text { Temperature } \\
\left({ }^{\circ} \mathrm{C}\right)\end{array}$ & PICT $\left({ }^{\circ} \mathrm{C}\right)$ \\
\hline & \multicolumn{10}{|c|}{ Mid-Cycle 160B Outage - 5 Day, 14 Hour Down Time } \\
\hline & & 263.5 & 11.16 & 223.5 & $3.67977 \mathrm{E}+13$ & $1.55488 \mathrm{E}+13$ & $7.68058 \mathrm{E}+13$ & $2.75145 \mathrm{E}+20$ & 1221.2 & 424.3 \\
\hline & $\operatorname{EOC}(2 / 23 / 2017,1400)$ & 283.0 & 12.13 & 224.6 & $3.82109 \mathrm{E}+13$ & $1.58728 \mathrm{E}+13$ & $7.85509 \mathrm{E}+13$ & $2.99113 \mathrm{E}+20$ & 1226.9 & 425.4 \\
\hline \multirow[t]{8}{*}{$161 \mathrm{~A}$} & \multicolumn{10}{|c|}{ Cycle 161A Outage - 95 Day, 13 Hour Down Time } \\
\hline & $\mathrm{BOC}(5 / 30 / 2017,0300)$ & 284.7 & 12.22 & 195.2 & $3.31875 \mathrm{E}+13$ & $1.36143 \mathrm{E}+13$ & $6.79554 \mathrm{E}+13$ & $3.01281 \mathrm{E}+20$ & 1066.6 & 391.8 \\
\hline & & 285.0 & 12.23 & 181.3 & $3.10199 \mathrm{E}+13$ & $1.27640 \mathrm{E}+13$ & $6.39434 \mathrm{E}+13$ & $3.01522 \mathrm{E}+20$ & 992.2 & 374.9 \\
\hline & & 289.0 & 12.41 & 181.0 & $3.09408 \mathrm{E}+13$ & $1.28323 \mathrm{E}+13$ & $6.36992 \mathrm{E}+13$ & $3.06098 \mathrm{E}+20$ & 990.2 & 374.4 \\
\hline & & 291.0 & 12.51 & 185.2 & $3.17831 \mathrm{E}+13$ & $1.29553 \mathrm{E}+13$ & $6.47743 \mathrm{E}+13$ & $3.08447 \mathrm{E}+20$ & 1012.7 & 379.6 \\
\hline & & 293.0 & 12.61 & 187.9 & $3.22630 \mathrm{E}+13$ & $1.30933 \mathrm{E}+13$ & $6.58069 \mathrm{E}+13$ & $3.10856 \mathrm{E}+20$ & 1027.5 & 383.0 \\
\hline & & 295.0 & 12.70 & 190.1 & $3.27189 \mathrm{E}+13$ & $1.33541 \mathrm{E}+13$ & $6.68357 \mathrm{E}+13$ & $3.13204 \mathrm{E}+20$ & 1039.2 & 385.7 \\
\hline & $\operatorname{EOC}(6 / 11 / 2017,1600)$ & 295.5 & 12.72 & 192.2 & $3.31458 \mathrm{E}+13$ & $1.36529 \mathrm{E}+13$ & $6.79497 \mathrm{E}+13$ & $3.13686 \mathrm{E}+20$ & 1050.3 & 388.2 \\
\hline \multirow[t]{9}{*}{$162 \mathrm{~A}$} & \multicolumn{10}{|c|}{ Cycle 162A Outage } \\
\hline & $\mathrm{BOC}$ & 296.5 & 12.76 & 178.6 & $3.13217 \mathrm{E}+13$ & $1.27289 \mathrm{E}+13$ & $6.39027 \mathrm{E}+13$ & $3.14529 \mathrm{E}+20$ & 977.4 & 371.4 \\
\hline & & 298.5 & 12.84 & 166.7 & $2.88553 \mathrm{E}+13$ & 1.17697E+13 & $5.92906 \mathrm{E}+13$ & $3.16697 \mathrm{E}+20$ & 914.4 & 356.4 \\
\hline & & 305.5 & 13.11 & 167.2 & $2.87472 \mathrm{E}+13$ & $1.16622 \mathrm{E}+13$ & $5.90116 \mathrm{E}+13$ & $3.23261 \mathrm{E}+20$ & 916.8 & 356.9 \\
\hline & & 325.5 & 13.90 & 173.2 & $3.00953 \mathrm{E}+13$ & $1.21464 \mathrm{E}+13$ & $6.13469 \mathrm{E}+13$ & $3.42652 \mathrm{E}+20$ & 949.0 & 364.7 \\
\hline & & 335.5 & 14.29 & 173.9 & $3.05587 \mathrm{E}+13$ & $1.21400 \mathrm{E}+13$ & $6.18985 \mathrm{E}+13$ & $3.52347 \mathrm{E}+20$ & 952.7 & 365.6 \\
\hline & & 345.5 & 14.71 & 185.0 & $3.31723 \mathrm{E}+13$ & $1.30171 \mathrm{E}+13$ & $6.68730 \mathrm{E}+13$ & $3.62645 \mathrm{E}+20$ & 1011.8 & 379.4 \\
\hline & & 354.5 & 15.11 & 191.6 & $3.51775 \mathrm{E}+13$ & $1.36067 \mathrm{E}+13$ & $7.02909 \mathrm{E}+13$ & $3.72461 \mathrm{E}+20$ & 1047.4 & 387.5 \\
\hline & EOC & 357.5 & 15.24 & 189.7 & $3.51222 \mathrm{E}+13$ & $1.34587 \mathrm{E}+13$ & $7.00007 \mathrm{E}+13$ & $3.75713 \mathrm{E}+20$ & 1037.1 & 385.2 \\
\hline
\end{tabular}


TEM-10300-1

$03 / 01 / 2012$

Rev. 03

Title: $\quad$ ATF-1 Power Histories

\begin{tabular}{lllll} 
TEV No.: & 3119 & Rev. No.: & 0 & Project No.: \\
\hline
\end{tabular}

Table 19. ATF-18 Power History

\begin{tabular}{|c|c|c|c|c|c|c|c|c|c|c|}
\hline Cycle & Critical Dates & EFPD & $\begin{array}{c}\text { Burnup } \\
\text { (GWd/MTU) }\end{array}$ & $\begin{array}{l}\text { LHGR } \\
(\mathrm{W} / \mathrm{cm})\end{array}$ & $\begin{array}{l}\text { Thermal Flux } \\
\left(\mathrm{n} / \mathrm{cm}^{2 *} \mathrm{~s}\right)\end{array}$ & $\begin{array}{c}\text { Fast Flux } \\
\left(\mathrm{n} / \mathrm{cm}^{2 *} \mathrm{~s}>1\right. \\
\mathrm{MeV})\end{array}$ & $\begin{array}{l}\text { Total Flux } \\
\left(\mathrm{n} / \mathrm{cm}^{2 *} \mathrm{~s}\right)\end{array}$ & $\begin{array}{l}\text { Fission Density } \\
\left(\text { Fissions } / \mathrm{cm}^{3}\right)\end{array}$ & $\begin{array}{c}\text { Fuel Centerline } \\
\text { Temperature } \\
\left({ }^{\circ} \mathrm{C}\right)\end{array}$ & PICT $\left({ }^{\circ} \mathrm{C}\right)$ \\
\hline \multirow[t]{2}{*}{$157 \mathrm{C}$} & BOC $(2 / 10 / 2015,0600)$ & 0.0 & 0.00 & 201.1 & $2.67025 \mathrm{E}+13$ & $1.68032 E+13$ & $6.93337 E+13$ & $0.00000 \mathrm{E}+00$ & 1067.4 & 385.3 \\
\hline & EOC $(2 / 15 / 2015,0800)$ & 5.4 & 0.27 & 206.6 & $2.75325 \mathrm{E}+13$ & $1.71705 E+13$ & $7.08161 \mathrm{E}+13$ & $6.74464 \mathrm{E}+18$ & 1096.6 & 391.6 \\
\hline \multirow[t]{13}{*}{$157 \mathrm{D}$} & \multicolumn{10}{|c|}{ Cycle 157D Outage - 104 Day, 3 Hour Down Time } \\
\hline & $\operatorname{BOC}(5 / 30 / 2015,1100)$ & 5.4 & 0.27 & 169.4 & $2.25402 \mathrm{E}+13$ & $1.39630 \mathrm{E}+13$ & $5.77818 \mathrm{E}+13$ & $6.74464 \mathrm{E}+18$ & 901.3 & 347.1 \\
\hline & & 8.4 & 0.41 & 192.8 & $2.57277 \mathrm{E}+13$ & $1.59219 \mathrm{E}+13$ & $6.57903 E+13$ & $1.01772 \mathrm{E}+19$ & 1023.4 & 375.6 \\
\hline & & 15.4 & 0.73 & 190.5 & $2.54898 \mathrm{E}+13$ & $1.57856 \mathrm{E}+13$ & $6.54360 \mathrm{E}+13$ & $1.78251 \mathrm{E}+19$ & 1011.1 & 372.8 \\
\hline & & 22.4 & 1.04 & 190.6 & $2.56581 \mathrm{E}+13$ & $1.56266 \mathrm{E}+13$ & $6.54082 \mathrm{E}+13$ & $2.54731 \mathrm{E}+19$ & 1011.7 & 372.9 \\
\hline & & 29.4 & 1.36 & 194.3 & $2.62734 \mathrm{E}+13$ & $1.60962 \mathrm{E}+13$ & $6.69187 E+13$ & 3.33017E+19 & 1031.1 & 377.3 \\
\hline & & 36.4 & 1.67 & 196.9 & $2.68110 \mathrm{E}+13$ & $1.62613 \mathrm{E}+13$ & $6.78721 \mathrm{E}+13$ & 4.11303E+19 & 1045.3 & 380.4 \\
\hline & & 43.4 & 2.00 & 205.1 & $2.81691 \mathrm{E}+13$ & $1.68868 \mathrm{E}+13$ & $7.09318 \mathrm{E}+13$ & 4.91997E+19 & 1088.5 & 389.9 \\
\hline & & 50.4 & 2.34 & 207.2 & $2.85993 E+13$ & $1.70974 \mathrm{E}+13$ & $7.18250 \mathrm{E}+13$ & $5.74499 \mathrm{E}+19$ & 1100.0 & 392.3 \\
\hline & & 57.4 & 2.68 & 214.6 & $2.98064 \mathrm{E}+13$ & $1.78558 \mathrm{E}+13$ & $7.51914 \mathrm{E}+13$ & $6.58807 \mathrm{E}+19$ & 1139.5 & 400.7 \\
\hline & & 60.4 & 2.83 & 214.0 & $2.99728 \mathrm{E}+13$ & $1.77043 E+13$ & $7.52736 \mathrm{E}+13$ & $6.95541 \mathrm{E}+19$ & 1136.5 & 400.0 \\
\hline & \multicolumn{10}{|c|}{ Mid-Cycle 157D Outage - 14 Day, 10 Hour Down Time $(7 / 23 / 2015,1800$ - 8/7/2015, 0400) } \\
\hline & EOC $(8 / 12 / 2015,0400)$ & 65.1 & 3.06 & 214.0 & $3.01629 \mathrm{E}+13$ & $1.76391 \mathrm{E}+13$ & $7.53619 \mathrm{E}+13$ & $7.50943 \mathrm{E}+19$ & 1136.2 & 400.0 \\
\hline \multirow[t]{8}{*}{$158 \mathrm{~A}$} & \multicolumn{10}{|c|}{ Cycle 158A Outage - 91 Day, 11 Hour Down Time } \\
\hline & $\operatorname{BOC}(11 / 11 / 2015,1500)$ & 65.1 & 3.06 & 171.7 & $2.43561 \mathrm{E}+13$ & $1.45396 \mathrm{E}+13$ & $6.40392 \mathrm{E}+13$ & $7.50943 \mathrm{E}+19$ & 913.3 & 350.0 \\
\hline & & 75.1 & 3.47 & 175.5 & $2.48601 \mathrm{E}+13$ & $1.48466 \mathrm{E}+13$ & $6.51505 E+13$ & $8.52113 \mathrm{E}+19$ & 932.9 & 354.6 \\
\hline & & 85.1 & 3.89 & 180.7 & $2.58130 \mathrm{E}+13$ & $1.53867 E+13$ & $6.72752 \mathrm{E}+13$ & $9.56294 \mathrm{E}+19$ & 960.2 & 361.1 \\
\hline & & 95.1 & 4.35 & 198.0 & $2.85140 \mathrm{E}+13$ & $1.65750 \mathrm{E}+13$ & $7.25231 \mathrm{E}+13$ & $1.06770 \mathrm{E}+20$ & 1050.8 & 381.7 \\
\hline & & 105.1 & 4.81 & 199.7 & $2.90063 \mathrm{E}+13$ & $1.66433 E+13$ & $7.29159 \mathrm{E}+13$ & $1.18091 \mathrm{E}+20$ & 1060.2 & 383.7 \\
\hline & & 117.1 & 5.37 & 200.5 & $2.93166 \mathrm{E}+13$ & $1.68588 \mathrm{E}+13$ & $7.34956 \mathrm{E}+13$ & $1.31882 \mathrm{E}+20$ & 1063.9 & 384.5 \\
\hline & $\operatorname{EOC}(1 / 2 / 2016,0400)$ & 117.3 & 5.38 & 202.1 & $2.98456 \mathrm{E}+13$ & $1.70534 \mathrm{E}+13$ & $7.45587 E+13$ & $1.32062 \mathrm{E}+20$ & 1072.6 & 386.4 \\
\hline \multirow[t]{5}{*}{$158 \mathrm{~B}$} & \multicolumn{10}{|c|}{ Cycle 158B Outage - 39 Day, 2 Hour Down Time } \\
\hline & BOC $(2 / 10 / 2016,0600)$ & 118.3 & 5.41 & 192.7 & $2.84661 \mathrm{E}+13$ & $1.61871 \mathrm{E}+13$ & $7.13066 \mathrm{E}+13$ & $1.33026 \mathrm{E}+20$ & 1022.7 & 375.4 \\
\hline & & 119.3 & 5.45 & 177.4 & $2.63426 \mathrm{E}+13$ & $1.49891 \mathrm{E}+13$ & $6.61450 \mathrm{E}+13$ & $1.33869 \mathrm{E}+20$ & 942.7 & 357.0 \\
\hline & & 122.3 & 5.57 & 172.1 & $2.54481 \mathrm{E}+13$ & $1.45810 \mathrm{E}+13$ & $6.40289 \mathrm{E}+13$ & $1.36940 \mathrm{E}+20$ & 915.2 & 350.4 \\
\hline & & 127.3 & 5.77 & 176.0 & $2.61607 \mathrm{E}+13$ & $1.49503 E+13$ & $6.57143 \mathrm{E}+13$ & $1.41698 \mathrm{E}+20$ & 935.4 & 355.2 \\
\hline
\end{tabular}


TEM-10300-1

$03 / 01 / 2012$

Rev. 03

Title: $\quad$ ATF-1 Power Histories

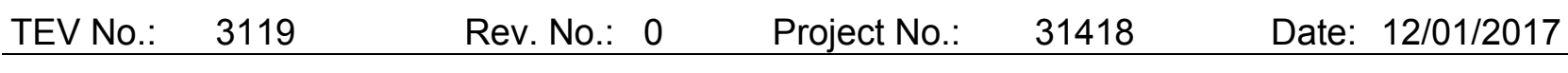

Table 19. ATF-18 Power History

\begin{tabular}{|c|c|c|c|c|c|c|c|c|c|c|}
\hline Cycle & Critical Dates & EFPD & $\begin{array}{c}\text { Burnup } \\
\text { (GWd/MTU) }\end{array}$ & $\begin{array}{l}\text { LHGR } \\
(\mathrm{W} / \mathrm{cm})\end{array}$ & $\begin{array}{l}\text { Thermal Flux } \\
\left(\mathrm{n} / \mathrm{cm}^{2 *} \mathrm{~s}\right)\end{array}$ & $\begin{array}{c}\text { Fast Flux } \\
\left(\mathrm{n} / \mathrm{cm}^{2 *} \mathrm{~s}>1\right. \\
\mathrm{MeV})\end{array}$ & $\begin{array}{l}\text { Total Flux } \\
\left(\mathrm{n} / \mathrm{cm}^{2 *} \mathrm{~s}\right)\end{array}$ & $\begin{array}{l}\text { Fission Density } \\
\text { (Fissions/cm³) }\end{array}$ & $\begin{array}{c}\text { Fuel Centerline } \\
\text { Temperature } \\
\left({ }^{\circ} \mathrm{C}\right)\end{array}$ & PICT $\left({ }^{\circ} \mathrm{C}\right)$ \\
\hline & & 132.3 & 5.97 & 182.2 & $2.72096 \mathrm{E}+13$ & $1.53263 \mathrm{E}+13$ & $6.77329 \mathrm{E}+13$ & $1.46636 \mathrm{E}+20$ & 967.9 & 362.9 \\
\hline & & 137.3 & 6.17 & 185.0 & $2.75586 \mathrm{E}+13$ & $1.54805 \mathrm{E}+13$ & $6.82608 \mathrm{E}+13$ & $1.51574 \mathrm{E}+20$ & 982.2 & 366.2 \\
\hline & & 147.3 & 6.62 & 198.2 & $2.97871 \mathrm{E}+13$ & $1.63473 \mathrm{E}+13$ & $7.29659 \mathrm{E}+13$ & $1.62714 \mathrm{E}+20$ & 1052.0 & 381.9 \\
\hline & & 157.3 & 7.08 & 199.9 & $3.02848 \mathrm{E}+13$ & $1.63806 \mathrm{E}+13$ & $7.35614 \mathrm{E}+13$ & $1.73976 \mathrm{E}+20$ & 1061.0 & 383.9 \\
\hline & EOC $(4 / 1 / 2016,0900)$ & 168.7 & 7.62 & 204.8 & $3.16178 \mathrm{E}+13$ & $1.67407 \mathrm{E}+13$ & $7.54148 \mathrm{E}+13$ & $1.87284 \mathrm{E}+20$ & 1087.2 & 389.6 \\
\hline \multirow[t]{7}{*}{$159 \mathrm{~A}$} & \multicolumn{10}{|c|}{ Cycle 159A Outage - 77 Day, 2 Hour Down Time } \\
\hline & $\operatorname{BOC}(6 / 17 / 2016,1100)$ & 169.7 & 7.64 & 92.5 & $1.43818 \mathrm{E}+13$ & $7.79402 \mathrm{E}+12$ & $3.43176 \mathrm{E}+13$ & $1.87706 \mathrm{E}+20$ & 516.8 & 241.8 \\
\hline & & 170.7 & 7.66 & 94.3 & $1.45865 \mathrm{E}+13$ & $7.73941 \mathrm{E}+12$ & $3.45156 \mathrm{E}+13$ & $1.88188 \mathrm{E}+20$ & 525.1 & 244.4 \\
\hline & & 174.6 & 7.72 & 75.4 & 1.17016E+13 & $6.39990 \mathrm{E}+12$ & $2.81940 \mathrm{E}+13$ & 1.89573E+20 & 434.7 & 216.0 \\
\hline & \multicolumn{10}{|c|}{ Mid-Cycle 159A Outage - 3 Day, 21 Hour Down Time (6/23/2016, 1000 - 6/27/2016, 0700) } \\
\hline & & 176.0 & 7.74 & 154.7 & $2.40423 \mathrm{E}+13$ & 1.29280E+13 & $5.74392 \mathrm{E}+13$ & 1.90175E+20 & 825.7 & 328.3 \\
\hline & EOC $(6 / 28 / 2016,1800)$ & 176.2 & 7.75 & 215.1 & $3.33436 \mathrm{E}+13$ & 1.79197E+13 & $7.96139 \mathrm{E}+13$ & $1.90416 \mathrm{E}+20$ & 1142.4 & 401.3 \\
\hline \multirow[t]{11}{*}{$160 \mathrm{~A}$} & \multicolumn{10}{|c|}{ Cycle 160A Outage - 80 Day, 1 Hour Down Time } \\
\hline & $\operatorname{BOC}(9 / 16 / 2016,1900)$ & 177.2 & 7.79 & 211.0 & $3.26949 \mathrm{E}+13$ & $1.77480 \mathrm{E}+13$ & $7.97718 \mathrm{E}+13$ & $1.91439 \mathrm{E}+20$ & 1120.0 & 396.6 \\
\hline & & 179.2 & 7.89 & 199.5 & $3.11625 \mathrm{E}+13$ & $1.68141 \mathrm{E}+13$ & $7.62404 \mathrm{E}+13$ & $1.93908 \mathrm{E}+20$ & 1058.9 & 383.4 \\
\hline & & 186.2 & 8.22 & 207.6 & $3.24381 \mathrm{E}+13$ & $1.75607 \mathrm{E}+13$ & $7.86096 \mathrm{E}+13$ & $2.01918 \mathrm{E}+20$ & 1102.1 & 392.8 \\
\hline & & 196.2 & 8.70 & 214.3 & $3.37868 \mathrm{E}+13$ & $1.80682 \mathrm{E}+13$ & $8.12957 \mathrm{E}+13$ & $2.13781 \mathrm{E}+20$ & 1138.0 & 400.3 \\
\hline & & 206.2 & 9.24 & 232.5 & $3.69569 \mathrm{E}+13$ & $1.96108 \mathrm{E}+13$ & $8.82111 \mathrm{E}+13$ & $2.27029 \mathrm{E}+20$ & 1236.6 & 420.2 \\
\hline & & 216.2 & 9.76 & 221.8 & $3.57487 \mathrm{E}+13$ & $1.85543 \mathrm{E}+13$ & $8.41896 \mathrm{E}+13$ & $2.39796 \mathrm{E}+20$ & 1178.4 & 408.7 \\
\hline & & 222.2 & 10.07 & 229.4 & $3.74108 \mathrm{E}+13$ & $1.90698 \mathrm{E}+13$ & $8.73437 \mathrm{E}+13$ & $2.47504 \mathrm{E}+20$ & 1219.5 & 416.9 \\
\hline & & 223.8 & 10.17 & 230.3 & $3.78546 \mathrm{E}+13$ & $1.91216 \mathrm{E}+13$ & $8.79281 \mathrm{E}+13$ & $2.49853 E+20$ & 1224.2 & 417.8 \\
\hline & & 226.2 & 10.30 & 233.6 & $3.85361 \mathrm{E}+13$ & $1.94418 \mathrm{E}+13$ & $8.93454 \mathrm{E}+13$ & $2.53044 \mathrm{E}+20$ & 1242.4 & 421.4 \\
\hline & EOC§§ $(11 / 8 / 2016,1100)$ & 228.9 & 10.44 & 229.7 & $3.81388 \mathrm{E}+13$ & $1.91326 \mathrm{E}+13$ & 8.79617E+13 & $2.56537 \mathrm{E}+20$ & 1221.3 & 417.2 \\
\hline
\end{tabular}

$\$$ ATF-18 was discharged for PIE following Cycle 160A irradiation. 
TEM-10300-1

$03 / 01 / 2012$

Rev. 03

Title: $\quad$ ATF-1 Power Histories

\begin{tabular}{lllll} 
TEV No.: & 3119 & Rev. No.: & 0 & Project No.: \\
\hline
\end{tabular}

Table 20. ATF-20 Power History

\begin{tabular}{|c|c|c|c|c|c|c|c|c|c|c|}
\hline Cycle & Critical Dates & EFPD & $\begin{array}{c}\text { Burnup } \\
\text { (GWd/MTU) }\end{array}$ & $\begin{array}{l}\text { LHGR } \\
(\mathrm{W} / \mathrm{cm})\end{array}$ & $\begin{array}{l}\text { Thermal Flux } \\
\left(\mathrm{n} / \mathrm{cm}^{2 *} \mathrm{~s}\right)\end{array}$ & $\begin{array}{c}\text { Fast Flux } \\
\left(\mathrm{n} / \mathrm{cm}^{2 *} \mathrm{~s}>1\right. \\
\mathrm{MeV})\end{array}$ & $\begin{array}{l}\text { Total Flux } \\
\left(\mathrm{n} / \mathrm{cm}^{2 *} \mathrm{~s}\right)\end{array}$ & $\begin{array}{l}\text { Fission Density } \\
\text { (Fissions } / \mathrm{cm}^{3} \text { ) }\end{array}$ & $\begin{array}{c}\text { Fuel Centerline } \\
\text { Temperature } \\
\left({ }^{\circ} \mathrm{C}\right)\end{array}$ & PICT $\left({ }^{\circ} \mathrm{C}\right)$ \\
\hline \multirow[t]{2}{*}{$157 \mathrm{C}$} & $\mathrm{BOC}(2 / 10 / 2015,0600)$ & 0.0 & 0.00 & 193.1 & $2.51587 \mathrm{E}+13$ & 1.21067E+13 & $5.44625 \mathrm{E}+13$ & $0.00000 \mathrm{E}+00$ & 1087.5 & 419.0 \\
\hline & EOC $(2 / 15 / 2015,0800)$ & 5.4 & 0.25 & 204.1 & $2.66629 \mathrm{E}+13$ & $1.27171 \mathrm{E}+13$ & $5.69708 \mathrm{E}+13$ & $6.14244 \mathrm{E}+18$ & 1148.8 & 433.1 \\
\hline \multirow[t]{13}{*}{ 157D } & \multicolumn{10}{|c|}{ Cycle 157D Outage - 104 Day, 3 Hour Down Time } \\
\hline & $\mathrm{BOC}(5 / 30 / 2015,1100)$ & 5.4 & 0.25 & 148.3 & $1.94440 \mathrm{E}+13$ & $1.02322 \mathrm{E}+13$ & $4.44282 \mathrm{E}+13$ & $6.14244 \mathrm{E}+18$ & 842.9 & 357.0 \\
\hline & & 8.4 & 0.37 & 170.2 & $2.23358 \mathrm{E}+13$ & 1.16763E+13 & $5.08708 \mathrm{E}+13$ & $9.21366 \mathrm{E}+18$ & 961.8 & 388.3 \\
\hline & & 15.4 & 0.66 & 169.3 & $2.22469 \mathrm{E}+13$ & $1.16035 \mathrm{E}+13$ & $5.07757 \mathrm{E}+13$ & $1.61992 \mathrm{E}+19$ & 956.5 & 386.9 \\
\hline & & 22.4 & 0.94 & 168.9 & $2.23646 \mathrm{E}+13$ & $1.15918 \mathrm{E}+13$ & $5.09282 E+13$ & $2.31847 E+19$ & 954.2 & 386.3 \\
\hline & & 29.4 & 1.23 & 173.8 & $2.31605 E+13$ & 1.19268E+13 & $5.25980 \mathrm{E}+13$ & 3.02907E+19 & 981.5 & 393.2 \\
\hline & & 36.4 & 1.52 & 175.9 & $2.35662 \mathrm{E}+13$ & $1.20028 \mathrm{E}+13$ & $5.32450 \mathrm{E}+13$ & $3.75171 \mathrm{E}+19$ & 992.6 & 396.0 \\
\hline & & 43.4 & 1.82 & 181.9 & $2.44649 \mathrm{E}+13$ & $1.24809 \mathrm{E}+13$ & $5.53638 \mathrm{E}+13$ & $4.49241 \mathrm{E}+19$ & 1025.7 & 404.2 \\
\hline & & 50.4 & 2.13 & 184.8 & $2.49561 \mathrm{E}+13$ & $1.27101 \mathrm{E}+13$ & $5.64064 \mathrm{E}+13$ & $5.23914 \mathrm{E}+19$ & 1041.5 & 408.0 \\
\hline & & 57.4 & 2.45 & 194.0 & $2.63628 \mathrm{E}+13$ & $1.33014 \mathrm{E}+13$ & $5.94830 \mathrm{E}+13$ & $6.02200 \mathrm{E}+19$ & 1092.7 & 420.2 \\
\hline & & 60.4 & 2.58 & 195.6 & $2.69228 \mathrm{E}+13$ & $1.34912 \mathrm{E}+13$ & $6.04470 \mathrm{E}+13$ & $6.36525 \mathrm{E}+19$ & 1101.7 & 422.3 \\
\hline & \multicolumn{10}{|c|}{ Mid-Cycle 157D Outage - 14 Day, 10 Hour Down Time $(7 / 23 / 2015,1800$ - 8/7/2015, 0400) } \\
\hline & EOC $(8 / 12 / 2015,0400)$ & 65.1 & 2.79 & 197.3 & $2.73537 \mathrm{E}+13$ & $1.36093 \mathrm{E}+13$ & $6.09250 \mathrm{E}+13$ & $6.88315 \mathrm{E}+19$ & 1111.3 & 424.5 \\
\hline \multirow[t]{8}{*}{$158 \mathrm{~A}$} & \multicolumn{10}{|c|}{ Cycle 158A Outage - 91 Day, 11 Hour Down Time } \\
\hline & $\mathrm{BOC}(11 / 11 / 2015,1500)$ & 65.1 & 2.79 & 145.9 & $2.02800 \mathrm{E}+13$ & $1.04544 \mathrm{E}+13$ & $4.88533 \mathrm{E}+13$ & $6.88315 \mathrm{E}+19$ & 829.6 & 353.4 \\
\hline & & 75.1 & 3.13 & 149.8 & $2.08030 \mathrm{E}+13$ & 1.06703E+13 & 4.97419E+13 & $7.71418 \mathrm{E}+19$ & 851.0 & 359.2 \\
\hline & & 85.1 & 3.49 & 160.2 & $2.24435 \mathrm{E}+13$ & 1.15320E+13 & $5.31898 \mathrm{E}+13$ & $8.59942 E+19$ & 907.3 & 374.2 \\
\hline & & 95.1 & 3.91 & 176.2 & $2.47900 \mathrm{E}+13$ & $1.26604 \mathrm{E}+13$ & $5.76543 E+13$ & $9.62316 \mathrm{E}+19$ & 994.5 & 396.5 \\
\hline & & 105.1 & 4.34 & 185.4 & $2.62554 \mathrm{E}+13$ & $1.30076 \mathrm{E}+13$ & $6.00990 \mathrm{E}+13$ & $1.06891 \mathrm{E}+20$ & 1045.2 & 408.9 \\
\hline & & 117.1 & 4.87 & 194.0 & $2.76344 \mathrm{E}+13$ & $1.36121 \mathrm{E}+13$ & $6.25782 \mathrm{E}+13$ & $1.19838 \mathrm{E}+20$ & 1092.6 & 420.2 \\
\hline & EOC $(1 / 2 / 2016,0400)$ & 117.3 & 4.87 & 196.0 & $2.83125 \mathrm{E}+13$ & $1.38068 \mathrm{E}+13$ & $6.38540 \mathrm{E}+13$ & $1.20018 \mathrm{E}+20$ & 1103.9 & 422.8 \\
\hline \multirow[t]{4}{*}{$158 \mathrm{~B}$} & \multicolumn{10}{|c|}{ Cycle 158B Outage - 39 Day, 2 Hour Down Time } \\
\hline & $\mathrm{BOC}(2 / 10 / 2016,0600)$ & 118.3 & 4.90 & 163.5 & $2.36824 \mathrm{E}+13$ & 1.16597E+13 & $5.38520 \mathrm{E}+13$ & $1.20741 \mathrm{E}+20$ & 924.8 & 378.8 \\
\hline & & 119.3 & 4.93 & 153.1 & $2.21199 \mathrm{E}+13$ & $1.07929 \mathrm{E}+13$ & $5.04104 \mathrm{E}+13$ & $1.21464 \mathrm{E}+20$ & 868.4 & 363.9 \\
\hline & & 122.3 & 5.04 & 148.6 & $2.14529 \mathrm{E}+13$ & $1.05668 \mathrm{E}+13$ & $4.89686 \mathrm{E}+13$ & 1.24234E+20 & 844.2 & 357.3 \\
\hline
\end{tabular}


TEM-10300-1

$03 / 01 / 2012$

Rev. 03

Title: $\quad$ ATF-1 Power Histories

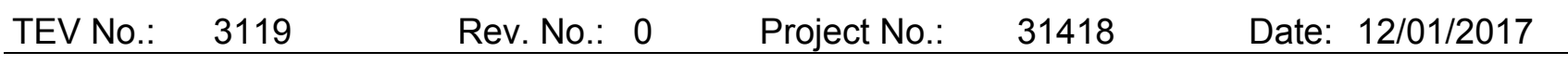

Table 20. ATF-20 Power History

\begin{tabular}{|c|c|c|c|c|c|c|c|c|c|c|}
\hline Cycle & Critical Dates & EFPD & $\begin{array}{c}\text { Burnup } \\
\text { (GWd/MTU) }\end{array}$ & $\begin{array}{l}\text { LHGR } \\
(\mathrm{W} / \mathrm{cm})\end{array}$ & $\begin{array}{l}\text { Thermal Flux } \\
\left(\mathrm{n} / \mathrm{cm}^{2 *} \mathrm{~s}\right)\end{array}$ & $\begin{array}{c}\text { Fast Flux } \\
\left(\mathrm{n} / \mathrm{cm}^{2 *} \mathrm{~s}>1\right. \\
\mathrm{MeV})\end{array}$ & $\begin{array}{l}\text { Total Flux } \\
\left(\mathrm{n} / \mathrm{cm}^{2 *} \mathrm{~s}\right)\end{array}$ & $\begin{array}{l}\text { Fission Density } \\
\text { (Fissions/cm³) }\end{array}$ & $\begin{array}{c}\text { Fuel Centerline } \\
\text { Temperature } \\
\left({ }^{\circ} \mathrm{C}\right)\end{array}$ & $\mathrm{PICT}\left({ }^{\circ} \mathrm{C}\right)$ \\
\hline & & 127.3 & 5.22 & 151.0 & $2.19530 \mathrm{E}+13$ & $1.06827 \mathrm{E}+13$ & $4.99931 \mathrm{E}+13$ & $1.28449 \mathrm{E}+20$ & 857.2 & 360.9 \\
\hline & & 132.3 & 5.39 & 158.4 & $2.30959 \mathrm{E}+13$ & $1.09681 \mathrm{E}+13$ & $5.22215 E+13$ & $1.32785 \mathrm{E}+20$ & 897.1 & 371.5 \\
\hline & & 137.3 & 5.57 & 164.1 & $2.40636 \mathrm{E}+13$ & $1.13858 \mathrm{E}+13$ & $5.36073 \mathrm{E}+13$ & $1.37302 \mathrm{E}+20$ & 928.5 & 379.7 \\
\hline & & 147.3 & 5.99 & 177.6 & $2.60248 \mathrm{E}+13$ & $1.24630 \mathrm{E}+13$ & $5.77688 \mathrm{E}+13$ & $1.47539 \mathrm{E}+20$ & 1002.1 & 398.4 \\
\hline & & 157.3 & 6.41 & 180.6 & $2.66581 \mathrm{E}+13$ & $1.27688 \mathrm{E}+13$ & $5.93769 \mathrm{E}+13$ & 1.57957E+20 & 1018.6 & 402.4 \\
\hline & EOC $(4 / 1 / 2016,0900)$ & 168.7 & 6.90 & 194.5 & $2.94085 \mathrm{E}+13$ & $1.37632 \mathrm{E}+13$ & $6.38744 \mathrm{E}+13$ & $1.69941 \mathrm{E}+20$ & 1095.7 & 420.9 \\
\hline \multirow[t]{7}{*}{$159 \mathrm{~A}$} & \multicolumn{10}{|c|}{ Cycle 159A Outage - 77 Day, 2 Hour Down Time } \\
\hline & $\mathrm{BOC}(6 / 17 / 2016,1100)$ & 169.7 & 6.92 & 89.5 & $1.34248 \mathrm{E}+13$ & $6.14004 \mathrm{E}+12$ & $2.88768 \mathrm{E}+13$ & $1.70362 \mathrm{E}+20$ & 533.0 & 264.6 \\
\hline & & 170.7 & 6.93 & 91.8 & $1.37974 \mathrm{E}+13$ & $6.43987 \mathrm{E}+12$ & $2.96952 \mathrm{E}+13$ & $1.70784 \mathrm{E}+20$ & 544.8 & 268.4 \\
\hline & & 174.6 & 6.99 & 75.9 & $1.13615 \mathrm{E}+13$ & $5.24122 \mathrm{E}+12$ & $2.46279 \mathrm{E}+13$ & $1.72229 \mathrm{E}+20$ & 463.1 & 241.4 \\
\hline & \multicolumn{10}{|c|}{ Mid-Cycle 159A Outage - 3 Day, 21 Hour Down Time $(6 / 23 / 2016,1000$ - 6/27/2016, 0700) } \\
\hline & & 176.0 & 7.02 & 147.1 & $2.22257 \mathrm{E}+13$ & $1.02393 E+13$ & $4.89101 \mathrm{E}+13$ & $1.72771 \mathrm{E}+20$ & 836.2 & 355.2 \\
\hline & EOC $(6 / 28 / 2016,1800)$ & 176.2 & 7.02 & 204.2 & $3.09011 \mathrm{E}+13$ & $1.42602 \mathrm{E}+13$ & $6.76245 E+13$ & $1.72952 \mathrm{E}+20$ & 1149.5 & 433.3 \\
\hline $160 \mathrm{~A}$ & \multicolumn{10}{|c|}{ Cycle 160A Outage - 80 Day, 1 Hour Down Time } \\
\hline & $\operatorname{BOC}(9 / 16 / 2016,1900)$ & 177.2 & 7.06 & 180.9 & $2.73944 \mathrm{E}+13$ & $1.30200 \mathrm{E}+13$ & $6.08378 \mathrm{E}+13$ & $1.73795 \mathrm{E}+20$ & 1020.0 & 402.8 \\
\hline & & 179.2 & 7.15 & 175.0 & $2.65185 \mathrm{E}+13$ & $1.23541 \mathrm{E}+13$ & $5.90136 \mathrm{E}+13$ & $1.76083 \mathrm{E}+20$ & 987.9 & 394.8 \\
\hline & & 186.2 & 7.44 & 181.8 & $2.75719 \mathrm{E}+13$ & $1.28590 \mathrm{E}+13$ & $6.09532 \mathrm{E}+13$ & $1.83310 \mathrm{E}+20$ & 1025.1 & 404.0 \\
\hline & & 196.2 & 7.89 & 195.2 & $2.97286 \mathrm{E}+13$ & $1.38001 \mathrm{E}+13$ & $6.53893 E+13$ & $1.94390 \mathrm{E}+20$ & 1099.2 & 421.7 \\
\hline & & 206.2 & 8.38 & 215.3 & $3.30288 \mathrm{E}+13$ & $1.52048 \mathrm{E}+13$ & $7.17173 \mathrm{E}+13$ & $2.06374 \mathrm{E}+20$ & 1212.1 & 447.2 \\
\hline & & 216.2 & 8.86 & 212.5 & $3.32522 \mathrm{E}+13$ & $1.50073 E+13$ & $7.16204 \mathrm{E}+13$ & $2.18237 \mathrm{E}+20$ & 1196.3 & 443.7 \\
\hline & & 222.2 & 9.17 & 223.7 & $3.53064 \mathrm{E}+13$ & $1.56768 \mathrm{E}+13$ & $7.55562 \mathrm{E}+13$ & $2.25765 \mathrm{E}+20$ & 1259.2 & 457.2 \\
\hline & & 223.8 & 9.26 & 225.8 & $3.58256 \mathrm{E}+13$ & $1.58778 \mathrm{E}+13$ & $7.62036 E+13$ & $2.28113 \mathrm{E}+20$ & 1271.3 & 459.8 \\
\hline & & 226.2 & 9.39 & 229.0 & $3.64482 \mathrm{E}+13$ & $1.60645 E+13$ & $7.74537 E+13$ & $2.31305 E+20$ & 1289.6 & 463.6 \\
\hline & EOC $(11 / 8 / 2016,1100)$ & 228.9 & 9.53 & 226.9 & $3.62997 \mathrm{E}+13$ & $1.57281 \mathrm{E}+13$ & $7.66545 E+13$ & $2.34738 \mathrm{E}+20$ & 1277.5 & 461.1 \\
\hline \multirow[t]{5}{*}{$160 \mathrm{~B}$} & \multicolumn{10}{|c|}{ Cycle 160B Outage - 42 Day, 1 Hour Down Time Outage } \\
\hline & $\mathrm{BOC}(12 / 20 / 2016,1200)$ & 229.9 & 9.57 & 177.5 & $2.84268 \mathrm{E}+13$ & $1.25951 \mathrm{E}+13$ & $6.10294 \mathrm{E}+13$ & $2.35641 \mathrm{E}+20$ & 1001.3 & 398.2 \\
\hline & & 231.9 & 9.66 & 178.4 & $2.86229 \mathrm{E}+13$ & $1.28398 \mathrm{E}+13$ & $6.18257 E+13$ & $2.37869 \mathrm{E}+20$ & 1006.5 & 399.5 \\
\hline & & 238.9 & 9.95 & 180.6 & $2.89289 \mathrm{E}+13$ & $1.28103 E+13$ & $6.18380 \mathrm{E}+13$ & $2.45035 \mathrm{E}+20$ & 1018.5 & 402.4 \\
\hline & & 248.9 & 10.39 & 194.8 & $3.14387 \mathrm{E}+13$ & $1.37681 \mathrm{E}+13$ & $6.66814 \mathrm{E}+13$ & $2.55995 \mathrm{E}+20$ & 1097.2 & 421.3 \\
\hline
\end{tabular}


TEM-10300-1

03/01/2012

Rev. 03

Title: $\quad$ ATF-1 Power Histories

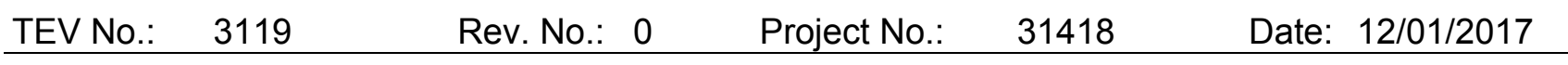

Table 20. ATF-20 Power History

\begin{tabular}{|c|c|c|c|c|c|c|c|c|c|c|}
\hline Cycle & Critical Dates & EFPD & $\begin{array}{c}\text { Burnup } \\
\text { (GWd/MTU) }\end{array}$ & $\begin{array}{l}\text { LHGR } \\
(\mathrm{W} / \mathrm{cm})\end{array}$ & $\begin{array}{l}\text { Thermal Flux } \\
\left(\mathrm{n} / \mathrm{cm}^{2 *} \mathrm{~s}\right)\end{array}$ & $\begin{array}{c}\text { Fast Flux } \\
\left(\mathrm{n} / \mathrm{cm}^{2 *} \mathrm{~s}>1\right. \\
\mathrm{MeV})\end{array}$ & $\begin{array}{l}\text { Total Flux } \\
\left(\mathrm{n} / \mathrm{cm}^{2 *} \mathrm{~s}\right)\end{array}$ & $\begin{array}{c}\text { Fission Density } \\
\left(\text { Fissions } / \mathrm{cm}^{3}\right)\end{array}$ & $\begin{array}{c}\text { Fuel Centerline } \\
\text { Temperature } \\
\left({ }^{\circ} \mathrm{C}\right)\end{array}$ & $\mathrm{PICT}\left({ }^{\circ} \mathrm{C}\right)$ \\
\hline & & 258.1 & 10.82 & 202.2 & $3.34135 \mathrm{E}+13$ & $1.44061 \mathrm{E}+13$ & $7.03280 \mathrm{E}+13$ & $2.66594 \mathrm{E}+20$ & 1138.3 & 430.7 \\
\hline & \multicolumn{10}{|c|}{ Mid-Cycle 160B Outage - 5 Day, 14 Hour Down Time } \\
\hline & & 268.9 & 11.34 & 220.8 & $3.66314 \mathrm{E}+13$ & $1.55721 \mathrm{E}+13$ & $7.65453 \mathrm{E}+13$ & $2.79361 \mathrm{E}+20$ & 1243.0 & 453.8 \\
\hline & & 278.9 & 11.83 & 220.4 & $3.69175 \mathrm{E}+13$ & $1.56060 \mathrm{E}+13$ & $7.68831 \mathrm{E}+13$ & $2.91465 \mathrm{E}+20$ & 1240.8 & 453.3 \\
\hline & EOC $(2 / 23 / 2017,1400)$ & 288.4 & 12.31 & 223.0 & $3.81461 \mathrm{E}+13$ & $1.57897 \mathrm{E}+13$ & $7.84548 \mathrm{E}+13$ & $3.03208 \mathrm{E}+20$ & 1255.4 & 456.4 \\
\hline $161 \mathrm{~A}$ & \multicolumn{10}{|c|}{ Cycle 161A Outage - 95 Day, 13 Hour Down Time } \\
\hline & BOC $(5 / 30 / 2017,0300)$ & 290.1 & 12.40 & 194.5 & $3.32747 \mathrm{E}+13$ & $1.36783 E+13$ & $6.81225 E+13$ & $3.05376 \mathrm{E}+20$ & 1095.5 & 420.9 \\
\hline & & 290.4 & 12.41 & 180.0 & $3.08284 \mathrm{E}+13$ & $1.27652 E+13$ & $6.34395 E+13$ & $3.05677 \mathrm{E}+20$ & 1015.2 & 401.6 \\
\hline & & 292.4 & 12.50 & 181.4 & $3.10416 \mathrm{E}+13$ & $1.27098 \mathrm{E}+13$ & $6.37255 E+13$ & $3.07965 \mathrm{E}+20$ & 1022.8 & 403.5 \\
\hline & & 294.4 & 12.60 & 179.6 & $3.09056 \mathrm{E}+13$ & $1.28558 \mathrm{E}+13$ & $6.35908 \mathrm{E}+13$ & $3.10253 \mathrm{E}+20$ & 1013.2 & 401.1 \\
\hline & & 296.4 & 12.69 & 183.7 & $3.16707 \mathrm{E}+13$ & $1.29305 E+13$ & $6.49071 \mathrm{E}+13$ & $3.12602 \mathrm{E}+20$ & 1035.5 & 406.5 \\
\hline & & 298.4 & 12.79 & 185.5 & $3.21526 \mathrm{E}+13$ & $1.31041 \mathrm{E}+13$ & $6.56533 \mathrm{E}+13$ & $3.14890 \mathrm{E}+20$ & 1045.5 & 409.0 \\
\hline & & 300.4 & 12.88 & 188.3 & $3.26143 E+13$ & $1.32969 E+13$ & $6.66689 \mathrm{E}+13$ & $3.17299 \mathrm{E}+20$ & 1061.2 & 412.7 \\
\hline & EOC $(6 / 11 / 2017,1600)$ & 300.9 & 12.90 & 190.7 & $3.31132 \mathrm{E}+13$ & $1.34690 \mathrm{E}+13$ & $6.77912 \mathrm{E}+13$ & $3.17781 \mathrm{E}+20$ & 1074.6 & 415.9 \\
\hline $162 \mathrm{~A}$ & \multicolumn{10}{|c|}{ Cycle 162A Outage } \\
\hline & $\mathrm{BOC}$ & 301.9 & 12.94 & 178.2 & $3.14166 \mathrm{E}+13$ & $1.28962 \mathrm{E}+13$ & $6.40456 \mathrm{E}+13$ & $3.18624 \mathrm{E}+20$ & 1005.1 & 399.1 \\
\hline & & 303.9 & 13.03 & 165.9 & $2.88634 \mathrm{E}+13$ & $1.17718 \mathrm{E}+13$ & $5.90833 E+13$ & $3.20792 E+20$ & 937.9 & 382.2 \\
\hline & & 310.9 & 13.29 & 166.3 & $2.86622 \mathrm{E}+13$ & $1.16477 \mathrm{E}+13$ & $5.87947 E+13$ & $3.27296 \mathrm{E}+20$ & 940.2 & 382.8 \\
\hline & & 320.9 & 13.69 & 172.2 & $2.99534 \mathrm{E}+13$ & $1.19986 \mathrm{E}+13$ & $6.07779 \mathrm{E}+13$ & $3.37051 \mathrm{E}+20$ & 972.5 & 391.0 \\
\hline & & 330.9 & 14.08 & 174.0 & $3.03742 \mathrm{E}+13$ & $1.20672 \mathrm{E}+13$ & $6.15056 \mathrm{E}+13$ & $3.46747 \mathrm{E}+20$ & 982.1 & 393.4 \\
\hline & & 340.9 & 14.47 & 174.2 & $3.08256 \mathrm{E}+13$ & $1.21312 \mathrm{E}+13$ & $6.21397 E+13$ & $3.56442 \mathrm{E}+20$ & 983.6 & 393.8 \\
\hline & & 350.9 & 14.89 & 183.8 & $3.31130 \mathrm{E}+13$ & $1.29820 E+13$ & $6.65300 \mathrm{E}+13$ & $3.66680 \mathrm{E}+20$ & 1036.1 & 406.7 \\
\hline & & 359.9 & 15.29 & 192.0 & $3.54269 \mathrm{E}+13$ & $1.36943 E+13$ & $7.07865 E+13$ & $3.76495 \mathrm{E}+20$ & 1081.8 & 417.6 \\
\hline & EOC & 362.9 & 15.42 & 189.4 & $3.52188 \mathrm{E}+13$ & $1.34806 \mathrm{E}+13$ & $7.01618 \mathrm{E}+13$ & $3.79687 \mathrm{E}+20$ & 1067.1 & 414.2 \\
\hline
\end{tabular}


TEM-10300-1

$03 / 01 / 2012$

Rev. 03

Title:

ATF-1 Power Histories

\begin{tabular}{lllll} 
TEV No.: & 3119 & Rev. No.: & 0 & Project No.: \\
\hline
\end{tabular}

Table 21. ATF-29 Power History

\begin{tabular}{|c|c|c|c|c|c|c|c|c|c|c|}
\hline Cycle & Critical Dates & EFPD & $\begin{array}{c}\text { Burnup } \\
\text { (GWd/MTU) }\end{array}$ & $\begin{array}{l}\text { LHGR } \\
(\mathrm{W} / \mathrm{cm})\end{array}$ & $\begin{array}{l}\text { Thermal Flux } \\
\left(\mathrm{n} / \mathrm{cm}^{2 *} \mathrm{~s}\right)\end{array}$ & $\begin{array}{c}\text { Fast Flux } \\
\left(\mathrm{n} / \mathrm{cm}^{2 *} \mathrm{~s}>1\right. \\
\mathrm{MeV})\end{array}$ & $\begin{array}{l}\text { Total Flux } \\
\left(\mathrm{n} / \mathrm{cm}^{2 *} \mathrm{~s}\right)\end{array}$ & $\begin{array}{l}\text { Fission Density } \\
\text { (Fissions } / \mathrm{cm}^{3} \text { ) }\end{array}$ & $\begin{array}{c}\text { Fuel Centerline } \\
\text { Temperature } \\
\left({ }^{\circ} \mathrm{C}\right)\end{array}$ & $\mathrm{PICT}\left({ }^{\circ} \mathrm{C}\right)$ \\
\hline \multirow{10}{*}{$160 \mathrm{~A}$} & BOC $(9 / 16 / 2016,1900)$ & 1.0 & 0.04 & 250.9 & $2.81779 \mathrm{E}+13$ & $1.73750 \mathrm{E}+13$ & $6.99894 \mathrm{E}+13$ & $1.20440 \mathrm{E}+18$ & 624.2 & 300.2 \\
\hline & & 3.0 & 0.12 & 243.6 & $2.73653 \mathrm{E}+13$ & $1.70401 \mathrm{E}+13$ & $6.82520 \mathrm{E}+13$ & $4.03474 \mathrm{E}+18$ & 611.9 & 294.6 \\
\hline & & 10.0 & 0.43 & 249.7 & $2.81335 \mathrm{E}+13$ & $1.73092 \mathrm{E}+13$ & $7.04009 E+13$ & $1.40915 \mathrm{E}+19$ & 622.2 & 299.3 \\
\hline & & 20.0 & 0.88 & 255.0 & $2.88475 \mathrm{E}+13$ & $1.76318 \mathrm{E}+13$ & $7.13042 \mathrm{E}+13$ & $2.84841 \mathrm{E}+19$ & 630.9 & 303.4 \\
\hline & & 30.0 & 1.32 & 255.0 & $2.91182 \mathrm{E}+13$ & $1.76139 \mathrm{E}+13$ & $7.18400 \mathrm{E}+13$ & $4.28164 \mathrm{E}+19$ & 630.8 & 303.3 \\
\hline & & 40.0 & 1.76 & 256.7 & $2.95522 \mathrm{E}+13$ & $1.79169 \mathrm{E}+13$ & $7.27609 \mathrm{E}+13$ & $5.71488 \mathrm{E}+19$ & 633.6 & 304.6 \\
\hline & & 46.0 & 2.03 & 271.4 & $3.16931 \mathrm{E}+13$ & $1.90759 \mathrm{E}+13$ & $7.78879 \mathrm{E}+13$ & $6.59409 \mathrm{E}+19$ & 657.7 & 315.8 \\
\hline & & 47.6 & 2.11 & 249.4 & $2.93896 \mathrm{E}+13$ & $1.74800 \mathrm{E}+13$ & $7.15216 \mathrm{E}+13$ & $6.84701 \mathrm{E}+19$ & 621.5 & 299.0 \\
\hline & & 50.0 & 2.21 & 250.1 & $2.93565 \mathrm{E}+13$ & $1.74549 \mathrm{E}+13$ & $7.15584 \mathrm{E}+13$ & $7.18425 \mathrm{E}+19$ & 622.8 & 299.6 \\
\hline & EOC $(11 / 8 / 2016,1100)$ & 52.7 & 2.32 & 239.5 & $2.83620 \mathrm{E}+13$ & $1.69052 \mathrm{E}+13$ & $6.97280 \mathrm{E}+13$ & 7.53954E+19 & 605.0 & 291.4 \\
\hline $160 \mathrm{~B}$ & \multicolumn{10}{|c|}{ Cycle 160B Outage - 42 Day, 1 Hour Down Time Outage } \\
\hline & $\mathrm{BOC}(12 / 20 / 2016,1200)$ & 53.7 & 2.37 & 287.1 & $3.38323 \mathrm{E}+13$ & $2.00054 \mathrm{E}+13$ & 8.29427E+13 & 7.67805E+19 & 682.6 & 327.4 \\
\hline & & 55.7 & 2.47 & 279.2 & $3.29949 \mathrm{E}+13$ & $1.95501 \mathrm{E}+13$ & $8.10080 E+13$ & 7.99722E+19 & 670.2 & 321.6 \\
\hline & & 62.7 & 2.80 & 273.6 & $3.24065 \mathrm{E}+13$ & $1.91815 \mathrm{E}+13$ & $7.98669 \mathrm{E}+13$ & $9.06913 \mathrm{E}+19$ & 661.2 & 317.4 \\
\hline & & 72.7 & 3.24 & 263.3 & $3.14474 \mathrm{E}+13$ & $1.85568 \mathrm{E}+13$ & $7.72581 \mathrm{E}+13$ & $1.05265 \mathrm{E}+20$ & 644.5 & 309.6 \\
\hline & & 81.9 & 3.67 & 263.5 & $3.21358 \mathrm{E}+13$ & $1.86158 \mathrm{E}+13$ & $7.82026 \mathrm{E}+13$ & $1.18995 \mathrm{E}+20$ & 644.8 & 309.8 \\
\hline & \multicolumn{10}{|c|}{ Mid-Cycle 160B Outage - 5 Day, 14 Hour Down Time } \\
\hline & & 92.7 & 4.18 & 273.1 & $3.33641 \mathrm{E}+13$ & $1.91813 \mathrm{E}+13$ & $8.09237 E+13$ & $1.35676 \mathrm{E}+20$ & 660.3 & 317.0 \\
\hline & & 102.7 & 4.65 & 279.3 & $3.44362 \mathrm{E}+13$ & $1.96238 \mathrm{E}+13$ & $8.30133 \mathrm{E}+13$ & $1.50911 \mathrm{E}+20$ & 670.3 & 321.6 \\
\hline & EOC $(2 / 23 / 2017,1400)$ & 112.2 & 5.12 & 287.2 & $3.61849 \mathrm{E}+13$ & $2.02598 \mathrm{E}+13$ & $8.61695 \mathrm{E}+13$ & 1.66027E+20 & 682.8 & 327.5 \\
\hline $162 \mathrm{~A}$ & \multicolumn{10}{|c|}{ Withheld from Cycle 161A PALM } \\
\hline & $\mathrm{BOC}$ & 113.2 & 5.15 & 252.7 & $3.23919 \mathrm{E}+13$ & $1.80983 \mathrm{E}+13$ & $7.73773 E+13$ & $1.67231 \mathrm{E}+20$ & 627.1 & 301.6 \\
\hline & & 115.2 & 5.24 & 241.9 & $2.99581 \mathrm{E}+13$ & $1.67380 \mathrm{E}+13$ & $7.19744 E+13$ & $1.70001 \mathrm{E}+20$ & 609.0 & 293.2 \\
\hline & & 122.2 & 5.54 & 243.5 & $3.05274 \mathrm{E}+13$ & 1.71177E+13 & $7.33176 \mathrm{E}+13$ & $1.79576 \mathrm{E}+20$ & 611.7 & 294.5 \\
\hline & & 132.2 & 5.95 & 241.9 & $3.09309 E+13$ & $1.71723 E+13$ & $7.41213 E+13$ & $1.93126 \mathrm{E}+20$ & 609.1 & 293.3 \\
\hline & & 142.2 & 6.37 & 240.8 & $3.10862 \mathrm{E}+13$ & $1.71835 \mathrm{E}+13$ & $7.41017 E+13$ & $2.06555 \mathrm{E}+20$ & 607.2 & 292.4 \\
\hline & & 152.2 & 6.79 & 244.1 & $3.22321 \mathrm{E}+13$ & $1.74562 \mathrm{E}+13$ & $7.59948 E+13$ & $2.20345 E+20$ & 612.7 & 294.9 \\
\hline & & 162.2 & 7.21 & 241.7 & $3.18945 \mathrm{E}+13$ & $1.72825 \mathrm{E}+13$ & $7.50818 \mathrm{E}+13$ & $2.33774 \mathrm{E}+20$ & 608.7 & 293.1 \\
\hline & & 171.2 & 7.60 & 250.7 & $3.39420 \mathrm{E}+13$ & $1.80645 \mathrm{E}+13$ & $7.92076 \mathrm{E}+13$ & $2.46601 \mathrm{E}+20$ & 623.7 & 300.0 \\
\hline & EOC & 174.2 & 7.73 & 248.7 & $3.42641 \mathrm{E}+13$ & 1.81957E+13 & $7.97472 E+13$ & $2.50756 \mathrm{E}+20$ & 620.5 & 298.6 \\
\hline
\end{tabular}


TEM-10300-1

$03 / 01 / 2012$

Rev. 03

Title:

ATF-1 Power Histories

\begin{tabular}{lllll} 
TEV No.: & 3119 & Rev. No.: & 0 & Project No.: \\
\hline
\end{tabular}

Table 22. ATF-30 Power History

\begin{tabular}{|c|c|c|c|c|c|c|c|c|c|c|}
\hline Cycle & Critical Dates & EFPD & $\begin{array}{c}\text { Burnup } \\
\text { (GWd/MTU) }\end{array}$ & $\begin{array}{l}\text { LHGR } \\
(\mathrm{W} / \mathrm{cm})\end{array}$ & $\begin{array}{l}\text { Thermal Flux } \\
\left(\mathrm{n} / \mathrm{cm}^{2 *} \mathrm{~s}\right)\end{array}$ & $\begin{array}{c}\text { Fast Flux } \\
\left(\mathrm{n} / \mathrm{cm}^{2 *} \mathrm{~s}>1\right. \\
\mathrm{MeV})\end{array}$ & $\begin{array}{l}\text { Total Flux } \\
\left(\mathrm{n} / \mathrm{cm}^{2 *} \mathrm{~s}\right)\end{array}$ & $\begin{array}{l}\text { Fission Density } \\
\text { (Fissions } / \mathrm{cm}^{3} \text { ) }\end{array}$ & $\begin{array}{c}\text { Fuel Centerline } \\
\text { Temperature } \\
\left({ }^{\circ} \mathrm{C}\right)\end{array}$ & $\operatorname{PICT}\left({ }^{\circ} \mathrm{C}\right)$ \\
\hline \multirow[t]{10}{*}{$160 \mathrm{~A}$} & BOC (9/16/2016, 1900) & 1.0 & 0.08 & 414.5 & $4.64243 \mathrm{E}+13$ & $2.86281 \mathrm{E}+13$ & $1.14759 \mathrm{E}+14$ & $2.58946 \mathrm{E}+18$ & 847.4 & 404.8 \\
\hline & & 3.0 & 0.22 & 403.9 & $4.53053 \mathrm{E}+13$ & $2.77164 \mathrm{E}+13$ & $1.12084 \mathrm{E}+14$ & $6.98552 \mathrm{E}+18$ & 833.4 & 398.1 \\
\hline & & 10.0 & 0.70 & 409.9 & $4.61946 \mathrm{E}+13$ & $2.83668 \mathrm{E}+13$ & $1.14383 \mathrm{E}+14$ & $2.28234 \mathrm{E}+19$ & 841.3 & 401.9 \\
\hline & & 20.0 & 1.41 & 411.9 & $4.69216 \mathrm{E}+13$ & $2.86680 \mathrm{E}+13$ & $1.15583 \mathrm{E}+14$ & $4.56468 \mathrm{E}+19$ & 844.0 & 403.2 \\
\hline & & 30.0 & 2.10 & 408.1 & $4.72974 \mathrm{E}+13$ & $2.83261 \mathrm{E}+13$ & $1.15432 \mathrm{E}+14$ & $6.81690 \mathrm{E}+19$ & 839.0 & 400.8 \\
\hline & & 40.0 & 2.80 & 407.4 & $4.78035 \mathrm{E}+13$ & $2.82564 \mathrm{E}+13$ & $1.15576 \mathrm{E}+14$ & $9.07515 \mathrm{E}+19$ & 838.1 & 400.4 \\
\hline & & 46.0 & 3.24 & 431.3 & $5.14653 \mathrm{E}+13$ & $2.99560 \mathrm{E}+13$ & $1.23433 \mathrm{E}+14$ & $1.05204 \mathrm{E}+20$ & 869.0 & 415.2 \\
\hline & & 47.6 & 3.35 & 390.8 & $4.69995 \mathrm{E}+13$ & $2.71690 \mathrm{E}+13$ & $1.12507 \mathrm{E}+14$ & $1.08757 \mathrm{E}+20$ & 816.1 & 389.8 \\
\hline & & 50.0 & 3.50 & 391.0 & $4.73173 \mathrm{E}+13$ & $2.73899 \mathrm{E}+13$ & $1.13238 \mathrm{E}+14$ & $1.13635 \mathrm{E}+20$ & 816.4 & 390.0 \\
\hline & EOC $(11 / 8 / 2016,1100)$ & 52.7 & 3.68 & 374.0 & $4.55630 \mathrm{E}+13$ & $2.62167 \mathrm{E}+13$ & $1.09281 \mathrm{E}+14$ & 1.19537E+20 & 793.6 & 379.0 \\
\hline $160 \mathrm{~B}$ & \multicolumn{10}{|c|}{ Cycle 160B Outage - 42 Day, 1 Hour Down Time Outage } \\
\hline & $\mathrm{BOC}(12 / 20 / 2016,1200)$ & 53.7 & 3.76 & 396.0 & $4.82146 \mathrm{E}+13$ & $2.81101 \mathrm{E}+13$ & $1.19756 \mathrm{E}+14$ & $1.22006 \mathrm{E}+20$ & 823.1 & 393.2 \\
\hline & & 55.7 & 3.89 & 385.1 & $4.70119 \mathrm{E}+13$ & $2.73091 \mathrm{E}+13$ & $1.16981 \mathrm{E}+14$ & $1.26101 \mathrm{E}+20$ & 808.6 & 386.2 \\
\hline & & 62.7 & 4.34 & 378.4 & $4.63856 \mathrm{E}+13$ & $2.70618 \mathrm{E}+13$ & $1.15534 \mathrm{E}+14$ & $1.40674 \mathrm{E}+20$ & 799.5 & 381.9 \\
\hline & & 72.7 & 4.96 & 365.5 & $4.53058 \mathrm{E}+13$ & $2.59758 \mathrm{E}+13$ & $1.12081 \mathrm{E}+14$ & $1.60968 \mathrm{E}+20$ & 782.0 & 373.5 \\
\hline & & 81.9 & 5.54 & 361.2 & $4.60644 \mathrm{E}+13$ & $2.57646 \mathrm{E}+13$ & $1.12747 \mathrm{E}+14$ & 1.79817E+20 & 776.0 & 370.6 \\
\hline & \multicolumn{10}{|c|}{ Mid-Cycle 160B Outage - 5 Day, 14 Hour Down Time } \\
\hline & & 92.7 & 6.22 & 372.1 & $4.76726 \mathrm{E}+13$ & $2.67994 \mathrm{E}+13$ & $1.16498 \mathrm{E}+14$ & $2.01737 \mathrm{E}+20$ & 790.9 & 377.8 \\
\hline & & 102.7 & 6.86 & 380.9 & $4.93614 \mathrm{E}+13$ & $2.72352 \mathrm{E}+13$ & $1.19369 \mathrm{E}+14$ & $2.22513 \mathrm{E}+20$ & 802.8 & 383.5 \\
\hline & EOC $(2 / 23 / 2017,1400)$ & 112.2 & 7.49 & 386.6 & $5.16819 \mathrm{E}+13$ & $2.77741 \mathrm{E}+13$ & $1.22833 \mathrm{E}+14$ & $2.42867 \mathrm{E}+20$ & 810.5 & 387.1 \\
\hline $162 \mathrm{~A}$ & \multicolumn{10}{|c|}{ Withheld from Cycle 161A PALM } \\
\hline & $\mathrm{BOC}$ & 113.2 & 7.55 & 341.1 & $4.63967 \mathrm{E}+13$ & $2.50849 \mathrm{E}+13$ & $1.11706 \mathrm{E}+14$ & $2.45035 \mathrm{E}+20$ & 748.1 & 357.3 \\
\hline & & 115.2 & 7.66 & 325.9 & $4.29201 \mathrm{E}+13$ & $2.32273 \mathrm{E}+13$ & $1.03638 \mathrm{E}+14$ & $2.48588 \mathrm{E}+20$ & 726.6 & 347.1 \\
\hline & & 122.2 & 8.05 & 330.8 & $4.40135 \mathrm{E}+13$ & $2.37813 \mathrm{E}+13$ & $1.05879 \mathrm{E}+14$ & $2.61295 \mathrm{E}+20$ & 733.6 & 350.4 \\
\hline & & 132.2 & 8.62 & 331.6 & $4.51446 \mathrm{E}+13$ & $2.40418 \mathrm{E}+13$ & $1.07802 \mathrm{E}+14$ & $2.79782 \mathrm{E}+20$ & 734.7 & 351.0 \\
\hline & & 142.2 & 9.18 & 323.4 & $4.47635 \mathrm{E}+13$ & $2.36459 \mathrm{E}+13$ & $1.06570 \mathrm{E}+14$ & $2.97848 \mathrm{E}+20$ & 723.1 & 345.4 \\
\hline & & 152.2 & 9.75 & 328.5 & $4.66622 \mathrm{E}+13$ & $2.44032 \mathrm{E}+13$ & $1.10224 \mathrm{E}+14$ & $3.16336 \mathrm{E}+20$ & 730.3 & 348.9 \\
\hline & & 162.2 & 10.31 & 324.1 & $4.64199 \mathrm{E}+13$ & $2.37465 \mathrm{E}+13$ & $1.08699 \mathrm{E}+14$ & $3.34462 \mathrm{E}+20$ & 724.1 & 345.9 \\
\hline & & 171.2 & 10.84 & 331.2 & $4.86791 \mathrm{E}+13$ & $2.47432 \mathrm{E}+13$ & $1.13328 \mathrm{E}+14$ & $3.51504 \mathrm{E}+20$ & 734.1 & 350.7 \\
\hline & EOC & 174.2 & 11.01 & 325.5 & $4.90926 \mathrm{E}+13$ & $2.44984 \mathrm{E}+13$ & $1.13470 \mathrm{E}+14$ & $3.57225 \mathrm{E}+20$ & 726.0 & 346.8 \\
\hline
\end{tabular}


TEM-10300-1

$03 / 01 / 2012$

Rev. 03

Title:

ATF-1 Power Histories

\begin{tabular}{lllll} 
TEV No.: & 3119 & Rev. No.: & 0 & Project No.: \\
\hline
\end{tabular}

Table 23. ATF-31 Power History

\begin{tabular}{|c|c|c|c|c|c|c|c|c|c|c|}
\hline Cycle & Critical Dates & EFPD & $\begin{array}{c}\text { Burnup } \\
\text { (GWd/MTU) }\end{array}$ & $\begin{array}{l}\text { LHGR } \\
(\mathrm{W} / \mathrm{cm})\end{array}$ & $\begin{array}{l}\text { Thermal Flux } \\
\left(\mathrm{n} / \mathrm{cm}^{2 *} \mathrm{~s}\right)\end{array}$ & $\begin{array}{c}\text { Fast Flux } \\
\left(\mathrm{n} / \mathrm{cm}^{2 *} \mathrm{~s}>1\right. \\
\mathrm{MeV})\end{array}$ & $\begin{array}{l}\text { Total Flux } \\
\left(\mathrm{n} / \mathrm{cm}^{2 *} \mathrm{~s}\right)\end{array}$ & $\begin{array}{c}\text { Fission Density } \\
\text { (Fissions/cm³) }\end{array}$ & $\begin{array}{c}\text { Fuel Centerline } \\
\text { Temperature } \\
\left({ }^{\circ} \mathrm{C}\right)\end{array}$ & $\mathrm{PICT}\left({ }^{\circ} \mathrm{C}\right)$ \\
\hline \multirow[t]{10}{*}{$160 \mathrm{~A}$} & $\mathrm{BOC}(9 / 16 / 2016,1900)$ & 1.0 & 0.08 & 445.1 & $4.99690 \mathrm{E}+13$ & $3.07625 E+13$ & $1.23264 \mathrm{E}+14$ & $2.70990 \mathrm{E}+18$ & 883.6 & 411.1 \\
\hline & & 3.0 & 0.23 & 432.2 & $4.86664 \mathrm{E}+13$ & $2.97875 E+13$ & $1.20218 \mathrm{E}+14$ & $7.40706 \mathrm{E}+18$ & 867.5 & 403.6 \\
\hline & & 10.0 & 0.74 & 438.3 & $4.95012 \mathrm{E}+13$ & $3.02170 \mathrm{E}+13$ & $1.22395 \mathrm{E}+14$ & $2.41482 \mathrm{E}+19$ & 875.1 & 407.1 \\
\hline & & 20.0 & 1.51 & 440.2 & $5.02059 \mathrm{E}+13$ & $3.06782 \mathrm{E}+13$ & $1.23609 \mathrm{E}+14$ & $4.88384 \mathrm{E}+19$ & 877.6 & 408.3 \\
\hline & & 30.0 & 2.27 & 442.4 & $5.13611 \mathrm{E}+13$ & $3.03158 \mathrm{E}+13$ & $1.24675 \mathrm{E}+14$ & $7.36491 \mathrm{E}+19$ & 880.4 & 409.6 \\
\hline & & 40.0 & 3.02 & 436.2 & $5.13951 \mathrm{E}+13$ & $3.02908 \mathrm{E}+13$ & $1.24699 \mathrm{E}+14$ & $9.80382 \mathrm{E}+19$ & 872.5 & 406.0 \\
\hline & & 46.0 & 3.49 & 461.0 & $5.52152 \mathrm{E}+13$ & $3.19978 \mathrm{E}+13$ & $1.32149 \mathrm{E}+14$ & $1.13274 \mathrm{E}+20$ & 903.4 & 420.2 \\
\hline & & 47.6 & 3.61 & 420.0 & $5.09079 \mathrm{E}+13$ & $2.94740 \mathrm{E}+13$ & $1.21646 \mathrm{E}+14$ & $1.17068 \mathrm{E}+20$ & 851.9 & 396.4 \\
\hline & & 50.0 & 3.79 & 419.1 & $5.08850 \mathrm{E}+13$ & $2.93738 \mathrm{E}+13$ & $1.21250 \mathrm{E}+14$ & $1.22909 \mathrm{E}+20$ & 850.7 & 395.9 \\
\hline & EOC $(11 / 8 / 2016,1100)$ & 52.7 & 3.98 & 398.7 & $4.88241 \mathrm{E}+13$ & $2.82072 \mathrm{E}+13$ & 1.17075E+14 & $1.29051 \mathrm{E}+20$ & 824.3 & 383.7 \\
\hline 160B & \multicolumn{10}{|c|}{ Cycle 160B Outage - 42 Day, 1 Hour Down Time Outage } \\
\hline & $\mathrm{BOC}(12 / 20 / 2016,1200)$ & 53.7 & 4.05 & 411.5 & $5.04746 \mathrm{E}+13$ & $2.93872 \mathrm{E}+13$ & $1.25366 \mathrm{E}+14$ & $1.31520 \mathrm{E}+20$ & 841.0 & 391.4 \\
\hline & & 55.7 & 4.19 & 401.0 & $4.92820 \mathrm{E}+13$ & $2.86530 \mathrm{E}+13$ & $1.22710 \mathrm{E}+14$ & $1.35796 \mathrm{E}+20$ & 827.2 & 385.0 \\
\hline & & 62.7 & 4.67 & 398.9 & $4.91733 \mathrm{E}+13$ & $2.84210 \mathrm{E}+13$ & $1.22167 E+14$ & $1.51574 \mathrm{E}+20$ & 824.5 & 383.8 \\
\hline & & 72.7 & 5.33 & 391.8 & $4.89580 \mathrm{E}+13$ & $2.79248 \mathrm{E}+13$ & $1.20843 \mathrm{E}+14$ & $1.73012 \mathrm{E}+20$ & 815.1 & 379.4 \\
\hline & & 81.9 & 5.95 & 384.9 & $4.95257 \mathrm{E}+13$ & $2.76108 \mathrm{E}+13$ & $1.21057 E+14$ & $1.92885 \mathrm{E}+20$ & 806.0 & 375.2 \\
\hline & \multicolumn{10}{|c|}{ Mid-Cycle 160B Outage - 5 Day, 14 Hour Down Time } \\
\hline & & 92.7 & 6.70 & 397.3 & $5.13899 \mathrm{E}+13$ & $2.84619 E+13$ & $1.25091 \mathrm{E}+14$ & $2.17214 \mathrm{E}+20$ & 822.4 & 382.8 \\
\hline & & 102.7 & 7.39 & 406.1 & $5.30921 \mathrm{E}+13$ & $2.91495 E+13$ & $1.28192 \mathrm{E}+14$ & $2.39736 \mathrm{E}+20$ & 834.0 & 388.1 \\
\hline & EOC $(2 / 23 / 2017,1400)$ & 112.2 & 8.07 & 409.8 & $5.54618 \mathrm{E}+13$ & $2.94044 \mathrm{E}+13$ & $1.31063 E+14$ & $2.61716 \mathrm{E}+20$ & 838.8 & 390.3 \\
\hline $162 \mathrm{~A}$ & \multicolumn{10}{|c|}{ Withheld from Cycle 161A PALM } \\
\hline & $\mathrm{BOC}$ & 113.2 & 8.14 & 355.2 & $4.89333 \mathrm{E}+13$ & $2.61543 E+13$ & $1.16998 \mathrm{E}+14$ & $2.63944 \mathrm{E}+20$ & 765.7 & 356.7 \\
\hline & & 115.2 & 8.25 & 338.0 & $4.50795 \mathrm{E}+13$ & $2.42520 \mathrm{E}+13$ & $1.08633 \mathrm{E}+14$ & $2.67497 \mathrm{E}+20$ & 741.7 & 345.7 \\
\hline & & 122.2 & 8.65 & 345.2 & $4.65691 \mathrm{E}+13$ & $2.47859 \mathrm{E}+13$ & $1.11373 \mathrm{E}+14$ & $2.80685 \mathrm{E}+20$ & 751.9 & 350.3 \\
\hline & & 132.2 & 9.25 & 351.9 & $4.87155 \mathrm{E}+13$ & $2.56056 \mathrm{E}+13$ & $1.15765 \mathrm{E}+14$ & $3.00016 \mathrm{E}+20$ & 761.1 & 354.6 \\
\hline & & 142.2 & 9.83 & 344.8 & $4.83923 \mathrm{E}+13$ & $2.51985 \mathrm{E}+13$ & $1.14433 \mathrm{E}+14$ & $3.18985 \mathrm{E}+20$ & 751.2 & 350.0 \\
\hline & & 152.2 & 10.44 & 347.1 & $5.02118 \mathrm{E}+13$ & $2.57312 \mathrm{E}+13$ & $1.17980 \mathrm{E}+14$ & $3.38798 \mathrm{E}+20$ & 754.5 & 351.5 \\
\hline & & 162.2 & 11.03 & 342.8 & $4.99676 \mathrm{E}+13$ & $2.53088 \mathrm{E}+13$ & $1.16427 E+14$ & $3.57707 \mathrm{E}+20$ & 748.5 & 348.8 \\
\hline & & 171.2 & 11.57 & 348.0 & $5.22072 \mathrm{E}+13$ & $2.60123 E+13$ & $1.20464 \mathrm{E}+14$ & $3.75351 \mathrm{E}+20$ & 755.7 & 352.1 \\
\hline & EOC & 174.2 & 11.75 & 341.9 & $5.25954 \mathrm{E}+13$ & $2.57268 \mathrm{E}+13$ & $1.20686 \mathrm{E}+14$ & $3.81193 \mathrm{E}+20$ & 747.3 & 348.2 \\
\hline
\end{tabular}


TEM-10300-1

$03 / 01 / 2012$

Rev. 03

Title:

ATF-1 Power Histories

\begin{tabular}{lllll} 
TEV No.: & 3119 & Rev. No.: & 0 & Project No.: \\
\hline
\end{tabular}

Table 24. ATF-32 Power History

\begin{tabular}{|c|c|c|c|c|c|c|c|c|c|c|}
\hline Cycle & Critical Dates & EFPD & $\begin{array}{c}\text { Burnup } \\
\text { (GWd/MTU) }\end{array}$ & $\begin{array}{l}\text { LHGR } \\
(\mathrm{W} / \mathrm{cm})\end{array}$ & $\begin{array}{l}\text { Thermal Flux } \\
\left(\mathrm{n} / \mathrm{cm}^{2 *} \mathrm{~s}\right)\end{array}$ & $\begin{array}{c}\text { Fast Flux } \\
\left(\mathrm{n} / \mathrm{cm}^{2 *} \mathrm{~s}>1\right. \\
\mathrm{MeV})\end{array}$ & $\begin{array}{l}\text { Total Flux } \\
\left(\mathrm{n} / \mathrm{cm}^{2 *} \mathrm{~s}\right)\end{array}$ & $\begin{array}{l}\text { Fission Density } \\
(\text { Fissions/cm³) }\end{array}$ & $\begin{array}{c}\text { Fuel Centerline } \\
\text { Temperature } \\
\left({ }^{\circ} \mathrm{C}\right)\end{array}$ & PICT $\left({ }^{\circ} \mathrm{C}\right)$ \\
\hline \multirow[t]{10}{*}{$160 \mathrm{~A}$} & BOC $(9 / 16 / 2016,1900)$ & 1.0 & 0.04 & 252.3 & $2.82821 \mathrm{E}+13$ & $1.75073 E+13$ & $7.04304 \mathrm{E}+13$ & $1.20440 \mathrm{E}+18$ & 629.7 & 296.1 \\
\hline & & 3.0 & 0.13 & 245.3 & $2.75170 \mathrm{E}+13$ & $1.69699 \mathrm{E}+13$ & $6.85591 \mathrm{E}+13$ & 4.09496E+18 & 618.0 & 290.9 \\
\hline & & 10.0 & 0.44 & 252.4 & $2.84428 \mathrm{E}+13$ & 1.73847E+13 & $7.07405 E+13$ & 1.42119E+19 & 629.9 & 296.3 \\
\hline & & 20.0 & 0.88 & 254.6 & $2.88531 \mathrm{E}+13$ & $1.75631 \mathrm{E}+13$ & $7.14916 \mathrm{E}+13$ & $2.84841 \mathrm{E}+19$ & 633.6 & 297.9 \\
\hline & & 30.0 & 1.32 & 256.5 & $2.94177 \mathrm{E}+13$ & $1.76730 \mathrm{E}+13$ & $7.21225 \mathrm{E}+13$ & $4.29369 \mathrm{E}+19$ & 636.7 & 299.3 \\
\hline & & 40.0 & 1.77 & 256.1 & $2.96310 \mathrm{E}+13$ & $1.78345 \mathrm{E}+13$ & $7.30345 \mathrm{E}+13$ & $5.72692 \mathrm{E}+19$ & 636.1 & 299.0 \\
\hline & & 46.0 & 2.06 & 276.6 & $3.21640 \mathrm{E}+13$ & $1.92700 \mathrm{E}+13$ & $7.87736 \mathrm{E}+13$ & $6.67840 \mathrm{E}+19$ & 669.4 & 314.0 \\
\hline & & 47.6 & 2.13 & 251.2 & $2.95032 \mathrm{E}+13$ & 1.73737E+13 & $7.15564 \mathrm{E}+13$ & $6.92530 \mathrm{E}+19$ & 627.9 & 295.3 \\
\hline & & 50.0 & 2.24 & 251.7 & $2.95874 \mathrm{E}+13$ & $1.75354 \mathrm{E}+13$ & $7.21672 \mathrm{E}+13$ & $7.26855 \mathrm{E}+19$ & 628.6 & 295.7 \\
\hline & EOC $(11 / 8 / 2016,1100)$ & 52.7 & 2.35 & 242.1 & $2.86668 \mathrm{E}+13$ & $1.70582 \mathrm{E}+13$ & $7.02118 \mathrm{E}+13$ & $7.63590 \mathrm{E}+19$ & 612.6 & 288.5 \\
\hline 160B & \multicolumn{10}{|c|}{ Cycle 160B Outage - 42 Day, 1 Hour Down Time Outage } \\
\hline & $\mathrm{BOC}(12 / 20 / 2016,1200)$ & 53.7 & 2.39 & 286.3 & $3.38297 \mathrm{E}+13$ & $2.00359 \mathrm{E}+13$ & $8.30463 E+13$ & 7.76236E+19 & 684.9 & 321.0 \\
\hline & & 55.7 & 2.49 & 279.5 & $3.30965 \mathrm{E}+13$ & $1.95829 \mathrm{E}+13$ & $8.14336 \mathrm{E}+13$ & $8.08152 \mathrm{E}+19$ & 674.0 & 316.1 \\
\hline & & 62.7 & 2.82 & 274.2 & $3.25262 \mathrm{E}+13$ & $1.93309 \mathrm{E}+13$ & $8.01789 \mathrm{E}+13$ & $9.15344 \mathrm{E}+19$ & 665.6 & 312.3 \\
\hline & & 72.7 & 3.27 & 262.5 & $3.13740 \mathrm{E}+13$ & $1.83956 \mathrm{E}+13$ & $7.69898 \mathrm{E}+13$ & $1.06108 \mathrm{E}+20$ & 646.5 & 303.7 \\
\hline & & 81.9 & 3.69 & 264.2 & $3.22173 E+13$ & $1.86634 \mathrm{E}+13$ & $7.83828 \mathrm{E}+13$ & $1.19838 \mathrm{E}+20$ & 649.4 & 305.0 \\
\hline & \multicolumn{10}{|c|}{ Mid-Cycle 160B Outage - 5 Day, 14 Hour Down Time } \\
\hline & & 92.7 & 4.20 & 272.3 & $3.34003 E+13$ & $1.91713 \mathrm{E}+13$ & $8.08849 \mathrm{E}+13$ & $1.36398 \mathrm{E}+20$ & 662.5 & 310.9 \\
\hline & & 102.7 & 4.67 & 278.4 & $3.42898 \mathrm{E}+13$ & 1.96719E+13 & $8.27967 \mathrm{E}+13$ & $1.51634 \mathrm{E}+20$ & 672.3 & 315.3 \\
\hline & EOC $(2 / 23 / 2017,1400)$ & 112.2 & 5.14 & 287.3 & $3.62239 \mathrm{E}+13$ & $2.01316 \mathrm{E}+13$ & $8.61781 \mathrm{E}+13$ & $1.66749 \mathrm{E}+20$ & 686.4 & 321.7 \\
\hline $162 \mathrm{~A}$ & \multicolumn{10}{|c|}{ Withheld from Cycle 161A PALM } \\
\hline & $\mathrm{BOC}$ & 113.2 & 5.18 & 253.4 & $3.24316 \mathrm{E}+13$ & $1.82752 \mathrm{E}+13$ & $7.77175 \mathrm{E}+13$ & $1.67954 \mathrm{E}+20$ & 631.6 & 297.0 \\
\hline & & 115.2 & 5.26 & 242.2 & $2.99857 \mathrm{E}+13$ & $1.68859 \mathrm{E}+13$ & $7.21637 \mathrm{E}+13$ & $1.70724 \mathrm{E}+20$ & 612.7 & 288.5 \\
\hline & & 122.2 & 5.56 & 243.5 & $3.05570 \mathrm{E}+13$ & 1.71107E+13 & $7.33293 E+13$ & $1.80299 \mathrm{E}+20$ & 614.9 & 289.5 \\
\hline & & 132.2 & 5.98 & 243.3 & $3.10708 \mathrm{E}+13$ & $1.72928 \mathrm{E}+13$ & $7.43253 \mathrm{E}+13$ & $1.93908 \mathrm{E}+20$ & 614.5 & 289.4 \\
\hline & & 142.2 & 6.39 & 240.3 & $3.10547 \mathrm{E}+13$ & $1.71970 \mathrm{E}+13$ & $7.39251 \mathrm{E}+13$ & $2.07277 \mathrm{E}+20$ & 609.6 & 287.1 \\
\hline & & 152.2 & 6.81 & 242.9 & $3.21383 \mathrm{E}+13$ & 1.75147E+13 & $7.60007 \mathrm{E}+13$ & $2.20887 \mathrm{E}+20$ & 614.0 & 289.1 \\
\hline & & 162.2 & 7.22 & 242.4 & $3.20342 \mathrm{E}+13$ & $1.73608 \mathrm{E}+13$ & $7.54259 \mathrm{E}+13$ & $2.34376 \mathrm{E}+20$ & 613.0 & 288.7 \\
\hline & & 171.2 & 7.62 & 249.8 & $3.38948 \mathrm{E}+13$ & $1.82123 \mathrm{E}+13$ & $7.91988 \mathrm{E}+13$ & $2.47203 \mathrm{E}+20$ & 625.5 & 294.3 \\
\hline & EOC & 174.2 & 7.74 & 247.0 & $3.41192 \mathrm{E}+13$ & $1.81051 \mathrm{E}+13$ & $7.94540 \mathrm{E}+13$ & $2.51238 \mathrm{E}+20$ & 620.9 & 292.2 \\
\hline
\end{tabular}


TEM-10300-1

$03 / 01 / 2012$

Rev. 03

Title:

ATF-1 Power Histories

\begin{tabular}{lllll} 
TEV No.: & 3119 & Rev. No.: & 0 & Project No.: \\
\hline
\end{tabular}

Table 25. ATF-33 Power History

\begin{tabular}{|c|c|c|c|c|c|c|c|c|c|c|}
\hline Cycle & Critical Dates & EFPD & $\begin{array}{c}\text { Burnup } \\
\text { (GWd/MTU) }\end{array}$ & $\begin{array}{l}\text { LHGR } \\
(\mathrm{W} / \mathrm{cm})\end{array}$ & $\begin{array}{l}\text { Thermal Flux } \\
\left(\mathrm{n} / \mathrm{cm}^{2 *} \mathrm{~s}\right)\end{array}$ & $\begin{array}{c}\text { Fast Flux } \\
\left(\mathrm{n} / \mathrm{cm}^{2 *} \mathrm{~s}>1\right. \\
\mathrm{MeV})\end{array}$ & $\begin{array}{l}\text { Total Flux } \\
\left(\mathrm{n} / \mathrm{cm}^{2 *} \mathrm{~s}\right)\end{array}$ & $\begin{array}{c}\text { Fission Density } \\
\text { (Fissions/cm³) }\end{array}$ & $\begin{array}{c}\text { Fuel Centerline } \\
\text { Temperature } \\
\left({ }^{\circ} \mathrm{C}\right)\end{array}$ & $\mathrm{PICT}\left({ }^{\circ} \mathrm{C}\right)$ \\
\hline \multirow[t]{10}{*}{$160 \mathrm{~A}$} & BOC (9/16/2016, 1900) & 1.0 & 0.08 & 414.7 & $4.64668 \mathrm{E}+13$ & $2.84629 \mathrm{E}+13$ & $1.14876 \mathrm{E}+14$ & $2.58946 \mathrm{E}+18$ & 869.8 & 407.3 \\
\hline & & 3.0 & 0.22 & 403.8 & $4.53833 \mathrm{E}+13$ & $2.79692 \mathrm{E}+13$ & $1.12409 \mathrm{E}+14$ & $6.98552 \mathrm{E}+18$ & 854.7 & 400.3 \\
\hline & & 10.0 & 0.71 & 409.6 & $4.61068 \mathrm{E}+13$ & $2.81458 \mathrm{E}+13$ & $1.14016 \mathrm{E}+14$ & $2.28836 \mathrm{E}+19$ & 862.8 & 404.1 \\
\hline & & 20.0 & 1.41 & 413.8 & $4.72029 \mathrm{E}+13$ & $2.86209 E+13$ & $1.16106 \mathrm{E}+14$ & $4.57672 \mathrm{E}+19$ & 868.6 & 406.7 \\
\hline & & 30.0 & 2.11 & 411.2 & $4.75308 \mathrm{E}+13$ & $2.85631 \mathrm{E}+13$ & $1.16112 \mathrm{E}+14$ & $6.84701 \mathrm{E}+19$ & 865.0 & 405.1 \\
\hline & & 40.0 & 2.80 & 407.5 & $4.78611 \mathrm{E}+13$ & $2.81541 \mathrm{E}+13$ & $1.15813 \mathrm{E}+14$ & $9.09924 \mathrm{E}+19$ & 859.9 & 402.7 \\
\hline & & 46.0 & 3.25 & 430.9 & $5.14288 \mathrm{E}+13$ & $2.98099 \mathrm{E}+13$ & $1.23473 \mathrm{E}+14$ & $1.05445 \mathrm{E}+20$ & 891.7 & 417.5 \\
\hline & & 47.6 & 3.36 & 394.0 & $4.74457 \mathrm{E}+13$ & $2.72135 \mathrm{E}+13$ & 1.13393E+14 & $1.09058 \mathrm{E}+20$ & 841.1 & 394.0 \\
\hline & & 50.0 & 3.51 & 392.4 & 4.74079E+13 & $2.69498 \mathrm{E}+13$ & $1.12826 \mathrm{E}+14$ & $1.13996 \mathrm{E}+20$ & 838.9 & 393.0 \\
\hline & EOC $(11 / 8 / 2016,1100)$ & 52.7 & 3.70 & 374.7 & $4.56453 \mathrm{E}+13$ & $2.58857 \mathrm{E}+13$ & $1.09226 \mathrm{E}+14$ & $1.19898 \mathrm{E}+20$ & 813.9 & 381.5 \\
\hline $160 \mathrm{~B}$ & \multicolumn{10}{|c|}{ Cycle 160B Outage - 42 Day, 1 Hour Down Time Outage } \\
\hline & $\mathrm{BOC}(12 / 20 / 2016,1200)$ & 53.7 & 3.77 & 394.6 & $4.80783 \mathrm{E}+13$ & $2.79928 \mathrm{E}+13$ & $1.19634 \mathrm{E}+14$ & $1.22307 \mathrm{E}+20$ & 841.9 & 394.4 \\
\hline & & 55.7 & 3.90 & 384.9 & $4.69996 \mathrm{E}+13$ & $2.73554 \mathrm{E}+13$ & $1.17147 \mathrm{E}+14$ & $1.26462 \mathrm{E}+20$ & 828.3 & 388.1 \\
\hline & & 62.7 & 4.35 & 379.7 & $4.64964 \mathrm{E}+13$ & $2.68844 \mathrm{E}+13$ & $1.15408 \mathrm{E}+14$ & $1.41035 \mathrm{E}+20$ & 821.0 & 384.7 \\
\hline & & 72.7 & 4.97 & 366.1 & $4.53834 \mathrm{E}+13$ & $2.62252 \mathrm{E}+13$ & $1.12228 \mathrm{E}+14$ & $1.61390 \mathrm{E}+20$ & 801.6 & 375.8 \\
\hline & & 81.9 & 5.56 & 360.2 & $4.59887 \mathrm{E}+13$ & $2.59274 \mathrm{E}+13$ & $1.12703 \mathrm{E}+14$ & $1.80238 \mathrm{E}+20$ & 793.1 & 371.9 \\
\hline & \multicolumn{10}{|c|}{ Mid-Cycle 160B Outage - 5 Day, 14 Hour Down Time } \\
\hline & & 92.7 & 6.23 & 372.3 & $4.77665 \mathrm{E}+13$ & $2.68296 \mathrm{E}+13$ & 1.16586E+14 & $2.02098 \mathrm{E}+20$ & 810.4 & 379.8 \\
\hline & & 102.7 & 6.87 & 380.3 & $4.93239 \mathrm{E}+13$ & $2.73534 \mathrm{E}+13$ & 1.19517E+14 & $2.22934 \mathrm{E}+20$ & 821.8 & 385.1 \\
\hline & EOC $(2 / 23 / 2017,1400)$ & 112.2 & 7.50 & 384.6 & $5.14519 \mathrm{E}+13$ & $2.76130 \mathrm{E}+13$ & $1.22422 \mathrm{E}+14$ & $2.43229 \mathrm{E}+20$ & 827.9 & 387.9 \\
\hline $162 \mathrm{~A}$ & \multicolumn{10}{|c|}{ Withheld from Cycle 161A PALM } \\
\hline & $\mathrm{BOC}$ & 113.2 & 7.56 & 343.5 & $4.65517 \mathrm{E}+13$ & $2.51875 E+13$ & $1.11567 E+14$ & $2.45397 \mathrm{E}+20$ & 768.6 & 360.6 \\
\hline & & 115.2 & 7.67 & 327.8 & $4.31360 \mathrm{E}+13$ & $2.35652 \mathrm{E}+13$ & $1.04259 \mathrm{E}+14$ & $2.48889 \mathrm{E}+20$ & 745.5 & 349.9 \\
\hline & & 122.2 & 8.06 & 331.1 & $4.41415 \mathrm{E}+13$ & $2.38640 \mathrm{E}+13$ & $1.05910 \mathrm{E}+14$ & $2.61596 \mathrm{E}+20$ & 750.3 & 352.1 \\
\hline & & 132.2 & 8.63 & 330.2 & $4.50114 \mathrm{E}+13$ & $2.40387 E+13$ & $1.07361 \mathrm{E}+14$ & $2.80083 \mathrm{E}+20$ & 749.0 & 351.5 \\
\hline & & 142.2 & 9.19 & 324.2 & $4.48669 \mathrm{E}+13$ & $2.36810 \mathrm{E}+13$ & $1.06599 \mathrm{E}+14$ & $2.98209 \mathrm{E}+20$ & 740.0 & 347.4 \\
\hline & & 152.2 & 9.76 & 326.7 & $4.65140 \mathrm{E}+13$ & $2.42991 \mathrm{E}+13$ & $1.09738 \mathrm{E}+14$ & $3.16577 \mathrm{E}+20$ & 743.9 & 349.2 \\
\hline & & 162.2 & 10.31 & 321.7 & $4.61307 \mathrm{E}+13$ & $2.37580 \mathrm{E}+13$ & $1.08139 \mathrm{E}+14$ & $3.34522 \mathrm{E}+20$ & 736.4 & 345.7 \\
\hline & & 171.2 & 10.84 & 329.8 & $4.85947 \mathrm{E}+13$ & $2.46997 E+13$ & $1.12951 \mathrm{E}+14$ & $3.51564 \mathrm{E}+20$ & 748.4 & 351.3 \\
\hline & EOC & 174.2 & 11.01 & 325.8 & $4.91527 \mathrm{E}+13$ & $2.45182 \mathrm{E}+13$ & $1.13419 \mathrm{E}+14$ & $3.57225 \mathrm{E}+20$ & 742.5 & 348.5 \\
\hline
\end{tabular}


TEM-10300-1

$03 / 01 / 2012$

Rev. 03

Title:

ATF-1 Power Histories

\begin{tabular}{lllll} 
TEV No.: & 3119 & Rev. No.: & 0 & Project No.: \\
\hline
\end{tabular}

Table 26. ATF-34 Power History

\begin{tabular}{|c|c|c|c|c|c|c|c|c|c|c|}
\hline Cycle & Critical Dates & EFPD & $\begin{array}{c}\text { Burnup } \\
\text { (GWd/MTU) }\end{array}$ & $\begin{array}{c}\text { LHGR } \\
\text { (W/cm) }\end{array}$ & $\begin{array}{l}\text { Thermal Flux } \\
\left(\mathrm{n} / \mathrm{cm}^{2 *} \mathrm{~s}\right)\end{array}$ & $\begin{array}{c}\text { Fast Flux } \\
\left(\mathrm{n} / \mathrm{cm}^{2 *} \mathrm{~s}>1\right. \\
\mathrm{MeV})\end{array}$ & $\begin{array}{l}\text { Total Flux } \\
\left(\mathrm{n} / \mathrm{cm}^{2 *} \mathrm{~s}\right)\end{array}$ & $\begin{array}{c}\text { Fission Density } \\
\text { (Fissions } / \mathrm{cm}^{3} \text { ) }\end{array}$ & $\begin{array}{c}\text { Fuel Centerline } \\
\text { Temperature } \\
\left({ }^{\circ} \mathrm{C}\right)\end{array}$ & PICT $\left({ }^{\circ} \mathrm{C}\right)$ \\
\hline \multirow[t]{10}{*}{$160 \mathrm{~A}$} & $\operatorname{BOC}(9 / 16 / 2016,1900)$ & 1.0 & 0.08 & 444.8 & $4.98285 \mathrm{E}+13$ & $3.04837 \mathrm{E}+13$ & $1.22724 \mathrm{E}+14$ & $2.70990 \mathrm{E}+18$ & 1271.9 & 437.8 \\
\hline & & 3.0 & 0.23 & 432.8 & $4.85387 \mathrm{E}+13$ & $2.96806 \mathrm{E}+13$ & $1.20050 \mathrm{E}+14$ & $7.40706 \mathrm{E}+18$ & 1254.9 & 430.1 \\
\hline & & 10.0 & 0.74 & 437.9 & $4.93981 \mathrm{E}+13$ & $3.01660 \mathrm{E}+13$ & $1.22077 \mathrm{E}+14$ & $2.41482 \mathrm{E}+19$ & 1262.2 & 433.4 \\
\hline & & 20.0 & 1.51 & 443.6 & $5.04916 \mathrm{E}+13$ & $3.06331 \mathrm{E}+13$ & $1.24053 \mathrm{E}+14$ & $4.90191 \mathrm{E}+19$ & 1270.2 & 437.1 \\
\hline & & 30.0 & 2.27 & 440.8 & $5.10997 \mathrm{E}+13$ & $3.08087 \mathrm{E}+13$ & $1.24958 \mathrm{E}+14$ & $7.37695 \mathrm{E}+19$ & 1266.4 & 435.3 \\
\hline & & 40.0 & 3.03 & 436.3 & $5.13998 \mathrm{E}+13$ & $3.00896 \mathrm{E}+13$ & $1.23944 \mathrm{E}+14$ & $9.82188 \mathrm{E}+19$ & 1259.9 & 432.3 \\
\hline & & 46.0 & 3.50 & 462.7 & $5.53353 \mathrm{E}+13$ & $3.21381 \mathrm{E}+13$ & $1.32615 \mathrm{E}+14$ & $1.13454 \mathrm{E}+20$ & 1296.8 & 449.9 \\
\hline & & 47.6 & 3.61 & 419.1 & $5.07229 \mathrm{E}+13$ & $2.90699 \mathrm{E}+13$ & $1.20812 \mathrm{E}+14$ & $1.17248 \mathrm{E}+20$ & 1235.0 & 421.4 \\
\hline & & 50.0 & 3.79 & 418.1 & $5.08183 \mathrm{E}+13$ & $2.89873 E+13$ & $1.20703 \mathrm{E}+14$ & $1.23090 \mathrm{E}+20$ & 1233.6 & 420.8 \\
\hline & EOC $(11 / 8 / 2016,1100)$ & 52.7 & 3.98 & 397.3 & $4.87316 \mathrm{E}+13$ & $2.76621 \mathrm{E}+13$ & $1.16191 \mathrm{E}+14$ & $1.29232 \mathrm{E}+20$ & 1202.5 & 408.2 \\
\hline 160B & \multicolumn{10}{|c|}{ Cycle 160B Outage - 42 Day, 1 Hour Down Time Outage } \\
\hline & $\mathrm{BOC}(12 / 20 / 2016,1200)$ & 53.7 & 4.06 & 413.5 & $5.06622 \mathrm{E}+13$ & $2.94981 \mathrm{E}+13$ & $1.25719 \mathrm{E}+14$ & $1.31701 \mathrm{E}+20$ & 1226.8 & 417.9 \\
\hline & & 55.7 & 4.19 & 401.6 & $4.93455 \mathrm{E}+13$ & $2.88225 \mathrm{E}+13$ & $1.22797 \mathrm{E}+14$ & $1.35977 \mathrm{E}+20$ & 1209.0 & 410.8 \\
\hline & & 62.7 & 4.68 & 398.9 & $4.92421 \mathrm{E}+13$ & $2.84261 \mathrm{E}+13$ & $1.22319 \mathrm{E}+14$ & $1.51815 \mathrm{E}+20$ & 1205.0 & 409.2 \\
\hline & & 72.7 & 5.34 & 391.2 & $4.89344 \mathrm{E}+13$ & $2.78940 \mathrm{E}+13$ & $1.20894 \mathrm{E}+14$ & $1.73193 \mathrm{E}+20$ & 1193.2 & 404.6 \\
\hline & & 81.9 & 5.95 & 385.4 & $4.96545 \mathrm{E}+13$ & $2.76808 \mathrm{E}+13$ & $1.21362 \mathrm{E}+14$ & $1.93065 \mathrm{E}+20$ & 1184.3 & 401.3 \\
\hline & \multicolumn{10}{|c|}{ Mid-Cycle 160B Outage - 5 Day, 14 Hour Down Time } \\
\hline & & 92.7 & 6.70 & 398.5 & $5.15660 \mathrm{E}+13$ & $2.84785 \mathrm{E}+13$ & $1.25192 \mathrm{E}+14$ & $2.17394 \mathrm{E}+20$ & 1204.3 & 408.9 \\
\hline & & 102.7 & 7.40 & 407.5 & $5.33611 \mathrm{E}+13$ & $2.91893 E+13$ & $1.28450 \mathrm{E}+14$ & $2.39977 \mathrm{E}+20$ & 1217.8 & 414.3 \\
\hline & EOC $(2 / 23 / 2017,1400)$ & 112.2 & 8.07 & 408.6 & $5.53428 \mathrm{E}+13$ & $2.93713 \mathrm{E}+13$ & $1.31101 \mathrm{E}+14$ & $2.61837 \mathrm{E}+20$ & 1219.6 & 415.0 \\
\hline $162 \mathrm{~A}$ & \multicolumn{10}{|c|}{ Withheld from Cycle 161A PALM } \\
\hline & $\mathrm{BOC}$ & 113.2 & 8.14 & 354.7 & $4.89046 \mathrm{E}+13$ & $2.61696 \mathrm{E}+13$ & $1.16909 \mathrm{E}+14$ & $2.64065 \mathrm{E}+20$ & 1135.6 & 384.2 \\
\hline & & 115.2 & 8.25 & 337.7 & $4.50451 \mathrm{E}+13$ & $2.40942 \mathrm{E}+13$ & $1.08363 \mathrm{E}+14$ & $2.67678 \mathrm{E}+20$ & 1107.7 & 375.4 \\
\hline & & 122.2 & 8.65 & 342.1 & $4.62728 \mathrm{E}+13$ & $2.47485 \mathrm{E}+13$ & $1.11022 \mathrm{E}+14$ & $2.80685 \mathrm{E}+20$ & 1115.0 & 377.7 \\
\hline & & 132.2 & 9.25 & 350.6 & $4.85529 \mathrm{E}+13$ & $2.57169 \mathrm{E}+13$ & $1.15645 \mathrm{E}+14$ & $3.00016 \mathrm{E}+20$ & 1129.0 & 382.1 \\
\hline & & 142.2 & 9.83 & 343.3 & $4.82633 \mathrm{E}+13$ & $2.50751 \mathrm{E}+13$ & $1.14302 \mathrm{E}+14$ & $3.18925 \mathrm{E}+20$ & 1116.9 & 378.3 \\
\hline & & 152.2 & 10.44 & 347.2 & $5.01218 \mathrm{E}+13$ & $2.57207 E+13$ & $1.17921 \mathrm{E}+14$ & $3.38798 \mathrm{E}+20$ & 1123.4 & 380.3 \\
\hline & & 162.2 & 11.02 & 342.0 & $4.98121 \mathrm{E}+13$ & $2.51523 \mathrm{E}+13$ & $1.16280 \mathrm{E}+14$ & $3.57586 \mathrm{E}+20$ & 1114.8 & 377.6 \\
\hline & & 171.2 & 11.57 & 347.6 & $5.21139 \mathrm{E}+13$ & $2.59863 \mathrm{E}+13$ & $1.20682 \mathrm{E}+14$ & $3.75231 \mathrm{E}+20$ & 1124.1 & 380.5 \\
\hline & EOC & 174.2 & 11.75 & 343.4 & $5.27602 \mathrm{E}+13$ & $2.57257 E+13$ & $1.20976 \mathrm{E}+14$ & $3.81132 \mathrm{E}+20$ & 1117.2 & 378.3 \\
\hline
\end{tabular}


TEM-10300-1

$03 / 01 / 2012$

Rev. 03

Title: $\quad$ ATF-1 Power Histories

\begin{tabular}{lllll} 
TEV No.: & 3119 & Rev. No.: & 0 & Project No.: \\
\hline
\end{tabular}

Table 27. ATF-41 Power History

\begin{tabular}{|c|c|c|c|c|c|c|c|c|c|c|}
\hline Cycle & Critical Dates & EFPD & $\begin{array}{l}\text { Burnup } \\
\text { (GWd/MTU) }\end{array}$ & $\begin{array}{l}\text { LHGR } \\
(\mathrm{W} / \mathrm{cm})\end{array}$ & $\begin{array}{l}\text { Thermal Flux } \\
\left(\mathrm{n} / \mathrm{cm}^{2 *} \mathrm{~s}\right)\end{array}$ & $\begin{array}{c}\text { Fast Flux } \\
\left(\mathrm{n} / \mathrm{cm}^{2 *} \mathrm{~s}>1\right. \\
\mathrm{MeV})\end{array}$ & $\begin{array}{l}\text { Total Flux } \\
\left(\mathrm{n} / \mathrm{cm}^{2 *} \mathrm{~s}\right)\end{array}$ & $\begin{array}{l}\text { Fission Density } \\
\left(\text { Fissions/cm }{ }^{3}\right)\end{array}$ & $\begin{array}{c}\text { Fuel Centerline } \\
\text { Temperature } \\
\left({ }^{\circ} \mathrm{C}\right)\end{array}$ & PICT $\left({ }^{\circ} \mathrm{C}\right)$ \\
\hline \multirow[t]{10}{*}{$160 \mathrm{~A}$} & BOC $(9 / 16 / 2016,1900)$ & 1.0 & 0.04 & 259.8 & $2.46928 \mathrm{E}+13$ & $1.77087 \mathrm{E}+13$ & $6.66039 \mathrm{E}+13$ & $1.26462 \mathrm{E}+18$ & 747.1 & 378.1 \\
\hline & & 3.0 & 0.14 & 258.1 & $2.45485 \mathrm{E}+13$ & $1.75842 \mathrm{E}+13$ & $6.64663 \mathrm{E}+13$ & $4.33584 \mathrm{E}+18$ & 743.7 & 376.6 \\
\hline & & 10.0 & 0.45 & 260.0 & $2.48539 \mathrm{E}+13$ & $1.77440 \mathrm{E}+13$ & $6.67713 \mathrm{E}+13$ & 1.43324E+19 & 747.4 & 378.2 \\
\hline & & 20.0 & 0.90 & 258.0 & $2.48469 \mathrm{E}+13$ & $1.77273 \mathrm{E}+13$ & $6.68696 \mathrm{E}+13$ & $2.84238 \mathrm{E}+19$ & 743.6 & 376.5 \\
\hline & & 30.0 & 1.35 & 260.3 & $2.51431 \mathrm{E}+13$ & 1.80997E+13 & $6.82261 \mathrm{E}+13$ & $4.26960 \mathrm{E}+19$ & 747.9 & 378.5 \\
\hline & & 40.0 & 1.82 & 266.8 & $2.60101 \mathrm{E}+13$ & $1.84860 \mathrm{E}+13$ & $6.97711 \mathrm{E}+13$ & $5.73294 \mathrm{E}+19$ & 760.3 & 383.9 \\
\hline & & 46.0 & 2.10 & 262.8 & $2.59486 \mathrm{E}+13$ & $1.82057 \mathrm{E}+13$ & $6.91216 \mathrm{E}+13$ & $6.60613 E+19$ & 752.7 & 380.6 \\
\hline & & 47.6 & 2.18 & 247.0 & $2.44766 \mathrm{E}+13$ & 1.70147E+13 & $6.48875 E+13$ & $6.85906 \mathrm{E}+19$ & 722.2 & 366.9 \\
\hline & & 50.0 & 2.28 & 243.7 & $2.40862 \mathrm{E}+13$ & $1.66418 \mathrm{E}+13$ & $6.39748 \mathrm{E}+13$ & $7.20231 \mathrm{E}+19$ & 715.6 & 364.0 \\
\hline & $\operatorname{EOC}(11 / 8 / 2016,1100)$ & 52.7 & 2.40 & 233.7 & $2.32213 \mathrm{E}+13$ & $1.60171 \mathrm{E}+13$ & $6.19500 \mathrm{E}+13$ & $7.55761 \mathrm{E}+19$ & 696.0 & 355.1 \\
\hline $160 \mathrm{~B}$ & \multicolumn{10}{|c|}{ Cycle 160B Outage - 42 Day, 1 Hour Down Time Outage } \\
\hline & $\mathrm{BOC}(12 / 20 / 2016,1200)$ & 53.7 & 2.44 & 243.8 & $2.43040 \mathrm{E}+13$ & $1.68234 \mathrm{E}+13$ & $6.48198 \mathrm{E}+13$ & $7.67805 \mathrm{E}+19$ & 715.9 & 364.1 \\
\hline & & 55.7 & 2.53 & 242.6 & $2.42204 \mathrm{E}+13$ & $1.67899 \mathrm{E}+13$ & $6.47434 \mathrm{E}+13$ & 7.97313E+19 & 713.7 & 363.1 \\
\hline & & 62.7 & 2.82 & 239.4 & $2.39556 \mathrm{E}+13$ & $1.67908 \mathrm{E}+13$ & $6.40398 \mathrm{E}+13$ & $8.88847 \mathrm{E}+19$ & 707.2 & 360.2 \\
\hline & & 72.7 & 3.22 & 229.9 & $2.31247 \mathrm{E}+13$ & $1.59412 \mathrm{E}+13$ & $6.17304 \mathrm{E}+13$ & $1.01651 \mathrm{E}+20$ & 688.2 & 351.6 \\
\hline & & 81.9 & 3.60 & 228.6 & $2.34202 E+13$ & $1.58620 \mathrm{E}+13$ & $6.18814 \mathrm{E}+13$ & $1.13454 \mathrm{E}+20$ & 685.7 & 350.5 \\
\hline & \multicolumn{10}{|c|}{ Mid-Cycle 160B Outage - 5 Day, 14 Hour Down Time } \\
\hline & & 92.7 & 4.04 & 236.3 & $2.41191 \mathrm{E}+13$ & $1.64116 \mathrm{E}+13$ & $6.36353 E+13$ & $1.27426 \mathrm{E}+20$ & 701.1 & 357.4 \\
\hline & & 102.7 & 4.44 & 230.8 & $2.38561 \mathrm{E}+13$ & $1.63292 \mathrm{E}+13$ & $6.45080 \mathrm{E}+13$ & $1.40132 \mathrm{E}+20$ & 690.1 & 352.4 \\
\hline & EOC $(2 / 23 / 2017,1400)$ & 112.2 & 4.85 & 245.5 & $2.57504 \mathrm{E}+13$ & $1.72774 \mathrm{E}+13$ & $6.79249 \mathrm{E}+13$ & $1.53019 \mathrm{E}+20$ & 719.3 & 365.6 \\
\hline $161 \mathrm{~A}$ & \multicolumn{10}{|c|}{ Cycle 161A Outage - 95 Day, 13 Hour Down Time } \\
\hline & BOC $(5 / 30 / 2017,0300)$ & 113.9 & 4.90 & 203.8 & $2.13356 \mathrm{E}+13$ & $1.43445 \mathrm{E}+13$ & $5.59931 \mathrm{E}+13$ & $1.54645 \mathrm{E}+20$ & 634.8 & 327.3 \\
\hline & & 114.2 & 4.91 & 182.3 & $1.91221 \mathrm{E}+13$ & $1.27384 \mathrm{E}+13$ & $5.00591 \mathrm{E}+13$ & $1.54886 \mathrm{E}+20$ & 589.4 & 306.3 \\
\hline & & 116.2 & 4.99 & 188.2 & $1.97436 \mathrm{E}+13$ & $1.31996 \mathrm{E}+13$ & $5.16196 \mathrm{E}+13$ & $1.57295 \mathrm{E}+20$ & 602.0 & 312.1 \\
\hline & & 118.2 & 5.07 & 189.3 & $1.99106 \mathrm{E}+13$ & $1.33536 \mathrm{E}+13$ & $5.22179 \mathrm{E}+13$ & $1.59764 \mathrm{E}+20$ & 604.2 & 313.2 \\
\hline & & 120.2 & 5.14 & 192.2 & $2.01762 \mathrm{E}+13$ & $1.33812 \mathrm{E}+13$ & $5.26511 \mathrm{E}+13$ & $1.62112 \mathrm{E}+20$ & 610.6 & 316.1 \\
\hline & & 122.2 & 5.22 & 195.6 & $2.06056 \mathrm{E}+13$ & $1.36747 \mathrm{E}+13$ & $5.36582 \mathrm{E}+13$ & $1.64581 \mathrm{E}+20$ & 617.7 & 319.4 \\
\hline & & 124.2 & 5.30 & 199.3 & $2.10527 \mathrm{E}+13$ & $1.38934 E+13$ & $5.49065 \mathrm{E}+13$ & $1.67111 \mathrm{E}+20$ & 625.4 & 322.9 \\
\hline
\end{tabular}


TEM-10300-1

03/01/2012

TECHNICAL EVALUATION

Page 66 of 110

Rev. 03

ATF-1 Power Histories

$\begin{array}{lllll}\text { TEV No.: } & 3119 & \text { Rev. No.: } & 0 & \text { Project No.: }\end{array} 31418$

Table 27. ATF-41 Power History

\begin{tabular}{|c|c|c|c|c|c|c|c|c|c|c|}
\hline Cycle & Critical Dates & EFPD & $\begin{array}{l}\text { Burnup } \\
\text { (GWd/MTU) }\end{array}$ & $\begin{array}{l}\text { LHGR } \\
(\mathrm{W} / \mathrm{cm})\end{array}$ & $\begin{array}{l}\text { Thermal Flux } \\
\left(\mathrm{n} / \mathrm{cm}^{2 *} \mathrm{~s}\right)\end{array}$ & $\begin{array}{c}\text { Fast Flux } \\
\left(\mathrm{n} / \mathrm{cm}^{2 *} \mathrm{~s}>1\right. \\
\mathrm{MeV})\end{array}$ & $\begin{array}{l}\text { Total Flux } \\
\left(\mathrm{n} / \mathrm{cm}^{2 *} \mathrm{~s}\right)\end{array}$ & $\begin{array}{c}\text { Fission Density } \\
\text { (Fissions } / \mathrm{cm}^{3} \text { ) }\end{array}$ & $\begin{array}{c}\text { Fuel Centerline } \\
\text { Temperature } \\
\left({ }^{\circ} \mathrm{C}\right)\end{array}$ & $\operatorname{PICT}\left({ }^{\circ} \mathrm{C}\right)$ \\
\hline & $\operatorname{EOC}(6 / 11 / 2017,1600)$ & 124.7 & 5.32 & 202.6 & $2.13780 \mathrm{E}+13$ & $1.40713 \mathrm{E}+13$ & $5.55468 \mathrm{E}+13$ & $1.67592 \mathrm{E}+20$ & 632.4 & 326.2 \\
\hline \multirow[t]{10}{*}{$162 \mathrm{~A}$} & \multicolumn{10}{|c|}{ Cycle 162A Outage } \\
\hline & $\mathrm{BOC}$ & 125.7 & 5.35 & 266.5 & $2.73937 \mathrm{E}+13$ & $1.80610 \mathrm{E}+13$ & $7.12115 E+13$ & $1.68797 \mathrm{E}+20$ & 759.7 & 383.7 \\
\hline & & 127.7 & 5.45 & 251.1 & $2.61650 \mathrm{E}+13$ & $1.73422 \mathrm{E}+13$ & $6.80843 E+13$ & $1.71747 \mathrm{E}+20$ & 730.1 & 370.5 \\
\hline & & 134.7 & 5.75 & 250.6 & $2.61959 \mathrm{E}+13$ & $1.72729 \mathrm{E}+13$ & $6.79280 \mathrm{E}+13$ & $1.81202 \mathrm{E}+20$ & 729.2 & 370.1 \\
\hline & & 144.7 & 6.17 & 253.5 & $2.61972 \mathrm{E}+13$ & $1.72929 \mathrm{E}+13$ & $6.80721 \mathrm{E}+13$ & $1.94571 \mathrm{E}+20$ & 734.8 & 372.6 \\
\hline & & 154.7 & 6.60 & 253.3 & $2.65060 \mathrm{E}+13$ & $1.73159 \mathrm{E}+13$ & $6.83816 \mathrm{E}+13$ & $2.08000 \mathrm{E}+20$ & 734.4 & 372.4 \\
\hline & & 164.7 & 7.02 & 255.4 & $2.68282 \mathrm{E}+13$ & $1.72062 \mathrm{E}+13$ & $6.87739 \mathrm{E}+13$ & $2.21429 \mathrm{E}+20$ & 738.5 & 374.3 \\
\hline & & 174.7 & 7.48 & 258.9 & $2.78483 \mathrm{E}+13$ & $1.78311 \mathrm{E}+13$ & $7.12384 \mathrm{E}+13$ & $2.35822 \mathrm{E}+20$ & 745.4 & 377.3 \\
\hline & & 183.7 & 7.87 & 253.0 & $2.73514 \mathrm{E}+13$ & $1.73872 \mathrm{E}+13$ & $7.00257 E+13$ & $2.48106 \mathrm{E}+20$ & 733.9 & 372.2 \\
\hline & EOC & 186.7 & 8.00 & 252.4 & $2.75840 \mathrm{E}+13$ & $1.73297 \mathrm{E}+13$ & $7.02447 \mathrm{E}+13$ & $2.52141 \mathrm{E}+20$ & 732.7 & 371.6 \\
\hline
\end{tabular}


TEM-10300-1

$03 / 01 / 2012$

TECHNICAL EVALUATION

Page 67 of 110

Rev. 03

Title: $\quad$ ATF-1 Power Histories

$\begin{array}{lllll}\text { TEV No.: } & 3119 & \text { Rev. No.: } & 0 & \text { Project No.: }\end{array}$

Table 28. ATF-44 Power History

\begin{tabular}{|c|c|c|c|c|c|c|c|c|c|c|}
\hline Cycle & Critical Dates & EFPD & $\begin{array}{c}\text { Burnup } \\
\text { (GWd/MTU) }\end{array}$ & $\begin{array}{c}\text { LHGR } \\
(\mathrm{W} / \mathrm{cm})\end{array}$ & $\begin{array}{l}\text { Thermal Flux } \\
\left(\mathrm{n} / \mathrm{cm}^{2 *} \mathrm{~s}\right)\end{array}$ & $\begin{array}{c}\text { Fast Flux } \\
\left(\mathrm{n} / \mathrm{cm}^{2 *} \mathrm{~s}>1\right. \\
\mathrm{MeV})\end{array}$ & $\begin{array}{l}\text { Total Flux } \\
\left(\mathrm{n} / \mathrm{cm}^{2 *} \mathrm{~s}\right)\end{array}$ & $\begin{array}{c}\text { Fission Density } \\
\left(\text { Fissions } / \mathrm{cm}^{3}\right)\end{array}$ & $\begin{array}{c}\text { Fuel Centerline } \\
\text { Temperature } \\
\left({ }^{\circ} \mathrm{C}\right)\end{array}$ & PICT $\left({ }^{\circ} \mathrm{C}\right)$ \\
\hline \multirow[t]{9}{*}{ 160B } & $\operatorname{BOC}(12 / 20 / 2016,1200)$ & 1.0 & 0.05 & 230.7 & $4.09589 \mathrm{E}+13$ & $1.71838 E+13$ & $8.71000 E+13$ & $1.56572 E+18$ & 668.3 & 329.6 \\
\hline & & 3.0 & 0.13 & 223.1 & $3.97527 \mathrm{E}+13$ & $1.66357 \mathrm{E}+13$ & $8.50041 \mathrm{E}+13$ & $4.09496 \mathrm{E}+18$ & 653.7 & 323.1 \\
\hline & & 10.0 & 0.41 & 222.2 & $3.97295 \mathrm{E}+13$ & $1.65219 \mathrm{E}+13$ & $8.46686 \mathrm{E}+13$ & $1.30677 \mathrm{E}+19$ & 652.0 & 322.3 \\
\hline & & 20.0 & 0.78 & 218.8 & $3.93997 \mathrm{E}+13$ & $1.62807 \mathrm{E}+13$ & $8.36574 \mathrm{E}+13$ & $2.52322 \mathrm{E}+19$ & 645.2 & 319.3 \\
\hline & & 29.2 & 1.14 & 217.7 & $4.00693 E+13$ & $1.63538 \mathrm{E}+13$ & $8.48079 \mathrm{E}+13$ & $3.66740 \mathrm{E}+19$ & 643.0 & 318.3 \\
\hline & \multicolumn{10}{|c|}{ Mid-Cycle 160B Outage - 5 Day, 14 Hour Down Time } \\
\hline & & 40.0 & 1.57 & 225.0 & $4.16406 \mathrm{E}+13$ & $1.69630 \mathrm{E}+13$ & $8.78500 \mathrm{E}+13$ & $5.04041 \mathrm{E}+19$ & 657.3 & 324.7 \\
\hline & & 50.0 & 1.96 & 229.6 & $4.27944 \mathrm{E}+13$ & $1.71845 E+13$ & $8.94754 \mathrm{E}+13$ & $6.28697 \mathrm{E}+19$ & 666.3 & 328.7 \\
\hline & EOC $(2 / 23 / 2017,1400)$ & 59.5 & 2.34 & 234.6 & $4.47565 \mathrm{E}+13$ & $1.76710 \mathrm{E}+13$ & $9.26579 \mathrm{E}+13$ & $7.52148 \mathrm{E}+19$ & 675.9 & 332.9 \\
\hline $162 \mathrm{~A}$ & \multicolumn{10}{|c|}{ Withheld From Cycle 161A PALM } \\
\hline & $\mathrm{BOC}$ & 60.5 & 2.37 & 200.9 & $3.89311 \mathrm{E}+13$ & $1.55261 \mathrm{E}+13$ & $8.11820 \mathrm{E}+13$ & $7.60579 \mathrm{E}+19$ & 609.7 & 303.5 \\
\hline & & 62.5 & 2.43 & 191.9 & $3.60235 \mathrm{E}+13$ & $1.42741 \mathrm{E}+13$ & $7.53880 \mathrm{E}+13$ & $7.81656 \mathrm{E}+19$ & 591.3 & 295.2 \\
\hline & & 69.5 & 2.66 & 195.5 & $3.70926 \mathrm{E}+13$ & $1.46325 E+13$ & $7.74430 \mathrm{E}+13$ & $8.55726 \mathrm{E}+19$ & 598.6 & 298.5 \\
\hline & & 79.5 & 3.01 & 200.6 & $3.88859 \mathrm{E}+13$ & $1.51433 E+13$ & $8.07555 E+13$ & $9.67735 \mathrm{E}+19$ & 609.0 & 303.1 \\
\hline & & 89.5 & 3.35 & 196.7 & $3.85751 \mathrm{E}+13$ & $1.50316 \mathrm{E}+13$ & $8.01460 \mathrm{E}+13$ & $1.07794 \mathrm{E}+20$ & 601.0 & 299.6 \\
\hline & & 99.5 & 3.70 & 199.7 & $4.01264 \mathrm{E}+13$ & $1.53567 \mathrm{E}+13$ & $8.26900 \mathrm{E}+13$ & $1.19055 \mathrm{E}+20$ & 607.3 & 302.4 \\
\hline & & 109.5 & 4.05 & 197.2 & $3.96806 \mathrm{E}+13$ & $1.51531 \mathrm{E}+13$ & $8.16504 \mathrm{E}+13$ & $1.30075 \mathrm{E}+20$ & 602.2 & 300.1 \\
\hline & & 118.5 & 4.37 & 202.9 & $4.19209 E+13$ & $1.57591 \mathrm{E}+13$ & $8.55792 E+13$ & $1.40614 \mathrm{E}+20$ & 613.7 & 305.3 \\
\hline & EOC & 121.5 & 4.49 & 201.0 & $4.22931 \mathrm{E}+13$ & $1.57337 E+13$ & $8.59336 \mathrm{E}+13$ & $1.44287 \mathrm{E}+20$ & 609.7 & 303.5 \\
\hline
\end{tabular}


TEM-10300-1

$03 / 01 / 2012$

Rev. 03

Title: $\quad$ ATF-1 Power Histories

\begin{tabular}{lllll} 
TEV No.: & 3119 & Rev. No.: & 0 & Project No.: \\
\hline
\end{tabular}

Table 29. ATF-45 Power History

\begin{tabular}{|c|c|c|c|c|c|c|c|c|c|c|}
\hline Cycle & Critical Dates & EFPD & $\begin{array}{c}\text { Burnup } \\
\text { (GWd/MTU) }\end{array}$ & $\begin{array}{c}\text { LHGR } \\
\text { (W/cm) }\end{array}$ & $\begin{array}{l}\text { Thermal Flux } \\
\left(\mathrm{n} / \mathrm{cm}^{2 *} \mathrm{~s}\right)\end{array}$ & $\begin{array}{c}\text { Fast Flux } \\
\left(\mathrm{n} / \mathrm{cm}^{2 *} \mathrm{~s}>1\right. \\
\mathrm{MeV})\end{array}$ & $\begin{array}{l}\text { Total Flux } \\
\left(\mathrm{n} / \mathrm{cm}^{2 *} \mathrm{~s}\right)\end{array}$ & $\begin{array}{l}\text { Fission Density } \\
\text { (Fissions/cm }{ }^{3} \text { ) }\end{array}$ & $\begin{array}{c}\text { Fuel Centerline } \\
\text { Temperature } \\
\left({ }^{\circ} \mathrm{C}\right)\end{array}$ & PICT $\left({ }^{\circ} \mathrm{C}\right)$ \\
\hline \multirow[t]{10}{*}{$160 \mathrm{~A}$} & BOC $(9 / 16 / 2016,1900)$ & 1.0 & 0.07 & 268.9 & $2.45843 E+13$ & $1.84960 \mathrm{E}+13$ & $6.73827 E+13$ & $1.38506 \mathrm{E}+18$ & 808.9 & 386.2 \\
\hline & & 3.0 & 0.21 & 266.7 & $2.44783 \mathrm{E}+13$ & $1.83425 \mathrm{E}+13$ & $6.71762 \mathrm{E}+13$ & $4.15518 \mathrm{E}+18$ & 804.8 & 384.4 \\
\hline & & 10.0 & 0.72 & 268.3 & $2.46726 \mathrm{E}+13$ & $1.84804 \mathrm{E}+13$ & $6.77812 E+13$ & $1.42721 \mathrm{E}+19$ & 807.8 & 385.8 \\
\hline & & 20.0 & 1.47 & 265.0 & $2.45040 \mathrm{E}+13$ & $1.83524 \mathrm{E}+13$ & $6.73171 \mathrm{E}+13$ & $2.89658 \mathrm{E}+19$ & 801.6 & 383.0 \\
\hline & & 30.0 & 2.22 & 271.0 & $2.53080 \mathrm{E}+13$ & $1.85916 \mathrm{E}+13$ & $6.86825 E+13$ & $4.39004 \mathrm{E}+19$ & 812.8 & 388.0 \\
\hline & & 40.0 & 2.99 & 275.9 & $2.61017 \mathrm{E}+13$ & $1.91081 \mathrm{E}+13$ & $7.05777 \mathrm{E}+13$ & $5.90758 \mathrm{E}+19$ & 821.9 & 392.1 \\
\hline & & 46.0 & 3.44 & 272.9 & $2.60210 \mathrm{E}+13$ & $1.87723 \mathrm{E}+13$ & $7.00017 \mathrm{E}+13$ & $6.79884 \mathrm{E}+19$ & 816.4 & 389.6 \\
\hline & & 47.6 & 3.55 & 256.3 & $2.45288 \mathrm{E}+13$ & 1.77970E+13 & $6.60359 \mathrm{E}+13$ & $7.00961 \mathrm{E}+19$ & 784.9 & 375.5 \\
\hline & & 50.0 & 3.71 & 253.6 & $2.42966 \mathrm{E}+13$ & $1.73646 \mathrm{E}+13$ & $6.51661 \mathrm{E}+13$ & $7.32275 \mathrm{E}+19$ & 779.8 & 373.2 \\
\hline & EOC $(11 / 8 / 2016,1100)$ & 52.7 & 3.91 & 242.0 & $2.33336 \mathrm{E}+13$ & $1.66583 \mathrm{E}+13$ & $6.29180 \mathrm{E}+13$ & $7.70816 \mathrm{E}+19$ & 757.0 & 363.0 \\
\hline 160B & \multicolumn{10}{|c|}{ Cycle 160B Outage - 42 Day, 1 Hour Down Time Outage } \\
\hline & $\operatorname{BOC}(12 / 20 / 2016,1200)$ & 53.7 & 3.97 & 253.5 & $2.44059 \mathrm{E}+13$ & $1.76198 \mathrm{E}+13$ & $6.58067 E+13$ & $7.83462 \mathrm{E}+19$ & 779.6 & 373.1 \\
\hline & & 55.7 & 4.10 & 252.2 & $2.43438 \mathrm{E}+13$ & $1.75482 \mathrm{E}+13$ & $6.56536 \mathrm{E}+13$ & $8.09357 \mathrm{E}+19$ & 777.0 & 372.0 \\
\hline & & 62.7 & 4.58 & 250.5 & $2.41727 \mathrm{E}+13$ & $1.72834 \mathrm{E}+13$ & $6.48978 \mathrm{E}+13$ & $9.03902 E+19$ & 773.7 & 370.5 \\
\hline & & 72.7 & 5.26 & 239.6 & $2.32984 \mathrm{E}+13$ & $1.67269 \mathrm{E}+13$ & $6.27717 \mathrm{E}+13$ & $1.03759 \mathrm{E}+20$ & 752.3 & 360.9 \\
\hline & & 81.9 & 5.88 & 237.3 & $2.34417 \mathrm{E}+13$ & $1.65536 \mathrm{E}+13$ & $6.25804 \mathrm{E}+13$ & $1.16044 \mathrm{E}+20$ & 747.7 & 358.8 \\
\hline & \multicolumn{10}{|c|}{ Mid-Cycle 160B Outage - 5 Day, 14 Hour Down Time } \\
\hline & & 92.7 & 6.62 & 245.4 & $2.42921 \mathrm{E}+13$ & 1.69057E+13 & $6.44848 E+13$ & 1.30677E+20 & 763.6 & 366.0 \\
\hline & & 102.7 & 7.29 & 240.7 & $2.40511 \mathrm{E}+13$ & $1.69531 \mathrm{E}+13$ & $6.53650 \mathrm{E}+13$ & $1.43986 \mathrm{E}+20$ & 754.4 & 361.8 \\
\hline & EOC $(2 / 23 / 2017,1400)$ & 112.2 & 7.98 & 254.3 & $2.58719 \mathrm{E}+13$ & $1.80710 \mathrm{E}+13$ & $6.89334 \mathrm{E}+13$ & $1.57415 \mathrm{E}+20$ & 781.0 & 373.8 \\
\hline $161 \mathrm{~A}$ & \multicolumn{10}{|c|}{ Cycle 161A Outage - 95 Day, 13 Hour Down Time } \\
\hline & BOC $(5 / 30 / 2017,0300)$ & 113.9 & 8.07 & 214.0 & $2.17166 \mathrm{E}+13$ & $1.49262 \mathrm{E}+13$ & $5.70568 \mathrm{E}+13$ & $1.59282 \mathrm{E}+20$ & 700.2 & 337.4 \\
\hline & & 114.2 & 8.09 & 189.9 & $1.92436 \mathrm{E}+13$ & $1.33198 \mathrm{E}+13$ & $5.08307 E+13$ & $1.59583 \mathrm{E}+20$ & 648.9 & 314.3 \\
\hline & & 116.2 & 8.19 & 195.7 & $1.98917 \mathrm{E}+13$ & $1.37478 \mathrm{E}+13$ & $5.24733 E+13$ & $1.61570 \mathrm{E}+20$ & 661.5 & 320.0 \\
\hline & & 118.2 & 8.29 & 199.2 & $2.02818 \mathrm{E}+13$ & $1.39471 \mathrm{E}+13$ & $5.34109 E+13$ & $1.63558 \mathrm{E}+20$ & 669.0 & 323.4 \\
\hline & & 120.2 & 8.39 & 198.5 & $2.01949 \mathrm{E}+13$ & $1.38267 \mathrm{E}+13$ & $5.33628 \mathrm{E}+13$ & $1.65545 \mathrm{E}+20$ & 667.5 & 322.7 \\
\hline & & 122.2 & 8.49 & 202.9 & $2.07041 \mathrm{E}+13$ & $1.41362 \mathrm{E}+13$ & $5.43670 \mathrm{E}+13$ & $1.67592 \mathrm{E}+20$ & 676.8 & 326.9 \\
\hline & & 124.2 & 8.60 & 206.9 & $2.11571 \mathrm{E}+13$ & $1.44341 \mathrm{E}+13$ & $5.56671 \mathrm{E}+13$ & $1.69700 \mathrm{E}+20$ & 685.4 & 330.8 \\
\hline
\end{tabular}


TEM-10300-1

03/01/2012

TECHNICAL EVALUATION

Page 69 of 110

Rev. 03

ATF-1 Power Histories

$\begin{array}{lllll}\text { TEV No.: } & 3119 & \text { Rev. No.: } & 0 & \text { Project No.: }\end{array} 31418$

Table 29. ATF-45 Power History

\begin{tabular}{|c|c|c|c|c|c|c|c|c|c|c|}
\hline Cycle & Critical Dates & EFPD & $\begin{array}{c}\text { Burnup } \\
\text { (GWd/MTU) }\end{array}$ & $\begin{array}{l}\text { LHGR } \\
(\mathrm{W} / \mathrm{cm})\end{array}$ & $\begin{array}{l}\text { Thermal Flux } \\
\left(\mathrm{n} / \mathrm{cm}^{2 *} \mathrm{~s}\right)\end{array}$ & $\begin{array}{c}\text { Fast Flux } \\
\left(\mathrm{n} / \mathrm{cm}^{2 *} \mathrm{~s}>1\right. \\
\mathrm{MeV})\end{array}$ & $\begin{array}{l}\text { Total Flux } \\
\left(\mathrm{n} / \mathrm{cm}^{2 *} \mathrm{~s}\right)\end{array}$ & $\begin{array}{c}\text { Fission Density } \\
\text { (Fissions } / \mathrm{cm}^{3} \text { ) }\end{array}$ & $\begin{array}{c}\text { Fuel Centerline } \\
\text { Temperature } \\
\left({ }^{\circ} \mathrm{C}\right)\end{array}$ & $\operatorname{PICT}\left({ }^{\circ} \mathrm{C}\right)$ \\
\hline & $\operatorname{EOC}(6 / 11 / 2017,1600)$ & 124.7 & 8.63 & 210.6 & $2.16056 \mathrm{E}+13$ & $1.46745 \mathrm{E}+13$ & $5.63605 \mathrm{E}+13$ & $1.70242 \mathrm{E}+20$ & 693.1 & 334.3 \\
\hline \multirow[t]{10}{*}{$162 \mathrm{~A}$} & \multicolumn{10}{|c|}{ Cycle 162A Outage } \\
\hline & $\mathrm{BOC}$ & 125.7 & 8.70 & 276.3 & $2.74735 \mathrm{E}+13$ & $1.89023 \mathrm{E}+13$ & $7.22431 \mathrm{E}+13$ & $1.71627 \mathrm{E}+20$ & 822.6 & 392.4 \\
\hline & & 127.7 & 8.83 & 261.0 & $2.63938 \mathrm{E}+13$ & $1.79515 \mathrm{E}+13$ & $6.89456 \mathrm{E}+13$ & $1.74216 \mathrm{E}+20$ & 794.0 & 379.6 \\
\hline & & 134.7 & 9.31 & 259.9 & $2.63558 \mathrm{E}+13$ & $1.79166 \mathrm{E}+13$ & $6.88477 E+13$ & $1.83852 \mathrm{E}+20$ & 791.8 & 378.6 \\
\hline & & 144.7 & 10.03 & 264.0 & $2.64320 \mathrm{E}+13$ & $1.80020 \mathrm{E}+13$ & $6.91295 E+13$ & $1.98003 \mathrm{E}+20$ & 799.6 & 382.1 \\
\hline & & 154.7 & 10.74 & 262.0 & $2.66517 \mathrm{E}+13$ & $1.78851 \mathrm{E}+13$ & $6.92274 \mathrm{E}+13$ & $2.12035 \mathrm{E}+20$ & 795.7 & 380.4 \\
\hline & & 164.7 & 11.46 & 264.5 & $2.70329 \mathrm{E}+13$ & $1.79882 \mathrm{E}+13$ & $6.98780 \mathrm{E}+13$ & $2.26186 \mathrm{E}+20$ & 800.5 & 382.5 \\
\hline & & 174.7 & 12.19 & 267.8 & $2.80891 \mathrm{E}+13$ & $1.85662 \mathrm{E}+13$ & $7.22423 E+13$ & $2.40639 \mathrm{E}+20$ & 806.7 & 385.3 \\
\hline & & 183.7 & 12.84 & 260.2 & $2.74402 \mathrm{E}+13$ & $1.81372 \mathrm{E}+13$ & $7.08995 E+13$ & $2.53346 \mathrm{E}+20$ & 792.3 & 378.8 \\
\hline & EOC & 186.7 & 13.06 & 259.5 & $2.77104 \mathrm{E}+13$ & $1.80921 \mathrm{E}+13$ & $7.11761 \mathrm{E}+13$ & $2.57802 \mathrm{E}+20$ & 791.0 & 378.2 \\
\hline
\end{tabular}


TEM-10300-1

03/01/2012

Rev. 03

Title:

ATF-1 Power Histories

\begin{tabular}{lllll} 
TEV No.: & 3119 & Rev. No.: & 0 & Project No.: \\
\hline
\end{tabular}

Table 30. ATF-73 (Pellet 1 - Top) Power History

\begin{tabular}{|c|c|c|c|c|c|c|c|c|}
\hline Cycle & Critical Dates & EFPD & $\begin{array}{c}\text { Burnup } \\
\text { (GWd/MTU) }\end{array}$ & $\begin{array}{l}\text { LHGR } \\
(\mathrm{W} / \mathrm{cm})\end{array}$ & $\begin{array}{l}\text { Thermal Flux } \\
\left(\mathrm{n} / \mathrm{cm}^{2 *} \mathrm{~s}\right)\end{array}$ & $\begin{array}{c}\text { Fast Flux } \\
\left(\mathrm{n} / \mathrm{cm}^{2 *} \mathrm{~s}>1\right. \\
\mathrm{MeV})\end{array}$ & $\begin{array}{l}\text { Total Flux } \\
\left(\mathrm{n} / \mathrm{cm}^{2 *} \mathrm{~s}\right)\end{array}$ & $\begin{array}{c}\text { Fission Density } \\
\text { (Fissions } / \mathrm{cm}^{3} \text { ) }\end{array}$ \\
\hline \multirow[t]{9}{*}{$160 \mathrm{~B}$} & $\operatorname{BOC}(12 / 20 / 2016,1200)$ & 1.0 & 0.07 & 206.0 & $4.35515 \mathrm{E}+13$ & $9.15001 \mathrm{E}+12$ & $7.25558 \mathrm{E}+13$ & $1.68616 \mathrm{E}+18$ \\
\hline & & 3.0 & 0.23 & 204.2 & $4.32771 \mathrm{E}+13$ & $9.11102 \mathrm{E}+12$ & $7.24926 \mathrm{E}+13$ & $5.66068 \mathrm{E}+18$ \\
\hline & & 10.0 & 0.79 & 211.4 & $4.46452 E+13$ & $9.13590 \mathrm{E}+12$ & $7.32583 E+13$ & $1.95113 \mathrm{E}+19$ \\
\hline & & 20.0 & 1.64 & 220.4 & $4.73675 \mathrm{E}+13$ & $9.95742 \mathrm{E}+12$ & $7.85756 \mathrm{E}+13$ & $4.04678 \mathrm{E}+19$ \\
\hline & & 29.2 & 2.46 & 229.9 & $5.08539 \mathrm{E}+13$ & $1.01715 \mathrm{E}+13$ & $8.23710 \mathrm{E}+13$ & $6.08824 \mathrm{E}+19$ \\
\hline & \multicolumn{8}{|c|}{ Mid-Cycle 160B Outage - 5 Day, 14 Hour Down Time } \\
\hline & & 40.0 & 3.44 & 235.2 & $5.26147 \mathrm{E}+13$ & $1.03049 E+13$ & $8.52885 E+13$ & $8.49704 \mathrm{E}+19$ \\
\hline & & 50.0 & 4.34 & 238.9 & $5.38967 \mathrm{E}+13$ & $1.08621 \mathrm{E}+13$ & $8.75422 E+13$ & $1.07372 \mathrm{E}+20$ \\
\hline & EOC $(2 / 23 / 2017,1400)$ & 59.5 & 5.20 & 232.8 & $5.50148 \mathrm{E}+13$ & $1.07146 \mathrm{E}+13$ & $8.81823 E+13$ & $1.28449 \mathrm{E}+20$ \\
\hline $161 \mathrm{~A}$ & \multicolumn{8}{|c|}{ Cycle 161A Outage - 95 Day, 13 Hour Down Time } \\
\hline & BOC $(5 / 30 / 2017,0300)$ & 61.2 & 5.33 & 202.8 & $4.74507 \mathrm{E}+13$ & $8.96546 \mathrm{E}+12$ & $7.53126 \mathrm{E}+13$ & $1.31882 \mathrm{E}+20$ \\
\hline & & 61.5 & 5.35 & 188.9 & $4.42475 \mathrm{E}+13$ & $8.67921 \mathrm{E}+12$ & $7.02833 E+13$ & $1.32303 \mathrm{E}+20$ \\
\hline & & 63.5 & 5.50 & 184.9 & $4.40480 \mathrm{E}+13$ & $8.35460 \mathrm{E}+12$ & $7.04148 E+13$ & $1.35917 \mathrm{E}+20$ \\
\hline & & 65.5 & 5.65 & 185.6 & $4.42002 E+13$ & $8.20180 \mathrm{E}+12$ & $7.00315 E+13$ & $1.39590 \mathrm{E}+20$ \\
\hline & & 67.5 & 5.80 & 190.9 & $4.56266 \mathrm{E}+13$ & $8.59399 \mathrm{E}+12$ & $7.15986 \mathrm{E}+13$ & $1.43324 \mathrm{E}+20$ \\
\hline & & 69.5 & 5.95 & 194.3 & $4.59630 \mathrm{E}+13$ & $9.01757 \mathrm{E}+12$ & $7.42717 \mathrm{E}+13$ & $1.47057 \mathrm{E}+20$ \\
\hline & & 71.5 & 6.10 & 194.5 & $4.65186 \mathrm{E}+13$ & $8.75739 E+12$ & $7.43285 E+13$ & $1.50851 \mathrm{E}+20$ \\
\hline & $\operatorname{EOC}(6 / 11 / 2017,1600)$ & 72.0 & 6.13 & 200.4 & $4.85395 \mathrm{E}+13$ & $9.20532 E+12$ & $7.67597 \mathrm{E}+13$ & $1.51634 \mathrm{E}+20$ \\
\hline \multirow[t]{10}{*}{$162 \mathrm{~A}$} & \multicolumn{8}{|c|}{ Cycle 162A Outage } \\
\hline & $\mathrm{BOC}$ & 73.0 & 6.20 & 190.9 & $4.67767 \mathrm{E}+13$ & $8.98341 \mathrm{E}+12$ & $7.49572 \mathrm{E}+13$ & $1.53200 \mathrm{E}+20$ \\
\hline & & 75.0 & 6.34 & 180.2 & $4.37932 \mathrm{E}+13$ & $8.44875 E+12$ & $7.07112 \mathrm{E}+13$ & $1.56753 \mathrm{E}+20$ \\
\hline & & 82.0 & 6.83 & 184.4 & $4.42171 \mathrm{E}+13$ & $8.15825 \mathrm{E}+12$ & $7.06693 \mathrm{E}+13$ & $1.68797 \mathrm{E}+20$ \\
\hline & & 92.0 & 7.52 & 186.4 & $4.55172 \mathrm{E}+13$ & $8.22222 E+12$ & $7.16211 \mathrm{E}+13$ & $1.85959 \mathrm{E}+20$ \\
\hline & & 102.0 & 8.20 & 184.5 & $4.53934 \mathrm{E}+13$ & 8.47067E+12 & $7.22127 E+13$ & $2.02821 \mathrm{E}+20$ \\
\hline & & 112.0 & 8.88 & 183.4 & $4.58306 \mathrm{E}+13$ & $8.29573 E+12$ & $7.23910 \mathrm{E}+13$ & $2.19622 \mathrm{E}+20$ \\
\hline & & 122.0 & 9.61 & 189.7 & $4.86686 \mathrm{E}+13$ & $8.90935 E+12$ & $7.79067 E+13$ & $2.37447 \mathrm{E}+20$ \\
\hline & & 131.0 & 10.29 & 196.9 & $5.15939 \mathrm{E}+13$ & $9.33794 \mathrm{E}+12$ & $8.18789 E+13$ & $2.54430 \mathrm{E}+20$ \\
\hline & EOC & 134.0 & 10.52 & 192.8 & $5.15402 \mathrm{E}+13$ & $9.19457 \mathrm{E}+12$ & $8.11919 \mathrm{E}+13$ & $2.60150 \mathrm{E}+20$ \\
\hline
\end{tabular}


TEM-10300-1

03/01/2012

Rev. 03

Title:

ATF-1 Power Histories

$\begin{array}{lllll}\text { TEV No.: } & 3119 & \text { Rev. No.: } & 0 & \text { Project No.: } 31418\end{array}$

Table 31. ATF-73 (Pellet 2) Power History

\begin{tabular}{|c|c|c|c|c|c|c|c|c|}
\hline Cycle & Critical Dates & EFPD & $\begin{array}{c}\text { Burnup } \\
\text { (GWd/MTU) }\end{array}$ & $\begin{array}{l}\text { LHGR } \\
(\mathrm{W} / \mathrm{cm})\end{array}$ & $\begin{array}{l}\text { Thermal Flux } \\
\left(\mathrm{n} / \mathrm{cm}^{2 *} \mathrm{~s}\right)\end{array}$ & $\begin{array}{c}\text { Fast Flux } \\
\left(\mathrm{n} / \mathrm{cm}^{2 *} \mathrm{~s}>1\right. \\
\mathrm{MeV})\end{array}$ & $\begin{array}{l}\text { Total Flux } \\
\left(\mathrm{n} / \mathrm{cm}^{2 *} \mathrm{~s}\right)\end{array}$ & $\begin{array}{l}\text { Fission Density } \\
\text { (Fissions } / \mathrm{cm}^{3} \text { ) }\end{array}$ \\
\hline \multirow[t]{9}{*}{$160 \mathrm{~B}$} & $\operatorname{BOC}(12 / 20 / 2016,1200)$ & 1.0 & 0.06 & 191.0 & $4.09046 \mathrm{E}+13$ & $9.03842 \mathrm{E}+12$ & $6.86925 \mathrm{E}+13$ & $1.56572 \mathrm{E}+18$ \\
\hline & & 3.0 & 0.22 & 197.8 & $4.22837 \mathrm{E}+13$ & $9.17925 \mathrm{E}+12$ & $7.08165 E+13$ & $5.41980 \mathrm{E}+18$ \\
\hline & & 10.0 & 0.75 & 197.3 & $4.21480 \mathrm{E}+13$ & $9.41472 \mathrm{E}+12$ & $7.10178 \mathrm{E}+13$ & $1.84273 \mathrm{E}+19$ \\
\hline & & 20.0 & 1.56 & 211.1 & $4.54268 \mathrm{E}+13$ & $1.01026 \mathrm{E}+13$ & $7.64982 \mathrm{E}+13$ & $3.84806 \mathrm{E}+19$ \\
\hline & & 29.2 & 2.30 & 212.9 & $4.74600 \mathrm{E}+13$ & $1.04542 \mathrm{E}+13$ & $7.90208 \mathrm{E}+13$ & $5.69681 \mathrm{E}+19$ \\
\hline & \multicolumn{8}{|c|}{ Mid-Cycle 160B Outage - 5 Day, 14 Hour Down Time } \\
\hline & & 40.0 & 3.25 & 229.7 & $5.15548 \mathrm{E}+13$ & $1.12552 \mathrm{E}+13$ & $8.53820 \mathrm{E}+13$ & $8.04539 \mathrm{E}+19$ \\
\hline & & 50.0 & 4.10 & 219.7 & $4.96845 \mathrm{E}+13$ & $1.09366 \mathrm{E}+13$ & $8.26253 \mathrm{E}+13$ & $1.01230 \mathrm{E}+20$ \\
\hline & EOC $(2 / 23 / 2017,1400)$ & 59.5 & 4.92 & 222.4 & $5.22315 \mathrm{E}+13$ & $1.06063 E+13$ & $8.54281 \mathrm{E}+13$ & $1.21584 \mathrm{E}+20$ \\
\hline \multirow[t]{9}{*}{$161 \mathrm{~A}$} & \multicolumn{8}{|c|}{ Cycle 161A Outage - 95 Day, 13 Hour Down Time } \\
\hline & BOC $(5 / 30 / 2017,0300)$ & 61.2 & 5.05 & 191.8 & $4.51425 \mathrm{E}+13$ & $8.77831 \mathrm{E}+12$ & $7.32389 \mathrm{E}+13$ & $1.24836 \mathrm{E}+20$ \\
\hline & & 61.5 & 5.07 & 178.6 & $4.19582 \mathrm{E}+13$ & $8.51068 \mathrm{E}+12$ & $6.80897 E+13$ & $1.25318 \mathrm{E}+20$ \\
\hline & & 63.5 & 5.21 & 184.2 & $4.33393 \mathrm{E}+13$ & $8.49204 \mathrm{E}+12$ & $7.04883 E+13$ & $1.28871 \mathrm{E}+20$ \\
\hline & & 65.5 & 5.36 & 181.4 & $4.26358 \mathrm{E}+13$ & $8.35122 \mathrm{E}+12$ & $6.86808 \mathrm{E}+13$ & $1.32424 \mathrm{E}+20$ \\
\hline & & 67.5 & 5.50 & 180.6 & $4.26943 \mathrm{E}+13$ & $8.69700 \mathrm{E}+12$ & $6.98875 \mathrm{E}+13$ & $1.35977 \mathrm{E}+20$ \\
\hline & & 69.5 & 5.65 & 185.8 & $4.41594 \mathrm{E}+13$ & $9.13325 E+12$ & $7.20807 E+13$ & $1.39650 \mathrm{E}+20$ \\
\hline & & 71.5 & 5.80 & 184.2 & $4.39409 \mathrm{E}+13$ & $9.27965 E+12$ & $7.18495 E+13$ & $1.43263 \mathrm{E}+20$ \\
\hline & EOC $(6 / 11 / 2017,1600)$ & 72.0 & 5.83 & 188.3 & $4.51010 \mathrm{E}+13$ & $9.05698 \mathrm{E}+12$ & $7.31635 \mathrm{E}+13$ & $1.44046 \mathrm{E}+20$ \\
\hline \multirow[t]{10}{*}{$162 \mathrm{~A}$} & \multicolumn{8}{|c|}{ Cycle 162A Outage } \\
\hline & $\mathrm{BOC}$ & 73.0 & 5.89 & 183.3 & $4.49583 \mathrm{E}+13$ & $9.35558 \mathrm{E}+12$ & $7.27792 \mathrm{E}+13$ & $1.45492 \mathrm{E}+20$ \\
\hline & & 75.0 & 6.02 & 168.2 & $4.08352 \mathrm{E}+13$ & $8.26832 \mathrm{E}+12$ & $6.68504 \mathrm{E}+13$ & $1.48804 \mathrm{E}+20$ \\
\hline & & 82.0 & 6.48 & 173.8 & $4.18106 \mathrm{E}+13$ & $8.12923 E+12$ & $6.74640 \mathrm{E}+13$ & $1.60125 \mathrm{E}+20$ \\
\hline & & 92.0 & 7.14 & 176.7 & $4.28000 \mathrm{E}+13$ & $8.55564 \mathrm{E}+12$ & $6.91629 E+13$ & $1.76445 \mathrm{E}+20$ \\
\hline & & 102.0 & 7.80 & 178.6 & $4.35214 \mathrm{E}+13$ & $8.57963 E+12$ & $7.02765 E+13$ & $1.92764 \mathrm{E}+20$ \\
\hline & & 112.0 & 8.45 & 177.0 & $4.37591 \mathrm{E}+13$ & $8.66612 \mathrm{E}+12$ & $7.00924 \mathrm{E}+13$ & $2.08903 \mathrm{E}+20$ \\
\hline & & 122.0 & 9.15 & 182.6 & $4.68971 \mathrm{E}+13$ & $9.22197 E+12$ & $7.49936 \mathrm{E}+13$ & $2.26186 \mathrm{E}+20$ \\
\hline & & 131.0 & 9.80 & 188.6 & $4.95092 \mathrm{E}+13$ & $9.29694 \mathrm{E}+12$ & $7.89286 E+13$ & $2.42145 E+20$ \\
\hline & EOC & 134.0 & 10.00 & 187.1 & $4.95990 \mathrm{E}+13$ & $9.13480 \mathrm{E}+12$ & $7.87757 E+13$ & $2.47263 \mathrm{E}+20$ \\
\hline
\end{tabular}


TEM-10300-1

03/01/2012

Rev. 03

Title:

ATF-1 Power Histories

$\begin{array}{lllll}\text { TEV No.: } & 3119 & \text { Rev. No.: } & 0 & \text { Project No.: } 31418\end{array}$

Table 32. ATF-73 (Pellet 3) Power History

\begin{tabular}{|c|c|c|c|c|c|c|c|c|}
\hline Cycle & Critical Dates & EFPD & $\begin{array}{c}\text { Burnup } \\
\text { (GWd/MTU) }\end{array}$ & $\begin{array}{l}\text { LHGR } \\
(\mathrm{W} / \mathrm{cm})\end{array}$ & $\begin{array}{l}\text { Thermal Flux } \\
\left(\mathrm{n} / \mathrm{cm}^{2 *} \mathrm{~s}\right)\end{array}$ & $\begin{array}{c}\text { Fast Flux } \\
\left(\mathrm{n} / \mathrm{cm}^{2 *} \mathrm{~s}>1\right. \\
\mathrm{MeV})\end{array}$ & $\begin{array}{l}\text { Total Flux } \\
\left(\mathrm{n} / \mathrm{cm}^{2 *} \mathrm{~s}\right)\end{array}$ & $\begin{array}{l}\text { Fission Density } \\
\text { (Fissions } / \mathrm{cm}^{3} \text { ) }\end{array}$ \\
\hline \multirow[t]{9}{*}{$160 \mathrm{~B}$} & $\operatorname{BOC}(12 / 20 / 2016,1200)$ & 1.0 & 0.06 & 187.6 & $4.01246 \mathrm{E}+13$ & $9.06909 \mathrm{E}+12$ & $6.79274 \mathrm{E}+13$ & $1.56572 \mathrm{E}+18$ \\
\hline & & 3.0 & 0.21 & 185.3 & $3.97476 \mathrm{E}+13$ & $9.23681 \mathrm{E}+12$ & $6.83694 \mathrm{E}+13$ & $5.17892 \mathrm{E}+18$ \\
\hline & & 10.0 & 0.73 & 191.6 & $4.08159 \mathrm{E}+13$ & $9.21739 \mathrm{E}+12$ & $6.89978 \mathrm{E}+13$ & $1.79456 \mathrm{E}+19$ \\
\hline & & 20.0 & 1.48 & 201.0 & $4.34390 \mathrm{E}+13$ & $9.92193 E+12$ & $7.34932 E+13$ & $3.66740 \mathrm{E}+19$ \\
\hline & & 29.2 & 2.21 & 204.0 & $4.54841 \mathrm{E}+13$ & $1.02779 \mathrm{E}+13$ & $7.67954 \mathrm{E}+13$ & $5.45593 \mathrm{E}+19$ \\
\hline & \multicolumn{8}{|c|}{ Mid-Cycle 160B Outage - 5 Day, 14 Hour Down Time } \\
\hline & & 40.0 & 3.11 & 222.4 & $4.96660 \mathrm{E}+13$ & $1.07270 \mathrm{E}+13$ & $8.23053 \mathrm{E}+13$ & $7.67805 \mathrm{E}+19$ \\
\hline & & 50.0 & 3.94 & 219.6 & $4.98570 \mathrm{E}+13$ & $1.10500 \mathrm{E}+13$ & $8.29496 \mathrm{E}+13$ & $9.74962 \mathrm{E}+19$ \\
\hline & EOC $(2 / 23 / 2017,1400)$ & 59.5 & 4.75 & 217.3 & $5.06518 \mathrm{E}+13$ & $1.04301 \mathrm{E}+13$ & $8.23548 E+13$ & 1.17369E+20 \\
\hline \multirow[t]{9}{*}{$161 \mathrm{~A}$} & \multicolumn{8}{|c|}{ Cycle 161A Outage - 95 Day, 13 Hour Down Time } \\
\hline & BOC $(5 / 30 / 2017,0300)$ & 61.2 & 4.87 & 183.1 & $4.34794 \mathrm{E}+13$ & $8.69358 \mathrm{E}+12$ & $7.10866 \mathrm{E}+13$ & $1.20500 \mathrm{E}+20$ \\
\hline & & 61.5 & 4.89 & 173.7 & $4.06081 \mathrm{E}+13$ & $8.85918 \mathrm{E}+12$ & $6.71339 \mathrm{E}+13$ & $1.20862 \mathrm{E}+20$ \\
\hline & & 63.5 & 5.04 & 181.7 & $4.22529 \mathrm{E}+13$ & $8.83476 \mathrm{E}+12$ & $6.98345 E+13$ & $1.24475 \mathrm{E}+20$ \\
\hline & & 65.5 & 5.18 & 176.4 & $4.13902 E+13$ & $9.28634 \mathrm{E}+12$ & $6.80508 \mathrm{E}+13$ & $1.27968 \mathrm{E}+20$ \\
\hline & & 67.5 & 5.32 & 176.6 & $4.13561 \mathrm{E}+13$ & $9.29041 \mathrm{E}+12$ & $6.86349 \mathrm{E}+13$ & $1.31520 \mathrm{E}+20$ \\
\hline & & 69.5 & 5.46 & 179.4 & $4.23705 \mathrm{E}+13$ & $9.13399 \mathrm{E}+12$ & $6.89232 \mathrm{E}+13$ & $1.35073 \mathrm{E}+20$ \\
\hline & & 71.5 & 5.61 & 181.5 & $4.26474 \mathrm{E}+13$ & $9.19197 \mathrm{E}+12$ & $6.96182 \mathrm{E}+13$ & $1.38626 \mathrm{E}+20$ \\
\hline & EOC $(6 / 11 / 2017,1600)$ & 72.0 & 5.64 & 191.7 & $4.50707 \mathrm{E}+13$ & $9.48617 \mathrm{E}+12$ & $7.33035 E+13$ & $1.39409 \mathrm{E}+20$ \\
\hline \multirow[t]{10}{*}{$162 \mathrm{~A}$} & \multicolumn{8}{|c|}{ Cycle 162A Outage } \\
\hline & $\mathrm{BOC}$ & 73.0 & 5.70 & 181.3 & $4.39235 \mathrm{E}+13$ & $9.04658 \mathrm{E}+12$ & $7.15348 \mathrm{E}+13$ & $1.40915 \mathrm{E}+20$ \\
\hline & & 75.0 & 5.83 & 167.2 & $4.04460 \mathrm{E}+13$ & $8.29601 \mathrm{E}+12$ & $6.60533 E+13$ & $1.44227 \mathrm{E}+20$ \\
\hline & & 82.0 & 6.30 & 172.4 & $4.07583 \mathrm{E}+13$ & $8.29960 \mathrm{E}+12$ & $6.60975 \mathrm{E}+13$ & $1.55608 \mathrm{E}+20$ \\
\hline & & 92.0 & 6.96 & 174.8 & $4.17386 \mathrm{E}+13$ & $8.44459 \mathrm{E}+12$ & $6.80178 \mathrm{E}+13$ & $1.71928 \mathrm{E}+20$ \\
\hline & & 102.0 & 7.61 & 176.3 & $4.23932 \mathrm{E}+13$ & $8.44506 \mathrm{E}+12$ & $6.88268 \mathrm{E}+13$ & $1.88188 \mathrm{E}+20$ \\
\hline & & 112.0 & 8.27 & 174.6 & $4.29268 \mathrm{E}+13$ & $8.51526 \mathrm{E}+12$ & $6.94644 \mathrm{E}+13$ & $2.04387 \mathrm{E}+20$ \\
\hline & & 122.0 & 8.95 & 181.5 & $4.59017 \mathrm{E}+13$ & $9.00830 \mathrm{E}+12$ & $7.33342 E+13$ & $2.21248 \mathrm{E}+20$ \\
\hline & & 131.0 & 9.60 & 188.7 & $4.90542 \mathrm{E}+13$ & $1.01230 \mathrm{E}+13$ & $7.90741 \mathrm{E}+13$ & $2.37267 E+20$ \\
\hline & EOC & 134.0 & 9.80 & 184.4 & $4.88043 \mathrm{E}+13$ & $9.67112 \mathrm{E}+12$ & $7.83309 \mathrm{E}+13$ & $2.42265 \mathrm{E}+20$ \\
\hline
\end{tabular}


TEM-10300-1

03/01/2012

Rev. 03

Title:

ATF-1 Power Histories

\begin{tabular}{lllll} 
TEV No.: & 3119 & Rev. No.: & 0 & Project No.: \\
\hline
\end{tabular}

Table 33. ATF-73 (Pellet 4) Power History

\begin{tabular}{|c|c|c|c|c|c|c|c|c|}
\hline Cycle & Critical Dates & EFPD & $\begin{array}{c}\text { Burnup } \\
\text { (GWd/MTU) }\end{array}$ & $\begin{array}{l}\text { LHGR } \\
(\mathrm{W} / \mathrm{cm})\end{array}$ & $\begin{array}{l}\text { Thermal Flux } \\
\left(\mathrm{n} / \mathrm{cm}^{2 *} \mathrm{~s}\right)\end{array}$ & $\begin{array}{c}\text { Fast Flux } \\
\left(\mathrm{n} / \mathrm{cm}^{2 *} \mathrm{~s}>1\right. \\
\mathrm{MeV})\end{array}$ & $\begin{array}{l}\text { Total Flux } \\
\left(\mathrm{n} / \mathrm{cm}^{2 *} \mathrm{~s}\right)\end{array}$ & $\begin{array}{l}\text { Fission Density } \\
\text { (Fissions } / \mathrm{cm}^{3} \text { ) }\end{array}$ \\
\hline \multirow[t]{9}{*}{$160 \mathrm{~B}$} & $\operatorname{BOC}(12 / 20 / 2016,1200)$ & 1.0 & 0.06 & 182.5 & $3.90771 \mathrm{E}+13$ & $8.95667 \mathrm{E}+12$ & $6.66253 E+13$ & $1.50550 \mathrm{E}+18$ \\
\hline & & 3.0 & 0.21 & 183.9 & $3.93796 \mathrm{E}+13$ & $9.08838 \mathrm{E}+12$ & $6.73430 \mathrm{E}+13$ & $5.17892 \mathrm{E}+18$ \\
\hline & & 10.0 & 0.71 & 184.7 & $3.93487 \mathrm{E}+13$ & $9.11509 \mathrm{E}+12$ & $6.79109 \mathrm{E}+13$ & $1.75240 \mathrm{E}+19$ \\
\hline & & 20.0 & 1.46 & 197.8 & $4.26810 \mathrm{E}+13$ & $1.00209 \mathrm{E}+13$ & $7.27931 \mathrm{E}+13$ & $3.60116 \mathrm{E}+19$ \\
\hline & & 29.2 & 2.17 & 200.4 & $4.44971 \mathrm{E}+13$ & $9.89973 \mathrm{E}+12$ & $7.47288 \mathrm{E}+13$ & $5.36560 \mathrm{E}+19$ \\
\hline & \multicolumn{8}{|c|}{ Mid-Cycle 160B Outage - 5 Day, 14 Hour Down Time } \\
\hline & & 40.0 & 3.06 & 219.5 & $4.89076 \mathrm{E}+13$ & $1.08434 \mathrm{E}+13$ & $8.15932 \mathrm{E}+13$ & $7.57568 \mathrm{E}+19$ \\
\hline & & 50.0 & 3.87 & 213.6 & $4.79082 \mathrm{E}+13$ & $1.04249 \mathrm{E}+13$ & $7.94138 \mathrm{E}+13$ & $9.56294 \mathrm{E}+19$ \\
\hline & EOC $(2 / 23 / 2017,1400)$ & 59.5 & 4.65 & 207.7 & $4.85775 \mathrm{E}+13$ & $1.05623 E+13$ & $8.05845 E+13$ & $1.14840 \mathrm{E}+20$ \\
\hline \multirow[t]{9}{*}{$161 \mathrm{~A}$} & \multicolumn{8}{|c|}{ Cycle 161A Outage - 95 Day, 13 Hour Down Time } \\
\hline & BOC $(5 / 30 / 2017,0300)$ & 61.2 & 4.77 & 183.8 & $4.25965 \mathrm{E}+13$ & $8.97602 \mathrm{E}+12$ & $7.05098 \mathrm{E}+13$ & $1.17971 \mathrm{E}+20$ \\
\hline & & 61.5 & 4.79 & 169.3 & $3.99124 \mathrm{E}+13$ & $8.62097 E+12$ & $6.65305 E+13$ & $1.18332 \mathrm{E}+20$ \\
\hline & & 63.5 & 4.92 & 168.9 & $3.98952 \mathrm{E}+13$ & $8.47395 \mathrm{E}+12$ & $6.52882 \mathrm{E}+13$ & $1.21644 \mathrm{E}+20$ \\
\hline & & 65.5 & 5.06 & 168.6 & $3.96059 \mathrm{E}+13$ & $7.82060 \mathrm{E}+12$ & $6.48918 \mathrm{E}+13$ & $1.24957 \mathrm{E}+20$ \\
\hline & & 67.5 & 5.19 & 172.0 & $4.10905 \mathrm{E}+13$ & $8.26763 E+12$ & $6.75871 \mathrm{E}+13$ & $1.28389 \mathrm{E}+20$ \\
\hline & & 69.5 & 5.34 & 179.6 & $4.29458 \mathrm{E}+13$ & $8.32578 \mathrm{E}+12$ & $6.94748 E+13$ & $1.31942 \mathrm{E}+20$ \\
\hline & & 71.5 & 5.48 & 180.2 & $4.29643 \mathrm{E}+13$ & $8.47946 \mathrm{E}+12$ & $6.97310 \mathrm{E}+13$ & $1.35495 \mathrm{E}+20$ \\
\hline & EOC $(6 / 11 / 2017,1600)$ & 72.0 & 5.51 & 182.8 & 4.36177E+13 & $8.47941 \mathrm{E}+12$ & $7.09608 \mathrm{E}+13$ & $1.36218 \mathrm{E}+20$ \\
\hline \multirow[t]{10}{*}{$162 \mathrm{~A}$} & \multicolumn{8}{|c|}{ Cycle 162A Outage } \\
\hline & $\mathrm{BOC}$ & 73.0 & 5.57 & 174.6 & $4.25365 \mathrm{E}+13$ & $8.50024 \mathrm{E}+12$ & $6.91836 \mathrm{E}+13$ & $1.37663 \mathrm{E}+20$ \\
\hline & & 75.0 & 5.70 & 163.2 & $3.92435 \mathrm{E}+13$ & $7.89612 \mathrm{E}+12$ & $6.38064 \mathrm{E}+13$ & $1.40915 \mathrm{E}+20$ \\
\hline & & 82.0 & 6.14 & 165.0 & $3.90155 \mathrm{E}+13$ & $8.29191 \mathrm{E}+12$ & $6.49405 E+13$ & $1.51875 \mathrm{E}+20$ \\
\hline & & 92.0 & 6.78 & 168.5 & $4.00679 \mathrm{E}+13$ & $7.87381 \mathrm{E}+12$ & $6.52163 E+13$ & $1.67652 \mathrm{E}+20$ \\
\hline & & 102.0 & 7.41 & 168.2 & $4.04909 \mathrm{E}+13$ & $7.98036 \mathrm{E}+12$ & $6.64462 \mathrm{E}+13$ & $1.83249 \mathrm{E}+20$ \\
\hline & & 112.0 & 8.04 & 168.9 & $4.16896 \mathrm{E}+13$ & $8.03661 \mathrm{E}+12$ & $6.74721 \mathrm{E}+13$ & $1.98786 \mathrm{E}+20$ \\
\hline & & 122.0 & 8.70 & 175.3 & $4.40404 \mathrm{E}+13$ & $8.77708 \mathrm{E}+12$ & $7.12376 \mathrm{E}+13$ & $2.15166 \mathrm{E}+20$ \\
\hline & & 131.0 & 9.33 & 179.9 & $4.65756 \mathrm{E}+13$ & $8.81243 E+12$ & $7.48749 E+13$ & $2.30522 \mathrm{E}+20$ \\
\hline & EOC & 134.0 & 9.53 & 178.1 & $4.70704 \mathrm{E}+13$ & $8.91180 \mathrm{E}+12$ & $7.52587 E+13$ & $2.35460 \mathrm{E}+20$ \\
\hline
\end{tabular}


TEM-10300-1

03/01/2012

Rev. 03

Title:

ATF-1 Power Histories

$\begin{array}{lllll}\text { TEV No.: } & 3119 & \text { Rev. No.: } & 0 & \text { Project No.: } \\ & & & \end{array}$

Table 34. ATF-73 (Pellet 5) Power History

\begin{tabular}{|c|c|c|c|c|c|c|c|c|}
\hline Cycle & Critical Dates & EFPD & $\begin{array}{c}\text { Burnup } \\
\text { (GWd/MTU) }\end{array}$ & $\begin{array}{l}\text { LHGR } \\
(\mathrm{W} / \mathrm{cm})\end{array}$ & $\begin{array}{l}\text { Thermal Flux } \\
\left(\mathrm{n} / \mathrm{cm}^{2 *} \mathrm{~s}\right)\end{array}$ & $\begin{array}{c}\text { Fast Flux } \\
\left(\mathrm{n} / \mathrm{cm}^{2 *} \mathrm{~s}>1\right. \\
\mathrm{MeV})\end{array}$ & $\begin{array}{l}\text { Total Flux } \\
\left(\mathrm{n} / \mathrm{cm}^{2 *} \mathrm{~s}\right)\end{array}$ & $\begin{array}{c}\text { Fission Density } \\
\text { (Fissions } / \mathrm{cm}^{3} \text { ) }\end{array}$ \\
\hline \multirow[t]{9}{*}{$160 \mathrm{~B}$} & $\mathrm{BOC}(12 / 20 / 2016,1200)$ & 1.0 & 0.06 & 175.5 & $3.71657 \mathrm{E}+13$ & $8.69166 \mathrm{E}+12$ & $6.41623 E+13$ & $1.44528 \mathrm{E}+18$ \\
\hline & & 3.0 & 0.20 & 180.4 & $3.83415 \mathrm{E}+13$ & $8.54354 \mathrm{E}+12$ & $6.57295 E+13$ & $4.99826 \mathrm{E}+18$ \\
\hline & & 10.0 & 0.69 & 179.2 & $3.86253 \mathrm{E}+13$ & $9.01358 \mathrm{E}+12$ & $6.53589 \mathrm{E}+13$ & $1.71025 \mathrm{E}+19$ \\
\hline & & 20.0 & 1.42 & 191.6 & $4.13661 \mathrm{E}+13$ & $8.98094 \mathrm{E}+12$ & $6.98551 \mathrm{E}+13$ & $3.50480 \mathrm{E}+19$ \\
\hline & & 29.2 & 2.11 & 195.9 & $4.34962 E+13$ & $9.38705 E+12$ & $7.31172 \mathrm{E}+13$ & $5.20903 E+19$ \\
\hline & \multicolumn{8}{|c|}{ Mid-Cycle 160B Outage - 5 Day, 14 Hour Down Time } \\
\hline & & 40.0 & 2.96 & 206.5 & $4.59828 \mathrm{E}+13$ & $9.79890 \mathrm{E}+12$ & $7.69694 \mathrm{E}+13$ & $7.30469 \mathrm{E}+19$ \\
\hline & & 50.0 & 3.74 & 203.5 & $4.56499 E+13$ & $1.00183 E+13$ & $7.66348 \mathrm{E}+13$ & $9.24377 \mathrm{E}+19$ \\
\hline & EOC $(2 / 23 / 2017,1400)$ & 59.5 & 4.49 & 205.8 & $4.81039 \mathrm{E}+13$ & $9.91470 \mathrm{E}+12$ & $7.87229 \mathrm{E}+13$ & $1.10925 \mathrm{E}+20$ \\
\hline \multirow[t]{9}{*}{$161 \mathrm{~A}$} & \multicolumn{8}{|c|}{ Cycle 161A Outage - 95 Day, 13 Hour Down Time } \\
\hline & BOC $(5 / 30 / 2017,0300)$ & 61.2 & 4.61 & 177.4 & $4.16266 \mathrm{E}+13$ & $8.85740 \mathrm{E}+12$ & $6.85757 \mathrm{E}+13$ & 1.13996E+20 \\
\hline & & 61.5 & 4.63 & 169.4 & $3.96712 \mathrm{E}+13$ & $8.31857 \mathrm{E}+12$ & $6.49148 \mathrm{E}+13$ & $1.14358 \mathrm{E}+20$ \\
\hline & & 63.5 & 4.76 & 165.5 & $3.85875 \mathrm{E}+13$ & $8.76754 \mathrm{E}+12$ & $6.49504 \mathrm{E}+13$ & $1.17670 \mathrm{E}+20$ \\
\hline & & 65.5 & 4.89 & 163.8 & $3.89569 \mathrm{E}+13$ & $8.45855 E+12$ & $6.48356 \mathrm{E}+13$ & $1.20922 \mathrm{E}+20$ \\
\hline & & 67.5 & 5.02 & 169.0 & $3.95757 \mathrm{E}+13$ & $8.53842 \mathrm{E}+12$ & $6.59215 \mathrm{E}+13$ & $1.24174 \mathrm{E}+20$ \\
\hline & & 69.5 & 5.16 & 170.7 & $4.03794 \mathrm{E}+13$ & $8.79655 \mathrm{E}+12$ & $6.67754 \mathrm{E}+13$ & $1.27546 \mathrm{E}+20$ \\
\hline & & 71.5 & 5.30 & 174.4 & $4.13125 \mathrm{E}+13$ & $8.74781 \mathrm{E}+12$ & $6.80544 \mathrm{E}+13$ & $1.30918 \mathrm{E}+20$ \\
\hline & EOC (6/11/2017, 1600) & 72.0 & 5.33 & 173.9 & $4.08576 \mathrm{E}+13$ & $8.72284 \mathrm{E}+12$ & $6.73707 \mathrm{E}+13$ & $1.31641 \mathrm{E}+20$ \\
\hline \multirow[t]{10}{*}{$162 \mathrm{~A}$} & \multicolumn{8}{|c|}{ Cycle 162A Outage } \\
\hline & $\mathrm{BOC}$ & 73.0 & 5.38 & 172.3 & $4.10220 \mathrm{E}+13$ & $8.23784 \mathrm{E}+12$ & $6.65826 \mathrm{E}+13$ & $1.33086 \mathrm{E}+20$ \\
\hline & & 75.0 & 5.51 & 157.1 & $3.71049 \mathrm{E}+13$ & $7.69886 \mathrm{E}+12$ & $6.17978 \mathrm{E}+13$ & $1.36157 \mathrm{E}+20$ \\
\hline & & 82.0 & 5.95 & 163.5 & $3.83853 E+13$ & $7.62235 \mathrm{E}+12$ & $6.26537 \mathrm{E}+13$ & $1.47178 \mathrm{E}+20$ \\
\hline & & 92.0 & 6.59 & 166.5 & $3.93999 \mathrm{E}+13$ & $7.88895 \mathrm{E}+12$ & $6.44258 \mathrm{E}+13$ & $1.62895 \mathrm{E}+20$ \\
\hline & & 102.0 & 7.21 & 164.9 & $3.97095 E+13$ & $7.86328 \mathrm{E}+12$ & $6.51365 E+13$ & $1.78311 \mathrm{E}+20$ \\
\hline & & 112.0 & 7.83 & 163.9 & $3.97952 \mathrm{E}+13$ & $7.98142 \mathrm{E}+12$ & $6.49714 \mathrm{E}+13$ & $1.93607 \mathrm{E}+20$ \\
\hline & & 122.0 & 8.49 & 173.5 & $4.32415 E+13$ & 8.30333E+12 & $6.95711 \mathrm{E}+13$ & $2.09806 \mathrm{E}+20$ \\
\hline & & 131.0 & 9.11 & 180.2 & $4.63060 \mathrm{E}+13$ & $8.77658 \mathrm{E}+12$ & $7.39874 \mathrm{E}+13$ & $2.25163 \mathrm{E}+20$ \\
\hline & EOC & 134.0 & 9.31 & 177.8 & $4.59649 \mathrm{E}+13$ & $8.70608 E+12$ & $7.35088 E+13$ & $2.30040 \mathrm{E}+20$ \\
\hline
\end{tabular}


TEM-10300-1

03/01/2012

Rev. 03

Title:

ATF-1 Power Histories

$\begin{array}{lllll}\text { TEV No.: } & 3119 & \text { Rev. No.: } & 0 & \text { Project No.: } \\ & & & \end{array}$

Table 35. ATF-73 (Pellet 6) Power History

\begin{tabular}{|c|c|c|c|c|c|c|c|c|}
\hline Cycle & Critical Dates & EFPD & $\begin{array}{c}\text { Burnup } \\
\text { (GWd/MTU) }\end{array}$ & $\begin{array}{l}\text { LHGR } \\
(\mathrm{W} / \mathrm{cm})\end{array}$ & $\begin{array}{l}\text { Thermal Flux } \\
\left(\mathrm{n} / \mathrm{cm}^{2 *} \mathrm{~s}\right)\end{array}$ & $\begin{array}{c}\text { Fast Flux } \\
\left(\mathrm{n} / \mathrm{cm}^{2 *} \mathrm{~s}>1\right. \\
\mathrm{MeV})\end{array}$ & $\begin{array}{l}\text { Total Flux } \\
\left(\mathrm{n} / \mathrm{cm}^{2 *} \mathrm{~s}\right)\end{array}$ & $\begin{array}{l}\text { Fission Density } \\
\text { (Fissions } / \mathrm{cm}^{3} \text { ) }\end{array}$ \\
\hline \multirow[t]{9}{*}{$160 \mathrm{~B}$} & $\operatorname{BOC}(12 / 20 / 2016,1200)$ & 1.0 & 0.06 & 170.7 & $3.60641 \mathrm{E}+13$ & $8.31340 \mathrm{E}+12$ & $6.17983 E+13$ & $1.44528 \mathrm{E}+18$ \\
\hline & & 3.0 & 0.20 & 178.1 & $3.80073 E+13$ & $8.53725 E+12$ & $6.41139 \mathrm{E}+13$ & $4.99826 \mathrm{E}+18$ \\
\hline & & 10.0 & 0.68 & 174.4 & $3.70041 \mathrm{E}+13$ & $8.26048 \mathrm{E}+12$ & $6.33176 \mathrm{E}+13$ & $1.67412 \mathrm{E}+19$ \\
\hline & & 20.0 & 1.38 & 183.9 & $3.98401 \mathrm{E}+13$ & $8.84029 \mathrm{E}+12$ & $6.76651 \mathrm{E}+13$ & $3.40845 \mathrm{E}+19$ \\
\hline & & 29.2 & 2.06 & 187.9 & $4.19407 \mathrm{E}+13$ & $9.14918 \mathrm{E}+12$ & $7.02956 \mathrm{E}+13$ & $5.08257 \mathrm{E}+19$ \\
\hline & \multicolumn{8}{|c|}{ Mid-Cycle 160B Outage - 5 Day, 14 Hour Down Time } \\
\hline & & 40.0 & 2.89 & 202.7 & $4.50431 \mathrm{E}+13$ & $9.97411 \mathrm{E}+12$ & $7.58262 \mathrm{E}+13$ & $7.15414 \mathrm{E}+19$ \\
\hline & & 50.0 & 3.65 & 200.6 & $4.49584 \mathrm{E}+13$ & $9.68358 \mathrm{E}+12$ & $7.52397 \mathrm{E}+13$ & $9.01493 \mathrm{E}+19$ \\
\hline & EOC $(2 / 23 / 2017,1400)$ & 59.5 & 4.38 & 200.7 & $4.64784 \mathrm{E}+13$ & $9.88944 \mathrm{E}+12$ & $7.65126 \mathrm{E}+13$ & $1.08215 \mathrm{E}+20$ \\
\hline \multirow[t]{9}{*}{$161 \mathrm{~A}$} & \multicolumn{8}{|c|}{ Cycle 161A Outage - 95 Day, 13 Hour Down Time } \\
\hline & BOC $(5 / 30 / 2017,0300)$ & 61.2 & 4.50 & 174.3 & $4.02972 \mathrm{E}+13$ & $8.28147 \mathrm{E}+12$ & $6.69210 \mathrm{E}+13$ & $1.11287 \mathrm{E}+20$ \\
\hline & & 61.5 & 4.52 & 164.8 & $3.81683 \mathrm{E}+13$ & $7.51114 \mathrm{E}+12$ & $6.25008 \mathrm{E}+13$ & $1.11708 \mathrm{E}+20$ \\
\hline & & 63.5 & 4.65 & 163.5 & $3.86078 \mathrm{E}+13$ & $8.04871 \mathrm{E}+12$ & $6.37423 E+13$ & $1.14900 \mathrm{E}+20$ \\
\hline & & 65.5 & 4.78 & 164.3 & $3.83079 \mathrm{E}+13$ & $7.75846 \mathrm{E}+12$ & $6.31040 \mathrm{E}+13$ & $1.18152 \mathrm{E}+20$ \\
\hline & & 67.5 & 4.91 & 165.3 & $3.82983 E+13$ & $7.64072 \mathrm{E}+12$ & $6.28134 \mathrm{E}+13$ & $1.21404 \mathrm{E}+20$ \\
\hline & & 69.5 & 5.05 & 168.8 & $3.94828 \mathrm{E}+13$ & $7.79206 \mathrm{E}+12$ & $6.50019 \mathrm{E}+13$ & $1.24776 \mathrm{E}+20$ \\
\hline & & 71.5 & 5.18 & 169.1 & $3.97261 \mathrm{E}+13$ & $8.03394 \mathrm{E}+12$ & $6.53270 \mathrm{E}+13$ & $1.28088 \mathrm{E}+20$ \\
\hline & EOC $(6 / 11 / 2017,1600)$ & 72.0 & 5.21 & 173.0 & $4.07314 \mathrm{E}+13$ & 7.92777E+12 & $6.62170 \mathrm{E}+13$ & $1.28811 \mathrm{E}+20$ \\
\hline \multirow[t]{10}{*}{$162 \mathrm{~A}$} & \multicolumn{8}{|c|}{ Cycle 162A Outage } \\
\hline & $\mathrm{BOC}$ & 73.0 & 5.26 & 164.6 & $3.95689 \mathrm{E}+13$ & $8.27085 E+12$ & $6.49270 E+13$ & $1.30135 \mathrm{E}+20$ \\
\hline & & 75.0 & 5.39 & 151.5 & $3.60424 \mathrm{E}+13$ & $7.31176 \mathrm{E}+12$ & $5.97711 \mathrm{E}+13$ & $1.33207 \mathrm{E}+20$ \\
\hline & & 82.0 & 5.78 & 151.7 & $3.56958 \mathrm{E}+13$ & $7.76926 \mathrm{E}+12$ & $5.93080 \mathrm{E}+13$ & $1.42902 \mathrm{E}+20$ \\
\hline & & 92.0 & 6.38 & 158.9 & $3.77261 \mathrm{E}+13$ & 7.98997E+12 & $6.29812 \mathrm{E}+13$ & $1.57656 \mathrm{E}+20$ \\
\hline & & 102.0 & 6.97 & 157.9 & $3.81454 \mathrm{E}+13$ & $7.97533 E+12$ & $6.32056 \mathrm{E}+13$ & $1.72289 \mathrm{E}+20$ \\
\hline & & 112.0 & 7.56 & 158.9 & $3.85325 \mathrm{E}+13$ & $7.50101 \mathrm{E}+12$ & $6.28361 \mathrm{E}+13$ & $1.86983 \mathrm{E}+20$ \\
\hline & & 122.0 & 8.19 & 166.2 & $4.17301 \mathrm{E}+13$ & $8.22081 \mathrm{E}+12$ & $6.76108 \mathrm{E}+13$ & $2.02399 \mathrm{E}+20$ \\
\hline & & 131.0 & 8.78 & 171.2 & $4.38698 \mathrm{E}+13$ & 8.99336E+12 & $7.16937 E+13$ & $2.17093 E+20$ \\
\hline & EOC & 134.0 & 8.97 & 167.4 & $4.34073 \mathrm{E}+13$ & $8.50438 \mathrm{E}+12$ & $7.03519 \mathrm{E}+13$ & $2.21730 \mathrm{E}+20$ \\
\hline
\end{tabular}


TEM-10300-1

03/01/2012

Rev. 03

Title:

ATF-1 Power Histories

$\begin{array}{lllll}\text { TEV No.: } & 3119 & \text { Rev. No.: } & 0 & \text { Project No.: } \\ & & & \end{array}$

Table 36. ATF-73 (Pellet 7) Power History

\begin{tabular}{|c|c|c|c|c|c|c|c|c|}
\hline Cycle & Critical Dates & EFPD & $\begin{array}{c}\text { Burnup } \\
\text { (GWd/MTU) }\end{array}$ & $\begin{array}{l}\text { LHGR } \\
(\mathrm{W} / \mathrm{cm})\end{array}$ & $\begin{array}{l}\text { Thermal Flux } \\
\left(\mathrm{n} / \mathrm{cm}^{2 *} \mathrm{~s}\right)\end{array}$ & $\begin{array}{c}\text { Fast Flux } \\
\left(\mathrm{n} / \mathrm{cm}^{2 *} \mathrm{~s}>1\right. \\
\mathrm{MeV})\end{array}$ & $\begin{array}{l}\text { Total Flux } \\
\left(\mathrm{n} / \mathrm{cm}^{2 *} \mathrm{~s}\right)\end{array}$ & $\begin{array}{l}\text { Fission Density } \\
\text { (Fissions/cm }{ }^{3} \text { ) }\end{array}$ \\
\hline \multirow[t]{9}{*}{$160 \mathrm{~B}$} & $\operatorname{BOC}(12 / 20 / 2016,1200)$ & 1.0 & 0.06 & 165.3 & $3.49976 \mathrm{E}+13$ & $7.99230 \mathrm{E}+12$ & $6.02728 \mathrm{E}+13$ & $1.38506 \mathrm{E}+18$ \\
\hline & & 3.0 & 0.19 & 168.9 & $3.59759 \mathrm{E}+13$ & $8.02473 E+12$ & $6.21753 \mathrm{E}+13$ & $4.75738 \mathrm{E}+18$ \\
\hline & & 10.0 & 0.63 & 168.3 & $3.62302 \mathrm{E}+13$ & 8.47697E+12 & $6.16980 \mathrm{E}+13$ & $1.56572 \mathrm{E}+19$ \\
\hline & & 20.0 & 1.32 & 180.8 & $3.89534 \mathrm{E}+13$ & $8.67490 \mathrm{E}+12$ & $6.65128 \mathrm{E}+13$ & $3.26995 \mathrm{E}+19$ \\
\hline & & 29.2 & 1.99 & 186.1 & $4.12117 \mathrm{E}+13$ & $9.11280 \mathrm{E}+12$ & $6.90840 \mathrm{E}+13$ & $4.91997 \mathrm{E}+19$ \\
\hline & \multicolumn{8}{|c|}{ Mid-Cycle 160B Outage - 5 Day, 14 Hour Down Time } \\
\hline & & 40.0 & 2.82 & 201.3 & $4.45780 \mathrm{E}+13$ & $9.73947 \mathrm{E}+12$ & $7.49290 \mathrm{E}+13$ & $6.96745 \mathrm{E}+19$ \\
\hline & & 50.0 & 3.56 & 196.0 & $4.38852 \mathrm{E}+13$ & $9.49081 \mathrm{E}+12$ & $7.35592 \mathrm{E}+13$ & $8.79212 \mathrm{E}+19$ \\
\hline & EOC $(2 / 23 / 2017,1400)$ & 59.5 & 4.27 & 194.8 & $4.52494 \mathrm{E}+13$ & $9.44544 \mathrm{E}+12$ & $7.48638 \mathrm{E}+13$ & $1.05445 \mathrm{E}+20$ \\
\hline \multirow[t]{9}{*}{$161 \mathrm{~A}$} & \multicolumn{8}{|c|}{ Cycle 161A Outage - 95 Day, 13 Hour Down Time } \\
\hline & BOC $(5 / 30 / 2017,0300)$ & 61.2 & 4.38 & 171.3 & $3.96918 \mathrm{E}+13$ & $8.68792 \mathrm{E}+12$ & $6.65635 \mathrm{E}+13$ & $1.08396 \mathrm{E}+20$ \\
\hline & & 61.5 & 4.40 & 156.7 & $3.59853 \mathrm{E}+13$ & $7.75139 \mathrm{E}+12$ & $5.96916 \mathrm{E}+13$ & $1.08818 \mathrm{E}+20$ \\
\hline & & 63.5 & 4.53 & 155.3 & $3.65169 \mathrm{E}+13$ & $7.71651 \mathrm{E}+12$ & $6.04693 E+13$ & 1.11949E+20 \\
\hline & & 65.5 & 4.66 & 158.7 & $3.69971 \mathrm{E}+13$ & $7.78916 \mathrm{E}+12$ & $6.12399 \mathrm{E}+13$ & $1.15080 \mathrm{E}+20$ \\
\hline & & 67.5 & 4.79 & 161.8 & $3.73771 \mathrm{E}+13$ & $8.10839 E+12$ & $6.28896 \mathrm{E}+13$ & $1.18332 \mathrm{E}+20$ \\
\hline & & 69.5 & 4.92 & 160.7 & $3.73689 \mathrm{E}+13$ & $7.83900 \mathrm{E}+12$ & $6.22964 \mathrm{E}+13$ & $1.21524 \mathrm{E}+20$ \\
\hline & & 71.5 & 5.05 & 162.8 & $3.78036 \mathrm{E}+13$ & $7.79844 \mathrm{E}+12$ & $6.28495 E+13$ & $1.24716 \mathrm{E}+20$ \\
\hline & EOC $(6 / 11 / 2017,1600)$ & 72.0 & 5.07 & 165.8 & $3.93402 \mathrm{E}+13$ & 7.78727E+12 & $6.43340 \mathrm{E}+13$ & $1.25438 \mathrm{E}+20$ \\
\hline \multirow[t]{10}{*}{$162 \mathrm{~A}$} & \multicolumn{8}{|c|}{ Cycle 162A Outage } \\
\hline & $\mathrm{BOC}$ & 73.0 & 5.13 & 158.9 & $3.79459 \mathrm{E}+13$ & $7.59260 \mathrm{E}+12$ & $6.25389 \mathrm{E}+13$ & $1.26703 \mathrm{E}+20$ \\
\hline & & 75.0 & 5.25 & 150.6 & $3.53663 \mathrm{E}+13$ & $7.16950 \mathrm{E}+12$ & $5.82929 \mathrm{E}+13$ & $1.29774 \mathrm{E}+20$ \\
\hline & & 82.0 & 5.63 & 148.8 & $3.49400 \mathrm{E}+13$ & $7.27118 \mathrm{E}+12$ & $5.78044 \mathrm{E}+13$ & $1.39229 \mathrm{E}+20$ \\
\hline & & 92.0 & 6.20 & 153.0 & $3.57991 \mathrm{E}+13$ & $7.52546 \mathrm{E}+12$ & $5.93689 E+13$ & $1.53260 \mathrm{E}+20$ \\
\hline & & 102.0 & 6.79 & 155.5 & $3.70280 \mathrm{E}+13$ & $7.72559 \mathrm{E}+12$ & $6.14953 E+13$ & $1.67954 \mathrm{E}+20$ \\
\hline & & 112.0 & 7.38 & 152.9 & $3.66668 \mathrm{E}+13$ & $7.57315 \mathrm{E}+12$ & $6.02751 \mathrm{E}+13$ & $1.82406 \mathrm{E}+20$ \\
\hline & & 122.0 & 8.00 & 163.5 & $4.02760 \mathrm{E}+13$ & $8.14118 \mathrm{E}+12$ & $6.61680 \mathrm{E}+13$ & $1.97762 \mathrm{E}+20$ \\
\hline & & 131.0 & 8.60 & 170.0 & 4.27619E+13 & $8.33918 E+12$ & $6.96729 E+13$ & $2.12577 E+20$ \\
\hline & EOC & 134.0 & 8.78 & 165.5 & $4.23599 \mathrm{E}+13$ & $8.16734 \mathrm{E}+12$ & $6.89477 \mathrm{E}+13$ & $2.17153 \mathrm{E}+20$ \\
\hline
\end{tabular}


TEM-10300-1

03/01/2012

Rev. 03

Title:

ATF-1 Power Histories

$\begin{array}{lllll}\text { TEV No.: } & 3119 & \text { Rev. No.: } & 0 & \text { Project No.: } 31418\end{array}$

Table 37. ATF-73 (Pellet 8) Power History

\begin{tabular}{|c|c|c|c|c|c|c|c|c|}
\hline Cycle & Critical Dates & EFPD & $\begin{array}{c}\text { Burnup } \\
\text { (GWd/MTU) }\end{array}$ & $\begin{array}{l}\text { LHGR } \\
(\mathrm{W} / \mathrm{cm})\end{array}$ & $\begin{array}{l}\text { Thermal Flux } \\
\left(\mathrm{n} / \mathrm{cm}^{2 *} \mathrm{~s}\right)\end{array}$ & $\begin{array}{c}\text { Fast Flux } \\
\left(\mathrm{n} / \mathrm{cm}^{2 *} \mathrm{~s}>1\right. \\
\mathrm{MeV})\end{array}$ & $\begin{array}{l}\text { Total Flux } \\
\left(\mathrm{n} / \mathrm{cm}^{2 *} \mathrm{~s}\right)\end{array}$ & $\begin{array}{l}\text { Fission Density } \\
\text { (Fissions/cm }{ }^{3} \text { ) }\end{array}$ \\
\hline \multirow[t]{9}{*}{$160 \mathrm{~B}$} & $\operatorname{BOC}(12 / 20 / 2016,1200)$ & 1.0 & 0.05 & 157.6 & $3.33464 \mathrm{E}+13$ & $7.86917 \mathrm{E}+12$ & $5.87539 \mathrm{E}+13$ & $1.26462 \mathrm{E}+18$ \\
\hline & & 3.0 & 0.18 & 160.4 & $3.42018 \mathrm{E}+13$ & $8.00047 E+12$ & $5.92317 \mathrm{E}+13$ & $4.51650 \mathrm{E}+18$ \\
\hline & & 10.0 & 0.61 & 161.3 & $3.40831 \mathrm{E}+13$ & $7.90915 \mathrm{E}+12$ & $5.91620 \mathrm{E}+13$ & $1.49948 \mathrm{E}+19$ \\
\hline & & 20.0 & 1.29 & 176.2 & $3.76457 \mathrm{E}+13$ & $8.24302 \mathrm{E}+12$ & $6.40915 \mathrm{E}+13$ & $3.18564 \mathrm{E}+19$ \\
\hline & & 29.2 & 1.90 & 176.1 & $3.88505 E+13$ & $9.06304 \mathrm{E}+12$ & $6.64172 \mathrm{E}+13$ & $4.70318 \mathrm{E}+19$ \\
\hline & \multicolumn{8}{|c|}{ Mid-Cycle 160B Outage - 5 Day, 14 Hour Down Time } \\
\hline & & 40.0 & 2.67 & 190.2 & $4.20660 \mathrm{E}+13$ & $9.56190 \mathrm{E}+12$ & $7.16278 \mathrm{E}+13$ & $6.60011 \mathrm{E}+19$ \\
\hline & & 50.0 & 3.39 & 189.8 & $4.23997 \mathrm{E}+13$ & $9.69125 \mathrm{E}+12$ & 7.20537E+13 & $8.37058 \mathrm{E}+19$ \\
\hline & EOC $(2 / 23 / 2017,1400)$ & 59.5 & 4.07 & 184.9 & $4.27810 \mathrm{E}+13$ & $9.20257 E+12$ & $7.14556 \mathrm{E}+13$ & $1.00507 \mathrm{E}+20$ \\
\hline \multirow[t]{9}{*}{$161 \mathrm{~A}$} & \multicolumn{8}{|c|}{ Cycle 161A Outage - 95 Day, 13 Hour Down Time } \\
\hline & BOC $(5 / 30 / 2017,0300)$ & 61.2 & 4.18 & 160.7 & $3.69785 E+13$ & $7.97843 \mathrm{E}+12$ & $6.18943 \mathrm{E}+13$ & $1.03277 \mathrm{E}+20$ \\
\hline & & 61.5 & 4.19 & 153.3 & $3.54441 \mathrm{E}+13$ & $7.31359 \mathrm{E}+12$ & $5.87384 \mathrm{E}+13$ & $1.03639 \mathrm{E}+20$ \\
\hline & & 63.5 & 4.32 & 151.2 & $3.50137 \mathrm{E}+13$ & $7.89364 \mathrm{E}+12$ & $5.93769 \mathrm{E}+13$ & $1.06710 \mathrm{E}+20$ \\
\hline & & 65.5 & 4.44 & 150.9 & $3.49066 \mathrm{E}+13$ & $7.63982 E+12$ & $5.92513 \mathrm{E}+13$ & $1.09721 \mathrm{E}+20$ \\
\hline & & 67.5 & 4.57 & 156.6 & $3.61724 \mathrm{E}+13$ & $7.53279 \mathrm{E}+12$ & $6.03589 \mathrm{E}+13$ & $1.12913 \mathrm{E}+20$ \\
\hline & & 69.5 & 4.70 & 159.9 & $3.73886 \mathrm{E}+13$ & $8.01839 E+12$ & $6.19934 \mathrm{E}+13$ & $1.16104 \mathrm{E}+20$ \\
\hline & & 71.5 & 4.83 & 161.7 & $3.75813 \mathrm{E}+13$ & $8.31236 \mathrm{E}+12$ & $6.26427 \mathrm{E}+13$ & 1.19296E+20 \\
\hline & EOC $(6 / 11 / 2017,1600)$ & 72.0 & 4.85 & 158.3 & $3.73165 \mathrm{E}+13$ & $7.91854 \mathrm{E}+12$ & $6.23858 \mathrm{E}+13$ & $1.19898 \mathrm{E}+20$ \\
\hline \multirow[t]{10}{*}{$162 \mathrm{~A}$} & \multicolumn{8}{|c|}{ Cycle 162A Outage } \\
\hline & $\mathrm{BOC}$ & 73.0 & 4.90 & 151.2 & $3.60106 \mathrm{E}+13$ & $7.97419 \mathrm{E}+12$ & $6.03763 \mathrm{E}+13$ & $1.21163 \mathrm{E}+20$ \\
\hline & & 75.0 & 5.02 & 143.6 & $3.34683 \mathrm{E}+13$ & $7.46781 \mathrm{E}+12$ & $5.69563 \mathrm{E}+13$ & $1.24113 \mathrm{E}+20$ \\
\hline & & 82.0 & 5.40 & 145.1 & $3.37369 \mathrm{E}+13$ & $7.17429 E+12$ & $5.63020 \mathrm{E}+13$ & $1.33448 \mathrm{E}+20$ \\
\hline & & 92.0 & 5.97 & 147.7 & $3.47854 \mathrm{E}+13$ & $7.52974 \mathrm{E}+12$ & $5.79060 \mathrm{E}+13$ & $1.47479 \mathrm{E}+20$ \\
\hline & & 102.0 & 6.53 & 149.3 & $3.52419 \mathrm{E}+13$ & $7.64076 \mathrm{E}+12$ & 5.92357E+13 & $1.61510 \mathrm{E}+20$ \\
\hline & & 112.0 & 7.10 & 149.7 & $3.60905 \mathrm{E}+13$ & $7.40951 \mathrm{E}+12$ & $5.95224 \mathrm{E}+13$ & $1.75602 \mathrm{E}+20$ \\
\hline & & 122.0 & 7.71 & 157.8 & $3.85456 \mathrm{E}+13$ & $8.06533 E+12$ & $6.41841 \mathrm{E}+13$ & $1.90536 \mathrm{E}+20$ \\
\hline & & 131.0 & 8.28 & 163.6 & $4.11180 \mathrm{E}+13$ & $8.50683 E+12$ & $6.78714 \mathrm{E}+13$ & $2.04688 E+20$ \\
\hline & EOC & 134.0 & 8.46 & 161.4 & $4.09957 \mathrm{E}+13$ & $8.30478 \mathrm{E}+12$ & $6.73519 \mathrm{E}+13$ & $2.09144 \mathrm{E}+20$ \\
\hline
\end{tabular}


TEM-10300-1

03/01/2012

Rev. 03

Title:

ATF-1 Power Histories

$\begin{array}{lllll}\text { TEV No.: } & 3119 & \text { Rev. No.: } & 0 & \text { Project No.: } \\ & & & \end{array}$

Table 38. ATF-73 (Pellet 9) Power History

\begin{tabular}{|c|c|c|c|c|c|c|c|c|}
\hline Cycle & Critical Dates & EFPD & $\begin{array}{c}\text { Burnup } \\
\text { (GWd/MTU) }\end{array}$ & $\begin{array}{l}\text { LHGR } \\
(\mathrm{W} / \mathrm{cm})\end{array}$ & $\begin{array}{l}\text { Thermal Flux } \\
\left(\mathrm{n} / \mathrm{cm}^{2 *} \mathrm{~s}\right)\end{array}$ & $\begin{array}{c}\text { Fast Flux } \\
\left(\mathrm{n} / \mathrm{cm}^{2 *} \mathrm{~s}>1\right. \\
\mathrm{MeV})\end{array}$ & $\begin{array}{l}\text { Total Flux } \\
\left(\mathrm{n} / \mathrm{cm}^{2 *} \mathrm{~s}\right)\end{array}$ & $\begin{array}{l}\text { Fission Density } \\
\text { (Fissions } / \mathrm{cm}^{3} \text { ) }\end{array}$ \\
\hline \multirow[t]{9}{*}{$160 \mathrm{~B}$} & $\operatorname{BOC}(12 / 20 / 2016,1200)$ & 1.0 & 0.05 & 152.6 & $3.22636 \mathrm{E}+13$ & $7.55112 \mathrm{E}+12$ & $5.62633 \mathrm{E}+13$ & $1.26462 \mathrm{E}+18$ \\
\hline & & 3.0 & 0.18 & 154.5 & $3.27893 E+13$ & $7.40771 \mathrm{E}+12$ & $5.63572 \mathrm{E}+13$ & $4.33584 \mathrm{E}+18$ \\
\hline & & 10.0 & 0.58 & 155.3 & $3.29821 \mathrm{E}+13$ & $7.75739 \mathrm{E}+12$ & $5.76902 \mathrm{E}+13$ & $1.44528 \mathrm{E}+19$ \\
\hline & & 20.0 & 1.24 & 169.5 & $3.62837 \mathrm{E}+13$ & $8.49001 \mathrm{E}+12$ & $6.24351 \mathrm{E}+13$ & $3.05918 \mathrm{E}+19$ \\
\hline & & 29.2 & 1.84 & 171.5 & $3.78841 \mathrm{E}+13$ & $8.77231 \mathrm{E}+12$ & $6.48578 \mathrm{E}+13$ & $4.54059 \mathrm{E}+19$ \\
\hline & \multicolumn{8}{|c|}{ Mid-Cycle 160B Outage - 5 Day, 14 Hour Down Time } \\
\hline & & 40.0 & 2.61 & 185.5 & $4.08470 \mathrm{E}+13$ & $9.08727 \mathrm{E}+12$ & $6.94100 \mathrm{E}+13$ & $6.44956 \mathrm{E}+19$ \\
\hline & & 50.0 & 3.30 & 181.1 & $4.04095 \mathrm{E}+13$ & $8.85428 \mathrm{E}+12$ & $6.81613 \mathrm{E}+13$ & $8.15379 \mathrm{E}+19$ \\
\hline & EOC $(2 / 23 / 2017,1400)$ & 59.5 & 3.97 & 184.9 & $4.21613 \mathrm{E}+13$ & $8.67684 \mathrm{E}+12$ & $6.99197 \mathrm{E}+13$ & $9.82188 \mathrm{E}+19$ \\
\hline \multirow[t]{9}{*}{$161 \mathrm{~A}$} & \multicolumn{8}{|c|}{ Cycle 161A Outage - 95 Day, 13 Hour Down Time } \\
\hline & BOC $(5 / 30 / 2017,0300)$ & 61.2 & 4.09 & 156.7 & $3.65717 \mathrm{E}+13$ & $7.81602 \mathrm{E}+12$ & $6.09888 \mathrm{E}+13$ & $1.00989 \mathrm{E}+20$ \\
\hline & & 61.5 & 4.10 & 151.0 & $3.47754 \mathrm{E}+13$ & $7.32562 \mathrm{E}+12$ & $5.73900 \mathrm{E}+13$ & $1.01350 \mathrm{E}+20$ \\
\hline & & 63.5 & 4.22 & 151.1 & $3.47395 \mathrm{E}+13$ & $7.30525 E+12$ & $5.76203 E+13$ & $1.04421 \mathrm{E}+20$ \\
\hline & & 65.5 & 4.35 & 151.3 & $3.50965 \mathrm{E}+13$ & $7.12090 \mathrm{E}+12$ & $5.73859 \mathrm{E}+13$ & $1.07493 \mathrm{E}+20$ \\
\hline & & 67.5 & 4.48 & 154.9 & $3.54597 \mathrm{E}+13$ & $7.47167 \mathrm{E}+12$ & $5.87988 \mathrm{E}+13$ & $1.10624 \mathrm{E}+20$ \\
\hline & & 69.5 & 4.60 & 154.2 & $3.61078 \mathrm{E}+13$ & $7.66630 \mathrm{E}+12$ & $5.93788 \mathrm{E}+13$ & $1.13756 \mathrm{E}+20$ \\
\hline & & 71.5 & 4.73 & 157.7 & $3.67904 \mathrm{E}+13$ & $7.86477 \mathrm{E}+12$ & $6.06484 \mathrm{E}+13$ & 1.16887E+20 \\
\hline & EOC $(6 / 11 / 2017,1600)$ & 72.0 & 4.76 & 160.7 & $3.75634 \mathrm{E}+13$ & $7.82040 \mathrm{E}+12$ & $6.22017 \mathrm{E}+13$ & $1.17610 \mathrm{E}+20$ \\
\hline \multirow[t]{10}{*}{$162 \mathrm{~A}$} & \multicolumn{8}{|c|}{ Cycle 162A Outage } \\
\hline & $\mathrm{BOC}$ & 73.0 & 4.81 & 149.9 & $3.54019 \mathrm{E}+13$ & $7.63634 \mathrm{E}+12$ & $5.84692 \mathrm{E}+13$ & $1.18814 \mathrm{E}+20$ \\
\hline & & 75.0 & 4.92 & 140.0 & $3.27916 \mathrm{E}+13$ & $7.01609 \mathrm{E}+12$ & $5.45859 \mathrm{E}+13$ & $1.21705 \mathrm{E}+20$ \\
\hline & & 82.0 & 5.29 & 140.2 & $3.30128 \mathrm{E}+13$ & $7.14378 E+12$ & $5.48406 \mathrm{E}+13$ & $1.30738 \mathrm{E}+20$ \\
\hline & & 92.0 & 5.83 & 143.6 & $3.36413 E+13$ & $7.21182 \mathrm{E}+12$ & $5.58923 E+13$ & $1.44106 \mathrm{E}+20$ \\
\hline & & 102.0 & 6.37 & 145.0 & $3.41913 \mathrm{E}+13$ & $7.31562 \mathrm{E}+12$ & $5.67326 \mathrm{E}+13$ & $1.57415 \mathrm{E}+20$ \\
\hline & & 112.0 & 6.90 & 144.2 & $3.44303 E+13$ & $7.34178 \mathrm{E}+12$ & $5.72555 \mathrm{E}+13$ & $1.70603 \mathrm{E}+20$ \\
\hline & & 122.0 & 7.48 & 150.8 & $3.71354 \mathrm{E}+13$ & $7.85964 \mathrm{E}+12$ & $6.13717 \mathrm{E}+13$ & $1.84936 \mathrm{E}+20$ \\
\hline & & 131.0 & 8.05 & 161.4 & $4.01110 \mathrm{E}+13$ & $8.18836 \mathrm{E}+12$ & $6.54690 \mathrm{E}+13$ & 1.98907E+20 \\
\hline & EOC & 134.0 & 8.22 & 159.6 & $4.00281 \mathrm{E}+13$ & $8.08210 \mathrm{E}+12$ & $6.52690 \mathrm{E}+13$ & $2.03303 \mathrm{E}+20$ \\
\hline
\end{tabular}


TEM-10300-1

03/01/2012

Rev. 03

Title:

ATF-1 Power Histories

\begin{tabular}{lllll} 
TEV No.: & 3119 & Rev. No.: & 0 & Project No.: \\
\hline
\end{tabular}

Table 39. ATF-73 (Pellet 10) Power History

\begin{tabular}{|c|c|c|c|c|c|c|c|c|}
\hline Cycle & Critical Dates & EFPD & $\begin{array}{c}\text { Burnup } \\
\text { (GWd/MTU) }\end{array}$ & $\begin{array}{l}\text { LHGR } \\
(\mathrm{W} / \mathrm{cm})\end{array}$ & $\begin{array}{l}\text { Thermal Flux } \\
\left(\mathrm{n} / \mathrm{cm}^{2 *} \mathrm{~s}\right)\end{array}$ & $\begin{array}{c}\text { Fast Flux } \\
\left(\mathrm{n} / \mathrm{cm}^{2 *} \mathrm{~s}>1\right. \\
\mathrm{MeV})\end{array}$ & $\begin{array}{l}\text { Total Flux } \\
\left(\mathrm{n} / \mathrm{cm}^{2 *} \mathrm{~s}\right)\end{array}$ & $\begin{array}{l}\text { Fission Density } \\
\left(\text { Fissions } / \mathrm{cm}^{3}\right)\end{array}$ \\
\hline \multirow[t]{9}{*}{$160 \mathrm{~B}$} & BOC $(12 / 20 / 2016,1200)$ & 1.0 & 0.05 & 146.8 & $3.10870 \mathrm{E}+13$ & $7.16265 E+12$ & $5.37960 E+13$ & $1.20440 \mathrm{E}+18$ \\
\hline & & 3.0 & 0.17 & 149.6 & $3.19694 \mathrm{E}+13$ & $7.23974 \mathrm{E}+12$ & $5.48833 E+13$ & $4.27562 \mathrm{E}+18$ \\
\hline & & 10.0 & 0.56 & 146.0 & $3.15250 \mathrm{E}+13$ & $7.14581 \mathrm{E}+12$ & $5.45730 \mathrm{E}+13$ & $1.37904 \mathrm{E}+19$ \\
\hline & & 20.0 & 1.18 & 160.6 & $3.45771 \mathrm{E}+13$ & $8.22149 E+12$ & $5.92993 E+13$ & $2.92669 \mathrm{E}+19$ \\
\hline & & 29.2 & 1.76 & 163.7 & $3.63375 \mathrm{E}+13$ & $8.45595 \mathrm{E}+12$ & $6.22435 E+13$ & $4.35391 \mathrm{E}+19$ \\
\hline & \multicolumn{8}{|c|}{ Mid-Cycle 160B Outage - 5 Day, 14 Hour Down Time } \\
\hline & & 40.0 & 2.48 & 178.1 & $3.95406 \mathrm{E}+13$ & $8.26834 \mathrm{E}+12$ & $6.61385 E+13$ & $6.13642 \mathrm{E}+19$ \\
\hline & & 50.0 & 3.16 & 176.1 & $3.94198 \mathrm{E}+13$ & $8.65424 \mathrm{E}+12$ & $6.63339 \mathrm{E}+13$ & $7.81053 \mathrm{E}+19$ \\
\hline & EOC $(2 / 23 / 2017,1400)$ & 59.5 & 3.81 & 176.7 & $4.07670 \mathrm{E}+13$ & $8.52693 \mathrm{E}+12$ & $6.71821 \mathrm{E}+13$ & $9.43045 \mathrm{E}+19$ \\
\hline \multirow[t]{9}{*}{$161 \mathrm{~A}$} & \multicolumn{8}{|c|}{ Cycle 161A Outage - 95 Day, 13 Hour Down Time } \\
\hline & BOC $(5 / 30 / 2017,0300)$ & 61.2 & 3.92 & 152.2 & $3.49633 \mathrm{E}+13$ & $7.39951 \mathrm{E}+12$ & $5.76311 \mathrm{E}+13$ & $9.70144 \mathrm{E}+19$ \\
\hline & & 61.5 & 3.94 & 147.5 & $3.33174 \mathrm{E}+13$ & $7.03335 E+12$ & $5.52796 \mathrm{E}+13$ & $9.73757 \mathrm{E}+19$ \\
\hline & & 63.5 & 4.06 & 142.1 & $3.26594 \mathrm{E}+13$ & $6.59701 \mathrm{E}+12$ & $5.40286 \mathrm{E}+13$ & $1.00266 \mathrm{E}+20$ \\
\hline & & 65.5 & 4.18 & 145.2 & $3.34615 \mathrm{E}+13$ & $7.10581 \mathrm{E}+12$ & $5.56449 \mathrm{E}+13$ & $1.03217 \mathrm{E}+20$ \\
\hline & & 67.5 & 4.29 & 144.9 & $3.34475 \mathrm{E}+13$ & $6.93481 \mathrm{E}+12$ & $5.55138 \mathrm{E}+13$ & $1.06168 \mathrm{E}+20$ \\
\hline & & 69.5 & 4.42 & 147.5 & $3.41014 \mathrm{E}+13$ & $7.03426 \mathrm{E}+12$ & $5.57776 \mathrm{E}+13$ & $1.09179 \mathrm{E}+20$ \\
\hline & & 71.5 & 4.54 & 151.7 & $3.48598 \mathrm{E}+13$ & $7.11370 \mathrm{E}+12$ & $5.74205 E+13$ & $1.12250 \mathrm{E}+20$ \\
\hline & EOC $(6 / 11 / 2017,1600)$ & 72.0 & 4.57 & 151.1 & $3.54436 \mathrm{E}+13$ & $7.07116 \mathrm{E}+12$ & $5.81457 \mathrm{E}+13$ & $1.12852 \mathrm{E}+20$ \\
\hline \multirow[t]{10}{*}{$162 \mathrm{~A}$} & \multicolumn{8}{|c|}{ Cycle 162A Outage } \\
\hline & $\mathrm{BOC}$ & 73.0 & 4.62 & 144.9 & $3.41532 \mathrm{E}+13$ & $7.56765 E+12$ & $5.67269 \mathrm{E}+13$ & 1.14117E+20 \\
\hline & & 75.0 & 4.73 & 133.7 & $3.09688 \mathrm{E}+13$ & $6.69690 \mathrm{E}+12$ & $5.18191 \mathrm{E}+13$ & $1.16827 \mathrm{E}+20$ \\
\hline & & 82.0 & 5.08 & 136.0 & $3.15938 \mathrm{E}+13$ & $6.50135 \mathrm{E}+12$ & $5.26236 \mathrm{E}+13$ & $1.25679 \mathrm{E}+20$ \\
\hline & & 92.0 & 5.61 & 140.7 & $3.27164 \mathrm{E}+13$ & $6.91917 E+12$ & $5.41858 \mathrm{E}+13$ & $1.38747 \mathrm{E}+20$ \\
\hline & & 102.0 & 6.15 & 144.7 & $3.39535 \mathrm{E}+13$ & $7.32900 \mathrm{E}+12$ & $5.67367 \mathrm{E}+13$ & $1.51995 \mathrm{E}+20$ \\
\hline & & 112.0 & 6.67 & 139.7 & $3.33672 \mathrm{E}+13$ & $6.95550 \mathrm{E}+12$ & $5.56678 \mathrm{E}+13$ & $1.64882 \mathrm{E}+20$ \\
\hline & & 122.0 & 7.24 & 149.3 & $3.64207 \mathrm{E}+13$ & $7.32720 \mathrm{E}+12$ & $5.98165 E+13$ & $1.79034 \mathrm{E}+20$ \\
\hline & & 131.0 & 7.76 & 153.3 & $3.84315 \mathrm{E}+13$ & $8.13627 E+12$ & $6.28667 E+13$ & $1.91861 \mathrm{E}+20$ \\
\hline & EOC & 134.0 & 7.93 & 151.6 & $3.84278 E+13$ & $8.03695 E+12$ & $6.26595 E+13$ & $1.96137 \mathrm{E}+20$ \\
\hline
\end{tabular}


TEM-10300-1

03/01/2012

Rev. 03

Title:

ATF-1 Power Histories

\begin{tabular}{lllll} 
TEV No.: & 3119 & Rev. No.: & 0 & Project No.: \\
\hline
\end{tabular}

Table 40. ATF-73 (Pellet 11) Power History

\begin{tabular}{|c|c|c|c|c|c|c|c|c|}
\hline Cycle & Critical Dates & EFPD & $\begin{array}{c}\text { Burnup } \\
\text { (GWd/MTU) }\end{array}$ & $\begin{array}{l}\text { LHGR } \\
(\mathrm{W} / \mathrm{cm})\end{array}$ & $\begin{array}{l}\text { Thermal Flux } \\
\left(\mathrm{n} / \mathrm{cm}^{2 *} \mathrm{~s}\right)\end{array}$ & $\begin{array}{c}\text { Fast Flux } \\
\left(\mathrm{n} / \mathrm{cm}^{2 *} \mathrm{~s}>1\right. \\
\mathrm{MeV})\end{array}$ & $\begin{array}{l}\text { Total Flux } \\
\left(\mathrm{n} / \mathrm{cm}^{2 *} \mathrm{~s}\right)\end{array}$ & $\begin{array}{l}\text { Fission Density } \\
\left(\text { Fissions } / \mathrm{cm}^{3}\right)\end{array}$ \\
\hline \multirow[t]{9}{*}{$160 \mathrm{~B}$} & BOC $(12 / 20 / 2016,1200)$ & 1.0 & 0.05 & 143.6 & $3.05365 \mathrm{E}+13$ & $7.51501 \mathrm{E}+12$ & $5.30948 E+13$ & $1.20440 \mathrm{E}+18$ \\
\hline & & 3.0 & 0.17 & 143.7 & $3.06402 E+13$ & $7.27717 \mathrm{E}+12$ & $5.32588 E+13$ & $4.21540 \mathrm{E}+18$ \\
\hline & & 10.0 & 0.55 & 144.9 & $3.08221 \mathrm{E}+13$ & $6.99648 E+12$ & $5.31393 E+13$ & $1.36097 \mathrm{E}+19$ \\
\hline & & 20.0 & 1.14 & 153.9 & $3.32222 \mathrm{E}+13$ & $7.56728 \mathrm{E}+12$ & $5.60004 \mathrm{E}+13$ & $2.80625 \mathrm{E}+19$ \\
\hline & & 29.2 & 1.70 & 158.9 & $3.50460 \mathrm{E}+13$ & $7.74430 \mathrm{E}+12$ & $5.94722 \mathrm{E}+13$ & $4.20336 \mathrm{E}+19$ \\
\hline & \multicolumn{8}{|c|}{ Mid-Cycle 160B Outage - 5 Day, 14 Hour Down Time } \\
\hline & & 40.0 & 2.39 & 171.9 & $3.78263 \mathrm{E}+13$ & $8.49874 \mathrm{E}+12$ & $6.45478 \mathrm{E}+13$ & $5.91963 \mathrm{E}+19$ \\
\hline & & 50.0 & 3.05 & 168.5 & $3.79261 \mathrm{E}+13$ & $8.63019 \mathrm{E}+12$ & $6.44168 \mathrm{E}+13$ & $7.53954 \mathrm{E}+19$ \\
\hline & EOC $(2 / 23 / 2017,1400)$ & 59.5 & 3.66 & 169.5 & $3.88480 \mathrm{E}+13$ & $8.48695 \mathrm{E}+12$ & $6.45560 \mathrm{E}+13$ & $9.04504 \mathrm{E}+19$ \\
\hline \multirow[t]{9}{*}{$161 \mathrm{~A}$} & \multicolumn{8}{|c|}{ Cycle 161A Outage - 95 Day, 13 Hour Down Time } \\
\hline & BOC $(5 / 30 / 2017,0300)$ & 61.2 & 3.77 & 149.8 & $3.40156 \mathrm{E}+13$ & $7.18979 \mathrm{E}+12$ & $5.61989 E+13$ & $9.31001 \mathrm{E}+19$ \\
\hline & & 61.5 & 3.78 & 137.3 & $3.18272 E+13$ & $6.77051 \mathrm{E}+12$ & $5.30865 E+13$ & $9.34012 \mathrm{E}+19$ \\
\hline & & 63.5 & 3.90 & 139.2 & $3.18275 \mathrm{E}+13$ & $6.85452 \mathrm{E}+12$ & $5.32277 \mathrm{E}+13$ & $9.62918 \mathrm{E}+19$ \\
\hline & & 65.5 & 4.01 & 137.4 & $3.19963 \mathrm{E}+13$ & $6.67466 \mathrm{E}+12$ & $5.29829 \mathrm{E}+13$ & $9.91221 \mathrm{E}+19$ \\
\hline & & 67.5 & 4.13 & 139.8 & $3.24893 E+13$ & $6.93081 \mathrm{E}+12$ & $5.43500 \mathrm{E}+13$ & $1.02073 \mathrm{E}+20$ \\
\hline & & 69.5 & 4.25 & 142.2 & $3.31042 E+13$ & $6.94349 E+12$ & $5.46124 \mathrm{E}+13$ & $1.04963 \mathrm{E}+20$ \\
\hline & & 71.5 & 4.37 & 143.4 & $3.32145 \mathrm{E}+13$ & $7.02418 \mathrm{E}+12$ & $5.51893 E+13$ & $1.07914 \mathrm{E}+20$ \\
\hline & EOC $(6 / 11 / 2017,1600)$ & 72.0 & 4.39 & 149.7 & $3.47830 \mathrm{E}+13$ & $7.47431 \mathrm{E}+12$ & $5.76632 \mathrm{E}+13$ & $1.08516 \mathrm{E}+20$ \\
\hline \multirow[t]{10}{*}{$162 \mathrm{~A}$} & \multicolumn{8}{|c|}{ Cycle 162A Outage } \\
\hline & $\mathrm{BOC}$ & 73.0 & 4.44 & 140.1 & $3.25969 \mathrm{E}+13$ & $6.98383 E+12$ & $5.40904 \mathrm{E}+13$ & $1.09721 \mathrm{E}+20$ \\
\hline & & 75.0 & 4.55 & 129.5 & $2.98611 \mathrm{E}+13$ & $6.51054 \mathrm{E}+12$ & $5.08166 \mathrm{E}+13$ & $1.12431 \mathrm{E}+20$ \\
\hline & & 82.0 & 4.89 & 129.2 & $2.98873 \mathrm{E}+13$ & $6.28820 \mathrm{E}+12$ & $5.02560 \mathrm{E}+13$ & $1.20801 \mathrm{E}+20$ \\
\hline & & 92.0 & 5.40 & 134.5 & $3.12470 \mathrm{E}+13$ & $6.66998 \mathrm{E}+12$ & $5.24041 \mathrm{E}+13$ & $1.33387 \mathrm{E}+20$ \\
\hline & & 102.0 & 5.91 & 136.8 & $3.19560 \mathrm{E}+13$ & $6.83343 \mathrm{E}+12$ & $5.34950 \mathrm{E}+13$ & $1.46034 \mathrm{E}+20$ \\
\hline & & 112.0 & 6.41 & 135.6 & $3.22000 \mathrm{E}+13$ & $6.73761 \mathrm{E}+12$ & $5.37033 E+13$ & $1.58499 \mathrm{E}+20$ \\
\hline & & 122.0 & 6.94 & 143.0 & $3.48420 \mathrm{E}+13$ & $7.08858 \mathrm{E}+12$ & $5.74259 \mathrm{E}+13$ & 1.71627E+20 \\
\hline & & 131.0 & 7.46 & 151.4 & $3.71114 \mathrm{E}+13$ & $7.36246 \mathrm{E}+12$ & $6.08521 \mathrm{E}+13$ & $1.84333 \mathrm{E}+20$ \\
\hline & EOC & 134.0 & 7.62 & 147.5 & $3.70266 \mathrm{E}+13$ & $7.07611 \mathrm{E}+12$ & $6.04826 E+13$ & $1.88428 \mathrm{E}+20$ \\
\hline
\end{tabular}


TEM-10300-1

03/01/2012

Rev. 03

Title:

ATF-1 Power Histories

\begin{tabular}{lllll} 
TEV No.: & 3119 & Rev. No.: & 0 & Project No.: \\
\hline
\end{tabular}

Table 41. ATF-73 (Pellet 12) Power History

\begin{tabular}{|c|c|c|c|c|c|c|c|c|}
\hline Cycle & Critical Dates & EFPD & $\begin{array}{c}\text { Burnup } \\
\text { (GWd/MTU) }\end{array}$ & $\begin{array}{l}\text { LHGR } \\
(\mathrm{W} / \mathrm{cm})\end{array}$ & $\begin{array}{l}\text { Thermal Flux } \\
\left(\mathrm{n} / \mathrm{cm}^{2 *} \mathrm{~s}\right)\end{array}$ & $\begin{array}{c}\text { Fast Flux } \\
\left(\mathrm{n} / \mathrm{cm}^{2 *} \mathrm{~s}>1\right. \\
\mathrm{MeV})\end{array}$ & $\begin{array}{l}\text { Total Flux } \\
\left(\mathrm{n} / \mathrm{cm}^{2 *} \mathrm{~s}\right)\end{array}$ & $\begin{array}{l}\text { Fission Density } \\
\left(\text { Fissions } / \mathrm{cm}^{3}\right)\end{array}$ \\
\hline \multirow[t]{9}{*}{$160 \mathrm{~B}$} & BOC $(12 / 20 / 2016,1200)$ & 1.0 & 0.05 & 134.6 & $2.85752 \mathrm{E}+13$ & $6.73233 E+12$ & $4.96186 \mathrm{E}+13$ & $1.14418 \mathrm{E}+18$ \\
\hline & & 3.0 & 0.16 & 138.8 & $2.96751 \mathrm{E}+13$ & $6.96791 \mathrm{E}+12$ & $5.11702 E+13$ & $3.97452 E+18$ \\
\hline & & 10.0 & 0.54 & 140.5 & $2.98218 \mathrm{E}+13$ & $6.70833 E+12$ & $5.05155 \mathrm{E}+13$ & $1.33688 \mathrm{E}+19$ \\
\hline & & 20.0 & 1.10 & 149.0 & $3.20657 \mathrm{E}+13$ & $7.09565 \mathrm{E}+12$ & $5.43932 E+13$ & $2.71592 \mathrm{E}+19$ \\
\hline & & 29.2 & 1.66 & 156.3 & $3.44510 \mathrm{E}+13$ & $7.69250 \mathrm{E}+12$ & $5.87207 E+13$ & $4.09496 \mathrm{E}+19$ \\
\hline & \multicolumn{8}{|c|}{ Mid-Cycle 160B Outage - 5 Day, 14 Hour Down Time } \\
\hline & & 40.0 & 2.31 & 166.1 & $3.64108 \mathrm{E}+13$ & $7.99393 \mathrm{E}+12$ & $6.13013 E+13$ & $5.72090 \mathrm{E}+19$ \\
\hline & & 50.0 & 2.95 & 162.6 & $3.64660 \mathrm{E}+13$ & $7.69774 \mathrm{E}+12$ & $6.14468 \mathrm{E}+13$ & $7.28662 \mathrm{E}+19$ \\
\hline & EOC $(2 / 23 / 2017,1400)$ & 59.5 & 3.54 & 161.9 & $3.71078 \mathrm{E}+13$ & $7.96425 \mathrm{E}+12$ & $6.24686 \mathrm{E}+13$ & $8.73792 \mathrm{E}+19$ \\
\hline \multirow[t]{9}{*}{$161 \mathrm{~A}$} & \multicolumn{8}{|c|}{ Cycle 161A Outage - 95 Day, 13 Hour Down Time } \\
\hline & BOC $(5 / 30 / 2017,0300)$ & 61.2 & 3.64 & 148.4 & $3.34717 \mathrm{E}+13$ & $7.10331 \mathrm{E}+12$ & $5.50867 \mathrm{E}+13$ & $9.00289 \mathrm{E}+19$ \\
\hline & & 61.5 & 3.65 & 133.1 & $3.06880 \mathrm{E}+13$ & $6.52324 \mathrm{E}+12$ & $5.05335 E+13$ & $9.03300 \mathrm{E}+19$ \\
\hline & & 63.5 & 3.77 & 135.4 & $3.07973 \mathrm{E}+13$ & $6.19938 \mathrm{E}+12$ & $5.10883 E+13$ & $9.31603 \mathrm{E}+19$ \\
\hline & & 65.5 & 3.88 & 134.1 & $3.09860 \mathrm{E}+13$ & $6.40343 E+12$ & $5.09834 \mathrm{E}+13$ & $9.59907 \mathrm{E}+19$ \\
\hline & & 67.5 & 4.00 & 135.1 & $3.10925 \mathrm{E}+13$ & $6.78638 \mathrm{E}+12$ & $5.19940 \mathrm{E}+13$ & $9.87608 \mathrm{E}+19$ \\
\hline & & 69.5 & 4.11 & 139.1 & $3.18029 E+13$ & $7.09283 E+12$ & $5.31302 \mathrm{E}+13$ & $1.01591 \mathrm{E}+20$ \\
\hline & & 71.5 & 4.23 & 140.3 & $3.19754 \mathrm{E}+13$ & $6.88229 E+12$ & $5.29033 E+13$ & $1.04482 \mathrm{E}+20$ \\
\hline & EOC $(6 / 11 / 2017,1600)$ & 72.0 & 4.25 & 144.2 & $3.29566 \mathrm{E}+13$ & $6.99335 E+12$ & $5.47871 \mathrm{E}+13$ & $1.05084 \mathrm{E}+20$ \\
\hline \multirow[t]{10}{*}{$162 \mathrm{~A}$} & \multicolumn{8}{|c|}{ Cycle 162A Outage } \\
\hline & $\mathrm{BOC}$ & 73.0 & 4.30 & 138.1 & $3.22935 E+13$ & $6.68523 E+12$ & $5.34286 \mathrm{E}+13$ & $1.06168 \mathrm{E}+20$ \\
\hline & & 75.0 & 4.40 & 125.6 & $2.91334 \mathrm{E}+13$ & $6.43662 \mathrm{E}+12$ & $4.95994 \mathrm{E}+13$ & $1.08818 \mathrm{E}+20$ \\
\hline & & 82.0 & 4.73 & 126.1 & $2.86606 \mathrm{E}+13$ & $6.35272 \mathrm{E}+12$ & $4.82311 \mathrm{E}+13$ & $1.17007 \mathrm{E}+20$ \\
\hline & & 92.0 & 5.23 & 129.1 & $2.98878 \mathrm{E}+13$ & $6.15593 E+12$ & $5.01781 \mathrm{E}+13$ & $1.29172 \mathrm{E}+20$ \\
\hline & & 102.0 & 5.73 & 133.0 & $3.07181 \mathrm{E}+13$ & $6.46066 \mathrm{E}+12$ & $5.13405 E+13$ & $1.41517 \mathrm{E}+20$ \\
\hline & & 112.0 & 6.22 & 133.0 & $3.15080 \mathrm{E}+13$ & $6.38597 \mathrm{E}+12$ & $5.16901 \mathrm{E}+13$ & $1.53802 \mathrm{E}+20$ \\
\hline & & 122.0 & 6.74 & 138.9 & $3.33212 \mathrm{E}+13$ & $7.21997 \mathrm{E}+12$ & $5.54872 \mathrm{E}+13$ & $1.66629 \mathrm{E}+20$ \\
\hline & & 131.0 & 7.24 & 145.6 & $3.58569 \mathrm{E}+13$ & $7.33650 \mathrm{E}+12$ & $5.89749 \mathrm{E}+13$ & $1.78914 \mathrm{E}+20$ \\
\hline & EOC & 134.0 & 7.40 & 143.5 & $3.55417 \mathrm{E}+13$ & $7.21652 E+12$ & $5.87150 E+13$ & $1.82948 \mathrm{E}+20$ \\
\hline
\end{tabular}


TEM-10300-1

03/01/2012

Rev. 03

Title:

ATF-1 Power Histories

$\begin{array}{cccccc}\text { TEV No.: } & 3119 & \text { Rev. No.: } & 0 & \text { Project No.: } & 31418 \\ & & \text { Table 42. ATF-73 (Pellet } 13 \text { - Bottom) Power History }\end{array}$

\begin{tabular}{|c|c|c|c|c|c|c|c|c|}
\hline Cycle & Critical Dates & EFPD & $\begin{array}{c}\text { Burnup } \\
\text { (GWd/MTU) }\end{array}$ & $\begin{array}{l}\text { LHGR } \\
(\mathrm{W} / \mathrm{cm})\end{array}$ & $\begin{array}{l}\text { Thermal Flux } \\
\left(\mathrm{n} / \mathrm{cm}^{2 *} \mathrm{~s}\right)\end{array}$ & $\begin{array}{c}\text { Fast Flux } \\
\left(\mathrm{n} / \mathrm{cm}^{2 *} \mathrm{~s}>1\right. \\
\mathrm{MeV})\end{array}$ & $\begin{array}{l}\text { Total Flux } \\
\left(\mathrm{n} / \mathrm{cm}^{2 *} \mathrm{~s}\right)\end{array}$ & $\begin{array}{c}\text { Fission Density } \\
\text { (Fissions } / \mathrm{cm}^{3} \text { ) }\end{array}$ \\
\hline \multirow[t]{9}{*}{$160 \mathrm{~B}$} & $\operatorname{BOC}(12 / 20 / 2016,1200)$ & 1.0 & 0.05 & 133.8 & $2.81538 \mathrm{E}+13$ & $6.04829 E+12$ & $4.76395 \mathrm{E}+13$ & $1.14418 \mathrm{E}+18$ \\
\hline & & 3.0 & 0.16 & 137.6 & $2.89422 \mathrm{E}+13$ & $6.11117 \mathrm{E}+12$ & $4.90072 \mathrm{E}+13$ & $3.97452 \mathrm{E}+18$ \\
\hline & & 10.0 & 0.53 & 136.4 & $2.89416 \mathrm{E}+13$ & $6.26048 \mathrm{E}+12$ & $4.87647 \mathrm{E}+13$ & $1.30075 \mathrm{E}+19$ \\
\hline & & 20.0 & 1.09 & 147.3 & $3.11993 \mathrm{E}+13$ & $6.78373 \mathrm{E}+12$ & $5.28312 \mathrm{E}+13$ & $2.69183 \mathrm{E}+19$ \\
\hline & & 29.2 & 1.63 & 153.7 & $3.32886 \mathrm{E}+13$ & $7.19264 \mathrm{E}+12$ & $5.56669 \mathrm{E}+13$ & $4.04076 \mathrm{E}+19$ \\
\hline & \multicolumn{8}{|c|}{ Mid-Cycle 160B Outage - 5 Day, 14 Hour Down Time } \\
\hline & & 40.0 & 2.28 & 161.1 & $3.56305 \mathrm{E}+13$ & $7.26273 E+12$ & $5.94728 \mathrm{E}+13$ & $5.62455 \mathrm{E}+19$ \\
\hline & & 50.0 & 2.90 & 159.8 & $3.54756 \mathrm{E}+13$ & $7.59968 \mathrm{E}+12$ & $5.86365 \mathrm{E}+13$ & $7.16016 \mathrm{E}+19$ \\
\hline & EOC $(2 / 23 / 2017,1400)$ & 59.5 & 3.48 & 158.7 & $3.62842 \mathrm{E}+13$ & $7.44889 E+12$ & $6.00831 \mathrm{E}+13$ & $8.59339 \mathrm{E}+19$ \\
\hline \multirow[t]{9}{*}{$161 \mathrm{~A}$} & \multicolumn{8}{|c|}{ Cycle 161A Outage - 95 Day, 13 Hour Down Time } \\
\hline & $\mathrm{BOC}(5 / 30 / 2017,0300)$ & 61.2 & 3.58 & 140.1 & $3.18222 \mathrm{E}+13$ & $6.55431 \mathrm{E}+12$ & $5.27179 \mathrm{E}+13$ & $8.84632 \mathrm{E}+19$ \\
\hline & & 61.5 & 3.59 & 131.9 & $3.06083 E+13$ & $6.29102 \mathrm{E}+12$ & $4.95310 \mathrm{E}+13$ & $8.87643 \mathrm{E}+19$ \\
\hline & & 63.5 & 3.70 & 130.3 & $2.97471 \mathrm{E}+13$ & $6.07938 \mathrm{E}+12$ & $4.91241 \mathrm{E}+13$ & $9.14742 \mathrm{E}+19$ \\
\hline & & 65.5 & 3.81 & 128.4 & $2.95107 \mathrm{E}+13$ & $5.90289 \mathrm{E}+12$ & $4.84949 \mathrm{E}+13$ & $9.41841 \mathrm{E}+19$ \\
\hline & & 67.5 & 3.92 & 135.5 & $3.11143 \mathrm{E}+13$ & $6.04960 \mathrm{E}+12$ & $5.04360 \mathrm{E}+13$ & $9.68940 \mathrm{E}+19$ \\
\hline & & 69.5 & 4.03 & 137.8 & $3.14852 \mathrm{E}+13$ & $6.49477 \mathrm{E}+12$ & $5.17952 \mathrm{E}+13$ & 9.97243E+19 \\
\hline & & 71.5 & 4.15 & 138.5 & $3.20673 E+13$ & $6.37837 \mathrm{E}+12$ & $5.25442 \mathrm{E}+13$ & $1.02555 \mathrm{E}+20$ \\
\hline & EOC (6/11/2017, 1600) & 72.0 & 4.17 & 141.4 & $3.23541 \mathrm{E}+13$ & $6.63224 \mathrm{E}+12$ & $5.28627 \mathrm{E}+13$ & $1.03157 \mathrm{E}+20$ \\
\hline \multirow[t]{10}{*}{$162 \mathrm{~A}$} & \multicolumn{8}{|c|}{ Cycle 162A Outage } \\
\hline & $\mathrm{BOC}$ & 73.0 & 4.21 & 127.3 & $2.98753 \mathrm{E}+13$ & $6.06486 \mathrm{E}+12$ & $4.96319 \mathrm{E}+13$ & $1.04181 \mathrm{E}+20$ \\
\hline & & 75.0 & 4.32 & 122.3 & $2.80534 \mathrm{E}+13$ & $5.42337 \mathrm{E}+12$ & $4.65252 \mathrm{E}+13$ & $1.06770 \mathrm{E}+20$ \\
\hline & & 82.0 & 4.65 & 124.0 & $2.81496 \mathrm{E}+13$ & $5.62057 E+12$ & $4.69859 \mathrm{E}+13$ & $1.15020 \mathrm{E}+20$ \\
\hline & & 92.0 & 5.13 & 125.8 & $2.85251 \mathrm{E}+13$ & $5.69442 \mathrm{E}+12$ & $4.73478 \mathrm{E}+13$ & $1.26884 \mathrm{E}+20$ \\
\hline & & 102.0 & 5.62 & 127.7 & $2.94592 \mathrm{E}+13$ & $5.98570 \mathrm{E}+12$ & $4.91872 \mathrm{E}+13$ & $1.38867 \mathrm{E}+20$ \\
\hline & & 112.0 & 6.10 & 127.8 & $2.99350 \mathrm{E}+13$ & $5.97805 \mathrm{E}+12$ & $4.98187 \mathrm{E}+13$ & $1.50791 \mathrm{E}+20$ \\
\hline & & 122.0 & 6.61 & 133.9 & $3.18780 \mathrm{E}+13$ & $6.30568 \mathrm{E}+12$ & $5.32916 \mathrm{E}+13$ & $1.63317 \mathrm{E}+20$ \\
\hline & & 131.0 & 7.09 & 138.6 & $3.40016 \mathrm{E}+13$ & $6.86980 \mathrm{E}+12$ & $5.55927 \mathrm{E}+13$ & $1.75240 \mathrm{E}+20$ \\
\hline & EOC & 134.0 & 7.25 & 138.1 & $3.40604 \mathrm{E}+13$ & $6.96355 E+12$ & $5.55665 \mathrm{E}+13$ & $1.79155 \mathrm{E}+20$ \\
\hline
\end{tabular}


TEM-10300-1

03/01/2012

Rev. 03

Title:

ATF-1 Power Histories

\begin{tabular}{lllll} 
TEV No.: & 3119 & Rev. No.: & 0 & Project No.: \\
\hline
\end{tabular}

Table 43. ATF-74 (Pellet 1 -Top) Power History

\begin{tabular}{|c|c|c|c|c|c|c|c|c|}
\hline Cycle & Critical Dates & EFPD & $\begin{array}{c}\text { Burnup } \\
\text { (GWd/MTU) }\end{array}$ & $\begin{array}{c}\text { LHGR } \\
(\mathrm{W} / \mathrm{cm})\end{array}$ & $\begin{array}{l}\text { Thermal Flux } \\
\left(\mathrm{n} / \mathrm{cm}^{2 *} \mathrm{~s}\right)\end{array}$ & $\begin{array}{c}\text { Fast Flux } \\
\left(\mathrm{n} / \mathrm{cm}^{2 *} \mathrm{~s}>1\right. \\
\mathrm{MeV})\end{array}$ & $\begin{array}{l}\text { Total Flux } \\
\left(\mathrm{n} / \mathrm{cm}^{2 *} \mathrm{~s}\right)\end{array}$ & $\begin{array}{l}\text { Fission Density } \\
\text { (Fissions } / \mathrm{cm}^{3} \text { ) }\end{array}$ \\
\hline \multirow[t]{9}{*}{$160 \mathrm{~B}$} & $\operatorname{BOC}(12 / 20 / 2016,1200)$ & 1.0 & 0.07 & 206.0 & $4.35561 \mathrm{E}+13$ & $1.08331 \mathrm{E}+13$ & $7.80850 \mathrm{E}+13$ & $1.68616 \mathrm{E}+18$ \\
\hline & & 3.0 & 0.23 & 206.8 & $4.38948 \mathrm{E}+13$ & $1.08546 \mathrm{E}+13$ & $7.84794 \mathrm{E}+13$ & $5.66068 \mathrm{E}+18$ \\
\hline & & 10.0 & 0.77 & 201.7 & $4.29933 E+13$ & $1.10361 \mathrm{E}+13$ & $7.73579 \mathrm{E}+13$ & $1.89091 \mathrm{E}+19$ \\
\hline & & 20.0 & 1.50 & 192.1 & $4.13187 \mathrm{E}+13$ & $1.03000 \mathrm{E}+13$ & $7.36708 \mathrm{E}+13$ & $3.70353 \mathrm{E}+19$ \\
\hline & & 29.2 & 2.17 & 186.8 & $4.15228 \mathrm{E}+13$ & $9.81052 \mathrm{E}+12$ & $7.31984 \mathrm{E}+13$ & $5.35958 \mathrm{E}+19$ \\
\hline & \multicolumn{8}{|c|}{ Mid-Cycle 160B Outage - 5 Day, 14 Hour Down Time } \\
\hline & & 40.0 & 2.96 & 191.0 & $4.26066 \mathrm{E}+13$ & $1.01435 \mathrm{E}+13$ & $7.52578 \mathrm{E}+13$ & $7.32877 \mathrm{E}+19$ \\
\hline & & 50.0 & 3.66 & 182.9 & $4.13473 \mathrm{E}+13$ & $9.74817 \mathrm{E}+12$ & $7.44305 E+13$ & $9.04504 \mathrm{E}+19$ \\
\hline & EOC $(2 / 23 / 2017,1400)$ & 59.5 & 4.36 & 192.4 & $4.46732 \mathrm{E}+13$ & $1.05414 \mathrm{E}+13$ & $7.90925 E+13$ & $1.07734 \mathrm{E}+20$ \\
\hline \multirow[t]{9}{*}{$161 \mathrm{~A}$} & \multicolumn{8}{|c|}{ Cycle 161A Outage - 95 Day, 13 Hour Down Time } \\
\hline & BOC $(5 / 30 / 2017,0300)$ & 61.2 & 4.47 & 148.1 & $3.47677 \mathrm{E}+13$ & $8.24360 \mathrm{E}+12$ & $6.03128 \mathrm{E}+13$ & $1.10383 \mathrm{E}+20$ \\
\hline & & 61.5 & 4.48 & 134.0 & $3.09246 \mathrm{E}+13$ & $7.04167 \mathrm{E}+12$ & $5.36434 \mathrm{E}+13$ & $1.10684 \mathrm{E}+20$ \\
\hline & & 63.5 & 4.59 & 136.4 & $3.19807 \mathrm{E}+13$ & $7.51739 \mathrm{E}+12$ & $5.60617 \mathrm{E}+13$ & $1.13515 \mathrm{E}+20$ \\
\hline & & 65.5 & 4.71 & 136.2 & $3.21176 \mathrm{E}+13$ & $7.54994 \mathrm{E}+12$ & $5.60844 \mathrm{E}+13$ & $1.16345 \mathrm{E}+20$ \\
\hline & & 67.5 & 4.82 & 138.7 & $3.25825 \mathrm{E}+13$ & $7.93702 \mathrm{E}+12$ & $5.70089 \mathrm{E}+13$ & $1.19175 \mathrm{E}+20$ \\
\hline & & 69.5 & 4.94 & 141.8 & $3.31366 \mathrm{E}+13$ & $7.76348 \mathrm{E}+12$ & $5.77778 \mathrm{E}+13$ & $1.22066 \mathrm{E}+20$ \\
\hline & & 71.5 & 5.06 & 144.0 & $3.39763 \mathrm{E}+13$ & $7.82523 E+12$ & $5.88952 \mathrm{E}+13$ & $1.25017 \mathrm{E}+20$ \\
\hline & EOC $(6 / 11 / 2017,1600)$ & 72.0 & 5.08 & 145.7 & $3.47921 \mathrm{E}+13$ & 8.29719E+12 & $6.01384 \mathrm{E}+13$ & $1.25619 \mathrm{E}+20$ \\
\hline \multirow[t]{10}{*}{$162 \mathrm{~A}$} & \multicolumn{8}{|c|}{ Cycle 162A Outage } \\
\hline & $\mathrm{BOC}$ & 73.0 & 5.15 & 204.0 & $4.69333 \mathrm{E}+13$ & $1.15837 \mathrm{E}+13$ & $8.37238 \mathrm{E}+13$ & $1.27245 \mathrm{E}+20$ \\
\hline & & 75.0 & 5.30 & 199.6 & $4.69115 \mathrm{E}+13$ & $1.10842 \mathrm{E}+13$ & $8.24090 \mathrm{E}+13$ & $1.31039 \mathrm{E}+20$ \\
\hline & & 82.0 & 5.82 & 198.9 & $4.70084 \mathrm{E}+13$ & $1.07350 \mathrm{E}+13$ & $8.22789 \mathrm{E}+13$ & $1.43805 \mathrm{E}+20$ \\
\hline & & 92.0 & 6.58 & 203.1 & $4.76896 \mathrm{E}+13$ & $1.10410 \mathrm{E}+13$ & $8.32396 \mathrm{E}+13$ & $1.62534 \mathrm{E}+20$ \\
\hline & & 102.0 & 7.34 & 202.1 & $4.84033 \mathrm{E}+13$ & $1.11735 E+13$ & $8.40811 \mathrm{E}+13$ & $1.81383 \mathrm{E}+20$ \\
\hline & & 112.0 & 8.07 & 197.3 & $4.79337 \mathrm{E}+13$ & $1.09704 \mathrm{E}+13$ & $8.29535 E+13$ & $1.99509 \mathrm{E}+20$ \\
\hline & & 122.0 & 8.82 & 198.6 & $4.98287 \mathrm{E}+13$ & $1.11428 \mathrm{E}+13$ & $8.66625 E+13$ & $2.18057 E+20$ \\
\hline & & 131.0 & 9.43 & 183.1 & $4.66193 \mathrm{E}+13$ & $1.06861 \mathrm{E}+13$ & $8.18286 E+13$ & $2.32991 \mathrm{E}+20$ \\
\hline & EOC & 134.0 & 9.64 & 178.8 & $4.64187 \mathrm{E}+13$ & $1.05006 \mathrm{E}+13$ & $8.13203 E+13$ & $2.38291 \mathrm{E}+20$ \\
\hline
\end{tabular}


TEM-10300-1

03/01/2012

Rev. 03

Title:

ATF-1 Power Histories

$\begin{array}{lllll}\text { TEV No.: } & 3119 & \text { Rev. No.: } & 0 & \text { Project No.: } \\ & & & \end{array}$

Table 44. ATF-74 (Pellet 2) Power History

\begin{tabular}{|c|c|c|c|c|c|c|c|c|}
\hline Cycle & Critical Dates & EFPD & $\begin{array}{c}\text { Burnup } \\
\text { (GWd/MTU) }\end{array}$ & $\begin{array}{l}\text { LHGR } \\
(\mathrm{W} / \mathrm{cm})\end{array}$ & $\begin{array}{l}\text { Thermal Flux } \\
\left(\mathrm{n} / \mathrm{cm}^{2 *} \mathrm{~s}\right)\end{array}$ & $\begin{array}{c}\text { Fast Flux } \\
\left(\mathrm{n} / \mathrm{cm}^{2 *} \mathrm{~s}>1\right. \\
\mathrm{MeV})\end{array}$ & $\begin{array}{l}\text { Total Flux } \\
\left(\mathrm{n} / \mathrm{cm}^{2 *} \mathrm{~s}\right)\end{array}$ & $\begin{array}{l}\text { Fission Density } \\
\text { (Fissions } / \mathrm{cm}^{3} \text { ) }\end{array}$ \\
\hline \multirow[t]{9}{*}{$160 \mathrm{~B}$} & $\operatorname{BOC}(12 / 20 / 2016,1200)$ & 1.0 & 0.06 & 191.1 & $4.07608 \mathrm{E}+13$ & $1.17430 \mathrm{E}+13$ & $7.63095 \mathrm{E}+13$ & $1.56572 \mathrm{E}+18$ \\
\hline & & 3.0 & 0.21 & 191.8 & $4.09441 \mathrm{E}+13$ & $1.16518 \mathrm{E}+13$ & $7.67224 \mathrm{E}+13$ & $5.29936 \mathrm{E}+18$ \\
\hline & & 10.0 & 0.73 & 190.7 & $4.12938 \mathrm{E}+13$ & $1.13534 \mathrm{E}+13$ & $7.63065 \mathrm{E}+13$ & $1.80058 \mathrm{E}+19$ \\
\hline & & 20.0 & 1.42 & 182.0 & $3.93102 \mathrm{E}+13$ & $1.09088 \mathrm{E}+13$ & $7.33411 \mathrm{E}+13$ & $3.51083 E+19$ \\
\hline & & 29.2 & 2.05 & 177.2 & $3.94675 \mathrm{E}+13$ & $1.07349 \mathrm{E}+13$ & $7.30803 E+13$ & $5.07052 \mathrm{E}+19$ \\
\hline & \multicolumn{8}{|c|}{ Mid-Cycle 160B Outage - 5 Day, 14 Hour Down Time } \\
\hline & & 40.0 & 2.82 & 183.8 & $4.08400 \mathrm{E}+13$ & $1.13908 \mathrm{E}+13$ & $7.45433 \mathrm{E}+13$ & $6.97348 \mathrm{E}+19$ \\
\hline & & 50.0 & 3.48 & 172.9 & $3.92772 \mathrm{E}+13$ & $1.07826 \mathrm{E}+13$ & $7.40200 \mathrm{E}+13$ & $8.60544 \mathrm{E}+19$ \\
\hline & EOC $(2 / 23 / 2017,1400)$ & 59.5 & 4.14 & 179.9 & $4.19383 E+13$ & $1.11882 \mathrm{E}+13$ & $7.70082 E+13$ & $1.02434 \mathrm{E}+20$ \\
\hline \multirow[t]{9}{*}{$161 \mathrm{~A}$} & \multicolumn{8}{|c|}{ Cycle 161A Outage - 95 Day, 13 Hour Down Time } \\
\hline & BOC $(5 / 30 / 2017,0300)$ & 61.2 & 4.25 & 142.3 & $3.33780 \mathrm{E}+13$ & $9.10762 \mathrm{E}+12$ & $6.06757 \mathrm{E}+13$ & $1.05024 \mathrm{E}+20$ \\
\hline & & 61.5 & 4.26 & 127.9 & $2.99972 \mathrm{E}+13$ & $8.23032 \mathrm{E}+12$ & $5.42272 \mathrm{E}+13$ & $1.05325 \mathrm{E}+20$ \\
\hline & & 63.5 & 4.37 & 131.4 & $3.07832 \mathrm{E}+13$ & $8.13478 \mathrm{E}+12$ & $5.63741 \mathrm{E}+13$ & $1.08095 \mathrm{E}+20$ \\
\hline & & 65.5 & 4.48 & 130.1 & $3.08198 \mathrm{E}+13$ & 8.74207E+12 & $5.66596 \mathrm{E}+13$ & $1.10745 \mathrm{E}+20$ \\
\hline & & 67.5 & 4.59 & 132.2 & $3.09231 \mathrm{E}+13$ & $7.94822 \mathrm{E}+12$ & $5.53590 \mathrm{E}+13$ & $1.13515 \mathrm{E}+20$ \\
\hline & & 69.5 & 4.70 & 135.8 & $3.21633 \mathrm{E}+13$ & $8.54297 \mathrm{E}+12$ & $5.84842 \mathrm{E}+13$ & $1.16285 \mathrm{E}+20$ \\
\hline & & 71.5 & 4.82 & 136.0 & $3.23245 \mathrm{E}+13$ & $8.48978 \mathrm{E}+12$ & $5.85586 \mathrm{E}+13$ & 1.19055E+20 \\
\hline & EOC $(6 / 11 / 2017,1600)$ & 72.0 & 4.84 & 140.8 & $3.33310 \mathrm{E}+13$ & $9.01024 \mathrm{E}+12$ & $6.04431 \mathrm{E}+13$ & 1.19657E+20 \\
\hline \multirow[t]{10}{*}{$162 \mathrm{~A}$} & \multicolumn{8}{|c|}{ Cycle 162A Outage } \\
\hline & $\mathrm{BOC}$ & 73.0 & 4.90 & 200.1 & $4.61034 \mathrm{E}+13$ & $1.23133 \mathrm{E}+13$ & 8.40727E+13 & $1.21223 \mathrm{E}+20$ \\
\hline & & 75.0 & 5.05 & 191.7 & $4.46927 \mathrm{E}+13$ & 1.17936E+13 & $8.21149 \mathrm{E}+13$ & $1.24836 \mathrm{E}+20$ \\
\hline & & 82.0 & 5.56 & 192.8 & $4.52057 \mathrm{E}+13$ & $1.17756 \mathrm{E}+13$ & $8.26277 \mathrm{E}+13$ & $1.37422 \mathrm{E}+20$ \\
\hline & & 92.0 & 6.26 & 193.3 & $4.50476 \mathrm{E}+13$ & $1.16145 \mathrm{E}+13$ & $8.20217 \mathrm{E}+13$ & $1.54765 \mathrm{E}+20$ \\
\hline & & 102.0 & 6.98 & 190.8 & $4.53661 \mathrm{E}+13$ & $1.18836 \mathrm{E}+13$ & $8.20884 \mathrm{E}+13$ & $1.72530 \mathrm{E}+20$ \\
\hline & & 112.0 & 7.70 & 193.9 & $4.66320 \mathrm{E}+13$ & $1.17588 \mathrm{E}+13$ & $8.43025 E+13$ & $1.90416 \mathrm{E}+20$ \\
\hline & & 122.0 & 8.42 & 190.8 & $4.74049 \mathrm{E}+13$ & $1.23245 \mathrm{E}+13$ & $8.55767 \mathrm{E}+13$ & $2.08241 \mathrm{E}+20$ \\
\hline & & 131.0 & 9.02 & 179.6 & $4.50718 \mathrm{E}+13$ & 1.11787E+13 & $8.15561 \mathrm{E}+13$ & $2.23055 \mathrm{E}+20$ \\
\hline & EOC & 134.0 & 9.21 & 178.8 & $4.58055 \mathrm{E}+13$ & $1.11085 \mathrm{E}+13$ & $8.20848 \mathrm{E}+13$ & $2.27752 \mathrm{E}+20$ \\
\hline
\end{tabular}


TEM-10300-1

03/01/2012

Rev. 03

Title:

ATF-1 Power Histories

\begin{tabular}{lllll} 
TEV No.: & 3119 & Rev. No.: & 0 & Project No.: \\
\hline
\end{tabular}

Table 45. ATF-74 (Pellet 3) Power History

\begin{tabular}{|c|c|c|c|c|c|c|c|c|}
\hline Cycle & Critical Dates & EFPD & $\begin{array}{c}\text { Burnup } \\
\text { (GWd/MTU) }\end{array}$ & $\begin{array}{l}\text { LHGR } \\
(\mathrm{W} / \mathrm{cm})\end{array}$ & $\begin{array}{l}\text { Thermal Flux } \\
\left(\mathrm{n} / \mathrm{cm}^{2 *} \mathrm{~s}\right)\end{array}$ & $\begin{array}{c}\text { Fast Flux } \\
\left(\mathrm{n} / \mathrm{cm}^{2 *} \mathrm{~s}>1\right. \\
\mathrm{MeV})\end{array}$ & $\begin{array}{l}\text { Total Flux } \\
\left(\mathrm{n} / \mathrm{cm}^{2 *} \mathrm{~s}\right)\end{array}$ & $\begin{array}{l}\text { Fission Density } \\
\text { (Fissions } / \mathrm{cm}^{3} \text { ) }\end{array}$ \\
\hline \multirow[t]{9}{*}{$160 \mathrm{~B}$} & $\operatorname{BOC}(12 / 20 / 2016,1200)$ & 1.0 & 0.06 & 186.5 & $3.99337 \mathrm{E}+13$ & $1.17461 \mathrm{E}+13$ & 7.56357E+13 & $1.50550 \mathrm{E}+18$ \\
\hline & & 3.0 & 0.21 & 187.0 & $4.00276 \mathrm{E}+13$ & $1.15704 \mathrm{E}+13$ & $7.56559 \mathrm{E}+13$ & $5.17892 \mathrm{E}+18$ \\
\hline & & 10.0 & 0.70 & 182.6 & $3.96380 \mathrm{E}+13$ & $1.16771 \mathrm{E}+13$ & $7.45442 \mathrm{E}+13$ & $1.74036 \mathrm{E}+19$ \\
\hline & & 20.0 & 1.39 & 179.1 & $3.90743 \mathrm{E}+13$ & 1.14217E+13 & 7.36976E+13 & $3.43254 \mathrm{E}+19$ \\
\hline & & 29.2 & 2.00 & 173.3 & $3.87309 \mathrm{E}+13$ & $1.09983 \mathrm{E}+13$ & $7.23603 \mathrm{E}+13$ & $4.93202 \mathrm{E}+19$ \\
\hline & \multicolumn{8}{|c|}{ Mid-Cycle 160B Outage - 5 Day, 14 Hour Down Time } \\
\hline & & 40.0 & 2.71 & 176.0 & $3.94895 \mathrm{E}+13$ & $1.14471 \mathrm{E}+13$ & $7.48689 \mathrm{E}+13$ & $6.69646 \mathrm{E}+19$ \\
\hline & & 50.0 & 3.37 & 173.7 & $3.94342 \mathrm{E}+13$ & $1.12552 \mathrm{E}+13$ & $7.53340 \mathrm{E}+13$ & $8.32240 \mathrm{E}+19$ \\
\hline & EOC $(2 / 23 / 2017,1400)$ & 59.5 & 4.02 & 178.5 & $4.14195 \mathrm{E}+13$ & 1.14797E+13 & $7.71850 \mathrm{E}+13$ & $9.94834 \mathrm{E}+19$ \\
\hline \multirow[t]{9}{*}{$161 \mathrm{~A}$} & \multicolumn{8}{|c|}{ Cycle 161A Outage - 95 Day, 13 Hour Down Time } \\
\hline & BOC $(5 / 30 / 2017,0300)$ & 61.2 & 4.13 & 141.5 & $3.25677 \mathrm{E}+13$ & $9.59430 \mathrm{E}+12$ & $6.07806 \mathrm{E}+13$ & $1.02073 \mathrm{E}+20$ \\
\hline & & 61.5 & 4.14 & 127.2 & $2.94648 \mathrm{E}+13$ & 7.78887E+12 & $5.38870 \mathrm{E}+13$ & $1.02374 \mathrm{E}+20$ \\
\hline & & 63.5 & 4.25 & 127.7 & $2.97595 \mathrm{E}+13$ & $8.50001 \mathrm{E}+12$ & $5.57230 \mathrm{E}+13$ & $1.05084 \mathrm{E}+20$ \\
\hline & & 65.5 & 4.36 & 128.9 & $3.01040 \mathrm{E}+13$ & $8.60349 \mathrm{E}+12$ & $5.63374 \mathrm{E}+13$ & $1.07794 \mathrm{E}+20$ \\
\hline & & 67.5 & 4.47 & 131.0 & $3.08408 \mathrm{E}+13$ & $8.52461 \mathrm{E}+12$ & $5.65247 \mathrm{E}+13$ & $1.10504 \mathrm{E}+20$ \\
\hline & & 69.5 & 4.58 & 132.7 & $3.13158 \mathrm{E}+13$ & $8.60452 \mathrm{E}+12$ & $5.72861 \mathrm{E}+13$ & $1.13214 \mathrm{E}+20$ \\
\hline & & 71.5 & 4.69 & 134.2 & $3.15733 \mathrm{E}+13$ & $8.74034 \mathrm{E}+12$ & $5.78676 \mathrm{E}+13$ & $1.15984 \mathrm{E}+20$ \\
\hline & EOC $(6 / 11 / 2017,1600)$ & 72.0 & 4.72 & 140.5 & $3.27325 E+13$ & $9.04374 \mathrm{E}+12$ & $6.00608 \mathrm{E}+13$ & $1.16586 \mathrm{E}+20$ \\
\hline \multirow[t]{10}{*}{$162 \mathrm{~A}$} & \multicolumn{8}{|c|}{ Cycle 162A Outage } \\
\hline & $\mathrm{BOC}$ & 73.0 & 4.78 & 196.9 & $4.54158 \mathrm{E}+13$ & $1.23696 \mathrm{E}+13$ & $8.36188 \mathrm{E}+13$ & $1.18152 \mathrm{E}+20$ \\
\hline & & 75.0 & 4.93 & 189.5 & $4.44279 \mathrm{E}+13$ & $1.21691 \mathrm{E}+13$ & $8.11921 \mathrm{E}+13$ & $1.21765 \mathrm{E}+20$ \\
\hline & & 82.0 & 5.42 & 189.7 & $4.43886 \mathrm{E}+13$ & $1.19336 \mathrm{E}+13$ & $8.12622 E+13$ & $1.33990 \mathrm{E}+20$ \\
\hline & & 92.0 & 6.11 & 189.0 & $4.40096 \mathrm{E}+13$ & $1.18465 \mathrm{E}+13$ & $8.08933 E+13$ & $1.51152 \mathrm{E}+20$ \\
\hline & & 102.0 & 6.80 & 187.1 & $4.45745 \mathrm{E}+13$ & $1.23323 \mathrm{E}+13$ & $8.13803 E+13$ & $1.68194 \mathrm{E}+20$ \\
\hline & & 112.0 & 7.49 & 188.1 & $4.49337 \mathrm{E}+13$ & $1.20519 \mathrm{E}+13$ & $8.23547 \mathrm{E}+13$ & $1.85116 \mathrm{E}+20$ \\
\hline & & 122.0 & 8.21 & 190.4 & $4.72742 \mathrm{E}+13$ & $1.25563 \mathrm{E}+13$ & $8.58706 \mathrm{E}+13$ & $2.03002 \mathrm{E}+20$ \\
\hline & & 131.0 & 8.80 & 176.3 & $4.42299 \mathrm{E}+13$ & $1.14452 \mathrm{E}+13$ & $8.13572 E+13$ & $2.17635 \mathrm{E}+20$ \\
\hline & EOC & 134.0 & 8.99 & 173.8 & $4.46113 \mathrm{E}+13$ & $1.13082 \mathrm{E}+13$ & $8.15023 E+13$ & $2.22272 \mathrm{E}+20$ \\
\hline
\end{tabular}


TEM-10300-1

03/01/2012

Rev. 03

Title:

ATF-1 Power Histories

$\begin{array}{lllll}\text { TEV No.: } & 3119 & \text { Rev. No.: } & 0 & \text { Project No.: } \\ & & & \end{array}$

Table 46. ATF-74 (Pellet 4) Power History

\begin{tabular}{|c|c|c|c|c|c|c|c|c|}
\hline Cycle & Critical Dates & EFPD & $\begin{array}{c}\text { Burnup } \\
\text { (GWd/MTU) }\end{array}$ & $\begin{array}{l}\text { LHGR } \\
(\mathrm{W} / \mathrm{cm})\end{array}$ & $\begin{array}{l}\text { Thermal Flux } \\
\left(\mathrm{n} / \mathrm{cm}^{2 *} \mathrm{~s}\right)\end{array}$ & $\begin{array}{c}\text { Fast Flux } \\
\left(\mathrm{n} / \mathrm{cm}^{2 *} \mathrm{~s}>1\right. \\
\mathrm{MeV})\end{array}$ & $\begin{array}{l}\text { Total Flux } \\
\left(\mathrm{n} / \mathrm{cm}^{2 *} \mathrm{~s}\right)\end{array}$ & $\begin{array}{l}\text { Fission Density } \\
\text { (Fissions/cm }{ }^{3} \text { ) }\end{array}$ \\
\hline \multirow[t]{9}{*}{$160 \mathrm{~B}$} & $\operatorname{BOC}(12 / 20 / 2016,1200)$ & 1.0 & 0.06 & 184.8 & $3.98831 \mathrm{E}+13$ & $1.22934 \mathrm{E}+13$ & $7.62490 \mathrm{E}+13$ & $1.50550 \mathrm{E}+18$ \\
\hline & & 3.0 & 0.20 & 182.2 & $3.95047 \mathrm{E}+13$ & $1.20038 \mathrm{E}+13$ & $7.55551 \mathrm{E}+13$ & $5.05848 \mathrm{E}+18$ \\
\hline & & 10.0 & 0.69 & 178.8 & $3.87934 \mathrm{E}+13$ & 1.17193E+13 & $7.48538 \mathrm{E}+13$ & 1.70423E+19 \\
\hline & & 20.0 & 1.36 & 174.9 & $3.83432 \mathrm{E}+13$ & $1.13498 \mathrm{E}+13$ & $7.28236 \mathrm{E}+13$ & $3.35425 \mathrm{E}+19$ \\
\hline & & 29.2 & 1.97 & 170.8 & $3.83205 E+13$ & $1.07360 \mathrm{E}+13$ & $7.20446 \mathrm{E}+13$ & $4.87782 \mathrm{E}+19$ \\
\hline & \multicolumn{8}{|c|}{ Mid-Cycle 160B Outage - 5 Day, 14 Hour Down Time } \\
\hline & & 40.0 & 2.71 & 173.3 & $3.89571 \mathrm{E}+13$ & $1.14942 \mathrm{E}+13$ & $7.41592 \mathrm{E}+13$ & $6.70249 \mathrm{E}+19$ \\
\hline & & 50.0 & 3.35 & 167.5 & $3.81854 \mathrm{E}+13$ & 1.12623E+13 & $7.39676 \mathrm{E}+13$ & $8.27423 E+19$ \\
\hline & EOC $(2 / 23 / 2017,1400)$ & 59.5 & 3.99 & 175.9 & $4.10939 E+13$ & $1.14918 \mathrm{E}+13$ & $7.73100 \mathrm{E}+13$ & $9.86404 \mathrm{E}+19$ \\
\hline \multirow[t]{9}{*}{$161 \mathrm{~A}$} & \multicolumn{8}{|c|}{ Cycle 161A Outage - 95 Day, 13 Hour Down Time } \\
\hline & BOC $(5 / 30 / 2017,0300)$ & 61.2 & 4.09 & 136.3 & $3.16783 \mathrm{E}+13$ & 9.70707E+12 & $6.01089 \mathrm{E}+13$ & $1.01109 \mathrm{E}+20$ \\
\hline & & 61.5 & 4.10 & 124.0 & $2.88132 \mathrm{E}+13$ & $8.38235 \mathrm{E}+12$ & $5.39726 \mathrm{E}+13$ & $1.01410 \mathrm{E}+20$ \\
\hline & & 63.5 & 4.21 & 127.3 & $2.95900 \mathrm{E}+13$ & $8.50019 \mathrm{E}+12$ & $5.55446 \mathrm{E}+13$ & $1.04060 \mathrm{E}+20$ \\
\hline & & 65.5 & 4.31 & 125.9 & $2.95274 \mathrm{E}+13$ & $8.31416 \mathrm{E}+12$ & $5.51167 \mathrm{E}+13$ & $1.06650 \mathrm{E}+20$ \\
\hline & & 67.5 & 4.42 & 128.5 & $3.00072 E+13$ & $8.87438 \mathrm{E}+12$ & $5.67287 \mathrm{E}+13$ & $1.09299 \mathrm{E}+20$ \\
\hline & & 69.5 & 4.53 & 131.1 & $3.06779 \mathrm{E}+13$ & $8.80002 \mathrm{E}+12$ & $5.74304 \mathrm{E}+13$ & 1.11949E+20 \\
\hline & & 71.5 & 4.64 & 135.3 & $3.17773 \mathrm{E}+13$ & $9.18339 \mathrm{E}+12$ & $5.94572 \mathrm{E}+13$ & 1.14719E+20 \\
\hline & EOC $(6 / 11 / 2017,1600)$ & 72.0 & 4.66 & 132.6 & $3.12772 \mathrm{E}+13$ & $9.07442 \mathrm{E}+12$ & $5.86857 \mathrm{E}+13$ & $1.15261 \mathrm{E}+20$ \\
\hline \multirow[t]{10}{*}{$162 \mathrm{~A}$} & \multicolumn{8}{|c|}{ Cycle 162A Outage } \\
\hline & $\mathrm{BOC}$ & 73.0 & 4.72 & 187.7 & $4.34271 \mathrm{E}+13$ & $1.28136 \mathrm{E}+13$ & $8.22225 \mathrm{E}+13$ & $1.16706 \mathrm{E}+20$ \\
\hline & & 75.0 & 4.87 & 186.6 & $4.37023 \mathrm{E}+13$ & $1.21751 \mathrm{E}+13$ & $8.20433 E+13$ & $1.20320 \mathrm{E}+20$ \\
\hline & & 82.0 & 5.36 & 187.0 & $4.38209 \mathrm{E}+13$ & $1.19514 \mathrm{E}+13$ & $8.17812 \mathrm{E}+13$ & $1.32484 \mathrm{E}+20$ \\
\hline & & 92.0 & 6.03 & 182.6 & $4.24074 \mathrm{E}+13$ & 1.19507E+13 & $7.93851 \mathrm{E}+13$ & $1.49045 \mathrm{E}+20$ \\
\hline & & 102.0 & 6.70 & 182.4 & $4.33761 \mathrm{E}+13$ & 1.19187E+13 & $8.11413 E+13$ & $1.65605 \mathrm{E}+20$ \\
\hline & & 112.0 & 7.37 & 183.3 & $4.37804 \mathrm{E}+13$ & $1.22098 \mathrm{E}+13$ & $8.10711 \mathrm{E}+13$ & $1.82105 \mathrm{E}+20$ \\
\hline & & 122.0 & 8.06 & 182.0 & $4.52888 \mathrm{E}+13$ & $1.21350 \mathrm{E}+13$ & $8.39564 \mathrm{E}+13$ & $1.99208 \mathrm{E}+20$ \\
\hline & & 131.0 & 8.64 & 173.9 & $4.35424 \mathrm{E}+13$ & $1.13602 \mathrm{E}+13$ & $8.09255 E+13$ & $2.13661 \mathrm{E}+20$ \\
\hline & EOC & 134.0 & 8.83 & 172.0 & $4.39123 E+13$ & 1.15697E+13 & $8.12288 \mathrm{E}+13$ & $2.18298 \mathrm{E}+20$ \\
\hline
\end{tabular}


TEM-10300-1

03/01/2012

Rev. 03

Title:

ATF-1 Power Histories

\begin{tabular}{lllll} 
TEV No.: & 3119 & Rev. No.: & 0 & Project No.: \\
\hline
\end{tabular}

Table 47. ATF-74 (Pellet 5) Power History

\begin{tabular}{|c|c|c|c|c|c|c|c|c|}
\hline Cycle & Critical Dates & EFPD & $\begin{array}{c}\text { Burnup } \\
\text { (GWd/MTU) }\end{array}$ & $\begin{array}{l}\text { LHGR } \\
(\mathrm{W} / \mathrm{cm})\end{array}$ & $\begin{array}{l}\text { Thermal Flux } \\
\left(\mathrm{n} / \mathrm{cm}^{2 *} \mathrm{~s}\right)\end{array}$ & $\begin{array}{c}\text { Fast Flux } \\
\left(\mathrm{n} / \mathrm{cm}^{2 *} \mathrm{~s}>1\right. \\
\mathrm{MeV})\end{array}$ & $\begin{array}{l}\text { Total Flux } \\
\left(\mathrm{n} / \mathrm{cm}^{2 *} \mathrm{~s}\right)\end{array}$ & $\begin{array}{l}\text { Fission Density } \\
\text { (Fissions } / \mathrm{cm}^{3} \text { ) }\end{array}$ \\
\hline \multirow[t]{9}{*}{$160 \mathrm{~B}$} & $\operatorname{BOC}(12 / 20 / 2016,1200)$ & 1.0 & 0.06 & 175.4 & $3.81603 \mathrm{E}+13$ & $1.18235 E+13$ & $7.40343 E+13$ & $1.44528 \mathrm{E}+18$ \\
\hline & & 3.0 & 0.20 & 175.7 & $3.82085 \mathrm{E}+13$ & $1.19376 \mathrm{E}+13$ & $7.43159 \mathrm{E}+13$ & $4.93804 \mathrm{E}+18$ \\
\hline & & 10.0 & 0.69 & 180.4 & $3.92142 \mathrm{E}+13$ & $1.19484 \mathrm{E}+13$ & $7.50863 E+13$ & $1.70423 \mathrm{E}+19$ \\
\hline & & 20.0 & 1.34 & 169.3 & $3.71254 \mathrm{E}+13$ & 1.14907E+13 & $7.12650 \mathrm{E}+13$ & $3.31812 \mathrm{E}+19$ \\
\hline & & 29.2 & 1.91 & 162.8 & $3.64549 \mathrm{E}+13$ & $1.12613 \mathrm{E}+13$ & $7.02290 \mathrm{E}+13$ & $4.72727 \mathrm{E}+19$ \\
\hline & \multicolumn{8}{|c|}{ Mid-Cycle 160B Outage - 5 Day, 14 Hour Down Time } \\
\hline & & 40.0 & 2.63 & 168.9 & $3.79104 \mathrm{E}+13$ & $1.14187 \mathrm{E}+13$ & $7.22950 \mathrm{E}+13$ & $6.49172 \mathrm{E}+19$ \\
\hline & & 50.0 & 3.25 & 162.5 & $3.72672 \mathrm{E}+13$ & $1.11057 \mathrm{E}+13$ & $7.20882 E+13$ & $8.03937 \mathrm{E}+19$ \\
\hline & EOC $(2 / 23 / 2017,1400)$ & 59.5 & 3.87 & 168.1 & $3.93760 \mathrm{E}+13$ & $1.13899 E+13$ & $7.47702 \mathrm{E}+13$ & $9.56896 \mathrm{E}+19$ \\
\hline \multirow[t]{9}{*}{$161 \mathrm{~A}$} & \multicolumn{8}{|c|}{ Cycle 161A Outage - 95 Day, 13 Hour Down Time } \\
\hline & BOC $(5 / 30 / 2017,0300)$ & 61.2 & 3.97 & 135.7 & $3.15419 \mathrm{E}+13$ & $9.26758 \mathrm{E}+12$ & $5.94154 \mathrm{E}+13$ & $9.81586 \mathrm{E}+19$ \\
\hline & & 61.5 & 3.98 & 119.5 & $2.81170 \mathrm{E}+13$ & $8.09990 \mathrm{E}+12$ & $5.28234 \mathrm{E}+13$ & $9.84597 \mathrm{E}+19$ \\
\hline & & 63.5 & 4.09 & 123.3 & $2.87401 \mathrm{E}+13$ & $8.88756 \mathrm{E}+12$ & $5.46704 \mathrm{E}+13$ & $1.01049 \mathrm{E}+20$ \\
\hline & & 65.5 & 4.20 & 126.6 & $2.95374 \mathrm{E}+13$ & $8.89178 \mathrm{E}+12$ & $5.60100 \mathrm{E}+13$ & $1.03699 \mathrm{E}+20$ \\
\hline & & 67.5 & 4.30 & 127.9 & $2.99725 \mathrm{E}+13$ & $8.79740 \mathrm{E}+12$ & $5.61924 \mathrm{E}+13$ & $1.06349 \mathrm{E}+20$ \\
\hline & & 69.5 & 4.41 & 129.3 & $3.01976 \mathrm{E}+13$ & $9.03361 \mathrm{E}+12$ & $5.67409 \mathrm{E}+13$ & $1.09058 \mathrm{E}+20$ \\
\hline & & 71.5 & 4.52 & 133.7 & $3.14539 \mathrm{E}+13$ & $9.05625 E+12$ & $5.87848 \mathrm{E}+13$ & $1.11829 \mathrm{E}+20$ \\
\hline & EOC $(6 / 11 / 2017,1600)$ & 72.0 & 4.55 & 133.4 & $3.12269 \mathrm{E}+13$ & $8.62382 \mathrm{E}+12$ & $5.90588 \mathrm{E}+13$ & $1.12371 \mathrm{E}+20$ \\
\hline \multirow[t]{10}{*}{$162 \mathrm{~A}$} & \multicolumn{8}{|c|}{ Cycle 162A Outage } \\
\hline & $\mathrm{BOC}$ & 73.0 & 4.60 & 184.9 & $4.26994 \mathrm{E}+13$ & $1.28415 \mathrm{E}+13$ & $8.15259 \mathrm{E}+13$ & $1.13756 \mathrm{E}+20$ \\
\hline & & 75.0 & 4.74 & 180.5 & $4.22370 \mathrm{E}+13$ & $1.24351 \mathrm{E}+13$ & $8.04116 \mathrm{E}+13$ & $1.17248 \mathrm{E}+20$ \\
\hline & & 82.0 & 5.23 & 181.8 & $4.25236 \mathrm{E}+13$ & $1.23602 \mathrm{E}+13$ & $8.07002 E+13$ & $1.29172 \mathrm{E}+20$ \\
\hline & & 92.0 & 5.89 & 180.5 & $4.16591 \mathrm{E}+13$ & $1.20159 \mathrm{E}+13$ & $7.89312 \mathrm{E}+13$ & $1.45552 \mathrm{E}+20$ \\
\hline & & 102.0 & 6.56 & 180.2 & $4.26009 \mathrm{E}+13$ & $1.21435 E+13$ & $8.01542 \mathrm{E}+13$ & $1.62052 \mathrm{E}+20$ \\
\hline & & 112.0 & 7.22 & 181.0 & $4.33084 \mathrm{E}+13$ & $1.24120 \mathrm{E}+13$ & $8.10277 E+13$ & $1.78372 \mathrm{E}+20$ \\
\hline & & 122.0 & 7.89 & 180.7 & $4.46480 \mathrm{E}+13$ & $1.27365 E+13$ & $8.33236 E+13$ & $1.94992 \mathrm{E}+20$ \\
\hline & & 131.0 & 8.46 & 166.8 & 4.15903E+13 & $1.22175 E+13$ & $7.95968 E+13$ & $2.09024 \mathrm{E}+20$ \\
\hline & EOC & 134.0 & 8.64 & 165.0 & $4.19304 \mathrm{E}+13$ & $1.20003 E+13$ & $8.00470 \mathrm{E}+13$ & $2.13480 \mathrm{E}+20$ \\
\hline
\end{tabular}


TEM-10300-1

03/01/2012

Rev. 03

Title:

ATF-1 Power Histories

$\begin{array}{lllll}\text { TEV No.: } & 3119 & \text { Rev. No.: } & 0 & \text { Project No.: } \\ & & & \end{array}$

Table 48. ATF-74 (Pellet 6) Power History

\begin{tabular}{|c|c|c|c|c|c|c|c|c|}
\hline Cycle & Critical Dates & EFPD & $\begin{array}{c}\text { Burnup } \\
\text { (GWd/MTU) }\end{array}$ & $\begin{array}{l}\text { LHGR } \\
(\mathrm{W} / \mathrm{cm})\end{array}$ & $\begin{array}{l}\text { Thermal Flux } \\
\left(\mathrm{n} / \mathrm{cm}^{2 *} \mathrm{~s}\right)\end{array}$ & $\begin{array}{c}\text { Fast Flux } \\
\left(\mathrm{n} / \mathrm{cm}^{2 *} \mathrm{~s}>1\right. \\
\mathrm{MeV})\end{array}$ & $\begin{array}{l}\text { Total Flux } \\
\left(\mathrm{n} / \mathrm{cm}^{2 *} \mathrm{~s}\right)\end{array}$ & $\begin{array}{l}\text { Fission Density } \\
\text { (Fissions } / \mathrm{cm}^{3} \text { ) }\end{array}$ \\
\hline \multirow[t]{9}{*}{$160 \mathrm{~B}$} & $\operatorname{BOC}(12 / 20 / 2016,1200)$ & 1.0 & 0.06 & 174.7 & $3.80088 \mathrm{E}+13$ & $1.22945 \mathrm{E}+13$ & $7.49322 \mathrm{E}+13$ & $1.38506 \mathrm{E}+18$ \\
\hline & & 3.0 & 0.20 & 175.5 & $3.79964 \mathrm{E}+13$ & $1.22001 \mathrm{E}+13$ & $7.49521 \mathrm{E}+13$ & $4.87782 \mathrm{E}+18$ \\
\hline & & 10.0 & 0.67 & 175.2 & $3.75309 \mathrm{E}+13$ & $1.23602 \mathrm{E}+13$ & $7.40790 \mathrm{E}+13$ & $1.66207 \mathrm{E}+19$ \\
\hline & & 20.0 & 1.33 & 171.7 & $3.75975 \mathrm{E}+13$ & $1.17390 \mathrm{E}+13$ & $7.38362 \mathrm{E}+13$ & $3.28801 \mathrm{E}+19$ \\
\hline & & 29.2 & 1.91 & 165.0 & $3.71195 \mathrm{E}+13$ & $1.12771 \mathrm{E}+13$ & $7.23110 \mathrm{E}+13$ & $4.71523 \mathrm{E}+19$ \\
\hline & \multicolumn{8}{|c|}{ Mid-Cycle 160B Outage - 5 Day, 14 Hour Down Time } \\
\hline & & 40.0 & 2.60 & 168.8 & $3.75757 \mathrm{E}+13$ & $1.15774 \mathrm{E}+13$ & $7.28816 \mathrm{E}+13$ & $6.42547 \mathrm{E}+19$ \\
\hline & & 50.0 & 3.23 & 162.8 & $3.69716 \mathrm{E}+13$ & $1.13095 \mathrm{E}+13$ & $7.28001 \mathrm{E}+13$ & $7.97313 \mathrm{E}+19$ \\
\hline & EOC $(2 / 23 / 2017,1400)$ & 59.5 & 3.84 & 168.8 & $3.95132 \mathrm{E}+13$ & 1.15857E+13 & $7.63131 \mathrm{E}+13$ & $9.49669 \mathrm{E}+19$ \\
\hline \multirow[t]{9}{*}{$161 \mathrm{~A}$} & \multicolumn{8}{|c|}{ Cycle 161A Outage - 95 Day, 13 Hour Down Time } \\
\hline & BOC $(5 / 30 / 2017,0300)$ & 61.2 & 3.94 & 137.5 & $3.18405 E+13$ & $9.35708 \mathrm{E}+12$ & $5.97708 \mathrm{E}+13$ & $9.74360 \mathrm{E}+19$ \\
\hline & & 61.5 & 3.95 & 121.6 & $2.76042 \mathrm{E}+13$ & $8.37451 \mathrm{E}+12$ & 5.32417E+13 & $9.77371 \mathrm{E}+19$ \\
\hline & & 63.5 & 4.06 & 121.6 & $2.88083 \mathrm{E}+13$ & $8.42152 \mathrm{E}+12$ & $5.44380 \mathrm{E}+13$ & $1.00327 \mathrm{E}+20$ \\
\hline & & 65.5 & 4.16 & 123.8 & $2.93076 \mathrm{E}+13$ & 8.71177E+12 & $5.61689 \mathrm{E}+13$ & $1.02916 \mathrm{E}+20$ \\
\hline & & 67.5 & 4.27 & 125.7 & $2.94673 \mathrm{E}+13$ & $8.38139 \mathrm{E}+12$ & $5.51426 \mathrm{E}+13$ & $1.05566 \mathrm{E}+20$ \\
\hline & & 69.5 & 4.38 & 124.6 & $2.91768 \mathrm{E}+13$ & $8.46132 \mathrm{E}+12$ & $5.53759 \mathrm{E}+13$ & $1.08155 \mathrm{E}+20$ \\
\hline & & 71.5 & 4.48 & 125.7 & $2.96648 \mathrm{E}+13$ & $8.90528 \mathrm{E}+12$ & $5.68436 \mathrm{E}+13$ & 1.10805E+20 \\
\hline & EOC $(6 / 11 / 2017,1600)$ & 72.0 & 4.50 & 129.7 & $3.05693 \mathrm{E}+13$ & 9.29977E+12 & $5.84146 \mathrm{E}+13$ & $1.11347 \mathrm{E}+20$ \\
\hline \multirow[t]{10}{*}{$162 \mathrm{~A}$} & \multicolumn{8}{|c|}{ Cycle 162A Outage } \\
\hline & $\mathrm{BOC}$ & 73.0 & 4.56 & 184.1 & $4.21361 \mathrm{E}+13$ & $1.30084 \mathrm{E}+13$ & $8.12193 \mathrm{E}+13$ & $1.12792 \mathrm{E}+20$ \\
\hline & & 75.0 & 4.70 & 177.1 & $4.13643 \mathrm{E}+13$ & $1.21780 \mathrm{E}+13$ & $7.87702 \mathrm{E}+13$ & $1.16164 \mathrm{E}+20$ \\
\hline & & 82.0 & 5.17 & 176.9 & $4.15369 \mathrm{E}+13$ & $1.22773 \mathrm{E}+13$ & $7.90712 \mathrm{E}+13$ & $1.27666 \mathrm{E}+20$ \\
\hline & & 92.0 & 5.82 & 176.3 & $4.08060 \mathrm{E}+13$ & $1.17796 \mathrm{E}+13$ & $7.83372 \mathrm{E}+13$ & $1.43745 \mathrm{E}+20$ \\
\hline & & 102.0 & 6.46 & 177.2 & $4.20309 \mathrm{E}+13$ & 1.19873E+13 & 7.97537E+13 & $1.59764 \mathrm{E}+20$ \\
\hline & & 112.0 & 7.11 & 175.2 & $4.17320 \mathrm{E}+13$ & $1.20896 \mathrm{E}+13$ & 7.99311E+13 & $1.75662 \mathrm{E}+20$ \\
\hline & & 122.0 & 7.76 & 178.1 & $4.41754 \mathrm{E}+13$ & $1.25701 \mathrm{E}+13$ & $8.26092 \mathrm{E}+13$ & $1.91921 \mathrm{E}+20$ \\
\hline & & 131.0 & 8.33 & 168.7 & $4.17710 \mathrm{E}+13$ & $1.20454 \mathrm{E}+13$ & $8.01069 E+13$ & $2.05952 E+20$ \\
\hline & EOC & 134.0 & 8.51 & 166.6 & $4.18789 \mathrm{E}+13$ & $1.18815 \mathrm{E}+13$ & $7.96086 \mathrm{E}+13$ & $2.10409 \mathrm{E}+20$ \\
\hline
\end{tabular}


TEM-10300-1

03/01/2012

Rev. 03

Title:

ATF-1 Power Histories

\begin{tabular}{lllll} 
TEV No.: & 3119 & Rev. No.: & 0 & Project No.: \\
\hline
\end{tabular}

Table 49. ATF-74 (Pellet 7) Power History

\begin{tabular}{|c|c|c|c|c|c|c|c|c|}
\hline Cycle & Critical Dates & EFPD & $\begin{array}{c}\text { Burnup } \\
\text { (GWd/MTU) }\end{array}$ & $\begin{array}{l}\text { LHGR } \\
(\mathrm{W} / \mathrm{cm})\end{array}$ & $\begin{array}{l}\text { Thermal Flux } \\
\left(\mathrm{n} / \mathrm{cm}^{2 *} \mathrm{~s}\right)\end{array}$ & $\begin{array}{c}\text { Fast Flux } \\
\left(\mathrm{n} / \mathrm{cm}^{2 *} \mathrm{~s}>1\right. \\
\mathrm{MeV})\end{array}$ & $\begin{array}{l}\text { Total Flux } \\
\left(\mathrm{n} / \mathrm{cm}^{2 *} \mathrm{~s}\right)\end{array}$ & $\begin{array}{l}\text { Fission Density } \\
\text { (Fissions } / \mathrm{cm}^{3} \text { ) }\end{array}$ \\
\hline \multirow[t]{9}{*}{$160 \mathrm{~B}$} & $\operatorname{BOC}(12 / 20 / 2016,1200)$ & 1.0 & 0.06 & 173.6 & $3.74232 \mathrm{E}+13$ & $1.23465 E+13$ & 7.37754E+13 & $1.38506 \mathrm{E}+18$ \\
\hline & & 3.0 & 0.19 & 173.0 & $3.74964 \mathrm{E}+13$ & $1.20256 \mathrm{E}+13$ & $7.36746 \mathrm{E}+13$ & $4.81760 \mathrm{E}+18$ \\
\hline & & 10.0 & 0.67 & 174.2 & $3.75071 \mathrm{E}+13$ & $1.16895 E+13$ & $7.22474 \mathrm{E}+13$ & $1.65003 \mathrm{E}+19$ \\
\hline & & 20.0 & 1.32 & 166.9 & $3.65702 \mathrm{E}+13$ & $1.15114 \mathrm{E}+13$ & 7.07933E+13 & $3.25790 \mathrm{E}+19$ \\
\hline & & 29.2 & 1.89 & 163.5 & $3.66512 \mathrm{E}+13$ & $1.13552 \mathrm{E}+13$ & $7.08391 \mathrm{E}+13$ & $4.66705 \mathrm{E}+19$ \\
\hline & \multicolumn{8}{|c|}{ Mid-Cycle 160B Outage - 5 Day, 14 Hour Down Time } \\
\hline & & 40.0 & 2.57 & 165.2 & $3.72005 E+13$ & $1.13799 \mathrm{E}+13$ & $7.13232 \mathrm{E}+13$ & $6.35321 \mathrm{E}+19$ \\
\hline & & 50.0 & 3.18 & 158.6 & $3.62443 \mathrm{E}+13$ & $1.11710 \mathrm{E}+13$ & $7.15701 \mathrm{E}+13$ & $7.85871 \mathrm{E}+19$ \\
\hline & EOC $(2 / 23 / 2017,1400)$ & 59.5 & 3.79 & 165.3 & $3.88297 \mathrm{E}+13$ & $1.16396 \mathrm{E}+13$ & $7.51563 E+13$ & $9.35819 \mathrm{E}+19$ \\
\hline \multirow[t]{9}{*}{$161 \mathrm{~A}$} & \multicolumn{8}{|c|}{ Cycle 161A Outage - 95 Day, 13 Hour Down Time } \\
\hline & BOC $(5 / 30 / 2017,0300)$ & 61.2 & 3.88 & 133.6 & $3.08975 E+13$ & $9.07851 \mathrm{E}+12$ & $5.89610 \mathrm{E}+13$ & $9.59907 \mathrm{E}+19$ \\
\hline & & 61.5 & 3.89 & 117.0 & $2.74119 \mathrm{E}+13$ & $7.78408 \mathrm{E}+12$ & 5.17947E+13 & $9.62316 \mathrm{E}+19$ \\
\hline & & 63.5 & 4.00 & 125.5 & $2.91981 \mathrm{E}+13$ & $8.59337 \mathrm{E}+12$ & $5.55163 \mathrm{E}+13$ & $9.88812 \mathrm{E}+19$ \\
\hline & & 65.5 & 4.10 & 124.1 & $2.90321 \mathrm{E}+13$ & $8.67237 E+12$ & $5.58529 \mathrm{E}+13$ & $1.01471 \mathrm{E}+20$ \\
\hline & & 67.5 & 4.21 & 123.1 & $2.84075 \mathrm{E}+13$ & $8.52983 E+12$ & $5.44464 \mathrm{E}+13$ & $1.04120 \mathrm{E}+20$ \\
\hline & & 69.5 & 4.32 & 126.4 & $2.91925 \mathrm{E}+13$ & $8.80194 \mathrm{E}+12$ & $5.60283 E+13$ & $1.06770 \mathrm{E}+20$ \\
\hline & & 71.5 & 4.43 & 128.0 & $2.95769 \mathrm{E}+13$ & $8.91725 E+12$ & $5.73029 \mathrm{E}+13$ & $1.09420 \mathrm{E}+20$ \\
\hline & EOC $(6 / 11 / 2017,1600)$ & 72.0 & 4.45 & 128.9 & $3.02619 \mathrm{E}+13$ & $8.56394 \mathrm{E}+12$ & $5.72978 \mathrm{E}+13$ & $1.09962 \mathrm{E}+20$ \\
\hline \multirow[t]{10}{*}{$162 \mathrm{~A}$} & \multicolumn{8}{|c|}{ Cycle 162A Outage } \\
\hline & $\mathrm{BOC}$ & 73.0 & 4.51 & 181.7 & $4.17936 \mathrm{E}+13$ & $1.26260 \mathrm{E}+13$ & $8.02474 \mathrm{E}+13$ & $1.11407 \mathrm{E}+20$ \\
\hline & & 75.0 & 4.64 & 175.0 & $4.06565 \mathrm{E}+13$ & $1.20719 \mathrm{E}+13$ & $7.80681 \mathrm{E}+13$ & 1.14719E+20 \\
\hline & & 82.0 & 5.09 & 172.0 & $4.02780 \mathrm{E}+13$ & $1.19699 E+13$ & $7.75851 \mathrm{E}+13$ & $1.25920 \mathrm{E}+20$ \\
\hline & & 92.0 & 5.74 & 177.5 & $4.14479 \mathrm{E}+13$ & $1.23958 \mathrm{E}+13$ & 7.87775E+13 & $1.41939 \mathrm{E}+20$ \\
\hline & & 102.0 & 6.41 & 177.3 & $4.15779 \mathrm{E}+13$ & $1.21978 \mathrm{E}+13$ & $7.85892 \mathrm{E}+13$ & $1.58559 \mathrm{E}+20$ \\
\hline & & 112.0 & 7.06 & 176.9 & $4.24906 \mathrm{E}+13$ & $1.21310 \mathrm{E}+13$ & $7.92476 \mathrm{E}+13$ & $1.74457 \mathrm{E}+20$ \\
\hline & & 122.0 & 7.73 & 177.9 & $4.41198 \mathrm{E}+13$ & $1.22946 \mathrm{E}+13$ & $8.22135 \mathrm{E}+13$ & $1.91138 \mathrm{E}+20$ \\
\hline & & 131.0 & 8.30 & 165.9 & $4.18395 \mathrm{E}+13$ & 1.17977E+13 & 7.93283E+13 & $2.05049 E+20$ \\
\hline & EOC & 134.0 & 8.47 & 163.8 & $4.21591 \mathrm{E}+13$ & $1.14460 \mathrm{E}+13$ & $7.93246 \mathrm{E}+13$ & $2.09445 \mathrm{E}+20$ \\
\hline
\end{tabular}


TEM-10300-1

03/01/2012

Rev. 03

Title:

ATF-1 Power Histories

$\begin{array}{lllll}\text { TEV No.: } & 3119 & \text { Rev. No.: } & 0 & \text { Project No.: } \\ & & & \end{array}$

Table 50. ATF-74 (Pellet 8) Power History

\begin{tabular}{|c|c|c|c|c|c|c|c|c|}
\hline Cycle & Critical Dates & EFPD & $\begin{array}{c}\text { Burnup } \\
\text { (GWd/MTU) }\end{array}$ & $\begin{array}{l}\text { LHGR } \\
(\mathrm{W} / \mathrm{cm})\end{array}$ & $\begin{array}{l}\text { Thermal Flux } \\
\left(\mathrm{n} / \mathrm{cm}^{2 *} \mathrm{~s}\right)\end{array}$ & $\begin{array}{c}\text { Fast Flux } \\
\left(\mathrm{n} / \mathrm{cm}^{2 *} \mathrm{~s}>1\right. \\
\mathrm{MeV})\end{array}$ & $\begin{array}{l}\text { Total Flux } \\
\left(\mathrm{n} / \mathrm{cm}^{2 *} \mathrm{~s}\right)\end{array}$ & $\begin{array}{l}\text { Fission Density } \\
\text { (Fissions } / \mathrm{cm}^{3} \text { ) }\end{array}$ \\
\hline \multirow[t]{9}{*}{$160 \mathrm{~B}$} & $\operatorname{BOC}(12 / 20 / 2016,1200)$ & 1.0 & 0.06 & 171.8 & $3.70980 \mathrm{E}+13$ & $1.14002 \mathrm{E}+13$ & $7.27072 \mathrm{E}+13$ & $1.44528 \mathrm{E}+18$ \\
\hline & & 3.0 & 0.20 & 172.3 & $3.73164 \mathrm{E}+13$ & $1.14177 \mathrm{E}+13$ & $7.26692 \mathrm{E}+13$ & $4.87782 \mathrm{E}+18$ \\
\hline & & 10.0 & 0.67 & 173.7 & $3.75191 \mathrm{E}+13$ & $1.17628 \mathrm{E}+13$ & $7.31491 \mathrm{E}+13$ & $1.66207 \mathrm{E}+19$ \\
\hline & & 20.0 & 1.30 & 162.3 & $3.54503 \mathrm{E}+13$ & $1.09783 E+13$ & $6.89555 E+13$ & $3.21575 \mathrm{E}+19$ \\
\hline & & 29.2 & 1.86 & 160.9 & $3.60191 \mathrm{E}+13$ & $1.08381 \mathrm{E}+13$ & $6.94470 \mathrm{E}+13$ & $4.60081 \mathrm{E}+19$ \\
\hline & \multicolumn{8}{|c|}{ Mid-Cycle 160B Outage - 5 Day, 14 Hour Down Time } \\
\hline & & 40.0 & 2.54 & 161.4 & $3.62029 \mathrm{E}+13$ & $1.08688 \mathrm{E}+13$ & $7.05625 \mathrm{E}+13$ & $6.26890 \mathrm{E}+19$ \\
\hline & & 50.0 & 3.14 & 157.0 & $3.55354 \mathrm{E}+13$ & $1.08427 \mathrm{E}+13$ & $7.02020 \mathrm{E}+13$ & $7.76838 \mathrm{E}+19$ \\
\hline & EOC $(2 / 23 / 2017,1400)$ & 59.5 & 3.75 & 163.6 & $3.85870 \mathrm{E}+13$ & $1.10890 E+13$ & $7.38978 \mathrm{E}+13$ & $9.26184 \mathrm{E}+19$ \\
\hline \multirow[t]{9}{*}{$161 \mathrm{~A}$} & \multicolumn{8}{|c|}{ Cycle 161A Outage - 95 Day, 13 Hour Down Time } \\
\hline & BOC $(5 / 30 / 2017,0300)$ & 61.2 & 3.82 & 128.9 & $2.99301 \mathrm{E}+13$ & $8.94243 E+12$ & $5.70597 \mathrm{E}+13$ & $9.44250 \mathrm{E}+19$ \\
\hline & & 61.5 & 3.83 & 119.3 & $2.77011 \mathrm{E}+13$ & $8.16654 \mathrm{E}+12$ & $5.32503 E+13$ & $9.46658 \mathrm{E}+19$ \\
\hline & & 63.5 & 3.94 & 123.9 & $2.83814 \mathrm{E}+13$ & $8.42253 \mathrm{E}+12$ & $5.42762 \mathrm{E}+13$ & $9.73155 \mathrm{E}+19$ \\
\hline & & 65.5 & 4.04 & 123.0 & $2.85595 \mathrm{E}+13$ & $8.32849 E+12$ & $5.42269 \mathrm{E}+13$ & $9.98448 \mathrm{E}+19$ \\
\hline & & 67.5 & 4.14 & 124.8 & $2.88719 \mathrm{E}+13$ & $8.72790 \mathrm{E}+12$ & $5.54258 \mathrm{E}+13$ & $1.02434 \mathrm{E}+20$ \\
\hline & & 69.5 & 4.25 & 128.7 & $2.99727 \mathrm{E}+13$ & $8.88318 \mathrm{E}+12$ & $5.74827 E+13$ & $1.05144 \mathrm{E}+20$ \\
\hline & & 71.5 & 4.36 & 130.2 & $3.06580 \mathrm{E}+13$ & $9.00126 \mathrm{E}+12$ & $5.88270 \mathrm{E}+13$ & $1.07854 \mathrm{E}+20$ \\
\hline & EOC $(6 / 11 / 2017,1600)$ & 72.0 & 4.38 & 129.5 & $3.04217 \mathrm{E}+13$ & $8.86899 \mathrm{E}+12$ & $5.78211 \mathrm{E}+13$ & $1.08396 \mathrm{E}+20$ \\
\hline \multirow[t]{10}{*}{$162 \mathrm{~A}$} & \multicolumn{8}{|c|}{ Cycle 162A Outage } \\
\hline & $\mathrm{BOC}$ & 73.0 & 4.44 & 176.7 & $4.04316 \mathrm{E}+13$ & $1.23072 \mathrm{E}+13$ & $7.82611 \mathrm{E}+13$ & $1.09781 \mathrm{E}+20$ \\
\hline & & 75.0 & 4.57 & 175.1 & $4.04264 \mathrm{E}+13$ & $1.21980 \mathrm{E}+13$ & $7.69602 \mathrm{E}+13$ & $1.13093 \mathrm{E}+20$ \\
\hline & & 82.0 & 5.04 & 175.1 & $4.05288 \mathrm{E}+13$ & $1.23245 E+13$ & $7.74426 \mathrm{E}+13$ & $1.24655 \mathrm{E}+20$ \\
\hline & & 92.0 & 5.67 & 172.0 & $3.99909 \mathrm{E}+13$ & $1.18791 \mathrm{E}+13$ & $7.66175 \mathrm{E}+13$ & $1.40252 \mathrm{E}+20$ \\
\hline & & 102.0 & 6.31 & 172.7 & $4.05827 \mathrm{E}+13$ & $1.16664 \mathrm{E}+13$ & $7.67405 E+13$ & $1.56030 \mathrm{E}+20$ \\
\hline & & 112.0 & 6.94 & 170.2 & $4.07640 \mathrm{E}+13$ & $1.13470 \mathrm{E}+13$ & $7.70364 \mathrm{E}+13$ & $1.71507 \mathrm{E}+20$ \\
\hline & & 122.0 & 7.59 & 174.9 & $4.31250 \mathrm{E}+13$ & $1.16292 \mathrm{E}+13$ & $7.99908 E+13$ & $1.87646 \mathrm{E}+20$ \\
\hline & & 131.0 & 8.15 & 164.5 & $4.09383 E+13$ & $1.10777 \mathrm{E}+13$ & $7.75590 \mathrm{E}+13$ & $2.01556 \mathrm{E}+20$ \\
\hline & EOC & 134.0 & 8.33 & 162.9 & $4.11549 \mathrm{E}+13$ & $1.11866 \mathrm{E}+13$ & $7.80014 \mathrm{E}+13$ & $2.05952 \mathrm{E}+20$ \\
\hline
\end{tabular}


TEM-10300-1

03/01/2012

Rev. 03

Title:

ATF-1 Power Histories

\begin{tabular}{lllll} 
TEV No.: & 3119 & Rev. No.: & 0 & Project No.: \\
\hline
\end{tabular}

Table 51. ATF-74 (Pellet 9) Power History

\begin{tabular}{|c|c|c|c|c|c|c|c|c|}
\hline Cycle & Critical Dates & EFPD & $\begin{array}{c}\text { Burnup } \\
\text { (GWd/MTU) }\end{array}$ & $\begin{array}{l}\text { LHGR } \\
(\mathrm{W} / \mathrm{cm})\end{array}$ & $\begin{array}{l}\text { Thermal Flux } \\
\left(\mathrm{n} / \mathrm{cm}^{2 *} \mathrm{~s}\right)\end{array}$ & $\begin{array}{c}\text { Fast Flux } \\
\left(\mathrm{n} / \mathrm{cm}^{2 *} \mathrm{~s}>1\right. \\
\mathrm{MeV})\end{array}$ & $\begin{array}{l}\text { Total Flux } \\
\left(\mathrm{n} / \mathrm{cm}^{2 *} \mathrm{~s}\right)\end{array}$ & $\begin{array}{l}\text { Fission Density } \\
\text { (Fissions } / \mathrm{cm}^{3} \text { ) }\end{array}$ \\
\hline \multirow[t]{9}{*}{$160 \mathrm{~B}$} & $\operatorname{BOC}(12 / 20 / 2016,1200)$ & 1.0 & 0.06 & 171.1 & $3.65745 \mathrm{E}+13$ & $1.16078 \mathrm{E}+13$ & $7.15964 \mathrm{E}+13$ & $1.44528 \mathrm{E}+18$ \\
\hline & & 3.0 & 0.19 & 171.8 & $3.66786 \mathrm{E}+13$ & $1.16462 \mathrm{E}+13$ & $7.21895 \mathrm{E}+13$ & $4.81760 \mathrm{E}+18$ \\
\hline & & 10.0 & 0.65 & 167.4 & $3.62019 \mathrm{E}+13$ & $1.14547 \mathrm{E}+13$ & $7.10928 \mathrm{E}+13$ & $1.60787 \mathrm{E}+19$ \\
\hline & & 20.0 & 1.27 & 160.7 & $3.49802 \mathrm{E}+13$ & $1.11589 \mathrm{E}+13$ & 6.95907E+13 & $3.14951 \mathrm{E}+19$ \\
\hline & & 29.2 & 1.81 & 153.6 & $3.44536 \mathrm{E}+13$ & $1.10412 \mathrm{E}+13$ & $6.80466 \mathrm{E}+13$ & $4.47435 \mathrm{E}+19$ \\
\hline & \multicolumn{8}{|c|}{ Mid-Cycle 160B Outage - 5 Day, 14 Hour Down Time } \\
\hline & & 40.0 & 2.50 & 160.9 & $3.65136 \mathrm{E}+13$ & $1.09880 \mathrm{E}+13$ & $6.98139 \mathrm{E}+13$ & $6.17255 \mathrm{E}+19$ \\
\hline & & 50.0 & 3.11 & 156.5 & $3.55309 \mathrm{E}+13$ & $1.08633 \mathrm{E}+13$ & $7.00282 \mathrm{E}+13$ & $7.67805 \mathrm{E}+19$ \\
\hline & EOC $(2 / 23 / 2017,1400)$ & 59.5 & 3.72 & 163.3 & $3.79449 \mathrm{E}+13$ & $1.14230 \mathrm{E}+13$ & $7.31454 \mathrm{E}+13$ & $9.19559 \mathrm{E}+19$ \\
\hline \multirow[t]{9}{*}{$161 \mathrm{~A}$} & \multicolumn{8}{|c|}{ Cycle 161A Outage - 95 Day, 13 Hour Down Time } \\
\hline & BOC $(5 / 30 / 2017,0300)$ & 61.2 & 3.82 & 128.9 & $3.03802 E+13$ & $8.70296 \mathrm{E}+12$ & $5.74785 \mathrm{E}+13$ & $9.43647 \mathrm{E}+19$ \\
\hline & & 61.5 & 3.83 & 122.0 & $2.81143 \mathrm{E}+13$ & $8.47593 E+12$ & $5.35403 E+13$ & $9.46658 \mathrm{E}+19$ \\
\hline & & 63.5 & 3.93 & 123.5 & $2.87955 \mathrm{E}+13$ & $8.87377 \mathrm{E}+12$ & $5.48229 \mathrm{E}+13$ & $9.72553 \mathrm{E}+19$ \\
\hline & & 65.5 & 4.04 & 126.1 & $2.91869 \mathrm{E}+13$ & $8.99108 \mathrm{E}+12$ & $5.54038 \mathrm{E}+13$ & $9.99050 \mathrm{E}+19$ \\
\hline & & 67.5 & 4.15 & 124.2 & $2.88367 \mathrm{E}+13$ & $8.06498 \mathrm{E}+12$ & $5.44663 \mathrm{E}+13$ & $1.02555 \mathrm{E}+20$ \\
\hline & & 69.5 & 4.26 & 127.6 & $3.01266 \mathrm{E}+13$ & $8.54781 \mathrm{E}+12$ & $5.63333 \mathrm{E}+13$ & $1.05204 \mathrm{E}+20$ \\
\hline & & 71.5 & 4.36 & 128.3 & $3.00427 \mathrm{E}+13$ & $8.77705 E+12$ & $5.68704 \mathrm{E}+13$ & $1.07854 \mathrm{E}+20$ \\
\hline & EOC $(6 / 11 / 2017,1600)$ & 72.0 & 4.39 & 131.2 & $3.09235 \mathrm{E}+13$ & $8.24337 \mathrm{E}+12$ & $5.74677 \mathrm{E}+13$ & $1.08396 \mathrm{E}+20$ \\
\hline \multirow[t]{10}{*}{$162 \mathrm{~A}$} & \multicolumn{8}{|c|}{ Cycle 162A Outage } \\
\hline & $\mathrm{BOC}$ & 73.0 & 4.44 & 173.7 & $3.99730 \mathrm{E}+13$ & $1.19509 \mathrm{E}+13$ & $7.69542 \mathrm{E}+13$ & $1.09781 \mathrm{E}+20$ \\
\hline & & 75.0 & 4.58 & 171.0 & $3.99319 \mathrm{E}+13$ & $1.16789 \mathrm{E}+13$ & $7.63559 \mathrm{E}+13$ & 1.13093E+20 \\
\hline & & 82.0 & 5.03 & 169.0 & $3.95829 E+13$ & $1.14993 E+13$ & $7.53301 \mathrm{E}+13$ & $1.24234 \mathrm{E}+20$ \\
\hline & & 92.0 & 5.67 & 175.6 & $4.08051 \mathrm{E}+13$ & $1.17473 E+13$ & $7.66629 \mathrm{E}+13$ & $1.40132 \mathrm{E}+20$ \\
\hline & & 102.0 & 6.31 & 172.5 & $4.11615 \mathrm{E}+13$ & $1.12853 E+13$ & 7.71448E+13 & $1.55970 \mathrm{E}+20$ \\
\hline & & 112.0 & 6.94 & 171.8 & $4.13011 \mathrm{E}+13$ & $1.15318 \mathrm{E}+13$ & $7.69932 \mathrm{E}+13$ & $1.71507 \mathrm{E}+20$ \\
\hline & & 122.0 & 7.58 & 174.1 & $4.28289 \mathrm{E}+13$ & $1.15960 \mathrm{E}+13$ & $7.95690 \mathrm{E}+13$ & $1.87405 \mathrm{E}+20$ \\
\hline & & 131.0 & 8.13 & 163.0 & 4.07027E+13 & $1.09113 E+13$ & 7.65599E+13 & $2.01075 E+20$ \\
\hline & EOC & 134.0 & 8.31 & 161.6 & $4.10245 E+13$ & $1.06172 \mathrm{E}+13$ & $7.62715 \mathrm{E}+13$ & $2.05410 \mathrm{E}+20$ \\
\hline
\end{tabular}


TEM-10300-1

03/01/2012

Rev. 03

Title:

ATF-1 Power Histories

$\begin{array}{lllll}\text { TEV No.: } & 3119 & \text { Rev. No.: } & 0 & \text { Project No.: } \\ & & & \end{array}$

Table 52. ATF-74 (Pellet 10) Power History

\begin{tabular}{|c|c|c|c|c|c|c|c|c|}
\hline Cycle & Critical Dates & EFPD & $\begin{array}{c}\text { Burnup } \\
\text { (GWd/MTU) }\end{array}$ & $\begin{array}{l}\text { LHGR } \\
(\mathrm{W} / \mathrm{cm})\end{array}$ & $\begin{array}{l}\text { Thermal Flux } \\
\left(\mathrm{n} / \mathrm{cm}^{2 *} \mathrm{~s}\right)\end{array}$ & $\begin{array}{c}\text { Fast Flux } \\
\left(\mathrm{n} / \mathrm{cm}^{2 *} \mathrm{~s}>1\right. \\
\mathrm{MeV})\end{array}$ & $\begin{array}{l}\text { Total Flux } \\
\left(\mathrm{n} / \mathrm{cm}^{2 *} \mathrm{~s}\right)\end{array}$ & $\begin{array}{l}\text { Fission Density } \\
\text { (Fissions } / \mathrm{cm}^{3} \text { ) }\end{array}$ \\
\hline \multirow[t]{9}{*}{$160 \mathrm{~B}$} & $\operatorname{BOC}(12 / 20 / 2016,1200)$ & 1.0 & 0.06 & 172.6 & $3.71004 \mathrm{E}+13$ & $1.12459 \mathrm{E}+13$ & $7.15999 \mathrm{E}+13$ & $1.44528 \mathrm{E}+18$ \\
\hline & & 3.0 & 0.20 & 173.0 & $3.70354 \mathrm{E}+13$ & $1.15373 E+13$ & $7.21545 E+13$ & $4.87782 \mathrm{E}+18$ \\
\hline & & 10.0 & 0.64 & 168.1 & $3.60634 \mathrm{E}+13$ & $1.09207 E+13$ & $7.02896 \mathrm{E}+13$ & $1.57776 \mathrm{E}+19$ \\
\hline & & 20.0 & 1.28 & 164.9 & $3.58921 \mathrm{E}+13$ & $1.11017 E+13$ & $6.90962 E+13$ & $3.15553 \mathrm{E}+19$ \\
\hline & & 29.2 & 1.84 & 160.8 & $3.57886 \mathrm{E}+13$ & $1.07494 \mathrm{E}+13$ & $6.86827 E+13$ & $4.55263 \mathrm{E}+19$ \\
\hline & \multicolumn{8}{|c|}{ Mid-Cycle 160B Outage - 5 Day, 14 Hour Down Time } \\
\hline & & 40.0 & 2.52 & 162.0 & $3.61611 \mathrm{E}+13$ & $1.10088 \mathrm{E}+13$ & $6.92132 \mathrm{E}+13$ & $6.22073 \mathrm{E}+19$ \\
\hline & & 50.0 & 3.14 & 160.9 & $3.64817 \mathrm{E}+13$ & $1.06688 \mathrm{E}+13$ & $7.09822 E+13$ & $7.76838 \mathrm{E}+19$ \\
\hline & EOC $(2 / 23 / 2017,1400)$ & 59.5 & 3.73 & 163.7 & $3.78998 \mathrm{E}+13$ & $1.09617 \mathrm{E}+13$ & $7.24291 \mathrm{E}+13$ & $9.23173 \mathrm{E}+19$ \\
\hline \multirow[t]{9}{*}{$161 \mathrm{~A}$} & \multicolumn{8}{|c|}{ Cycle 161A Outage - 95 Day, 13 Hour Down Time } \\
\hline & BOC $(5 / 30 / 2017,0300)$ & 61.2 & 3.83 & 131.3 & $3.04587 E+13$ & $8.83229 \mathrm{E}+12$ & $5.73170 \mathrm{E}+13$ & $9.46658 \mathrm{E}+19$ \\
\hline & & 61.5 & 3.84 & 118.2 & $2.73553 \mathrm{E}+13$ & $8.32018 \mathrm{E}+12$ & $5.21400 \mathrm{E}+13$ & $9.49669 \mathrm{E}+19$ \\
\hline & & 63.5 & 3.94 & 121.1 & $2.79673 \mathrm{E}+13$ & $7.96353 \mathrm{E}+12$ & $5.31852 \mathrm{E}+13$ & $9.74962 \mathrm{E}+19$ \\
\hline & & 65.5 & 4.05 & 124.4 & $2.89969 \mathrm{E}+13$ & $7.93940 \mathrm{E}+12$ & $5.45866 \mathrm{E}+13$ & $1.00146 \mathrm{E}+20$ \\
\hline & & 67.5 & 4.16 & 124.4 & $2.86593 \mathrm{E}+13$ & $8.10221 \mathrm{E}+12$ & $5.42443 \mathrm{E}+13$ & $1.02735 \mathrm{E}+20$ \\
\hline & & 69.5 & 4.26 & 124.8 & $2.91701 \mathrm{E}+13$ & $8.26338 \mathrm{E}+12$ & $5.47600 \mathrm{E}+13$ & $1.05325 \mathrm{E}+20$ \\
\hline & & 71.5 & 4.37 & 129.3 & $3.03556 \mathrm{E}+13$ & $8.58853 E+12$ & $5.74925 E+13$ & $1.08035 \mathrm{E}+20$ \\
\hline & EOC $(6 / 11 / 2017,1600)$ & 72.0 & 4.39 & 129.6 & $3.03722 \mathrm{E}+13$ & $8.79649 E+12$ & $5.71273 E+13$ & $1.08577 \mathrm{E}+20$ \\
\hline \multirow[t]{10}{*}{$162 \mathrm{~A}$} & \multicolumn{8}{|c|}{ Cycle 162A Outage } \\
\hline & $\mathrm{BOC}$ & 73.0 & 4.45 & 178.2 & $4.07093 \mathrm{E}+13$ & $1.19907 \mathrm{E}+13$ & $7.80144 \mathrm{E}+13$ & $1.09962 \mathrm{E}+20$ \\
\hline & & 75.0 & 4.58 & 174.0 & $4.05070 \mathrm{E}+13$ & $1.14206 \mathrm{E}+13$ & $7.70426 \mathrm{E}+13$ & $1.13334 \mathrm{E}+20$ \\
\hline & & 82.0 & 5.04 & 173.5 & $4.03426 \mathrm{E}+13$ & $1.14963 E+13$ & $7.68109 \mathrm{E}+13$ & $1.24655 \mathrm{E}+20$ \\
\hline & & 92.0 & 5.68 & 171.5 & $3.98784 \mathrm{E}+13$ & $1.11039 \mathrm{E}+13$ & $7.59899 \mathrm{E}+13$ & $1.40373 \mathrm{E}+20$ \\
\hline & & 102.0 & 6.30 & 170.9 & $4.07611 \mathrm{E}+13$ & $1.11598 \mathrm{E}+13$ & $7.67038 \mathrm{E}+13$ & $1.55849 \mathrm{E}+20$ \\
\hline & & 112.0 & 6.93 & 169.9 & $4.03879 \mathrm{E}+13$ & $1.11311 \mathrm{E}+13$ & $7.62654 \mathrm{E}+13$ & $1.71386 \mathrm{E}+20$ \\
\hline & & 122.0 & 7.57 & 172.3 & $4.24706 \mathrm{E}+13$ & $1.18480 \mathrm{E}+13$ & $7.98930 \mathrm{E}+13$ & $1.87164 \mathrm{E}+20$ \\
\hline & & 131.0 & 8.12 & 163.1 & $4.06609 \mathrm{E}+13$ & $1.10746 \mathrm{E}+13$ & $7.69570 \mathrm{E}+13$ & $2.00834 \mathrm{E}+20$ \\
\hline & EOC & 134.0 & 8.30 & 161.1 & $4.07254 \mathrm{E}+13$ & $1.08678 \mathrm{E}+13$ & $7.65003 \mathrm{E}+13$ & $2.05170 \mathrm{E}+20$ \\
\hline
\end{tabular}


TEM-10300-1

03/01/2012

Rev. 03

Title:

ATF-1 Power Histories

\begin{tabular}{lllll} 
TEV No.: & 3119 & Rev. No.: & 0 & Project No.: \\
\hline
\end{tabular}

Table 53. ATF-74 (Pellet 11) Power History

\begin{tabular}{|c|c|c|c|c|c|c|c|c|}
\hline Cycle & Critical Dates & EFPD & $\begin{array}{c}\text { Burnup } \\
\text { (GWd/MTU) }\end{array}$ & $\begin{array}{l}\text { LHGR } \\
(\mathrm{W} / \mathrm{cm})\end{array}$ & $\begin{array}{l}\text { Thermal Flux } \\
\left(\mathrm{n} / \mathrm{cm}^{2 *} \mathrm{~s}\right)\end{array}$ & $\begin{array}{c}\text { Fast Flux } \\
\left(\mathrm{n} / \mathrm{cm}^{2 *} \mathrm{~s}>1\right. \\
\mathrm{MeV})\end{array}$ & $\begin{array}{l}\text { Total Flux } \\
\left(\mathrm{n} / \mathrm{cm}^{2 *} \mathrm{~s}\right)\end{array}$ & $\begin{array}{l}\text { Fission Density } \\
\text { (Fissions } / \mathrm{cm}^{3} \text { ) }\end{array}$ \\
\hline \multirow[t]{9}{*}{$160 \mathrm{~B}$} & $\operatorname{BOC}(12 / 20 / 2016,1200)$ & 1.0 & 0.06 & 171.8 & $3.71579 \mathrm{E}+13$ & $1.10878 \mathrm{E}+13$ & $7.15777 \mathrm{E}+13$ & $1.44528 \mathrm{E}+18$ \\
\hline & & 3.0 & 0.19 & 171.8 & $3.69768 \mathrm{E}+13$ & $1.10983 E+13$ & $7.13205 E+13$ & $4.81760 \mathrm{E}+18$ \\
\hline & & 10.0 & 0.64 & 169.5 & $3.63671 \mathrm{E}+13$ & $1.08068 \mathrm{E}+13$ & $6.94599 \mathrm{E}+13$ & $1.57776 \mathrm{E}+19$ \\
\hline & & 20.0 & 1.27 & 164.4 & $3.55602 \mathrm{E}+13$ & $1.06508 \mathrm{E}+13$ & $6.86273 E+13$ & $3.14348 \mathrm{E}+19$ \\
\hline & & 29.2 & 1.83 & 159.7 & $3.54941 \mathrm{E}+13$ & $9.89825 \mathrm{E}+12$ & $6.74408 \mathrm{E}+13$ & $4.51650 \mathrm{E}+19$ \\
\hline & \multicolumn{8}{|c|}{ Mid-Cycle 160B Outage - 5 Day, 14 Hour Down Time } \\
\hline & & 40.0 & 2.51 & 164.2 & $3.69126 \mathrm{E}+13$ & $1.08887 \mathrm{E}+13$ & $7.00901 \mathrm{E}+13$ & $6.20266 \mathrm{E}+19$ \\
\hline & & 50.0 & 3.13 & 160.2 & $3.62554 \mathrm{E}+13$ & $1.02061 \mathrm{E}+13$ & $6.92600 \mathrm{E}+13$ & $7.72623 \mathrm{E}+19$ \\
\hline & EOC $(2 / 23 / 2017,1400)$ & 59.5 & 3.74 & 166.2 & $3.83933 E+13$ & $1.06910 \mathrm{E}+13$ & $7.26214 \mathrm{E}+13$ & $9.25581 \mathrm{E}+19$ \\
\hline \multirow[t]{9}{*}{$161 \mathrm{~A}$} & \multicolumn{8}{|c|}{ Cycle 161A Outage - 95 Day, 13 Hour Down Time } \\
\hline & BOC $(5 / 30 / 2017,0300)$ & 61.2 & 3.84 & 129.4 & $3.01899 \mathrm{E}+13$ & $8.34547 \mathrm{E}+12$ & $5.57569 \mathrm{E}+13$ & $9.49669 \mathrm{E}+19$ \\
\hline & & 61.5 & 3.85 & 117.6 & $2.72357 E+13$ & $7.63986 \mathrm{E}+12$ & $5.12156 \mathrm{E}+13$ & $9.52078 \mathrm{E}+19$ \\
\hline & & 63.5 & 3.95 & 120.1 & $2.81126 \mathrm{E}+13$ & $7.95417 \mathrm{E}+12$ & $5.24566 \mathrm{E}+13$ & $9.77371 \mathrm{E}+19$ \\
\hline & & 65.5 & 4.06 & 123.3 & $2.89772 \mathrm{E}+13$ & $7.82386 \mathrm{E}+12$ & $5.32069 \mathrm{E}+13$ & $1.00387 \mathrm{E}+20$ \\
\hline & & 67.5 & 4.17 & 123.8 & $2.84675 \mathrm{E}+13$ & $7.96575 \mathrm{E}+12$ & $5.35466 \mathrm{E}+13$ & $1.02976 \mathrm{E}+20$ \\
\hline & & 69.5 & 4.27 & 122.5 & $2.83783 \mathrm{E}+13$ & $8.24205 E+12$ & $5.39354 \mathrm{E}+13$ & $1.05566 \mathrm{E}+20$ \\
\hline & & 71.5 & 4.38 & 125.1 & $2.89305 E+13$ & $8.05822 \mathrm{E}+12$ & $5.47850 \mathrm{E}+13$ & $1.08215 \mathrm{E}+20$ \\
\hline & EOC $(6 / 11 / 2017,1600)$ & 72.0 & 4.40 & 131.1 & $3.11193 E+13$ & $8.36195 E+12$ & $5.67122 \mathrm{E}+13$ & $1.08697 \mathrm{E}+20$ \\
\hline \multirow[t]{10}{*}{$162 \mathrm{~A}$} & \multicolumn{8}{|c|}{ Cycle 162A Outage } \\
\hline & $\mathrm{BOC}$ & 73.0 & 4.46 & 176.3 & $4.06289 \mathrm{E}+13$ & $1.13527 \mathrm{E}+13$ & $7.64028 \mathrm{E}+13$ & $1.10142 \mathrm{E}+20$ \\
\hline & & 75.0 & 4.59 & 169.7 & $3.96372 \mathrm{E}+13$ & $1.11083 E+13$ & $7.41171 \mathrm{E}+13$ & $1.13394 \mathrm{E}+20$ \\
\hline & & 82.0 & 5.04 & 172.5 & $4.02345 \mathrm{E}+13$ & $1.12750 \mathrm{E}+13$ & $7.51305 \mathrm{E}+13$ & $1.24655 \mathrm{E}+20$ \\
\hline & & 92.0 & 5.69 & 172.2 & $4.00620 \mathrm{E}+13$ & $1.07649 \mathrm{E}+13$ & $7.42795 \mathrm{E}+13$ & $1.40553 \mathrm{E}+20$ \\
\hline & & 102.0 & 6.31 & 169.2 & $3.99262 \mathrm{E}+13$ & $1.10100 \mathrm{E}+13$ & $7.43049 \mathrm{E}+13$ & $1.56030 \mathrm{E}+20$ \\
\hline & & 112.0 & 6.95 & 171.0 & $4.06927 \mathrm{E}+13$ & $1.06730 \mathrm{E}+13$ & $7.46876 \mathrm{E}+13$ & $1.71808 \mathrm{E}+20$ \\
\hline & & 122.0 & 7.58 & 169.4 & $4.15109 \mathrm{E}+13$ & $1.14210 \mathrm{E}+13$ & $7.70795 E+13$ & $1.87344 \mathrm{E}+20$ \\
\hline & & 131.0 & 8.13 & 162.5 & $4.03610 \mathrm{E}+13$ & $1.05683 E+13$ & $7.45254 \mathrm{E}+13$ & $2.00954 \mathrm{E}+20$ \\
\hline & EOC & 134.0 & 8.30 & 159.3 & $4.01841 \mathrm{E}+13$ & $1.03083 E+13$ & $7.39311 \mathrm{E}+13$ & $2.05230 \mathrm{E}+20$ \\
\hline
\end{tabular}


TEM-10300-1

03/01/2012

Rev. 03

Title:

ATF-1 Power Histories

\begin{tabular}{lllll} 
TEV No.: & 3119 & Rev. No.: & 0 & Project No.: \\
\hline
\end{tabular}

Table 54. ATF-74 (Pellet 12) Power History

\begin{tabular}{|c|c|c|c|c|c|c|c|c|}
\hline Cycle & Critical Dates & EFPD & $\begin{array}{c}\text { Burnup } \\
\text { (GWd/MTU) }\end{array}$ & $\begin{array}{l}\text { LHGR } \\
(\mathrm{W} / \mathrm{cm})\end{array}$ & $\begin{array}{l}\text { Thermal Flux } \\
\left(\mathrm{n} / \mathrm{cm}^{2 *} \mathrm{~s}\right)\end{array}$ & $\begin{array}{c}\text { Fast Flux } \\
\left(\mathrm{n} / \mathrm{cm}^{2 *} \mathrm{~s}>1\right. \\
\mathrm{MeV})\end{array}$ & $\begin{array}{l}\text { Total Flux } \\
\left(\mathrm{n} / \mathrm{cm}^{2 *} \mathrm{~s}\right)\end{array}$ & $\begin{array}{l}\text { Fission Density } \\
\left(\text { Fissions } / \mathrm{cm}^{3}\right)\end{array}$ \\
\hline \multirow[t]{9}{*}{$160 \mathrm{~B}$} & BOC $(12 / 20 / 2016,1200)$ & 1.0 & 0.06 & 169.5 & $3.68086 \mathrm{E}+13$ & $1.02262 E+13$ & $6.92536 \mathrm{E}+13$ & $1.38506 \mathrm{E}+18$ \\
\hline & & 3.0 & 0.19 & 168.6 & $3.64925 E+13$ & $1.02496 \mathrm{E}+13$ & $6.89577 \mathrm{E}+13$ & $4.75738 \mathrm{E}+18$ \\
\hline & & 10.0 & 0.67 & 172.1 & $3.67736 \mathrm{E}+13$ & $1.04502 E+13$ & $6.97626 \mathrm{E}+13$ & $1.64401 \mathrm{E}+19$ \\
\hline & & 20.0 & 1.27 & 161.4 & $3.49146 \mathrm{E}+13$ & $1.00687 E+13$ & $6.72802 E+13$ & $3.14348 \mathrm{E}+19$ \\
\hline & & 29.2 & 1.83 & 155.4 & $3.46186 \mathrm{E}+13$ & $1.00004 \mathrm{E}+13$ & $6.66229 E+13$ & $4.51650 \mathrm{E}+19$ \\
\hline & \multicolumn{8}{|c|}{ Mid-Cycle 160B Outage - 5 Day, 14 Hour Down Time } \\
\hline & & 40.0 & 2.48 & 160.3 & $3.58505 \mathrm{E}+13$ & $1.01046 \mathrm{E}+13$ & $6.72783 \mathrm{E}+13$ & $6.11835 \mathrm{E}+19$ \\
\hline & & 50.0 & 3.09 & 158.7 & $3.59132 \mathrm{E}+13$ & $9.97124 \mathrm{E}+12$ & $6.81559 \mathrm{E}+13$ & $7.64192 \mathrm{E}+19$ \\
\hline & EOC $(2 / 23 / 2017,1400)$ & 59.5 & 3.68 & 164.7 & $3.85990 \mathrm{E}+13$ & $1.05586 \mathrm{E}+13$ & $7.13740 \mathrm{E}+13$ & $9.10526 \mathrm{E}+19$ \\
\hline \multirow[t]{9}{*}{$161 \mathrm{~A}$} & \multicolumn{8}{|c|}{ Cycle 161A Outage - 95 Day, 13 Hour Down Time } \\
\hline & BOC $(5 / 30 / 2017,0300)$ & 61.2 & 3.78 & 133.8 & $3.12626 \mathrm{E}+13$ & $8.47568 \mathrm{E}+12$ & $5.68963 E+13$ & $9.34614 \mathrm{E}+19$ \\
\hline & & 61.5 & 3.79 & 114.2 & $2.64967 E+13$ & $7.34625 E+12$ & $4.97382 E+13$ & $9.37023 E+19$ \\
\hline & & 63.5 & 3.90 & 120.6 & $2.84324 \mathrm{E}+13$ & $7.61836 \mathrm{E}+12$ & $5.27526 \mathrm{E}+13$ & $9.62918 \mathrm{E}+19$ \\
\hline & & 65.5 & 4.00 & 123.8 & $2.92886 \mathrm{E}+13$ & $7.65436 \mathrm{E}+12$ & $5.30172 \mathrm{E}+13$ & $9.89415 \mathrm{E}+19$ \\
\hline & & 67.5 & 4.11 & 120.5 & $2.83083 E+13$ & $7.48312 \mathrm{E}+12$ & $5.18811 \mathrm{E}+13$ & $1.01471 \mathrm{E}+20$ \\
\hline & & 69.5 & 4.21 & 124.4 & $2.93366 \mathrm{E}+13$ & $7.91146 \mathrm{E}+12$ & $5.37084 \mathrm{E}+13$ & $1.04120 \mathrm{E}+20$ \\
\hline & & 71.5 & 4.32 & 125.6 & $2.98493 E+13$ & $7.91559 E+12$ & $5.45412 \mathrm{E}+13$ & $1.06710 \mathrm{E}+20$ \\
\hline & EOC $(6 / 11 / 2017,1600)$ & 72.0 & 4.34 & 131.6 & $3.06833 E+13$ & $8.21736 \mathrm{E}+12$ & $5.60664 \mathrm{E}+13$ & $1.07252 \mathrm{E}+20$ \\
\hline \multirow[t]{10}{*}{$162 \mathrm{~A}$} & \multicolumn{8}{|c|}{ Cycle 162A Outage } \\
\hline & $\mathrm{BOC}$ & 73.0 & 4.40 & 179.3 & $4.09118 \mathrm{E}+13$ & $1.10886 E+13$ & $7.68246 E+13$ & $1.08697 \mathrm{E}+20$ \\
\hline & & 75.0 & 4.53 & 169.9 & $3.95135 \mathrm{E}+13$ & $1.08298 \mathrm{E}+13$ & $7.36592 E+13$ & $1.12009 \mathrm{E}+20$ \\
\hline & & 82.0 & 4.99 & 170.3 & $3.97410 \mathrm{E}+13$ & $1.06703 E+13$ & $7.38702 \mathrm{E}+13$ & $1.23270 \mathrm{E}+20$ \\
\hline & & 92.0 & 5.63 & 172.6 & $3.97391 \mathrm{E}+13$ & $1.06950 \mathrm{E}+13$ & $7.37201 \mathrm{E}+13$ & $1.39108 \mathrm{E}+20$ \\
\hline & & 102.0 & 6.26 & 170.0 & $3.98688 \mathrm{E}+13$ & $1.08343 \mathrm{E}+13$ & $7.40899 \mathrm{E}+13$ & $1.54826 \mathrm{E}+20$ \\
\hline & & 112.0 & 6.89 & 171.0 & $4.04669 \mathrm{E}+13$ & $1.05850 \mathrm{E}+13$ & $7.38589 \mathrm{E}+13$ & $1.70423 \mathrm{E}+20$ \\
\hline & & 122.0 & 7.53 & 170.4 & $4.16177 \mathrm{E}+13$ & $1.10624 \mathrm{E}+13$ & $7.69333 E+13$ & $1.86140 \mathrm{E}+20$ \\
\hline & & 131.0 & 8.09 & 162.2 & $4.01088 \mathrm{E}+13$ & $1.01179 \mathrm{E}+13$ & $7.38307 E+13$ & $1.99930 \mathrm{E}+20$ \\
\hline & EOC & 134.0 & 8.26 & 160.9 & $4.03369 \mathrm{E}+13$ & $9.92256 \mathrm{E}+12$ & $7.39085 E+13$ & $2.04266 \mathrm{E}+20$ \\
\hline
\end{tabular}


TEM-10300-1

03/01/2012

Rev. 03

Title:

ATF-1 Power Histories

$\begin{array}{cccccc}\text { TEV No.: } & 3119 & \text { Rev. No.: } & 0 & \text { Project No.: } & 31418 \\ & & \text { Table 55. ATF-74 (Pellet 13 - Bottom) Power History }\end{array}$

\begin{tabular}{|c|c|c|c|c|c|c|c|c|}
\hline Cycle & Critical Dates & EFPD & $\begin{array}{c}\text { Burnup } \\
\text { (GWd/MTU) }\end{array}$ & $\begin{array}{l}\text { LHGR } \\
(\mathrm{W} / \mathrm{cm})\end{array}$ & $\begin{array}{l}\text { Thermal Flux } \\
\left(\mathrm{n} / \mathrm{cm}^{2 *} \mathrm{~s}\right)\end{array}$ & $\begin{array}{c}\text { Fast Flux } \\
\left(\mathrm{n} / \mathrm{cm}^{2 *} \mathrm{~s}>1\right. \\
\mathrm{MeV})\end{array}$ & $\begin{array}{l}\text { Total Flux } \\
\left(\mathrm{n} / \mathrm{cm}^{2 *} \mathrm{~s}\right)\end{array}$ & $\begin{array}{c}\text { Fission Density } \\
\text { (Fissions } / \mathrm{cm}^{3} \text { ) }\end{array}$ \\
\hline \multirow[t]{9}{*}{$160 \mathrm{~B}$} & $\operatorname{BOC}(12 / 20 / 2016,1200)$ & 1.0 & 0.06 & 177.2 & $3.80257 \mathrm{E}+13$ & $1.01575 E+13$ & $6.94269 \mathrm{E}+13$ & $1.44528 \mathrm{E}+18$ \\
\hline & & 3.0 & 0.20 & 179.9 & $3.85817 \mathrm{E}+13$ & $1.02798 \mathrm{E}+13$ & $7.01173 \mathrm{E}+13$ & $4.99826 \mathrm{E}+18$ \\
\hline & & 10.0 & 0.65 & 172.0 & $3.70272 \mathrm{E}+13$ & $9.46194 \mathrm{E}+12$ & $6.72459 \mathrm{E}+13$ & $1.60787 \mathrm{E}+19$ \\
\hline & & 20.0 & 1.30 & 167.1 & $3.63475 \mathrm{E}+13$ & $9.32317 \mathrm{E}+12$ & $6.61568 \mathrm{E}+13$ & 3.20973E+19 \\
\hline & & 29.2 & 1.87 & 162.1 & $3.58796 \mathrm{E}+13$ & $9.10016 \mathrm{E}+12$ & $6.54223 \mathrm{E}+13$ & $4.61887 \mathrm{E}+19$ \\
\hline & \multicolumn{8}{|c|}{ Mid-Cycle 160B Outage - 5 Day, 14 Hour Down Time } \\
\hline & & 40.0 & 2.54 & 163.5 & $3.66074 \mathrm{E}+13$ & $9.23303 E+12$ & $6.66261 \mathrm{E}+13$ & $6.27492 \mathrm{E}+19$ \\
\hline & & 50.0 & 3.16 & 161.7 & $3.64140 \mathrm{E}+13$ & $9.34211 \mathrm{E}+12$ & $6.68087 \mathrm{E}+13$ & $7.80451 \mathrm{E}+19$ \\
\hline & EOC $(2 / 23 / 2017,1400)$ & 59.5 & 3.78 & 169.2 & $3.92491 \mathrm{E}+13$ & $1.00165 E+13$ & $7.09645 E+13$ & $9.33410 \mathrm{E}+19$ \\
\hline \multirow[t]{9}{*}{$161 \mathrm{~A}$} & \multicolumn{8}{|c|}{ Cycle 161A Outage - 95 Day, 13 Hour Down Time } \\
\hline & $\mathrm{BOC}(5 / 30 / 2017,0300)$ & 61.2 & 3.88 & 136.2 & $3.15536 \mathrm{E}+13$ & $7.55210 \mathrm{E}+12$ & $5.58059 \mathrm{E}+13$ & $9.58702 E+19$ \\
\hline & & 61.5 & 3.89 & 122.4 & $2.78254 \mathrm{E}+13$ & $6.96546 \mathrm{E}+12$ & $5.08356 \mathrm{E}+13$ & $9.61713 \mathrm{E}+19$ \\
\hline & & 63.5 & 3.99 & 122.7 & $2.86331 \mathrm{E}+13$ & $7.31515 \mathrm{E}+12$ & $5.16034 \mathrm{E}+13$ & $9.87006 \mathrm{E}+19$ \\
\hline & & 65.5 & 4.10 & 123.8 & $2.87512 \mathrm{E}+13$ & $6.89315 \mathrm{E}+12$ & $5.14170 \mathrm{E}+13$ & $1.01290 \mathrm{E}+20$ \\
\hline & & 67.5 & 4.20 & 126.6 & $2.94196 \mathrm{E}+13$ & $7.20805 E+12$ & $5.25778 \mathrm{E}+13$ & $1.03940 \mathrm{E}+20$ \\
\hline & & 69.5 & 4.31 & 128.4 & $2.97279 \mathrm{E}+13$ & $7.61872 \mathrm{E}+12$ & $5.39021 \mathrm{E}+13$ & $1.06650 \mathrm{E}+20$ \\
\hline & & 71.5 & 4.42 & 129.0 & $3.00974 \mathrm{E}+13$ & $7.58773 \mathrm{E}+12$ & $5.43715 \mathrm{E}+13$ & $1.09299 \mathrm{E}+20$ \\
\hline & EOC (6/11/2017, 1600) & 72.0 & 4.44 & 133.8 & $3.14402 \mathrm{E}+13$ & $7.70214 \mathrm{E}+12$ & $5.59334 \mathrm{E}+13$ & $1.09841 \mathrm{E}+20$ \\
\hline \multirow[t]{10}{*}{$162 \mathrm{~A}$} & \multicolumn{8}{|c|}{ Cycle 162A Outage } \\
\hline & $\mathrm{BOC}$ & 73.0 & 4.50 & 179.3 & $4.08105 \mathrm{E}+13$ & $1.02332 \mathrm{E}+13$ & $7.43264 \mathrm{E}+13$ & 1.11287E+20 \\
\hline & & 75.0 & 4.64 & 177.2 & $4.08628 \mathrm{E}+13$ & $9.95601 \mathrm{E}+12$ & $7.24323 \mathrm{E}+13$ & $1.14659 \mathrm{E}+20$ \\
\hline & & 82.0 & 5.10 & 176.8 & $4.09783 \mathrm{E}+13$ & $9.82460 \mathrm{E}+12$ & $7.29765 E+13$ & $1.26101 \mathrm{E}+20$ \\
\hline & & 92.0 & 5.75 & 173.8 & $4.01944 \mathrm{E}+13$ & $9.75085 E+12$ & $7.19070 \mathrm{E}+13$ & $1.42119 \mathrm{E}+20$ \\
\hline & & 102.0 & 6.39 & 174.6 & $4.13854 \mathrm{E}+13$ & $9.87762 \mathrm{E}+12$ & $7.33399 \mathrm{E}+13$ & $1.58017 \mathrm{E}+20$ \\
\hline & & 112.0 & 7.02 & 171.3 & $4.09163 E+13$ & $9.58446 \mathrm{E}+12$ & $7.28501 \mathrm{E}+13$ & $1.73614 \mathrm{E}+20$ \\
\hline & & 122.0 & 7.67 & 173.9 & $4.29253 \mathrm{E}+13$ & $9.95316 \mathrm{E}+12$ & $7.57316 \mathrm{E}+13$ & $1.89512 \mathrm{E}+20$ \\
\hline & & 131.0 & 8.23 & 164.3 & $4.09113 \mathrm{E}+13$ & $9.82285 E+12$ & $7.30608 \mathrm{E}+13$ & $2.03423 \mathrm{E}+20$ \\
\hline & EOC & 134.0 & 8.41 & 163.1 & $4.12218 \mathrm{E}+13$ & $9.82236 \mathrm{E}+12$ & $7.31288 \mathrm{E}+13$ & $2.07879 \mathrm{E}+20$ \\
\hline
\end{tabular}


TEM-10300-1

03/01/2012

Rev. 03

Title:

ATF-1 Power Histories

\begin{tabular}{lllll} 
TEV No.: & 3119 & Rev. No.: & 0 & Project No.: \\
\hline
\end{tabular}

Table 56. ATF-75 (Pellet 1 - Top) Power History

\begin{tabular}{|c|c|c|c|c|c|c|c|c|}
\hline Cycle & Critical Dates & EFPD & $\begin{array}{c}\text { Burnup } \\
\text { (GWd/MTU) }\end{array}$ & $\begin{array}{c}\text { LHGR } \\
(\mathrm{W} / \mathrm{cm})\end{array}$ & $\begin{array}{l}\text { Thermal Flux } \\
\left(\mathrm{n} / \mathrm{cm}^{2 *} \mathrm{~s}\right)\end{array}$ & $\begin{array}{c}\text { Fast Flux } \\
\left(\mathrm{n} / \mathrm{cm}^{2 *} \mathrm{~s}>1\right. \\
\mathrm{MeV})\end{array}$ & $\begin{array}{l}\text { Total Flux } \\
\left(\mathrm{n} / \mathrm{cm}^{2 *} \mathrm{~s}\right)\end{array}$ & $\begin{array}{l}\text { Fission Density } \\
\text { (Fissions } / \mathrm{cm}^{3} \text { ) }\end{array}$ \\
\hline \multirow[t]{9}{*}{$160 \mathrm{~B}$} & $\operatorname{BOC}(12 / 20 / 2016,1200)$ & 1.0 & 0.07 & 206.3 & $4.33558 \mathrm{E}+13$ & $9.55841 \mathrm{E}+12$ & $7.28956 \mathrm{E}+13$ & $1.68616 \mathrm{E}+18$ \\
\hline & & 3.0 & 0.23 & 204.3 & $4.31476 \mathrm{E}+13$ & $9.58655 \mathrm{E}+12$ & $7.28361 \mathrm{E}+13$ & $5.60046 \mathrm{E}+18$ \\
\hline & & 10.0 & 0.78 & 207.6 & $4.41764 \mathrm{E}+13$ & $9.15323 E+12$ & $7.33372 \mathrm{E}+13$ & $1.92102 \mathrm{E}+19$ \\
\hline & & 20.0 & 1.63 & 222.5 & $4.74833 \mathrm{E}+13$ & $9.97209 E+12$ & $7.77108 \mathrm{E}+13$ & $4.03474 \mathrm{E}+19$ \\
\hline & & 29.2 & 2.43 & 226.3 & $4.99569 \mathrm{E}+13$ & $1.03986 \mathrm{E}+13$ & $8.22206 \mathrm{E}+13$ & $5.99791 \mathrm{E}+19$ \\
\hline & \multicolumn{8}{|c|}{ Mid-Cycle 160B Outage - 5 Day, 14 Hour Down Time } \\
\hline & & 40.0 & 3.41 & 241.2 & $5.34006 \mathrm{E}+13$ & $1.12707 \mathrm{E}+13$ & $8.74337 \mathrm{E}+13$ & $8.43682 \mathrm{E}+19$ \\
\hline & & 50.0 & 4.32 & 237.6 & $5.34675 \mathrm{E}+13$ & $1.09576 \mathrm{E}+13$ & $8.67393 \mathrm{E}+13$ & $1.06830 \mathrm{E}+20$ \\
\hline & EOC $(2 / 23 / 2017,1400)$ & 59.5 & 5.18 & 233.1 & $5.48831 \mathrm{E}+13$ & $1.06635 E+13$ & $8.80041 \mathrm{E}+13$ & $1.27968 \mathrm{E}+20$ \\
\hline \multirow[t]{9}{*}{$161 \mathrm{~A}$} & \multicolumn{8}{|c|}{ Cycle 161A Outage - 95 Day, 13 Hour Down Time } \\
\hline & BOC $(5 / 30 / 2017,0300)$ & 61.2 & 5.31 & 199.6 & $4.65097 \mathrm{E}+13$ & $9.16058 \mathrm{E}+12$ & $7.53475 \mathrm{E}+13$ & $1.31280 \mathrm{E}+20$ \\
\hline & & 61.5 & 5.33 & 186.1 & $4.33720 \mathrm{E}+13$ & $8.34070 \mathrm{E}+12$ & $6.90840 \mathrm{E}+13$ & $1.31761 \mathrm{E}+20$ \\
\hline & & 63.5 & 5.48 & 187.6 & $4.44231 \mathrm{E}+13$ & $8.35092 \mathrm{E}+12$ & $7.10061 \mathrm{E}+13$ & $1.35435 \mathrm{E}+20$ \\
\hline & & 65.5 & 5.62 & 184.8 & $4.37328 \mathrm{E}+13$ & $8.64971 \mathrm{E}+12$ & $7.05349 \mathrm{E}+13$ & $1.39048 \mathrm{E}+20$ \\
\hline & & 67.5 & 5.78 & 192.2 & $4.51028 \mathrm{E}+13$ & $8.79048 \mathrm{E}+12$ & $7.22047 E+13$ & $1.42782 \mathrm{E}+20$ \\
\hline & & 69.5 & 5.93 & 199.4 & $4.74076 \mathrm{E}+13$ & $8.90142 \mathrm{E}+12$ & $7.42097 E+13$ & $1.46636 \mathrm{E}+20$ \\
\hline & & 71.5 & 6.09 & 199.0 & $4.76143 \mathrm{E}+13$ & $8.79191 \mathrm{E}+12$ & $7.50182 E+13$ & $1.50430 \mathrm{E}+20$ \\
\hline & EOC $(6 / 11 / 2017,1600)$ & 72.0 & 6.12 & 195.3 & $4.69883 E+13$ & $9.50342 \mathrm{E}+12$ & $7.52164 \mathrm{E}+13$ & $1.51273 \mathrm{E}+20$ \\
\hline \multirow[t]{10}{*}{$162 \mathrm{~A}$} & \multicolumn{8}{|c|}{ Cycle 162A Outage } \\
\hline & $\mathrm{BOC}$ & 73.0 & 6.18 & 194.2 & $4.72379 \mathrm{E}+13$ & $9.22366 \mathrm{E}+12$ & $7.55582 \mathrm{E}+13$ & $1.52838 \mathrm{E}+20$ \\
\hline & & 75.0 & 6.33 & 184.1 & $4.40833 \mathrm{E}+13$ & $8.91950 \mathrm{E}+12$ & $7.14544 \mathrm{E}+13$ & $1.56391 \mathrm{E}+20$ \\
\hline & & 82.0 & 6.81 & 182.7 & $4.38160 \mathrm{E}+13$ & $8.82159 \mathrm{E}+12$ & $7.05053 \mathrm{E}+13$ & $1.68435 \mathrm{E}+20$ \\
\hline & & 92.0 & 7.50 & 185.1 & $4.48399 \mathrm{E}+13$ & $8.41260 \mathrm{E}+12$ & $7.15392 \mathrm{E}+13$ & $1.85478 \mathrm{E}+20$ \\
\hline & & 102.0 & 8.20 & 184.9 & $4.52338 \mathrm{E}+13$ & $8.31641 \mathrm{E}+12$ & $7.20515 E+13$ & $2.02580 \mathrm{E}+20$ \\
\hline & & 112.0 & 8.87 & 182.2 & $4.50853 \mathrm{E}+13$ & $8.09368 \mathrm{E}+12$ & $7.16323 E+13$ & $2.19261 \mathrm{E}+20$ \\
\hline & & 122.0 & 9.60 & 191.5 & $4.92861 \mathrm{E}+13$ & $8.99078 \mathrm{E}+12$ & $7.76996 \mathrm{E}+13$ & $2.37387 E+20$ \\
\hline & & 131.0 & 10.27 & 194.9 & $5.12603 \mathrm{E}+13$ & $9.34965 E+12$ & $8.07805 E+13$ & $2.53827 E+20$ \\
\hline & EOC & 134.0 & 10.48 & 190.4 & $5.08687 \mathrm{E}+13$ & $9.28418 \mathrm{E}+12$ & $8.04795 E+13$ & $2.59006 \mathrm{E}+20$ \\
\hline
\end{tabular}


TEM-10300-1

03/01/2012

Rev. 03

Title:

ATF-1 Power Histories

$\begin{array}{lllll}\text { TEV No.: } & 3119 & \text { Rev. No.: } & 0 & \text { Project No.: } \\ & & & \end{array}$

Table 57. ATF-75 (Pellet 2) Power History

\begin{tabular}{|c|c|c|c|c|c|c|c|c|}
\hline Cycle & Critical Dates & EFPD & $\begin{array}{c}\text { Burnup } \\
\text { (GWd/MTU) }\end{array}$ & $\begin{array}{l}\text { LHGR } \\
(\mathrm{W} / \mathrm{cm})\end{array}$ & $\begin{array}{l}\text { Thermal Flux } \\
\left(\mathrm{n} / \mathrm{cm}^{2 *} \mathrm{~s}\right)\end{array}$ & $\begin{array}{c}\text { Fast Flux } \\
\left(\mathrm{n} / \mathrm{cm}^{2 *} \mathrm{~s}>1\right. \\
\mathrm{MeV})\end{array}$ & $\begin{array}{l}\text { Total Flux } \\
\left(\mathrm{n} / \mathrm{cm}^{2 *} \mathrm{~s}\right)\end{array}$ & $\begin{array}{l}\text { Fission Density } \\
\text { (Fissions } / \mathrm{cm}^{3} \text { ) }\end{array}$ \\
\hline \multirow[t]{9}{*}{$160 \mathrm{~B}$} & $\operatorname{BOC}(12 / 20 / 2016,1200)$ & 1.0 & 0.07 & 193.0 & $4.06877 \mathrm{E}+13$ & $9.62134 \mathrm{E}+12$ & $6.99333 E+13$ & $1.62594 \mathrm{E}+18$ \\
\hline & & 3.0 & 0.22 & 195.8 & $4.15448 \mathrm{E}+13$ & $9.37150 \mathrm{E}+12$ & $7.07196 \mathrm{E}+13$ & $5.41980 \mathrm{E}+18$ \\
\hline & & 10.0 & 0.75 & 196.8 & $4.18699 \mathrm{E}+13$ & $9.47233 E+12$ & $7.06202 E+13$ & $1.84875 \mathrm{E}+19$ \\
\hline & & 20.0 & 1.56 & 212.7 & $4.56775 \mathrm{E}+13$ & $9.87021 \mathrm{E}+12$ & $7.61161 \mathrm{E}+13$ & $3.86010 \mathrm{E}+19$ \\
\hline & & 29.2 & 2.31 & 212.9 & $4.75566 \mathrm{E}+13$ & $9.88850 \mathrm{E}+12$ & $7.87157 \mathrm{E}+13$ & $5.71488 \mathrm{E}+19$ \\
\hline & \multicolumn{8}{|c|}{ Mid-Cycle 160B Outage - 5 Day, 14 Hour Down Time } \\
\hline & & 40.0 & 3.25 & 224.7 & $5.04465 E+13$ & $1.12198 \mathrm{E}+13$ & $8.44097 \mathrm{E}+13$ & $8.02733 \mathrm{E}+19$ \\
\hline & & 50.0 & 4.09 & 222.5 & $5.03788 \mathrm{E}+13$ & $1.07462 \mathrm{E}+13$ & $8.33601 \mathrm{E}+13$ & $1.01170 \mathrm{E}+20$ \\
\hline & EOC $(2 / 23 / 2017,1400)$ & 59.5 & 4.92 & 224.9 & $5.27884 \mathrm{E}+13$ & $1.04816 \mathrm{E}+13$ & $8.53483 E+13$ & $1.21705 \mathrm{E}+20$ \\
\hline \multirow[t]{9}{*}{$161 \mathrm{~A}$} & \multicolumn{8}{|c|}{ Cycle 161A Outage - 95 Day, 13 Hour Down Time } \\
\hline & BOC $(5 / 30 / 2017,0300)$ & 61.2 & 5.06 & 198.4 & $4.60955 E+13$ & $9.05961 \mathrm{E}+12$ & $7.46533 \mathrm{E}+13$ & $1.25077 \mathrm{E}+20$ \\
\hline & & 61.5 & 5.08 & 182.5 & $4.31816 \mathrm{E}+13$ & $8.81003 E+12$ & $6.98698 \mathrm{E}+13$ & $1.25559 \mathrm{E}+20$ \\
\hline & & 63.5 & 5.22 & 182.2 & $4.28711 \mathrm{E}+13$ & $8.60101 \mathrm{E}+12$ & $7.00033 E+13$ & $1.29112 \mathrm{E}+20$ \\
\hline & & 65.5 & 5.37 & 182.8 & $4.27032 \mathrm{E}+13$ & $8.51563 E+12$ & $6.92726 \mathrm{E}+13$ & $1.32665 \mathrm{E}+20$ \\
\hline & & 67.5 & 5.51 & 181.8 & $4.28873 \mathrm{E}+13$ & $8.39441 \mathrm{E}+12$ & $6.94892 \mathrm{E}+13$ & $1.36157 \mathrm{E}+20$ \\
\hline & & 69.5 & 5.65 & 187.4 & $4.43001 \mathrm{E}+13$ & $8.65888 \mathrm{E}+12$ & $7.11521 \mathrm{E}+13$ & $1.39771 \mathrm{E}+20$ \\
\hline & & 71.5 & 5.81 & 193.0 & $4.54051 \mathrm{E}+13$ & $8.84714 \mathrm{E}+12$ & $7.30847 E+13$ & $1.43504 \mathrm{E}+20$ \\
\hline & EOC $(6 / 11 / 2017,1600)$ & 72.0 & 5.83 & 193.4 & $4.61872 \mathrm{E}+13$ & $9.21397 \mathrm{E}+12$ & $7.37525 \mathrm{E}+13$ & $1.44227 \mathrm{E}+20$ \\
\hline \multirow[t]{10}{*}{$162 \mathrm{~A}$} & \multicolumn{8}{|c|}{ Cycle 162A Outage } \\
\hline & $\mathrm{BOC}$ & 73.0 & 5.90 & 187.5 & $4.56368 \mathrm{E}+13$ & $8.80065 E+12$ & $7.34691 \mathrm{E}+13$ & $1.45793 \mathrm{E}+20$ \\
\hline & & 75.0 & 6.04 & 172.8 & $4.16010 \mathrm{E}+13$ & $8.15914 \mathrm{E}+12$ & $6.73897 E+13$ & $1.49225 \mathrm{E}+20$ \\
\hline & & 82.0 & 6.49 & 173.2 & $4.14194 \mathrm{E}+13$ & $8.13306 \mathrm{E}+12$ & $6.72844 \mathrm{E}+13$ & $1.60547 \mathrm{E}+20$ \\
\hline & & 92.0 & 7.16 & 178.1 & $4.30209 \mathrm{E}+13$ & $8.87916 \mathrm{E}+12$ & $6.97078 E+13$ & $1.77047 \mathrm{E}+20$ \\
\hline & & 102.0 & 7.82 & 178.7 & $4.38190 \mathrm{E}+13$ & $9.02389 \mathrm{E}+12$ & $7.11474 \mathrm{E}+13$ & $1.93427 \mathrm{E}+20$ \\
\hline & & 112.0 & 8.48 & 178.8 & $4.42814 \mathrm{E}+13$ & $8.81190 \mathrm{E}+12$ & $7.17327 \mathrm{E}+13$ & $2.09746 \mathrm{E}+20$ \\
\hline & & 122.0 & 9.17 & 183.1 & $4.67257 \mathrm{E}+13$ & $9.10133 E+12$ & $7.50276 \mathrm{E}+13$ & $2.26608 \mathrm{E}+20$ \\
\hline & & 131.0 & 9.83 & 192.9 & $5.04820 \mathrm{E}+13$ & $8.72674 \mathrm{E}+12$ & $7.92639 E+13$ & $2.42927 E+20$ \\
\hline & EOC & 134.0 & 10.03 & 188.4 & $5.00297 \mathrm{E}+13$ & $8.60561 \mathrm{E}+12$ & $7.88173 \mathrm{E}+13$ & $2.48046 \mathrm{E}+20$ \\
\hline
\end{tabular}


TEM-10300-1

03/01/2012

Rev. 03

Title:

ATF-1 Power Histories

$\begin{array}{lllll}\text { TEV No.: } & 3119 & \text { Rev. No.: } & 0 & \text { Project No.: } \\ & & & \end{array}$

Table 58. ATF-75 (Pellet 3) Power History

\begin{tabular}{|c|c|c|c|c|c|c|c|c|}
\hline Cycle & Critical Dates & EFPD & $\begin{array}{c}\text { Burnup } \\
\text { (GWd/MTU) }\end{array}$ & $\begin{array}{l}\text { LHGR } \\
(\mathrm{W} / \mathrm{cm})\end{array}$ & $\begin{array}{l}\text { Thermal Flux } \\
\left(\mathrm{n} / \mathrm{cm}^{2 *} \mathrm{~s}\right)\end{array}$ & $\begin{array}{c}\text { Fast Flux } \\
\left(\mathrm{n} / \mathrm{cm}^{2 *} \mathrm{~s}>1\right. \\
\mathrm{MeV})\end{array}$ & $\begin{array}{l}\text { Total Flux } \\
\left(\mathrm{n} / \mathrm{cm}^{2 *} \mathrm{~s}\right)\end{array}$ & $\begin{array}{l}\text { Fission Density } \\
\text { (Fissions } / \mathrm{cm}^{3} \text { ) }\end{array}$ \\
\hline \multirow[t]{9}{*}{$160 \mathrm{~B}$} & $\operatorname{BOC}(12 / 20 / 2016,1200)$ & 1.0 & 0.06 & 188.3 & $4.02737 \mathrm{E}+13$ & $9.17066 \mathrm{E}+12$ & $6.75810 \mathrm{E}+13$ & $1.56572 \mathrm{E}+18$ \\
\hline & & 3.0 & 0.21 & 187.8 & $3.99306 \mathrm{E}+13$ & $9.11954 \mathrm{E}+12$ & $6.80110 \mathrm{E}+13$ & $5.23914 \mathrm{E}+18$ \\
\hline & & 10.0 & 0.73 & 193.5 & $4.11152 \mathrm{E}+13$ & $9.26073 \mathrm{E}+12$ & $6.94511 \mathrm{E}+13$ & $1.81262 \mathrm{E}+19$ \\
\hline & & 20.0 & 1.52 & 208.7 & $4.47450 \mathrm{E}+13$ & $1.00580 \mathrm{E}+13$ & $7.51033 E+13$ & $3.76375 \mathrm{E}+19$ \\
\hline & & 29.2 & 2.27 & 209.6 & $4.65642 \mathrm{E}+13$ & $9.74179 \mathrm{E}+12$ & $7.73910 \mathrm{E}+13$ & $5.61250 \mathrm{E}+19$ \\
\hline & \multicolumn{8}{|c|}{ Mid-Cycle 160B Outage - 5 Day, 14 Hour Down Time } \\
\hline & & 40.0 & 3.19 & 221.4 & 4.94377E+13 & $1.08397 \mathrm{E}+13$ & $8.22558 \mathrm{E}+13$ & $7.88882 \mathrm{E}+19$ \\
\hline & & 50.0 & 4.03 & 218.2 & $4.94755 \mathrm{E}+13$ & $1.06977 \mathrm{E}+13$ & $8.19316 \mathrm{E}+13$ & $9.96039 \mathrm{E}+19$ \\
\hline & EOC $(2 / 23 / 2017,1400)$ & 59.5 & 4.84 & 218.9 & $5.11672 \mathrm{E}+13$ & $1.02320 E+13$ & $8.29041 \mathrm{E}+13$ & 1.19717E+20 \\
\hline \multirow[t]{9}{*}{$161 \mathrm{~A}$} & \multicolumn{8}{|c|}{ Cycle 161A Outage - 95 Day, 13 Hour Down Time } \\
\hline & BOC $(5 / 30 / 2017,0300)$ & 61.2 & 4.97 & 193.1 & $4.49731 \mathrm{E}+13$ & $9.03318 \mathrm{E}+12$ & $7.30978 \mathrm{E}+13$ & $1.22909 \mathrm{E}+20$ \\
\hline & & 61.5 & 4.99 & 177.9 & $4.11602 \mathrm{E}+13$ & $8.44736 \mathrm{E}+12$ & $6.74113 \mathrm{E}+13$ & $1.23391 \mathrm{E}+20$ \\
\hline & & 63.5 & 5.13 & 176.8 & $4.18006 \mathrm{E}+13$ & $8.76402 \mathrm{E}+12$ & $6.84817 \mathrm{E}+13$ & $1.26823 \mathrm{E}+20$ \\
\hline & & 65.5 & 5.27 & 177.2 & $4.16214 \mathrm{E}+13$ & $8.19786 \mathrm{E}+12$ & $6.71762 \mathrm{E}+13$ & $1.30256 \mathrm{E}+20$ \\
\hline & & 67.5 & 5.41 & 182.2 & $4.29003 \mathrm{E}+13$ & $8.47501 \mathrm{E}+12$ & $7.00947 \mathrm{E}+13$ & $1.33809 \mathrm{E}+20$ \\
\hline & & 69.5 & 5.56 & 185.0 & $4.35239 \mathrm{E}+13$ & $8.80345 E+12$ & $7.04317 \mathrm{E}+13$ & $1.37422 \mathrm{E}+20$ \\
\hline & & 71.5 & 5.71 & 186.0 & $4.40270 \mathrm{E}+13$ & $8.93876 \mathrm{E}+12$ & $7.14378 \mathrm{E}+13$ & $1.41035 \mathrm{E}+20$ \\
\hline & EOC $(6 / 11 / 2017,1600)$ & 72.0 & 5.74 & 190.0 & $4.53438 \mathrm{E}+13$ & $8.91292 \mathrm{E}+12$ & $7.24536 \mathrm{E}+13$ & $1.41818 \mathrm{E}+20$ \\
\hline \multirow[t]{10}{*}{$162 \mathrm{~A}$} & \multicolumn{8}{|c|}{ Cycle 162A Outage } \\
\hline & $\mathrm{BOC}$ & 73.0 & 5.80 & 178.2 & $4.34015 \mathrm{E}+13$ & $8.66975 \mathrm{E}+12$ & $7.12089 \mathrm{E}+13$ & $1.43324 \mathrm{E}+20$ \\
\hline & & 75.0 & 5.93 & 170.2 & $4.05550 \mathrm{E}+13$ & $8.21960 \mathrm{E}+12$ & $6.69472 \mathrm{E}+13$ & $1.46636 \mathrm{E}+20$ \\
\hline & & 82.0 & 6.39 & 169.3 & $4.04446 \mathrm{E}+13$ & $7.95816 \mathrm{E}+12$ & $6.56029 \mathrm{E}+13$ & $1.57837 \mathrm{E}+20$ \\
\hline & & 92.0 & 7.04 & 173.8 & $4.16498 \mathrm{E}+13$ & $7.79451 \mathrm{E}+12$ & $6.75703 E+13$ & $1.74036 \mathrm{E}+20$ \\
\hline & & 102.0 & 7.69 & 174.4 & $4.20439 \mathrm{E}+13$ & $7.80790 \mathrm{E}+12$ & $6.82542 \mathrm{E}+13$ & $1.89994 \mathrm{E}+20$ \\
\hline & & 112.0 & 8.34 & 174.6 & $4.28647 \mathrm{E}+13$ & $8.02171 \mathrm{E}+12$ & $6.92247 E+13$ & $2.06073 \mathrm{E}+20$ \\
\hline & & 122.0 & 9.03 & 181.1 & $4.53292 \mathrm{E}+13$ & $8.93599 \mathrm{E}+12$ & $7.32770 \mathrm{E}+13$ & $2.23236 \mathrm{E}+20$ \\
\hline & & 131.0 & 9.66 & 181.6 & $4.74293 E+13$ & $9.16898 \mathrm{E}+12$ & $7.63806 \mathrm{E}+13$ & $2.38712 \mathrm{E}+20$ \\
\hline & EOC & 134.0 & 9.86 & 179.9 & $4.78871 \mathrm{E}+13$ & $9.22254 \mathrm{E}+12$ & $7.70002 \mathrm{E}+13$ & $2.43590 \mathrm{E}+20$ \\
\hline
\end{tabular}


TEM-10300-1

03/01/2012

Rev. 03

Title:

ATF-1 Power Histories

$\begin{array}{lllll}\text { TEV No.: } & 3119 & \text { Rev. No.: } & 0 & \text { Project No.: } \\ & & \end{array}$

Table 59. ATF-75 (Pellet 4) Power History

\begin{tabular}{|c|c|c|c|c|c|c|c|c|}
\hline Cycle & Critical Dates & EFPD & $\begin{array}{c}\text { Burnup } \\
\text { (GWd/MTU) }\end{array}$ & $\begin{array}{l}\text { LHGR } \\
(\mathrm{W} / \mathrm{cm})\end{array}$ & $\begin{array}{l}\text { Thermal Flux } \\
\left(\mathrm{n} / \mathrm{cm}^{2 *} \mathrm{~s}\right)\end{array}$ & $\begin{array}{c}\text { Fast Flux } \\
\left(\mathrm{n} / \mathrm{cm}^{2 *} \mathrm{~s}>1\right. \\
\mathrm{MeV})\end{array}$ & $\begin{array}{l}\text { Total Flux } \\
\left(\mathrm{n} / \mathrm{cm}^{2 *} \mathrm{~s}\right)\end{array}$ & $\begin{array}{l}\text { Fission Density } \\
\text { (Fissions } / \mathrm{cm}^{3} \text { ) }\end{array}$ \\
\hline \multirow[t]{9}{*}{$160 \mathrm{~B}$} & $\operatorname{BOC}(12 / 20 / 2016,1200)$ & 1.0 & 0.06 & 179.3 & $3.80132 \mathrm{E}+13$ & $8.85942 \mathrm{E}+12$ & $6.52291 \mathrm{E}+13$ & $1.44528 \mathrm{E}+18$ \\
\hline & & 3.0 & 0.21 & 183.0 & $3.89493 E+13$ & $9.00910 \mathrm{E}+12$ & $6.70675 \mathrm{E}+13$ & $5.11870 \mathrm{E}+18$ \\
\hline & & 10.0 & 0.70 & 183.2 & $3.89110 \mathrm{E}+13$ & $8.77188 \mathrm{E}+12$ & $6.67692 \mathrm{E}+13$ & $1.74036 \mathrm{E}+19$ \\
\hline & & 20.0 & 1.46 & 197.6 & $4.26246 \mathrm{E}+13$ & $9.47718 \mathrm{E}+12$ & $7.22793 \mathrm{E}+13$ & $3.60718 \mathrm{E}+19$ \\
\hline & & 29.2 & 2.17 & 201.1 & $4.50447 \mathrm{E}+13$ & $9.41865 E+12$ & $7.47867 E+13$ & $5.37162 \mathrm{E}+19$ \\
\hline & \multicolumn{8}{|c|}{ Mid-Cycle 160B Outage - 5 Day, 14 Hour Down Time } \\
\hline & & 40.0 & 3.05 & 213.6 & 4.79667E+13 & $1.03730 \mathrm{E}+13$ & $8.00619 \mathrm{E}+13$ & $7.52750 \mathrm{E}+19$ \\
\hline & & 50.0 & 3.85 & 211.7 & $4.77013 \mathrm{E}+13$ & $1.01601 \mathrm{E}+13$ & $7.87621 \mathrm{E}+13$ & $9.52680 \mathrm{E}+19$ \\
\hline & EOC $(2 / 23 / 2017,1400)$ & 59.5 & 4.64 & 212.4 & $4.98397 \mathrm{E}+13$ & $1.02370 \mathrm{E}+13$ & $8.18241 \mathrm{E}+13$ & 1.14719E+20 \\
\hline \multirow[t]{9}{*}{$161 \mathrm{~A}$} & \multicolumn{8}{|c|}{ Cycle 161A Outage - 95 Day, 13 Hour Down Time } \\
\hline & BOC $(5 / 30 / 2017,0300)$ & 61.2 & 4.77 & 184.7 & $4.30166 \mathrm{E}+13$ & $8.59685 E+12$ & $6.98964 \mathrm{E}+13$ & $1.17911 \mathrm{E}+20$ \\
\hline & & 61.5 & 4.79 & 176.5 & $4.05751 \mathrm{E}+13$ & $8.48523 E+12$ & $6.64148 \mathrm{E}+13$ & 1.18393E+20 \\
\hline & & 63.5 & 4.93 & 177.6 & $4.12268 \mathrm{E}+13$ & $8.40389 \mathrm{E}+12$ & $6.77512 \mathrm{E}+13$ & $1.21885 \mathrm{E}+20$ \\
\hline & & 65.5 & 5.07 & 171.7 & $4.00666 \mathrm{E}+13$ & $7.95550 \mathrm{E}+12$ & $6.64751 \mathrm{E}+13$ & $1.25258 \mathrm{E}+20$ \\
\hline & & 67.5 & 5.21 & 175.5 & $4.08619 \mathrm{E}+13$ & $8.65447 E+12$ & $6.75707 E+13$ & $1.28750 \mathrm{E}+20$ \\
\hline & & 69.5 & 5.35 & 179.0 & $4.17921 \mathrm{E}+13$ & $9.17766 \mathrm{E}+12$ & $6.86768 \mathrm{E}+13$ & $1.32243 \mathrm{E}+20$ \\
\hline & & 71.5 & 5.49 & 182.0 & $4.28140 \mathrm{E}+13$ & $9.24638 \mathrm{E}+12$ & $6.97515 E+13$ & $1.35796 \mathrm{E}+20$ \\
\hline & EOC $(6 / 11 / 2017,1600)$ & 72.0 & 5.53 & 184.3 & $4.34995 \mathrm{E}+13$ & $8.86224 \mathrm{E}+12$ & $7.13442 \mathrm{E}+13$ & $1.36579 \mathrm{E}+20$ \\
\hline \multirow[t]{10}{*}{$162 \mathrm{~A}$} & \multicolumn{8}{|c|}{ Cycle 162A Outage } \\
\hline & $\mathrm{BOC}$ & 73.0 & 5.58 & 179.3 & $4.32447 \mathrm{E}+13$ & $8.51103 E+12$ & $6.96767 \mathrm{E}+13$ & $1.38024 \mathrm{E}+20$ \\
\hline & & 75.0 & 5.72 & 160.1 & $3.80464 \mathrm{E}+13$ & $8.24712 \mathrm{E}+12$ & $6.34174 \mathrm{E}+13$ & $1.41276 \mathrm{E}+20$ \\
\hline & & 82.0 & 6.16 & 166.1 & $3.92683 E+13$ & $8.23002 E+12$ & $6.47891 \mathrm{E}+13$ & $1.52176 \mathrm{E}+20$ \\
\hline & & 92.0 & 6.79 & 169.5 & $4.03521 \mathrm{E}+13$ & $8.10438 E+12$ & $6.57757 E+13$ & $1.67773 \mathrm{E}+20$ \\
\hline & & 102.0 & 7.42 & 169.6 & $4.09204 \mathrm{E}+13$ & $7.88154 \mathrm{E}+12$ & $6.65287 E+13$ & $1.83430 \mathrm{E}+20$ \\
\hline & & 112.0 & 8.05 & 169.0 & $4.14114 \mathrm{E}+13$ & $7.90831 \mathrm{E}+12$ & $6.67747 \mathrm{E}+13$ & $1.99087 \mathrm{E}+20$ \\
\hline & & 122.0 & 8.72 & 177.5 & $4.45722 \mathrm{E}+13$ & $8.49422 \mathrm{E}+12$ & $7.17677 E+13$ & $2.15467 \mathrm{E}+20$ \\
\hline & & 131.0 & 9.34 & 183.8 & $4.71360 \mathrm{E}+13$ & $8.95068 E+12$ & $7.58830 E+13$ & $2.30944 \mathrm{E}+20$ \\
\hline & EOC & 134.0 & 9.54 & 179.3 & $4.70421 \mathrm{E}+13$ & $8.91562 E+12$ & $7.50288 \mathrm{E}+13$ & $2.35822 \mathrm{E}+20$ \\
\hline
\end{tabular}


TEM-10300-1

03/01/2012

Rev. 03

Title:

ATF-1 Power Histories

$\begin{array}{lllll}\text { TEV No.: } & 3119 & \text { Rev. No.: } & 0 & \text { Project No.: } \\ & & & \end{array}$

Table 60. ATF-75 (Pellet 5) Power History

\begin{tabular}{|c|c|c|c|c|c|c|c|c|}
\hline Cycle & Critical Dates & EFPD & $\begin{array}{c}\text { Burnup } \\
\text { (GWd/MTU) }\end{array}$ & $\begin{array}{l}\text { LHGR } \\
(\mathrm{W} / \mathrm{cm})\end{array}$ & $\begin{array}{l}\text { Thermal Flux } \\
\left(\mathrm{n} / \mathrm{cm}^{2 *} \mathrm{~s}\right)\end{array}$ & $\begin{array}{c}\text { Fast Flux } \\
\left(\mathrm{n} / \mathrm{cm}^{2 *} \mathrm{~s}>1\right. \\
\mathrm{MeV})\end{array}$ & $\begin{array}{l}\text { Total Flux } \\
\left(\mathrm{n} / \mathrm{cm}^{2 *} \mathrm{~s}\right)\end{array}$ & $\begin{array}{l}\text { Fission Density } \\
\text { (Fissions/cm }{ }^{3} \text { ) }\end{array}$ \\
\hline \multirow[t]{9}{*}{$160 \mathrm{~B}$} & $\operatorname{BOC}(12 / 20 / 2016,1200)$ & 1.0 & 0.06 & 177.1 & $3.78077 \mathrm{E}+13$ & $8.72347 \mathrm{E}+12$ & $6.48111 \mathrm{E}+13$ & $1.44528 \mathrm{E}+18$ \\
\hline & & 3.0 & 0.20 & 181.0 & $3.83666 \mathrm{E}+13$ & $8.61860 \mathrm{E}+12$ & $6.54461 \mathrm{E}+13$ & $5.05848 \mathrm{E}+18$ \\
\hline & & 10.0 & 0.69 & 179.1 & $3.82450 \mathrm{E}+13$ & $8.43528 \mathrm{E}+12$ & $6.45615 \mathrm{E}+13$ & $1.69820 \mathrm{E}+19$ \\
\hline & & 20.0 & 1.42 & 192.6 & $4.17395 \mathrm{E}+13$ & $9.07861 \mathrm{E}+12$ & $7.03978 \mathrm{E}+13$ & $3.51083 E+19$ \\
\hline & & 29.2 & 2.12 & 196.7 & $4.36301 \mathrm{E}+13$ & $9.43736 \mathrm{E}+12$ & $7.24731 \mathrm{E}+13$ & $5.23312 \mathrm{E}+19$ \\
\hline & \multicolumn{8}{|c|}{ Mid-Cycle 160B Outage - 5 Day, 14 Hour Down Time } \\
\hline & & 40.0 & 2.98 & 211.5 & $4.71509 \mathrm{E}+13$ & $1.03532 \mathrm{E}+13$ & $7.84973 \mathrm{E}+13$ & $7.36491 \mathrm{E}+19$ \\
\hline & & 50.0 & 3.78 & 207.0 & $4.66820 \mathrm{E}+13$ & $1.02735 \mathrm{E}+13$ & $7.79278 \mathrm{E}+13$ & $9.33410 \mathrm{E}+19$ \\
\hline & EOC $(2 / 23 / 2017,1400)$ & 59.5 & 4.53 & 205.9 & $4.78385 \mathrm{E}+13$ & $9.90904 \mathrm{E}+12$ & $7.89274 \mathrm{E}+13$ & 1.11889E+20 \\
\hline \multirow[t]{9}{*}{$161 \mathrm{~A}$} & \multicolumn{8}{|c|}{ Cycle 161A Outage - 95 Day, 13 Hour Down Time } \\
\hline & BOC $(5 / 30 / 2017,0300)$ & 61.2 & 4.65 & 177.9 & $4.15324 \mathrm{E}+13$ & $8.98439 \mathrm{E}+12$ & $6.92437 \mathrm{E}+13$ & $1.15020 \mathrm{E}+20$ \\
\hline & & 61.5 & 4.67 & 170.8 & $3.97447 \mathrm{E}+13$ & $8.41188 \mathrm{E}+12$ & $6.51052 \mathrm{E}+13$ & $1.15382 \mathrm{E}+20$ \\
\hline & & 63.5 & 4.80 & 168.8 & $3.99365 \mathrm{E}+13$ & $8.23581 \mathrm{E}+12$ & $6.58183 E+13$ & $1.18754 \mathrm{E}+20$ \\
\hline & & 65.5 & 4.94 & 165.0 & $3.88008 \mathrm{E}+13$ & $7.99814 \mathrm{E}+12$ & $6.46508 \mathrm{E}+13$ & $1.22066 \mathrm{E}+20$ \\
\hline & & 67.5 & 5.07 & 169.1 & $4.01860 \mathrm{E}+13$ & $8.68529 E+12$ & $6.61245 E+13$ & $1.25438 \mathrm{E}+20$ \\
\hline & & 69.5 & 5.22 & 175.2 & $4.08996 \mathrm{E}+13$ & $8.62047 E+12$ & $6.70230 \mathrm{E}+13$ & $1.28931 \mathrm{E}+20$ \\
\hline & & 71.5 & 5.35 & 173.2 & $4.08308 \mathrm{E}+13$ & $8.74493 E+12$ & $6.74328 \mathrm{E}+13$ & $1.32303 \mathrm{E}+20$ \\
\hline & EOC $(6 / 11 / 2017,1600)$ & 72.0 & 5.38 & 179.1 & $4.24729 \mathrm{E}+13$ & $8.94366 \mathrm{E}+12$ & $6.97586 \mathrm{E}+13$ & $1.33026 \mathrm{E}+20$ \\
\hline \multirow[t]{10}{*}{$162 \mathrm{~A}$} & \multicolumn{8}{|c|}{ Cycle 162A Outage } \\
\hline & $\mathrm{BOC}$ & 73.0 & 5.44 & 169.5 & $4.06664 \mathrm{E}+13$ & $8.37555 \mathrm{E}+12$ & $6.69728 \mathrm{E}+13$ & $1.34411 \mathrm{E}+20$ \\
\hline & & 75.0 & 5.57 & 157.3 & $3.74058 \mathrm{E}+13$ & $8.20688 \mathrm{E}+12$ & $6.21631 \mathrm{E}+13$ & $1.37603 \mathrm{E}+20$ \\
\hline & & 82.0 & 6.00 & 159.1 & $3.73319 \mathrm{E}+13$ & $7.78187 E+12$ & $6.19038 E+13$ & $1.48262 \mathrm{E}+20$ \\
\hline & & 92.0 & 6.62 & 164.4 & $3.89045 \mathrm{E}+13$ & $7.78319 \mathrm{E}+12$ & $6.40909 E+13$ & $1.63738 \mathrm{E}+20$ \\
\hline & & 102.0 & 7.24 & 163.8 & $3.92277 \mathrm{E}+13$ & $7.65696 \mathrm{E}+12$ & $6.50551 \mathrm{E}+13$ & $1.79034 \mathrm{E}+20$ \\
\hline & & 112.0 & 7.87 & 164.8 & $3.98113 \mathrm{E}+13$ & $7.64603 \mathrm{E}+12$ & $6.52779 \mathrm{E}+13$ & $1.94450 \mathrm{E}+20$ \\
\hline & & 122.0 & 8.51 & 172.6 & $4.29896 \mathrm{E}+13$ & $8.48052 \mathrm{E}+12$ & $7.02348 E+13$ & $2.10469 \mathrm{E}+20$ \\
\hline & & 131.0 & 9.13 & 177.0 & $4.57676 \mathrm{E}+13$ & $9.06622 E+12$ & $7.39321 \mathrm{E}+13$ & $2.25584 \mathrm{E}+20$ \\
\hline & EOC & 134.0 & 9.32 & 173.0 & $4.53006 \mathrm{E}+13$ & $8.99235 E+12$ & $7.30785 E+13$ & $2.30342 \mathrm{E}+20$ \\
\hline
\end{tabular}


TEM-10300-1

03/01/2012

Rev. 03

Title:

ATF-1 Power Histories

$\begin{array}{lllll}\text { TEV No.: } & 3119 & \text { Rev. No.: } & 0 & \text { Project No.: } 31418\end{array}$

Table 61. ATF-75 (Pellet 6) Power History

\begin{tabular}{|c|c|c|c|c|c|c|c|c|}
\hline Cycle & Critical Dates & EFPD & $\begin{array}{c}\text { Burnup } \\
\text { (GWd/MTU) }\end{array}$ & $\begin{array}{c}\text { LHGR } \\
(\mathrm{W} / \mathrm{cm})\end{array}$ & $\begin{array}{l}\text { Thermal Flux } \\
\left(\mathrm{n} / \mathrm{cm}^{2 *} \mathrm{~s}\right)\end{array}$ & $\begin{array}{c}\text { Fast Flux } \\
\left(\mathrm{n} / \mathrm{cm}^{2 *} \mathrm{~s}>1\right. \\
\mathrm{MeV})\end{array}$ & $\begin{array}{l}\text { Total Flux } \\
\left(\mathrm{n} / \mathrm{cm}^{2 *} \mathrm{~s}\right)\end{array}$ & $\begin{array}{l}\text { Fission Density } \\
\text { (Fissions } / \mathrm{cm}^{3} \text { ) }\end{array}$ \\
\hline \multirow[t]{9}{*}{$160 \mathrm{~B}$} & $\operatorname{BOC}(12 / 20 / 2016,1200)$ & 1.0 & 0.06 & 168.8 & $3.57976 \mathrm{E}+13$ & $8.10343 E+12$ & $6.22224 \mathrm{E}+13$ & $1.38506 \mathrm{E}+18$ \\
\hline & & 3.0 & 0.19 & 171.9 & $3.65271 \mathrm{E}+13$ & $8.32450 \mathrm{E}+12$ & $6.34233 E+13$ & $4.75738 \mathrm{E}+18$ \\
\hline & & 10.0 & 0.63 & 169.1 & $3.60761 \mathrm{E}+13$ & $8.51212 \mathrm{E}+12$ & $6.18759 \mathrm{E}+13$ & $1.56572 \mathrm{E}+19$ \\
\hline & & 20.0 & 1.33 & 182.1 & $3.94343 \mathrm{E}+13$ & $8.76251 \mathrm{E}+12$ & $6.74890 \mathrm{E}+13$ & $3.28199 \mathrm{E}+19$ \\
\hline & & 29.2 & 2.01 & 188.3 & $4.17228 \mathrm{E}+13$ & $9.25665 \mathrm{E}+12$ & $7.07689 \mathrm{E}+13$ & $4.96815 \mathrm{E}+19$ \\
\hline & \multicolumn{8}{|c|}{ Mid-Cycle 160B Outage - 5 Day, 14 Hour Down Time } \\
\hline & & 40.0 & 2.84 & 201.2 & $4.48239 \mathrm{E}+13$ & $9.87460 \mathrm{E}+12$ & $7.57187 \mathrm{E}+13$ & $7.00961 \mathrm{E}+19$ \\
\hline & & 50.0 & 3.58 & 196.8 & $4.41560 \mathrm{E}+13$ & $9.63074 \mathrm{E}+12$ & $7.45278 \mathrm{E}+13$ & $8.85234 \mathrm{E}+19$ \\
\hline & EOC $(2 / 23 / 2017,1400)$ & 59.5 & 4.32 & 201.7 & $4.65456 \mathrm{E}+13$ & $9.99565 E+12$ & $7.73717 \mathrm{E}+13$ & $1.06770 \mathrm{E}+20$ \\
\hline \multirow[t]{9}{*}{$161 \mathrm{~A}$} & \multicolumn{8}{|c|}{ Cycle 161A Outage - 95 Day, 13 Hour Down Time } \\
\hline & BOC $(5 / 30 / 2017,0300)$ & 61.2 & 4.44 & 174.2 & $4.02158 \mathrm{E}+13$ & $8.38261 \mathrm{E}+12$ & $6.67664 \mathrm{E}+13$ & $1.09781 \mathrm{E}+20$ \\
\hline & & 61.5 & 4.46 & 164.7 & $3.78413 \mathrm{E}+13$ & $8.22603 E+12$ & $6.31509 E+13$ & 1.10203E+20 \\
\hline & & 63.5 & 4.60 & 170.0 & $3.88909 \mathrm{E}+13$ & $8.22616 \mathrm{E}+12$ & $6.43505 E+13$ & $1.13635 \mathrm{E}+20$ \\
\hline & & 65.5 & 4.73 & 163.4 & $3.81810 \mathrm{E}+13$ & $7.84151 \mathrm{E}+12$ & $6.28508 \mathrm{E}+13$ & $1.16887 \mathrm{E}+20$ \\
\hline & & 67.5 & 4.86 & 163.5 & $3.80936 \mathrm{E}+13$ & $8.34299 E+12$ & $6.39936 \mathrm{E}+13$ & $1.20079 \mathrm{E}+20$ \\
\hline & & 69.5 & 5.00 & 172.0 & $3.99934 \mathrm{E}+13$ & $8.55079 \mathrm{E}+12$ & $6.60340 \mathrm{E}+13$ & $1.23511 \mathrm{E}+20$ \\
\hline & & 71.5 & 5.13 & 171.4 & $4.00463 E+13$ & $8.76226 \mathrm{E}+12$ & $6.66688 \mathrm{E}+13$ & $1.26884 \mathrm{E}+20$ \\
\hline & EOC $(6 / 11 / 2017,1600)$ & 72.0 & 5.16 & 176.7 & $4.14187 \mathrm{E}+13$ & $8.01065 \mathrm{E}+12$ & $6.74479 \mathrm{E}+13$ & $1.27606 \mathrm{E}+20$ \\
\hline \multirow[t]{10}{*}{$162 \mathrm{~A}$} & \multicolumn{8}{|c|}{ Cycle 162A Outage } \\
\hline & $\mathrm{BOC}$ & 73.0 & 5.22 & 165.8 & $3.96787 \mathrm{E}+13$ & $8.15643 E+12$ & $6.54653 E+13$ & $1.28931 \mathrm{E}+20$ \\
\hline & & 75.0 & 5.34 & 152.7 & $3.59493 \mathrm{E}+13$ & $7.73198 \mathrm{E}+12$ & $5.96883 E+13$ & $1.32002 \mathrm{E}+20$ \\
\hline & & 82.0 & 5.74 & 153.5 & $3.59057 \mathrm{E}+13$ & $7.40987 E+12$ & $6.00140 \mathrm{E}+13$ & $1.41818 \mathrm{E}+20$ \\
\hline & & 92.0 & 6.32 & 156.9 & $3.69488 \mathrm{E}+13$ & $7.77718 \mathrm{E}+12$ & $6.17703 E+13$ & $1.56211 \mathrm{E}+20$ \\
\hline & & 102.0 & 6.91 & 156.6 & $3.75415 \mathrm{E}+13$ & $7.77201 \mathrm{E}+12$ & $6.21625 E+13$ & $1.70904 \mathrm{E}+20$ \\
\hline & & 112.0 & 7.49 & 155.1 & $3.78509 \mathrm{E}+13$ & $7.64957 E+12$ & $6.24803 E+13$ & $1.85056 \mathrm{E}+20$ \\
\hline & & 122.0 & 8.11 & 163.8 & $4.04174 \mathrm{E}+13$ & $8.04079 E+12$ & $6.63073 E+13$ & $2.00472 \mathrm{E}+20$ \\
\hline & & 131.0 & 8.71 & 172.2 & $4.36301 \mathrm{E}+13$ & $8.39122 \mathrm{E}+12$ & $7.04826 E+13$ & $2.15226 \mathrm{E}+20$ \\
\hline & EOC & 134.0 & 8.90 & 169.4 & $4.34676 \mathrm{E}+13$ & $8.31413 \mathrm{E}+12$ & $7.00836 \mathrm{E}+13$ & $2.19923 \mathrm{E}+20$ \\
\hline
\end{tabular}


TEM-10300-1

03/01/2012

Rev. 03

Title:

ATF-1 Power Histories

\begin{tabular}{lllll} 
TEV No.: & 3119 & Rev. No.: & 0 & Project No.: \\
\hline
\end{tabular}

Table 62. ATF-75 (Pellet 7) Power History

\begin{tabular}{|c|c|c|c|c|c|c|c|c|}
\hline Cycle & Critical Dates & EFPD & $\begin{array}{c}\text { Burnup } \\
\text { (GWd/MTU) }\end{array}$ & $\begin{array}{l}\text { LHGR } \\
(\mathrm{W} / \mathrm{cm})\end{array}$ & $\begin{array}{l}\text { Thermal Flux } \\
\left(\mathrm{n} / \mathrm{cm}^{2 *} \mathrm{~s}\right)\end{array}$ & $\begin{array}{c}\text { Fast Flux } \\
\left(\mathrm{n} / \mathrm{cm}^{2 *} \mathrm{~s}>1\right. \\
\mathrm{MeV})\end{array}$ & $\begin{array}{l}\text { Total Flux } \\
\left(\mathrm{n} / \mathrm{cm}^{2 *} \mathrm{~s}\right)\end{array}$ & $\begin{array}{l}\text { Fission Density } \\
\text { (Fissions/cm }{ }^{3} \text { ) }\end{array}$ \\
\hline \multirow[t]{9}{*}{$160 \mathrm{~B}$} & $\operatorname{BOC}(12 / 20 / 2016,1200)$ & 1.0 & 0.05 & 164.2 & $3.48985 \mathrm{E}+13$ & $7.81270 \mathrm{E}+12$ & $5.97816 \mathrm{E}+13$ & $1.32484 \mathrm{E}+18$ \\
\hline & & 3.0 & 0.19 & 167.5 & $3.53853 \mathrm{E}+13$ & $8.39066 \mathrm{E}+12$ & $6.12084 \mathrm{E}+13$ & $4.69716 \mathrm{E}+18$ \\
\hline & & 10.0 & 0.65 & 166.6 & $3.56081 \mathrm{E}+13$ & $8.46641 \mathrm{E}+12$ & $6.13209 E+13$ & $1.60185 \mathrm{E}+19$ \\
\hline & & 20.0 & 1.33 & 178.2 & $3.88012 \mathrm{E}+13$ & $8.98898 \mathrm{E}+12$ & $6.66461 \mathrm{E}+13$ & $3.28801 \mathrm{E}+19$ \\
\hline & & 29.2 & 1.97 & 183.6 & $4.09682 \mathrm{E}+13$ & $9.37017 \mathrm{E}+12$ & $6.95469 \mathrm{E}+13$ & $4.87180 \mathrm{E}+19$ \\
\hline & \multicolumn{8}{|c|}{ Mid-Cycle 160B Outage - 5 Day, 14 Hour Down Time } \\
\hline & & 40.0 & 2.79 & 197.6 & $4.40170 \mathrm{E}+13$ & $9.71290 \mathrm{E}+12$ & $7.36119 \mathrm{E}+13$ & $6.88917 \mathrm{E}+19$ \\
\hline & & 50.0 & 3.52 & 193.5 & $4.35980 \mathrm{E}+13$ & $9.64103 E+12$ & $7.29162 \mathrm{E}+13$ & $8.70179 \mathrm{E}+19$ \\
\hline & EOC $(2 / 23 / 2017,1400)$ & 59.5 & 4.23 & 195.7 & $4.53362 \mathrm{E}+13$ & $1.00922 E+13$ & $7.55984 \mathrm{E}+13$ & $1.04482 \mathrm{E}+20$ \\
\hline \multirow[t]{9}{*}{$161 \mathrm{~A}$} & \multicolumn{8}{|c|}{ Cycle 161A Outage - 95 Day, 13 Hour Down Time } \\
\hline & BOC $(5 / 30 / 2017,0300)$ & 61.2 & 4.34 & 163.1 & $3.79458 \mathrm{E}+13$ & $8.55787 \mathrm{E}+12$ & $6.33042 \mathrm{E}+13$ & $1.07312 \mathrm{E}+20$ \\
\hline & & 61.5 & 4.36 & 159.6 & $3.71233 \mathrm{E}+13$ & $7.61767 \mathrm{E}+12$ & $6.09570 \mathrm{E}+13$ & $1.07734 \mathrm{E}+20$ \\
\hline & & 63.5 & 4.49 & 161.9 & $3.72175 \mathrm{E}+13$ & $8.17035 E+12$ & $6.19340 \mathrm{E}+13$ & $1.10985 \mathrm{E}+20$ \\
\hline & & 65.5 & 4.62 & 157.8 & $3.66738 \mathrm{E}+13$ & $7.96099 \mathrm{E}+12$ & $6.12436 \mathrm{E}+13$ & $1.14177 \mathrm{E}+20$ \\
\hline & & 67.5 & 4.75 & 162.8 & $3.80037 \mathrm{E}+13$ & $8.20045 E+12$ & $6.28880 \mathrm{E}+13$ & $1.17429 \mathrm{E}+20$ \\
\hline & & 69.5 & 4.88 & 162.4 & $3.81138 \mathrm{E}+13$ & $7.93530 \mathrm{E}+12$ & $6.33283 E+13$ & $1.20681 \mathrm{E}+20$ \\
\hline & & 71.5 & 5.01 & 167.3 & $3.88471 \mathrm{E}+13$ & $8.33291 \mathrm{E}+12$ & $6.50600 \mathrm{E}+13$ & $1.23933 \mathrm{E}+20$ \\
\hline & EOC $(6 / 11 / 2017,1600)$ & 72.0 & 5.04 & 167.3 & $3.95215 \mathrm{E}+13$ & $8.24659 \mathrm{E}+12$ & $6.51770 \mathrm{E}+13$ & $1.24595 \mathrm{E}+20$ \\
\hline \multirow[t]{10}{*}{$162 \mathrm{~A}$} & \multicolumn{8}{|c|}{ Cycle 162A Outage } \\
\hline & $\mathrm{BOC}$ & 73.0 & 5.09 & 160.9 & $3.84141 \mathrm{E}+13$ & $8.08487 E+12$ & $6.35065 E+13$ & $1.25920 \mathrm{E}+20$ \\
\hline & & 75.0 & 5.22 & 148.7 & $3.52299 \mathrm{E}+13$ & $7.53504 \mathrm{E}+12$ & $5.91846 \mathrm{E}+13$ & $1.28931 \mathrm{E}+20$ \\
\hline & & 82.0 & 5.60 & 147.0 & $3.47090 \mathrm{E}+13$ & $7.46306 \mathrm{E}+12$ & $5.81648 \mathrm{E}+13$ & $1.38446 \mathrm{E}+20$ \\
\hline & & 92.0 & 6.18 & 156.3 & $3.67817 \mathrm{E}+13$ & $7.91035 E+12$ & $6.08254 \mathrm{E}+13$ & $1.52778 \mathrm{E}+20$ \\
\hline & & 102.0 & 6.78 & 158.0 & $3.73173 \mathrm{E}+13$ & $8.09194 \mathrm{E}+12$ & $6.21363 E+13$ & $1.67713 \mathrm{E}+20$ \\
\hline & & 112.0 & 7.38 & 157.5 & $3.77842 \mathrm{E}+13$ & $7.92363 E+12$ & $6.26556 \mathrm{E}+13$ & $1.82527 \mathrm{E}+20$ \\
\hline & & 122.0 & 7.99 & 158.6 & $3.95128 \mathrm{E}+13$ & $8.15987 E+12$ & $6.52963 E+13$ & $1.97522 \mathrm{E}+20$ \\
\hline & & 131.0 & 8.59 & 170.3 & $4.33455 \mathrm{E}+13$ & $8.64629 E+12$ & $7.05891 \mathrm{E}+13$ & $2.12215 E+20$ \\
\hline & EOC & 134.0 & 8.77 & 166.5 & $4.27271 \mathrm{E}+13$ & $8.35720 \mathrm{E}+12$ & $6.99433 E+13$ & $2.16792 \mathrm{E}+20$ \\
\hline
\end{tabular}


TEM-10300-1

03/01/2012

Rev. 03

Title:

ATF-1 Power Histories

$\begin{array}{lllll}\text { TEV No.: } & 3119 & \text { Rev. No.: } & 0 & \text { Project No.: } 31418\end{array}$

Table 63. ATF-75 (Pellet 8) Power History

\begin{tabular}{|c|c|c|c|c|c|c|c|c|}
\hline Cycle & Critical Dates & EFPD & $\begin{array}{c}\text { Burnup } \\
\text { (GWd/MTU) }\end{array}$ & $\begin{array}{l}\text { LHGR } \\
(\mathrm{W} / \mathrm{cm})\end{array}$ & $\begin{array}{l}\text { Thermal Flux } \\
\left(\mathrm{n} / \mathrm{cm}^{2 *} \mathrm{~s}\right)\end{array}$ & $\begin{array}{c}\text { Fast Flux } \\
\left(\mathrm{n} / \mathrm{cm}^{2 *} \mathrm{~s}>1\right. \\
\mathrm{MeV})\end{array}$ & $\begin{array}{l}\text { Total Flux } \\
\left(\mathrm{n} / \mathrm{cm}^{2 *} \mathrm{~s}\right)\end{array}$ & $\begin{array}{l}\text { Fission Density } \\
\text { (Fissions } / \mathrm{cm}^{3} \text { ) }\end{array}$ \\
\hline \multirow[t]{9}{*}{$160 \mathrm{~B}$} & $\operatorname{BOC}(12 / 20 / 2016,1200)$ & 1.0 & 0.05 & 161.5 & $3.42253 \mathrm{E}+13$ & $7.79516 \mathrm{E}+12$ & $5.88282 \mathrm{E}+13$ & $1.32484 \mathrm{E}+18$ \\
\hline & & 3.0 & 0.19 & 161.3 & $3.42407 \mathrm{E}+13$ & $7.88827 E+12$ & $5.91910 \mathrm{E}+13$ & $4.63694 \mathrm{E}+18$ \\
\hline & & 10.0 & 0.61 & 159.4 & $3.42052 \mathrm{E}+13$ & $7.85583 E+12$ & $5.84070 \mathrm{E}+13$ & $1.49948 \mathrm{E}+19$ \\
\hline & & 20.0 & 1.27 & 171.0 & $3.66363 \mathrm{E}+13$ & $8.56412 \mathrm{E}+12$ & $6.34771 \mathrm{E}+13$ & $3.13746 \mathrm{E}+19$ \\
\hline & & 29.2 & 1.91 & 178.9 & $3.91350 \mathrm{E}+13$ & $8.82597 E+12$ & $6.63609 \mathrm{E}+13$ & $4.72727 \mathrm{E}+19$ \\
\hline & \multicolumn{8}{|c|}{ Mid-Cycle 160B Outage - 5 Day, 14 Hour Down Time } \\
\hline & & 40.0 & 2.68 & 189.6 & $4.18469 \mathrm{E}+13$ & $9.37246 \mathrm{E}+12$ & $7.09165 \mathrm{E}+13$ & $6.62420 \mathrm{E}+19$ \\
\hline & & 50.0 & 3.39 & 187.6 & $4.20848 \mathrm{E}+13$ & $9.29480 \mathrm{E}+12$ & $7.08424 \mathrm{E}+13$ & $8.37058 \mathrm{E}+19$ \\
\hline & EOC $(2 / 23 / 2017,1400)$ & 59.5 & 4.08 & 187.7 & $4.31268 \mathrm{E}+13$ & $9.79516 \mathrm{E}+12$ & $7.20540 \mathrm{E}+13$ & $1.00869 \mathrm{E}+20$ \\
\hline \multirow[t]{9}{*}{$161 \mathrm{~A}$} & \multicolumn{8}{|c|}{ Cycle 161A Outage - 95 Day, 13 Hour Down Time } \\
\hline & BOC $(5 / 30 / 2017,0300)$ & 61.2 & 4.20 & 165.1 & $3.80154 \mathrm{E}+13$ & $8.04936 \mathrm{E}+12$ & $6.25524 \mathrm{E}+13$ & $1.03759 \mathrm{E}+20$ \\
\hline & & 61.5 & 4.21 & 153.2 & $3.55044 \mathrm{E}+13$ & $7.42825 \mathrm{E}+12$ & $5.91584 \mathrm{E}+13$ & $1.04120 \mathrm{E}+20$ \\
\hline & & 63.5 & 4.34 & 154.2 & $3.55097 \mathrm{E}+13$ & $7.63226 \mathrm{E}+12$ & $5.92255 \mathrm{E}+13$ & $1.07252 \mathrm{E}+20$ \\
\hline & & 65.5 & 4.47 & 153.7 & $3.58247 \mathrm{E}+13$ & $7.67333 \mathrm{E}+12$ & $5.96223 \mathrm{E}+13$ & $1.10383 \mathrm{E}+20$ \\
\hline & & 67.5 & 4.59 & 153.8 & $3.57929 \mathrm{E}+13$ & $7.74368 \mathrm{E}+12$ & $5.94658 \mathrm{E}+13$ & $1.13454 \mathrm{E}+20$ \\
\hline & & 69.5 & 4.72 & 156.4 & $3.64956 \mathrm{E}+13$ & $7.79002 E+12$ & $6.04867 \mathrm{E}+13$ & $1.16646 \mathrm{E}+20$ \\
\hline & & 71.5 & 4.85 & 159.1 & $3.74494 \mathrm{E}+13$ & $8.40489 \mathrm{E}+12$ & $6.22382 \mathrm{E}+13$ & 1.19778E+20 \\
\hline & EOC $(6 / 11 / 2017,1600)$ & 72.0 & 4.87 & 161.6 & $3.79115 \mathrm{E}+13$ & $8.06161 \mathrm{E}+12$ & $6.29812 \mathrm{E}+13$ & $1.20500 \mathrm{E}+20$ \\
\hline \multirow[t]{10}{*}{$162 \mathrm{~A}$} & \multicolumn{8}{|c|}{ Cycle 162A Outage } \\
\hline & $\mathrm{BOC}$ & 73.0 & 4.92 & 153.4 & $3.64314 \mathrm{E}+13$ & $7.80737 \mathrm{E}+12$ & $6.06736 \mathrm{E}+13$ & $1.21705 \mathrm{E}+20$ \\
\hline & & 75.0 & 5.05 & 143.3 & $3.38545 \mathrm{E}+13$ & $7.68015 \mathrm{E}+12$ & $5.66739 \mathrm{E}+13$ & $1.24716 \mathrm{E}+20$ \\
\hline & & 82.0 & 5.41 & 142.1 & $3.28253 E+13$ & $7.12864 \mathrm{E}+12$ & $5.51966 \mathrm{E}+13$ & $1.33749 \mathrm{E}+20$ \\
\hline & & 92.0 & 5.97 & 150.8 & $3.55009 \mathrm{E}+13$ & $7.40528 \mathrm{E}+12$ & $5.83564 \mathrm{E}+13$ & $1.47659 \mathrm{E}+20$ \\
\hline & & 102.0 & 6.54 & 153.0 & $3.62549 \mathrm{E}+13$ & $7.42928 \mathrm{E}+12$ & $5.93268 \mathrm{E}+13$ & $1.61691 \mathrm{E}+20$ \\
\hline & & 112.0 & 7.10 & 151.4 & $3.63996 \mathrm{E}+13$ & $7.35174 \mathrm{E}+12$ & $6.00757 \mathrm{E}+13$ & $1.75481 \mathrm{E}+20$ \\
\hline & & 122.0 & 7.70 & 156.9 & $3.83484 \mathrm{E}+13$ & $8.13664 \mathrm{E}+12$ & $6.34263 E+13$ & $1.90295 \mathrm{E}+20$ \\
\hline & & 131.0 & 8.26 & 161.9 & $4.10344 \mathrm{E}+13$ & $8.21044 E+12$ & $6.68641 \mathrm{E}+13$ & $2.04206 E+20$ \\
\hline & EOC & 134.0 & 8.44 & 159.4 & $4.08665 \mathrm{E}+13$ & $8.17914 \mathrm{E}+12$ & $6.67051 \mathrm{E}+13$ & $2.08542 \mathrm{E}+20$ \\
\hline
\end{tabular}


TEM-10300-1

03/01/2012

Rev. 03

Title:

ATF-1 Power Histories

\begin{tabular}{lllll} 
TEV No.: & 3119 & Rev. No.: & 0 & Project No.: \\
\hline
\end{tabular}

Table 64. ATF-75 (Pellet 9) Power History

\begin{tabular}{|c|c|c|c|c|c|c|c|c|}
\hline Cycle & Critical Dates & EFPD & $\begin{array}{c}\text { Burnup } \\
\text { (GWd/MTU) }\end{array}$ & $\begin{array}{l}\text { LHGR } \\
(\mathrm{W} / \mathrm{cm})\end{array}$ & $\begin{array}{l}\text { Thermal Flux } \\
\left(\mathrm{n} / \mathrm{cm}^{2 *} \mathrm{~s}\right)\end{array}$ & $\begin{array}{c}\text { Fast Flux } \\
\left(\mathrm{n} / \mathrm{cm}^{2 *} \mathrm{~s}>1\right. \\
\mathrm{MeV})\end{array}$ & $\begin{array}{l}\text { Total Flux } \\
\left(\mathrm{n} / \mathrm{cm}^{2 *} \mathrm{~s}\right)\end{array}$ & $\begin{array}{l}\text { Fission Density } \\
\text { (Fissions } / \mathrm{cm}^{3} \text { ) }\end{array}$ \\
\hline \multirow[t]{9}{*}{$160 \mathrm{~B}$} & $\operatorname{BOC}(12 / 20 / 2016,1200)$ & 1.0 & 0.05 & 155.8 & $3.28425 \mathrm{E}+13$ & $7.62489 \mathrm{E}+12$ & $5.68725 \mathrm{E}+13$ & $1.32484 \mathrm{E}+18$ \\
\hline & & 3.0 & 0.18 & 156.8 & $3.33642 E+13$ & $7.79110 \mathrm{E}+12$ & $5.80019 \mathrm{E}+13$ & $4.45628 \mathrm{E}+18$ \\
\hline & & 10.0 & 0.59 & 155.7 & $3.31954 \mathrm{E}+13$ & $7.74176 \mathrm{E}+12$ & $5.69938 \mathrm{E}+13$ & $1.45732 \mathrm{E}+19$ \\
\hline & & 20.0 & 1.24 & 166.0 & $3.57205 E+13$ & $8.28468 \mathrm{E}+12$ & $6.08366 \mathrm{E}+13$ & $3.05315 \mathrm{E}+19$ \\
\hline & & 29.2 & 1.85 & 170.1 & $3.76312 \mathrm{E}+13$ & $8.66747 E+12$ & $6.38320 \mathrm{E}+13$ & $4.57672 \mathrm{E}+19$ \\
\hline & \multicolumn{8}{|c|}{ Mid-Cycle 160B Outage - 5 Day, 14 Hour Down Time } \\
\hline & & 40.0 & 2.61 & 186.6 & $4.07898 \mathrm{E}+13$ & $8.80955 E+12$ & $6.82588 \mathrm{E}+13$ & $6.44354 \mathrm{E}+19$ \\
\hline & & 50.0 & 3.30 & 180.7 & $4.07176 \mathrm{E}+13$ & $9.32399 \mathrm{E}+12$ & $6.86693 \mathrm{E}+13$ & $8.15379 \mathrm{E}+19$ \\
\hline & EOC $(2 / 23 / 2017,1400)$ & 59.5 & 3.97 & 183.4 & $4.18369 \mathrm{E}+13$ & $9.13734 \mathrm{E}+12$ & $7.03353 E+13$ & $9.81586 \mathrm{E}+19$ \\
\hline \multirow[t]{9}{*}{$161 \mathrm{~A}$} & \multicolumn{8}{|c|}{ Cycle 161A Outage - 95 Day, 13 Hour Down Time } \\
\hline & BOC $(5 / 30 / 2017,0300)$ & 61.2 & 4.08 & 159.7 & $3.64356 \mathrm{E}+13$ & $8.09019 \mathrm{E}+12$ & $6.00409 \mathrm{E}+13$ & $1.00929 \mathrm{E}+20$ \\
\hline & & 61.5 & 4.10 & 149.2 & $3.47072 \mathrm{E}+13$ & $6.63141 \mathrm{E}+12$ & $5.68635 \mathrm{E}+13$ & $1.01290 \mathrm{E}+20$ \\
\hline & & 63.5 & 4.22 & 150.2 & $3.47826 \mathrm{E}+13$ & $7.26305 E+12$ & $5.68738 \mathrm{E}+13$ & $1.04301 \mathrm{E}+20$ \\
\hline & & 65.5 & 4.34 & 149.6 & $3.49598 \mathrm{E}+13$ & $7.32957 E+12$ & $5.76975 \mathrm{E}+13$ & $1.07312 \mathrm{E}+20$ \\
\hline & & 67.5 & 4.47 & 154.6 & $3.58619 \mathrm{E}+13$ & $7.44511 \mathrm{E}+12$ & $5.96156 \mathrm{E}+13$ & $1.10443 \mathrm{E}+20$ \\
\hline & & 69.5 & 4.59 & 153.9 & $3.61472 \mathrm{E}+13$ & $8.06655 E+12$ & $6.03279 \mathrm{E}+13$ & $1.13575 \mathrm{E}+20$ \\
\hline & & 71.5 & 4.72 & 157.4 & $3.71225 \mathrm{E}+13$ & $8.12681 \mathrm{E}+12$ & $6.15279 \mathrm{E}+13$ & 1.16767E+20 \\
\hline & EOC $(6 / 11 / 2017,1600)$ & 72.0 & 4.75 & 157.8 & $3.71295 \mathrm{E}+13$ & $8.07398 \mathrm{E}+12$ & $6.15341 \mathrm{E}+13$ & $1.17429 \mathrm{E}+20$ \\
\hline \multirow[t]{10}{*}{$162 \mathrm{~A}$} & \multicolumn{8}{|c|}{ Cycle 162A Outage } \\
\hline & $\mathrm{BOC}$ & 73.0 & 4.80 & 149.0 & $3.52209 \mathrm{E}+13$ & $7.35091 \mathrm{E}+12$ & $5.90577 \mathrm{E}+13$ & $1.18633 \mathrm{E}+20$ \\
\hline & & 75.0 & 4.91 & 138.8 & $3.26686 \mathrm{E}+13$ & $7.24117 \mathrm{E}+12$ & $5.44672 \mathrm{E}+13$ & $1.21464 \mathrm{E}+20$ \\
\hline & & 82.0 & 5.27 & 138.0 & $3.22476 \mathrm{E}+13$ & $6.80030 \mathrm{E}+12$ & $5.41206 \mathrm{E}+13$ & $1.30376 \mathrm{E}+20$ \\
\hline & & 92.0 & 5.82 & 146.3 & $3.44129 \mathrm{E}+13$ & $7.34415 E+12$ & $5.68577 \mathrm{E}+13$ & $1.43986 \mathrm{E}+20$ \\
\hline & & 102.0 & 6.38 & 148.3 & $3.50230 \mathrm{E}+13$ & $7.40424 \mathrm{E}+12$ & $5.81606 \mathrm{E}+13$ & $1.57596 \mathrm{E}+20$ \\
\hline & & 112.0 & 6.93 & 148.5 & $3.52628 \mathrm{E}+13$ & $7.70132 \mathrm{E}+12$ & $5.86465 \mathrm{E}+13$ & $1.71205 \mathrm{E}+20$ \\
\hline & & 122.0 & 7.52 & 152.2 & $3.73873 \mathrm{E}+13$ & $7.66096 \mathrm{E}+12$ & $6.15307 \mathrm{E}+13$ & $1.85779 \mathrm{E}+20$ \\
\hline & & 131.0 & 8.05 & 158.4 & $3.98058 \mathrm{E}+13$ & $7.70879 E+12$ & $6.49217 E+13$ & $1.99027 \mathrm{E}+20$ \\
\hline & EOC & 134.0 & 8.23 & 156.8 & $3.99248 \mathrm{E}+13$ & $7.91415 \mathrm{E}+12$ & $6.50657 \mathrm{E}+13$ & $2.03483 \mathrm{E}+20$ \\
\hline
\end{tabular}


TEM-10300-1

03/01/2012

Rev. 03

Title:

ATF-1 Power Histories

\begin{tabular}{lllll} 
TEV No.: & 3119 & Rev. No.: & 0 & Project No.: \\
\hline
\end{tabular}

Table 65. ATF-75 (Pellet 10) Power History

\begin{tabular}{|c|c|c|c|c|c|c|c|c|}
\hline Cycle & Critical Dates & EFPD & $\begin{array}{c}\text { Burnup } \\
\text { (GWd/MTU) }\end{array}$ & $\begin{array}{l}\text { LHGR } \\
(\mathrm{W} / \mathrm{cm})\end{array}$ & $\begin{array}{l}\text { Thermal Flux } \\
\left(\mathrm{n} / \mathrm{cm}^{2 *} \mathrm{~s}\right)\end{array}$ & $\begin{array}{c}\text { Fast Flux } \\
\left(\mathrm{n} / \mathrm{cm}^{2 *} \mathrm{~s}>1\right. \\
\mathrm{MeV})\end{array}$ & $\begin{array}{l}\text { Total Flux } \\
\left(\mathrm{n} / \mathrm{cm}^{2 *} \mathrm{~s}\right)\end{array}$ & $\begin{array}{l}\text { Fission Density } \\
\text { (Fissions/cm }{ }^{3} \text { ) }\end{array}$ \\
\hline \multirow[t]{9}{*}{ 160B } & $\operatorname{BOC}(12 / 20 / 2016,1200)$ & 1.0 & 0.05 & 145.8 & $3.13271 \mathrm{E}+13$ & $7.09954 \mathrm{E}+12$ & $5.37055 \mathrm{E}+13$ & $1.20440 \mathrm{E}+18$ \\
\hline & & 3.0 & 0.17 & 149.9 & $3.21088 \mathrm{E}+13$ & $7.56126 \mathrm{E}+12$ & $5.56762 \mathrm{E}+13$ & $4.21540 \mathrm{E}+18$ \\
\hline & & 10.0 & 0.57 & 149.3 & $3.20231 \mathrm{E}+13$ & 7.71013E+12 & $5.53250 \mathrm{E}+13$ & $1.39710 \mathrm{E}+19$ \\
\hline & & 20.0 & 1.17 & 161.4 & $3.45537 \mathrm{E}+13$ & $8.24255 \mathrm{E}+12$ & $5.96353 \mathrm{E}+13$ & $2.89658 \mathrm{E}+19$ \\
\hline & & 29.2 & 1.74 & 163.9 & $3.61790 \mathrm{E}+13$ & $7.97790 \mathrm{E}+12$ & $6.15241 \mathrm{E}+13$ & $4.31175 \mathrm{E}+19$ \\
\hline & \multicolumn{8}{|c|}{ Mid-Cycle 160B Outage - 5 Day, 14 Hour Down Time } \\
\hline & & 40.0 & 2.46 & 176.9 & $3.93280 \mathrm{E}+13$ & $8.95050 \mathrm{E}+12$ & $6.71895 \mathrm{E}+13$ & $6.08824 \mathrm{E}+19$ \\
\hline & & 50.0 & 3.14 & 176.1 & $3.94800 \mathrm{E}+13$ & 8.72037E+12 & $6.63914 \mathrm{E}+13$ & $7.75031 \mathrm{E}+19$ \\
\hline & EOC $(2 / 23 / 2017,1400)$ & 59.5 & 3.79 & 177.7 & $4.09589 \mathrm{E}+13$ & $8.81002 E+12$ & $6.70100 \mathrm{E}+13$ & $9.37625 \mathrm{E}+19$ \\
\hline \multirow[t]{9}{*}{$161 \mathrm{~A}$} & \multicolumn{8}{|c|}{ Cycle 161A Outage - 95 Day, 13 Hour Down Time } \\
\hline & $\mathrm{BOC}(5 / 30 / 2017,0300)$ & 61.2 & 3.90 & 152.9 & $3.50593 \mathrm{E}+13$ & $7.49299 \mathrm{E}+12$ & $5.80169 \mathrm{E}+13$ & $9.64122 \mathrm{E}+19$ \\
\hline & & 61.5 & 3.91 & 142.3 & $3.29685 \mathrm{E}+13$ & $6.93837 \mathrm{E}+12$ & $5.52621 \mathrm{E}+13$ & $9.67735 \mathrm{E}+19$ \\
\hline & & 63.5 & 4.04 & 142.9 & $3.27444 \mathrm{E}+13$ & $7.34620 \mathrm{E}+12$ & $5.52949 \mathrm{E}+13$ & $9.97845 \mathrm{E}+19$ \\
\hline & & 65.5 & 4.16 & 142.3 & $3.29210 \mathrm{E}+13$ & $7.02285 \mathrm{E}+12$ & $5.47111 \mathrm{E}+13$ & $1.02735 \mathrm{E}+20$ \\
\hline & & 67.5 & 4.28 & 147.1 & $3.41571 \mathrm{E}+13$ & $7.19262 \mathrm{E}+12$ & $5.64600 \mathrm{E}+13$ & $1.05686 \mathrm{E}+20$ \\
\hline & & 69.5 & 4.40 & 147.2 & $3.43751 \mathrm{E}+13$ & $7.08126 \mathrm{E}+12$ & $5.76698 \mathrm{E}+13$ & 1.08697E+20 \\
\hline & & 71.5 & 4.52 & 146.1 & $3.42553 \mathrm{E}+13$ & $7.14077 \mathrm{E}+12$ & $5.77835 \mathrm{E}+13$ & $1.11648 \mathrm{E}+20$ \\
\hline & EOC $(6 / 11 / 2017,1600)$ & 72.0 & 4.54 & 152.8 & $3.60262 \mathrm{E}+13$ & $7.82162 \mathrm{E}+12$ & $5.99521 \mathrm{E}+13$ & $1.12310 \mathrm{E}+20$ \\
\hline \multirow[t]{10}{*}{$162 \mathrm{~A}$} & \multicolumn{8}{|c|}{ Cycle 162A Outage } \\
\hline & $\mathrm{BOC}$ & 73.0 & 4.59 & 147.8 & $3.49174 \mathrm{E}+13$ & $7.24901 \mathrm{E}+12$ & $5.74404 \mathrm{E}+13$ & $1.13515 \mathrm{E}+20$ \\
\hline & & 75.0 & 4.70 & 133.6 & $3.09254 \mathrm{E}+13$ & $6.63604 \mathrm{E}+12$ & $5.21307 \mathrm{E}+13$ & $1.16285 \mathrm{E}+20$ \\
\hline & & 82.0 & 5.06 & 134.5 & $3.14046 \mathrm{E}+13$ & $6.88286 \mathrm{E}+12$ & $5.23855 \mathrm{E}+13$ & 1.25017E+20 \\
\hline & & 92.0 & 5.58 & 140.5 & $3.29146 \mathrm{E}+13$ & 7.40287E+12 & $5.54572 \mathrm{E}+13$ & 1.37964E+20 \\
\hline & & 102.0 & 6.11 & 142.3 & $3.34839 \mathrm{E}+13$ & $7.16076 \mathrm{E}+12$ & $5.58492 \mathrm{E}+13$ & $1.50972 \mathrm{E}+20$ \\
\hline & & 112.0 & 6.63 & 140.7 & $3.35881 \mathrm{E}+13$ & $7.23286 \mathrm{E}+12$ & $5.59966 \mathrm{E}+13$ & 1.63979E+20 \\
\hline & & 122.0 & 7.19 & 147.7 & $3.61870 \mathrm{E}+13$ & $7.25815 \mathrm{E}+12$ & $5.92895 \mathrm{E}+13$ & $1.77649 \mathrm{E}+20$ \\
\hline & & 131.0 & 7.72 & 155.1 & $3.85313 E+13$ & $7.81412 \mathrm{E}+12$ & $6.31260 \mathrm{E}+13$ & 1.90717E+20 \\
\hline & EOC & 134.0 & 7.89 & 154.7 & $3.90208 E+13$ & 7.75908E+12 & $6.33067 E+13$ & $1.95113 \mathrm{E}+20$ \\
\hline
\end{tabular}


TEM-10300-1

03/01/2012

Rev. 03

Title:

ATF-1 Power Histories

\begin{tabular}{lllll} 
TEV No.: & 3119 & Rev. No.: & 0 & Project No.: \\
\hline
\end{tabular}

Table 66. ATF-75 (Pellet 11) Power History

\begin{tabular}{|c|c|c|c|c|c|c|c|c|}
\hline Cycle & Critical Dates & EFPD & $\begin{array}{c}\text { Burnup } \\
\text { (GWd/MTU) }\end{array}$ & $\begin{array}{l}\text { LHGR } \\
(\mathrm{W} / \mathrm{cm})\end{array}$ & $\begin{array}{l}\text { Thermal Flux } \\
\left(\mathrm{n} / \mathrm{cm}^{2 *} \mathrm{~s}\right)\end{array}$ & $\begin{array}{c}\text { Fast Flux } \\
\left(\mathrm{n} / \mathrm{cm}^{2 *} \mathrm{~s}>1\right. \\
\mathrm{MeV})\end{array}$ & $\begin{array}{l}\text { Total Flux } \\
\left(\mathrm{n} / \mathrm{cm}^{2 *} \mathrm{~s}\right)\end{array}$ & $\begin{array}{l}\text { Fission Density } \\
\left(\text { Fissions } / \mathrm{cm}^{3}\right)\end{array}$ \\
\hline \multirow[t]{9}{*}{$160 \mathrm{~B}$} & BOC $(12 / 20 / 2016,1200)$ & 1.0 & 0.05 & 143.6 & $3.03192 \mathrm{E}+13$ & $7.03170 \mathrm{E}+12$ & $5.25366 \mathrm{E}+13$ & $1.20440 \mathrm{E}+18$ \\
\hline & & 3.0 & 0.17 & 143.6 & $3.05385 E+13$ & $6.92105 E+12$ & $5.26290 E+13$ & $4.21540 \mathrm{E}+18$ \\
\hline & & 10.0 & 0.55 & 142.2 & $3.04598 \mathrm{E}+13$ & $7.03979 \mathrm{E}+12$ & $5.23825 E+13$ & $1.35495 \mathrm{E}+19$ \\
\hline & & 20.0 & 1.14 & 157.3 & $3.42590 \mathrm{E}+13$ & $7.58549 \mathrm{E}+12$ & $5.78300 \mathrm{E}+13$ & $2.82432 \mathrm{E}+19$ \\
\hline & & 29.2 & 1.70 & 158.7 & $3.51895 \mathrm{E}+13$ & $7.74762 \mathrm{E}+12$ & $5.94791 \mathrm{E}+13$ & $4.19733 \mathrm{E}+19$ \\
\hline & \multicolumn{8}{|c|}{ Mid-Cycle 160B Outage - 5 Day, 14 Hour Down Time } \\
\hline & & 40.0 & 2.41 & 175.1 & $3.86653 \mathrm{E}+13$ & $8.44773 \mathrm{E}+12$ & $6.49181 \mathrm{E}+13$ & $5.95576 \mathrm{E}+19$ \\
\hline & & 50.0 & 3.06 & 169.5 & $3.77137 \mathrm{E}+13$ & $8.51470 \mathrm{E}+12$ & $6.38548 \mathrm{E}+13$ & $7.56363 \mathrm{E}+19$ \\
\hline & EOC $(2 / 23 / 2017,1400)$ & 59.5 & 3.67 & 169.7 & $3.87338 \mathrm{E}+13$ & $7.99059 \mathrm{E}+12$ & $6.48551 \mathrm{E}+13$ & $9.08118 \mathrm{E}+19$ \\
\hline \multirow[t]{9}{*}{$161 \mathrm{~A}$} & \multicolumn{8}{|c|}{ Cycle 161A Outage - 95 Day, 13 Hour Down Time } \\
\hline & BOC $(5 / 30 / 2017,0300)$ & 61.2 & 3.78 & 145.0 & $3.33531 \mathrm{E}+13$ & $7.01938 \mathrm{E}+12$ & $5.57127 \mathrm{E}+13$ & $9.34614 \mathrm{E}+19$ \\
\hline & & 61.5 & 3.80 & 144.0 & $3.31338 E+13$ & $7.06808 E+12$ & $5.48019 E+13$ & $9.38228 \mathrm{E}+19$ \\
\hline & & 63.5 & 3.91 & 139.1 & $3.20161 \mathrm{E}+13$ & $6.89073 E+12$ & $5.32100 E+13$ & $9.67133 \mathrm{E}+19$ \\
\hline & & 65.5 & 4.03 & 139.3 & $3.19718 \mathrm{E}+13$ & $7.37702 \mathrm{E}+12$ & $5.43256 \mathrm{E}+13$ & $9.96039 \mathrm{E}+19$ \\
\hline & & 67.5 & 4.15 & 143.9 & $3.33496 \mathrm{E}+13$ & $7.31841 \mathrm{E}+12$ & $5.54783 E+13$ & $1.02494 \mathrm{E}+20$ \\
\hline & & 69.5 & 4.26 & 142.2 & $3.33390 \mathrm{E}+13$ & $6.79715 E+12$ & $5.50296 \mathrm{E}+13$ & $1.05385 \mathrm{E}+20$ \\
\hline & & 71.5 & 4.38 & 143.4 & $3.39094 \mathrm{E}+13$ & $6.95158 \mathrm{E}+12$ & $5.58856 \mathrm{E}+13$ & $1.08336 \mathrm{E}+20$ \\
\hline & EOC $(6 / 11 / 2017,1600)$ & 72.0 & 4.40 & 147.8 & $3.44972 E+13$ & $7.61498 \mathrm{E}+12$ & $5.71634 \mathrm{E}+13$ & $1.08878 \mathrm{E}+20$ \\
\hline \multirow[t]{10}{*}{$162 \mathrm{~A}$} & \multicolumn{8}{|c|}{ Cycle 162A Outage } \\
\hline & $\mathrm{BOC}$ & 73.0 & 4.45 & 142.6 & $3.35322 E+13$ & $7.25867 E+12$ & $5.57499 \mathrm{E}+13$ & $1.10022 \mathrm{E}+20$ \\
\hline & & 75.0 & 4.56 & 129.2 & $3.01918 \mathrm{E}+13$ & $6.22603 E+12$ & $4.99278 \mathrm{E}+13$ & $1.12732 \mathrm{E}+20$ \\
\hline & & 82.0 & 4.91 & 130.8 & $3.01275 \mathrm{E}+13$ & $6.47088 \mathrm{E}+12$ & $5.04201 \mathrm{E}+13$ & $1.21283 \mathrm{E}+20$ \\
\hline & & 92.0 & 5.41 & 134.3 & $3.11250 \mathrm{E}+13$ & $6.26545 \mathrm{E}+12$ & $5.13997 E+13$ & $1.33809 \mathrm{E}+20$ \\
\hline & & 102.0 & 5.92 & 135.2 & $3.16563 E+13$ & $6.21567 E+12$ & $5.20168 \mathrm{E}+13$ & $1.46395 \mathrm{E}+20$ \\
\hline & & 112.0 & 6.43 & 134.5 & $3.22126 \mathrm{E}+13$ & $6.64971 \mathrm{E}+12$ & $5.33019 E+13$ & $1.58921 \mathrm{E}+20$ \\
\hline & & 122.0 & 6.97 & 143.6 & $3.47779 \mathrm{E}+13$ & $6.86241 \mathrm{E}+12$ & $5.70743 E+13$ & $1.72229 \mathrm{E}+20$ \\
\hline & & 131.0 & 7.48 & 149.9 & $3.72066 \mathrm{E}+13$ & $7.40832 \mathrm{E}+12$ & $6.09135 E+13$ & $1.84875 \mathrm{E}+20$ \\
\hline & EOC & 134.0 & 7.64 & 146.2 & $3.66693 E+13$ & $7.44709 E+12$ & $6.05348 E+13$ & $1.88970 \mathrm{E}+20$ \\
\hline
\end{tabular}


TEM-10300-1

03/01/2012

Rev. 03

Title:

ATF-1 Power Histories

\begin{tabular}{lllll} 
TEV No.: & 3119 & Rev. No.: & 0 & Project No.: \\
\hline
\end{tabular}

Table 67. ATF-75 (Pellet 12) Power History

\begin{tabular}{|c|c|c|c|c|c|c|c|c|}
\hline Cycle & Critical Dates & EFPD & $\begin{array}{c}\text { Burnup } \\
\text { (GWd/MTU) }\end{array}$ & $\begin{array}{l}\text { LHGR } \\
(\mathrm{W} / \mathrm{cm})\end{array}$ & $\begin{array}{l}\text { Thermal Flux } \\
\left(\mathrm{n} / \mathrm{cm}^{2 *} \mathrm{~s}\right)\end{array}$ & $\begin{array}{c}\text { Fast Flux } \\
\left(\mathrm{n} / \mathrm{cm}^{2 *} \mathrm{~s}>1\right. \\
\mathrm{MeV})\end{array}$ & $\begin{array}{l}\text { Total Flux } \\
\left(\mathbf{n} / \mathrm{cm}^{2 *} \mathrm{~s}\right)\end{array}$ & $\begin{array}{c}\text { Fission Density } \\
\text { (Fissions/cm }{ }^{3} \text { ) }\end{array}$ \\
\hline \multirow[t]{9}{*}{$160 \mathrm{~B}$} & $\operatorname{BOC}(12 / 20 / 2016,1200)$ & 1.0 & 0.04 & 135.7 & $2.89153 \mathrm{E}+13$ & $6.56013 E+12$ & $4.96959 \mathrm{E}+13$ & $1.08396 \mathrm{E}+18$ \\
\hline & & 3.0 & 0.16 & 140.4 & $2.98899 \mathrm{E}+13$ & $6.56349 \mathrm{E}+12$ & $5.08609 \mathrm{E}+13$ & $4.03474 \mathrm{E}+18$ \\
\hline & & 10.0 & 0.53 & 137.7 & $2.94336 \mathrm{E}+13$ & $6.51418 \mathrm{E}+12$ & $4.98567 \mathrm{E}+13$ & $1.30075 \mathrm{E}+19$ \\
\hline & & 20.0 & 1.09 & 148.7 & $3.21383 \mathrm{E}+13$ & $7.38798 \mathrm{E}+12$ & $5.45998 \mathrm{E}+13$ & $2.70388 \mathrm{E}+19$ \\
\hline & & 29.2 & 1.63 & 152.1 & $3.33894 \mathrm{E}+13$ & $7.61847 \mathrm{E}+12$ & $5.73332 \mathrm{E}+13$ & $4.04076 \mathrm{E}+19$ \\
\hline & \multicolumn{8}{|c|}{ Mid-Cycle 160B Outage - 5 Day, 14 Hour Down Time } \\
\hline & & 40.0 & 2.29 & 166.6 & $3.65762 \mathrm{E}+13$ & $8.29153 E+12$ & $6.15199 \mathrm{E}+13$ & $5.66068 \mathrm{E}+19$ \\
\hline & & 50.0 & 2.91 & 162.0 & $3.63316 \mathrm{E}+13$ & $8.32233 E+12$ & $6.16981 \mathrm{E}+13$ & $7.20231 \mathrm{E}+19$ \\
\hline & EOC $(2 / 23 / 2017,1400)$ & 59.5 & 3.52 & 163.7 & $3.73626 \mathrm{E}+13$ & $8.22882 E+12$ & $6.32252 E+13$ & $8.70781 \mathrm{E}+19$ \\
\hline \multirow[t]{9}{*}{$161 \mathrm{~A}$} & \multicolumn{8}{|c|}{ Cycle 161A Outage - 95 Day, 13 Hour Down Time } \\
\hline & $\mathrm{BOC}(5 / 30 / 2017,0300)$ & 61.2 & 3.63 & 144.6 & $3.29159 \mathrm{E}+13$ & $7.06916 \mathrm{E}+12$ & $5.45269 \mathrm{E}+13$ & $8.97278 \mathrm{E}+19$ \\
\hline & & 61.5 & 3.64 & 134.3 & $3.05802 \mathrm{E}+13$ & $6.93868 \mathrm{E}+12$ & $5.14406 \mathrm{E}+13$ & $9.00289 \mathrm{E}+19$ \\
\hline & & 63.5 & 3.75 & 133.5 & $3.05545 \mathrm{E}+13$ & $6.68518 \mathrm{E}+12$ & $5.06968 \mathrm{E}+13$ & $9.27388 \mathrm{E}+19$ \\
\hline & & 65.5 & 3.87 & 136.1 & $3.08823 \mathrm{E}+13$ & $7.05145 \mathrm{E}+12$ & $5.23254 \mathrm{E}+13$ & $9.55691 \mathrm{E}+19$ \\
\hline & & 67.5 & 3.98 & 134.9 & $3.06600 \mathrm{E}+13$ & $6.43733 E+12$ & $5.18019 \mathrm{E}+13$ & $9.83995 E+19$ \\
\hline & & 69.5 & 4.10 & 139.6 & $3.22742 \mathrm{E}+13$ & $6.53084 \mathrm{E}+12$ & $5.29229 \mathrm{E}+13$ & $1.01290 \mathrm{E}+20$ \\
\hline & & 71.5 & 4.21 & 142.0 & $3.28702 \mathrm{E}+13$ & $6.82366 \mathrm{E}+12$ & $5.37261 \mathrm{E}+13$ & $1.04181 \mathrm{E}+20$ \\
\hline & EOC $(6 / 11 / 2017,1600)$ & 72.0 & 4.24 & 143.6 & $3.30549 \mathrm{E}+13$ & 7.47973E+12 & $5.57797 \mathrm{E}+13$ & $1.04723 \mathrm{E}+20$ \\
\hline \multirow[t]{10}{*}{$162 \mathrm{~A}$} & \multicolumn{8}{|c|}{ Cycle 162A Outage } \\
\hline & $\mathrm{BOC}$ & 73.0 & 4.28 & 130.3 & $3.07023 E+13$ & $6.89923 E+12$ & $5.18844 \mathrm{E}+13$ & $1.05807 \mathrm{E}+20$ \\
\hline & & 75.0 & 4.39 & 124.6 & $2.88927 \mathrm{E}+13$ & $6.48346 \mathrm{E}+12$ & $4.90623 E+13$ & $1.08396 \mathrm{E}+20$ \\
\hline & & 82.0 & 4.72 & 127.7 & $2.93502 \mathrm{E}+13$ & $5.92965 E+12$ & 4.84207E+13 & 1.16767E+20 \\
\hline & & 92.0 & 5.22 & 130.7 & $3.01891 \mathrm{E}+13$ & $6.49912 \mathrm{E}+12$ & $5.03391 \mathrm{E}+13$ & $1.28931 \mathrm{E}+20$ \\
\hline & & 102.0 & 5.71 & 132.6 & $3.07286 \mathrm{E}+13$ & $6.29385 \mathrm{E}+12$ & $5.11006 \mathrm{E}+13$ & $1.41216 \mathrm{E}+20$ \\
\hline & & 112.0 & 6.21 & 132.1 & $3.10586 \mathrm{E}+13$ & $6.52682 E+12$ & $5.16391 \mathrm{E}+13$ & $1.53441 \mathrm{E}+20$ \\
\hline & & 122.0 & 6.73 & 138.7 & $3.32668 \mathrm{E}+13$ & $7.02779 \mathrm{E}+12$ & $5.59416 \mathrm{E}+13$ & $1.66267 \mathrm{E}+20$ \\
\hline & & 131.0 & 7.22 & 145.1 & $3.56420 \mathrm{E}+13$ & $7.39850 \mathrm{E}+12$ & $5.84523 E+13$ & $1.78552 \mathrm{E}+20$ \\
\hline & EOC & 134.0 & 7.39 & 145.1 & $3.59791 \mathrm{E}+13$ & $7.42058 E+12$ & $5.87765 E+13$ & $1.82647 \mathrm{E}+20$ \\
\hline
\end{tabular}


TEM-10300-1

03/01/2012

Rev. 03

Title:

ATF-1 Power Histories

$\begin{array}{cccccc}\text { TEV No.: } & 3119 & \text { Rev. No.: } & 0 & \text { Project No.: } & 31418 \\ & & \text { Table 68. ATF-75 (Pellet 13 - Bottom) Power History }\end{array}$

\begin{tabular}{|c|c|c|c|c|c|c|c|c|}
\hline Cycle & Critical Dates & EFPD & $\begin{array}{c}\text { Burnup } \\
\text { (GWd/MTU) }\end{array}$ & $\begin{array}{l}\text { LHGR } \\
(\mathrm{W} / \mathrm{cm})\end{array}$ & $\begin{array}{l}\text { Thermal Flux } \\
\left(\mathrm{n} / \mathrm{cm}^{2 *} \mathrm{~s}\right)\end{array}$ & $\begin{array}{c}\text { Fast Flux } \\
\left(\mathrm{n} / \mathrm{cm}^{2 *} \mathrm{~s}>1\right. \\
\mathrm{MeV})\end{array}$ & $\begin{array}{l}\text { Total Flux } \\
\left(\mathrm{n} / \mathrm{cm}^{2 *} \mathrm{~s}\right)\end{array}$ & $\begin{array}{c}\text { Fission Density } \\
\text { (Fissions } / \mathrm{cm}^{3} \text { ) }\end{array}$ \\
\hline \multirow[t]{9}{*}{$160 \mathrm{~B}$} & $\operatorname{BOC}(12 / 20 / 2016,1200)$ & 1.0 & 0.05 & 133.9 & $2.82169 \mathrm{E}+13$ & $6.13442 E+12$ & $4.78578 \mathrm{E}+13$ & $1.14418 \mathrm{E}+18$ \\
\hline & & 3.0 & 0.16 & 132.9 & $2.81810 \mathrm{E}+13$ & $6.59566 \mathrm{E}+12$ & $4.82463 \mathrm{E}+13$ & $3.85408 \mathrm{E}+18$ \\
\hline & & 10.0 & 0.52 & 136.3 & $2.89182 \mathrm{E}+13$ & $6.37728 \mathrm{E}+12$ & $4.89650 \mathrm{E}+13$ & $1.28269 \mathrm{E}+19$ \\
\hline & & 20.0 & 1.08 & 146.3 & $3.10842 \mathrm{E}+13$ & $7.17009 \mathrm{E}+12$ & $5.28848 \mathrm{E}+13$ & $2.66775 \mathrm{E}+19$ \\
\hline & & 29.2 & 1.62 & 151.1 & $3.28814 \mathrm{E}+13$ & $7.44319 \mathrm{E}+12$ & $5.58089 \mathrm{E}+13$ & $3.99259 \mathrm{E}+19$ \\
\hline & \multicolumn{8}{|c|}{ Mid-Cycle 160B Outage - 5 Day, 14 Hour Down Time } \\
\hline & & 40.0 & 2.28 & 162.5 & $3.57238 \mathrm{E}+13$ & $7.38546 \mathrm{E}+12$ & $5.89680 \mathrm{E}+13$ & $5.63659 \mathrm{E}+19$ \\
\hline & & 50.0 & 2.89 & 158.0 & $3.50802 \mathrm{E}+13$ & $7.61660 \mathrm{E}+12$ & $5.87776 \mathrm{E}+13$ & $7.14209 \mathrm{E}+19$ \\
\hline & EOC $(2 / 23 / 2017,1400)$ & 59.5 & 3.47 & 159.9 & $3.60525 E+13$ & $7.49036 \mathrm{E}+12$ & $5.96843 E+13$ & $8.56931 \mathrm{E}+19$ \\
\hline \multirow[t]{9}{*}{$161 \mathrm{~A}$} & \multicolumn{8}{|c|}{ Cycle 161A Outage - 95 Day, 13 Hour Down Time } \\
\hline & $\mathrm{BOC}(5 / 30 / 2017,0300)$ & 61.2 & 3.57 & 138.4 & $3.11760 \mathrm{E}+13$ & $6.30338 \mathrm{E}+12$ & $5.18074 \mathrm{E}+13$ & $8.82223 E+19$ \\
\hline & & 61.5 & 3.58 & 132.1 & $3.00687 \mathrm{E}+13$ & $6.14097 E+12$ & $4.99932 \mathrm{E}+13$ & $8.85234 \mathrm{E}+19$ \\
\hline & & 63.5 & 3.69 & 132.1 & $3.04108 \mathrm{E}+13$ & $6.38066 \mathrm{E}+12$ & $5.00255 \mathrm{E}+13$ & $9.12935 \mathrm{E}+19$ \\
\hline & & 65.5 & 3.81 & 128.9 & $2.98505 \mathrm{E}+13$ & $5.82712 \mathrm{E}+12$ & $4.87837 \mathrm{E}+13$ & $9.40636 \mathrm{E}+19$ \\
\hline & & 67.5 & 3.92 & 137.3 & $3.12021 \mathrm{E}+13$ & $6.55517 \mathrm{E}+12$ & $5.17487 \mathrm{E}+13$ & $9.68940 \mathrm{E}+19$ \\
\hline & & 69.5 & 4.04 & 137.2 & $3.13525 \mathrm{E}+13$ & $6.46864 \mathrm{E}+12$ & $5.15124 \mathrm{E}+13$ & $9.97845 \mathrm{E}+19$ \\
\hline & & 71.5 & 4.15 & 139.5 & $3.21760 \mathrm{E}+13$ & $6.35730 \mathrm{E}+12$ & $5.25323 \mathrm{E}+13$ & $1.02675 \mathrm{E}+20$ \\
\hline & EOC (6/11/2017, 1600) & 72.0 & 4.18 & 139.3 & $3.20086 \mathrm{E}+13$ & $6.57855 E+12$ & $5.19798 \mathrm{E}+13$ & 1.03277E+20 \\
\hline \multirow[t]{10}{*}{$162 \mathrm{~A}$} & \multicolumn{8}{|c|}{ Cycle 162A Outage } \\
\hline & $\mathrm{BOC}$ & 73.0 & 4.22 & 125.5 & $2.96006 \mathrm{E}+13$ & $5.99592 \mathrm{E}+12$ & $4.90752 \mathrm{E}+13$ & $1.04301 \mathrm{E}+20$ \\
\hline & & 75.0 & 4.32 & 121.8 & $2.80421 \mathrm{E}+13$ & $5.79751 \mathrm{E}+12$ & $4.63978 \mathrm{E}+13$ & $1.06891 \mathrm{E}+20$ \\
\hline & & 82.0 & 4.66 & 123.7 & $2.84349 \mathrm{E}+13$ & $5.67703 \mathrm{E}+12$ & $4.68352 \mathrm{E}+13$ & $1.15080 \mathrm{E}+20$ \\
\hline & & 92.0 & 5.13 & 124.3 & $2.90290 \mathrm{E}+13$ & $6.07045 E+12$ & $4.81759 \mathrm{E}+13$ & $1.26763 \mathrm{E}+20$ \\
\hline & & 102.0 & 5.61 & 127.0 & $2.97059 \mathrm{E}+13$ & $6.23383 E+12$ & $4.95708 \mathrm{E}+13$ & $1.38626 \mathrm{E}+20$ \\
\hline & & 112.0 & 6.09 & 127.4 & $3.00738 \mathrm{E}+13$ & $6.24765 E+12$ & $4.95561 \mathrm{E}+13$ & $1.50490 \mathrm{E}+20$ \\
\hline & & 122.0 & 6.59 & 132.5 & $3.18320 \mathrm{E}+13$ & $6.66918 \mathrm{E}+12$ & $5.28038 \mathrm{E}+13$ & $1.62895 \mathrm{E}+20$ \\
\hline & & 131.0 & 7.08 & 140.4 & $3.41203 E+13$ & $6.63111 \mathrm{E}+12$ & $5.56488 \mathrm{E}+13$ & $1.74999 \mathrm{E}+20$ \\
\hline & EOC & 134.0 & 7.24 & 136.9 & $3.35801 \mathrm{E}+13$ & $6.42591 \mathrm{E}+12$ & $5.51409 E+13$ & $1.78914 \mathrm{E}+20$ \\
\hline
\end{tabular}


Rev. 03

Title: $\quad$ ATF-1 Power Histories

TEV No.: $\quad 3119$

Rev. No.: 0

Project No:

31418

Date: $12 / 01 / 2017$

\section{PAGE INTENTIONALLY BLANK}


TEM-10300-1

$03 / 01 / 2012$

TECHNICAL EVALUATION

Page 110 of 110

Rev. 03

Title: $\quad$ ATF-1 Power Histories

TEV No.: 3119

Rev. No.: 0

Project No::

31418

Date: $12 / 01 / 2017$

\section{Appendix B Request for Analysis}

\section{ATF-1 Cycle 160B-1/161A-1 As-Runs and Cycle 162A-1 Projections}

Core, Gregory M <gregory.core@inl.gov>

Tue, Jun 27,2017 at 8:11 AM

To: Daniel B Chapman <daniel.chapman@inl.gov>, Cody Hale <cody.hale@inl.gov>, "Curnutt, Bryon J"

$<$ bryon.curnutt@inl.gov>

Cc: "Lillo, Misti A" <misti.lillo@inl.gov>

Dan, Bryon, Cody,

As we discussed yesterday, this email serves as your engineering input per LWP-10000 to perform the subject calculations and deliverables. Scope items and other pertinent details follow:

- Neutronics ECAR which documents the relevant as-run and projection values for the subject cycles, performed in a similar manner as past as-run and projection ECARs

- Thermal ECAR which documents the as-run and projection values for the ORNL-FCCI experiment for the subject cycles, performed in a similar manner as past as-run and projection ECARs

- TEV documenting as-runs for all capsules which includes data similar to the recent ATF-13 and ATF-15 As-run ECAR as well as data which were included in previous as-run ECARs; It is planned that this TEV will be revised to append additional as-run data as they are generated

- If at all possible, the TEV should include a tech-checked spreadsheet of as-run data, similar to what was done previously for the ATF-13 \& ATF-15 capsules

- Bryon Curnutt will be the performer of the neutronics ECAR and the As-Run TEV; if available, Brian Gross would be a preferred checker due to familiarity with calculations

- Cody Hale will be the performer of the thermal ECAR

- Dan Chapman will be the checker of the thermal ECAR

- The neutronics ECAR must be approved by $7 / 27 / 17$

- The thermal ECAR should be tech-checked by $7 / 27 / 17$

- Charge Number is 102395402

- Please see me with any questions

Thanks all.

Greg Core

Experiment Manager

Idaho National Laboratory

EROB IF-654 3EL 504

Email: gregory.core@inl.gov

Phone: 208-526-1892 